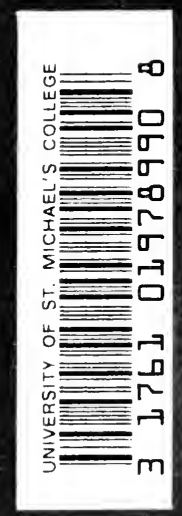



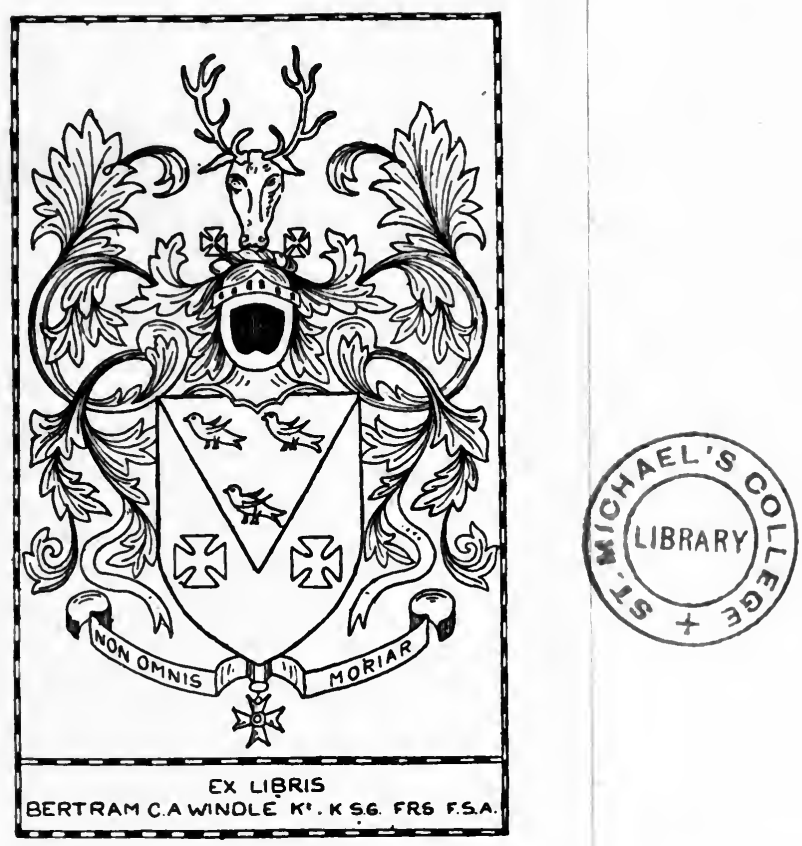

tion now than in some earlier ages, how is it that if, as you tell us, the earth is liable to be grossly over-populated at the present rate of increase in a few hundred years, it has not, in the three to five hundred thousand years cheerfully postulated by so many writers, long ago become destitute of any square yard of land unoccupied by a human being? Yet it would appear that there is room for twice or three times the existing population. 
THE PROBLEM OF POPULATION. By Harold Cox. New York: G. P. Putnam's Sons. $\$ 2.50$.

THE POPULATION PROBLEM. By A. M. Carr-Saunders. New York: Oxford University Press. $\$ 7.00$.

As to the first of these books we have only to say that in our opinion it is nothing short of lamentable that such a work, even by a person occupying so prominent a position as the Editor of The Edinburgh Review, should have been published in the United States by a respectable firm. From start to finish it is nothing but a purely materialistic plea for contraceptive measures, and the two following passages will afford some idea of what its morality and theology are. "It is a far less evil that a hundred women should indulge in irregular intercourse free from the fear of conception than that one illegitimate child should be born." ( $P$. 166.) "The very story of the virgin birth of Christ itself implies that there is something immoral in the ordinary method of conception." (P. 213.) The present Archbishop of New York and Monsignor Brown, of Southwark, England, strong supporters of morality, are the chief objects of the writer's abuse. They may be proud of it. The author of the second book only once alludes to this particular matter and that in a note where he expresses his agreement with the Laodicean policy of Dr. Inge, the wellknown Dean of St. Paul's, which amounts to this: "If you cannot be continent, I suppose you must be contraceptive, but you ought to be ashamed of yourself." Video meliora proboque-it is not a very high level of morality but better than the carnality, naked and unashamed, of Mr. Cox.

Without doubt this second work is the most important contribution to the question of population which has appeared for many years. It is scientific, erudite, well documented, and cannot be neglected by biologists, sociologists, or politicians, nor should any library fail to possess so valuable a work of reference. Incidentally it may be mentioned that it demolishes the foundation stone of Mr. Cox's argument, by showing that England never yet has been over-populated and further that, as Leroy Beaulieu points out, "if the degree of skill in production now found in Western Europe was extended throughout the world, the population economically desirable"-note, please, not merely possible, but desirable- "would be from two to three times the population of the world." So much for the bed-rock argument of those who urge that civilization can only be saved by Malthusian methods.

One would like to ask one question of these persons, most of whom claim an enormous antiquity for the human race, and it is this: Granted that there are less checks on the increase of popula- 
Digitized by the Internet Archive in 2007 with funding from Microsoft Corporation 


\section{THE POPULATION P ROBLEM}





\title{
THE POPULATION
}

\section{PROBLEM}

\author{
A Stuop in
}

\section{HUMAN EVOLUTION}

BY

A. M. CARR-SAUNDERS

O X F O R D

AT THE CLARENDON PRESS

1922 


\section{OXFORD UNIVERSITY PRESS}

London Edinburgh Glasgow Copenhagen

New York Toronto Melbourne Cape Town

Bombay Calcutta Madras Shanghai

HUMPHREY MILFORD

Publisher to the University

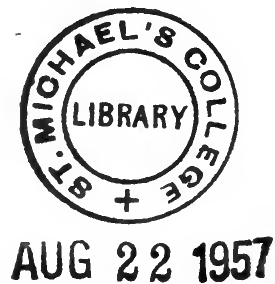




\section{PREFACE}

MaNy different questions connected with population are frequently discussed at the present day. This book is designed not so much as a contribution to the study of any one of these questions in particular as an attempt to trace back to their origin the main problems which now attract attention and to indicate their relation one to the other-to view the whole problem in fact from an historical and evolutionary standpoint. The book is an accident of the war. When war broke out I was engaged in collecting material which is incorporated in this book with the object of discussing certain aspects of the population problem. It had occurred to me that I might write an introduction showing how these aspects are related to the problem as a whole. Further than that I had not gone when there followed five years of active service during which the project of writing an introductory sketch became converted into the far more ambitious project of treating at some length the population problem as a whole. Had it thus not been for these years during which it was possible only to elaborate schemes for future work, I should never have embarked on so ambitious a task. The task is not an easy one, involving as it does both difficulties of proportion and difficulties arising from the necessity of touching upon biological, anthropological and economic problems, with regard to all of which no one man can pretend to have extensive knowledge. Nevertheless, quite apart from the question as to whether this book does in any measure achieve its purpose, those best acquainted with modern population literature will probably agree that there is room for an attempt to view the whole population problem in historical perspective. If therefore this book does no more than draw the attention of those interested in particular aspects of the matter to the necessity of so doing, it will not altogether have failed of its purpose. 
I wish to express my very great obligations to Professor L. T. Hobhouse, who read through the whole book in manuscript and furnished me with numerous most valuable suggestions and criticisms. To Mr. Julian Huxley, who read the chapters which deal most directly with biological problems, I am also indebted for valuable help. The calculations which appear in the last section of the fifth chapter I owe to Mr. H. T. Tizard. 


\title{
CONTENTS
}

\author{
CHAPTER I
}

HISTORICAL

(1) Problems of population fall under two headings-quantitative and qualitative; the former have long attracted attention; the latter have, until recently, only sporadically aroused interest. (2) Numbers were discussed in a restricted sense in Greece and Rome, but it was not until the sixteenth century (3) that quantitative problems were considered from a modern point of view when (4) a dense population was generally held to be advantageous. (5) This view was not everywhere accepted, and (6) the relation between numbers and the food-supply was from time to time discussed, and the position of Malthus more or less anticipated. (7) In 1798 Malthus published his book, which (8) was favourably received. (9) Neomalthusian propaganda, though not approved by Malthus, began early. (10) Darwin and Wallace were influenced by Malthus in founding the hypothesis of natural selection, and from 1858 onwards qualitative problems have come to assume an equally important place.

\section{CHAPTER II}

THE BASIS OF THE POPULATION PROBLEM : (1) THE QUANTITATIVE ASPECT $. \quad . . \quad . \quad . \quad . \quad . \quad$.

(1) The ancestors of man were once subject to the conditions prevailing among species in a state of nature. These conditions are studied. (2) Reproduction, the necessity for which can be explained, (3) always consists in the fusion of two gametes, but (4) is accomplished by different methods. (5) Whatever the method, most ripe ova are fertilized, though among lower forms failures may not be infrequent. A reference to the nature of (6) reflex action, (7) instinct, (8) intelligence, and (9) reason shows that, (10) whatever the stage of mental evolution reached among species in a state of nature, reproduction is essentially 'mechanical'. (11) The vast number of ova (12) is only partially called for by the fact that a certain proportion only is fertilized. The vast number is necessary because (13) owing to the interdependence of all species a large proportion of the young of (14) both animals (15) and plants perish before maturity (16) and is determined by the sum of the dangers to which the young are exposed. 


\section{CHAPTER III}

\section{THE BASIS OF THE POPULATION PROBLEM: (2) THE} QUALITATIVE ASPECT . . . . . . .

(1) In the chromatin of the nucleus we have apparently to recognize the physical basis of the inherited qualities which (2) take the form of predispositions towards the development of certain characters. (3) Difference between modifications and mutations. (4) 'Pure Line' and (5) Mendelian investigations indicate nature and behaviour of latter on crossing. (6) Germinal change consists in the apparent addition, dropping out, or modification of ' factors', the causes of which (7) are unknown, and are not due to the inheritance of acquired characters. (8) Owing to the death-rate being selective, different germinal constitutions are differently favoured, and thus (9) permanent change among species in a state of nature comes about.

\section{CHAPTER IV}

THE POPULATION PROBLEM AMONG MEN . . . '

(1) The population problem among men has, owing to the evolution of reason, assumed in both aspects a wholly different form. (2) To follow the nature, causes, and results of these changes a sketch is required of human evolution-especially of social evolution. History only provides a partial record for a few thousand years, and (3) we are thus dependent for social history upon our knowledge of primitive races which may be applied to prehistoric races. (4) Outline of future chapters.

\section{CHAPTER V}

HUMAN FECUNDITY . . . . . . . . .

(1) We have to ask whether human fecundity has changed and what the effect of certain customs is upon fecundity. (2) It is not to factors influencing the male (3) but to factors influencing the female that we have to look. (4) The length of the mature period increases with good conditions; (5) the interval between births has decreased in mankind, (6) while the number at a birth has probably increased (7) owing to good conditions. (8) That human fecundity has increased is supported by other evidence, (9) including the number of children, and (10) was held by Darwin and others. (11) Polygamy has had no influence, but lactation, age at marriage, early intercourse, and development of fat adversely affect fecundity. (12) Calculations of possible increase. 


\section{CHAPTER VI}

(1) The outline of human history can be roughly dated by (2) estimating the length of the geological eras. (3) Little is known as to the evolution of the Primate stock, but (4) the association of geological data, fossils, and cultural data give a table of human history. (5) Fossil remains of man before the fourth glacial epoch. (6) Neanderthal man in the fourth glacial epoch. (7). Numerous remains in the post-glacial epoch. (8) Putting aside eoliths, (9) we have Lower, Middle, and Upper Palaeolithic cultures, followed by (10) Neolithic culture and (11) the age of metals. (12). Putting together fossil and cultural evidence, we reach a scheme the details of which can be filled in by (13) our knowledge of existing primitive races with (14) certain reservations.

\section{CHAPTER VII}

\section{HUNTING AND FISHING RACES}

(1) A survey of these races is undertaken to show the prevalence of factors bearing upon fertility and elimination, and evidence of (2) pre-puberty intercourse, (3) prolonged lactation, (4) initiation ceremonies, (5) postponement of marriage, and (6) abstention from intercourse is given. (7) Evidence of small average size of family. (8) Among factors of elimination, abortion, (9) infanticide, (10) warfare, (11) feuds, (12) killing of old and sick, $(13,14)$ disease, and (15) child mortality are investigated.

\section{CHAPTER VIII}

\section{PRIMITIVE AGRICULTURAL RACES}

(1) A similar survey of these races is undertaken, and evidence is given regarding the prevalence in America (2) of pre-puberty intercourse, (3) postponement of marriage, (4) prolonged lactation, (5) abstention from intercourse, (6) small size of the family, (7) abortion, (8) infanticide, (9) war, (10) feuds, (11) disease, (12) and child mortality. (13) In Africa similarly regarding pre-puberty intercourse, (14) lactation, (15) marriage, (16) abstention from intercourse, (17) contraceptive methods, (18) size of family, (19) abortion, (20) infanticide, (21) warfare, (22) feuds, (23) disease, (24) and child mortality. (25) In Oceania regarding pre-puberty intercourse, (26) lactation, (27) marriage, (28) abstention from intercourse, (29) contraceptive practices, (30) size of family, (31) abortion, (32) infanticide, (33) warfare, (34) feuds, (35) and child mortality. (36) In Asia of the same practices. 


\section{CHAPTER IX}

(1) The theory of Malthus has long been disproved, and (2) has been replaced by the modern theory of an optimum density of population. (3) Territories are strictly defined among hunting and fishing races, and (4) among primitive agricultural races. (5) Among all these races there is co-operation in the search for food. (6) The principle of the optimum number holds good for all of them, and by the natural selection of customs, practices restrictive of increase come everywhere into use in the shape of abstention from intercourse, abortion, and infanticide. (7) Evidence of these practices is incomplete, (8) but shows that they are so practised as to keep numbers down to a certain level, (9) and, though they may be adjusted solely by natural selection, yet some semi-conscious adjustment may take place. (10) That adjustment should take place, a certain standard of skill is necessary on the part of those who set up new families and is ensured by marriage customs. (11) General conditions of savage life afford evidence that customs are effective. (12) There is no correlation between these practices and economic stages; though evidence is lacking, the presumption is that some such practices were everywhere in use (13) not only among primitive but also among prehistoric races, (14) who have moved slowly away from the conditions prevalent in the intermediate stage.

\section{CHAPTER X}

\section{HISTORICAL RACES .}

(1) These races fall under four sub-headings. For all of them the evidence is discussed concerning (2) disease, (3) warfare, (4) and child mortality. (5) In sub-groups 1 and 2 celibacy and postponement of marriage, (6) pre-puberty marriage, (7) lactation, (8) restraint from intercourse, (9) contraceptive practices, (10) size of family, (11) abortion, (12) and infanticide. (13) In sub-groups 3 and 4 prepuberty intercourse and lactation, (14) celibacy and marriage, (15) contraceptive practices, (16) abortion and infanticide, (17) and venereal disease.

\section{CHAPTER XI -}

\section{THE REGULATION OF NUMBERS AMONG HISTORICAL RACES}

(1) Among these races, in spite of certain complications, the principle of the optimum number holds good.. (2) The evidence for the first sub-group is too scanty to base conclusions upon. (3) Among the races of the second sub-group we see how the pressure is felt, and (4) we have knowledge of the factors at work, but (5) in 
India, China, and Egypt there is evidence that the factors are not effective, and that over-population occurs. (6) In the third subgroup, where the factors are different, (7) we can see in disabilities placed upon serfs, and (8) in difficulties, (9) restrictions, and (10) Poor Law provisions hindrances to marriage. (11) The system was generally effective, though over-population occasionally occurred. (12) In the fourth sub-group there is no evidence of over-population; (13) the factors are rather different, and (14) pressure comes about by unconscious response to economic conditions. (15) Summarizing the position for all groups we find that owing to the great power of increase, customs restrictive of increase are always necessary, and (16) have taken various forms (17) with varying effectiveness. (18) Changes in quantity are a result rather than a primary cause of historical events. (19) Neither migration (20) nor war is due directly to overpopulation, though the position as regards quantity may be an element in the situation predisposing nations towards migration and war.

\section{CHAPTER XII}

SOME MODERN PROBLEMS . . . . . . .

(1) The recent increase in numbers was in response to economic requirements, and affords no ground for pessimism as to the future. (2) The density desirable may be considered from the point of view of moral welfare (3) and of national safety, as well as from the economic standpoint. (4) The importance of minor fluctuations. (5) Methods of limiting increase at the present day. (6) Different ratios of increase as between different classes, (7) between different races in the same country, and (8) between different countries.

\section{CHAPTER XIII}

THE QUALITATIVE PROBLEM . • • • • • •

We have to ask how far those changes which constitute history are due to germinal changes, and are therefore comparable to changes among species in a state of nature. Analysis of remaining chapters.

\section{CHAPTER XIV}

THE INFLUENCE OF THE ENVIRONMENT AMONG ANIMALS AND PLANTS. • • • • . . .

(1) Observations show that environment and heredity are complementary one to the other. (2) Abnormal stimuli may produce almost any result, but (3) for each specics there is a normal environ. 
ment, to certain elements in which marked responses may be made, though (4) generally speaking sessile organisms are far more responsive to differences in the environment than are free-living organisms. (5) Among free-living species in a state of nature closely related to man, modifications are not of great importance in producing variations.

\section{CHAPTER XV}

THE INFLUENCE OF THE ENVIRONMENT UPON MAN . •

(1) Among men there is no normal environment. (2) The effect of exercise, (3) of general conditions, (4) and of climate. (5) Huntington's theory of optimum climatic conditions. (6) The effect of food, altitude, \&c. (7) Statistical results. (8) Effect of disease. (9) Evidence derived from twins. (10) Conclusion that modifications, with the exception of those produced by disease, are not of great importance, though of more importance than in the case of species closely allied to man.

\section{CHAPTER XVI}

HEREDITY IN MAN . . . . . . . .

(1) Recent work in heredity leads to the conclusion that at the basis of all human characters lies a vast number of unit-factors; but few have yet been recognized, and (2) we have to depend upon statistical data, which show that all mental and physical characters are inherited. Further discussion of the inheritance of (3) disease, (4) temperament, (5) instinct, and (6) intellect. (7) Conclusion.

\section{CHAPTER XVII}

THE EVOLUTION OF PHYSICAL CHARACTERS . . .

(1) Preliminary notice of certain difficulties-cause of mutation, strength of selection. (2) During the intermediate period great changes took place, but (3) in general during the first and second periods the tendency was towards the evolution and preservation of certain types, (4) which is comprehensible when the conditions among primitive races are studied. (5) In the third period more variations have been allowed to persist; disease becomes of importance. (6) At all times warfare, migration, and crossing have influenced physical characters. (7) Factors of possible importance other than selection. (8) Conclusion. 


\section{CHAPTER XVIII}

THE EVOLUTION OF MENTAL CHARACTERS .

(1) We have to inquire into the stage of mental evolution reached at different times. (2) After discussing evidence derived from fossils, (3) from the study of primitive races, (4) from Binet-Simon tests, and (5) elsewhere, we conclude that by far the greater part of mental evolution had been accomplished in Palaeolithic times. (6) Conditions in the intermediate period strongly favoured intellect, (7) but in the first and second periods there ceased to be any considerable premium upon intellect, (8) while, though at the opening of the third period some advance was favoured, later conditions merely allowed of the existence of greater diversity. (9) Definite conclusions postponed until tradition has been studied.

\section{CHAPTER XIX}

THE NATURE OF TRADITION . • . . . .

(1) The evolution of conceptual thought went hand in hand with that of language, (2) and has passed through a series of stages. (3) The products of conceptual thought are stored up in various ways, (4) are transmitted by language and by the operation of suggestion and sympathy, and (5) are retained by habit. (6) In a relatively unimportant way tradition is present among the higher animals. (7) Tradition moulds the degree and direction of the use of mental faculties, and (8) is naturally selected.

\section{CHAPTER XX}

THE ORIGIN OF TRADITION . • . • . . .

(1) Supposing other things to be equal, we have to study the influence of differences in fertility and contact upon tradition. (2) Fertility is a purely relative term. (3) The greater the fertility, the greater the incentive to increase in skill and to the transmission of skilled processes. (4) Since fertility is relative there is a shifting of the centres of progress. (5) Contact varies in quantity and quality, and facilitates the transmission and encourages the formation of skill. (6) Contact is hindered by isolation by sea, deserts, and mountains, and is facilitated by rivers. Importance of location and language. (7) Contact also influenced by economic factors which have brought about (8) the evolution from the segmentary to the organic form of social organization. (9) Thus the origin and transmission of tradition have been very greatly encouraged in the third period, (10) though certain influences work against the full realization of the organic type in modern communities. (11) Differences in economic organization are correlated with other differences in social organization. 


\section{CHAPTER XXI}

(1) Definite conclusions are difficult to reach. (2) History in the intermediate period was due to germinal change. (3) The natural endowment of America, (4) Asia, (5) Africa, (6) and of other regions, (7) and their geographical features, (8) suggests that history in the subsequent periods was chiefly of the nature of traditional change. (9) Though germinal changes are important they do not account for the outstanding events, though they have contributed to them. (10) Lesser germinal changes colour tradition, but are not determining factors in history. (11) Known germinal changes, such as those produced by crossing, are less important than accompanying traditional changes, (12) of which there are many instances in the third period. (13) The direct effect of the environment.is not negligible, and has influenced the course of history. (14) The cyclic course of civilization, which has been attributed to germinal change, is in the main due to traditional changes. (15) Tradition at the present day overlays the manifestation of mental characters in so great a degree that (16) differences between classes may well seem wholly traditional, but (17) psychological tests and (18) other evidence show that innate mental differences are present, (19) though it is difficult to appraise their value and the importance of modern differential fertility.

\section{CHAPTER XXII}

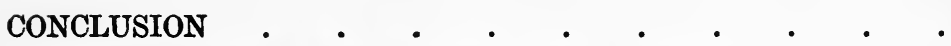

Summary of the argument and of the conclusions.

\section{APPENDIX I}

Summary of evidence as to restriction of increase amongst Primitive Races.

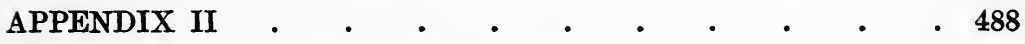

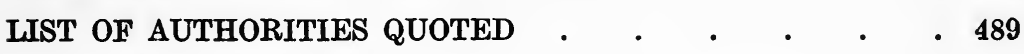

INDEX 


\section{LIST OF ABBREVIATIONS}

A. R. B. $E$. = Annual Report, Bureau of Ethnology, Smithsonian Institution. A.R.S.I. = Annual Report of the Smithsonian Institution.

Am. Anth. = American Anthropologist.

Am. Jour. Obstet. $=$ American Journal of Obstetrics.

Am. Stat. Ass. $=$ Publications of the American Statistical Association.

Ann. de Pal. $=$ Annales de Paléontologie.

Ann. Mus. Congo Belge = Annales du Musée du Congo Belge.

Arch. für Anth. =Archiv für Anthropologie.

Aust. Med. Jour. =Australian Medical Journal.

Biol. Bull. $=$ Biological Bulletin.

Bull. Soc. Anth. = Bulletin de la Société d'Anthropologie.

C. I. $A$. = Congrès International d'Anthropologie.

Cent. für Min., Geol. und Pal. =Centralblatt für Mineralogie, Geologie und Paläontologie.

Coll. Mon. Eth. = Collection de Monographies Ethnographiques.

Eug. Lab. Mem. =Eugenic Laboratory Memoirs.

Eug. Rev. = Eugenics Review.

Geog. Jour. = Geographical Journal.

Int. Arch. Eth. = Internationales Archiv für Ethnographie.

$J$. A. $I .=$ Journal of the Anthropological Institute.

Jour. Eth. Soc. = Journal of the Ethnological Society.

Jour. Pol. Soc. = Journal of the Polynesian Society.

Jour. Roy. Agric. Soc. = Journal of the Royal Agricultural Society.

$J . R . S . S .=$ Journal of the Royal Statistical Society.

Mem. Am. Anth. Soc. $=$ Memoirs of the American Anthropological and Ethno-

logical Societies.

Mem. Anth. Soc. $=$ Memoirs of the Anthropological Society.

Mem. \& Proc. Man. Lit. \& Phil. Soc. = Memoirs and Proceedings of the Manchester Literary and Philosophical Society.

Mém. Soc. Anth. = Mémoires de la Société d'Anthropologie.

Phil. Trans. $=$ Philosophical Transactions of the Royal Society.

Quart. Jour. Geol. Soc. = Quarterly Journal of the Geological Society.

Q.J.M. S. = Quarterly Journal of Microscopical Science.

Rev. Col. Inter. $=$ Revue Coloniale Internationale.

Rev. $d^{\prime}$ Anth. $=$ Revue d'Anthropologie.

Rev. $d^{\prime} E t h .=$ Revue d'Ethnographie.

S. I. B. $E .=$ Smithsonian Institute, Bureau of Ethnology.

Soc. Rev. $=$ Sociological Review.

Trans. Amer. Gym. Soc. =Transactions of the American Gynecological Society. 
Trans. \& Proc. N. Z. Inst. = Transactions and Proceedings of the New Zealand Institute.

Trans. Edin. Obstet. Soc. = Transactions of the Edinburgh Obstetrical Society. Trans. Eth. Soc. = Transactions of the Ethnological Society.

U. S. Geog. \& Geol. Surrey = United States Geographical and Geological Survey of the Rocky Mountain Region.

Z. G. $E$. = Zeitschrift der Gesellschaft für Erdkunde.

Zeit. für all. Erd. =Zeitschrift für allgemeine Erdkunde.

Zeit. für Eth. = Zeitschrift für Ethnologie.

Zeit. für Morph. und Anth. =Zeitschrift für Morphologie und Anthropologie.

Zeit. für Soc. =Zeitschrift für Socialwissenschaft. 


\section{HISTORICAL}

1. Problems of population fall into two main groups-those connected with the quantity and those connected with the quality of the population. Considerations of the population problem are commonly devoted to one of these chief aspects to the exclusion of the other with the result that the relation between them is seldom appreciated. It is one of the objects of this book to show that all problems of population have the same origin. The development of biological knowledge in the last century, and in particular the discussion of evolution, have made it clear that the whole population question and all the problems arising therefrom have their origin in the fact that mankind has a definite position in the animal kingdom. In the next two chapters an attempt is made to set out the basis of the whole question in the light of modern research, and in the fourth chapter are discussed the nature of the various problems and their relation to one another; first those which are connected with the quantity and then those that are connected with the quality of the population.

This was not, however, the manner in which the question was first approached. There was not, to begin with, an understanding of the zoological position of man leading to a growing comprehension of the problems to which it gave rise. From early days attention was particularly directed to the question of numbers. Between the fifth century B.c. and the eighteenth century A.D. -between, that is to say, the time of Plato and that of Malthusopinions were often expressed regarding the desirability or otherwise of a dense population. The work of Malthus focussed opinion upon this point. In a restricted sense this problem can be solved without an understanding of the biological origin of the whole question, and the discussion which followed the publication of the Essay on Population has resulted in the general acceptance of a solution by students of political economy. In a wider sense the solution of this problem depends upon a comprehension of its biological origin, and it was not therefore until after the middle 
of the last century that all the problems connected with quantity fell into their proper setting owing to the work of Darwin and Wallace, both of whom acknowledged their debt to Malthus. The latter, though he did not in any way realize the fact, was discussing a point which is most intimately connected with evolution, and both Darwin and Wallace were influenced by their acquaintance with the work of Malthus in arriving at their explanation of the process of evolution. Thus from the time of Darwin and Wallace it has been possible to view the population problem as a whole, though the discussions of the problems connected with quantity have been pursued independently not only before but also after the publication of the Origin of Species.

Problems of quality did not arouse the same early interest. It is, of course, well known that Plato was occupied with this aspect of the whole problem $;^{1}$ Roman authors also commented upon the eugenic bearing of certain practices. ${ }^{2}$ In later days in a remarkable book Campanella dwelt upon the importance of good breeding. ${ }^{3}$ But the interest in quality was not, as was the interest in quantity, widespread or long maintained; it was not until the origin of living species by evolution had been generally accepted and some knowledge of inheritance had been gained, that problems of quality came to occupy anything more than the passing attention of mankind.4 J. S. Mill and Buckle, for instance, both of whose opinions were formed just before the time when the importance of heredity came to be appreciated, deny that it is in any way relevant to the study of social problems. ${ }^{5}$

2. In the preface to the greatly enlarged second edition of his famous book Malthus stated that he had found many references to the subject of which he had not been aware when he published the first volume. ${ }^{6}$ He acknowledged that several authors had shown themselves to have possessed a grasp of the 'principle'

1 On this subject see Roper, Ancient Eugenics.

2 Roper (loc. cit., p. 12) quotes Seneca as follows: "We drown the weakling and the monstrosity. It is not passion, but reason, to separate the useless from the fit.' Civitas Solis.

- Mr. Roper in his book quoted above exaggerates the attention paid in early times to quality. He says, for instance, when speaking of infanticide and similar practices, that 'these barbaric eugenics... were concerned with questions both of quantity and of quality' (p. 11). As will be pointed out later, these practices were in fact primarily concerned with quantity; such bearing as they had upon. quality was incidental.

s See, for instance, Mill, Principles of Political Economy, vol. i, p. 389. Darwin commented upon this aspect of Mill's work (Descent of Man, p. 98).

- Malthus, Essay on the Principle of Population, vol. i, p. 5. 
which it was the object of his book to demonstrate. Among these he mentions Plato and Aristotle. In these cases Malthus was too anxious to attribute the first enunciation of the 'principle' to others. It was only owing to the very peculiar circumstances of Greek life that the problem ever arose at all. A consideration of the ideal city state involved the question as to what was the most desirable number of citizens. This point occupied Plato's attention, and in one place he deals with it in detail. There should be, he says, 5,040 citizens in the state. ${ }^{*}$ In the Republic he explained that the number of citizens was to be kept about the same by a strict regulation of unions. ${ }^{2}$ In the Laws, however, no such system is advocated, and he discusses the possibility of a failure to maintain the number. ${ }^{3}$ He appears to think that various checks such as infanticide and 'inundations' will keep numbers close to the desirable level; if too great an increase takes place, then recourse must be had to emigration. It does not seem that the problem was ever approached more closely in Greek literature.

References to this subject by Roman authors are confined for the most part to laments over the infertility of the old Roman stock. That which drew the attention of the Romans to the subject was thus a peculiar phase of the problem, and the views expressed by them were in consequence usually limited to the search for a remedy for a particular weakness in national life. As we shall point out later, it is doubtful how far infertility was characteristic of all classes ; that it was not widespread seems incidentally to be indicated in the following curious passage f 1 om Tertullian in which he is led to express views reminiscent of many modern contributions to the subject. Tertullian is confuting the Pythagorean theory of the transmigration of souls, and argues that, if it were true, the number of men must remain unchanged. But, he says, this is obviously not so ; population continually increases. This leads him to refer to the state of contemporary civilization. 'We find ', he says, ' in the records of the Antiquities of Man ${ }^{4}$ that the human race has progressed with a gradual growth of population. ... Surely it is obvious enough, if one looks at the whole world, that it is becoming better cultivated and more fully peopled than anciently. All places are now accessible, all are well known, all open to commerce; most pleasant farms have obliterated

\footnotetext{
1 Plato, Laws, v. 737.

2 Plato, Republic, v. 460.

- This is apparently a reference to a work by Varro.

${ }^{3}$ Plato, Laws, v. 740.
} 
all traces of what were once dreary and dangerous wastes; cultivated fields have subdued forests; flocks and herds have expelled wild beasts ; sandy deserts are sown ; rocks are planted ; marshes are drained; and where once were hardly solitary cottages are now large cities. No longer are savage islands dreaded, nor their rocky shores feared ; everywhere are houses, and inhabitants, and settled government, and civilized life. What most frequently meets our view is our teeming population; our numbers are burdensome to the world, which can hardly supply us from its natural elements ; our wants grow more and more keen, and our complaints more bitter in all mouths, whilst nature fails in affording us her usual sustenance. In very deed, pestilence, and famine, and wars, and earthquakes have to be regarded as a remedy for nations, as a means of pruning the luxuriance of the human race.' 1

3. It is not until we arrive at the sixteenth century that we discover any considerable interest in the matter. From thence onwards references are very frequent. In a general way it is not difficult to understand how this comes about. The movement of thought at that time turned to problems of practical importance. The interest in questions connected with population was above all things due to the problems created by the rise and consolidation of the great European states. In some measure also the voyages of discovery and the foundation of colonies drew men's thoughts to these questions. It is, as we shall see, in the works of the new class of political writers and theorists, and in the descriptions of travel, that we find most references. ${ }^{2}$

These references take for the most part the form of discussions as to the desirability or otherwise of a large population. There is, indeed, often but little discussion; the benefits of a large population seemed so obvious. Men were mostly of the same opinion as the author of the book of Proverbs, "in the multitude of the people is the king's glory ; but in the want of people is the destruction of the prince.' 3 The reasons are not far to seek. A large population appeared to mean both power and riches. The advan-

1 Tertullian, De Anima (Ante-Nicene Christian Library), p. 481.

2 The realization of the limitation of the surface of the globe-following upon the circumnavigation of the world-had no doubt an influence. The fact that the earth is spherical-or at least that it is not flat-(as a matter of fact it is somewhat tetrahedral) was of course known to Aristotle (De Caelo, ii. 14). It has been remarked that this conception was always present to the minds of the authors of all those speculations which have come down from antiquity (Bosanquet, Philosophical Theory of the State, p. 330). But it was not forced upon men's notice until it was practically demonstrated.

a Prov. xiv. 28. 
tage of a large population in supplying a large army proved most attractive and greatly influenced the authors of the eighteenth century. ${ }^{1}$ It has not altogether lost its attraction for some people at the present day, as we shall have reason to notice in a later chapter. This simple view of the benefits of a large population coincided with the development of the mercantile theory of trade. Authors of this school of thought also concluded that upon the whole the larger the population the better. Protests against this point of view are, however, met with from time to time. Those responsible for this opposition to the prevailing opinion were influenced in some cases more by observation than by theory and in other cases more by theory than by observation. As instances of the former several English writers of the end of the sixteenth and beginning of the seventeenth centuries may be cited; as instances of the latter several authors chiefly belonging to the later part of the eighteenth century. The views of the lastmentioned authors are often influenced by a conception of the relation between population and subsistence closely similar to that of Malthus. But they are not alone in their anticipation of his position. From the sixteenth century onwards we find in passages dealing with population more or less clear statements of the Malthusian position. These authors, however, are not always led to an unfavourable view of an increase in population. Many of them, indeed, support the current view as to the advantages of large numbers. Nevertheless on the whole a consideration of the connexion between population and the food-supply is usually associated with a fear of increase. We may now review in more detail the trend of opinion sketched above. This will bring us to the publication of the Essay on Population. ${ }^{2}$

4. Long before the sixteenth century men gave expression to the view that large numbers are beneficial. 'Quae familia plus multiplicatur in prolem, amplius cedit ad firmamentum politiae,' said Saint Thomas Aquinas. ${ }^{3}$ But it is only later that we meet with a marked insistence on this view. 'In my opinion', says Bodin, ' they erre much who doubt of a scarcitie by the multitude of

1 Thus Montesquieu wrote: 'Il n'y a que les grandes nations qui aient des armées' (Grandeur et Décadence des Romains, p. 130).

2 The literature has been several times reviewed. I am chiefly indebted to Stangeland, 'Pre-Malthusian Doctrines of Population', Studies in History, Economics and Public Law-Columbia University; vol. xxi, No. 3, 1901.

3 Thomas Aquinas, De Regimine Principum, Bk. IV, ch. ix. (The fourth book is supposititious; nevertheless it probably represents the views of St. Thomas.) 
citizens and children, when as no cities are more rich nor more famous in arts and discipline than those which abound most with citizens.' 1 According to Botero 'the greatness of a citty is sayd not to be the largeness of the citty or the circuit of the walls, but in the multitude and the number of the inhabitants and their power'.2 Machiavelli held very similar views, and Henry IV of France is reported as saying that 'the strength and riches of kings consist in the number and opulence of their subjects ' 3 In accordance with the views thus widely and strongly held, examples of which might be multiplied almost to any extent, practical measures were taken to encourage the increase of population. Of these the best known is the law of Colbert passed in 1664. Like most of such laws it was modelled on the laws of the later Roman Empire. Similar laws were at one time or another in force in most European countries.

Towards the end of the seventeenth century there arose a school of writers who might be classed as political economists, though the term was not in use. In accordance with the mercantile theory of commerce then predominant a large population was held to be advantageous. The older and vaguer theory that a dense population was necessary if the state was to be powerful was found to coincide with the newer theories as to conditions of prosperous trade. Of these authors Petty and Graunt are celebrated as among the pioneers of statistics. According to the former: 'fewness of people is real poverty; and a nation in which are eight millions of people are more than twice as rich as the same scope of land in which are but four.' 4 'Forasmuch as princes', says Graunt, 'are not only powerful but rich according to the number of their people (hands being the father as lands are the mother and womb of wealth) it is no wonder that states by encouraging marriage and hindering licentiousness, advance their own interest, as well as preserve the laws of God from contempt and violation.' 5 Sir Charles Davenant and Sir Josiah Child are two of the foremost authors of this school. The former writes as follows: 'people are the real strength and

1 Bodin, Six Books of a Commonweale, Bk. V, ch.ii, p. 575. $\quad{ }^{2}$ Botero, Delle Cause della Grandezza della Città, p. 5.

${ }_{3}$ Quoted by Stangeland, loc. cit., p. 103, from the Nouveau Dictionnaire d'Economie Politique, article 'Population'. According to Vauban (La Dîme Royale, p. 18) 'La grandeur des rois se mesure par le nombre de leurs sujets'. 'Petty, 'Treatise of Taxes and Con. tributions' in Economic Writings, vol. i, p. 34. 'Graunt, National and Political Observations upon the Bills of Mortality, ch. viii, section 14. 
riches of a country ; we see how impotent Spain is for want of inhabitants with their mines of gold and silver and the best ports and soil in the world; and we see how powerful their numbers make the United Provinces with bad harbours and the worst climate upon earth. It is perhaps better that a people should want country than that a country should want people. When there are but few inhabitants and a large territory, there is nothing but sloth and poverty; but when great numbers are confined to a narrow compass of ground, necessity puts upon them invention, frugality, and industry ; which in a nation are always recompensed with power and riches.' 1 The latter refers more than once to the subject. 'Whatever tends to the population of a country tends to the improvement of it.' 2 'Most nations in the civilized parts of the world are more or less rich or poor proportionable to the poverty or plenty of their people and not to the sterility of their lands.' 3 To these quotations we may add one from Sir William Temple: ' I believe the true and original ground of trade to be a great multitude of people crowded into a small compass of land.' 4

It would be possible to quote many similar remarks from authors who wrote in the following century. Frederick the Great had very decided opinions. In a letter to Voltaire he says : 'Je regarde les hommes comme une horde de cerfs dans le parc d'un grand seigneur et qui n'ont pas d'autre fonction que de peupler et remplir l'enclos.' 5 Hume speaks of 'the general rule that the happiness of any society and its populousness are necessary attendants' ${ }^{6}$ Adam Smith says 'that the most decisive mark of the prosperity of any country is the increase of the number of its inhabitants ' ${ }^{6}$ Nevertheless that which interests us most in the authors of this period is the growing interest in other aspects of the subject.

5. Before referring to the more or less close anticipations of the views of Malthus we may note that these optimistic views were not everywhere accepted. In England particularly, towards the end of the sixteenth century, several writers expressed a dread of overpopulation. According to Holinshed there were some

1 Davenant, Political and Commercial Works, vol. i, p. 16.

Discourse upon Trade, ch. $\mathrm{x}, \mathrm{p} .181$.

Observations upon the United Provinces, p. 164.

künstliche Beschränkung der Kinderzahl, p. 85.

of the Theories of Production and Distribution, p. 124.

Temple,

2 Child, New
'Temple,
y Ferdy, Die

- Quoted by Cannan, History 
men ' affirming that we had already too great a store of people in England; and that youth, by marrying too soon, do nothing profit the countrie, but fill it full of beggars, to the hurt and utter undoing (they saie) of the commonwealth'.1 Later we find Brückner speaking as follows: " there are some who believe that a people can never be too numerous and who speak of increase as if it always contributed to happiness, and who consequently continually urge the sovereign to encourage multiplication. The truth of the matter is, however, often far different, depending on the country and circumstances. In a free and enlightened nation, which has great natural advantages, and which is protected from the invasions of less fortunate neighbours, increasing numbers are a good.... In countries not so circumstanced increase is worse than useless ; it is, as a matter of fact, impossible, and attempts in this direction can but result in added suffering and an increased number of deaths.' 2 Arthur Young said the same in more emphatic terms. 'Of all the subjects of political economy I know not one that has given rise to such a crowd of errors as that of population. It seems for centuries to have been considered as the only test of national prosperity. The politicians of those times, and the majority of them in the present, have been of the opinion that to enumerate the people was the only step to be taken in order to ascertain the degree in which a country was flourishing. In my tour through the North of England, 1769, I entered my caveat against such a doctrine, and presumed to assert " that no nation is rich or powerful by mere numbers of people; it is the industrious class that constitutes a nation's strength"; that assertion I repeated in my Political Arithmetic in 1774.' 3 About the same time Rousseau remarked that 'il y a pire disette pour un état que celle d'hommes '.4

6. In the eighteenth century the problem was often discussed with reference to the connexion between the population and the food supply. The contrast between the vast possibilities of increase and the smallness of the actual growth in population also often attracted attention. 'Through various causes', says Wallace, 'there has never been such a number of inhabitants on the earth at any one point of time as might have been raised by the prolific

1 Quoted by Stangeland, loc. cit., p. 110. cit., p. 234. Brückner's work was published in 1769.

2 Quoted by Stangeland, loc. France, vol. i, p. 481. lation, p. 31.

- Quoted by Leroy-Beaulieu, La Question de la Popu- 
virtue of mankind. The causes of this paucity of inhabitants and the irregularity of increase are manifold. Some may be called physical, as they depend entirely on course of nature, and are independent of mankind. Others are moral and depend on the affections, passions, and institutions of men. . . . To this last we may refer so many destructive wars which men have waged against one another ; great poverty, corrupt institutions, either of a civil or religious kind, intemperance, debauchery, irregular amours, idleness, luxury, and whatever prevents marriage, weakens the generating faculties of men, or renders them negligent or incapable of educating their children, and cultivating the earth to advantage. 'Tis to such destructive causes that we must ascribe the small number of men.' 1 Sir James Steuart devotes a considerable amount of space to this subject. "The generative faculty', he says, ' resembles a spring loaded with a weight, which always exerts itself in proportion to the diminution of resistance ; when food has remained for some time without augmentation or diminution, generation will carry numbers as high as possible; if then food come to be diminished, the spring is overpowered; the force of it becomes less than nothing. Inhabitants will diminish, at least in proportion to the overcharge. If, upon the other hand, food be increased, the spring, which stood at 0 , will begin to exert itself in proportion as the resistance diminishes ; people will begin to be better fed; they will multiply, and in proportion as they increase in numbers, the food will become scarce again.' ${ }^{2}$

Malthus tells us that when he wrote his first edition the only authors ' from whose writings he had deduced the principle which formed the main argument of the essay' were Hume, Wallace, Adam Smith, and Price. ${ }^{3}$ In the interval between the publication of the first and second editions of the Essay he found that he had been anticipated more or less by many others. Subsequently many other similar passages have been brought to light. It is not worth while to discuss how far Malthus had been anticipated. It is enough to note some of the opinions that had been expressed. In the eighteenth century the fact that there is a connexion between the population and the food-supply had beeeme a commonplace. 'La mesure de la subsistance est celle de la population,' 4

1 Wallace, Dissertation on the Numbers of Mankind, p. 12.

2 Steuart, Principles of Political Economy, vol. i, p. 20. p. 3.

${ }^{3}$ Malthus, loc. cit., vol. i, ${ }^{4}$ Mirabeau, L'Ami des Hommes, ch ii, p. 14. 
said Mirabeau, and the remark could be paralleled many times over. ${ }^{1}$ Frequent references to the vast power of human increase may be found much earlier. In his History of the World Raleigh had remarked that were it not for wars, famines, pestilences, and so on, a teeming population all the world over would have resulted long ago. ${ }^{2}$ Machiavelli refers more than once to the existence of checks. He attributed the barbarian invasions in the later days of Rome to an unusually rapid increase of the tribes beyond the Rhine and the Danube. ${ }^{3}$ One of the most interesting references to the subject is to be found in the work of Botero from which we have already quoted. 'I say, then, that the augmentation of Cities proceedeth partly out of the virtue generative of men, and partly out of the virtue nutritive of Citties. The virtue generative is without doubt to this day the very same, or at least such as it was three thousand years past. So that if there were no other impediment or let therein, the propagation of mankind would increase without end and the augmentation of Citties would be without terme. And if it do not increase in infinite, I must needs say, it proceedeth out of the defect of nutriment and sustenance sufficient for it.' 4 Later on he continues: 'although men were as apt to generation in the height and pride of the Roman Empire, as in the first beginning thereof ; yet, for all that, the people increased not proportionately. For the virtue nutritive of Citties had no power to go further. . . . By the selfe same reason, mankinde growne to a certain complete number, hath growne no further. And it is three thousand years agone and more, that the world was replenished as full with people as it is at present.' 5

What are perhaps more remarkable than these references to the relation of numbers to the food-supply, so far as anticipations of Malthus are concerned, are discussions mentioning the ratios which are found from time to time. In the Primitive Origination of Mankind, Sir Matthew Hale endeavours to show that mankind must have had a beginning and must have an end, and finds support for this thesis in the facts regarding human fecundity.

1 'There is a principle in human society by which population is perpetually kept down to the level of the means of subsistence,' said Godwin in 1798 (Political Justice, Bk. VIII, p. 518). Many similar remarks are to be found in the writings of the Physiocrats. See, for instance, Turgot, Sur le Commerce, section 7, and Quesnay, Analyse du Tableau Economique, chs. Xxv and xxvi.

2 Raleigh, History of the World, in collected Works, vol. ii, p. 25.

3 Machiavelli, History of Florence, Bk. I, p. 5. "Botero, loc. cit., p. 73.

b Botero, loc. cit., p. 75. 
$\mathrm{He}$ calculated that the numbers of mankind must increase in a geometrical ratio unless hindered by checks. Obviously it has not done so chiefly because, owing to absence of sufficient food, large numbers are always being removed by various agencies which he enumerates as plagues, famines, wars, floods, and inundations. ${ }^{1}$ Sir William Petty deals with the geometrical ratio at some length. $\mathrm{He}$ drew up an elaborate table which, assuming four thousand years to have passed since the flood, shows how an estimated world population of $320,000,000$ could have been arrived at. $^{2}$ The ratios, however, never played a very prominent part in the discussion until after the publication of Malthus's book. They reappear now and again, as for instance in a book by Saxe. ${ }^{3}$

7. Malthus published his first edition in 1798 . He was born in 1766 at the 'Rookery', a country house of some size near Dorking. In 1784 he went up to Cambridge. In early days he had shown signs of ability and at the university fulfilled this promise; he gained some prizes and was placed as ninth wrangler in the mathematical tripos of 1788 . In 1793 he was elected fellow of his college, but only resided occasionally. In 1798 he was curate at Albury, close to his birthplace; in $\mathbf{1 7 9 9}$ he travelled through Sweden, Norway, and Russia, to collect information for his second edition; in 1802, owing to the Peace of Amiens, he was enabled to travel through Switzerland and France. The second edition appeared in 1803. It was virtually a new book. The four succeeding editions that were published in his lifetime were mainly reprints of this edition with some new matter added. In 1804 he married, and in 1805 became Professor of History and Political Economy at Haileybury College, which post he held until his death in $1834 .^{4}$

1 Hale, Primitive Origination of Mankind, ch. viii, section 2.

2 Petty, Essay concerning the Increase of Mankind, p. 21.

${ }^{3}$ See Stangeland, loc. cit., p. 230 . There are two valuable and important books which appeared in the eighteenth century dealing with population. They do not lend themselves to quotation, but deserve mention here. Benjamin Franklin, in his Observations concerning the Increase of Mankind and the Peopling of Countries, pointed out the differences in the marriage-rate as between Europe and the more recently colonized countries and analysed the causes. It is interesting to note that he was led to a more or less clear recognition of the presence of the struggle for existence among all living organisms (p. 21). The second book referred to above is Adam Ferguson's Essay on the History of Civil Society. The name of Ortes should perhaps be mentioned. His book Riflessioni sulla Popolazione, published in 1792, anticipated Malthus in almost every respect and yet aroused practically no attention.

- According to Leslie Stephen's article in the Dictionary of National Biography, 
It would seem that Malthus and his father used often to discuss new books and questions of the day. His father, Daniel Malthus, was inclined to favour the revolutionary school; Robert Malthus thought that he saw serious objections to Godwin's views on the subject of 'perfectibility', and was so much impressed by them that he decided to put his ideas in writing. Whereas in the first edition Malthus was chiefly concerned with controverting Godwin and his school, by the time of the second edition he had lost interest in this side of the matter and his attention was almost entirely devoted to the problem of population.

Such was the origin of the book which focused the attention of men on the population question. In the sixth chapter we shall have to review the position which it sets out and to trace the subsequent development of the theory. It is sufficient to say here that according to Malthus, whereas population tends to increase in a geometrical ratio, food tends to increase in an arithmetical ratio. As a result population is checked by the operation of vice and misery, to which factors he added, in the second edition, moral restraint. Statistics showed, exen in the lifetimo of Malthus, that the increase of food was not limited to an increase in an arithmetical ratio, and thus in reality the theory fell to the ground. It was, however, only gradually realized that this was so and that the relation between population and the food-supply is connected with the question of the return to industry, which may at any one time be increasing or decreasing according to the operation of many factors, of which the increase in skill is the chief. The points which are of interest in this historical sketch are the strong feelings which the book aroused among the adherents of the various schools of thought, the practical movement to which it gave shape, and finally the influence which it had upon Darwin and Wallace.

8. From what has been said, it is clear that the ground had been well prepared for a favourable reception. Further the " populousness of ancient nations ' was a famous theme for controversy in the

the chief authorities for the life of Malthus are (1) a notice by Otter, afterwards Bishop of Chichester, prefixed to the second edition of the Political Economy published in 1836 and (2) an article by Empsen in the Edinburgh Review. Jan. 1837, p. 469 to p. 506. See also Bonar's Malthus and his Work, to which in this and the following sections I am indebted. For some personal reminiscences see Martineau's Autobiography, vol. i, p. 211. He is described as possessing the 'most pleasing manners and the most benevolent heart' (Manning, History and Antiquities of Surrey, p. 578). 
eighteenth century. There had also been much discussion as to whether the population of England was increasing or decreasing. ${ }^{1}$ Again, in part owing to the industrial revolution, and in part owing to the war, questions as to poverty had attracted much attention, and to them Malthus's book had direct reference. Thus it is not surprising that the success of the first edition was great and immediate. ${ }^{2}$ Godwin admitted that it had ' converted friends of progress by the hundred '.3 Pitt dropped his Poor Law Bill in 1800 partly because of the opposition of those who had been influenced by the Essay. The earlier supporters of Malthus belonged, for the most part, to the Whig and Utilitarian schools. Pitt, Paley, Copleston, James Mill, Senior, Ricardo, Macintosh, and Whitbread all adhered to Malthus. In 1819 Brougham referred to the "principle of population as one of the soundest principles of political economy '.4 Support for Malthus was especially strong among the Utilitarians. Referring to about the year $1825 \mathrm{~J} . \mathrm{S}$. Mill says: 'Malthus's principle was quite as much a banner and a point of union among us as any opinion especially belonging to Bentham.' 5

Opposition was chiefly to be found among the Tories, Revolutionaries, and Radicals. Godwin published a reply to Malthus in 1801, and several years later, finding that the 'principle' still gained ground, made another attempt to refute it, which met with as little success as the earlier attempt. As an example of the attitude of the followers of Godwin towards Malthus, the following remarks by Shelley may be quoted. 'Metaphysics and inquiries into moral and political science have become little else than vain attempts to revive exploded superstitions, or sophisms like those of Mr. Malthus, calculated to lull the oppressors of mankind into a security of everlasting triumph.' 6 The Tory element of the opposition was influenced by the feeling that the ordering of human affairs could not be so innately bad as the Essay seemed

1 In the eighteenth century it was commonly supposed that the population of England was decreasing. Arthur Young was one of the few who did not share this view. (Mirabeau indeed thought that the population of the whole of Europe was increasing.) All kinds of reasons were brought forward to explain the supposed decrease. Price thought that it was due to the enclosures; Horace Walpole suggested excessive drinking as the cause.

2 It is not correct to say that 'the essay attracted comparatively little attention until 1803' (Political History of England, vol. xi, p. 421). ' Bonar, loc. cit., p. 43. 'Bonar, loc. cit., p. 363. ${ }^{5}$ Mill, Autobiography, p. 105. It is interesting to note that one of the reasons why Coleridge left the Utilitarians was the popularity of the Essay among them. See Benn, History of Rationalism, vol. i, p. 238.

Shelley, Preface to the Revolt of Islam, p. xi. 
to suggest. ${ }^{1}$ Southey and Coleridge represent this view; the former attacked the Essay more than once with considerable violence. $^{2}$ Cobbett made himself prominent among the critics by inventing the sobriquet 'Parson' Malthus. It occurs in the following passage.

'Why,' said I, ' how many children do you reckon to have had at last?

'I do not care how many,' said the man, 'God never sends mouths without sending meat.'

'Did you never hear,' said I, ' of one Parson Malthus ?'

'No, sir.'

'If he were to hear of your works, he would be outrageous, for he wants an Act of Parliament to prevent poor people from marrying young, and from having such lots of children.'

' Oh, the brute,' exclaimed the wife, while the husband laughed, thinking I was joking. ${ }^{3}$

Hazlitt, who may be classed as a radical, was one of the most violent of the opponents. Indeed, as Mr. Bonar says, 'Malthus was the best abused man of his age.' 4

The Essay early became known on the Continent. As far as Germany was concerned, this was attributable, according to von Mohl; to a work by Luden. ${ }^{5}$ During the lifetime of Malthus the violence of the opposition gradually weakened and the principle was very generally accepted. For many years such opposition as there was was based rather on religious grounds than upon an understanding of true weaknesses of the theory. Nevertheless Sumner, who was afterwards Archbishop of Canterbury, and Thomas Chalmers in early days both proclaimed their adherence to the principle. ${ }^{6}$ In 1840 three books were published which each attacked the principle on religious grounds. Of the authors, Sadler was a Churchman without apparently any strong political

1 It is thus curious to find that the Essay was highly praised by Joseph de Maistre-one of the greatest of conservatives of this or of any age : see Du Pape, Bk. III, ch. iii, section 3, where the Essay is called ' un profond ouvrage '... ' un des livres rares après lesquels tout le monde est dispensé de traiter le même sujet'. Yet in 1856 the Dictionnaire de l'Economie Politique was put upon the Index because it supported the conclusions of the Essay.

2 See, for instance, Aitken's Annual Review, vol. ii, 1803, p. $292 . \quad{ }^{3}$ Quoted by Leslie Stephen, English Utilitarians, vol. ii, p. 255. ' Bonar, loc. cit., p. 1.

- Luden, Handbuch der Staatsweisheit oder Politik. Von Mohl gives a useful review of the literature following upon the publication of the Essay (Die Geschichte und Literatur der Staatswissenschaften, vol. iii, p. 480, and Handwörterbuch der Staatswissenschaften, vol. ii, p. 955).

- Sumner, A Treatise on the Records of Creation and the Moral Attributes of the Creator; Chalmers, On Political Economy in connexion with the Moral State and Moral Prospects of Society. 
feelings, Alison a Tory, and Doubleday a Radical. ${ }^{1}$ That the religious motive continued to inspire opposition may be gathered from an essay by W. P. Greg which appeared at a much later date. $^{2}$

It may be noticed that strong opposition was nearly always exhibited to the views of Malthus by socialistic authors. ${ }^{3}$ According to Proudhon, ' la théorie de Malthus, e'est la théorie de l'assassinat politique, de l'assassinat par philanthropie, pour l'amour de Dieu.' 4 Karl Marx in a well-known passage attacked Malthus in most violent and offensive language. ${ }^{5}$

9. As a result of the Essay, there began within the lifetime of Malthus the advocacy of Neomalthusianism. Malthus himself definitely disapproved of this practical application of his "principle' ${ }^{6}$ The practical application of which he approved was made by Miss Martineau in one of her Illustrations to Political Economy, which read so strangely at the present day. ${ }^{7}$ As regards Neomalthusianism it is very generally supposed that active propaganda only began about the time of the famous BradlaughBesant trial in 1876. This, however, is a complete mistake. ${ }^{8}$ The first publication of importance in which these ideas were put forward was an article by James Mill in the Encyclopaedia Britannica. His language was very guarded but the drift of his remarks unmistakable. Four years later, in 1822, Francis Place wrote a reply to Godwin. He covered much other ground but openly and deliberately advocated these practices. For the next ten years there followed an active propaganda. The events which first brought the whole question into public notice are somewhat curious. In 1823 a number of handbills were sent to Mrs. Fildes, well known in the North for her work among the poor ; these bills contained descriptions of the methods which the new school of

1 Leslic Stephen, loc. cit., vol. iii, p. 180.

2 Greg, Enigmas of Life, ch. ii. 3 On this subject see Soetbeer, Die. Stellung der Sozialisten zur Malthusischen Bevolkerungslehre. Soetbeer includes in his review the writings of Godwin, Henry George, and others who cannot properly be regarded as socialists "Soetbeer, loc. cit., p. 20. 'Marx, Capital, vol. ii, p. 629.

- There does not seem to be any foundation whatever for the statement made by Place to the effect that Malthus recognized the advocacy of Neomalthusian methods to be the logical outcome of his position but that he shrank from their advocacy for fear of prejudice. Place, Illustrations and Proofs of the Principle of Population, p. 173.

7 Harriet Martineau, Illustrations to Political Economy, No. VI, Weal and Woe in Graveloch. $\quad{ }^{8}$ On this subject see Field, 'The Early History of the Popula. tion Movement', American Economic Review, April, 1911. I am indebted to this valuable article in what follows. 
writers wished to see adopted by the working classes ; an anonymous letter accompanied them asking her to help in distributing them. Mrs. Fildes was very indignant, and the whole story was published in the journal called the Black Dwarf. The bills attained a great notoriety and became known as the ' diabolical handbills'. Suspicion seems to have rested upon Robert Owen; it was stated in the Black Dwarf, and has often been repeated since, that Neomalthusian methods were in use at New Lanark. It is far more probable that Francis Place was the author both of the handbill and of the letter. Whether this is so or not, Place was occupied for the next few years in doing all hecould to spread the new opinions, for the sake of which he was prepared to sacrifice much popularity. ${ }^{1}$ In 1834 the Society for Promoting Useful Knowledge refused his help because of his opinions on the subject. He was violently attacked by Cobbett and Richard Carlile; the latter was afterwards converted and published in 1825 articles advocating these practices. The articles were reprinted in 1826 as Every Woman's Book, which went through several editions. At this early date it is clear that on the one hand these books and pamphlets were widely read among the working classes ; Carlile in the Republican, for instance, asserted that they were 'circulating by thousands in the populous districts of the north'. On the other hand Neomalthusian ideas were held by many eminent men of the day. The Utilitarian leaders, if not actually concerned in the propagation of these views, made their approval known. J. S. Mill in his youthful days got into trouble with the police through distributing some of these pamphlets. Grote somewhat later presented to London University a copy of the famous Fruits of Philosophy, of which there will be more to be said below.

After a while the propaganda died down, and for some fifty years there was little heard of it. ${ }^{2}$ Some books were, however, published in the interval which subsequent events made famous. [R. D. Owen's Moral Physiology appeared in 1830. Knowlton's Fruits of Philosophy and Drysdale's Elements of Social Science appeared in 1833 and in 1854 respectively. It was many years, however, before they became well known. For many years no objection was taken to the Fruits of Philosophy, or any similar work ; such books were

1 Graham Wallas, Life of Francis Place, p. 169.

2 For the later history of this movement see Hans Ferdy, loc. cit.; Garnier, Du Principe de la Population; and Leroy-Beaulieu, loc. cit. 
allowed to circulate freely. In 1876 a Bristol bookseller, Cook by name, was condemned to two years' imprisonment for selling an illustrated edition of Knowlton's work. Soon after another bookseller was also fined. Thereupon Mr. Bradlaugh and Mrs. Besant determined to take up the subject and fight for what they considered to be the right of freedom of discussion in these matters. They had an edition of the Fruits of Philosophy printed, and took a small shop where it was openly exposed for sale. A prosecution followed. In 1877 they were tried before Sir Alexander Cockburn, the Lord Chief Justice; the Attorney-General prosecuted. The summing-up was distinctly in favour of the accused; the jury, however, returned a verdict of guilty, adding a rider to the effect that they considered the defendants to be innocent of any immoral intention. Judgement was reserved. But in the interval Mr. Bradlaugh and Mrs. Besant continued to sell the book, and the result was that, instead of merely being bound over, as had been the intention of the judge, they were condemned to six months' imprisonment and to a fine of six hundred pounds. The judgement was subsequently quashed in a higher court.

The trial was a huge advertisement for Neomalthusianism. For some years spasmodic efforts to punish the sale of Neomalthusian books only served to encourage the propagandists. In 1877 the 'Neomalthusian League ' was founded and a vigorous propaganda was carried on. The activity of the league in England gradually declined, but not before the movement had spread to foreign countries. Propaganda in other countries followed a very similar course ; in 1888 there was a trial in Australia which is as famous in that country as the Bradlaugh-Besant trial in England. Prosecutions occurred in India and in America. In 1891 a remarkable dispute took place in Norway, and a law was finally passed to prohibit the sale of Neomalthusian books. As lately as 1908 a Belgian doctor was condemned to imprisonment for spreading the knowledge of Neomalthusian methods. ${ }^{1}$

10. With this notice of the history of the development of Neomalthusianism we may leave this side of the subject. The history of speculation regarding evolution has been so often described that there is no need to go into the facts here.

1 Still more recently prosecutions have taken place in the United States. The publications of the eminent Danish economist Pierson may be consulted for an able advocacy of Neomalthusian methods put forward at a time when birthcontrol received little scientific support. See Principles of Economics, vol. ii, p. 107. 
It is well known that before Darwin and Wallace turned their attention to the subject, the origin of organisms by evolution as opposed to origin by separate acts of creation had often been suggested. There was already at the beginning of the nineteenth century a considerable accumulation of facts regarding the structure and relation of organisms to one another and regarding the fossil remains of organisms. This evidence was seen to point to evolution as against creation, but until Darwin and Wallace produced their theories no one had been able to formulate a satisfactory hypothesis as to how evolution could have come about. It is of great interest to observe that it was after reading Malthus that both Darwin and Wallace independently formulated their theories. Malthus had, in fact, when taking up for consideration the quantitative aspect of the problem, so far as man was concerned, been dealing with that class of facts upon which, not only the quantitative, but also the qualitative aspect of the problem is based. When Darwin and Wallace, with the problem of evolution before them; which is essentially the problem of qualitythe problem of the manner in which one type of organic form can be derived from another type-chanced to read Malthus, their attention was called to the class of facts connected with the birthrate, the death-rate, and allied phenomena. They realized that among species in a state of nature a somewhat similar state of things existed to that which Malthus, with another aspect of the problem in view, was investigating in the case of man, and from a consideration of these facts they founded independently the hypothesis of natural selection.

It is of interest to note what Darwin and Wallace themselves say as to their indebtedness to Malthus. In a passage in the wellknown autobiographical sketch Darwin writes as follows: ' in October 1838, that is fifteen months after I had begun my systematic inquiry, I happened to read for amusement Malthus' 'Population', and being well prepared to appreciate the struggle for existence which everywhere goes on from long-continued observation of animals and plants it at once struck me that under these circumstances favourable variations would tend to be preserved, and unfavourable ones to be destroyed. The result of this would be the formation of a new species. Here then I had at last got hold of a theory by which to work.' 1 In two letters that have

1 Life and Letters of Charles Darwin, vol. i, p. 83. 
been published-one to Haeckel ${ }^{1}$ and one to Wallace ${ }^{2}$-Darwin also recorded in very similar words his debt to Malthus, and in the Origin of Species, after describing what is meant by the struggle for existence and how it comes about, says, "it is the doctrine of Malthus applied with manifold force to the animal and vegetable kingdoms.' ${ }^{3}$ So too Wallace, in his reply, after having been presented with the first Darwin-Wallace medal by the Linnean Society in 1908, acknowledged his debt to Malthus in the following words : ' both Darwin and myself, at the critical period when our minds were fully stored with a considerable body of preserved information and reflection bearing upon the problem to be solved, had our attention directed to the system of positive checks as expounded by Malthus in his Principle of Population. It is an unimportant detail that Darwin read this book two years after his return from his voyage, while I read it before I went abroad and it was a sudden recollection of its teachings that caused the solution to flash upon me.' 4

Since the publication of the Origin of Species much attention has been paid to the problem of quality as regards the human race. It was obvious that if the human race had evolved from some lower type, it was probably still in process of evolution, and that the direction of evolution was by no means necessarily upwards. It was also obvious that the further evolution of the race was to some extent at least within human control, if and when men chose to use the means of control that lay within their power. Great impetus was given to this side of the problem by the work of Sir Francis Galton, who coined the word ' eugenics '.5 Societies for the study of eugenics and for the advocacy of eugenic ideals have been started in England, in many European countries, in America and elsewhere, and at the present day the educated classes in every country are at least aware of the existence of the problem of the quality, as they have long been aware of the existence of the problem of the quantity, of population.

The development of opinion on this subject has thus run a peculiar course. Attention was drawn in early days to the existence of the problem of numbers, but men were long satisfied with asserting on comparatively simple grounds that a dense

1 Haeckel, History of Creation, vol. i, p. 134. Letters and Reminiscences, vol. $\mathrm{i}, \mathrm{p} .136$.

4 Marchant, loc. cit., vol. i, p. 116.

2 Marchant, A. R. Wallace, first proposed to use the word 'stirpiculture'.

3 Darwin, Origin of Species, p. 50. 5 See Sociological Papers, p. 45. Galton 
population was desirable or undesirable, and they usually came to the conclusion that the more inhabitants in a country, the better for the country. Long before the time of Malthus, and by many different writers, attention was paid to the connexion between numbers and the food-supply. It was Malthus, however, who first aroused public interest in the subject. His book, indeed, made an impression that few books have ever made. Since his time every educated man has had the problem of numbers in mind, and current opinion still often enough reflects the view put forward by Malthus if not in detail-for it is difficult to say what exactly the views of Malthus were-at least in the general and on the whole gloomy and fatalistic manner of regarding the problem. It is owing to the fact that those contributions which, after the time of Malthus, attracted most attention, added little to the development of thought, and owing to the influence of the writings of J. S. Mill, who himself never shook off the profound impression made upon him early in life by the Essay, that the views, or perhaps we should rather say the outlook, of Malthus so long maintained their sway. The development of the modern view as expounded by political economists was not set out in a form that attracted public attention, and it was thus possible for the late Governor of South Africa, when answering a question in the House of Commons a few years ago, to refer to the problem as though no important contribution had been made to the subject since the time of Malthus.

Of the development of opinion regarding the qualitative aspect of the problem there is little to be said. Popular interest in this subject is of comparatively recent birth. That which is interesting to observe is that, as a result of the course which thought has taken, the population problem is seldom seen as a whole, and that the relations one to another of the very numerous questions arising therefrom are scarcely ever appreciated. 
THE BASIS OF THE POPULATION PROBLEM: (1) THE QUANTITATIVE ASPECT

1. In what follows the evolutionary position is taken for granted. It is assumed, that is to say, that the higher forms of life have evolved from lower forms. If we make this assumption, we must suppose that at one time the ancestors of man were living under those conditions to which species in a state of nature are now subject. By species in a state of nature are meant all species of animals and plants with the exception of man and those species which have been domesticated by him. It is the object of this and the following chapter to show that among species in a state of nature the population problem exists both in its quantitative and qualitative aspects, and further that in certain particulars of fundamental importance the position is the same for all such species. Certain propositions may be laid down which hold good for any one of them, and in these chapters it is proposed to show what it is that may be affirmed with regard to all of them. It follows that, since among the ancestors of man the population problem must at one time have taken this form, we shall, as the result of the discussion, arrive at a basis from which we can proceed to study the shape which the problem in its double aspect has assumed in the case of man.

It is necessary to emphasize the fact that this argument does not take the form of developing an analogy between the position of man and that of species in a state of nature. What we are doing is merely to attempt to ascertain the conditions under which the ancestors of man lived, in order that we may follow the changes which have taken place. Further, it may be well to point out that the argument in these chapters will follow rather an unusual course. It may seem that certain facts of considerable general importance are passed over and others of little obvious importance emphasized. The justification for this course will be apparent later when we come to deal with the position with regard to man; for this sketch is not introduced 
primarily with the object of throwing light upon the position of species in a state of nature, but in order to prepare the way for a discussion of the shape which the problem has assumed in the case of man.

2. At the basis of both aspects of the problem lies the fact of reproduction. All organisms reproduce their kind. Reproduction is clearly a necessity, because all living things are liable to meet death by accident and, unless there was reproduction, every species would soon be extinguished. It is worth while observing that among all higher forms of life a more or less clearly defined limit to the duration of life has been evolved. In other words, natural death after a certain period ensues. It would seem that natural death has been evolved in the following manner. All organisms are subject to the wear and tear of daily life, to meet which there is a faculty of recuperation. Among the lower forms this faculty is very pronounced. The whole animal can be regenerated from a small fragment and, as a result, very serious accidents are not fatal. Among the higher forms there goes, generally speaking, with the advance in structure a loss of the power of regeneration, until among the highest there is little or no power of regeneration; for a certain average length of time the highest organisms withstand the batterings of daily life, gradually fail to recuperate, and finally die. Given the necessity of reproduction to provide for the continuance of the species aganst accident to its members, this further evolution is not difficult to understand. A power of recovery from all but the most extreme forms of accident is present among the simpler types; the retention of such a power of recovery tends to be incompatible with increasing complexity of structure, and it has, so to speak, been more economical to evolve organisns provided with a power of recuperation only against normal wear and tear over the period during which they remain capable of reproduction. The higher types therefore, after reproducing their kind, die and thus the battle of life is always being resumed by fresh generations.

3. That in some such fashion reproduction has to be explained seems clear ; but we are concerned here with the facts of reproduction. It will be necessary later to go into the nature of reproduction in greater detail ; all that needs to be said here can be stated quite shortly. There are two forms of reproduction, known respec- 
tively as the sexual and asexual ; the latter type of reproduction occurs only among plants and the lower animals. As sexual reproduction occurs among all groups of animals and plants, whether asexual reproduction occurs or not (except among the bacteria where the latter type alone is known) and as it is the only type which occurs among the higher groups, asexual reproduction may be disregarded here. The asexual type of reproduction, it may be remarked, introduces no new principle, and those groups among whom it is found are not on that account to be set apart from other groups as though the general conclusions at which we shall arrive were not applicable to them.

To make the process of sexual reproduction clear it is necessary to say something regarding the structure of organisms. Excluding the Protista for the moment, all organisms exhibit a cellular structure. Upon examination under a microscope the living tissues are found to consist of a number of compartments or cells. Among plants the typical cell is in the nature of a small box with thick sides; among animals the sides or cell-walls are very thin. Within the cell-walls there are to be distinguished two chief elements, a central body, the nucleus, and the surrounding cytoplasm. This cytoplasm consists of ordinary granular protoplasm and the nucleus of a special kind of protoplasm. Only protoplasm can properly be spoken of as alive. Those elements in the body which are not protoplasm are either in the course of being turned into protoplasm or are the products of protoplasmic activity. Examples of the latter in man are the hair and the nails, and in the case of other animals, feathers, shells, and so on. As a general rule, each cell contains a single nucleus, but there are cells without a nucleus and cells with more than one nucleus. The name Protista, mentioned above, is given to the lowest animals and plants which are commonly said to consist of a single cell. They may be thus thought of ; but the group includes forms with many nuclei of which it can only be said that cell structure has not yet been differentiated. It is therefore possible that the term cell should not be used at all in respect of this group.

Among the Protista sexual reproduction takes very different forms. Essentially, however, it consists in the fusion of two individuals, and if we regard each individual as a cell, it may be said to consist in the fusion of two cells. Among the higher 
forms the essential feature of sexual reproduction is the same; it consists, that is to say, in the fusion of two cells. It no longer consists in the fusion of two individuals, but in the fusion of two cells, one given off by one individual, and one by the other.

The manner in which these cells, known as gametes, are given off by the male and the female and the manner in which they fuse require some further explanation. What happens in plants is in all important features similar to that which happens among animals, and we may confine our attention to the latter. In every normal member of every species there is a generative organ. ${ }^{1}$ It consists of a surrounding wall within which is a mass of developing germ-cells. In the female the fully developed gamete, called the egg or ovum, is, relative to the male gamete, of large size. It is typically a motionless cell containing a varying quantity of food substance which may, as in the case of the bird's egg, reach a large amount. The male gamete is a much smaller cell. With a few exceptions it consists of a small oval. shaped head, to which is attached, by means of an intervening neck or middle-piece, a long vibratile tail. The head is the nucleus of the cell ; if there is any cytoplasm, it is reduced to a very small amount and its presence cannot be readily demonstrated. ${ }^{2}$ In strong contrast to the ovum the spermatozoon is typically motile and can swim in those fluids in which fertilization usually takes place.

Fertilization consists in the penetration of an ovum by a spermatozoon. The tail does not always enter the egg; as soon as the head has entered, a change takes place in the egg which sometimes prevents the tail, and usually other spermatozoa, from entering the egg. The head or nucleus of the spermatozoon approaches the nucleus of the egg and fuses with it. At this point, therefore, we have a single cell, known as the zygote, formed by the fusion of two cells, one derived from the male and one

1 Among many plants and not infrequently among lower animals the sexes are not separate-both male and female generative organs being found on the same individual. This condition occurs in some fish, though examples of hermaphroditism are rare in the Vertebrates. As a general rule in such cases the two generative organs found in the same individual ripen at different times and cross. fertilization is effected. In such cases the description given above applies. Where self-fertilization occurs, the above account does not apply in detail ; the occurrence of self-fertilization, however, in no way invalidates the general conclusions that will be drawn in what follows.

${ }_{2}$ 'The nucleus [of the spermatozoon] is probably enclosed within sheaths of cytoplasm, although this is not usually readily visible '(Doncaster, Cytology, p. 92). 
from the female. The zygote grows and divides and ultimately gives rise to an adult ; into this process of growth it is not necessary to go for the moment. The zygote is to be regarded as a new member of the species the existence of which dates from the fusion of the two nuclei. Whether the zygote lives an independent existence from the beginning, or is retained within the body of the mother for a longer or shorter period, is immaterial so far as the dating of the beginning of the existence of a new individual is concerned. Every man and woman is thus in reality some nine months older than his or her nominal age.

4. Such are the essential features of the process of sexual reproduction through which new members of the species arise. We have now to consider the outward features of the process, which vary very considerably. So various are they, that at first sight it may scarcely seem likely that there is any fundamental generalization that can be made regarding the process as a whole among all species in a state of nature. When we have glanced at the facts and at their interpretation we shall be in a position to discuss what it is that holds good for all such species.

Among all multicellular animals and plants the number of spermatozoa produced is infinitely greater than the number of ova. A single normal sexual emission in man is said to contain about 226,000,000 spermatozoa. This immense production of male gametes makes it likely that a male gamete will meet and fuse with each female gamete. Among the higher animals there are certain instincts which further ensure that the male cells will be brought into the proximity of the female cells. Among other multicellular animals and plants there are no such instincts. Generally speaking, among the latter types fertilization may be thought of as fortuitous. In anemophilous plants, for example, such as the Pines, the male cells or pollen-grains are specially adapted so that they are caught and carried by the wind for long distances. Many million times more male cells than female cells are formed in these plants, and some of them, wafted by the wind, eventually light upon the ovule. So too among such lowly animals as the sea-urchin the male and female cells are extruded into the surrounding sea-water; as these animals live close together, and as the male and female cells ripen and are extruded at the same time, the chance that any egg will remain 
unfertilized is small, the number of the active spermatozoa being so many times greater than the number of eggs. This simple form of fertilization is characteristic of most plants and of many animals. In plants there are certain complications, in particular those connected with fertilization with the help of insects, into which it is not necessary to go.

The increasing complexity of animal structure is only roughly correlated with increasing complexity in the processes connected with fertilization. Though the most complex form of the process is found among the highest animals, yet some animals, which stand relatively high in the scale, exhibit a simple method of fertilization. Of this the fish are an example, and, further, the more highly developed or bony fish exhibit a simpler form than some of the more lowly-organized cartilaginous fish. In fact, the stage next above that of which the sea-urchin was given as an example can be illustrated from the bony fish. The process in this group consists in the approach of the male to the female alongside of whom he swims, led by a rudimentary development of the sexual instinct. When the female ejects her gametes into the water, the male does the same and the vastly greater number of the male gametes ensures that all or nearly all of the eggs are penetrated by a spermatozoon. ${ }^{1}$

The next stage is that characterized by external copulation. The male clasps the female and when the latter ejects her eggs, he extrudes his spermatozoa at the same time. The meeting of the gametes thus still takes place outside the body of the female. This form of fertilization is found among the Amphibia; the male frog, for example, has specially developed pads on his front feet with which he embraces the female. Internal copulation, though it is to be regarded as the most complex form of the process, is found among such low forms as flat worms, ${ }^{2}$ of which the common tapeworm is a member, among many higher Invertebrates such as snails and insects, as well as among cartilaginous fish, birds, and mammals. The essential feature of internal copulation is that the male is provided with a special copulatory organ or penis which he inserts into the female. The male gametes pass through the penis, which takes the form of

\footnotetext{
1 A few bony fish, e.g. Anableps, copulate internally.

2 Internal copulation among the flat worms is of a very primitive type-the penis apparently, at least at times, penetrating the female at any point.
} 
a tube, directly into the body of the female, and fertilization thus takes place within the body of the female.

5. When fertilization takes place without copulation it might be thought that a small proportion of eggs only would be fertilized, that, so long as fertilization, for instance, depends on the wafting by the wind of a pollen-grain over a considerable distance until it alights on one small spot, or upon the chance meeting of a spermatozoon and an egg in the water, there would be but a small proportion of eggs fertilized. This, however, is not so ; observation shows that in a state of nature failure is comparatively rare, and that the majority of female gametes are fertilized. This is in the main due to the vast number of male cells compared with the number of eggs. Among those forms in which copulation takes place, fertilization is clearly dependent upon the strength of the sexual instinct, which impels the male to seek the female. That this instinct is very powerful is well known. It may for the time overcome all other instincts. There is a frenzy of desire among many animals. Male frogs and toads will remain clasping the female for many days waiting for the extrusion of the eggs. Female toads have been discovered smothered by the male in the sexual embrace. ${ }^{1}$. It has been noticed that birds, which under usual circumstances are frightened by the sound of a gun, will take no notice when in pursuit of a female. ${ }^{2}$ All the evidence points to the fact that, owing to the strength of the sexual instincts, females are rarely left undiscovered by a male in the sexual season. Jenner records that one of a pair of magpies was killed and that on the following day the survivor appeared with another mate. One of this pair was killed and on the next day the survivor again appeared with another mate. This was repeated for seven days and on each occasion the survivor always appeared with a new mate. ${ }^{3}$ In this connexion the great development of those forms of secondary sexual characters may be referred to which enable the two sexes to find and recognize one another. Such are recognition marks, call-notes of many insects, birds, and mammals, and the strong odours given off by many animals during the sexual period.

Generally speaking, the male is always prepared for the act of copulation and the act takes place when the female is ready

1 Letourneau, Evolution of Marriage, p. 8.

3 Letourneau, loc. cit., p. 28.

${ }^{2}$ Darwin, Descent of Man, p. 384. 
to receive the male. This is so among mammals whether the male experiences a sexual season known as the 'rut', as among stags, or whether he does not, as among dogs; for the period of ' rut' lasts longer than the period during which the female is ready to receive the male. The facts, however, regarding the sexual season in mammals are somewhat complicated owing to the nature of the sexual season experienced by the female. A number of different types of mammalian sexual season have been distinguished; it is not necessary, however, to go into these distinctions. It is sufficient to say that each sexual season consists of one or more sexual cycles, known as oestrous cycles. An oestrous cycle may be divided into four periods ; the first, known as the pro-oestrous, is the period of preparation which ends in the rupture of the blood-vessels in the mucous membrane of the uterus. When the blood passes to the exterior, it is known as the menstrual flow. The second period or oestrous is the period of desire. This period is always short; in the sheep it lasts about twelve hours and often in other species does not last as long. Only during this latter period will the female receive the male $;^{1}$ copulation never takes place at any other period, and yet in spite of the fact that the opportunity for copulation is narrowly restricted, so strong is the sexual instinct in the male, that it is very rare for a female, so far as observation goes, not to engage in copulation at each oestrous. The period of oestrous is followed by the period of metoestrous during which the activity of generation subsides, and the metoestrous is followed by the anoestrous or period of rest, after which another pro-oestrous period begins a new cycle.

The period of oestrous, during which copulation takes place, is usually marked by the presence of ripe female gametes which are therefore at once fertilized. Ripe ova are, however, not always present at oestrous ; in the rabbit ovulation takes place an hour and a half after copulation. In the bat there is a very marked want of coincidence between oestrous and ovulation; copulation takes place in the autumn, but ovulation does not take place until the following spring. In such cases the spermatozoa remain alive in the uterus until the female gametes are ripe, when fertilization is achieved; thus in the bat the spermatozoa remain alive for several months. When such cases occur, therefore, it does not

1 Except in man-the importance of which fact is dealt with in Chapter IV. 
mean that the ova are not fertilized. In the monkeys, however, ovulation may occur independently of oestrous and in such a manner that the ova then liberated may not be fertilized.

What has so far been said all goes to show that the majority of ripe ova are always fertilized among species in a state of nature. There must be a certain number of failures among those species which copulate, as when a mammalian female, as must now and again happen, does not engage in copulation during oestrous. Among species which do not copulate, failures are doubtless more frequent. There is probably a greater wastage of eggs when they are adhesive and fixed to some object than when they are pelagic, as is most often the case among marine animals ; for when eggs are adhesive the seminal fluid may drift away.

We are now approaching a point at which it is possible to establish the first generalization regarding the process of reproduction among species in a state of nature. It is, however, not possible to make clear what it is intended to convey until something has been said regarding animal behaviour. Some of the most striking differences between one class of animal and another as regards the process of reproduction are connected with the evolution of animal behaviour. So far we have spoken of instinct without defining what is meant, and until something has been said regarding the course and limit of mental evolution among animals, the nature of the common element in all forms of reproduction among all species in a state of nature and the manner in which it differs from what is found among men cannot be set out. It may seem that the following review is taking us somewhat out of our path. It so happens, however, that in view of the questions that will come up later for discussion, such a sketch will be necessary and it may therefore be now undertaken so as to render further amplification unnecessary.

6. Putting aside the behaviour of plants, we find among the lower animals a type of behaviour which, though simple in a certain sense, cannot be adequately explained without a prolonged discussion. Some idea can be obtained of its nature if we consider in what a reflex action consists. When a definite and simple stimulus is followed directly by a definite and simple reaction we have what is called a reflex action. This term, it may be noticed, is usually restricted to cases where a nervous system is present. When similar reactions occur among organisms in 
which the nervous system has not been differentiated, it has been suggested that the term 'autotype' should be used.'

Reactions, however, probably never follow invariably upon the repetition of the same stimulus. Among the lowest class of animals, the Protozoa, a free-swimming ciliate Infusorian, will, if it comes into contact with something in the medium which is abnormal, it may be, for example, an alkaline solution, stop and go backwards. This is an example of a reflex action. An analysis of its subsequent movements shows that, if it again and again meets the alkaline solution, some modification of the reaction takes place. The animal may show increased activity until either it is overcome by the disturbing medium or it has freed itself from it. So again after the digestion of food, such an organism shows a different response to the presence of food.

More definite instances of different reactions following upon the same stimulus are provided by experiments of changing the nature of the medium. An Infusorian will react violently against certain new elements in the medium to which after a time it becomes habituated, when the reaction no longer follows. Very interesting experiments have shown that even among the simplest class of organisms there is a certain learning from experience. 'A Stentor (one of the ciliate Infusorians) if gently touched upon one side will contract upon its stalk, but will soon open out again. Touched once more, it will perhaps bend to one side, and if continually molested in this manner, it will uproot itself in pardonable dudgeon and swim away. That is to say it has several ways of reacting to the stimulus and seeking equilibrium, and, if one fails, it tries another. But now when it anchors itself again, it seems to have learnt something, for if again touched it does not go through the stages of contracting and of bending aside. It keeps to its more radical remedy and moves off again.' 2 It is doubtful whether there is any further complication of this type of behaviour among organisms which do not attain to the kind of behaviour that we have now to describe.

7. Herbert Spencer, as is well known, defined instinct as compound reflex action. Though this definition cannot, for various

1 Jennings, Behaviour of the Lower Organisms, p. 277.

2 Hobhouse, Development and Purpose, p. 62. In this and the following sections I am indebted to Professor Hobhouse's review of the evolution of animal behaviour. 
reasons, be accepted as adequate, it does suggest the essential nature of instinct, which consists in the response to a given but often vague stimulus of a more or less complicated series of reactions. Instinct is more than compound reflex action because it involves the organism as a whole, and is accompanied by, or is the outcome of, a mental process. All mental process is said to involve three aspects-the cognitive or the knowing of an object, the affective or feeling in regard to an object, and the conative or striving to or from an object-and these three aspects are to be found in all instinctive actions. The instinctive action is initiated by a sense-impression and is followed by results so important because the nervous system is innately organized to respond to certain sense-impressions. It is presumed that some kind of emotional excitement, however faint, always follows and that it gives rise to the striving that we see in the form of movement. Instinct has been defined as ' an inherited or innate psychophysical disposition which determines its possessor to perceive and to pay attention to objects of a certain class, to experience an emotional excitement of a particular quality upon perceiving such an object, and to act in regard to it in a particular manner, or, at least, to experience an impulse to such action ' ${ }^{1}$

Every one is acquainted with many examples of instinctive action. "There are many instances of insects that invariably lay their eggs in the only place where the grubs, when hatched, will find the food they need and can eat, or where the larvae will be able to attach themselves as parasites to some host in a way that is necessary to their survival. In such cases it is clear that the behaviour of the parent is determined by the impression made on its senses by the appropriate objects or places : e.g. the smell of decaying fish leads the Carrion fly to deposit its eggs upon it ; the sight or odour of some particular flower leads another to lay its eggs among the ovules of the flower, which serve as food to the grubs. Others go through more elaborate traits of action, as when the Mason-wasp lays its eggs in a mud nest, fills up the space with caterpillars, which it paralyses by means of well-directed stings, and seals it up ; so that the caterpillars remain as a supply of fresh animal food for the young which the parent will never see and of whose needs it can have no knowledge or idea.' 2 To take some examples from

1 McDougall, Social Psychology, p. 29.

2 McDougall, loc. cit., p. 25. 
among the Vertebrates, "pheasants, plovers, moor-hen, domestic chicks and ducklings, with many others, are active soon after birth, and exhibit powers of complex co-ordination, with little or no practice of the necessary limb movements. They walk and balance the body so soon and so well as to show that this mode of procedure is congenital, and has not to be gradually acquired through the guidance of experience. Young water birds swim with neat orderly strokes the first time they are gently placed in the water. Even little chicks a day or two old can swim well.' ${ }^{1}$

Enough has been said in the way of illustration, as numerous examples are familiar to every one. It is possible that instinctive behaviour may have to be attributed to so lowly a group of organisms as the flat worms. Instinct reaches its greatest development among the insects, and some examples have been given above of the amazingly intricate series of actions which are performed by insects, under the guidance of instinct. There have been two lines of mental evolution among animals, one culminating in the insects and the other in the Vertebrates. Among the former instincts have become very specialized; among the latter they have remained far more generalized. Among the latter again there has been a far higher development of intelligence than among the former, thus further distinguishing the two lines of mental evolution. It is probable, however, that intelligence, though certainly at times in a very primitive form, always accompanies instinct, and to the discussion of intelligence we must now turn.

8. We saw how as lowly an animal as an Infusorian can in a sense learn from experience. It is only when learning from experience reaches a more advanced stage that we speak of intelligent action. If we watch one of the higher animals which, under the influence of desire, is striving to satisfy this desire, we find that it behaves in the following manner. An animal, for instance, is shut up in a box with food outside. It is led by instinct to all kinds of sporadic activities ; it will clutch and claw and make every kind of effort to extricate itself. If some simple catch has been contrived which opens the door and offers a way of escape, the animal will probably sooner or later accidentally operate the catch and escape. If the animal is replaced in the box many times, it is found to escape on the average sooner. It

1 Lloyd Morgan, Animal Behaviour, p. 84. 
learns in fact in some degree to operate the eatch and thus to gain freedom and food. The question which arises is what degree of mental development we have to assume in order to account for these facts.

The stage in learning from experience which follows next upon that present among some protozoa is exemplified by the chiok which at first instinctively pecks at various objects. If it pecks at a yellow caterpillar with an unpleasant taste it will drop it. The next time, or after a series of such experiences, it will avoid the caterpillar. The explanation seems to be that a modified response arises directly from the sight of the caterpillar. The sense-impression has become charged with feeling that first arose as the result of experience. This simple explanation is to be preferred to that which would assume the realization by the chick of the relation between the position when it again finds itself with a yellow caterpillar before it and the nastiness which it previously experienced. The process is thus one of the revival of acquired meaning, and we have now to ask if a higher stage of mental process is ever to be attributed to animals or whether the behaviour of the animal in the cage is to be explained on the same lines as the behaviour of the chick.

'Let us suppose', says Professor Hobhouse, 'revival to operate in a mind capable of perceiving three objects $\mathrm{A} \mathrm{B} \mathrm{C}$ in definite space and time relations, $\mathrm{C}$ being something desirable, e. g. food. If the three objects are present to the senses, the first two leading up to the third (e.g. as intervening objects in space), conation will be definitely directed to $\mathrm{C}$ via $\mathrm{A}$ and $\mathrm{B}$. Let this have happened and then let $\mathrm{A}$ alone be given. If the animal is hungry, i. e. if there is a conational basis to go upon, A will, according to the law of revival, excite a conation corresponding to the previous one, but this was a conation definitely directed to $\mathrm{B}$ and $\mathrm{C}$ in succession as things standing in a definite relation to $\mathrm{A}$. The animal then directs its efforts to a point where, in accordance with the first experience, $\mathrm{B}$ and $\mathrm{C}$ should be. It looks for them, or if $\mathrm{B}$ is some change which brings $\mathrm{C}$ about, sets itself to perform $\mathrm{B}$ and so obtain $\mathrm{C}$. Its action is directed to something not given, and this appears to be the germ of a conation or practical idea.' 1 It is thus possible that in the behaviour of the animal in the box we may have to recognize the first step towards a higher

1 Hobhouse, loc. cit., p. 76. 
mental process. Fffort may be directed to something not given and thus there may be the first sign of the emergence of an idea. Whether this is so or not, such an idea is certainly not a general idea ; it is merely a reference to something to come, and that is all. This is the highest degree of mental development that we can attribute to animals and it may be noticed that, this being so, there can probably be no true memory among animals. Explicit ideas, therefore, among animals, so far as they exist at all, do not give rise to other ideas following one another in sequence. They are isolated and serve merely to guide action.

9. The highest form of mental process attributable to animals reaches a fuller development among men. This stage of mental development has been called the stage of perceptual correlation. How far the apprehensions of direct relationships in consciousness are developed among animals is doubtful; there is no doubt that among men such relations are apprehended. Action, therefore, is not merely connected indirectly with the result, as in the example of the chick; action is undertaken with an end in view. If the chick came to apprehend the relation between the caterpillar and the unpleasant taste, it would have reached the fully developed stage of perceptual correlation; we have seen that we have to assume in this case a simpler state of mental process, though in certain cases a study of animal behaviour does suggest some approach to the higher stage. At this stage, which is fully developed only in man, the world ceases to be presented merely as sense-impressions charged with feeling and takes the shape of a mass of objects of perception related together and underlying the sense-impressions and the feelings evoked by them.

In man there is developed a still higher stage of mental process which is his peculiar possession and chief distinguishing characteristic. This is the stage of conceptual thought. In the perceptual stage activity is guided solely by the presence of the objects perceived. If there is any anticipation of the end, the action from moment to moment is still always guided by what is actually given. In the conceptual stage action is guided by an ideal anticipation of the end. What underlies mental process at this stage is generalization. The situation as given is broken up and analysed; elements common to it and to previous situations are recognized and synthesized. These two processes of analysis and synthesis go on side by side and concepts are formed which are 
outside the world of perception. Common elements in the perceptual order are recognized and there is thus made possible a grasp of the continuity running through experience.

Man is thus no longer guided by what is immediately given in experience; he can make plans and shape his actions with an ideal end in view. With the development of conceptual thought goes the development of language, whereby man learns from others and passes on to others what is in his mind. Of the stage of conceptual thought there will be more to say in a later chapter. This further development is only mentioned here in order to contrast mental process in its highest form in man with the process in animals.

10. We have now to consider the bearing of mental development upon the process of reproduction. All animals are endowed with a certain power of reproduction which we shall call fecundity. Fecundity is measured by the number of ripe ova produced, the number of spermatozoa having no direct bearing on fecundity. We have seen that the highest animals - those most nearly related to man-are gifted with instinct and intelligence. The value of intelligence lies in the fact that it enables instinct to adapt itself to the special circumstances of the moment and thus to bring about its end more surely. The sexual instinct is in this manner assisted by intelligence, and among animals which copulate the power of reproduction is thus able to realize itself to the full or almost to the full. There may be certain failures to achieve reproduction and certain cases of perverted instinct ; to some of these cases we have already referred. Broadly speaking, however, it is true that the highest development in animals of instinct and intelligence works towards the fulfilment of that degree of fecundity which is innately given. It follows, therefore, that in this sense mental developmient among animals has not in any fashion changed the position of the higher animals when compared with that of the lower animals. Reproduction in the sea-urchin and reproduction among the mammals-vastly as the mental processes differ-is still similar in this respect. The power of reproduction is realized to the full or almost to the full. All that instinct and intelligence do is to ensure that in this more complex process of fertilization the full power of reproduction is as nearly as possible realized-that is to say, that nearly all ova are fertilized. 
Among men, even in the lowest stage in which they have been studied, the position is entirely different. Owing to the development of conceptual thought men act with some ideal object in view. Customs grow up which in their origin must be traced to some process of reasoning, however obscure, and action deliberately undertaken, as well as custom, may affect the realization of the power of reproduction. Thus among the lowest of primitive races we find that men abstain from intercourse for various motives which we must regard as due to the presence of reason. Or again, they may practise certain forms of mutilation of the sexual organs which may affect the power of reproduction. The origin of such a custom may be hidden; it may be almost certain that it was not originated with any understanding of its effect upon reproduction and even that its effect has never been recognized; nevertheless originally such a custom could only have arisen if reason was present. Similarly, among the lowest races there are abundant examples of the practices of abortion and infanticide which, though they do not affect fecundity, have an important bearing upon the quantitative aspect of the population problem and are again the products of reason.

Among men, therefore, owing to the development of a higher stage of mental power, fecundity is not realized to the full, and we have to distinguish between the power of reproduction, which we have called fecundity, and the actual degree of reproduction, which we shall call fertility. It may, perhaps, assist to emphasize what is meant if for a moment we think of the reproductive process among animals as 'mechanical'. The introduction of this term should not be taken to have any ultimate significanceany reference whatever to the true nature of mental process. It is only used as a convenient term to illustrate the difference between reproduction among animals and among men. Among all species in a state of nature reproduction may be thought of as 'mechanical', whereas reproduction among men is never 'mechanical'. The number of young produced in the case of the human species is far from being completely correlated with the fecundity. There may be all degrees of difference between fecundity and fertility. Among speciesina state of nature fecundity and fertility are for all practical purposes one and the same thing because reproduction is 'mechanical' whatever may be the stage which mental development has reached. Further, such differences 
as exist among species in a state of nature between fecunditythe number of ripe ova-and fertility--the number of fertilized ova-are due to failures of the gametes to meet and may be called, just as the whole process may be called, ' mechanical'. Such 'mechanical' differences between fecundity and fertility may also be found in man but the chief cause of the differences in the case of man is altogether of another lind and is due, as we have seen, directly to the development of conceptual thought.

It was said above that it was proposed to show that certain generalizations can be made regarding the process of reproduction among species in a state of nature. This then is the first generalization. Fecundity and fertility are closely correlated and, compared with the position among men, reproduction may be thought of as ' mechanical' 'and even the failures to realize the full power of fecundity may be thought of as 'mechanical'.

11. It has been mentioned that fecundity is very large among all species in a state of nature and we have now to ask what it is that determines how large it shall be. As we have seen, the fecundity is roughly measured by the number of ova produced, and this number clearly depends upon many factors, such as the beginning and duration of the mature period, the number of eggs produced at any one time, and the length of the period between the epochs of egg production. Into details of the proximate causes of the differences in fecundity it is not necessary to go. What it is desired to know is what factor or factors in general ultimately determine the strength of fecundity in each species. We may first take some examples of the degree of fecundity. drawn from various groups.

The common whelk lays its eggs in capsules of which a great number are produced. It has been calculated that a small elump of such capsules of about two cubic inches in size contains about 200,000 eggs. Another molluse, Aplysia, may lay from 2,000,000 to 3,000,000 at a time. 'An oyster may have sixty million eggs and the average American yield is sixteen millions.' 1 The number of eggs found attached to the edible crab in the breeding season varies between half a million and three millions. A single pair of flies may produce 20,000 larvae. The number of eggs produced by parasites may very largely exceed these numbers. Among the vertebrates the fish are the most prolific class. 'In a Ling

1 Thomson, Darwinism and Human Life, p. 81. 
61 inches long and weighing 54 pounds the ovaries contained $28,361,000$ eggs ; a cod of $21 \frac{1}{2}$ pounds 6,$652 ; 000$. The least prolific of British food fishes is the herring, in which the number of ovarian eggs varied from 21,000 to 47,000 in four specimens examined.' 1

These vast numbers of eggs produced at any one time make theoretically possible a prodigious rate of increase. It has been calculated that a single cholera bacillus can give rise to sixteen hundred trillion of bacilli in a day, forming a solid mass weighing a hundred tons. "Wallace quotes Kerner to the effect that a common British weed (Sisymbrium sophia) often has threequarters of a million seeds ; if all grew to maturity for only three years the whole of the land surface of the globe would not hold them. An annual plant with only two seeds would be represented by $1,048,576$ in the twenty-first year. . . . If all the progeny of one oyster survived and multiplied, its great-great-grand-children would number thirty-six with thirty-three noughts after it, and the heap of the shells would be eight times the size of the world. Huxley calculated that if the descendants of a single green-fly all survived and multiplied they would, at the end of summer, weigh down the population of China. The common house-fly lays eggs in batches of 120 to 150 at a time, and may lay five or six of these batches during its life of about three weeks in very hot weather. At the end of summer, if all developed, and if there were six generations, the progeny of a single pair, pressed together into a solid mass, would occupy a space of something like a quarter of a million cubic feet, allowing 200,000 flies to a cubic foot.' 2 'There is no exception', says Darwin, in a wellknown passage, 'to the rule that every organic being naturally increases at so high a rate, that, if not destroyed, the earth would soon be covered by the progeny of a single pair. Even slowbreeding man has doubled in twenty years, and at this rate in less than a thousand years there would literally not be standing room for his progeny. Linnaeus has calculated that if an annual plant produced only two seeds--and there is no plant so unproductive as this-and their seedlings next year produced two, and so on, then in twenty years there would be a million plants. The elephant is reckoned the slowest breeder of all known animals,

1 Bridge, Cambridge Natural History, vol. Fishes, p. 412. cit., p. 82 .

2 Thomson, loc. 
and I have taken some pains to estimate its probable minimum rate of natural increase ; it will be safest to assume that it begins breeding when thirty years old, and goes on breeding until ninety years old, bringing forth six young in the interval, and surviving till 100 years old; if this be so, after a period of 740 to 750 years there would be nearly ninety million elephants alive, descended from the first pair.' 1

12. These examples show that the power of fecundity, which is always huge, is in many instances much greater than in others. Were it not for the fact that normally all but a small proportion of eggs are always fertilized, it might be suggested that in those cases in which there was no copulation a much larger number of eggs was necessary than among the higher forms, in order that a sufficient number should be fertilized. This, however, can only be a partial explanation of the larger number of eggs among those lower forms where there is no copulation.

In order to obtain an answer to the question as to what it is which determines the fecundity of any species, it is necessary to look into certain features of the life of animals and plants in a state of nature. Observation and deduction bring one remarkable fact to light. The number of adults of any species at any one season of the year, when compared with the number in the corresponding period in other years, remains upon the whole constant. This fact cannot be based upon statistics, for we cannot take anything approaching to a consus. Nevertheless, it is an unavoidable deduction from the known facts. The more emphasis that is laid upon variations in numbers from season to season, the more apparent does it become that such differences are trivial when compared with the possible rate of increase. But we know that all but a small proportion of eggs are fertilized, and as we date the existence of a new member of the species from the moment of fertilization, it is clear that the numbers composing every new generation greatly exceed the number of adults to which the new generation owes its existence. It follows, therefore, that all but a small proportion of the young of each generation perish before the adult stage is reached. The most remarkable increases in the adults of any species ever recorded are negligible compared with the possible increase, and observation shows that as a general rule there is no increase at all.

1 Darwin, Origin of Species, p. 51. 
Fecundity is, therefore, in some manner connected with this fact that the great majority of fertilized eggs do not give rise to adults, and in order to throw further light upon this connexion we must ask how it is that the young perish.

13. To make clear how it is that the young of every species perish on so large a scale, it is necessary to refer to the interdependence of all living organisms. This can perhaps' best be illustrated by reference to the chief distinction between animals and plants. A difference in the mode of nutrition is that which chiefly distinguishes animals from plants. There are other differences but their importance is small compared with that we may now describe. The need of food is common to all living things and is due to the nature of the living substance called protoplasmthe physical basis of all life. Protoplasm is of a very complex constitution. It is for ever wasting away and if life is to be preserved food must be supplied to compensate for the loss. The need is as great among plants as it is among animals, but the means of supplying it are fundamentally different.

Plants feed upon very simple substances-salts of nitric acid, salts of ammonia, and carbonic acid. Among green plants carbonic acid is taken in from the air through small apertures in the leaves known as stomata ; the salts of nitric acid and ammonia are absorbed from solution in the water of the soil. Carbonic acid and water are synthesized within the cells of the plant into starch ; starch is converted into sugar and the sugar is combined with the salts of nitric acid and ammonia to form amino-acids which are eventually transformed into proteids. Thus the plant makes good the unavoidable waste of its living substance by elaborating the highly complex protoplasm from the simplest elements.

The method pursued by animals is entirely different. They feed upon complicated substances, which may be divided into proteids, fats, and carbo-hydrates. These substances do not take their places directly in the living cells of the body; they first undergo a process of digestion, after which they are assimilated. Digestion involves the breaking down of these complex foods to a certain stage; proteids, for example, are reduced to aminoacids, and starches to sugars. In these forms they are soluble and are taken up by the walls of the alimentary canal and are afterwards resynthesized into proteids and starches. 
The importance of this distinction lies in the fact that the only method of obtaining the highly complex substances necessary for animals is to feed upon the tissues of other animals or plants. It is obvious that every animal species cannot feed upon some other animal species; in the end animals as a whole must depend upon plants because plants alone are able to elaborate the substances which animals need. In a sense, therefore, animals are parasitic upon plants ; in any case the existence of animals is bound up with the continued existence of plants. This interdependence of living organisms runs all through the conditions under which species in a state of nature live and takes a variety of shapes. The dependence of one organism upon another is largely connected with the question of the provision of appropriate surroundings which are often only found in the proximity of certain other species. Many species can only flourish in the neighbourhood of trees. The interdependence between certain species is very intimate. There are many examples of what is known as symbiosis, as when a certain species of sea anemone lives on the back of a particular species of crab. Again, parasites, which alternate between one host and another, are dependent upon finding a member of a particular species at a certain time in their life-history, as otherwise they perish. Many examples of interdependence are within common knowledge, and, bearing this feature of organic life in mind, we may go on to ask how it is that, through the elimination of a great proportion of the young of every species, the number of adults remains upon the whole constant.

14. Taking animals first, it is probable that the most common cause of elimination lies in the fact that the young of all species are consumed by members of other species. It is difficult to estimate even roughly the relative importance of the various causes of elimination; 'the causes', says Darwin, ' which check the natural tendency of each species to increase are most obscure '. The particular factor mentioned, however, certainly takes a very prominent place. The young of marine and fresh-water animals almost all form food for other species and are obviously exposed to attack. So too, though perhaps not to so great a degree, are the young of terrestrial animals; whether we think of the larvae of insects or the eggs of birds, we find that they are in most cases

- Darwin, Origin of Species, p. 49. 
liable to be consumed by enemies. Even where copulation is internal, the developing embryo is, except among mammals, seldom long retained within the body of the mother, and once exposed to the outside world it almost invariably becomes an object of prey to many enemies. In fact, wherever we look, this cause of elimination plays a very great part, arising immediately from that aspect of the interdependence of species which is derived from the mode among animals of making up for the wastage of protoplasm.

Elimination again is largely due to failure to find those conditions under which alone life can continue. These conditions may be connected with the nature of the organic or of the inorganic surroundings. When the young of the flat worm, known as the Liver Fluke, which infects sheep and causes a serious disease, passes from the sheep to the exterior, it can only maintain itself for a certain time in the free-living form. Unless within this time it meets with a certain species of snail into which it penetrates, it will die. Besides such failures to find suitable organic surroundings, there may be failures to find suitable inorganic surroundings. The larvae of such species as the mussel, which require suitable surroundings to which to attach themselves in order that the adult form may develop, will perish if such surroundings are not available. There is a further class of factors connected with the inorganic surroundings which bring about elimination. They may be summed up under the heading of external circumstances. Variations in temperature, moisture and so on, when they pass beyond a certain limit, which is more or less clearly marked for each species, are followed by death. Under this heading comes also death from accident, as when animals perish from the violence of storms and in any similar fashion. It is of interest to note that starvation is seldom the primary cause of death. It may be a secondary result of abnormal external circumstances; extreme cold, though not affecting directly the members of one species, may be fatal to members of another species upon which the former feed. But when circumstances are normal, so far as observation goes, starvation is rare.

15. Among plants the same three groups of factors can be traced, though their relative importance is not the same as among animals. Elimination through consumption as food, for instance, by other species is not so important. To a large extent plants 
can serve as food for animals and survive. Nevertheless the young, especially in the form of seeds, are very subject to attack by animals; seeds are one of the principal forms of food for many animal species, and this form of elimination plays a large part among plants. Dependence upon suitable organic and inorganic surroundings plays much the same rôle as it does among animals. Some species can only flourish in the shade of trees and others in the open. Some species require one kind of soil and others another. The importance of external circumstances again is very similar.

There is another factor of a somewhat different nature which is of great importance. 'With plants', says Darwin, "there is a vast destruction of seeds, but, from some observations which I have made, it appears that the seedlings suffer most from germinating in ground already thickly stocked with other plants.' ${ }^{1}$ In order that a seed may germinate, it must not only fall upon suitable soil but must find enough suitable soil unoccupied. Otherwise it will not germinate or will not develop into an adult. This is different from anything which happens among animals, and is clearly an approach to starvation. It is, however, better thought of as the result of the inability of any given area to support more than a given amount of life. The endowment of any area may be such as to render it incapable of supporting life at all, or it may be such as to render it capable of supporting any degree of life up to and beyond that for which there is space. In the sea the amount of nitrogen is the limiting factor, and the deficiency of nitrogen is such that the question of space does not arise. 2 In many parts of the world's surface, however, the endowment is such that more plants could be supported than there is space for.

In this connexion it may be noted that the limitation of the surface of the earth is not a cause of elimination in the true sense. - This is best seen if we imagine the surface to be extended. If to a continent already inhabited there is added an unoccupied area, there will, if the new area is generally of the same nature as that alone formerly existing, be a spreading of organic life over the new area. It will only be at the fringe of the occupied area that there will be any difference in the amount of elimina-

'Darwin, Origin of Species, p. 49.

2 Johnstone, Conditions of Life in the Sea, p. 235. 
tion of plant life. Unless the occupied area is of very small extent, there will be no difference except within this narrow fringe. In the fringe there will be a lessening of elimination, other things being equal, because there will not be the same number of seeds which fail to grow into plants owing to the previous occupation of suitable soil, as in the more central parts of the area. When the new area has been entirely occupied, the same conditions as existed in the smaller area will exist throughout the larger area. Further, if the surface available for occupation were indefinite in extent, there would, except at the fringe, which would in this case be permanent, be no difference as regards the amount of elimination. All that has been said applies equally well if, instead of imagining these additions to take place after evolution had reached its present stage, we imagine evolution to have taken place from the beginning on an area of indefinite extent.

16. We have, therefore, some idea of the manner in which the young of animals and plants perish. Let us consider any animal species ; we find that the young are faced with a large number of dangers. The new members of the species may be consumed by some enemy before they have developed beyond the stage of the fertilized egg or at any stage in their development. They may in general not meet with favourable organic and inorganic surroundings, or at some particular stage they may not meet with the environment necessary. At any time they may perish from unfavourable external circumstances. The position of young plants is similar, and in addition they may fail to find sufficient space in which to live.

The dangers which any species encounters remain both in kind and in degree fairly constant over a considerable period of time, and, unless in each generation a number of young survives at least equal to the number of adults in the generation to which it owes its birth, the species will decline. It follows that the power of reproduction must be such as to ensure that at least this number of young will survive. The power of reproduction in any species is therefore connected with the sum of all the dangers which the young of the species encounter. But it is not true that the greater the fecundity the better for the species. Reproduction over and above this degree would place the young in a less favourable position. Competition bringing no corresponding advantages 
would be increased, and starvation or injurious semi-starvation would result. Any considerable increase in the strength of fecundity beyond that which is essential could not therefore be beneficial. ${ }^{1}$

The facts regarding the conditions of life and the dangers to which the young of different species are exposed coincide with the view that the strength of reproduction is in the main determined by the sum of these dangers. The greater strength of fecundity among those species which shed their eggs into the water cannot be attributed, as we have seen, to any failure of fertilization on a large scale; it may be attributed, however, to the numerous dangers which the young of such species must encounter. The chance that any single fertilized egg will grow into an adult is far less in these cases than when the eggs are retained in the body of the mother and when the young are guarded by the parent, and, unless fecundity was on a large scale, a sufficient number would not survive. Among plants reproduction must always be on a large scale because it is necessary to ensure that a sufficient number of seeds will fall, not only on suitable ground, but on suitable ground that is not so occupied as to prevent growth to the adult stage, in addition to the necessity of providing such numbers as will ensure a sufficient number passing through all the other dangers. Eggs and young abandoned by the parents may be variously exposed to danger. The development of instincts common among insects which lead to the hiding of eggs in places where danger of destruction at the hand of enemies is decreased, and to the provision of food for the young when they develop from the eggs, is accompanied by a decrease in fecundity. Further developments of the parental instinct are accompanied by decreases in fecundity as the degree of danger lessens. Parental care for the young is not uncommon among the invertebrates. There are a number of cases in which the young are retained in brood-pouches, as for instance by the common water-flea. The fresh-water leech, Clepsine, broods over its young. Among the vertebrates the fish

1 For the sake of simplicity fecundity has been spoken of as though it was fixed at a certain strength for each species. As a matter of fact it varies within fairly wide limits-increasing with better conditions. In this fact lies the explanation of the increase of species under favourable conditions which has often been observed, although, when conditions are less favourable, there is little or no evidence of starvation among such species. 
for the most part take no care of their young. Some fish, however, such as Gasterosteus, the Stickleback, make nests. No great advance is found among the Amphibia or among the reptiles. One reptilian group, however, the Chelonia, shows a remarkable advance ; they live in pairs and guard their young with care. If marriage be defined as " a more or less durable connexion between male and female lasting beyond the mere act of propagation till after the birth of the offspring ', 1 then the beginning of marriage is discernable in this group. This more or less durable connexion between male and female is a well-known characteristic of birds, as is also care for the young. 'Most birds when they pair do so for good and all until one or the other dies.' 2 The connexion is not so durable, and the parental instinct is not so highly developed, among mammals as among birds. The retention of the young within the body of the mammalian mother, however, greatly decreases the dangers to which the young are exposed. It is also worthy of note that those species both of birds and mammals which prey upon others are on the whole less fecund than other species, a fact which is to be connected with the lower degree of danger to which their offspring are exposed.

The lower degree of danger to which the young are exposed, the less the fecundity; and the less the fecundity, provided that it reaches the strength necessary to preserve the species, the better on the whole for the species. In this manner we reach the second generalization regarding the quantitative aspect of the population problem among species in a state of nature, which may be stated by saying that the strength of fecundity in any species is determined by the sum of all the dangers to which the young of that species are exposed. This must be qualified, in so far as fecundity and fertility are not the same, by adding to the dangers to which the young are exposed the danger that a certain proportion of eggs will not be fertilized. It follows that among men, since fecundity and fertility are not the same for quite other than ' mechanical' reasons, fecundity is not directly related to the dangers to which the young are exposed. It is clear that, when men, as is now the case on a large scale, both abstain from intercourse and interfere with the natural result of intercourse, and at the same time increase in number, the strength of fecundity

1 Westermarck, History of Human Marriage, p. 19.

2 Brehm, Bird Life, p. 285. 
is considerably greater than that which would enable a sufficient number of young to escape the unavoidable dangers. But the ancestors of man must once have been subject to the same conditions as those to which species in a state of nature are now subject, and it will be into the causes and results of this progressive divergence from the former conditions that we shall look in the first part of this book. 


\section{THE BASIS OF THE POPULATION PROBLEM: (2) THE QUALITATIVE ASPECT}

1. We have now to consider the qualitative aspect of the population problem. There are two questions to which an answer is required. First we ask: What is the nature of the changes which occur? In other words, if we may speak of history among animals and plants, we want to know what kind of changes underlie the facts which go to make up that history. The second question is : How have these changes come about? There is no doubt about the answer to the first question, and it can be given very shortly. Though there is a certain consensus of agreement regarding the answer to the second question, there are considerable differences of opinion over points which are not unimportant, and in order to deal adequately with these points a long discussion would be required for which there is no space. All that can be done here is to set out the most important facts ; they will provide a satisfactory answer to the first question and enable some indication to be given of the lines which the answer to the second must follow.

However slight the treatment of the problem, it must begin with a reference to the physical basis of inheritance. Some description has been given of the process of fertilization. It was said that only the head and middle-piece of the spermatozoon penetrate the egg, and that no cytoplasm or ordinary granular protoplasm can be demonstrated in these parts of the spermatozoon. The head is the nucleus of the cell, while the middlepiece contains the centrosome, a body which is attached to the nucleus and plays an important part when the nucleus divides. From these facts a very important conclusion follows. It is known that both parents contribute equally to the offspring; therefore as the male parent contributes only a nucleus and its appanage the centrosome, the basis of the inherited qualities must be sought in the nucleus.

Attention is thus directed to the nucleus, which can sometimes 
be seen in the living cell. Its detailed structure can, however, only be made out in specimens which have been preserved and stained. The nucleus is then seen to consist of a thin wall within which is contained a colourless sap. In the sap are a number of beads of a darkly staining substance known as chromatin suspended on delicate threads of a substance known as linin. The division of a cell is always preceded by the division of the nucleus and, when the nucleus divides, the chromatin undergoes certain remarkable changes. The beads of chromatin become aggregated together into rods-the number of rods which appear being invariably constant in the same species, though varying from species to species. These rods are known as chromosomes. Beyond saying that the rods divide into two and that each daughter nucleus, and therefore each daughter cell, is provided with that number of chromosomes which is typical for the species, it is not necessary to follow the details of the process of division any farther. Stated in the briefest possible form, this is what happens during ordinary cell-division, such as that which takes place when a fertilized egg is growing from a single cell into a multicellular adult.

There is one remarkable exception to this type of nuclear division. In the last division but one of those series of divisions which lead to the formation of both male and female gametes, half, and not the full number of chromosomes, is transmitted to each daughter nucleus. If therefore the typical number of chromosomes in the nucleus of one species is eight and in another four, the number of chromosomes in the gametes will be four and two respectively. In the former species the nucleus of the egg will have four chromosomes, and that of the spermatozoon also four, and the full number typical of the species will only be restored when the nucleus of the spermatozoon fuses with the nucleus of the egg in fertilization.

The invariable reappearance of the same number of chromosomes in ordinary cell-division, their reduction to half that number in the last division but one which precedes the formation of the gametes, the complicated mechanism which is employed and other evidence all lead to the conclusion that the location of the basis of the hereditary qualities can be further narrowed and sought in the chromatin-in one element, that is to say, of the nucleus. ${ }^{1}$

1 That the basis of the inherited qualities is wholly situated in the chromatin 2498 
2. The nucleus must not be thought of as isolated from the rest of the cell ; there is a constant and active interchange between the nucleus and the cytoplasm. It has actually been demonstrated that at times particles stream out from the nucleus into the cytoplasm. The nucleus is the centre of activity; without the nucleus the cell cannot live. This being so, how are we to view the process of development from the fertilized egg to the adult? The process can be studied in detail. The egg divides into two cells, then into four, eight, sixteen, thirty-two cells, and so on. The gradual differentiation of the organs can be watched and their lineage to certain cells in earlier stages traced. At every stage the developing organism comes under the influence of certain stimuli provided by the surrounding conditions. Very many elements in the environment act as stimuli ; among them are light, temperature, gravitation, food, and so on. Provided that the environment is normal, provided, that is to say, that the variations in the stimuli do not exceed certain limits, an adult member of the species will be formed. What is implied in the term 'normal environment' will be more fully discussed in Chapter XIV, where the results of subjection to an abnormal environment will also be studied. That a large number of stimuli varying within only narrow limits are necessary, will there also be shown to have been proved.

The process of development, therefore, takes the shape of the growth of a particular organic form through the play of certain stimuli upon the germinal constitution. The germinal constitution has, as we have seen, apparently to be identified with the constitution of the chromosomes of the fertilized egg, of which half are derived from the father and half from the mother. It is known that the characters, which the organism exhibits, in some manner have a basis in the germinal constitution. The number of these separate characters is very large. What view then are we to take of the nature of the germinal constitution? In the first place every character as seen in the organism is the result of the play of certain stimuli upon something in the germinal constitution. This something can only be thought of as predispositions to the development of certain characters under certain stimuli and to the development of characters differing in

cannot be considered as established beyond doubt. The element of doubt, however, has no bearing upon the argument. 
degree or in kind from the former characters under other stimuli. It is altogether misleading to speak of anything but predispositions as present in the germinal constitution, though, of course, for the sake of brevity other phraseology may be employed once this point is understood. When, therefore, we speak of the inheritance of any character, we mean that there is a predisposition in the germinal constitutions both of the parent and of the offspring to develop this character under certain stimuli, which stimuli must play both upon the parent and upon the offspring if the character is to be manifested in both of them.

If we have to regard the germinal constitution as somehow containing very many separate predispositions or, as they are often called, factors, is it in the second place possible to say in what these factors consist? No definite answer to this question can as yet be given. Certain hypotheses have been put forward. Weismann, for instance, suggested that the factors were to be sought in groups of molecules of chromatin. It is not necessary, however, to postulate definite and separate particles as the physical basis of the predispositions. The protoplasmic molecule is a very complex structure consisting of a very large number of atoms. Similar atoms may be differently grouped within a molecule, and different predispositions may well be functions of different groupings.

3. In order that we may answer the first of the two problems set out at the beginning of the chapter we must consider in rather more detail in what the development of an organism consists. ${ }^{1}$ Every character is, as we have seen, the result of the influence of the environment upon what is innately given. If two individuals were endowed with precisely similar germinal constitutions, and if precisely similar stimuli played upon each of them, then the adult forms would be similar in respect of all their characters. But if the stimuli are not similar, if, for instance, more food is provided in one case than in another, then, though the germinal constitutions are similar, one adult may be larger than the other. Again, let us suppose that the germinal constitutions differ, that, for instance, there is in one case a predisposition to the development of greater size than in the other, then, even if the stimuli

${ }^{1}$ In this and the following sections I am indebted to Professor Goodrich's admirable treatment of these problems in his book, The Evolution of Living Organisms. 
are similar, the adult forms will differ. The larger members of each of these pairs may thus resemble one another very closely in outward characters ; but this close resemblance will not be due to similar germinal constitutions. It follows that by mere outward inspection no conclusion can be reached as to the germinal constitution. There are, therefore, two classes of influences at work, and alterations in either class of influence will bring about alterations in the resulting organism; similar characters may be the product of one kind of predisposition and one kind of stimulus, or of a different predisposition and a different stimulus. It also follows that we cannot speak of certain characters as inherited and of others as acquired. Let us suppose that some departure from the normal structure occurs. It may be due to a change in the environment, that is to say, to a new stimulus acting upon an unchanged germinal constitution, or it may be due to a change in the germinal constitution when no change in the environment takes place. It should not be said that in the former case the new departure is acquired and in the latter case that it is inherited. What has happened is that in the former case a new stimulus acting upon the old factors has brought forth a new character, and in the latter case that the old stimulus has brought forth a new character because it has acted upon a different factor.

Though the popular distinction between characters which are acquired and characters which are inherited is misleading, there are, nevertheless, two kinds of variation. A new departure may be due to a change in the germinal constitution. In this case the new character will reappear in future generations, provided that the changed germinal constitution remains and provided that the complex of stimuli which composed the environment does not change. Such a variation may be called a ' mutation'. A new departure may also be due to a new stimulus acting upon an unchanged germinal constitution. In this case the new character will only reappear in future generations provided that the new stimulus remains. Such a variation may be called a 'modification'. Mutations, therefore, are transmitted in the germinal constitution, while modifications are not so transmitted.

4. Already we have reached the answer to the first problem. Permanent change in organic form is due to germinal change. But before we go on and ask how germinal changes, that have arisen, become established, we must consider further the difficult 
problem of the manner in which germinal changes arise. A reference first to what are known as 'pure line' investigations and afterwards to Mendelian phenomena will illustrate what is known as to the nature of existing germinal differences; for until we have some such information, we cannot profitably ask how existing germinal differences arise. The best known 'pure line' experiments are those carried out by Johannsen with beans. The flowers of beans fertilize themselves; the offspring, therefore, have approximately the same germinal constitution. The offspring constitute a 'pure line', for by a 'pure line' is meant a group of children which are the offspring of a single parent. The character selected for investigation was weight and it was found that, if beans were collected from a bean-field and weighed, every gradation occurred between a minimum of about 20 centigrams and a maximum of about 90 centigrams. When the beans were separated into three classes-heavy, medium, and lightsown and plants raised from them, the average weight of the beans produced by the plants derived from the heavier seed was greater, though not proportionately greater, than the mean weight of all the beans, and that the average weight produced by the plants derived from the lighter seed was less, though not proportionately less, than the mean weight of all the beans. There was, in fact, a certain regression on the part of the heavy and light classes to the mean weight. A similar tendency to regression to the mean can be observed when other characters are similarly studied. The average stature, for example, of the offspring of tall parents is slightly nearer the mean stature of the race than the average stature of the parents.

This tendency to regression to the mean has long been known, but it was not until Johannsen proceeded farther and investigated inheritance within a 'pure line' that it was understood. In the experiment described, no attention was paid to the "pure line'. When Johannsen separated the beans produced by the self-fertilization of a single plant, divided them into heavier, medium, and lighter classes, sowed them and weighed their progeny, he discovered a very interesting fact. The average weight of the progeny of a heavy bean and of a light bean belonging to the same 'pure line' was the same. The conclusion to be drawn from this result is that the differences in weight between the offspring of a single self-fertilized plant are due to differences 
in the stimuli which play upon them. Obviously the stimuli do differ; the weight is affected by differences in light, shade, number, and position of the beans in a pod and so on. But since the offspring of a single plant have approximately the same germinal constitution, the average weight of the offspring of light beans, which are light because of subjection to less favourable stimuli than the average, will not be on that account lighter than the mean weight of the strain, and similarly mutatis mutandis with regard to the offspring of the heavy beans.

It follows, therefore, with regard to this particular character that there are a number of different strains in the population. The beans arising from any one strain are not of the same weight because they have been subjected to different stimuli. If each strain was pure and if differences in environmental stimuli could be removed, then beans gathered from a bean-field would not exhibit a simple gradation in weight; there would be a number of steps and as many steps as there were strains. In actual fact, the modifications produce the gradation which is observed. The fact that the nature of the germinal constitution cannot, as stated above, be determined by a mere inspection of the characters is exemplified by this experiment. If we take a bean weighing, say, 55 centigrams, it may belong to a strain which varies, say, between 20 and 65 centigrams, or to a strain which varies between 40 and 90 centigrams. It is only when this bean is sown, and the weight of the offspring calculated, that the strain to which it belongs can be ascertained. The experiment further shows why regression towards the mean is observed, when, instead of limiting investigation to a 'pure line', beans differing in a certain respect from the average, by, for instance, greater weight, are selected and sown. Such beans will belong to several different strains; they will, however, include more which have been favourably than unfavourably influenced by the surroundings. The average of the weight of all their offspring will therefore be less than the average weight of the parents. At this point we touch upon the problem of selection, but before we go on to deal with this question, it is necessary to say something more about variation and the origin of variations.

5. Self-fertilization is very exceptional, and what happens in the case of the beans, though illuminating, is not typical. As a general rule in reproduction, two parents contribute to the 
germinal constitution of the offspring. Strains, therefore, do not remain pure, as in the case of the bean, because they are continually crossed ; we require to know what happens when crossing takes place. This study, first successfully undertaken by Mendel, has been greatly extended in late years. We may first illustrate in the simplest form what it was that Mendel discovered and then go on to inquire what deductions bearing upon the question we have to answer are to be drawn from it.

A large number of experiments of the following kind has been made. Two strains in any species are chosen; these strains exhibit opposed characters. Such characters may be tallness and dwarfness, colour of the flower, shape of the comb in fowls, condition of the seed, whether smooth or wrinkled, and so on. We may call one character $\mathrm{A}$ and the other $a$. The two strains are crossed, and in the first generation the offspring are all alike and exhibit a character, $\mathrm{A}^{\prime}$. This character may be the same as either A or $a$, a blend between them or something wholly new. Whatever form it may take, it is produced by the interaction of $\mathrm{A}$ and $a$. The members of this first generation are then interbred, and of the second generation one quarter exhibit the character $\mathrm{A}$, one quarter the character $a$, and the remaining half the character $\mathrm{A}^{\prime}$. If the quarter exhibiting the character $\mathrm{A}$ are interbred, all the offspring exhibit the character $\mathrm{A}$, and the same holds good regarding the quarter exhibiting the character $a$; but if the half exhibiting $A^{\prime}$ are interbred, the offspring will split up in the same proportion as in the previous generation, one quarter exhibiting $\mathrm{A}$, one quarter $a$, and one half $\mathrm{A}^{\prime}$. This result holds good for any number of generations so long as interbreeding is continued.

Into the very numerous complications which occur it is not necessary to go. They are all interpreted by extensions of the simple explanation which is applied in the elementary case given above. It is supposed that all characters which behave on crossing as above are represented in the germinal constitution by factors which behave as separate units ; such characters are called 'unitcharacters', and such factors 'unit-factors'. It is further supposed that each gamete bears one unit-factor only in respect of each unit-character. If the strain is pure, as in the case of the two strains exhibiting characters $\mathrm{A}$ and $a$, then all the gametes will bear the unit-factors for $\mathrm{A}$ and $a$ respectively, and the fertilized eggs resulting from the crosses between them will therefore 
contain both unit-factors. The hybrids, which, as we have seen, exhibit the character $A^{\prime}$, will produce gametes, half of which bear the unit-factor for $\mathrm{A}$ and half the unit-factor for $a$. When the hybrids interbreed, on the average of chances one quarter of the fertilized eggs will have two factors for $\mathrm{A}$, one quarter two factors for $a$, and one half both a factor for $\mathrm{A}^{\prime}$ and a factor for a. In this manner the splitting up of the second generation is explained.

What is important in this explanation is the conception of unit-factors. The extension of the explanation to cover the more complicated cases does not involve any modification of principle. It follows that the germinal constitution contains a very large number of unit-factors ; what therefore is innately given in the germinal constitution is a collection of such unit-factors. Each unit-character based upon a unit-factor can theoretically be separately distinguished and isolated. The complications to which references have been made are in part due to the difficulty of distinguishing unit-characters. What is apparently a simple character may not be a unit-character, but a combination of unitcharacters. This apparently simple character cannot appear unless all the unit-factors, upon which these unit-characters are based, are present in the germinal constitution.

It may next be asked whether all unit-factors behave in this manner when crossed. To this no definite answer can yet be given. The successful analysis of apparently contradictory cases and the continual discovery of characters which do behave in this fashion seem to point to an affirmative answer. The suggestion is that, when this mode of behaviour cannot be demonstrated, it is because the unit-characters have not yet been distinguished and isolated.

This brief reference to 'pure line' investigations and to the Mendelian analysis of crossing leads therefore to the following conclusions. When, as in the case of the self-fertilizing bean, the strains are kept pure, existing germinal differences in respect of any character are found to be of the nature of steps which are usually small-though in outward manifestation the differences are smoothed over by the influence of environmental stimuli. This is the nature of the germinal differences, and they remain what they are-apart from the origin of new factors or of the loss of old factors, and apart from the effects of a differential 
death-rate. When, as is usually the case, biparental reproduction takes place, the position is more complicated : strains are being continually crossed, and, as the result of the chance mixture of factors in the germinal constitution of the children, the offspring of the same parents differ in their germinal constitution one from the other. Therefore, in any species in which biparental reproduction takes place, new combinations are constantly arising; but, though in this manner the germinal constitution may in a sense change, the change is due simply to a shuffling of factors. What we require now to ask is what is known as regards the manner in which new factors arise and are added to the germinal constitution, and the manner in which old factors drop out and are lost from the germinal constitution; for it is only owing to such additions and to such losses that true germinal change occurs, and that shuffling is rendered possible.

6. The interpretation of the results of the crossing of different strains has shown what kind of changes underlie the appearance of certain mutations. It has been shown that many of the varieties of domesticated species have originated by the apparent loss of one or more unit-factors. ${ }^{1}$ Thus the numerous varieties of domestic rabbits and of sweet-peas are all descended from a single wild species of rabbit and of pea, and differ from the wild stock not by an addition to, but by an apparent subtraction from, the total number of factors in the germinal composition of the wild stock. The reasoning which has led to this conclusion need not be followed here; one proof is that when certain varieties are crossed, characters of the original stock reappear, due to the fact that, one variety having apparently lost one factor and the other another, crossing results in the recombination of the factors necessary to the manifestation of the original character.

This is a strange conclusion, but it seems nevertheless to be true that in this manner many domestic varieties have arisen. If this was the only manner in which mutations could come about, then we should be driven to imagine that the most elementary form of life contained within it innumerable factors, and that evolution has merely consisted in the apparent dropping out of factors. This conclusion has indeed been tentatively suggested. But until it has been definitely shown that this is the only manner

1 There is probably no actual loss-no gap in a chromosome. It may be supposed that owing to a 'negative variation' a factor ceases to be functional. 
in which mutations originate, it must be supposed that changes in and additions to the complex of factors can and do occur. And we may note that certain distinguishing characters of domestic varieties of fowls and of pigeons appear to have arisen by the addition of factors; thus the 'single comb' of fowls is the original character of the wild stock which has been modified by the apparent addition of other factors and not by the dropping out of one or more existing factors. It is again not necessary to go into the reasons which have led to this conclusion. The conclusion is a deduction from the analysis of crosses between different breeds of fowls.

7. For the most part we are quite ignorant as to the causes which have led to the apparent losses and apparent additions of factors, though a few observations seem to indicate certain circumstances under which the dropping out of factors may take place. In this connexion some reference must be made to the problem as to the inheritance of acquired characters, though it follows from what has been said above regarding terminology, that we should more correctly speak of this problem as the question whether modifications in any one direction tend to be followed by mutations in the same direction. As the result of prolonged discussion and controversy it is now generally held that nothing of the kind takes place. It is almost universally agreed, for instance, that such modifications as are induced among men at the present day do not lead to mutations in the same direction. Therefore we may for the purposes of this book, so far as man is concerned, take it that acquired characters are not inherited. But looking at the problern as a whole it cannot be regarded as settled. There is, for example, some evidence of the parallel induction of modifications and of mutations; but the question may be raised whether such cases if substantiated fall under the heading of the inheritance of acquired characters. Again, some biologists regarding the problems of evolution generally find difficulty in arriving at an explanation unless under certain circumstances adaptive variations are followed by mutations.

We are thus left with the fact that mutations arise, and may be either large or small. Outwardly, variations in characters are usually continuous, because the environmental stimuli vary continuously, smooth over and obscure the differences due to mutation, as in the case of the beans mentioned above. But, discounting 
the influence of the environment, the mutations themselves may form a series separated by steps that are so small as to be searcely measurable or which may be very large. The weight of the beans is an example of the former kind of mutation; the so-called 'meristic' variations, when another member is added to a series, the addition, for instance, of a vertebra to the vertebral column, is an example of the latter kind.

Whenever a mutation occurs, we have to think of it as founded upon some change in the germinal constitution. Such changes are of the nature of modifications of factors-and may be positive (leading to the apparent addition of factors), negative (leading to the apparent loss of factors), or qualitative. But of the nature of these changes we know little, and of their causes less. What is important, however, is that these changes do occur. Further, they occur in all directions. The direction is, of course, in a sense determined by the starting-point-by what is already given in the germinal constitution-but, given the starting-point, mutations apparently occur in all directions. It is a matter of importance to know whether mutations ever tend to occur more in one direction than in another. Nothing definite has been ascertained as to this particular problem, though, as we shall note later, certain facts with regard to the evolution of animals suggest that there have been tendencies to change along certain lines. It may also be asked whether the continued selection of a character in any way affects the direction of the variation of the germinal constitution. To this again there is as yet no definite answer. On the whole it is not probable that selection has any such effect.

8. So far, therefore, as we have at present gone, we have found that permanent change is of the nature of germinal change. We have also discussed the nature of germinal differences as they exist between different individuals, and we have discussed the nature and causes of germinal change. With regard to variation there is much which is doubtful and obscure, but bearing in mind the essential features of what is certainly known, we may turn to consider what passes in the organic world. Upon this subject there is much less uncertainty. We have seen how huge is fecundity. Of the young in any generation, only about that number survives which equals the number of adults in the generation from which they are derived. For normally in the organic world the total number of adults of any species at corresponding periods 
in succeeding years remains much the same. The instances of a marked increase in the number of any species are rare, and are usually traceable to the intervention of man, as the result of which certain of the dangers which normally confront the young have been removed. It follows, therefore, that normally of the many thousands of fertilized eggs of a fish, for example, all but two perish. Even when the most rapid increase ever observed is taking place, the great majority of young perish. From a consideration of the circumstances it is evident that upon the whole those individuals which present certain characters will have a better chance of surviving than others which do not present these characters.

This point demands further consideration, because upon it turns the whole question of natural selection. What is involved in this theory is that the death-rate is selective, that those individuals which are best adapted to the surroundings which confront the species do, on the whole, have a better chance of survival. It is adaptation which determines fitness, but the concept of adaptation does not of necessity include any idea of progress. Given any complex of surroundings such as that which confronts any species, there may be a more or less close fitting of the organisms to this complex. The closeness of this fitting may have been obtained by a simplification of structure, a complication of structure, or it may be that for long ages the closeness of the fitting has been attained by the elimination of departures from the mean of the species in any dircction and the preservation of the average type.

In this connexion it has often been pointed out that the deathrate in certain cases is not selective, and does not therefore involve the survival of the more fit and the elimination of the less fit. When the whale opens its mouth and engulfs vast numbers of small organisms upon which it feeds, there is apparently no escaping of certain types of these organisms accompanied by a greater elimination of other types. But these cases are not on the whole common, and further there is nothing in such cases which counteracts selection; it merely means that sometimes selection is not operative. A consideration of the mode of operation of the factors of elimination, the general nature of which has been indicated, leads without any doubt to the conclusion that in the vast majority of cases, when any organism meets its death, 
either that organism does not possess some character which other organisms possess and which have enabled them to survive, or it possesses some character which other organisms do not possess, and which at some crucial moment has told against it. This subject could be considered at great length, but it must suffice to say here that the more experienced naturalists are, and the greater the knowledge they have of the conditions of organic life, the more it is borne in upon them that the death-rate is upon the whole selective, and that the best adapted types have a better chance of survival than other types.

From the nature of the case it must be difficult to obtain statistical evidence of natural selection. There are, however, certain cases known which are of great interest. Bumpus, for instance, after a storm in America collected 136 sparrows, all of which had been injured. Of these 72 recovered, while the remainder died. He weighed and measured all the specimens and compared the figures for the survivors with the figures for those which had perished. It was found that the average type of the latter was larger and heavier than the average type of the former. It was also observed that there was a less wide range of variability among the survivors than among the dead, showing that the favoured type approximated more closely than the others to the average type of the species. This favouring of the average type has been shown to occur by other observations, and it may be supposed that normally it is the average type of any species which is best fitted to the particular niche in nature occupied by the species, and that therefore adaptation will be measured by the nearness of approach to this type. When, on the other hand, circumstances are changing, some deviation from the average type will be favoured.

9. This leads us to ask in more detail what it is that happens in the process of selection. Selection has been likened in its action to a sieve separating the fit from the unfit. But fitness is measured by the characters exhibited, and these characters may be either of the nature of mutations or of modifications. What is exhibited is selected quite apart from the underlying nature of the character ; but it is only when variations of the nature of mutations are selected that there results any change in the composition of the germinal constitution of the species. So far as selection is merely that of modifications within a strain, there will be no change in 
the average germinal constitution of the progeny. It is only in so far as selection is a selection of strains that there will be any change. If the strains among the beans referred to above can be isolated, then the strain with the predisposition towards the greatest weight can be isolated, the other strains eliminated, and the average weight brought up to that found in this strain. If the strains cannot be isolated, then an approximation only to this result can be made by the continued selection of strains of the greatest weight, and this is what occurs in nature.

This leads to the most important conclusion of all. Selection can only act upon what is given. We may put aside the possible but quite unproved influence of selection upon the direction of mutation. Apart from this, selection can have no other effect than to eliminate certain strains and to favour others. Supposing that under changing circumstances a certain character is favoured, all that can happen through selection is that the strain exhibiting this character will be favoured and all other strains gradually eliminated. Further than this change cannot proceed until mutations have occurred in the direction of further increase in this character. As has been said, selection is no more than a sieve; as a rule it is occupied in separating out the extreme types and favouring the mean, but at times under certain circumstances it will favour some type that deviates from the mean, but it can do no more than favour what types are at any given moment in existence. ${ }^{1}$

10. We set out to answer two questions regarding quality. We asked in what change among species in a state of nature consisted. The answer to this is clear; it consists in changes in the germinal constitution. If we can speak of history in connexion with species in a state of nature, then their history is based solely upon germinal change. We also asked how change came about. It is less easy to answer in a few words. Obscure

1 A ccrtain amount of confusion has been introduced into the whole question of evolution by the manner in which in recent biological writings the function of selection has been depreciated. The followers of Darwin undoubtedly at times exaggerated the importance of selection. In particular selection has even been spoken of as though it caused variation and, as it were, drew organisms along certain paths. It is essential to remember, as has been emphasized above, that it can only act upon what is given. But without doubt there has been recently a tendency to underestimate the importance of selection. Though the process of selection is nothing more than a process of sifting, that process is of fundamental importance; unless due weight is given to it, evolution cannot be rendered comprehensible. 
as are many of the details connected with the process, there is a sufficient measure of agreement regarding the main facts to enable an answer shortly to be formulated. The answer rests upon two series of facts. The observed characters of organisms are based upon certain predispositions in the germinal constitution. These predispositions or factors under the stimuli of the environment give rise to the various characters. These factors are derived from both parents through the fusion of the gametes, and the complex of factors in the offspring is the result of a chance mixture of factors. There may further at times be a dropping out, an addition to, and perhaps a modification of the factors. Secondly, the death-rate is selective. Although the result of selection is confused by the effect of modifications which smooth over the differences due to differences in the germinal constitution, the effect is that individuals with certain factors are favoured and others with different factors are eliminated. Therefore the average nature of the factors may be changed should any type, other than that approximating to the normal type, be favoured.

It is possible in this manner to understand how change has come about. There are many difficulties, but they are all capable of fairly satisfactory explanation. None at least is insuperable. There is one problem which is perhaps somewhat difficult to solve unless a particular supposition is introduced. This problem is connected with the evolution of organs along certain lines, as, for instance, the evolution of horns and teeth in the vertebrates. This class of evidence has chiefly impressed itself upon palaeontologists, and one of the most distinguished of them has stated that from this class of evidence he concludes that "there are fundamental predispositions to vary in certain directions ' ${ }^{1}$ This is the supposition which it may be necessary to introduce, but which does not in any way conflict with what has been said.

1 Osborn, Evolution of Mammalian Molar Teeth, p. 228. One of the most valuable contributions to the discussion of orthogenesis has been made by Plate, Über die Bedeutung des Darwinischen Selectionsprinzips. Plate concludes that the evidence points to some kind of orthogenesis. 


\section{IV}

\section{THE POPULATION PROBLEM AMONG MEN}

1. IT has been shown in the last two chapters in what the population problem among species in a state of nature consists. With regard to the quantitative aspect in particular it has been shown that among such species mental evolution, greatly as the stage reached differs between the lowest and the highest forms, has not attained a point at which the essential features of the position have in consequence been affected. Enough has been said to show that in the case of the most primitive races of man now living mental evolution has reached a point in consequence of which the quantitative problem has assumed a totally different aspect.

With regard to the qualitative problem the question also arises as to how far mental evolution in man has affected the position. In one very important respect mental evolution has wholly altered the position of man as regards this aspect of the problem, as it has regarding the quantitative aspect. How this has come about may be considered after it has been observed that mental evolution has also been the cause of other changes which, while they do not alter the fundamental position of man as regards the qualitative aspect,-yet are of great importance.

As we have seen among species in a state of nature change is founded upon germinal change. So, too, among men there is germinal change, and so far as history (using the term in the widest sense to include what is often rather meaninglessly called 'prehistory ') is connected with germinal change, so far it is of essentially the same nature as change among other species. But both the direction and intensity of germinal change among men have been greatly influenced by mental evolution. Certain causes of elimination have been removed wholly or in part, others have been introduced. The facts are familiar; there is no need to labour the point. It is also worth noting that, should it be found that mutation frequently arises owing to alterations in the environment during the formation of the germ cells, such a discovery 
might have considerable bearing upon the position in man. Owing to human activity under the guidance of reason, the environment has been profoundly changed in many directions, and without question in consequence of such changes the germ cells of human beings are subject during their development to far more varied stimuli than are those of other species. This, however, is only a possibility. Nothing is known with certainty upon the subject. It may be that the great variety of foods, the absorption of alcohol and nicotin, the various occupations and customs, and many other factors, all ultimately traceable to reason, may, some of them in some way, tend to bring about mutation in man.

It is by making possible the development of tradition that the evolution of reason has fundamentally changed the nature of the qualitative problem among men. Into the nature and origin of tradition it will be necessary to go in another chapter. Here all that is required is an indication of its main features in order that its connexion with the problem of change may be understood. In the stage of conceptual thought reached by man, the formation of free ideas is the outstanding feature. With the development of conceptual thought went the development of language. By language ideas can be passed from one man to another and also from one generation to another. There are other ways in which the results of reasoning can be handed on, but they need not be considered at present. What is important is that they can be and are handed on. Let us consider for a moment the question of skill. Some improvement may be made in the methods of hunting or of fishing. It may, of course, be lost, but it may be, and perhaps usually is, transmitted to other men by the inventor and afterwards to succeeding generations. Tradition is, in fact, cumulative. Even among the most primitive races now existing there is a huge mass of tradition. Succeeding generations do not necessarily start at the beginning again. They start with the experience of the race behind them, so far as it has been preserved.

Among the higher animals there is a certain handing on of what has been learnt by experience ; to this extent there is tradition also among them. We shall return to this point later. So relatively unimportant, however, is tradition among other animals, that we may for the moment regard tradition as something which is found among men only. It is clear that tradition has played some part $\mathrm{i}^{\mathrm{n}}$ any case in producing the changes which we call history. 
We have only to think of the rise of Japan to the position of a great . power in the last half of the nineteenth century. The rise of Japan was an outstanding fact in the history of that period and it was clearly in the main, if not wholly, a traditional and not a germinal change. It took the form of a rapid absorption of European tradition. The evolution of reason has thus introduced into the problem of the causes of human history a factor which is not present in the case of other species ; it has also modified the course of selection, but this, as we have seen, has not made a fundamental difference between the position of man and that of species in a state of nature.

The problem before us is therefore as follows. Owing to the fact of reproduction the population problem in both its aspects exists for all species in a state of nature and further presents fundamentally the same features for all such species. The ancestors of man were at one time subject to the same conditions from which they have, step by step, moved away owing to the development of the faculty of reason. We have to trace the causes and results of this moving away-of the progressive modifications of the conditions existing among species in a state of nature.

Though the problem has two aspects, they are closely interwoven. Changes which affect numbers also influence the quality of population. The discussion has hitherto taken the form of an introduction to the problem as a whole, and the two next chapters, the subject-matter of which will be indicated in what follows, will also be devoted to certain problems which equally bear upon both aspects of the question. From the seventh chapter onwards the two aspects are treated independently; we deal first with the quantitative and then with the qualitative aspect. Nevertheless we shall, when dealing with the quantitative problem, present evidence which we shall consider again later when treating of the qualitative problem. The book thus falls into three parts; the first six chapters are introductory to the problem as a whole, the next six chapters are concerned with problems of quantity (though many of the facts brought forward will be found also to bear later upon quality), and the following nine chapters with problems of quality. The last chapter sums up our conclusions as to the whole problem.

2. We may next ask what data are required in order that we may look into the changes away from the conditions under which 
the pre-human ancestor lived towards the conditions which now exist. In order that we may consider the quantitative aspect of the problem, it is evident that we require some knowledge regarding fecundity and as full details as possible regarding the factors which bear upon fertility and elimination. It is only when we are provided with such information that we can hope to be able to determine how it is that numbers are regulated among men.

In addition to such information, it will be desirable to have information regarding various social customs and the general conditions of life. As regards the inquiry into the qualitative part of the problem, it is clear that facts regarding fertility and elimination are again of value inasmuch as they throw light upon the nature and intensity of selection. For the second part of the problem it is also necessary to have in mind the main facts regarding the changes which are summed up as history into the causes of which we have to inquire ; and in addition it will be necessary, in order that we may attempt some estimate of the relative importance of change in the germinal constitution on the one hand and of the other factor or factors of the nature indicated above on the other hand, to make some inquiry into the conditions which determine the nature of these latter factors. This will involve a reference to certain elementary facts of psychology together with some discussion of the origin, formation, preservation, and so on, of tradition.

If we first ask how far the data desired are available, we may afterwards go on to discuss how the facts are best presented. Our information is obviously very incomplete as regards the history of social habits and customs. Historical records do not take us farther back than at the most six thousand years, and the information available with respect to social customs, except for the latter part of the period, is very inadequate. If we take a broad view of history, written records have reference only to relatively modern times. There are two other sources of evidence upon which we have to rely and they are as follows.

Some indication of the bare outline of the course which history has taken can be gathered from a study of the fossil and cultural remains of man. The fossil remains are in the form of skeletons or portions of skeletons and the cultural remains in the shape of tools, weapons, and other traces of his mode of life that man has 
left behind him. It is interesting to observe that an attempt can be made to date these remains. They occur in certain strata, and estimates can be made of the length of time which has elapsed since these strata were deposited. Though a very large measure of doubt must surround any such estimates, nevertheless there is a certain measure of agreement concerning the facts which is of great interest. It is, therefore, possible to draw up a table showing these strata, together with what we should perhaps call guesses at the dates at which they began to be laid down. It can also be shown in the table what fossil and cultural remains are found in the respective strata.

The information, however, which we thus gain is very meagre. A study of fossil remains can only provide us with an outline of the facts connected with the evolution of the skeleton. More can be gathered from a study of the cultural remains, and certain deductions of considerable interest can be made especially in connexion with our present purpose so far as the evolution of skill is concerned. But, of course, we can in this manner gather nothing as to the nature of the social customs and institutions belonging to the races which practised these skilled methods. The question we have to face is whether this very bare outline of the facts of history can in any manner be filled in.

3. There is a further source of evidence which we have to consider. There has come under the observation of civilized man a large number of races in a low stage of culture. The evolution of culture or of control over the surroundings took place more quickly among certain races than among others. Some races got left out of the main stream of evolution, and finally in relatively recent years the more advanced races arrived at a stage where they were in a position completely to dominate the latter. The more primitive races were unable to offer any serious resistance to the civilized races. It was, therefore, possible to regard these primitive races dispassionately and scientifically; this study has led to the collection of a vast amount of information regarding these primitive races. These data are for many reasons often of a very unsatisfactory nature; of this the chief cause is that, before an accurate study was begun, contact with eivilized races had often had a considerable influence upon the former mode of life of the primitive races. What we require and what is difficult to obtain is information as to the conditions under which these races lived 
before they came into contact with Europeans. With regard to the unsatisfactory nature of the evidence there will be more to say later.

It is of primary importance to observe that these races are not to be regarded as actual representatives of certain stages through which civilized races have passed. We know that in the evolution of control over nature civilized races have passed through certain stages of culture; the first stage was marked by the use of unpolished stone implements and the obtaining of food by hunting and fishing only; the second stage was marked by the use of polished stone implements and the obtaining of food by agriculture and the domestication of animals. ${ }^{1}$ These two broad divisions - the Palaeolithic and the Neolithic-may be subdivided. But no primitive race can be regarded as representing any definite stage in exact detail, whether in the first or in the second of the two broad divisions through which civilized races have passed. While the culture of the ancestors of civilized man was evolving from that of the Palaeolithic stage to that of the present day, the culture of certain other races did not progress as far even as the Neolithic stage. But the culture of these races did not remain unmodified; it followed in each case a line of evolution of its own. These races are therefore to be regarded as specialized representatives of the earlier stages of culture through which the civilized races passed. Nevertheless, specialized though their culture may be, the general conditions of their existence are certainly similar to the general conditions which must have characterized the corresponding stage in the evolution of the culture of civilized races. That is to say, the broad features which characterize the conditions found among hunting and fishing races must also have

1 Except where otherwise stated, increase of skill-of power, that is to say, to control the environment-is taken as the criterion of 'progress'. Except, therefore, where otherwise stated, the terms 'civilized" and 'higher' races are merely intended to convey that races so denominated have a greater power of controlling the environment than 'uncivilized' and 'lower' races. The terms ' higher' and 'lower' are thus in this book merely convenient labels used in a special sense. In a fuller sense the terms 'higher' and 'lower' would take account of innate faculties, social organization, and moral tradition, as well as of the tradition of skilled methods. It is worthy of note, so far as the argument in this book is concerned, that there is a correlation between the tradition of skilled methods and the tradition of social organization and between both of these traditions and the innate faculties. The result, therefore, of a classification of races according to the special sense of the terms 'higher' and 'lower', as given above, does not conflict so directly as might be thought with a classification according to the fuller meaning of these terms. Further reference is made to the subject in the later part of this book. 
characterized the conditions under which our Palaeolithic ancestors lived.

There is here obviously suggested a method whereby the framework of history arrived at by the evidence of fossil and cultural remains may be filled in. The broad conclusions drawn from a study of hunting and fishing races may be applied to Palaeolithic races. But this method must be used with great caution; it has undoubtedly been pressed too far. It is possible to divide hunting and fishing races into groups and to compare these groups with stages in the evolution of Palaeolithic culture. How far it is in any way valid to attribute in this fashion particular social institutions which characterize lower, middle, and upper hunting races to lower, middle, and upper Palaeolithic races respectively is very doubtful. It is not proposed here to attempt to carry the method as far as this. For one thing, so far as the subject has been worked out, there is no clear correlation between different factors affecting fertility and elimination on the one hand and stages in culture among hunting races on the other hand. Thus, as it happens, any attempt to carry this method into more detail is not productive of results, and therefore it is not worth while to inquire whether results would be valid or not. All that it is proposed to do here is to examine the evidence regarding the prevalence of certain customs, habits, and so on, among hunting races, and to apply the broad conclusions founded on this evidence to the ancestors of civilized races who supported themselves by hunting. In this manner only is it at all possible to fill in the framework of history provided by the remains of culture and by fossils, and thus to arrive at any conclusions regarding the course of the movement away from the conditions found in the pre-human ancestor to those found among civilized races.

4. With regard to the question as to the way in which this evidence may be most conveniently set out, it is proposed to proceed in the following manner. Before we come to the evidence regarding fertility and elimination, something has to be said regarding fecundity in man, and that will be the subject of the fifth chapter. In the following chapter the evidence regarding fossil and cultural remains will be very briefly discussed, just so far as, in the first place, provides the necessary historical facts to form a basis for the discussion of the qualitative problem and as, in the second place, provides the framework into which we can fit 
the conclusions which we shall draw from a study of primitive races. In the seventh chapter the evidence drawn from a study of hunting races will be reviewed in so far as it throws light upon fertility and elimination. In the eighth chapter the evidence bearing upon these problems, so far as the agricultural races are concerned, will be similarly set out. In the ninth chapter the evidence will be discussed in its bearing upon the quantitative problem among these races-all reference to the qualitative problem being left for later chapters. In the tenth chapter the evidence for the historical races will be similarly reviewed, and in the eleventh chapter similarly discussed. The twelfth chapter will be devoted to a discussion of certain problems connected with the quantitative problem at the present day. The twelfth chapter therefore will complete the treatment of the first or quantitative aspect of the problem, and in the remaining chapters will be discussed the second or qualitative problem. Already there will have been presented a large part of the evidence necessary for the discussion of the second problem-namely, the evidence connected with the outline of the facts of history and that connected with fertility and elimination. What other evidence is necessary can be given as and when required. 


\section{V \\ HUMAN FECUNDITY}

1. As a preliminary to the separate inquiries into the two aspects of the problem, it is necessary briefly to consider the question of human fecundity.

We may ask, in the first place, whether, taking a broad view of human history, there is any reason to believe that human fecundity has changed since the epoch of the pre-human ancestor, and, if so, whether it has increased or diminished. Secondly, there are a number of habits and customs which have been practised in different degrees at different times, such as prolonged lactation, polygamy, and so on, and we may also ask what bearing, if any, such habits and customs have upon fecundity.

There is much connected with the subject which is difficult and obscure. It is, in particular, often difficult to ascertain whether any differences in fecundity, as may be found to exist, are of the nature of racial or of environmental differences. Into such points, however, we need not go. We are concerned only with the question as to whether there are differences in fecundity, and we may confine our attention to a notice of any broad differences which do exist and to the question whether certain habits and customs widely practised at one time or another and supposed to bear upon fecundity do or do not have the influence attributed to them. We may begin with the first question set out above. In order to obtain an answer to this question we have to rely upon evidence derived from various sources, partly from primitive races, which we assume in general to exhibit conditions prevalent among prehistoric races, and partly from evidence derived from a study of the mammalian species which have been subject to changes comparable with those to which man has in the course of his history been subject.

2. From what has been said regarding the nature of reproduction in Ohapter II, it follows that it is the female rather than the male that we have to. consider. The male gametes vastly outnumber the female, and, generally speaking, the male is always ready for 
intercourse. When studying the possibility of increasing the fecundity of domestic species, attention can, as pointed out by Marshall in the case of sheep, ${ }^{1}$ be limited to the female. Taking a broad view of the matter, it is clear that there has been no change in fecundity due to any change in male reproductive power.

There are, nevertheless, two points concerning the male which we may notice before we pass to the consideration of the female. First, with regard to the problem as to changes in fecundity during the course of history, we shall bring forward evidence to show that good conditions favour an increase in the reproductive power of the mammalian female. There is reason to think that good conditions have a similar effect on males. Thus among some mammalian species in a state of nature there is a special ' rutting season' for the male, and it is not infrequently found that in captivity the males of such species will rut all the year round. ${ }^{2}$ We shall also bring forward evidence to show that with improvements in conditions, sexual excitement is more easily aroused and that there has been an increase in the development of the generative organs, and we may note here that these observations apply to the male as well as to the female. As, however, any increase in male reproductive capacity can have had no general influence upon fecundity, we may, so far as the question of any general increase or decrease in fecundity is concerned, neglect the male and confine our attention to the female.

Secondly, with respect to the influence of certain factors at particular times, it is in nearly all cases the female and not the male which is affected. But it may be observed that sterile marriages are in part due to male sterility. Mayer estimates that this is so in a third of such marriages. ${ }^{3}$ Kelly gives the result of an investigation into 110 cases, of which 59 per cent. are attributed to the male.4 Male sterility is due either to inborn or acquired conditions. So far as sterility due to the former is concerned, there do not appear to be any grounds for thinking that it has to any important extent varied from one epoch to another. So far, however, as sterility due to disease is concerned, there have been in all probability certain changes. Gonorrhoea is perhaps the most important of those diseases which bring about sterility. Of

1 Marshall, Phil. Trans. B, cxcvi, 1904, p. 77. p. 12.
p. 354. See also Matthews Duncan, Sterility in Women, p. 3.

2 Heape, Q. J. M. S., vol. xliv, 
the 59 cases mentioned above 12 per cent. were due to gonorrhoea. Other diseases may cause sterility, such as tubercle and epididymitis. Relatively to the historical period as usually defined, gonorrhoea is an old disease; but we shall find reason for thinking that the great majority of diseases have evolved since the time of the pre-human ancestor, and to the extent to which this is true it must be allowed that male sterility due to disease is more or less recent. With these remarks we may leave the question of male sterility, as it is relatively of little importance.

3. It is therefore to factors which influence the female generative process that we must look for the causes of such increase or decrease of fecundity as are of importance. Certain factors may be put aside on the grounds that there is no reason to think that they have been of more importance at one time than at another. Such are malformations of any kind that prevent the meeting of the male and female gametes. 1 With regard to the factors of importance it is by their influence upon different aspects of the female generative process that they are best classified. There are three main aspects of this process, variations in any of which will influence fecundity. These are the length of the mature period, the interval between births, and the number at a birth.

Some reference to the nature of the female sexual cycle in mammals has been made. The beginning of maturity is usually measured by observing the beginning of menstruation. It is, perhaps, worthy of mention that the beginning of menstruation does not always coincide with the beginning of ovulation. Thus the estimation of the duration of the mature period made by watching for the beginning and end of menstruation is not always exact ; the difference is not of consequence here, but the fact that a difference is possible ernphasizes that what is essential in the whole process is ovulation. The true mature period is the period during which ovulation occurs. The interval between births is also dependent upon ovulation and so is number at a birth. ${ }^{2}$ In fact what we are asking is in the main what factors influence ovulation.

4. The information regarding the duration of the mature period is not satisfactory. Though there is a large amount of information about the beginning of menstruation, there is little information

- See Mosnier-Clauzel, De quelques Causes de Stérilité chez la Femme, for a suimmary of this question.

2 Except in the case of 'identical' twins. 
about the age at which it ends. There are at least three factors which influence the age at which menstruation begins-climate, race, and general nature of the surrounding conditions.

Generally speaking, the hotter the climate the earlier menstruation begins. Englemann gives the following figures : ${ }^{1}$

$\begin{array}{cccc}\text { Zone. } & & & \text { Average Age for Menst } \\ \text { Tropics . } & . & . & 12.9 \text { years } \\ \text { Temperate } & . & . & 15.5, \\ \text { Cold . } & \text {. } & \text {. } & 16.5 ~,\end{array}$

and Krieger gives the following figures: ${ }^{2}$

Place.

Christiania

16 years 9 months 25 days

Berlin . . . 15 , 7 , 25 ,

London . . . 15 " 1 " 14 ",

Lyons . . . 14 " 5 , 29 ,

Marseilles . . • $13,, 11$, , 11,

Calcutta . . . $12, \% 6, \quad 6,0$,

Sierra Leone . . $10,0,0,0$,

It further appears that there is a tendency for menstruation to end earlier in hot climates; so much so that upon the whole the mature period is shorter in hot than in cold climates.

There is, however, no very close or definite connexion between climate and menstruation. In the United States it has not been found possible to detect any influence of climate upon menstruation, though this country stretches from $29^{\circ}$ to $45^{\circ}$ of latitude, and has a temperature the annual average of which varies from $40^{\circ} \mathrm{F}$. to $70^{\circ} \mathrm{F}$. But if the conditions concerning the different racial elements in the population of the country are examined, it is found that in whatever part of the country they may be the average age for each racial element remains constant, and usually varies slightly from the average age for the whole population. ${ }^{3}$ There are other indications that the age at which menstruation begins are connected with differences in race. Krieger quotes the following from Joachim for girls in Hungary : ${ }^{4}$

Race.

Slavonic

Magyar

Jewish

Styrian
Average Age of Menstruation.

16 to 17 years

15,16 ,

$14,, 15$,

13,14, ,

1 Engelmann, Trans. Amer. Gyn. Soc., vol. xxvi, p. 87. ${ }^{2}$ Krieger, Die Menstruation, p. 52. See also Ploss and Bartels, Das Weib, vol. i, p. 421. 3 Engelmann, loc. cit., p. 98.

4 Krieger, loc. cit., p. 17. 
Other figures show that Jewesses menstruate earlier than the average age for menstruation in the country in which they live. ${ }^{1}$

What is of chief interest to us is that good conditions also influence the age at which menstruation begins. The better the conditions, the earlier does it begin. Further, it is also known that the mature period tends to be prolonged where conditions are good. It is known, for example, that the mature period comes to an end earlier among the labouring than among the richer classes. $^{2}$ Therefore good conditions tend to be connected not only with an earlier beginning but also with a longer duration of the mature period. Thus Mayer found the average age at which menstruation began among 3,000 women of the upper classes to be 14.69 years, and among 3,000 poor women to be $16: 0$ years. $^{3}$ Several observers have recorded the average age for the beginning of menstruation among the different classes in the population. The following table is given by Krieger : ${ }^{4}$

\begin{tabular}{|c|c|c|c|c|c|c|c|c|}
\hline \multirow[b]{3}{*}{ Upper Class } & \multicolumn{2}{|c|}{$\begin{array}{l}\text { Brienne de } \\
\text { Boisment. }\end{array}$} & \multicolumn{2}{|c|}{ Tilt. } & \multicolumn{2}{|c|}{ Irieger. } & \multirow{2}{*}{\multicolumn{2}{|c|}{$\begin{array}{l}\text { Ravn. } \\
\text { Yrs. Mths. }\end{array}$}} \\
\hline & Yrs. & Mths. & Yrs. & Mths. & Yrs. & Mths. & & \\
\hline & 13 & 8 & 13 & $5 \frac{1}{2}$ & 14 & $1 \frac{1}{6}$ & - 14 & 3 \\
\hline Middle Class & 14 & 5 & 14 & $3 \frac{1}{2}$ & 15 & $4 \frac{2}{6}$ & 15 & $5 \frac{1}{2}$ \\
\hline Lower Class & 14 & 1 & - & & 16 & $8 \frac{1}{6}$ & 16 & $.5 \frac{1}{4}$ \\
\hline
\end{tabular}

5. With regard to the question as to the interval between births some interesting indirect evidence is obtained from what we can learn regarding the former condition of the sexual cycle in man. A sketch was given in the second chapter of the main features of the mammalian sexual cycle. These cycles may follow one another without any prolonged interval, or there may be a lengthy period of rest. In monkeys and also in man the interval is short; but the former cannot breed at every heat period; special sexual seasons are experienced at definite times of the year when breeding takes place. It is probable that ovulation is limited to this special season, and that, although copulation may take place at other seasons of the year, conception can only follow copulation at this special season..$^{5}$ In civilized man, so far as is known; ovulation is

1 It is a remarkable fact that the Eskimo begin to menstruate at about thirteen years of age-at an earlier age, that is to say, than the average for the United States (Kelly, loc. cit., p. 83).

${ }^{3}$ Krieger, loc. cit., p. 21. 'Ibid., p. 24. ${ }^{5}$ Marshall,

2 Marshall, Physiology of Reproduction, p. 672. Physiology of Reproduction, p. 63. There are considerable variations in the conditions among monkeys; the above is only generally true. 
never confined to any particular season of the year ; conception can, therefore, follow copulation at any period. But we do find evidence of the former existence in man of a special sexual season, and this is of particular interest because it suggests that at one time there was in man, as there usually is in monkeys, one season only during which conception could follow copulation.

This evidence has been reviewed by Westermarck, by Ploss and Bartels, and by Havelock Ellis. ${ }^{1}$ Some examples of this evidence may be cited in order to show upon what facts this suggestion is founded. "According to Mr. Johnston, the Indians of California " have their rutting season as regularly as have the deer, the elk, the antelope, or any other animals ". And Mr. Powers confirms the correctness of this statement, at least with regard to some of these Indians, saying that spring 'is a literal St. Valentine's day with them, as with the natural birds and beasts of the forest'. As regards the Goddanes in Luzon, Mr. Foreman tells us that 'it is the custom of young men about to marry to vie with each other in presenting to the sires of their future brides all the scalps that they are able to take from their enemies, as proof of their manliness and courage. This practice prevails at a season of the year when the tree -popularly called by the Spaniards " the fire tree " - -is in bloom." Speaking of the Watch-an-dies in the western part of Australia, Mr. Oldfield remarks, 'Like the beasts of the field, the savage has but one time for copulation in the year. About the middle of the spring the Watch-an-dies begin to think of holding their grand semi-religious festival of Caa-ro, preparatory to the performance of the important duty of procreation. A similar feast was, according to Mr. Bonwick, celebrated by the Tasmanians at the same time of the year.' 2 Similar evidence is forthcoming as regards many other primitive races. It is interesting to note that among civilized races there are traces of customs which point to the former existence of a season of sexual licence. Such were the mediaeval Feast of Fools, and the classical festivals of Brumalia and Rosalia." 'Feasts, similar to the erotic feasts which were indulged in by the ancients . . . were still practised to some extent in Russia and in some parts of India at a much later date, while such customs as " gwneyd Bragod" and possibly our own "bean

1 Westermarck, History of Human Marriage, p. 25 ; Ploss and Bartels, loc. cit.. vol. i, ch. xxi ; Havelock Ellis, Stadies in the Psychology of Sex, vol. i, p. 85. 2 Westermarck, loc. cit., p. 28. $230 \mathrm{ff}$, where this subject is discussed at length. 
feasts " may not improbably be the modern representatives of these ancient customs in our own country.' 1

This evidence all points to a former condition when conceptions could only take place at one season of the year. We must suppose that it has gradually become possible for copulation to be followed by conception at any period, and that therefore fecundity has increased. ${ }^{2}$ Some light is thrown upon the causes that underlie these changes by a reference to certain facts regarding other species. Before we turn to this question reference may be made to the phenomenon of ' mittelschmerz' -or intermenstrual pain. It is not uncommon and is sometimes accompanied by a discharge. The interpretation of this phenomenon is doubtful. One explanation, however, has some interest for us. It is suggested that it may represent the first steps in the establishment of a different variety of sexual cycle in which the dioestrous periods occur fortnightly instead of monthly. If this interpretation is correct, it would mean that the sexual cycle is evolving in the direction of still greater fecundity. ${ }^{3}$

6. It has frequently been observed that when members of a wild species are brought into captivity there is a change in the sexual season. This change may be in the direction of an increase or of a decrease in the generative power. If the bringing into captivity involves such an alteration of conditions as to disorganize the generative process, then a decrease in fecundity may result. It frequently happens, for instance, that carnivores are infertile in captivity, and this may be due among other things to the absence of fresh meat. 4 Generally speaking, however, if the alterations do not produce disorganization, an increase in fecundity results, and as there is no reason to suppose that the change in human circum. stances was ever of such a nature as to produce disorganization, we must suppose, on the analogy of what happens among other mammalian species, that, if the betterment of the conditions has had any effect, it has been in the direction of an increase in fecundity.

A few examples of what is meant may be given. The wild dog of South America, the wolf, and the fox breed only once a year under

1 Heape, loc. cit., p. 34.

2 It may also be observed that among certain primitive races the menstrual flow is said only to occur at long intervals (Heape, loc. cit., p. 30). Among Eskimo women there is said to be no menstruation in winter (ibid., p. 29).

${ }^{3}$ For further details see Croom, Trans. Edin. Obstet. Soc., vol. xxi, 1896 ; Havelock Ellis, loc. cit., vol. i, p. 90 ; Marshall, loc. cit., p: 65 ; and Kelly, loc. cit., ch. v.

- Heape, loc. cit., p. 15. 
natural conditions; in captivity they experience two annual heats like the dog. ${ }^{1}$ The otter in the wild state breeds but once a year; in captivity oestrous may occur at regular monthly intervals all the year round. ${ }^{2}$ Bears are monoestrous in the wild state; in the Zoological Gardens oestrous may be experienced for two or three months. ${ }^{3}$ With regard to the Ungulata we have numerous facts of the same nature. The red deer offers the best example; under natural conditions there are two dioestrous cycles lasting three weeks; in captivity the sexual season extends over the whole year. ${ }^{4}$ The facts are somewhat similar in respect to cattle and horses. Examples might also be taken from other mammalian classes. The wild rabbit breeds from February to May ; in captivity the sexual season lasts nearly the whole year. Further it may be noticed that among lower groups good conditions are, as is well known, markedly favourable to increased fecundity.

Before we go on to look into the causes of this increase in fecundity, it may be noticed that the number of young produced at a birth among domestic species is on an average greater than that produced by the wild ancestor. This evidence, it will be observed, refers to the third factor which bears upon the increase in fecundity. The wild rabbit is said to produce at the most six young at a time; the tame rabbit has four to eleven, and a case is known in which eighteen were born, all of which survived. ${ }^{5}$ The wild sow bears from four to eight, and sometimes twelve, young. The domestic sow is considered to be of no value unless she produces at least eight at a birth. Equally remarkable examples are to be found outside the Mammals. "The hen of Gallus bankiva lays from six to ten eggs; the tame one in the course of a vear from eighty to one hundred.'

7. It is clear that one common feature in the conditions surrounding domesticated species and civilized man, as compared with species in a state of nature, is the increase in the richness and regularity of the food-supply, and the general betterment of the environment. In fact, just as this increase in the richness of the surroundings may increase the length of the mature period, so it may increase the number at a birth and also decrease the

1 Marshall, loc. cit., p. 57.

2 Ibid., p. 59.

3 Heape, loc. cit., p. 15.

Ibid., p. 15.

5 This and the following examples are taken from Darwin, Variations of Animals and Plants under Domestication, vol. ii, p. 90. 
interval between births. That this is the result of an improved environment in the case of the sheep has been shown by Marshall. $\mathrm{He}$ found, for instance, that in 'Scotch Blackfoot, Cheviot, and other Scottish sheep the normal percentage of ova discharged at any single oestrous is not appreciably in excess of the usual percentage of births at the lambing season ' 1 This, it may be noted, is an example of what was said in the second chapter regarding, in the first place, the measurement of fecundity by the number of ripe ova produced, and, in the second place, regarding the fertilization on the average of all, or nearly all, ripe ova. Marshall also found that " there was every reason for supposing that the processes of growth and maturation can be very largely influenced both by insufficiency of food supply on the one hand and by artificial stimulation on the other'? What apparently happens is that insufficiency of food retards the development of the ova and may cause the degeneration of some of them. Therefore if the food-supply is good, there will be more ripe ova at the sexual season, fewer sterile females, and a greater number of twin births. 'There is overwhelming evidence,' says Heape, 'that flocks in good condition at tupping time have a higher subsequent development of fertility than flocks in poor condition at tupping time.' 3 By ' good ' condition is meant not a 'fat' but a strong, healthy, and vigorous animal.

It may be asked whether there is any evidence as to the increase in numbers at a birth in the human female. Among civilized races about one birth in eighty to minety is on the average a twin birth. Our knowledge of primitive races is not sufficiently precise to enable any estimate to be made regarding the frequency of twin births among them. There are, however, very numerous references in the accounts of these races to the superstitions attaching to twin births. The nature of these superstitions, and in general the mystery felt to surround twin births, very strongly suggests that the phenomenon is rare. It can only be said that it is probable that twin births are rarer among primitive races than among civilized races.

So far, therefore, as we have gone, the evidence points to the conclusion that two of the three factors which determine fecundity have varied in the direction of increasing fecundity, and that 
possibly the third factor may have varied in the same direction. It has also been noted that similar changes have been observed to take place in animals which have been subject to better conditions. The fact that human conditions have certainly been bettered suggests that the same cause may have been at work in the case of man. There is, however, no doubt that changes in the case of man are not wholly explicable in this way. In part the differences are racial differences, and to the degree in which the differences are racial they cannot be attributed to the direct effect of the surroundings.

8. Additional support to the view that fecundity has increased with civilization is given by certain other types of evidence. In the first place it appears to be a fact that the reproductive organs of the more primitive races of mankind are smaller and in all respects less well-developed than those of civilized races. ${ }^{1}$ It is not meant that the organs differ qualitatively in any way; they are merely smaller relatively to the other organs than among civilized races. It is doubtful how we should interpret these facts ; but it does not seem unreasonable to assume a connexion between a lesser development of the reproductive organs and a lower degree of fecundity.

Secondly, we have a considerable body of evidence with respect to the strength of sexual desire among these races. ${ }^{2}$ Many observers have recorded their opinion that the members of these more primitive races do not experience sexual excitement to the same degree as do the members of the more civilized races. There are other observations which record the difficulty experienced in obtaining sexual erethism. It seems reasonable to associate these observations with a lower degree of fecundity than that found among civilized races.

In this connexion the fact that lack of sexual excitement renders fertilization less likely is relevant. As fertilization has been achieved with the female in a condition of narcosis, ${ }^{3}$ it is clear that sexual excitement is not necessary. Nevertheless, if the

1 The evidence is summarized by Ploss and Bartels, loc. cit., vol. i, ch. vi.

2 See Ploss and Bartels, loc. cit., vol. i, ch. xix, and Havelock Ellis, 'Studies in the Psychology of Sex', Analysis of the Sexual Impulse, p. 209. The fact is commented upon by Ratzel, History of Mankind, vol. ii, p. 17. As examples of the evidence for particular races see, for the Malays, Stevens, Zeit. für Eth., Bd. xxviii, 1896, p. 180 ; for the Fuegians, Hyades et Deniker, Mission scientifique du Cap Horn, Tome vii, p. 187; for the Andamanese, Portman, J A.I., vol. xxv, p. 369.

${ }^{3}$ Mayer, loc. cit., p. 409. 
question of sexual excitement has any bearing at all upon the degree of fecundity as between higher and lower races, it must be in the direction of rendering the lower races less fertile.

It was observed in the second chapter that the willingness of the female to receive the male at any time in the sexual cycle is a peculiarity of man. It may be observed in passing that evidence has lately been brought forward suggesting that the indifference of the female to the point in the sexual cycle at which intercourse takes place has been exaggerated, or rather that the existence of periods of desire has been somewhat overlooked.1 However this may be, what concerns us here is the peculiar fact that the male is received by the female at any time in the sexual cycle. It is possible that copulation at one time in the sexual cycle is more likely to result in conception than at other times, though the facts are obscure. But whether this is so or not is scarcely relevant to our purpose, because there is no reason to think that there have been any variations in the practice of copulation between one time and another or between one race and another, as could have sensibly affected fecundity even if the limitation of copulation to one period of the sexual cycle is of marked importance.

9. Lastly, we have to refer to what we may call the statistical evidence. The interpretation of the evidence as to number of children among primitive races is so difficult a matter that it cannot in itself be held to throw much light upon the question of fecundity. The trend of the evidence, however, certainly supports the conclusion that fecundity is less among primitive races than among civilized races. There are a vast number of observations recorded by travellers regarding the number of children among these races. For the most part these observations have reference only to the number of children seen alive, and therefore are not even a measure of the fertility-far less a measure of the fecundity.

Practically all such observations emphasize the small size of the families. When dealing with hunting and agricultural races in another connexion we shall have reason to refer in some detail to this evidence. Here we may give a few typical examples of this evidence, remembering that it is evidence for the most part of the number of children seen and not of the number of children born, though, in selecting the following examples for quotation, an effort has been made to pick out those in which the authors have

- Stopes, Married Love, p. 68. 
attempted to discount the effects of infanticide and other factors of elimination.

Of the Australians Curr remarks : 'I am of opinion that the Australian females bear on an average six children, or did before the advent of the whites and whilst living in their natural state.' 1 Spencer and Gillen state that sterility is common among the Australians ${ }^{2}$ and that the number of children rarely exceeds four or five and is generally two or three. ${ }^{3}$ Of the Eskimos we read : ' the women are not prolific. Although all the adults are or have been married, many of them are childless, and few have more than two children. One woman was known to have had at least four. Dr. Simpson heard of a "rare case" where one woman had borne seven children.' 4 'On the average the pure breed Greenlanders are not prolific. Two, three, or four children to each marriage is the general rule, though there are instances of families of six or eight or even more.' 5 Of the American Indians there is a large amount of evidence. Dr. Holder, who combined medical knowledge with exceptional opportunities for observation, says : 'With Indians large families are the exception. The Crow tribe, of less than 2,500 people, is divided into 630 families, which gives less than four to each family, and this includes parents and often grand-parents and relatives by affinity or adoption, leaving the average offspring to each child-bearing woman decidedly lower than in white communities.' 6 Speaking of the Indians of Vancouver Island, Sproat says : 'As a rule they have few children.' ? Bancroft reports of the Nootka tribe : 'Women rarely have more than two or three children.' 8 Of the Chinooks : 'Barrenness is common, the birth of twins rare, and families do not usually exceed two children.' 9 Catlin says : 'It is a very rare occurrence for an Indian woman to be "blessed" with more than four or five children during her life; generally they are contented with two or three.' 10

It is merely suggested here that evidence of this kind, which will be much amplified in the following chapters, may have to be interpreted as pointing to a lower degree of fecundity among these

1 Curr, The Australian Race, p. 70.

2 Spencer and Gillen, Native Tribes of Central Australia, p. 52.

${ }^{3}$ Ibid., p. 264.

4 Murdoch, Ethnological Results of the Port Barrow Expedition, 9th A.R.B.E., p. 38. Eskimo Life, p. 150.

6 . $\checkmark$ Sproat, Scenes and Studies of Savage Life, p. 94. Races of the Pacific States, vol. i, p. 197. North American Indians, vol. ii, p. 228.

G Bancroft, Native - Ibid., p. 242. 10 Catlin, 
lower races than among the civilized races. The trend of the evidence is at least suggestive when it is remembered that according to the estimate made by Matthews Duncan a normal woman among civilized races living in wedlock throughout the mature period under favourable circumstances should bear from ten to twelve children. ${ }^{1}$

If the view suggested is correct, we should expect to find in such countries as India and China a higher fecundity than among primitive races, but a lower fecundity than among European races. There is some indication that this is so. At first sight it might seem that the well-known fertility of these races indicated a higher fecundity than in Europe. When, however, in the case of India, not the crude fertility, but the fertility corrected for the number of married women of reproductive age is calculated, it is found to be lower than in Europe. The figures per 1,000 are 160 in India and 196 in England. ${ }^{2}$ It has, of course, to be remembered that there are in both countries certain factors bearing upon fertility-early marriage in India and restraint from intercourse and contraceptive methods in England. But it can hardly be supposed that the former is more effective than the latter in decreasing fecundity, and it is probable that we have here an indication of lower fecundity in India.

10. All the evidence, therefore, points to the same conclusion. If there has been any general change in the strength of human fecundity in the course of human history, using that phrase in the widest sense, it has been in the direction of an increase. It is not necessary for our present purpose to attempt to be more precise. This view is, as we shall show below, that of the best authorities. It may be noticed that different opinions are frequently expressed by authors who are not professional biologists. ${ }^{3}$ Such opinions are in most cases so vaguely worded that it is seldom clear what

1 Matthews Duncan, Fecundity, Fertility, and Sterility, p. 112.

2 Wattal, Population Problem in India, p. 7.

${ }^{3}$ See, for instance, Eucken, Currents of Modern Thought, p. 264. When Herbert Spencer says (Principles of Biology, vol. ii, p. 431) that 'advancing evolution must be accompanied by declining fertility ${ }^{2}$, he is apparently using the term fertility as equivalent to fecundity and is therefore misrepresenting the position. The Dean of St. Paul's is doubtless aware of the true state of the case ; never. theless his reference to the subject is likely to be misleading. After remarking upon the fact that fecundity (which he calls. fertility) decreases among animals with increasing care for the offspring, he goes on to say that ' man is no exception to these laws' (Outspoken Essays, p. 60). As a matter of fact, man is a remarkable exception to this 'law'; if anything, his fecundity has increased. His position has become assimilated to that of the animals because his fertility has decreasedquite another matter. 
precisely is meant. In many cases it is fairly evident that it is intended to imply that there is some connexion between growth of civilization and decrease in fecundity. So far as this is what is meant, there is no evidence for this view. There is no indication whatever that increasing intellectual activity is accompanied by decreasing fecundity. On the other hand, inasmuch as intellectual activity is connected with an amelioration in the conditions, to that degree there is a connexion between it and an increase in fecundity. Such views clearly owe their origin to the attention paid to the decline in the birth-rate. It is not always realized that a declining birth-rate may be due to a decline in fertility alone, wholly unconnected with a decline in fecundity.

That fecundity has increased was the opinion of Darwin. 'There is reason to suspect'; he says, 'that the reproductive power is actually less in barbarous than in civilized races. ... It is highly probable that savages, who often suffer much hardship and do not obtain so much nutritious food as civilized men, would be actually less prolific.' 1 According to Heape, 'it would seem highly probable that the reproductive power of man has increased with civilization, precisely as it may be increased in the lower animals by domestication; that the effect of a regular supply of good food, together with all the other stimulating factors available and exercised in modern civilized communities, has resulted in such great activity of the generative organs, and so great an increase in the supply of the reproductive elements, that conception in the healthy human female may be said to be possible almost at any time during the reproductive period.' 2

It is interesting to note that no differences in fecundity can be observed as between modern civilized races. ${ }^{3}$ The conclusion we have reached is merely that there has been, broadly speaking, an increase in fecundity, and that in large measure this is explicable as a result of the betterment of conditions, and there is nothing to lead us to expect that there would on this account be any difference between modern European races.

11. We may now pass to a brief consideration of certain habits, customs, and other factors which, operative from time to time, have, or are widely supposed to have, an influence upon fecundity,

1 Darwin, Descent of Man, p. 132.

2 Heape, loc. cit., p. 39. This is also the opinion of Havelock Ellis, see 'Studies in the Psychology of Sex', Analysis of the Sexual Impulse, p. 220. $\quad{ }^{3}$ Newsholme and Stevenson, J.R.S.S., vol. lix, p. 64. 
and in some cases upon fertility ; for though fertility is not, properly speaking, considered in this chapter, it is convenient to deal with certain matters here.

To polygamy has often been attributed a decrease in the number of children born to a woman. Whether it is supposed that the practice of polygamy in some way decreases fecundity or has an influence upon fertility is not clear. In any case there is no evidence that it has any influence either upon fecundity or upon fertility. There is no reason to think that polygamy is attended by any structural or physiological changes such as would influence fecundity, and the statistical evidence does not show any difference in the average number of children born, whether monogamy or polygamy is practised. Theal investigated this question as far as marriages among Bantu women are concerned. He collected a number of returns and sums up the returns as follows. - Altogether these returns embraced 393, the wives or widows of monogamists, mostly professing Christians, and 591 women, the wives or widows of polygamists. In a few instances it was noted that the women might not have passed the age of childbearing. The 393 women, wives of monogamists, had borne 2,223 children, that is on an average 5.65 children to a woman. The 591 women, wives of polygamists, had borne 3,298 children, that is on an average 5.58 children to each woman. Thus monogamy made hardly any appreciable difference in the birth-rate.' 1 The question of the influence of lactation on fecundity is of considerable importance, but unfortunately no very definite conclusions can be drawn from the facts known. There is a considerable amount of evidence to the effect that the continuance of lactation to some extent inhibits heat in animals and menstruation in women. Though the effect of continued lactation is doubtless in this direction, it cannot be said to be of any very definite strength. It is stated that mares giving suck are liable to miss a season. ' There can be no doubt that in the case of sows early weaning is conducive to a more frequent recurrence of oestrous and an increased number of litters.' 3 ' The return of menstruation during lactation in women has been dealt with recently by Heil and Dingwall Fordyce. Heil, who has studied the conditions of 200 nursing mothers, expresses the belief that

1 Theal, Yellow-and Dark-Skinned People of Africa, p. 348. loc. cit., p. $43 . \quad{ }^{3}$ Marshall, loc. cit., p. 400.

2 Heape, 
the recurrence of menstruation and not the condition of amenorrhoea is the normal state during lactation, but that menstruation is not so frequent in the later lactations as in the earlier ones. Fordyce has reached similar conclusions, finding that menstruation occurred during lactation in 40 per cent. of the cases in which suckling was performed, while in 92 per cent. of the cases its return was within nine months of parturition, and that menstruation during lactation was commoner with the earlier than with the later lactations, showing that age is an important factor.' 1

There is another question of importance. It may be asked what influence age at marriage has upon fecundity. As far as the age of the husband is concerned, there is scarcely any influence at all. For any given age of wife the fecundity remains nearly the same, whatever the age of the husband. The age of the wife, however, is of importance, apart from the fact that delay in marriage reduces the use made of the limited period of maturity. For a woman is not equally fecund throughout the mature period. The earlier years are the most fecund years, and therefore postponement of marriage reduces the fecundity, other things being equal, by more than the fraction of the mature period which is passed in celibacy. According to Dunlop's observations on marriage in Scotland ' the effect of one year's delay of marriage is to reduce the average family by fully one-third of a child, or that three years' delay may be expected to result in the family being one child less. This result may be fairly correct in general, but it cannot be strictly applied, for the crude observations show that the effect of one year's delay is not constant through the fertile period of the woman's life, but is greater for the younger and less for the later years. Thus a year's delay when the woman is aged from 20 to 25 averages 0.45 of a child, 0.37 when she is aged from 25 to $30,0.32$ when she is aged from 30 to $35,0.29$ when she is aged from 35 to 40 , and 0.19 when she is aged from 40 to $45 .^{2}$ It requires, it may be noticed, a delay of about forty years on the part of the husband to decrease the number of children by one child. ${ }^{3}$

A further problem is the influence of sexual intercourse before puberty upon fecundity. Exactly what influence early intercourse has upon the generative organs and their functions is not clear.

1 Marshall, loc. cit., p. 74. See also Heape, loc. cit., p. 43, and Matthews Duncan, Sterility in Women, p.85. $\quad 2$ Dunlop, J.R.S.S., vol. Ixxvi, p. 266. ${ }^{3}$ Ibid., p: 267. 
It is known, however, that early intercourse is injurious to the general health, and it is not difficult to understand in a general way how, if this is so, the reproductive functions would be adversely affected. In the Punjab Census Report 'it has been shown that the states which practise early marriage on an extensive scale have generally a smaller proportion of females at the age period 12 to 15. Inquiries into a large number of cases show that, when the marriage of young people is consummated at an early age, a fairly large number of wives dies of phthisis or some other disease of the respiratory organs or from some ovarian complication within ten years of the consummation of marriage.' 1 It is also known that when of two races both living a similar kind of life under similar conditions, one practises early marriage and the other does not, as for example the Hindus and Mohammedans in India, fertility is higher among the latter than among the former. ${ }^{2}$

Finally, it may be noted that the development of fat may lead to sterility. It is a fact well known to breeders that excessive fatness is accompanied by sterility. Animals that have been fattened for agricultural shows are often barren. There is no doubt that the development of fat may have the same effect in women. It is not quite clear why the development of fat should be inimical to fecundity; formerly it was supposed that the sperm was prevented from reaching the ovum owing to the presence of a mechanical obstruction. Although this may at times be the cause, it seems that the presence of an excessive amount of fat has a deleterious influence upon the metabolism of the organism and that the maturation of the ova must be in some way affected. Marshall found signs of abnormal ovarian metabolism in interstitial tissue of the ovaries of fat cows and heifers. ${ }^{3}$ Sterility, it may be remarked, is not to be regarded as an ultimate consequence of ' good ' conditions which have been made too ' good'. 'Good ' conditions do not merely consist in abundant food, but also in sufficient exercise and so on. The development of fat in such a degree as to cause sterility is due to an excessive amount of foodan abundant food-supply being one only of the factors going to make up ' good' conditions - to the exclusion of other factors.

1 Quoted by Wattal, loc. cit., p. 23. Duncan, Fecundity, Fertility, and Sterility, pp. $277 \mathrm{ff}$.

2 Ibid., p. 15. See also Matthews p. 505 , Marshall, loc. cit., p. 595. The corpus luteum persists in the ovary of fat animals and to this fact Fraenkel (Archiv für Gynaekologie, Bd. Ixviii, 1903) ascribes the barrenness that so often accompanies fatness. 
12. In conclusion, some calculations may be conveniently added to illustrate the strength of human fecundity, which, as we shall see, is constantly underestimated. Let us consider a population of a million born in the same year, half of whom are males and half females. Let us suppose that they all marry, each couple before the age of twenty producing two children, half of whom are girls and half boys. For the sake of simplicity we may imagine that at the end of each twenty-year period the parents die simultaneously with the birth of their offspring. Then, if the children marry and produce offspring as did their parents, we shall have a standard population of $1,000,000$, which will neither increase nor decrease so long as these conditions are fulfilled. If, however, the average number of children is $2 \frac{1}{2}$ per couple, then in 100 years the population will be $3,050,000$; if three, $7,954,000$; if four, $32,000,000$; if five, $97,650,000$. 


\section{VI \\ HUMAN HISTORY}

1. As a further preliminary to the separate inquiries into the two parts of the problein, we have to sketch the main outlines of history in its broadest aspect. The facts will in themselves form an important element in the subject-matter when we come in the second part of the book to discuss the qualitative problem. They also form, as explained in the third chapter, a framework into which such knowledge as we have of primitive races may be fitted with all the necessary qualifications and safeguards. The procedure will be in the first place to glance at the main subdivisions of the sedimentary deposits, and to ask how far they can be dated. In this fashion alone can we arrive at any chronological basis with regard to human evolution. We have then to refer to the evolution of the Primate branch of the mammals to which nan belongs, and afterwards to the evolution of the bodily form of man. As the fossils upon which the evidence is based are connected with certain geological strata, some vague indication of their date, or at least of their relative appearance in time, can be arrived at, in any case with regard to the later forms. We can then refer to the evidence of the cultural remains of man and correlate this evidence with that derived from the fossil remains. In this fashion some indication can be obtained of the evolution of culture and of the physical form of man anterior to the last three or four thousand years. For the last period written records are available, and supplement the evidence derived from cultural remains. Finally we have to discuss the manner in which we can use the evidence derived from primitive races to fill in the gaps in our knowledge.

2. The deposition of the sedimentary strata is usually divided into four main periods, each of which is subdivided into smaller eras. The following table shows the divisions most commonly adopted. 


Cainozoic $\begin{cases}\text { Quaternary } & \left\{\begin{array}{l}\text { Recent } \\ \text { Pleistocene }\end{array}\right. \\ \text { Tertiary } & \left\{\begin{array}{l}\text { Pliocene } \\ \text { Miocene } \\ \text { Oligocene } \\ \text { Eocene }\end{array}\right. \\ \text { Secondary } & \left\{\begin{array}{l}\text { Cretaceous } \\ \text { Jurassic } \\ \text { Triassic }\end{array}\right. \\ \text { Primary } & \left\{\begin{array}{l}\text { Permian } \\ \text { Carboniferous } \\ \text { Devonian } \\ \text { Silurian } \\ \text { Cambrian } \\ \text { Precambrian }\end{array}\right. \\ \text { Archaeozoic } & \end{cases}$

It is with the Cainozoic period that we are alone concerned, and chiefly with the Pleistocene and Recent subdivisions of that period. Before we consider the Quaternary epoch, on $\theta$ or two facts with regard to the dating of the other periods may be mentioned. However long it is supposed that the deposition of the sedimentary deposits has occupied, it is usually held that the Archaeozoic period occupied at least half of the whole length of time. This supposition is rendered necessary by the fact that already in the Cambrian era organisms of a high degree of complexity are found; thus in this era Crustaceans, Brachiopods, and Worms are common; Echinoderms, Coelenterates, and Sponges are also known. In the Silurian most of the classes of the animal kingdom are represented, the exceptions being amphibians, reptiles, birds, and mammals. The presence of fish shows that by this time vertebrates had already been evolved. Amphibians first appear in the Carboniferous and reptiles in the Permian. Birds first occur in the Upper Jurassic and mammals towards the close of the Triassic. The estimates as to the time occupied by the deposition of all these strata taken together vary very greatly-the average being about $100,000,000$ years, though it should be mentioned that as long a period as $1,000,000,000$ years has been proposed. ${ }^{1}$ With regard to the length of time

\footnotetext{
1 Jolly, Science Progress, 1914, No. 33, p. 41, states that there are four methods of estimating the duration of the whole period, by considering (1) the thickness of the sediment, (2) the mass of the sediment, (3) the sodium contained in the sea, and (4) radioactive transformation. (1) gives about 100 to 134 million years, (2) about 87 million years, (3) about 80 to 90 million years, and (4) a much longer period.
} 
which it is supposed that the later subdivisions of the Cainozoic period have covered, it may be noticed that Penck estimates the length of the Pliocene and Miocene periods at about two and three million years respectively.

It is the dating of the subdivisions of the Pleistocene epoch that is of interest here. This era is also known as the glacial epoch. During this period large areas both in the northern and southern hemispheres were covered by the formation of glaciers or by the advance of previously-existing glaciers. It is now almost universally admitted that in Europe at least there were four separate advances of the ice. After reaching on each occasion a point of maximum extension, the ice retreated, and there were thus three inter-glacial or genial epochs, while the period since the fourth glaciation is known as the Recent period. The limit of the extension of the ice varied in each glaciation, and was at its maximum in the second glaciation. These separate glaciations and intervening genial periods serve to subdivide the Pleistocene era, and when fossil and cultural remains are discovered an attempt is made to ascertain in which of these subdivisions they occur.

Nothing more than guesses can be made as to the length of the glacial period as a whole and of the subdivisions, and these guesses differ very widely. Osborn gives the following list of estimates : ${ }^{1}$

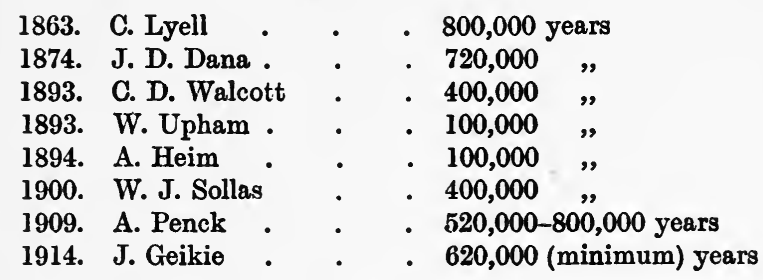

Osborn adopts the more conservative estimate of Penck for the duration of the whole period, and gives a subdivision of the period, which is as follows : ${ }^{2}$

1 Osborn, Old Stone Age, p. 22.

2 Ibid., p. 23. It is well to lay stress on the fact that all these figures are little more than guesses founded on various observations such as the rate of deposition of sediment by rivers. No conclusions with regard to evolution can be drawn from the supposed length of the whole period or from the supposed length of the subdivisions. Nevertheless the relative length of the different subdivisions as estimated has in all probability some approximation to fact, and thus, whatever may be the real length of the period, the time which elapsed between, say, the beginning of the Pleistocene and the middle of the third genial epoch was at least twice and perhaps three times longer than that whioh has elapsed since the beginning of the latter epoch to the present day. 
Period.

Pleistocene $\left\{\begin{array}{l}\text { Recent } \\ \text { Fourth Glacial } \\ \text { Third Genial } \\ \text { Third Glacial } \\ \text { Second Genial } \\ \text { Second Glacial } \\ \text { First Genial } \\ \text { First Glacial }\end{array}\right.$
Relative Duration.

25,000

25,000

100,000

25,000

200,000

25,000

75,000

25,000
Grand Totals.

25,000

50,000

150,000

175,000

375,000

400,000

475,000

500,000

3. With man are associated among marrumals more or less closely five groups of animals - the Anthropoid apes, the Platyrrhini or Old World monkeys, the Catarrhini or New World monkeys, the Lemurs, and the Tarsii. It is clear that all these groups diverged from a common stem; the first divergence, however, nust have occurred not later than the Eocene. To ascertain the interrelationship of these groups and the order of their divergence we have to rely chiefly upon the evidence provided by fossils. This evidence is very incomplete and, so far as the definitely pre-human ancestor is concerned, is lacking entirely until we come to a form known as Pithecanthropus, found in Java in a deposit attributed either to the late Pliocene or early Pleistocene. With regard to the evolution of the Primate stock it is usual to assume that the Lemurs ${ }^{1}$ and the Tarsii branched off from the main stem very early, and that the Catarrhini followed by the Platyrrhini branched off in the Eocene. It is usual, therefore, to assume that in Oligocene times there was existing a Primate stock ancestral both to the anthropoid apes and to man. It is possible that Propliopithecus from the Fayum is a representative of this common stock from which the gibbons branched off in the Miocene in one direction, and the orang, chimpanzee, gorilla, and man in another. Man, it is imagined, branched off from this latter stock in the early Miocene and the orang shortly afterwards, the separation of the chimpanzee and gorilla occurring somewhat later. Various ancestral gibbons (Pliopithecus and Pliohylobates) and ancestral anthropoids (Dryopithecus, Neopithecus, and Palaeopithecus) are known from the Miocene and Pliocene, but nothing has come to light as yet of the distinctively pre-human stock in these times.

1 The extinct Lemurs of the Lower Eocene were, it may be noticed, of a very generalized type and can only with difficulty be distinguished from Insectivores. At this time the chief features now characteristic of the various Mammalian classes had not yet appeared. 
Apart from the question as to how the distinctively human stock is related to the rest of the primate stock, there is much to be said for the view that the pre-human ancestor of Miocene times was a small arboreal primate which probably lived in a restricted area. But of the mode of life we have of course no direct knowledge. It is possible, however, that the mode of life of the anthropoid apes may give some indication of the con ditions under which the pre-human ancestor lived. That they are all confined to warm climates in the Old World is suggestive when it is remembered that Pithecanthropus was found in Java. They are all more or less arboreal. The orang seldorn comes to the ground; the chimpanzee, though more arboreal than the gorilla, is less so than the orang, while the gorilla is the least arboreal of all. The first two species construct nests in the trees, and the same is asserted of the gorilla, though this appears to be doubtful. They all adopt in varying degrees a semi-erect attitude from time to time. They exhibit a certain development of the social instinct; the gorilla is seen in bands, the gibbons congregate in the evening in groups. It seems certain that marriage in the sense in which Westermarck employs the term exists among them ; it is asserted that they are all polygamous, though the chimpanzee may be monogamous. In any case they are not promiscuous in their sexual relations.

If a guess is to be made at all as to the mode of life of the pre-human ancestor, it is most reasonable to assume that it was something after the same kind. But whereas the apes are powerful specialized animals, the pre-human ancestor must have been a weaker and more generalized primate, having specialized only in the increase of the brain. It was this character which undoubtedly enabled him to maintain himself, and which compensated for the relative absence of other means of defence. The apes, on the other hand, are well able to look after themselves. 'The orang, as Selenka informs us, is more than a match for the dangerous carnivora with which he has to contend, and the gorilla is monarch of the woods.' 1

When we survey the T'ertiary period as a whole we see that there was a rapid and varied evolution of the mammalian stock in Eocene and Oligocene times which culminated in the Miocene.

1 Sollas, 'Presidential Address to the Geological Society', 1910, p. 48 (Quart Journ. Geol. Soc., vol, lxvi). 
All classes of mammals were in process of rapid evolution. After this epoch of evolution, specialization, and adaptation, the way was opened to the taking of the lead by a species which exceeded others rather in intelligence than in bodily superiority. The prehuman ancestor, we must suppose, had somewhat fallen behind in this contest to achieve adaptation by means of specialization, and could not then have taken a prominent place among mammalian species. Nevertheless, somehow he managed to maintain himself, and ultimately, as we shall point out later on, the retention of a generalized bodily form became of a very distinct advantage in that it enabled the best use to be made of his growing intellect.

4. The fossil remains of man from the Pleistocene are few and incomplete. It is a matter of great interest to observe in what subdivision of the Pleistocene these fossils occur, in which glacial or genial epoch, that is to say, they are found, as in this manner some indication can be gained, if not of their absolute, then of their relative, distance from the present epoch in time. As already mentioned, abundant evidence of human cultural remains are also found in the Pleistocene, and these remains likewise can be associated with the subdivisions of the period. Various names have been given to these cultural periods, and we shall glance later at the evidence upon which these periods are based. But it will be of assistance now to give a table showing how the cultural periods are connected with the subdivisions of the epoch, as it will thus be apparent how the fossil remains stand in relation not only to the glacial and genial periods, but also to the cultural periods. ${ }^{1}$

With regard to this table, except in respect to epochs of the later post-glacial period, the dates are only intended to give an idea of relative distance in time. ${ }^{2}$ Some approximation to accuracy can be obtained for the Neolithic, Bronze, and Iron ages. In the case of the latter two epochs, different dates have been given for the beginning of the period in the Orient and in Europe. The remaining dates refer to Europe only. As many cultures, so far as can be deduced from the evidence, came from the Orient, the beginnings of such culture periods in the Orient are doubtless to be dated some considerable time before they first appeared in

1 The table is based on those given by Osborn (Old Stone Age, pp. 18 and 41).

2 To show how the estimates vary it may be mentioned that Sollas (Ancient Hunters, ch. xiv) only allows 27,000 years since the close of the Chellean period. 
Europe. The names of the cultural periods, it may be remarked, are taken from the places where either specimens of the culture were first found, or where they are seen at their best. Thus Chellean is derived from Chelles-a palaeolithic station close to Paris-Acheulean from St. Acheul in the valley of the Somme, Mousterian from Le Moustier on the right bank of the Vézère, and so on.

Period.

Date.

Culture.

Racial Type.

\begin{tabular}{|c|c|c|c|c|c|}
\hline Period. & Date. & & Culture. & & Racial Type. \\
\hline • & 1,000 & B.c. & $\dot{\text { Iron }} \cdot \cdot$ & $\{$ Europe & \\
\hline & 1,800 & , & • $\cdot$ • & Orient & \\
\hline & 2,000 & , & $\dot{\text { Bronze }}$ & Europe & \\
\hline - & 4,000 & ", & $\cdot \quad \cdot$ & lorient & \\
\hline Post-Glacial & 5,000 & , & $\therefore \quad \therefore$ & Copper & \\
\hline & 7,000 & , & Neolithic & Swiss Lake & - Modern racial types. \\
\hline & 10,000 & " & . $\cdot$ & (Early & \\
\hline & $\begin{array}{l}12,000 \\
16,000 \\
20,000\end{array}$ & ", & $\begin{array}{l}\text { Upper } \\
\text { Palaeolithic }\end{array}$ & $\begin{array}{l}\text { Azilian } \\
\text { Magdalenian } \\
\text { Solutrian }\end{array}$ & . Brünn and other races. \\
\hline & 25,000 & ", & $\cdot$. & Aurignacian & $\begin{array}{l}\text { Cro-Magnon and } \\
\text { Grimaldi. }\end{array}$ \\
\hline Fourth Glacial & 50,000 & $"$ & $\begin{array}{l}\text { Middle } \\
\text { Palaeolithic }\end{array}$ & Mousterian & . H. neanderthalensis. \\
\hline Third Genial & 150,000 & ", & $\begin{array}{l}\text { Lower } \\
\text { Palaeolithi }\end{array}$ & $\left\{\begin{array}{l}\text { Acheulean } \\
\text { Chellean }\end{array}\right.$ & \\
\hline Third Glacial & 175,000 & " & & (Pre-Chellcan & . Eoanthropus \\
\hline Second Genial & $\mathbf{3 7 5 , 0 0 0}$ & , & - & - & - H. heidelbergensis. \\
\hline Second Glacial & 400,000 & ", & & & \\
\hline First Genial & 475,000 & , & & & \\
\hline First Glacial & 500,000 & ", & & & \\
\hline $\begin{array}{l}\text { Beginning of } \\
\text { Pleistocene }\end{array}$ & $\mathbf{5 2 5 , 0 0 0}$ & . & & & \\
\hline & & " & & & \\
\hline
\end{tabular}

5. Turning to the fossil remains of man we have first to deal with Pithecanthropus. In September 1891 Dr. Eugene Dubois of Amsterdam discovered at Trinil in Java certain fossil remains ; he continued to excavate for some two years, and succeeded in finding other remains, all of which he attributed to the same individual. To this individual he gave the name of Pithecanthropus erectus. ${ }^{1}$ Dubois considered that the strata in which

1 Dubois, Pithecanthropus erectus : eine menschenähnliche Übergangsform aus Java. Dubois has published several other papers and the literature is very large. The earlier literature has been summarized by Klaatsch (Zoologisches Centralblatt, vol. vi, 1899, p. 217), and by Schwalbe (Zeit. für Morph. und Anth., Bd. 1, 1899, p. 16). For a concise account see Duckworth, Morphology and Anthropology, pp. $510 \mathrm{ff}$. The remains consist of the upper portion of a skull, a left femur, a second left upper molar, a third right upper molar and a second left lower pre-molar. The last tooth was not found by Dubois but by subsequent excavators. 
Pithecanthropus was found belonged to the Pliocene. Very great interest naturally attaches to this question; Dubois's view has been challenged: subsequent visitors to Trinil have very carefully considered the matter, and the weight of scientific opinion now favours the attribution of the strata to the early Pleistocene. ${ }^{1}$

The scantiness of the remains renders a reconstruction of the individual a difficult and doubtful matter. Nevértheless, after some twenty years of discussion there is a very general agreement that Pithecanthropus was in many respects intermediate in type between modern man and the hypothetical pre-human ancestor. The bones of the cranium are fused, the brow ridges massive, and there is a marked narrowing behind the orbit-all ape-like features. Further the cranium is flattened somewhat as among the apes, but not to so great a degree; the altitudinal index is 34.2 ; of the average European it is 52 ; of Neanderthal man 40.4. The cranial capacity is about 855 c.c., or 250 c.c. greater than the largest known skull of any of the Simiidae, whereas the average cranial capacity of the Australian, the lowest living race; is about 1190 c.c. The femur has only a slight curvature, and is decidedly human, indicating that its possessor was some 1650$1700 \mathrm{~mm}$. in height, and probably walked upright. The teeth are simian rather than human, the roots diverge and the crowns are large ; they exhibit, nevertheless, certain human features.

If we atternpt to reconstruct Pithecanthropus, we must picture a creature half ape, half man, which was probably terrestrial and erect. His body weight must have been about 70 kilograms. If this fact is correct, it provides a very useful method of estimating the relation of Pithecanthropus to the apes on one hand and to man on the other. We know roughly what proportion brain weight bears to body weight. If Pithecanthropus had been human-given the brain weight deduced from the cranial capacity - the body weight should be 19 kilograms. If Pithecanthropus had been simian, the body weight should be 230 kilcgrams. We have in fact reason to think that it was about 70 kilograms,

The skull and the femur were not found together but some fifty feet apart. The attribution of all the remains to one individual is, however, probably justified.

1 Elbert (Cent. für Min., Geol. und Pal., Bd. 17, 1909, p. 513) holds that the beds are on the border between the Pliocene and the Pleistocene. Volz (Neues Jahr. für Min., Geol. und Pal. ; Festband zur Feier des 100-jährigen Bestehens, 1907, p. 256) places the beds in the Middle Pleistocene. The whole problem is discussed by Selenka and Blanckenhorn. See Die Pithecanthropus-Schichten auf Java. 
which emphasizes the intermediate position of Dubois's famous discovery.

It is further probable that Pithecanthropus had free use of the arms, the legs having become specialized for progression. It is quite possible that he may have employed tools of wood or of stone, though there is no evidence to this effect. It is further possible that some rudimentary form of speech may have been employed. The motor centre for speech is found in a particular area of the brain known as Broca's area. This area can be identified in the brain cast of Pithecanthropus, and it is stated to be twice as great as in the apes, but only half as large as in man. ' The undeveloped forehead of Pithecanthropus and the diminutive frontal area of the brain indicate that the Trinil race had a limited faculty of profiting by experience and accumulated tradition, for in this prefrontal area of the brain are located the powers of attention and of control of the activities of all other parts of the brain. In the brain of the ape the sensory areas of touch, taste, and vision predominate, and these are well developed in Pithecanthropus. The central area of the brain, which is the storehouse of the memory of action and the foelings associated with them, is also well developed, but the prefrontal area, which is the seat of the faculty of profiting by experience or of recalling the consequences of previous responses to experience, is developed to a very limited degree.' 1

Pithecanthropus is thus a form of the highest interest in the history of man. Provisionally, it may be put at the dawn of the Pleistocene. Uncertainty also surrounds the position of the next human remains to be mentioned, which consist of a lower jaw from the Mauer Sands near Heidelberg. ${ }^{2}$ It is generally agreed that these sands were deposited in a genial epoch, and opinion now inclines to associate them with the second genial epoch. The jaw itself is very simian, scarcely showing any approach to human characters. The teeth, however, are remarkably human, and it is said that in some respects the teeth of existing primitive races approach the simian condition more nearly than do the teeth of the Heidelberg race. The relation of this form to Pithecanthropus and to those which follow may be left for consideration until we have completed our sketch of human fossil types.

1 Osborn, Old Stone Age, p. 83. H. heidelbergensis.

2 Schoetensack, Der Unterkiefer des 
There remains a third early form to be mentioned-Eoanthropus dawsoni from Piltdown near Lewis. ${ }^{1}$ The gravel in which Eoanthropus was found was certainly deposited in a warm period. It probably dates from either the second or the third genial epoch, but great uncertainty attaches to the correlation of these gravels with the phases of the glacial period. Some implements found near the bones have been referred to the pre-Chellean culture period, which would indicate the third genial epoch. The mammalian fauna seems also to favour the reference of these strata to the same period.

The remains of Eoanthropus consist of the greater part of the brain case and part of the right mandible with the first and second molars in sitı. The canine tooth was found later. The skull has been reconstructed from several fragments. There has been much controversy as to the correct method of performing this reconstruction. The first estimate of the cranial capacity put it at about 1,100 c.c. Woodward has later revised his estimate, and arrived at a figure approaching 1,300 c.c. Keith has suggested a higher figure. The bones of the skull are very thick, brow ridges are almost absent, and the forehead is very steep. The jaw, on the other hand, is even more ape-like than the jaw of Heidelberg man; it slopes away with scarcely any vestige of a chin, the pre-molar series of teeth is parallel, and the molars do not decrease in size backwards. It is owing to the simian features of the jaw that the authors felt justified in creating a new species for these remains. Although the absence of brow ridges and the presence of a steep forehead are characters which mark the higher human types, they are also found among all young apes. It is thus possible that Eoanthropus may represent in the general form of the skull the missing Miocene and Pliocene ancestor more nearly than Pithecanthropus. The brain, though very primitive, was decidedly human; there was a moderate development of those areas connected with speech.

6. It is a matter of doubt to what subdivisions of the Pleistocene the types hitherto mentioned should be assigned and with what cultures, if any, they should be connected. At this point we come to a region of less uncertainty. Associated with Mousterian implements and with the deposits of the fourth glacial period we find a distinct type of man whom we may call Mousterian or

${ }^{1}$ Dawson and Woodward, Quart. Jour. Geol. Soc., vol. lxix, 1913, p. 117. 
Neanderthal ; for it was in the Neanderthal near Düsseldorf that the first specimen of this race was discovered in $1856 .^{1}$ Since that date several other examples of this type have been found. Some of the specimens have been preserved almost complete, and Professor Boule has been able to make a detailed study of the skeleton from Ia Chapelle-aux-Saints. ${ }^{2}$ In the course of his memoir he institutes a comparison between this and other examples of Neanderthal man, and shows that they all agree in the possession of certain features of importance. Neanderthal man was small but massive ; the facial portion of the head was much developed in comparison with the cranial; the head was low and narrow, the forehead receding, the occiput protruding, the orbits large, and the brow ridges heavy. The lower jaw was strong, the chin rudimentary, and the hinder molar teeth primitive. The vertebral column indicates a less upright posture than that of modern man. With regard to one feature - the cranial capacity - there is great variability. The cranial capacity of the Gibraltar skull is only 1,296 c.c., whilst that of the Chapelle-aux-Saints specimen is 1,620 c.c. According to Boule, however, this variation is no greater than that found among modern European skulls. $\mathrm{He}$ makes out the average capacity of Neanderthal man as 1,400 c.c. ; this estimate is far lower than that usually given; it is often said that the average capacity approaches 1,600 c.c.

The Neanderthal race forms a very distinct type. It is found only in the Middle Palaeolithic period. ${ }^{3}$ Of its origin there will be something to be said later; it appears that it died out before the Upper Palaeolithic age began, for, as we shall see, apparently none of the types which occurs later descended from this race.

7. The Upper Palaeolithic culture is associated with the end of the fourth glacial epoch. In place of the remains of Neanderthal man we find the remains of several types, none of which shows any affinity to Neanderthal man. On the contrary they all approximate more or less closely to modern man. At one bound we seem, when looking at the fossil remains from this epoch in

1 The Gibraltar skull may have been discorered before the specimen from Neanderthal; it aroused no interest, however, until after the discovery of the latter. $\quad 2$ Boule, Ann. de Pal., vol. vi, 1911, vol. vii, 1912, vol. viii, 1913.

${ }^{3}$ In some cases the origin has not been clearly identified (e. g. the Gibraltar skull). It is said that remains of Neanderthal man are sometimes found associated with Acheulean instruments (in the case of the Krapina remains, for example). See Boule, loc. cit., vol. vi, p. 120, and vol. vii, p. 227, and also Anthony, Bull. et Mém. Soc. d'Anth., 1913, p. 189. 
Europe, to have passed into the modern period so far as human bodily form is concerned. As we shall see later, it is probable that the various types entered Europe from the East, and probably originated somewhere in Western Asia. The most important of these types is known as Cro-Magnon man. It is found throughout the Late Palaeolithic period. The first specimen was found at Cro-Magnon in the valley of the Vézère. Other specimens have been found in the Grotte de Grimaldi and assigned to the beginning of the Aurignacian, the first subdivision of the Upper Palaeolithic, and others again at Obercassel, and assigned to the Magdalenian, or last subdivision of the Upper Palaeolithic. In modern France in the region of Périgueux there is existing at the present day a type which in so many features resembles Cro-Magnon man that it may be a survival from Upper Palaeolithic times.

The general features of the chief types of the Upper Palaeolithic may be very briefly indicated. The skull of Cro-Magnon man was narrow, the face low and broad; the brow ridges project slightly ; the orbits are low and rectangular; the nose is long and narrow and the root broad. Cro-Magnon man was tall, approaching an average of $6 \mathrm{ft}$. in height ; the cranial capacity was large, being somewhere about 1,600 c.c. ${ }^{1}$

In the Grotte de Grimaldi were also found two skeletons of what has been called the Grimaldi race. No other skeletons of this type have been found in Europe. There are two points to notice about this type; first it is not relatively spoaking a low type; the cranial capacity was, for instance, 1,580 c.c.; secondly, it shows undoubted resemblance to the Negroid type. The skull is narrow, the face large and low, and prognathism is marked. The orbits are large and of low vertical height, the nose broad and flat.

The third type is that found at Brünn and again at Brux in connexion with Solutrian culture (middle of Upper Palaeolithic). The skull is very long and generally of a lower type than the Cro-Magnon skull. In certain respects there is a slight resem-

1 The remains found at the station of Raymonden, Chancelade, and described by Testut (Bull. Soc. d'Anth. de Lyon, vol. viii, 1889), are now generally classed with Cro-Magnon man. As Testut says, Chancelade man possesses 'tous les caractères propres aux races supérieures'. The cranial capacity was 1,710 c.c. But there are certain features in which it departs from the ordinary Cro-Magnon type and certain features in which it resembles the modern Eskimo typeparticularly in the shape of the cranium, the sides of which tend to slope up and meet at an angle. The possible significance of this will be referred to later. 
blance to the Neanderthal type. Finally from deposits at the close of the late Palaeolithic period at Offnet two further types are known. One of them is a long-headed race not unlike the Mediterranean type of European at the present day. The other is a broad-headed race, which is not unlike the Alpine type of modern European.

In a general sketch further details need not be added. There is no essential difference between the physical type existing in Neolithic times and that of modern man. Leaving the discussion of the inter-relationship and origin of these types until after we have spoken of the evolution of culture, what we have found so far is that in the beginning of the Pleistocene and in the early Palaeolithic there are three primitive types of man known, and that in the Middle Palaeolithic there is a peculiar type with many primitive features, whereas in the Upper Palaeolithic we are in the presence of types which for the most part differ but little from modern races, and even show affinity to certain modern races in particular cases. ${ }^{1}$

8. We have now to give some account of the evolution of culture as shown by the implements and other remains left by primitive man. The story of the first identification of stone implements by Boucher de Perthes is well known. Gradually a succession of periods has been recognized, and the names given to them have already been introduced in the preceding pages. Of the two main periods-Palaeolithic and Neolithic-the first is distinguished by the facts that stone implements were not polished, agriculture was not practised, pottery was not made, ${ }^{2}$ and animals were not domesticated. Within the Palaeolithic period several successive types of culture can be distinguished, but before we deal with them something must be said regarding the so-called Eolithic period.

There are found in many places stones supposed to exhibit a very primitive form of human workmanship. To them the name of eoliths has been given. They have been described from the

1 Thus Boule (loc. cit., vol. viii, p. 111), speaking of the Upper Palaeolithic types in general, says: 'Tous ces hommes fossiles ne sont pas plus différents des hommes actuels que ceux-ci ne diffèrent entre eux.'

2 Pottery stated to be Palaeolithic has been found in Belgium. There is nothing surprising in the fact, if it is a fact, that Palaeolithic man should have possessed this art, so far as his abilities are concerned. In general, however, nomadic races (and the Palaeolithic races were, of course, nomadic) have little use for pottery. See Déchelette, Manuel d'Archéologie, vol. i, pp. $169 \mathrm{ff}$. 
Oligocene, Miocene, and Pliocene. Many archaeologists believe that they are the remains of a primitive form of culture which preceded the Palaeolithic form of culture. The matter is still under debate.

We may note that eoliths exhibit very little variation. The same types appear from the Oligocene onwards, and if there was an eolithic culture it must have lasted some millions of years and been accompanied by little or no progress in the fashioning of implements. An exception must be made in the case of the socalled 'rostro-carinate' implements described by Mr. Reid Moir, and attributed to the late Pliocene. ${ }^{1}$ These stones are of a distinctive type, and apparently do not occur in the Oligocene and the Miocene. ${ }^{2}$ There are several considerations which make it difficult to accept eoliths as genuine remains of an early culture; some of these considerations do not apply to the rostro-carinate type, and some apply with less force. (a) Breuil has pointed out that as good eoliths occur in the Eocene as in the later strata. ${ }^{3}$ Apart from this, the occurrence of eoliths in the Oligocene, taken together with the immense length of the period during which no progress in skill was made, is almost incompatible with what we must suppose to have been the course of human evolution. The various classes of mammals were in rapid evolution at this time, and it is difficult to understand how, before this period of mamimalian expansion, man alone could have evolved to something akin to the type of the early Pleistocene, and then for so long a period have failed to progress. ${ }^{4}$ If indeed Propliopithecus,

1 Reid Moir, Pre-Palaeolithic Man. Rostro-carinates are found in the Red Crag which is a Pliocene formation. Mr. Reid Moir holds that they were evolved from eoliths (p. 66). L'Anthropologie, vol. xx, 1910.

2 Sollas, Ancient Hunters, ch. iii.

3 Breuil,

- Professor Wood Jones, basing his conclusions on comparative anatomy, has lately put forward a view of the course of human evolution somewhat at variance with that outlined above. He sums up his view by saying that 'Homo is not descended from the anthropoid apes preceded by a series of Primate forms represented by Old World Monkeys, New World Monkeys. and Lemurs. For we have seen that the anatomical characters of man demand rather a recognition of the finding that his stock branched off from the very root of the Primates; that man has evolved entirely by generalized development of the brain, and that he retains the bodily simplicity only found in some such far distant progenitor as the Tarsius stock; that no matter what may be the relation of the New World and Old World Monkeys, the human race combines, in some instances, a blend of their characters ; that the anthropoid apes retain a certain, and a varying, amount of the basal simplicity that belongs to man, but that the old World Monkeys have specialized far away from this simplicity. Regarded in this way we may say that the line of Homo springs from the base of the (non-Lemurine) Primate stem and not from its systematic apex' ('Origin of Man', p. 126, in Animal Life and Human Progress, edited by Dendy). In this view therefore the human 
or any similar form in the Oligocene, is a type from or near the line of human ascent, then we cannot allow Oligocene eoliths to be genuine artefacts, and there is no reason why, if we reject Oligocene eoliths, we should consider Miocene or Pliocene eoliths to be genuine. (b) Numerous observations have been made showing that stones similar to eoliths are formed under natural conditions of pressure, wave action, and so on. It is of particular interest to note that even the rostro-carinate type can be thus formed. ${ }^{1}$ (c) Upon the whole eoliths are only found where other stones of the same composition are found, whereas palaeoliths may be found anywhere-the deduction, of course, being that eoliths, inasmuch as they are formed under natural agencies, remain associated with other stones of the same composition whereas palaeoliths were widely distributed by those who used them. The question of eoliths is very far from being settled. Lest it be thought that the difficulties in the way of accepting eoliths as genuine have been exaggerated owing to some bias, it may be mentioned that the chief deduction we shall draw from human history as a whole would be considerably strengthened if eoliths could be regarded as genuine artefacts. If we cannot accept eoliths as without question genuine, it does not mean that before the Palaeolithic period man did not make and use stone implements. Undoubtedly he did so. But it may be from the nature of the case that it is impossible to recognize these instruments. ${ }^{2}$

9. In a warm period of the Pleistocene, now almost universally identified with the third genial epoch, oceur the first implements

stock has been separate from the rest of the primate stock since early in the Tertiary period. If this conclusion is well founded, the argument derived from the course of evolution as outlined above is certainly weakened.

1 Sollas, loc. cit., p. 72.

2 With reference to this subject Breuil says with great force that 'si la nature, exceptionnellement sans doute, peut produire des objets aussi semblables à des types industriels parfaitement définis et connus comme tels dans leurs milieux normaux en dehors de touts possibilité d'erreur, à combien plus forte raison doit-on se montrer circonspect à l'égard des manifestations les plus élémentaires de l'activité humaine, et se montrer exigeant avant de fonder sur leur constatation si problématique des théories dépassant si formidablement ce qui est acquis d'une manière définitive et en toute évidence !' (L'Anthropologie, vol. xxi, p. 407). For examples of the expression of similar views see Obermaier, Der Mensch der Vorzeit, p. 412, and Déchelette, loc. cit., vol. $i$, p. 22. The weight of expert opinion is against the acceptance of eoliths as genuine. Among the more distinguished supporters of the theory may be mentioned Rutot in Belgium, Ray Lankester and Reid Moir in England, Verworn in Germany, and de Mortillet in France. Among those who are sceptical are Boule, Breuil, and Déchelette in France, Schmidt and Obermaier in Germany, and Sollas in England. 
of undoubted human workmanship. Within this early Palaeolithic epoch three phases of culture are distinguished, the preChellean, Chellean, and Acheulean. It is worth while observing that M. Rutot, who is a foremost supporter of the eolithic theory, remarks, on arriving in a general review of the evidence at this pre-Chellean period, that there can be observed the 'apparition subite d'objets nouveaux'.$^{1}$ It is precisely these 'objets nouveaux' which many authorities consider as the first genuine artefacts, and not as mere improvements of a more primitive type of implement.

The typical instrument of the Chellean period was the hand axe-a stone shaped to fit the hand, and used for striking blows. ${ }^{2}$ Knives and scrapers were also common. In the Acheulean or latest period of the Palaeolithic there is seen a general improvement in workmanship. The hand axe is lighter and more carefully worked. There is a great variety of small implements, often showing a considerable degree of care and skill. For the first time there is in the Acheulean era evidence that man employed fire. It does not appear that bone was yet used for making implements. It can be shown that early Palaeolithic implements represent a steady and continual advance in skill with the improvement of old types and the development of new types.

During the late Acheulean period it was becoming colder, and the succeeding stage, or Middle Palaeolithic era, falls almost wholly within the fourth glacial period. Man began to make his home in caves, and we are well acquainted with the physical type under the name of Neanderthal man, described above. There are again traces of fire. Some kind of ceremonial burial was employed. A skeleton found at Ia Chapelle-aux-Saints showed that the body had been carefully disposed for burial; implements had been placed close to the body, one fine implement lying within reach of the left hand. There is a marked difference as regards the stone implements between this and the preceding period. A different method of shaping implements was employed. There is some inclination to regard the Mousterian stone industry as of a lower grade than the stage previously attained. With regard to this stage in general we may note that it is the first in

${ }^{1}$ Rutot, Revue de l'Université de Bruxelles, 1911, p. $258 . \quad 2$ Remains of Chellean industry are widespread and are found outside Europe-in Africa, for example, from the Cape to the Sahara. See Déchelette, loc. cit., vol. i, p. 88. 
which we have a detailed knowledge of the physical type and in which we have some indication of the stage of psychical development as shown by the employment of ceremonial burial.

This stage is sharply divided from that which follows it. So far as is known, Neanderthal man disappears at the end of the Middle Palaeolithic, and Mousterian industry is replaced by the Aurignacian, a wholly new type of culture, presumably introduced by some new racial element. As we have seen, there are several racial types in the Upper Palaeolithic, and the Cro-Magnon type, which occurs from the beginning of this period, may have brought the new culture into Europe. The art of stone implement making reached a height during the Aurignacian never previously approached; knives and scrapers were especially finely shaped. In the Solutrian, or middle period of the Upper Palaeolithic, stone implement making reached the highest point to which it attained in the Palaeolithic period. The workmanship exhibited in the well-known willow and laurel leaf lance-heads is very fine. Bone tools are first found in the Upper Palaeolithic. It is clear from the disposition of the skeletons that burials were again employed, and indicate, if not a belief in a future life in our meaning of the phrase, at least the presence of a body of tradition regarding the state of the deceased. It is not certain whether man at this stage possessed the bow and arrow. Nothing can, of course, be known with certainty regarding the degree to which perishable substances were manufactured; it can only be said that in all probability skins and furs were used as clothing, that basketwork was understood, and nets constructed. The most remarkable remains which have been preserved from this period are the drawings and paintings on the walls of caverns. They are found throughout the Upper Palaeolithic period, and indicate a high degree of skill and taste. ${ }^{1}$

That the Solutrian Age shows an improvement upon the Aurignacian from the point of view of stone implement making has been indicated. It also shows a similar improvement as regards bone implements ; bone needles with perforated eyes, for instance, occur for the first time. There is a distinct improvement in the drawings and paintings. In the next or Magdalenian period of

\footnotetext{
1 The admiration, justly enough aroused, for these works of art has undoubtedly led to an exaggeration of the level of social and intellectual development reached by Upper Palaeolithic man, whose times are sometimes referred to as though they were a kind of Golden Age.
} 
the Upper Palaeolithic there is a loss of skill so far as stone implements are concerned; there is no general decadence, however, as the making of implements from bone, horn, and ivory shows a considerable advance. Further, drawing and painting reached their highest development. The remains from this era are very abundant and well known, and exhibit a vast number of types of implements giving a general impression of a considerable degree of material wealth relatively to the fact that agriculture was not practised.

At the very end of the Upper Palaeolithic the Magdalenian culture is succeeded by a rather obscure stage known as the Azilian-Tardenoisian. There is again a sudden and very distinct break in the evolution of culture. In nearly all respects there is a failure to reach the previous level of skill. Both flint and bone implements are of inferior workmanship. The artistic spirit vanishes. Little is known about this culture; possibly man at this period was not so unskilled, as at first sight seems to have been the case, for he had apparently domesticated the dog. ${ }^{1}$

10. Neolithic culture is marked by the practice of agriculture, the domestication of animals, ${ }^{2}$ the polishing of stone implements, and the making of pottery. ${ }^{3}$ Though it is strongly contrasted with the preceding culture, there is considerable evidence of a transition period. This evidence, however, is generally interpreted as indicating the infiltration of the new culture from the East, and not the evolution of the new culture in Europe. Even at this relatively recent period in history the chronology is vague. The Swiss Lake Dwellings (Robehausian period) represent the typical fully developed European Neolithic culture, and are dated about 8000 to 7000 в. с. Farther East there is evidence of a far greater antiquity. Montelius on the basis of De Morgan's

1 Azilian remains are confined to the south of Europe; Tardenoisian remains are widely distributed in Europe. To this period also belongs the Maglemose culture from Zeeland. Some grain found in the cave of Mas d'Azil has been interpreted as showing that agriculture was practised; in all probability, however, the grain has been introduced recently-perhaps by rats. Certain curiously marked pebbles are known from Azilian times and have been taken by some authors to be evidence of the use of an alphabet. This interpretation cannot be accepted, though possibly these markings may have been signs intended to assist the memory - such as were in use in Peru, for example.

2 Palaeolithic man may have kept animals as pets. Many primitive races have a passion for keeping pets though they derive no economic advantage from so doing.

3 That agriculture is an art learnt but recently is not yet appreciated by some men of science. Thus Sir A. D. Hall in a book published in 1919 says that 'agriculture must be almost coeval with the human race' (Fertilizers and Manures, p. 2). 
observations at Susa calculated that the Neolithic Age began there about 20,000 years ago. ${ }^{1}$ Sir Arthur Evans estimates that at least 14,000 years have elapsed since the beginning of the Neolithic period at Cnossus. ${ }^{2}$ All that can be said is that the Neolithic culture was flourishing in the eighth millennium в. c. in many parts of Europe, having been brought from farther East, where it had originated at a considerably earlier date. It has proved very difficult to subdivide the Neolithic epoch into successive periods. In the Palaeolithic period superimposed deposits containing relics of various cultures are not infrequently found, thus enabling the succession of culture periods to be made out. In the Neolithic period evidence of this kind is for the most part lacking. It is not proposed to enter upon the subject here beyond saying that three stages are recognized by French archaeologists, the Campignienne, the Chasséo-Robenhaussienne, and the Carnacéenne. The first of these stages is markedly more primitive than the succeeding stages. The second is that of the Swiss Lake Dwellings, and the third that of Megalithic monuments-Dolmens, Menhirs, Cromlechs, Allées Couvertes, stone circles, and so on. ${ }^{3}$

What has been said regarding culture periods in generalnamely, that they are not definite periods in time in the history of mankind as a whole, but epochs through which different races have passed at different times-is well illustrated in the case of the Neolithic. The Neolithic culture had existed some thousands of years in Western Asia before it penetrated into Europe, and was practised in some parts of Europe, in Britain, for instance, up to about 2000 B. c. or later, for thousands of years after metals had been taken into use elsewhere. In general the Neolithic period has to be regarded as a period of great advance in material wealth. Relatively to Palaeolithic men, Neolithic men were rich, and often lived in villages of some size. Several animals were domesticated, several varieties of cereals cultivated, linen was manufactured, and the vine as well as a large number of fruittrees known. It appears that many at least of the well-known trading routes used in the Bronze Age were open in the Neolithic Age. Some of these routes brought the Baltic into communication with the Mediterranean, one began at Venice, passed over the

1 Montelius, C.I. A., 1906, vol. ii, p. 32.

2 Pottery from the earliest Neolithic at Cnossus was well made, and therefore this culture must have originated considerably more than 14,000 years ago. loc. cit., vol. i, p. 336.

${ }^{3}$ On this subject see Déchelette, 
Brenner, and reached the Danube by means of the Inn ; thence it traversed the Bohemian forest to the Moldau, and thus reached the Elbe, which was followed to its mouth. Another began at Trieste, and passing through Laibach and Grätz reached the Danube by means of the Leitha; thence it followed the March, traversed Moravia, passed through Silesia along the Oder, while the Vistula was reached by striking across Posen, the principal terminus being Danzig. ${ }^{1}$

11. Obscurity surrounds the location and date of the first use of metal. ${ }^{2}$ Gold was probably the first metal known to man; it is found in the pure state in many countries, and would attract attention owing to its lustre. It was not, however, of economic importance until much later. In all probability man learnt the art of metal working from the accidental reduction of ore that formed one of the ring of stones round the camp fire. Copper may have been first used where it appears at the surface in a pure state, having been beaten into implements, as was done by the Indians near Lake Superior; the use of this metal is, however, until heat is employed, to be regarded merely as a variation of the art of stone implement making, and does not form the first step in the art of metal working for which knowledge of reduction by heat is required. It is frequently said that an age of copper preceded the age of bronze, and in many places copper implements were used before bronze implements. Probably the occurrence of a Copper Age in any one place was dependent upon the nature of the ore found there. Copper is frequently associated with tin and other metals in a state of nature. We may suppose that the first metal workers were simply trying to get metal-being aware that metal could be obtained by heating certain kinds of stone-and that in some places they got copper and in other places bronze. Later they learnt to add a definite percentage of tin or some other metal, and thus to produce an alloy of definite composition. Thus a true Bronze Age followed either a Copper Age or an accidental Bronze Age. It is somewhat curious that iron was not taken sooner into use, for less heat is required than in the case of copper for reduction $-700^{\circ} \mathrm{C}$. to $800^{\circ} \mathrm{C}$. as against $1,100^{\circ} \mathrm{C}$.

1 Jade was found in the first city of Hissarlik, which must have come from the Kuen-lun (Gowland, J. A. I., vol. xlii, 1912, p. 260). see Gowland, loc. cit., p. 236.

${ }_{2}$ On this subject 
Whether there was more than one place of origin of the use of any metal in the Old World is not known. Copper was independently taken into use in the New World, and the use of copper may have originated independently in North and South America. ${ }^{1}$ Bronze was known in Peru. Heat was certainly employed for hammering and annealing copper, and casting may have been employed. Iron was sometimes used in North America to make ornaments. With regard to the Old World a claim is made for many countries as the original home of metal working. Reisner found copper daggers, spear heads, harpoons, pins, needles, and bracelets in the graves of the middle pre-Dynastic period of Egypt; by the time of the first dynasty the Egyptians were 'in possession of a full equipment of copper weapons '.2 In the early pre-Dynastic period metallic pigments containing copper were in use, and it is possible that the reduction of copper may have been first suggested by some accidental fusion of these substances. It is further claimed that there is evidence of the local evolution of copper implements since the earliest forms imitate stone implements, and the later forms are merely improvements of pre-existing types. $^{3}$ Reisner concludes that there is no reason to assume that the invention came from beyond the frontiers of Egypt. ${ }^{4}$ The Sumerians, on the other hand, when we first meet them at a very early date, must have been using copper for some considerable time. All that can be said with certainty is that the use of metals originated in the Orient, probably before 4000 в. с. -possibly some two or three thousand years earlier. Copper, it may be noticed, occurs in Armenia, in the upper basin of the Tigris and in Sinai amongst other places. The use of bronze gradually spread over Europe. It is found in Crete about 3000 в. с., in Southern Thessaly about 2500 в. c., and seems to have reached England about 2000 в. c. or somewhat later. Generally speaking. we can connect the taking into use of metals with the rise of the first civilizations. These civilizations reached their greatest development before the introduction of iron. The whole Minoan civilization, the Mycenean survival of Minoan civilization on the mainland, ${ }^{5}$ the most glorious period of Egyptian history up to

1 Handbook of American Indians : articles on Copper and Iron.

2 Reisner, 'Early Dynastic Cemeteries of Naga-ed-Dêr', University of California Publications, 1908, p. 117. 3 Reisner, loc. cit., p. 127. Ibid., p. 134. 5 Iron weapons are found in late Mycenean graves (Ridgeway, Early Age of Greece, p. 294). 
and including the greater partof the Second Empire, the Sumerian kingdom, the earlier periods of Babylonia and Assyria, all fall within the Bronze Age.

Though it is clear that a great advance took place in the Bronze Age, it is not evident how far this advance is attributable to the taking of metals into use and how far to other discoveries which were made about the same time. The first use of the wheel and of the plough, for instance, dates from this period, and very considerable importance without question is attributable to the plough. Still greater importance is in all probability to be attributed to writing, which again was first used about this time. At a far edrlier period various methods of marking sticks had doubtless been employed as aids to memory, as they are still employed by the Australians, and some progress may have been made towards such a system as that of the use of knotted cords in Peru. But the invention of writing properly speaking came much later. By the time of the fourth dynasty Egyptian writing had reached its final form, and the Sumerians, when we first meet them, were using cuneiform writing. This invention of such great import, whether it arose independently or not in Egypt, Mesopotamia, and China, was evidently roughly coincident with the first use of metals.

Estimates as to the date of the first use of iron in the Mesopotamian region vary considerably; 1500 в. с. to 1300 в. C. is the date most usually given. In Egypt the earliest known iron weapon dates from 1200 B.c. By 900 B.c. the use of iron had spread over most of Europe. It reached Scandinavia about 500 в.c. It was introduced into Greece by the Thresprotian invasion, which put an end to the Mycenean period, and inaugu. rated the Homeric Age. It is possible that the Etruscans brought the knowledge of iron to Italy where they arrived during the eleventh century B.c. Upon the whole, opinion inclines to the view that the use of iron originated in Western Asia. Claims have also been made for Europe (neighbourhood of Hallstatt) as the place of origin, ${ }^{1}$ for Egypt, and for Africa south of Egypt. ${ }^{2}$ With regard to Egyptian claims it may be noticed that iron does not become common until after 1200 B.c. But four examples of iron have been found in Egypt from periods before this date

1 Ridgeway, Beginnings of Iron, p. 644. (Zeit. für Eth., vol. xli, 1909, p. 52).

2 Von Luschan supports this view 
- some of them belonging to very early times. The finding of these pieces of iron presents a rather difficult problem, of which the solution seems to be that iron was very occasionally used, and probably only for ornamental purposes, long before 1200 в. с. in Egypt ; it was not, however, brought into general use at this early date.

It is not necessary to carry this sketch of cultural evolution any farther. The Bronze Age marks a turning-point in history. The outline at least of the subsequent course of events is sufficiently familiar. One point may be noticed. We have found reason to place the centre of progress somewhere in the Orient. After metal has been taken into use the centre of progress begins to shift westwards. Once metals had been introduced into Crete, for instance, Minoan civilization was in any case largely an autochthonous growth. Speaking of Crete, Hall says : 'We have nothing to show any intrusion of any other culture system which in any way suddenly modifies or alters the course of this development, which is that of the civilization of a single people raising itself on its own lines from Neolithic barbarism. Its first development from the static condition in which it had existed for many centuries in the Neolithic stage was sudden, and the dynamic influence which was given by the acquisition of metal speedily carried it to the great height of cultivation which we have seen.' 1 Hitherto--before, that is to say, the taking into use of metalthe centre of progress has been looked for in Central Asia. ${ }^{2}$ There had been progress in Europe within the culture periods, but the great steps in progress were made in the East.

12. Let us now review what has so far been said in this chapter. Such indications as the evidence provides point to the origin of man in the eastern portion of the Old World, and probably in a warm climate. The principal facts in favour of this view are the distribution of the anthropoids, the occurrence of Pithecanthropus in Java, and the failure to find any evidence of the antiquity of man in the New World. ${ }^{3}$ Unless Pithecanthropus is to be

1 Hall, Ancient History of the Near East, p. 254.

2 See on this point Breuil, 'Les Subdivisions du Paléolithique Supérieur, C. I. A., 1912. With regard to the Neolithic period Déchelette says : 'Il est permis de placer en Orient le principal centre de diffusion des accroissements successifs de la civilisation occidentale' (loc. cit., vol. i, p. 313).

${ }^{3}$ See Hrdlicka, 'Early Man in South America' (S. I. B. E., No. 52, 1912), 'Skeletal Remains suggesting or attributed to Early Man in North America' (ibid., No. 33, 1907), and 'Recent Discoveries attributed to Early Man in America' (ibid., No. 66, 1917). 
attributed to the Pliocene, we have no knowledge of man before the Pleistocene. It would seem that the pre-human ancestor of the Miocene and Pliocene was probably rather an obscure member of the primate stock retaining generalized features. Somehow without specialization he managed to survive-possibly in some very limited area. When the rapid evolution of bodily form among the mammals had slowed down, the only specialization in which the pre-human ancestor had indulged began to stand him in good stead. That it carried him as far as it did-to the dominion over all living things-was made possible by the fact that his bodily form was not specialized, but was capable of being turned into an instrument of the mind. The evidence seems to indicate the late Pliocene or early Pleistocene as the era. when man began to develop along this path.

Just as the East was probably the place of man's origin, so it remained the seat of both bodily and cultural evolution. There is no sign of the evolution of human physical form in Europe, nor is there any evidence of the evolution of the main stages of culture in Europe until after the taking of metals into use, though there is evidence both of progress and of decay within the culture periods. The evidence points to the conclusion that successive waves of immigrants brought with them new cultures from the East. Some may have entered Europe from Africa, as perhaps did the Cro-Magnons, bringing the Aurignacian culture. These waves of migration are not, however, to be regarded as comparable to migrations within the historical period-the Islamic migrations of the Arabs, for instance. These earlier movements were rather of the nature of slow driftings of peoples. They may have been connected with climatic changes and have occupied long periods of time. Quite possibly when we appear to see the extinction of one culture and the sudden replacement of it by another, what in fact happened was that, owing to climatic changes, those who practised the earlier culture had shifted elsewhere before the newcomers occupied the area in question.

With regard to the bodily evolution of man the first detailed knowledge we have is of the Neanderthal race. We know that Neanderthal man lived in the fourth glacial period. Before this epoch we know nothing of human bodily evolution except from three imperfect specimens which we cannot assign definitely to 
any period in the Pleistocene beyond placing them before the Middle Palaeolithic. No definite conclusions can be drawn as to the relation of these three forms one to another, or to the forms which follow.

Pithecanthropus may or may not be on the direct line leading to all the higher races. There is some probability that Heidelberg man is a forerunner of Neanderthal man, whereas Eoanthropus would seem to be related on the one hand to the hypothetical Pliocene ancestor, and on the other hand to the Late and not to the Middle Palaeolithic races. It is quite possible that, as Europe was not the scene of human evolution, Piltdown and Heidelberg men represent varieties which have died out, and not stages from the main line of human evolution. This is certainly the case with the Neanderthal race. In the Upper Palaeolithic we find races of as high a physical type as those of the present day, but we have no certain knowledge of their direct ancestors.

By far the most remarkable feature of the whole process has been the speeding up of the rate of progress of the evolution of culture. This speeding up would be very much emphasized if we could accept eoliths as genuine, as indicating, that is to say, the existence of an eolithic culture before the Palaeolithic culture extending over an immense period of time. As it is, the acceleration of the rate of progress is striking enough. The whole Palaeolithic period probably lasted at least 100,000 years. Regarding this period as a whole, we can observe a noteworthy speeding up of progress in the last part of the period. A few thousand years only separate the early from the late divisions of the Upper Palaeolithic and the progress exhibited is very considerable, whereas but relatively little progress is visible during the huge length of time which the Lower Palaeolithic must have occupied. The whole progress made, however, up to the opening of the Bronze Age is insignificant compared with that made since. Nevertheless, progress has not been uniform within the historical era. There have been periods of stagnation and even of decadence. Little progress in command over nature was made between the time of the later Egyptian civilization and the later Middle Ages as compared with the progress made in the last hundred years. Progress in skill gathered speed in the thirteenth century, and the speeding up became very marked in the eighteenth century. There has been a similar lack of uniformity in the evolution of 
culture where culture is judged by standards other than command over nature.

13. How far can we employ our knowledge of primitive races to fill in this framework? Some reference has been made in the fourth chapter to the position which these races occupy. It was pointed out that they represent ' relicts' of stages of culture through which the civilized races have passed, due allowance being made for the fact that they have developed to some degree on specialized lines of their own since their separation from the main stream of progress. In some few cases, as in the example of the Seri Indians, there may have been decadence.

Broadly speaking, these races fall into two groups. There are the hunting and fishing races, and there are those which practise a primitive form of agriculture. The former, it is to be presumed, ceased to be in any important manner influenced by, if they were not wholly cut off from, the main current of cultural evolution before the end of the Palaeolithic Age. The latter may have remained more or less in contact with the main stream of evolution until late Neolithic times, or they may have lost touch earlier and have acquired independently the art of agriculture, as was presumably the case with those American Indians who practise agriculture. The chief hunting and fishing races are the Tasmanians, Australians, Bushmen, Eskimos, and certain of the American Indians. Professor Sollas has drawn up a comparison between the culture of some of these races and that of certain epochs in the Palaeolithic era. He has shown that Tasmanian and Australian culture may be compared with early and late phases respectively of the Middle Palaeolithic. A very striking similarity indeed can be shown between the Aurignacian culture and that of the Bushmen on the one hand and between the Magdalenian culture and that of the Eskimo on the other. When we consider the osteological evidence, however, we do not find any striking similarity between Neanderthal man and Australians and Tasmanians. The existence of the negroid skeleton of Grimaldi is suggestive, but the likeness between it and the Bushmen type is not close. The skull found at Chancelade, however, and generally considered as an aberrant type of Cro-Magnon man does show a very curious resemblance to the Eskimo skull. Thus though we cannot think of the Tasmanians and Australians as actual relicts of the Neanderthal racial type, which we know in 
Europe, we may think of Australian culture in its broad general features as representative of the Middle Palaeolithic. ${ }^{1}$

As has been already indicated in the fourth chapter, it is not proposed to attempt to carry the method so far as to attribute to peoples of different Palaeolithic cultures peculiarities of certain primitive hunting and fishing races. All that it is proposed to do is to look into the general conditions which obtain among hunting and fishing races with the object of throwing light upon the conditions in the Palaeolithic era as a whole. Similarly it is proposed to throw light upon the Neolithic period by an inquiry into the conditions obtaining among prinitive agricultural races. This method is clearly legitimate. It may nevertheless be said at once that as a matter of fact, so far as the most important conclusions are concerned, we shall find no reasons for thinking that hunting and fishing races differ from agricultural races. The legitimacy of the method is therefore not strictly relevant. It follows from this that the splitting up of the data and the consideration separately of hunting and of agricultural races is in reality only a matter of convenience.

14. Before we embark upon this attempt to throw light upon such general characteristics of prehistoric races as are relevant to our particular purpose, there is another point to be considered. One supposition regarding the mode of life of man in early days is that he lived in groups of polygamous families. As monogamy occurs among some of the lowest of existing primitive races, he may have been monogamous. In any case it seems that early man was not promiscuous in his sexual relations. Whatever his primitive mode of life may have been, man gradually rose to the recognition of common bonds and to the observation in common of certain rules and restrictions. Primitive social organization in fact gradually came into being, and this step, slowly and painfully achieved as it no doubt was, represents one of the most important steps ever achieved by man. Then for the first time he began to reap the benefits of co-operation. Clearly we must imagine a primitive form of social organization to have existed in the late Palaeolithic; the attainment to so relatively high

1 It is interesting to note the cranial capacity of these races and to compare them with the figures given above for prehistorie races: Tasmanians, 1,225 c.c.; .Australians, 1,320 c.c. ; Bushmen, 1,244 c.c. ; Veddahs, 1,201 to 1,336 c.c.; American Indians, 1,300 to 1,450 c.c. See Hoernes, Natur und Urgeschichte der Menschen, vol. i, p. 60 . 
a degree of skill and achievement is not otherwise comprehensible. The achievements of late Palaeolithic man indicate the presence of a considerable body of tradition, and the presence of tradition in any considerable amount indicates social organization. It is difficult to come to any conclusion with regard to early and Middle Palaeolithic times. The mode of life of Neanderthal man is perhaps apt to be considered as more primitive than was in fact the case, owing to the somewhat brutal form attributed to his bodily features. It has to be remembered that he practised ceremonial burial, and was acquainted with the use of fire-very significant facts which render it on the whole probable that social organization of a kind existed. Possibly we may have to suppose that social organization existed among lower Palaeolithic men. They too employed fire, and it may well be that their mode of life was not very different from that of the Tasmanians.

Among all primitive races we find a form of social organization, and unless it has been achieved more than once they are all ' relicts' of the periods succeeding that in which men wandered in family groups. In the first place, therefore, primitive races are only comparable with prehistoric races which have achieved this great step forward. They throw little or no light upon the conditions anterior to the taking of this step. Further, when we look into the life of these races, we find that the form of social organization is rigid. Men are bound hand and foot by custom. ${ }^{1}$ It has been suggested that the peculiarly rigid nature of the social organization among these races-the fact that they are soaked in tradition-is due to their having been left out of the main stream of progress. It has been suggested in fact that, while other races have gone forward, they have more or less stagnated, and that-this is what is important-the relative stagnation has been the cause of the rigidness of the organization. It follows that, to the extent to which this is so, primitive races are not properly comparable with prehistoric races. But it may be doubted if the facts are altogether as sometimes alleged. Doubtless the earliest social organization was far less rigid than it afterwards became, and we must beware of attributing to those early races, which first became socially organized, the features characteristic of primitive races, at least in many aspects. But we must date back the origin of social organization in all pro-

${ }^{2}$ See J. J. Atkinson, Primal Law (in Social Origins, by Lang and Atkinson). 
bability nearer 100,000 than 50,000 years ago. In other words, later Palaeolithic races must have had some thousands of years of social organization behind them. Is then the peculiar rigidity of the organization of primitive races altogether to be regarded as an acquirement attained during the $15,000,20,000,30,000$ years, or whatever the period may have been, since they have been out of the main stream of progress ? It can scarcely be so. A hardening of the system may have taken place, but not to such an extent as to render it impossible to make comparisons between primitive races and prehistoric races, and, what concerns us, to render unacceptable the attribution to prehistoric races of those general conditions of life found among primitive races, which will form the subject-matter of the next two chapters. 


\section{VII \\ HUNTING AND FISHING RACES}

1. We now pass to a consideration of the quantitative problem. In this chapter we deal with hunting and fishing races. We require information first regarding those factors which hinder the realization to the full of the power of fecundity, and secondly regarding those factors which cause elimination. Not until we have this information can we hope to determine how numbers are regulated among these races. This inquiry is not intended to be exhaustive ; our aim is merely to gain an idea of the nature of the more important factors which are in operation. When we come to the interpretation of the evidence, there will be more to say both as to the incompleteness of the evidence as here presented and as to the inherent diffculties of ascertaining what the position was before European influence had made itself felt.

2. We may first consider sexual intercourse before maturity. It is perhaps more difficult to determine the prevalence of this custom than that of any other practice which we shall have to examine in the course of this chapter. The difficulty is due to the nature of the facts under discussion; first it is clearly not easy to ascertain them and comparatively few authors have the intimate knowledge necessary to enable definite statements to be made ; secondly such veiled and guarded language is often used that the true state of the case remains uncertain. We shall see that marriage, or at any rate cohabitation, very soon after puberty is the universal rule among these races. Statements therefore concerning 'very early' marriage must, in the absence of more exact information, be taken to mean no more than that marriage follows at once after puberty. Of the Tasmanians there is no certain information. Statements exist to the effect that marriages are sometimes consummated in Australia before puberty ${ }^{1}$ these statements are seldom specific or on good authority. The more trustworthy accounts state definitely that, no matter at what age betrothal occurs, the husband does not claim his wife until she is

1 Lumholtz, Among Cannibals, p. 164. 
mature. ${ }^{1}$ The existence of early betrothal as a widespread habit has no doubt betrayed some authors into an error. ${ }^{2}$ We know little of the customs of the Bushmen in this as in most other respects. Statements have been made, however, to the effect that marriage took place at so early an age that the girls could not have been mature. ${ }^{3}$

Passing to the Eskimos it would appear that pre-puberty intercourse is not uncommon. Of the Eskimos of Greenland we read as follows : 'Often indeed he marries before there is any chance of the union being productive.' 4 Murdoch says of the Port Barrow Eskimos that 'promiscuous sexual intercourse between married or unmarried people, or even among children, appears to be looked upon merely as a matter for amusement ' $;{ }^{5}$ similarly Turner says of the Eskimos of the Ungava district that 'many of the females are taken before that period' (i.e. before maturity). ${ }^{6}$ There are some indications that similar habits occur among the Indian tribes of the far north and of the Pacific Coast. Ross reports of the Eastern Tinneh that " they marry sometimes, but not often at ten, and have their menses at thirteen ' ${ }^{\prime}$ Bearing in mind the average age at which menstruation begins among American Indians, the following statement by Gibbs, referring to the tribes of Western Washington and North-western Oregon, points to the same conclusion. 'Cohabitation of unmarried females among their own people brings no disgrace if unaccompanied by childbirth. . . . This commences at a very early age, perhaps ten or twelve years.' 8 There does not seem to be any definite statement with regard to the Californians; nevertheless the common accounts of marriage at twelve or thereabouts, coupled with statements as to freedom before marriage, are suggestive. Speaking generally of the Californians, Powers says that ' marriage frequently takes place at the age of twelve or fourteen ',9 and that ' there is scarcely an attribute

${ }^{1}$ See, for example, Parker, Euahlayi Tribe, p. 56, and, for the tribes of Western Victoria, Brown, J. A. I., vol. xliii, p. 158.

2 Early betrothal is also common among other races of this group; but, unless otherwise stated, this should not be taken as meaning that cohabitation follows until after puberty. The more careful accounts usually state that it does not; for the Thompson Indians, for instance, see Teit, Jesup North Pacific Expedition, vol. i, p. 321 .

${ }^{3}$ Hartland, Primitive Paternity, vol. i, p. 267. This author has dealt at some length with the prevalence of this habit. Hansen, First Crossing of Greenland, p. 320.

5 Murdoch, 9th A. R. B. E., p. 419.

- Turner, 11 th $A . R . B . E$. p. 188 . See, for Behring Strait Eskimos, Nelson, 18th $A . R$. B. E. , p. 292. ${ }^{7}$ Ross, A.R.S.I., 1860, p. $305 . \quad{ }^{8}$ Gibbs, U.S. Geog. and Geol. Survey, vol. i, p. 199.

๑ Powers, ibid., vol. iii, p. 413. 
known as virtue or chastity in either sex before marriage'.1 Another observer speaks of very early marriage among the Comanches in such a manner as to suggest that it may be consummated before puberty. ${ }^{2}$ Lastly, there is some information for certain other races which belong to this group. We are told that among the Fuegians sexual relations begin at about ten to twelve years of age, ${ }^{3}$ whereas menstruation begins at about fourteen or fifteen years of age. ${ }^{4}$ Of the Veddahs we read that ' marriage takes place at an early age ; it was said that marriage sometimes takes place before puberty, and as we have heard of this at Herrebedda, Barrdaraduwa, and Omuni, we see no reason to doubt the truth of the statement '. ${ }^{5}$

3. There is ample evidence to the effect that the period of lactation is always prolonged among these races. Speaking of the Tasmanians, Ling Roth mentions two years ${ }^{6}$ and Bonwick three to four ${ }^{7}$ as the length of this period. Of the central Australians Eyre says that 'infants are not often weaned until between two and three years old '. 8 Of the Western Australians Grey says that 'the native women suckle their children until they are past the age of two or three years' ${ }^{9}$ For the Southern Australians, Eylmann mentions four years, ${ }^{10}$ for the Narrinyeri, Taplin two years, ${ }^{11}$ for the aborigines of Encounter Bay, Meyer 'a considerable time, sometimes to the age of five or six years', ${ }^{12}$ for the aborigines of Victoria, Curr three years, ${ }^{13}$ and for the natives of King George's Sound, Brown four to five years. ${ }^{14}$

From America the evidence is similar. Among the Port Barrow Eskimos the children are nursed until about three or four years of age. ${ }^{15}$ Nansen states that " Greenland mothers are very slow to wean their children. They often give suck until the child is three or four, and I have even heard of cases in which children of ten or over continue to take the breast.' 16 Crantz, speaking of the same country, corroborates this evidence, ${ }^{17}$ and Bessels mentions

1 Ibid., p. 157. and Deniker, Mission Scientifique, p. 188. (ibid., p. 96).

7 Bonwick, Daily Journals, vol. ii, p. 250. See also Spencer and Gillen, Native Australia, p. 51. Eingeborenen der South Australia, p. 15.

12 Meyer, Aborigines

${ }_{13}^{13}$ Curr, Recollections, p. 263 . See also Smyth,

${ }^{16}$ Nansen, Eskimo Life, p. 151.
2 Ten Kate, Rev. d' Eth., vol.iv, p. 129.

${ }^{4}$ Ibid., p. 187.

3 Hyades - Seligman,

${ }^{8}$ Ling Roth, Aborigines of Tasmania, p. 168, note. 8 , note. See also Spencer and Gillen, Native Tribes of Central
Grey, Journals, vol. ii, p. 250. ${ }_{10}$ Fylmann, Die
Südaustralien, p. 261. 9 Grey, Journals, vol. ii, p. 250. 10 Fylmann, Die
11 Taplin, Native Tribes of 11 Taplin, Native Tribes of
Encounter Bay Tribe, p. 187. Murdoch, loc. cit., p. 415. ${ }^{17}$ Crantz, History of Greenland, vol. i, p. 162. 
a case from Smith Sound in which a child was suckled up to seven years of age. ${ }^{1}$ Heriot, speaking generally of the Indian tribes of the North, mentions ' the length of time employed by the women in nursing their children, whom they nourish for three or four years', ${ }^{2}$ and Long gives four, five, and even six years. ${ }^{3}$ For the Ingaliks Dall mentions three years, ${ }^{4}$ for the Eastern Tinneh Ross three or four, ${ }^{5}$ for the Thlinkeet Krause four years, ${ }^{6}$ and Lord for the Indians of Puget Sound two or three years. ${ }^{7}$ Speaking of the Ahts of Vancouver Island, Sproat says he has seen ' a boy of four following his mother for her milk',8 and of the tribes of California Schoolcraft that " children are sometimes not weaned until five years of age'.9 Similarly the Nootkas suckle their children until they are three or four years old, ${ }^{10}$ and Chinooks their children ' until three, four, or five years old'.11 The facts are similar for the inland Salish and Shushwap ${ }^{12}$ as well as for the tribes of Oregon and Washington ${ }^{13}$ and the Chepewayans. ${ }^{14}$ So also in South America the Puelches ${ }^{15}$ and the Abipones ${ }^{16}$ suckle their children for three years, and the same is reported of the Fuegians ${ }^{17}$ and of the Andaman Islanders who 'never wean their babies so long as they are able to suckle them'.$^{18}$ Among the Ghiliaks suckling is continued up to the age of three years. 19

4. Initiation ceremonies at the period of maturity are of common occurrence among primitive races. These ceremonies are sometimes accompanied by mutilation of the genital organs; but there is apparently no reason to believe that this mutilation has any effect upon fecundity, except possibly among the Australians. ${ }^{20}$ It is unnecessary to describe the exact nature of the operations performed upon Australian boys and girls. The operations are severe and it seems very possible at first sight that they might affect the reproductive power. The opinion of authorities is divided on this point. Spencer and Gillen think that the opera-

1 Bessels, Arch. für Anth., vol. viii, p. 113.

2 Heriot, Travels, p. 339 . See also Weld, Travels, p. 373.

3 J. Long, Voyages, p. 60.

4 Dall, Alaska, p. 196. 5 Ross, loc. cit., p. 305. ${ }^{\circ}$ Krause, Die Thinklit-Indianer, p. 216. See also Bancroft, Native Races, vol. i, p. 111. TIord, Naturalist in Vancouver, vol. ii, p. 233.

Indian Tribes, vol. iii, p. 212. p. 242. p. 60 . 12 Ibid.

Sproat, loc. cit., p. 94. - Schoolcraft, 15 Guinnard, Three Years' Slavery among the Patagonians, 146. 18 Dobrizhoffer, Abipones, vol. ii, p. $195 . \quad 17$ Hyades and Deniker, loc. cit., vol. vii, p. 195. d'Eth., vol. ii, p. 303. 18 Man, J. A. I., vol. xii, p. 81. 19 Deniker, Rev. Anthropological Society of Bombay, vol. ii, 1890. 
tions may have an injurious effect ; $^{1}$ others, such as Roth ${ }^{2}$ and Mathews, ${ }^{3}$ hold the contrary view. Upon the whole, opinion inclines to the latter side.

Less doubt exists with regard to the assertion sometimes made that these ceremonies are practised with the intention of producing sterility, or in any case relative sterility. ${ }^{4}$ For this there is no good evidence and the best authorities definitely assert that it is not so. ${ }^{5}$ Another assertion has been made for which there is even less foundation; it is said, for instance, that 'in some tribes we find it a custom that every man submits to this operation after the birth of his second or third child ' ${ }^{6}$. It would appear that as a matter of fact the operation is performed at puberty and at no other time.

5. The three further factors to be considered which may have a bearing upon fecundity are all connected with marriage. It has been shown by Westermarck that marriage exists as an institution among all races, however primitive. ${ }^{7}$ What we have to ask is whether there is any postponement of marriage, whether, that is to say, there is any temporary or permanent celibacy and, so far as fecundity is concerned, the age of women at marriage alone is relevant. The evidence points to the fact that marriage of women takes place almost universally among these races if not before, then at or very soon after, puberty. This is Westermarck's conclusion, after a wide survey of the races belonging both to this and the next group. It will be sufficient to give some examples of the evidence and then to note a few cases in which some postponement is reported.

Summing up the evidence with regard to the Australians, Malinowski says that in general it is true that there are no unmarried girls under sixteen and no widows under forty-five, though there is some evidence with regard to the existence of unmarried females $;^{8}$ thus Smyth, speaking of the inhabitants of Victoria, records that girls were married between the ages of ten and twelve, ${ }^{9}$ and similar evidence is given by Taplin of the inhabitants of South Australia. ${ }^{10}$ In Australia there is no evidence worth mentioning

1 Spencer and Gillen, Native Tribes of Central Australia, p. 52. ${ }^{2}$ W. E. Roth, Ethnological Studies, p. $179 . \quad{ }_{3}$ R. H. Mathews, Ethnological Notes, p. 177.

${ }_{4}$ Curr, Australian Race, vol. ii, p. 19.

Spencer and Gillen, Native Tribes of Central Australia, p. 264.

Australian Bush, p. 234.

7 Westermarck, Human Marriage, p. 134.

${ }^{8}$ Malinowski, Family among Australian Aborigines, p. 134.

cit., vol. ii, p. 77.

${ }_{10}$ Taplin, loc. cit., p. 10.

$\checkmark$ Smyth, loc. 
of either temporary or permanent celibacy. Apart from the instances to be given below the evidence with regard to other races in this group is very similar to that given before of the Australians. Thus the average age for a girl to marry in Vancouver Island was sixteen, ${ }^{1}$ among the Californiars generally between twelve and fourteen, ${ }^{2}$ and among the Stseelis and Skaulits tribes fifteen. ${ }^{3}$ Veddah girls married between eleven and twelve. ${ }^{4}$

There is no doubt that, generally speaking, postponement of marriage is rare, though occasionally some slight delay is reported, as in the instances given in what follows. It should be mentioned, however, that from time to time there is quite a considerable delay upon the part of the man amongst these races ; to this point, we shall return later. As regards women, Crantz states that sometimes Eskimo girls do not marry until they are twenty. ${ }^{5}$ Hill Tout, speaking of the Salish and the Tinneh, says "with the exception of the Carrier tribes early marriages were everywhere the custom ' ${ }^{6}$ The same author mentions that among the Thompson tribes girls did not marry until seventeen or eighteen, ${ }^{7}$ and this is confirmed by Teit. ${ }^{8}$ The latter author gives somewhat similar evidence for the Shushwap, ${ }^{9}$ and also says that among the Lillooet Indians girls are isolated for two years after puberty, and only marry when from sixteen to twenty years old..$^{10}$ As regards South America we are told by Musters that among the Tehuelches the girls ' often remain several years spinsters', 11 and by Dobrizhoffer that among the Abipones girls do not marry before their twentieth year..$^{12}$

There is one other matter to which reference may be made here. There is often very considerable disparity in age between husband and wife at marriage among primitive races. It is frequently the practice for the girls on reaching the age of puberty to be taken in marriage by the older men, while the young men marry women twice their age. ${ }^{13}$ Since, however, as pointed out in Chapter IV the age of the husband has little or no effect upon fertility, this practice is of no importance from our present point of view. The important fact which results from the evidence, is that almost

1 Sproat, loc. cit., p. 94. vol. xxxiv, p. 319 .

Crantz, loc. cit., p. 158.

7 Hill Tout, loc. cit., p. 190. vol. i, p. 190.

Patagonians, p. 186. - Ibid., vol. ii, p. 591
2 Powers, loc. cit., p. 413.

3 Hill Tout, J. A. I., - Sarasin, Forschungen aus Ceylon, vol. iii, p. 469. - Hill Tout, British North America, p. 182. - Teit, Jesup North Pacific Expedition, 12 Dobrizhoffer, loc. cit., vol. ii, p. 42. ${ }_{13}$ See, for instance, as regards the Australians, Malinowski, loc. cit., p. 48 ; Tasmanians, Bonwick, loc. cit., p. 85; Bushmen, Theal, Yellow- and Dark-Skinned People, p. 46 ; and Chinooks, Bancroft, loc. cit., vol. i, p. 241. 
universally among these races the women are married at or soon after puberty and remain married throughout the mature period.

6. Among almost every uncivilized race there are numerous occasions upon which sexual intercourse is tabooed. Common instances are those of taboo during the preparations for a hunting party or a military expedition. It is not worth while to enumerate these occasions, because with one exception it does not seem that they can, even when considered together, have any marked effect upon fertility. ${ }^{1}$ The exception referred to is the prohibition upon intercourse that follows the birth of a child. This taboo is of very great importance among certain races that we shall consider in the eighth chapter. As regards hunting and fishing races there is no record of its existence in Tasmania, Australia, among the Bushmen, the Eskimos, or any other people of importance with the exception of the American Indians. The evidence in this case is scanty ; there are certain difficulties and the matter is better left for discussion in the next chapter when we come to deal with the agricultural races of this part of the world. It is enough to say here that certain early observers, speaking generally of the American Indians, refer to this custom, and that two early observers speaking specifically of two hunting tribes-one in North and one in South America-record this custom. Cabeça de Vaca says that the Yguazas abstain from intercourse for two years after childbirth, ${ }^{2}$ and Dobrizhoffer records of the Abipones that ' the mothers suckle their children for three years, during which time they have no conjugal intercourse with their husbands ' 3

7. At this point we may refer to the remarkable fullness and unanimity of the evidence with regard to the number of children observed among these races. The evidence is to the effect that the number of children is always small. ${ }^{4}$ Bonwick says that ' families were never large with the Tasmanians ',5 and that 'the native woman as a rule had very few children, and fewer still were ... permitted to live' $;{ }^{6}$ he attributes this to the fact that women did not begin to bear children until after several years of

${ }^{1}$ It is rare that the prohibition for such reasons extends over so long a period as a year. This is the period of abstinence sometimes enjoined upon the Thlinkeet Indians (Swanton, 26th A.R.B. E., p. 449). On this subject see Crawley, Mystic Rose, pp. 187, 215, and 342 ; Hubert and Mauss, Histoire des Religions ; Frazer, Golden Bough, vol. i, p. 29 ; and Westermarck, loc. cit., pp. $150 \mathrm{ff}$.

2 Cabeça de Vaca, Narrative, p. $62 . \quad 3$ Dobrizhoffer, loc. cit., vol. ii, p. 97. 4 This has, of course, often been remarked upon. See, for instance, Darwin, Descent of Man, vol. i, p. 132. p. 85 .

5 Bonwick, loc. cit., p. 79.

6 Ibid., 
marriage, ${ }^{1}$ and that women ceased to bear at or soon after the age of thirty-five. ${ }^{2}$ Abundant evidence is forthcoming from Australia. 'Large families of children are unusual among the aborigines,' says Dawson, ' however many may be born, rarely more than four are allowed to grow up.' 3 Lumholtz remarks that there are seldom more than three or four children.4 Mathew referring to two Queensland tribes records that " the number of children in a family was small on the average. Six would be rare. I know of no aboriginal family with more than five who survived infancy.' 5 Grey obtained some statistics in Western Australia with reference to the average number of children born by women who had passed child-bearing age. 'Forty-one females, of whose families I have obtained (from themselves and others) lists upon the accuracy of which I can rely, had 188 children, or about $4 \cdot 6$ children each.' ${ }^{6}$ Among the native tribes of Central Australia the number of children in a family rarely exceeds four or five, and is generally two or three ; ${ }^{7}$ at the same time sterility is common; the greatest fertility is noticed among the stout and the strong, whereas the thin and weak have scarcely any children. ${ }^{8}$ Eyre quotes some observations of Moorehouse, also made in Central Australia, and says that ' his investigations have led to the conclusion that each woman has on an average five children born (nine being the greatest number known) '; 9 Eyre adds that he agrees with this estimate. Schürmann records that 'the number of children reared by each family is variable, but in general very limited, rarely exceeding four'. He adds the interesting information that in spite of early marriage he has not observed that they have children at an earlier age than is common among the Europeans, and says that it is rare for a mother to have children 'in rapid succession '.10 Wilhelmi's evidence is similar; with reference to the aborigines of the Port Lincoln district he states that 'the number of children in a family varies considerably ; but, on the whole, it is limited-seldom exceeding four' ; he also adds that

1 Bonwick, loc. cit., p. 76.

2 Ibid., p. 85. See also Smyth, loc. cit., vol. ii, p. 387. ${ }^{3}$ Dawson, Australian Aborigines, p. 39. The interesting estimate of Curr has been given on p. 99. In another place he says: 'The number of children born, on the average, by Bangerang women I estimate at six or perhaps eight' (Recollections, p. 252).

5 J. Mathew, Eaglehawk and Crow, p. 165.

4 Lumholtz, loc. cit., p. 134. often commence before the age of sixteen' (ibid., p. 323). Gillen, Native Tribes of Central. Australia, p. 264.

? Spencer and 9 Eyre, Journals, vol. ii, p. 376.

10 Schürmann, Aboriginal Tribes, p. 223. 
it seldom happens that " children are born in a family quick after one another'.1

Stow remarks that 'the Bushmen seldom had large families ' ; 2 Theal, on the other hand, says that 'the earliest Dutch colonists observed that they were remarkably prolific ' 3 -one of the very few statements which attribute a high fertility to any of these races. Passing now to the Now World we find a similar abundance of evidence pointing to the same conclusion. 'The Greenlanders are not very prolific. A woman has commonly three or four children, but at most six ; they generally bear but one child in two or three years.' 4 Writing more than a hundred years later than Crantz, Nansen gives very similar testimony, which has already been quoted, ${ }^{5}$ as has that of Murdoch for the Port Barrow Eskimos. 6 The latter adds that 'they do not commonly bear children before the age of twenty'.7 According to Bessels the number in an Eskimo family near Smith Sound is on the average two ; this low figure is due to infanticide, he goes on to say. ${ }^{8}$ In the Ungava district ' the number of children born varies greatly, for, although these Eskimo are not a prolific race, a couple may occasionally claim parentage of as many as ten children. Two or three is the usual number.' 9 Armstrong notes of the Eskimos generally ' that they are not a prolific race from all that I could learn '.10 Of the Aleutes Ritter says that the average number in a family is two or three. ${ }^{11}$

Writing of the neighbourhood of Hudson Bay, Hearne says : ' Providence is very kind in causing these people to be less prolific than the inhabitants of civilized nations; it is very uncommon to see one woman have more than five or six children ; and these are always born at such a distance from one another, that the younger is generally two or three years old before another is born into the world.' ${ }^{12}$ Among the Eastern Tinneh to be confined once every three years is 'a high average' ${ }^{13}$ The author responsible for this statement goes on to say that " the women are capable of bearing children from fourteen to forty-five-a long period of their

${ }^{1}$ Wilhelmi,Transactions of the Royal Society of Victoria, vol.v, p. $180 . \quad{ }^{2}$ Stow, Native Races, p. $50 . \quad{ }_{3}$ Theal, loc. cit., p. $44 . \quad{ }^{4}$ Crantz, loc. cit., vol. i, p. 161. ${ }_{5}^{5}$ p. 99. Hutton, however, gives a high figure for the birth-rate (Eskinos of Labrador, p. 80). ${ }^{6}$ p. $99 .{ }_{7}^{7}$ Murdoch, loc. cit., p. 39. This author says further that 'all authors who have described Eskimos of unmixed descent agree in regard to the generally small number of their offspring ' (ibid., p. 419). B Bessels, loc. cit., p. 112. 10 Armstrong, Personal Narrative, p. 195. p. $265 . \quad{ }_{12}$ Hearne, Journey, p. 312.

9 Turner, loc. cit., p. 189.

1 Ritter, Zeit. für all. Erd., vol. xiii, 13 Ross, loc. cit., p. 305. 
lives-but in it very few infants are produced. Families on an average contain three children .... and ten is the greatest number I have seen.' In Alaska ' the females of the coast tribes are not fruitful, and to see four children of one mother is quite a rare occurrence, one or two being the common number of children to a family '.1 The evidence of Sproat for the Ahts and of Bancroft for the Nootkas and the Chinooks has already been quoted.2 Among the Omahas 'the usual number of children may be stated at from four to six in a family' 3 Of the Sound Indians Bancroft states that "the women.are not prolific, three or four being ordinarily the limit of their offspring ' .4 The woman of the interior of the Pacific Coast ' is not prolific ', ${ }^{5}$ and the number in a Chepewayan family is on the average four. ${ }^{6}$ In California ' barrenness was not infrequent, twins very uncommon, and the general average of families did not exceed two children '. people Baegert states that 'it is certain that many of their women are barren and that a great number of them bear not more than one child '.8 Of the Comanches we read : 'they are not a prolific race ; indeed it is but seldom that a woman has more than three or four children.' 9 Among the Puelches of South America 'children are not nearly so numerous as might be imagined '; this is due in part, however, according to the author, to infanticide. ${ }^{10}$ According to Bridges, in Tierra del Fuego 'few women have more than six children owing to the great length of time between the several births '.11 Of the same people, Hyades and Deniker record their opinion that four is the average number of children to a married woman. ${ }^{12}$ They add that in spite of early marriage very few young couples of eighteen to twenty years of age had any children, although sterility was rare.13

Of the other races which belong to this group the evidence points to a 'similar conclusion.14 Man thinks that three or four is an average number of children in an Andamanese family, and six is

1 Petroff, 'Report of the Population of Alaska', 10th Census of the U.S.A., p. 127. 2 p. $99 . \quad{ }^{3}$ S. H. Long, loc. cit., p. 19. See also Dorsey, 3rd A. R. B. E., p. 264. 4 Bancroft, loc. cit., vol. i, p. $218 .{ }^{5}$ Ibid., p. $156 .{ }^{6}$ Keating, Narrative, p. 156. 'Schoolcraft, loc. cit., vol. iii, p. $211 . \quad 8$ Baegert, A. R. B. E., 1863, p. 368 . Bancroft, loc. cit., vol. i, p. 513. 10 Guinnard, loc. cit., p. 143. 11 Bridges, $A$ Voice from South America, vol. xiii, p. 202. 12 Hyades and Deniker, loc. cit., vol. vii, p. $189 . \quad 13$ Ibid., p. 188.

14 It may be observed that according to Keane the Botocudos form an exception. 'Families', he says, 'are said to be comparatively large, four or five children being common enough" (J. A.I., vol. xiii). This is, however, contradicted by von Tschudi, Reisen durch Südamerika, vol. ii, p. 284. 
the largest number he met with. ${ }^{1}$ Portman mentions three as an average. ${ }^{2}$ The Sarasins are of opinion that Veddah women are fairly prolific and that the small families recorded are due to infant mortality. ${ }^{3}$ Of the Tuski tribes Dall says 'they are not prolific ', 4 and according to Deniker ' the Ghiliak women do not have many children; it is rare to find more than two or three to a family' .5

8. We now come to the consideration of the factors of elimination. The first practice, the extent of which we have to investigate, is that of abortion. The methods employed need not be specified in each case ; the use of violent physical means is perhaps the most common; among other methods the drinking of various decoctions is frequently referred to. Doubt is sometimes expressed as to the efficacy of some of these means-especially that of the last named. It may be that in some cases it is of the nature of a magical ceremony without any practical result ; as a general rule, however, there is no doubt that the methods used are effective.

Among the Tasmanians 'abortion was frequently practised '.6 Statements are sometimes made which seem to imply that abortion was not uncommon among the Australians. ${ }^{7}$ A survey of the literature does not support this view; the practice is indeed mentioned occasionally, but it is clearly negligible in comparison with the universal presence of infanticide. Curr says that it is occasionally employed. ${ }^{8}$ In Collins's account of the aborigines in the neighbourhood of Port Jackson there is a reference to "the horrid and cruel custom of endeavouring to cause a miscarriage '.9 Palmer records the practice as occurring among the Mythuggadi tribe, ${ }^{10}$ and Roth says it is common among certain districts of North-west and Central Queensland. ${ }^{11}$ Abortion has been noticed among the Eskimos, but never as a common practice..$^{12}$ It is among the Indian tribes of the North and the Pacific Coast that we find the most numerous references to this custom. ${ }^{13}$ Speaking of the tribes near Hudson Bay, Ellis mentions 'a very strange maxim of policy which prevails much amongst them, which is that of suffering

1 Man, loc. cit., p. 81. loc. cit., vol. iii, p. 169 . vol. $\mathrm{ii,}$ p. 302. Allgemeine Kut Race, vol. i, p. 76. 1803 , p. 34 . p. 183 . loc. cit., p. 112 America, vol. i, p. 297

2498
2 Portman, J. A. I., vol. xxv, p. $369 . \quad{ }^{3}$ Sarasins,

- Dall, loc. cit., p. 381 . 5 Deniker, Rev. d'Eth., 6 Bonwick, loc. cit., p. 76.
7 See, for example, Klemm,
\& Curr, Australian - Quoted by a reviewer in the Edinburgh Review, vol. ii, 10 Palmer, J. A. I. vol. xiii, 'p. 280.111 Roth, loc. cit.,
ells and Kelly, U.S.A. Bureau of Education, 1890, p. 19 ; Bessels, ${ }_{13}$ See Weld, loc. cit., p. 373, and Robertson, History of 
rather than of obliging their women to procure frequent abortions by the use of a certain herb common in that country '. 1 Similar facts are recorded of the Knisteneaux." Abortion is not ' uncommon among the Haidahs '. 3 The Nootkas ' frequently prevent the increase of their families by abortion' ${ }^{4}$ I ' causing of abortion' is ' not uncommon' among the natives of Puget Sound, ${ }^{5}$ while we find this practice spoken of as ' common' in Vancouver Island. ${ }^{6}$ Teit mentions abortion as being ' rarely practised ' among the Thompson Indians, ${ }^{7}$ and as ' rare' among the Shushwap. ${ }^{8}$ Gibbs speaks of abortion as being 'almost universal' among the tribes of Western Washington and Northwestern Oregon. 9 According to Bancroft abortion is of 'frequent occurrence' among the Chinooks, and 'not uncommon' among the inland tribes. ${ }^{\mathbf{1 0}}$ Powers also records the practice among the Kabinapek of California, ${ }^{11}$ and Castelnau among the Guaycurus ${ }^{12}$ and Rengger among the Payaguas. ${ }^{13}$ Cooper, who has reviewed the evidence so far as the Fuegians are concerned, states that 'deliberate abortion is common'. 14

9. There is one common form of infanticide-the killing of deformed children-which can have no importance so far as numbers are concerned. But this practice may be noted here because of its bearing upon quality. We may also note in particular when the killing is said to be of female children. ${ }^{15}$

Bonwick states that infanticide was fairly prevalent in Tasmania. ${ }^{16}$ Ling Roth, who summarizes the evidence of several authors, finds it somewhat conflicting; but it would seem that infanticide undoubtedly occurred though it is not known with any certainty whether it was practised on a large scale or not. ${ }^{17}$ In

1 H. Ellis, Voyage, p. 198.

3 Bancroft, loc. cit., vol. i, p. 169. in Vancouver, vol. ii, p. 231. loc. cit., vol. i, p. 305 . Ibid., vol. ii, p. 584 .
2 Mackenzie, Voyages, vol. i, p. 148.

I Ibid., p. 197. ' Lord, Naturalist

Sproat, loc. cit., p. 94

Gibbs, loc. cit., It also occurred among the Omahas but was not common (Dorsey, loc. cit., p. 263, and Long, loc. cit., p. 20). 11 Powers, loc. cit., p. 207. For New Mexico see Bancroft, loc. cit., vol. i, p. 590. 12 Castelnau, Expedition, vol. ii, p. 450. See also Azara, Voyages, p. 146. 13 Rengger, Reise, p. $329 . \quad$ is Cooper, S. I. B. E., Bull. No. 63, 1917, p. 171.

${ }^{18}$ Female infanticide was brought into great prominence by MacLennan. Although he asserted that female infanticide was "common among savages every. where' (Studies in Ancient History, p. 111), he did not bring forward any con. siderable body of evidence.

${ }^{16}$ Bonwick, loc. cit., pp. 79 and 85. See also Smyth, loc. cit., vol. ii, p. 386.

${ }^{17}{ }_{i}$ Ling Roth, loc. cit., p. 167. This author suggests that infanticide may have increased owing to the disturbances due to the arrival of the Europeans. There is no evidence that this was so, and for the reasons given later it is most improbable that this is ever the result of European influence. 
Australia infanticide is practised on a very large scale. Parker states that it was very frequent and that deformed children were always killed. ${ }^{1}$ Lumholtz's testimony is similar, ${ }^{2}$ while, according to Dawson, no matter how many children are born, ' rarely more than four are allowed to grow up'-the deformed children apparently always being destroyed. ${ }^{3}$ According to an estimate made by Curr of conditions in Victoria ' nearly one half [of the children born to any one married woman] fell victims to infanticide' ${ }^{4}$, and more girls than boys perished. ${ }^{5}$ According to Wilhelmi, if, as rarely happens, births follow one another quickly among the aborigines of the Port Lincoln district, ' the youngest is generally destroyed ' $;{ }^{6}$ Beveridge says that the practice prevails in Victoria and Riverina ' to a very considerable extent'? This last statement is supported by that of Mathews to the effect that 'infanticide is common' in New South Wales and Victoria. ${ }^{8}$ Among the inhabitants of the River Darling region 'it seems to have been the custom to kill many of the children directly after birth' ${ }^{9}$ and in Southern Australia infanticidewas very prevalent. ${ }^{10}$ According to Howitt infanticide was practised 'to some extent' among the Mining Tribe, ${ }^{11}$ in the Tongeranka tribe it was 'common' $;^{12}$ in the Mukjarawaint tribe 'the grandparents had to decide whether a child was to be kept alive or not ' $;{ }^{13}$ in all the tribes of the Wotjo nation and also the Tatuthi and other tribes of the Murray River frontage, when a child was weak and sickly they used to kill its infant brother and sister and feed it with the flesh to make it strong; ${ }^{\mathbf{1 4}}$ in the Wadthaurung tribe the practice was evidently not uncommon $;^{15}$ in the Narrinyeri tribe infanticide appears to have been very prevalent, so that ' more than one half of the children fell victims to this atrocious custom ', 16 whilst deformed children seem always to have been killed both in this case $^{17}$ and among the aborigines of Encounter Bay. ${ }^{18}$ Infanticide was common among the tribes of Port Lincoln-girls being less often spared than boys ${ }^{19}$-among the Dieyerie tribe, where

1 K. L. Parker, loc. cit., p. 23.

${ }^{2}$ Lumboltz, loc. cit., p. 134.

3 Dawson, loc. cit., p. 39.

Curr, Recollections, p. 252.

5 Ibid., p. 263.

6 Wilhelmi, loc. cit., p. 180. 7 P. Beveridge, Aborigines of Victoria, p. 26.

8 R. H. Mathews, Ethnological Notes, p. 17. 'Bonney, J. A. I., vol. xiii, p. 125. 10 Palmer, loc. cit., p. 280; Fison and Howitt, Kamilaroi ard Kurnai, p. 190 ; Eylmann, loc. cit., p. 261 ; and Smyth, loc. cit., vol. i, p. 52. 11 Howitt, Native Tribes, p. $748 . \quad{ }_{12}$ Howitt, loc. cit., p. $749 .{ }_{13}$ Ibid., p. 749. 14 Ibid., p. 750 . 15 Ibid., p. 750. 16 'Taplin, loc. cit., p. 12. 17 Ibid., p. 14. ${ }_{18}$ Meyer, loc. cit., p. 186. p. 223. 
deformed children were never spared, ${ }^{1}$ among two Queensland tribes, ${ }^{2}$ in the neighbourhood of Port Darwin, ${ }^{3}$ in Central Australia, ${ }^{4}$ and among the Northern tribes of Central Australia. ${ }^{5}$ In Western Australia deformed children were always killed. ${ }^{6}$

Our information with regard to the Bushmen suggests that infanticide was prevalent, though not confined to new-born children. 'The Bushmen will kill their children without remorse on various occasions; as when they are ill-shaped, when they are in want of food, when the father of a child has forsaken its mother, or when obliged to flee from the fathers of others.' ? Infanticide is more common than abortion among the Eskimos. ${ }^{8}$ Nelson says that in the neighbourhood of Behring Straits even girls of four to six years of age are at times killed. ${ }^{9}$ Among the Central Eskimos 'it is practised to some extent', though apparently only girls and the children of widows and widowers are destroyed. ${ }^{10}$ Murdoch never heard of infanticide in the Port Barrow region, ${ }^{11}$ but says that it is reported to be 'frequently practised among the Eskimo of Smith Sound without regard of sex ' and that female infanticide occurs among the people of King William Land.12 It is also recorded of the inhabitants of Smith Sound by Bessels that, after two children have been born, any others that may come are more often than not killed.13 In Greenland ' the heathen Eskimo killed deformed children, and those that seem too sickly to live, as well as those that lose their mothers at birth, when no one else can be found to take charge of them '.14 Killing of deformed children is also reported by Smith, ${ }^{15}$ and that of children who have lost their parents by Rink. ${ }^{16}$ Among the Aleuts infanticide is said to be rare, ${ }^{17}$ but among the Malemutes infanticide, especially of girls, was not infrequent. ${ }^{18}$

Infanticide is frequently practised among the Kutchins ${ }^{19}$ and the killing of deformed children by the inhabitants of the Copper

1 Gason, Manners and Customs, p. 258. Tribes, p. 165. vol. ii, p. 376. Northern Tribes, p. 608.

Missionary Labours, p. 58 Primitive Folk, p. 34 . Foelsche, J. A. I., vol. xxiv, p. 192.

2 Mathew, Two Representative p. 580. loc. cit., p. 112.

Life, p. 151. i1 Murd

58.

Nelson, loc. cit., p. 289. and Gillen, Native Tribes, p. 264.

${ }^{6}$ Grey, loc. cit., vol. ii, p. 251.
ee also Stow, Native Races, p. 51.

10 Boas, 6th A. R. B.E., ${ }_{16}$ Rink, loc. cit., p. $35 . \quad{ }_{17}$ Dall, loc. cit., p. $399 . \quad{ }_{18}$ Bancroft, loc. cit., vol. ii, p. 81. ${ }_{19}$ Kirkby, Church Missionary Intelligencer, vol. xiv, p. .115; Hardisty, A. R. S. I., 1866, p. 312 ; S. Jones, ibid., p. 327 ; MacKenzie, Voyages, vol. i, p. 148. 
River District is especially mentioned in the account of Jacobsen's journey. ${ }^{1}$ Among the Chinooks Lord says it is 'not uncommon ' 2 and Bancroft that it is of 'frequent occurrence '. 3 The Thompson Indians seldom kill their children, and a woman who did so was reprimanded. 4 With the Haidahs, on the other hand, infanticide is reported to be "not uncommon ', 5 and among the tribes between the Frazer and Columbia Rivers it was formerly common. ${ }^{6}$ The same impression is gained from accounts of the Chepewayans. ${ }^{7}$ The Koniagas 'prize boy babies, but frequently kill the girls ' .8 Powers, referring generally to the Californians, speaks of 'the prevalence of the crime of infanticide ' ; ${ }^{9}$ in particular he records that it was commion among the Gallinomero, who never spared deformed children, ${ }^{10}$ that among the Kabinapek ${ }^{11}$ and the Nishinan ${ }^{12}$ shildren who had lost their mother were killed, and that deformed children were destroyed by the Woruk. ${ }^{13}$ Among the Yguazes infanticide was fairly frequent. ${ }^{14}$ The Abipones of South America ' do not bring up more than two children to a family, the others being killed to save trouble;' 15 According to another observer ' they seldom rear but one child of each sex, murdering the rest as fast as they come into the world, till the eldest are strong enough to walk alone '.16 This practice, on the other hand, is said to be 'extremely rare' among the Botocudos..$^{17}$ It occurs among the Guaycurus. ${ }^{18}$ Among the Puelches infanticide is common and deformed children are always killed.19 - In spite of what has sometimes been said, infanticide 'only occurs occasionally' among the Fuegians. ${ }^{20}$ In the literature with regard to the Veddahs reviewed by the Sarasins, there is only one author who mentions infanticide. ${ }^{21}$

10. $\operatorname{Wars}^{22}$ were apparently common between the Tasmanian

1 Woldt, Kapitän Jacobsen's Reise, p. 393.

2 Lord, loc. cit., vol. ii, p. 231.

3 Bancroft, loc. cit., vol. i, p. 242.

loc. cit., p. 198 ; Bancroft, loc. cit., vol. i, p. 169.

p. 169.

7 Keating, loc. cit., p. 160 .

5 Gibbs,

9 Powers, loc. cit., p. 416; Bancroft, loc. cit., vol. i, pp. 390 and $413 .{ }_{10}$ Ibid.,
p. 177.
11 Ibid., p. 222.
12. Ibid., p. 328.
13 Ibid., p. 382.

${ }_{14}$ Cabeça de Vaca, loc. cit., p. 62. According to Ten Kate (Rev.d'Eth., vol. iv) the Comanches kill one of two twins. This is not an uncommon custom: in itself, however, it can have very little effect upon the quantity of the population. For the Californians see Bancroft, loc. cit., vol. i, pp. 390 and 413.

${ }_{15}$ Dobrizhoffer, loc. cit., vol. ii, p. $97 . \quad{ }^{16}$ Charlevoix, Histoire, vol. i, p. 405. 17 Keane, loc. cit., p. $206 . \quad{ }_{18}$ Church, loc. cit., p. $248 . \quad{ }_{19}$ Guinnard, loc. cit., p. 143. $\quad{ }_{20}$ Westermarck, loc. cit., p. 313, quoting a letter from Mr. Bridges who in another place (loc. cit., p. 181) says that deformed children are especially likely to be killed. $\quad 21$ Sarasins, loc. cit., vol. iii, p. 469.

${ }_{22}$ It should be remembered that elimination through war tells more upon males 
tribes, but we have no trustworthy information as to their character. ${ }^{1}$ Of conditions in Australia we have abundant evidence ; the literature has been recently studied by Wheeler. ${ }^{2}$ He distinguishes between regulated and unregulated warfare, of which the latter is rare. $^{3}$ By regulated warfare is meant not only that the use of heralds, messengers, and preliminary negotiations is recognized, but also that the fighting itself is regulated in such a manner as to restrict bloodshed. Most commonly, if it comes to fighting, the two opposing sides meet and throw boomerangs until one or two men are knocked down. Then, before further damage is done, fighting stops and peace is re-established-not a single life perhaps having been lost. 4 Unregulated warfare is a more serious matter ; a war party may attack and destroy a local group of some other tribe without observing any of the formalities described above. ${ }^{5}$ In the latter case women are sometimes slain, although this never happens in regulated warfare. 6 On the whole the loss of life owing to warfare in Australia must be very small.

The Bushmen gained the reputation of being vigorous fighters during the wars with the colonists and managed to inspire terror throughout a large part of Africa. We have, however, but little information as to their habits before they were so rudely disturbed and, it must be said, so barbarously treated. "They never appear', says Stow, 'to have had great wars against each other ; sudden quarrels among rival huntsmen, ending in lively skirmishes, which owing to their nimbleness and presence of mind, caused little damage to life or limb, appear to have been the extent of their individual and tribal differences.' 7 Of the Eskimos about the Behring Strait Nelson says that 'an almost continuous intertribal warfare' formerly existed ${ }^{8}$ such a state of things is clearly uncommon; in Greenland, for instance, we are told that war is rare, ${ }^{9}$ whereas among the Central Eskimos real wars have never happened. ${ }^{10}$ Nevertheless a good deal of fighting seems often to occur between the Eskimos and the Tinneh, whom they hate and fear. 'Along the line of contact with the Tinneh tribes of the

than females. Out of sixty-six cases in which there was the relevant information investigated by Professor Hobhouse and his collaborators, men only were slain in twenty cases (Hobhouse, Wheeler, and Ginsberg, Simpler Peoples, p. 232).

1 Ling Roth, loc. cit., p. $83 . \quad{ }_{2}^{2}$ Wheeler, Tribe in Australia. ${ }_{3}^{3}$ Ibid., pp. $116 \mathrm{ff}$. Curr (Recollections, p. 309) says that he never heard of any one being killed in a regular battle. 5 Wheeler, loc. cit., p. 151.6 Ibid., p. 154. 'Stow, loc. cit., p. 38. Letourneau, however, says that war was common (Ia Guerre, p. 54). ${ }^{8}$ Nelson, loc. cit., p. 327. ' Nansen, Eskimo Life, p. 162.10 Boas, loc. cit., p. 465. 
Interior a bitter feud was always in existence,' says Nelson, ${ }^{1}$ who is confirmed by Bancroft, ${ }^{2}$ Hall, ${ }^{3}$ and Ellis. ${ }^{4}$ Of the Malemutes we are told that "the occupants of the several islands are almost constantly at war'.5

It is among the American Indians both in the North and South that we find war to be more frequent and sanguinary than elsewhere. Certain tribes are more peaceful than others, but in the lives of only a few did war play an unimportant part. In addition to the evidence of actual fighting we have, what is suggestive in this connexion, many accounts of the large part which martial training played in the upbringing of these races. "The whole force of public opinion in our Indian communities', says Schoolcraft, ' is concentrated upon this point, its early Lodge teachings, its dances, its religious rites, the harangues of prominent actors made at public assemblies, all, in fact, that serves to awaken and fire ambition in the mind of the savage, is clustered about the idea of future distinction in war.' 6 'They are', says an eighteenthcentury traveller, ' early possessed with the notion that war ought to be the chief business of their lives.' " And war does play a very large part in their lives. 'All Indian tribes are frequently at war with one another,' says Harmon. ${ }^{8}$ The Thlinkeet are often at war ${ }^{9}$ and the same applies to all the tribes of the Pacific Coast, including the Haidahs, ${ }^{10}$ the Ahts, ${ }^{11}$ and the Kwakiutl. ${ }^{12}$ Among some of these tribes, however, as, for example, the Chinooks, ${ }^{13}$ frequent as fighting may be, it is not very sanguinary. The Northern tribes of the interior we have seen to be at war with the Eskimos from time to time, and we are told that among themselves it is practically continuous. ${ }^{14}$ The Inland tribes are perhaps somewhat less warlike, ${ }^{15}$ though the accounts of the Shushwap, ${ }^{16}$ Lillooets, ${ }^{17}$ and Thompson Indians ${ }^{\mathbf{1 8}}$ give the impression of tribes among whom fighting - and fighting of a severe kind-is by no means infrequent.

1 Nelson, loc. cit., p. 237. $\quad 2$ Bancroft, loc. cit., vol. i, p. $120 . \quad{ }^{3}$ Hall, loc. cit., p. 598. $\quad$ Ellis, loc. cit., p. 182. ${ }_{5}^{5}$ Bancroft, loc. cit., vol. i, p. 91. ${ }^{6}$ Schoolcraft, loc. cit., vol. ii, p. 57. See also ibid., vol. iii, p. 64. 7 Carver, Travels, p. 229. For accounts of early military training see Domenech, Seven Years' Residence, vol. ii, p. 229, and Dodge, Hunting Grounds of the Great West, p. 256. ${ }^{8}$ Harmon, Journal, p. 306. He is speaking in particular of the Carrier tribe ; a good account of the methods of fighting is given. ${ }^{2}$ Krause, loc. cit., p. 248 ; Swanton, 26th A. R. B. E., p. 449. ${ }_{10}$ Niblack, A. R. S. I., 1888, p. 340; Swanton, Jesup North Pacific Expedition, vol. v, pp. 55 ff. 1 Sproat, loc. cit., p. 59. ${ }_{12}$ Boas, A.R.B.E., 1895, p. $425 .{ }^{13}$ MacKenzie, loc. cit., vol. ii, pp. 123-30. ${ }^{14}$ Bancroft, loc. cit., vol. i, p. 236. ${ }_{15}$ Ibid., vol. i, p. $268 .{ }_{16}$ Teit, loc. cit., vol. ii, pp. $540 \mathrm{ff.}{ }^{17}$ Ibid., p. 234. ${ }^{16}$ Ibid., vol. i, p. 263. 
The Crees and the Blackfeet, for instance, waged 'unceasing war' ${ }^{1}{ }^{1}$ the warlike instinct of the Chepewayans is well known. ${ }^{2}$ The tribes of Western Washington and Northern Oregon engaged in constant warfare, though the actual encounters did not result in any great loss of life. ${ }^{3}$ In Central California 'battles, though frequent, were not attended with much loss of life '.4 In Southern California we hear of 'frequent and deadly wars' 5 from one author and of 'continual wars' from another, ${ }^{6}$ the last laying stress upon warfare as an important factor in the population question. The Seri Indians of Tiburon Island engage in practically continuous warfare," and the Comanches 'highly honour bravery on the battlefield'. From early youth they are taught the art of war. ${ }^{8}$ 'In consequence of continual hostilities' the Ygauzas ' cannot travel the country nor make many exchanges '. 9 Among the Puelches war is both common and sanguinary, ${ }^{10}$ and the same is the case with regard to the Guaycurus ${ }^{\mathbf{1 1}}$ and the Charruas. ${ }^{12}$ The Fuegians are 'almost always at war with adjoining tribes; they seldom meet, but a hostile encounter is the result '. ${ }^{13}$

11. Passing to the consideration of feuds it is found that they originate largely from motives of blood revenge. The expression blood revenge suggests retribution for a murder actually committed, but among primitive races the origin of blood revenge is far more often the natural death of some member of the tribe. Owing to the almost universal belief among these races that death is never natural in our sense of the word, the loss of a relation or fellowtribesman is attributed to magical influence, and the guilt fastened upon some individual by a process of magical divination. It appears that women are judged to be equally capable with men of causing death, and that therefore elimination from this cause falls equally upon both sexes.

' No such thing as natural death is recognized by the [Australian] natives; a man who dies has of necessity been killed by another man or perhaps even by a woman.' 14 'If an Abipon die from

1 Catlin, loc. cit., vol. i, p. 53.

2 MacKenzie, loc. cit., vol. i, p. 171. 3 Gibbs, loc: cit., p. 190.

- Bancroft, loc. cit., vol. i, p. 379.

5 Ibid. vol. i, p. 562. 'Baegert, loc. cit., p. 359. 'MacGee, 17th A.R. B. E., pp. 157 and 273. He speaks of a 'frequent decimation of the warriors' (p. 273). 8 Bancroft, loc. cit., vol. i, p. 499.

- Cabeça de Vaca, loc. cit., p. 54. 10 Guinnard, loc. cit., p. 125. ${ }^{11}$ Azara, vol. ii, p. $146 .{ }^{12}$ Ibid., p. 7. 13 King and Fitzroy, Narrative, vol. ii, p. 183. See also Featherman, Social History, vol. iii, p. 508 .

14 Spencer and Gillen, Native Tribes, p. 45. To show the length to which this 
being pierced by many wounds, or from having his bones broken, or his strength exhausted from extreme old age, his countrymen all deny that wounds or weakness occasioned his death, and anxiously try to discover by which of the jugglers and for what reason he was killed.' 1 Among different races the holding of this belief has different results. With regard to Australia, Messrs. Spencer and Gillen in the passage quoted above continue as follows : 'sooner or later that man or woman will be attacked. In the normal condition of the tribe every death meant the killing of another individual '-the guilty person being indicated by a medicine man. ${ }^{2}$ The avenging party is at times merely directed by the medicine man to proceed in a certain direction and after a march, it may be of a considerable distance, discovers another local group which it entirely wipes out. ${ }^{3}$ At other times an individual near by is indicated and at once dispatched. It is obvious that if such results commonly followed upon the death of every tribesman the population would rapidly diminish, and, as a matter of fact, serious as the consequences of this belief are, there are many indications that blood revenge is not carried out with the extreme rigour that the above passage would seem to indicate. Probably most deaths go unavenged, ${ }^{4}$ yet in spite of this blood revenge must be accounted as a factor of importance in Australia, ${ }^{5}$ especially as it affects women as well as men. ${ }^{6}$

Among the races belonging to the second group we shall meet with a somewhat similar state of affairs. But, widespread as this belief is, it does not appear to provoke bloodshed among the other races belonging to this group on any large scale. We hear of feuds among the Eskimos and the punishment of witchcraft by death. Murder is also reported as occurring among the Eskimos. "It is

belief is carried, the following story may be quoted. 'A woman, while clearing out a well, was bitten in the thumb by a black snake. It began to swell immediately, and in the short space of twenty-four hours the woman was a corpse. Still it was asserted that it was not an accident, but that the deceased had pointed out a certain aboriginee as her murderer' (Wilhelmi, loc. cit., p. 191). In this case no killing actually followed, though it nearly did so.

1 Dobrizhoffer, loc. cit., vol. ii,.p. 84. 2 Spencer and Gillen, Native Tribes, vol. ii, p. 45 . ${ }^{3}$ Curr, Australian Race, vol. i, p. 86.

- Spencer and Gillen, Native Tribes, p. 477. Wheeler (loc. cit., p. 149) says that ' warfare only arises as a result of a blood feud, due to the killing of a member of one local group by a member of another local group, nearly always by magical means. But even this ground of offence is most generally settled by one of the methods of regular procedure above described.' Since, as we have seen, the regular procedure seldom ends in the shedding of blood, many deaths thus pass unavenged.

5 Thomas, Natives of Australia, p. 47 ; Eyre, loc. cit., vol.ii, p. 379. $\quad{ }^{6}$ Curr, Recollections, p. 317. 
not a rare occurrence that a man who is offended by another man takes revenge by killing the offender,' and a feud then follows which may last for generations. ${ }^{1}$ Blood feuds originating in actual murder are also recorded of various Indian tribes $;^{2}$ but it does not appear that bloodshed, owing to belief in witeheraft, occurs on any significant scale either in North or South America. Of the Haidahs we are told that ' death is ascribed to the ill-will and malign influence of an enemy and one suspected of causing the death of a prominent individual must be ready to die ' ${ }^{3}$ Niblack, speaking of the Coast tribes from Southern Alaska to British Columbia and thus including the Haidahs, says: 'all severe diseases or illnesses are ascribed to the evil influence of enemies, and in the case of the death of an important personage, a victim is usually found who has presumably charmed away the life of the deceased.' 4 The implication is, of course, that a victim is only sought on rare occasions, as on the death of a prominent man.

12. The custom of killing the old and the sick is of sufficient importance to deserve mention. Among these races it is not uncommon and is connected with the nomadic nature of their existence. ${ }^{5}$ The Tasmanians would seem to have been in the habit of abandoning the sick and infirm. ${ }^{6}$ There is some evidence of this habit in Australia, ${ }^{7}$ but on the whole sick and aged are well looked after in that country.8 "The Bushmen frequently forsake their aged relations when removing from place to place for the sake of hunting. In this case they leave the old person with a piece of meat and an ostrich egg-shell full of water; as soon as this little stock is exhausted, the poor deserted creature must perish by hunger or become the prey of wild beasts.' 9 The aged are not treated well by the Eskimos. In the Ungava district they are put to death, ${ }^{10}$ and among the Central Eskimos a man may kill

1 Boas, 6th A. R. B. E., p. 582. Similar accounts are given by other authors: see Nelson, loc. cit., p. 293 ; Rink, loc. cit., p. 35 ; Klutschak, Als Eskimo unter den Eskimos, p. 228. 2 For instance, the Lillooet (Teit, loc. cit., vol. ii, p. 236) and the Shastika (Powers, loc. cit., p. 29). ${ }_{3}$ Bancroft, loc. cit., vol. i, p. 348. - Niblack, loc. cit., p. 348. A somewhat similar account is given of the Zaporos (Simson, J. A. I., vol. vii, p. 506).

- It is not only among these races that the aged are thought to be in the way. Bonnard, in an article in the Figaro on the Prince de Beauvais' travels in Canada, mentions that the latter saw an enterprising Canadian township recommended by the announcement that there were 'no old inhabitants to hinder progress' (Figaro, March 28, 1914). The evidence for the existence of this custom has been reviewed by Westermarck, Moral Ideas, vol. ii, pp. $386 \mathrm{ff}$.

6 Ling Roth, loc. cit., p. 73. 'Sartori, Globus, vol. Ixvii, p. $108 . \quad$ S See, for instance, Spencer and Gillen, Northern Tribes, p. 32, and Natives Tribes, p. 51 .

- Theal, loc. cit., p. $19 . \quad 10$ Turner, loc. cit., p. 186. 
his aged parents.' ' On the East coast (of Greenland) it sometimes happens that old people, who seem likely to die are drowned, or else drown themselves.' 2 For the most part the Indians treat their sick and aged well; but there are some instances to the contrary. The Queka Indians abandon the sick and aged. ${ }^{3}$ The Ahts, ${ }^{4}$ the tribes of Washington, ${ }^{5}$ and the Chepewayans ${ }^{6}$ did the same. The Central Californians killed the old people, ${ }^{7}$ and, according to MacGee the Seri ' often abandoned the sick and aged who cannot keep up with them in their constant wanderings to and fro' 8 In some of the accounts of the Fuegians similar habits are attributed to them ; there is, however, some reason to doubt their accuracy. ${ }^{9}$ These practices certainly occur among the Zaporos. ${ }^{10}$

13. We have now to mention certain other modes of elimination. That form of elimination, which we saw to be of great importance among animals in a state of nature whereby the young perish on a large scale because they are consumed by other species, is of little importance among men who have reached the stage of the lowest races known to us. ${ }^{11}$

The subject of elimination from disease can be approached from different aspects. We can ask what evidence there is regarding the prevalence of disease among primitive races when they first came under the observation of Europeans. This evidence is of somewhat doubtful value. It is also of assistance to ask how far these races were immune from diseases prevalent in Europe; for it is in general to be inferred that marked liability to disease is a sign that no immunity has been evolved against disease because it was previously absent. We can further consider the question of the evolution of disease in general and ask to what conclusions it points. The latter aspect of the matter can be taken first.

${ }^{1}$ Boas, 6th A. R. B. E., p. $165 . \quad{ }^{2}$ Nansen, Eskimo Life, p. 151. ${ }^{3}$ Woldt, loc. cit., p. 57. 'Bancroft, loc. cit., vol. i, p. 205. A. R. S. I., 1886, p. $274 . \quad{ }^{6}$ Long, loc. cit., p. 74.

7 Willoughby, sancroft, loc. cit., the Y. p. 390. vol. iii, p. 503.10 Simson, loc. cit., p. 507.

11 It is impossible to estimate the amount of elimination from this source. It is, as a rule, no doubt small. In India, although a higher degree of skill has been reached, elimination from this source is not inconsiderable owing to the prevalence of poisonous snakes. 'In 1910, 55 persons were killed by elephants, 25 by hyenas, 109 by bears, 319 by wolves, 853 by tigers, and 688 by other animals, including wild pigs. No less than 22,478 died from the bite of poisonous snakes. The grand total of mortality is 24,878 (Lull, Organic Evolution, p. 105). The amount of elimination will vary according to the nature of the fauna, the geographical surroundings, habits, and other factors. Thus among the Eskimo the use of the 'kayak' is responsible for a considerable number of deaths. See Crantz, loc. cit., p. 166 ; Nansen, loc. cit., p. 55 ; Boas, 6th A. R. B. E., p. 433. 
The organisms which cause disease do not belong all to one class. Some, such as the bacteria, are plants, others are animals. Further, those species which cause disease are closely related to other quite innocuous species. Non-virulent diphtheroid bacteria are, for instance, found in the throat. What has happened has been that certain species, belonging to different groups in the animal and vegetable kingdoms, have taken to a parasitic mode of life. It is not very difficult to imagine how this could occur. It is to be presumed that organisms now parasitic were once freeliving and saprophytic. There is a bacillus which lives on grass ; it is closely related to the tubercle bacillus but is harmless. Such an organism might be frequently swallowed; if liable to destruction, resistance might be evolved. ${ }^{1}$ Its presence might be innocuous, or it might not be so and a new disease might be the consequence. The invasion of a host by a parasite appears usually to be followed by a struggle - on the part of the host to get rid of the parasite, and on the part of the parasite to maintain itself within the host-in the course of which struggle the parasite may develop activities noxious to the host. To this struggle there are different possible solutions. But, when we find the struggle in progress, it is a fair assumption that the association of host and parasite is recent. The very fact of disease, therefore, suggests that it is of relatively recent origin.

There are two further facts which are relevant in this connexion. Diseases are very rare among species in a state of nature. Such diseases as we know of among animals and plants occur for the most part among domesticated species. Again, there are certain conditions which favour the evolution of disease ; these conditions are found among domesticated species and also among civilized man. There are many ways in which disease can be transmittedby insects, by liquid particles in the air, by drinking water, by water used for bathing, by water introduced within the tissues, and so on. Transmission by all these means, with the partial exception of transmission by insects, is obviously very greatly favoured by the aggregation of the members of the host species. In fact only when such aggregation occurs can we understand how disease on a large scale could be evolved. The aggregation of men, such as occurred within the third period, into villages and towns, gave rise to conditions under which bacteria could flourish

\footnotetext{
1 Cohen, Physiological Therapeutics, vol. v, p. 158.
} 
in close proximity to man; under these conditions there was far more opportunity for bacteria and other micro-organisms to become parasitic than before. The suggestion, therefore, is that most diseases evolved in that relatively recent period when, owing to the increase in skill, men came to live in close proximity under settled conditions.

This suggestion is supported by two other lines of evidence. It would appear that Australia and America were upon their discovery free from most of the diseases known in Europe. With regard to the former Davidson says: 'Australia presents us with a spectacle of a continent, from the pathology of which entire classes of diseases, prevalent in other divisions of the globe, were, until comparatively recent times, completely absent. Thus the whole class of eruptive fevers-small-pox, scarlet fever, and measles-so fatal elsewhere, were unknown. Epidemic cholera, relapsing fever, yellow fever, whooping cough, and diphtheria were equally absent, as also was syphilis. . . . Leprosy was absent from the southern continent.' 1 The facts with regard to America are very similar. It has been asserted that the only lethal disease of importance present in America before the visit of Columbus was malaria, and it is worthy of note that malaria is an insect-born disease. The question whether tuberculosis was known in America before the discovery has been studied by Hrdlicka, who concludes that, though it cannot be affirmed that it did not exist, it is highly improbable that it did. ${ }^{2}$ Again, the marked liability on the part of primitive races to common European diseases points strongly to the fact that no immunity had been evolved against these diseases because they were formerly unknown. Since contact with Europeans has been established, these races have been swept by epidemics, such as the epidemic of measles which carried off a large proportion of the population of Fiji in 1875 .

It is not to be concluded that most diseases are recent in the ordinary sense of the word, but only when a broad view is taken of human history. It is known, for instance, that tuberculosis, plague, leprosy, and bilharzia existed in ancient Egypt. Where and when diseases originated we cannot say, but the fact that certain widely-spread diseases can be traced to a previously limited and localized incidence-cholera, for instance, to the Ganges Valley-suggests that such diseases originated in those places in

1 Davidson, Geographical Pathology, vol. ii, p. 565. Bull. 42.

${ }^{2}$ Hrdlicka, S. I. B. E., 
relatively recent times and spread thence. But in spite of such indications the place of origin of most diseases is obscure ; syphilis was often said to have been introduced from America; the evidence, however, that it was apparently present in Germany in 1495 is against this view. ${ }^{1}$ It is further of great interest to note that certain diseases may have evolved in very recent days. Diphtheria may date from the beginning of the last century and trench fever may be a new disease.

Little as we may know regarding the facts concerning any one disease, the general conclusion is not doubtful. It was not until the third period, and, therefore, only among the races of the third group, that disease became a factor of the first importance in elimination. Some diseases may have reached the African races through Egypt; and to the extent to which this has occurred they are not typical representatives of the time preceding the last of the three periods. ${ }^{2}$

14. In further support of this conclusion some passages may be quoted regarding the races in this group when they had been little influenced by contact with Europeans. Such passages are perhaps not of great weight as evidence of the former absence of disease ; they are, however, worth notice because they also bear upon another subject that will come up for discussion in the ninth chapter. According to Bonwick 'there are strong reasons to believe that, before connexion with the whites, the aborigines [Tasmanians] were a healthy as well as a happy people ' ${ }^{3}$ Of the Australians Curr says that 'as a rule the health of the blacks in their wild state was excellent ' 4 Longevity may be considered as evidence of good health, and of the presence of aged people in Australia many observers speak. 'From numerous instances it would appear that the former generations were fairly long aged. Almost every small community would have in it two or three men or women over seventy years of age, and here and there some centenarians would be met with.' ${ }^{5}$ So, too, Burchell records having noticed many old people among the Bushmen. ${ }^{6}$ Writing of the Eskimos in a medical journal Smith calls them 'uncommonly healthy '.7 This is the opinion that one gains from other accounts, ${ }^{8}$

1 Hrdlicka, S. I. B. E., Bull. 34. See also Adami, Medical Contributions, pp. 1522. ${ }^{2}$ Among many races in this group-especially in tropical countries-certain non-lethal diseases are prevalent which are of great importance from another point of view. They will be referred to again in Chapter XV. ${ }^{3}$ Bonwick, loc. cit., p. 87. $\quad$ Curr, Recollections, p. $282 . \quad 5$ Mathew, loc. cit., p. 92. See also Eyre, loc. cit., vol. ii, p. 377, and Curr, Recollections, p. $297 .{ }^{\circ}$ Burchell, Travels, vol. ii, p. 57. ${ }^{7}$ Smith, loc. cit., p. 859. $\quad{ }^{8}$ Murdoch, loc. cit.,p. 39 
some of which specially mention longevity as a characteristic. ${ }^{1}$ 'The North Americans are in general robust and of a healthful temperament, calculated to live to an advanced age.' 2 Another author says that the Indians east of the Rocky Mountains are 'in general subject to few diseases ' ${ }^{3}$ Krause quotes the opinion of a doctor who lived among the Thlinkeets in the year 1836 to the effect that they were a strong, healthy people. ${ }^{4}$ Of the Shushwap we are told that they were formerly healthy and lived to a great age. $^{5}$ Hill Tout sums up the situation with regard to the Salish as follows: " the great age, to which both men and women formerly lived, shows the vigour of the race and the general wholesomeness of their lives and condition.' 6 'The Nootkas are generally a long-lived race, and from the beginning to the failing of manhood undergo little change in appearance. Jowitt states that during his captivity of three years at Nootka Sound, only five natural deaths occurred, and the people suffered scarcely any disease except the cholic.' 7 Powers, referring to the Californians as a whole, calls them 'a healthy, long-lived race ', 8 and Baegert speaks of them as 'strong, hardy, and much healthier than the many thousands who live in daily abundance' 9 [in civilized countries]. Among the Abipones 'the diseases which in Europe fill houses with sick persons and graves with the dead bodies are unknown here. . . . You scarce hear once in three years of any of them dying of a fever, pleurisy, or consumption.' 10 Hardt mentions the good health of the Botocudos ${ }^{11}$ and King and Fitrroy consider the Patagonians to be very healthy. ${ }^{\mathbf{1 2}}$

15. There remains one other matter to be mentioned. There is abundant evidence that the rate of child mortality is very high amongst all these races. The causes of death are various ; disease is seldom mentioned, and death is most often due to exposure as the result of improper treatment or of certain customs, or to want of suitable food. In Tasmania it was difficult to rear children largely owing to the fact that suitable food was not available. ${ }^{13}$ Turner says of the Eskimos of the Ungava District that ' many die in early childhood ',14 and this would seem to be generally true of

\footnotetext{
1 Crantz, loc. cit., p. 166.

2 Heriot, loc. cit., p. 350 .

3 Harmon, loc cit., p. $271 . \quad 4$ Krause, loc. cit., p. 148

6 Hill Tout, British North America, p. 252.

${ }_{5}^{5}$ Teit, loc. cit., vol. ii, p. 618 .

8 Powers, loc. cit., p. 416. loc. cit., vol. ii, p. 219.

$\checkmark$ Baegert, loc. cit., p. 385 . 11 Hardt Geography of Brazi Dobrizhoffer, and Fitzroy, loc. cit., vol. ii, p. 155.

13 Bonwick, loc. cit., pp. 78 and 85.

14 Turner, loc. cit., p. 189 and Hutton, loc. cit., p. 80.
} 
this race. The reason usually given is the absence of proper food ; 1 and it is not difficult to understand that the peculiar Eskimo diet should be unsuitable for children. Child mortality is heavy among the Indians, ' only a small proportion coming to maturity '.2 The reason given in this case is generally the absence not only of knowledge with regard to the simplest requirements of children, but of any reasonable care of them. ${ }^{3}$ To this we may add-as the evidence will show-the practice of extraordinary customs which seem designed to eliminate all but those with the strongest constitutions. 'With all the affection of the mother, the women are almost completely ignorant of ordinary sanitary rules as to feeding, exposure, \&c., with the result that infant mortality is something terrible in almost every tribe.' 4 Heriot speaks of 'incredible fatigues, whose excess occasions the death of many long before the age of maturity'.5 According to Domenech : ' many Indians die in infancy; their mothers, to inure them to suffering and to strengthen their constitution, do not take all the necessary care of them. . . . Till the age of ten or twelve years they are kept quite naked, having only in winter a garment which we would hardly call such in the warmest summer.' 6 Throughout America it is a common custom to bathe even new-born children in cold water at all seasons of the year, and to this Krause attributes the high child mortality that he records among the Thlinkeet." 'Many children [of the Eastern Tinneh] die at an early age,' according to one author, ${ }^{8}$ whilst from another we hear that ' the infant is not allowed food until four days after birth, in order to accustom it to fasting in the next world '.9 Nootka mothers ' roll their children in the snow to make them hardy ', ${ }^{10}$ and the Thompson Indians take small care of their children, allowing them to run about without any protection. ${ }^{11}$ Of the Californians the Jesuit missionary Baegert, who dwelt long among them when they were almost uninfluenced by European culture, says : " that many infants die among them is not surprising ; on the contrary, it would be a great

1 Crantz, loc. cit., p. 162 ; Murdoch, loc. cit., p. 415.

${ }^{2}$ Handbook of American Indians: Article, Child Iife.

3 The absence of care and the resulting mortality is emphasized by Gerland (Über das Aussterben der Natur. volker, p. 24 to p. 39). $\quad$ Handbook of American Indians: Article, Child Life. It may be noticed that, among the older authors, Robertson (loc. cit., vol. i, p. 297) has very similar remarks on the same subject. '5 Heriot, loc. cit. p. 344. - Domenech, loc. cit., vol. ii, p. $295 .{ }_{7}$ Krause, loc. cit., p. 217. Of these people Bancroft (loc. cit., vol. i, p. 111) says that "when the child is able to leave its cradle, it is bathed in the ocean every day without regard to season'. B Ross, loc. cit., p. $305 . \quad$ Bancroft, loc. cit., vol. i, p. 121.

p. 201 .

11 Teit, loc. cit., vol. i, p. 178.

10 Ibid., 
wonder if a great number remained alive. For when the poor child first sees the light of day, there is no other cradle provided for it but the hard soil, or the still harder shell of a turtle, in which the mother places it, without much covering, and drags it about wherever she goes. And in order to be unencumbered and enabled to use her limbs with greater freedom while running in the fields, she will leave it sometimes in charge of some old woman, and thus deprive the poor creature for ten hours or more of its natural nourishment. As soon as the child is a few months old the mother places it, perfectly naked, astraddle on her shoulders, its legs hanging down on both sides in front, and it has consequently to learn how to ride before it can stand on its feet. In this guise the mother roams about all day, exposing her helpless charge to the hot rays of the sun and the chilly winds that sweep over the inhospitable country.' 1 Conditions seem to be much the same in South America. Dobrizhoffer tells us that the Abipones plunge their new-born babies into a cold stream, ${ }^{2}$ and Guinnard says that very few diseases occur among the children of the Puelches, though child mortality is high. ${ }^{\mathbf{3}}$ ' Few [Fuegian] women save all their children ; most die in early infancy.' 4 So, too, among the Andamanese child mortality is said to be excessive ${ }^{5}$ and is ascribed to injudicious management on the part of the parents. ${ }^{6}$ According to the Sarasins it is the high death-rate that is the cause of the small size of the Veddah families which they observed. ${ }^{7}$ New-born Ghiliak children are bathed ' often when it is $40^{\circ}$ below zero. The children who can survive such an experience are necessarily very strong.' 8 'According to Schrenck, the Ghiliak woman never dares " to give birth to a child at home; she must, in spite of severity of season or of stormy weather, go out of the hut for the purpose. In late autumn or in winter they build a special hut for the woman, but a very uncomfortable one, so that the mother and the child suffer the cold and feel the wind "., 9

This concludes our survey of the races of the first group. Before we consider what conclusions are to be drawn from these facts, we have to deal in the same fashion with the races of the second group. This will form the subject-matter of the next chapter.

1 Baegert, loc cit., p. 368.

3 Guinnard, loc. cit., p. 147. loc. cit., p. 79. " 6 Ibid., p. 81.

8 Deniker, loc. cit., p. 303. a general sketch of the treatment of children by parents see Steinmetz, Entwick. lung der Strafe, vol. i, pp. $179 \mathrm{ff}$.

2498
${ }^{2}$ Dobrizhoffer, loc. cit., vol. ii, p. 43.

4 Bridges, loc. cit., p. $202 . \quad{ }_{5}$ Man, 7 Sarasins, loc. cit., vol. iii, p. 469 .

- Czaplicka, Aboriginal Siberia, p. 137. For $\mathbf{L}$ 


\section{VIII}

\section{PRIMITIVE AGRICUITURAL RACES}

1. IT is necessary first to explain what races will be considered. The lower limit has already been made clear. As regards the upper limit, some description has been given of the stage of Neolithic culture, and the races to be considered here are those which may be in general compared with the Neolithic races. The comparison can only be rough ; as we saw, the subsequent evolution of culture was rapid; metals came into use, and, generally speaking, about the time of the first taking of metals into use arose the first great empires. At this point we clearly enter upon a new epoch and the upper limit in time is defined by the rise of Eur-Asiatic civilization. We wish to study here the conditions anterior to this step in the evolution of culture. It so happens that this Eur-Asiatic culture has influenced in varying degrees many of the races of Africa, Oceania, and Asia. The upper limit among the races to be considered here is best defined by the degree to which they have been influenced by this culture. America stands apart as there has clearly been no influence of this culture upon the races in that continent. But it should be remembered that in some places in America a degree of culture was reached which was probably higher than that to which any Neolithic race attained. ${ }^{1}$ Nevertheless all the races of America not already dealt with may be considered here. Many African races are acquainted with the use of iron; it has been suggested that the knowledge of the use of this metal originated in Africa ; this, however, is doubtful and the existence of this practice is probably to be regarded as due to the spread of an element of Eur-Asiatic culture. The use of the plough is also an exaniple of the spread of an element of this culture $;^{2}$ roughly speaking

1 Reference has been made in Chapter $V$ to the use of metals in America. Mr. Joyce, however, says that in spite of progress in this direction the Central American races 'were living at the discovery practically in an age of stone' (Mexican Archaeology, p. 304).

2 The importance of the plough in agriculture has been emphasized by many authors. See, for instance, Hahn, Die Entstehung der Pflugkultur. 
the use of the plough does not spread towards the west, south of the latitude of Lake Chad ; towards the east it extends farther south, and on this account the Gallas, Somalis, and Abyssinians can scarcely be considered as representative of the second group of races. ${ }^{1}$ The peculiarity of the Oceanic region is that many of the races now found there undoubtedly migrated from some point on or close to the Asiatic coast after Eur-Asiatic culture had reached a high degree of development. Nevertheless they are for the most part ignorant of the use of metal and of the plough. ${ }^{2}$ The Dyaks are metal users and the plough is known in parts of Borneo. We shall not on this account, however, exclude the Dyaks from consideration here. The true Malays on the other hand will be excluded; they are Mohammedans and, originally located in Sumatra, spread in the twelfth century over a large part of this area. On the fringe of Asia are a number of races which, though in a broad survey are of no great importance, may be noticed here. Such are the Ostiaks, Yakuts, Chuckchees, Samoyeds, and so on. ${ }^{3}$ What races are dealt with here may be made more clear by a reference to those which will be considered in Chapters X and XI. In those chapters we shall consider the ancient empires, the classical races, mediaeval and modern Europe and its derivatives as well as the chief races of Asia, whether pastoral such as the Arabs or existing by a higher form of agriculture than that practised by the races to be noticed here. In this manner we shall obtain a broad view of the conditions both before and after the rise of Eur-Asiatic civilization.

It is obvious that we have before us a very large mass of material - too large, in fact, to be dealt with without further subdivision. It might seem that the only reasonable course is to distinguish between various grades of agricultural progress, and to consider in turn those races which fall into each grade. Such distinctions have been made by Professor Hobhouse and his collaborators, who recognize three grades of agriculture and two grades of pastoral nomadism. As has already been indicated, we do not

1 The influence of different phases of Eur-Asiatic culture upon the races of Africa must be borne in mind. Early Egyptian civilization exerted great influence and so at a later date did the Semitic-especially on the east coast; since the time of Mohammed the influence of Eur-Asiatic culture has been marked among the more northern of the negroid races. The inhabitants of Bornu were, for instance, converted to Islam in the eleventh century.

2 They may have had a knowledge of iron before the migration (see Thomson, Fijians, p. 11). art-obtaining what they want from the Russians. 
find any correlation between economic stages and the factors bearing upon fertility and elimination. What we do find is a certain correlation between these factors and large geographical areas, and in what follows the races of the second group will be considered according to the geographical areas in which they are situated, and not according to the stage of economic progress which they have reached. The areas selected are as follows. There is, in the first place, America; secondly, there is Africa, and thirdly Oceania. The first two areas are, on the whole, fairly homogeneous; though the third area is not so homogeneous, the level of agricultural skill is fairly uniform-nearly all these races falling into Hobhouse's second grade. Lastly, there are the Asiatic peoples among whom Eur-Asiatic culture has not penetrated ; many of them are inhabitants of the northern fringe of the continent. It will be noticed that the pastoral races chiefly fall within the last group. The so-called pastoral races of America - the Navahos, for instance-have only acquired domestic animals since coming into contact with Europeans. The pastoral races of Africa are with difficulty distinguished from their neighbours, who also raise cattle but practise some agriculture at the same time. It will thus be observed that this mode of classification is, as a matter of fact, roughly in correspondence with the mode of classification based upon the stage of economic progress reached.

\section{America}

2. We have now to examine in turn the races of these four regions. The procedure will be that followed when dealing with hunting and fishing races. As before we begin with examples of intercourse before maturity. This may take the form of marriage before puberty or of less regular connexions. As Mr. Hartland says, 'it would appear that sexual intercourse before puberty is either recognized by a formal marriage or tolerated as the gratification of a natural instinct among a great variety of peoples in all quarters of the globe ' .1 It will be sufficient here to note a few instances of this practice. Of the tribes of Guatemala, Bancroft says that ' marriages take place at an early age, often before puberty ', 2 and the same would seem to be the case among the Navahos. ${ }^{3}$ Accounts of the great laxity of manners and of

1 Hartland, Primitive Paternity, vol. i, p. 272.

2 Bancroft, loc. cit., vol. i, p. 702. $\quad 3$ Stephen, Am. Anth., vol. vi, p. 356. 
early intercourse are given of some North-American tribes-as, for instance, of the Hurons and the Illinois by Charlevoix. ${ }^{1}$ The same is also commonly reported of the Brazilian tribes in such a manner as to leave little doubt that intercourse before puberty is common among them. ${ }^{2}$

3. Generally speaking, throughout America lactation lasted two years or more. ${ }^{3}$ The suckling period indeed sometimes extended over several years; thus among the Sioux it might be continued until the child was five years old $;^{4}$ of the Lengua Indians of the Paraguayan Chaco, Hawtrey says that 'it is customary to suckle children till five or six years of age ' $;{ }^{5}$ Bancroft reports that a child might be suckled until eight years old among the Chichmics. ${ }^{6}$ Two years would appear to be about the minimum ; in Mexico ${ }^{7}$ and Guiana ${ }^{8}$ it was three years or more. D'Orbigny gives three years, ${ }^{9}$ and Forbes one or more, often two, for the Aymara Indians. ${ }^{10}$ In Mexico suckling was said to last three or four years. ${ }^{11}$

4. With regard to postponement of marriage the facts are very similar to those in respect to the races of the first group. As an almost universal rule girls are married soon after puberty, if not before. There is no postponement of marriage of a nature to affect fertility in any noticeable manner. There is at times a certain postponement of marriage among the male part of the population, and occasionally some evidence of lifelong celibacy. This, however, has no bearing upon fertility; it is of interest in another connexion, and we shall on that account return to it later.

5. As noticed in the last chapter there are among nearly all primitive races a number of occasions upon which sexual intercourse is prohibited. Alone among these prohibitions, that against intercourse for some time after the birth of a child is of importance as a factor bearing upon fertility. We shall find that

1 Charlevoix, loc. cit., vol. v, pp. 5 and 38. For the Creeks see Schoolcraft, loc. cit., vol. v, p. 272. $\quad 2$ Pöppig, Reise in Chile, vol. ii, p. 128; von Martius, Ethnographie Amerikas, vol. i, p. 112 ; Azara, loc. cit., vol. ii, p. 104. ${ }^{3}$ Hand. book of American Indians, vol. i, p. 265 . Heriot (loc. cit., p. 344) mentions six to seven years as not uncommon. Schoolcraft (loc. cit., vol. v, p. 655) gives eighteen months to two years for the Oregon Indians. 4 Keating, loc. cit., vol. i, p. 417. ${ }^{5}$ Hawtrey, J. A. I., vol. xxi, p. 295. Grubb gives three to four years (An Unknown People, p. 142). $\quad{ }^{6}$ Bancroft, loc. cit., vol. i, p. 633. 7 Ibid., vol. ii, p. 281.

im Thurn, Savages of Guiana, p. 219; Joest, Int. Arch. Eth., vol. v, p. 94. - D'Orbigny, L'Homme Américain, vol. i, p. 47. ${ }_{10}$ Forbes, Journ. Eth. Soc., vol. ii (new series), p. $224 . \quad 11$ Joyce, loc. cit., p. 162. 
in Africa this prohibition becomes of very great importance. The evidence as to the existence of this prohibition in America is somewhat puzzling. In the last chapter two instances were given in which intercourse during lactation was prohibited in two hunting races. Both these instances are reported by authors who made their observations many years ago when the customs of the American Indians had been less modified by contact with white men than is the case with most of the observations upon which we have to rely. We also find that two other observers, who wrote more than a hundred years ago, record the existence of this custom. It is not clear to what tribes these remarks refer -whether to hunting peoples or to agricultural peoples, or to both. Weld says: "They suckle the few children they have for several years, during which time, at least among many of the tribes, they avoid all connexion with their husbands.' 1 Heriot speaks of 'the length of time employed by the women in rearing their children, whom they nourish for three or four years, during which period they cohabit not with their husbands ${ }^{2}{ }^{2}$ In addition to this evidence we have the following records of the existence of this practice for various agricultural races. Two eighteenthcentury writers--Le Beau, speaking of the Iroquois, ${ }^{3}$ and Charle-

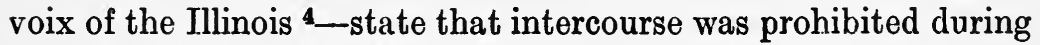
lactation. Speaking of the Crow tribe, Holder mentions that abstention from intercourse during lactation was observed. ${ }^{5}$ In Mexico, according to Bancroft, the suckling period lasted for three years or longer, and during this time there was often no intercourse. ${ }^{6}$ D'Orbigny says of the Moxos and Chiquitos that the mother 'invariably suckles her children for three years or more, during which time she has no relations whatever with her husband '? There do not seem to be any denials of the existence of this practice except in the following instance; in most individual cases there is no mention of the practice. The exception referred to is in the case of the Fuegians. Hyades and Deniker, referring to the statement of D'Orbigny mentioned above, remark that sexual relations are resumed within two months of the birth of a child among the Fuegians. ${ }^{8}$ It is not possible to arrive at

- Weld, loc. cit., p. $373 . \quad 2$ Heriot, loc. cit., p. 339. Aventures, ou Voyage curieux et nouveau, vol. ii, p. 200 . Charlevoix, loc.

3 Le Beau, cit., vol. vi, p. 5. Ashe (Travels, p. 276) says that the Shawnees abstain for nine weeks after birth. $\quad$ Holder, Am. Journ. Obstet., vol. xxv, p. 44. B Bancroft, loc. cit., vol. ii, p. $281 . \quad 7$ D'Orbigny, L'Homme Américain, vol. i, p. 47.

B Hyades and Deniker, loc. cit., vol. vii, p. 195. 
any conclusion as to what the condition of affairs was before the habits of the Indians had been much modified by contact with white men. The fact, however, that the habit is definitely recorded by various authors, who for the most part made their observations at a time when the habits of these races were far less modified than when the more detailed accounts were written from which these races are best known, strongly suggests that this custom was formerly widespread.

There is no evidence of the use of any effective methods to prevent fertilization. We are told that among the Shawnees 'the girls drink the juice of a certain herb which prevents conception, and often renders them barren throughout life' .1 But in those cases which have been carefully investigated it would seem that such methods are not in fact effective. Hrdlicka, for instance, referring to a number of tribes, among whom are the Apaches, Navahos, Pueblos, Pimas, Nahua, Aztec, and Uti, says that " there is a very general belief among the Indians visited that sterility may be artificially induced '.$^{2}$ On investigation, however, it was found that the substances used were quite ineffective.

6. Among agricultural, as among hunting and fishing races, we find numerous references to the small average number of children in a family. Speaking generally of the Indians of the North, Weld states that the number of children is small, ${ }^{3}$ and Le Beau that the number of children born is less than in Europe. ${ }^{4}$ Charlevoix comments on the small size of the family among the.Iroquois and attributes it to early intercourse, abstention from intercourse during lactation, and to prolonged lactation. ${ }^{5}$ Catlin, whose experience was chiefly obtained among the Mandans, has given the estimate quoted in the fourth chapter, ${ }^{6}$ and Holder's estimate for the Crow tribe has also been cited. ${ }^{7}$ Of the Sioux we are told that 'sterility among women is by no means uncommon', 8 and of the south-west Texas tribes 'they are not prolific-a woman seldom having more than three children'.9 In South America the facts are similar. Nordenskiöld visited the tribes between Peru and Bolivia. ' The families are not large,' he says, 'they live in monogamy, and one sees in each family one to three children; in the largest family that I saw (and that was among

1 Ashe, loc. cit., p. 272.

8 Weld, loc. cit., p. 373. voix, loc. cit., vol. vi, p. 5 . cit., vol. i, p. 415.
2 Hrdlicka, S. I. B. E., Bull. 34, p. 163. - Le Beau, loc. cit., vol. ii, p. 199. $p$. 99.

7 p. 99.

5 Charle-

8 Keating, loc. 
the Atsahuaca Indians) there were four children.' 1 Of the Aymara Indians of the same district we are told that 'there seem to be but few large families-very seldom more than four children, and often less than that number '.2 Spix and Martius, travelling in Brazil, 'seldom saw more than four children in a family '.3 Azara was much impressed by the small number of children that he observed in South America. He investigated the matter among the Guaranis with particular care, and found that the average number in a family was four. ${ }^{4}$ Taking into account various factors he came to the conclusion that the number of children born was less than among the Spaniards. ${ }^{5}$ Rengger, who also visited the Guaranis, is of the same general opinion though he estimates the average number in a family to be smaller than does Azara ; ${ }^{6}$ to the Guanas he allows only two or at the most three children. ${ }^{7}$ According to von Martius the marriages of the Macusis ' are not rich in children '.8 D'Orbigny carefully investigated the average number of children in a family among the Moxos and Chiquitos; he found it to be roughly about two in the case of the former and three in that of the latter. $\mathrm{He}$ attributed the small number to early intercourse and also to abstention from intercourse at certain periods. ${ }^{9}$ Two is the average number of children in a family of the tribes of the Upper Hualaga, and marriages are often sterile. ${ }^{10}$

7. We have now to examine the extent of the practices of abortion and infanticide; with regard to the former, we may repeat what was said above-namely, that there is every reason to suppose the methods used are usually effective. Among the Sioux, Keating says that 'married females frequently obtain miscarriages with the knowledge and consent of their husband ';11 this fact is confirmed by Schoolcraft. ${ }^{12}$ It was very common in the Crow tribe. ${ }^{13}$ In a report upon the tribes of the Southwestern United States and Northern Mexico, Hrdlicka states that ' artificial abortion is practised by all the tribes visited '.14 The tribes visited include, among others, the Apaches, Navahos, Pueblos, Pimas, Nahua, Otommi, and Aztec. The Pima Indians

1 Nordenskiöld, Zeit. für Eth., vol. xxxviii, p. 98.

Soc., vol. ii, p. 224.

loc. cit., vol. ii, p. 59.

7 Ibid., p. 335.

3 Spix and Martius,

2 Forbes, Journ. Eth. loc. cit., vol. i, pp. 46 and 47 .

1 Keating, loc. cit., vol. i, p. 394.

13 Holder, loc. cit., p. 44.

14 Hrdlicka, loc. cit., p. 163. , vol. ii, p. 246.

4. Azara, ' Rengger, loc. cit., p. 133. cit., vol. i, p. 642.
${ }_{10}$ Pöppig, loc. cit., vol. ii, p. 323 . 12 Schoolcraft, loc. cit., vol. iii, p. 243. 
practised abortion if conception took place before the previous child had been weaned; the suckling period among these people lasted until the child was six or seven years old. ${ }^{1}$ The habit is recorded of the Menomini tribe and of the Zunis, though it is rare among the latter. ${ }^{2}$ Among the Cheyennes 'it has long been the custom that a woman should not have a second child until her first is ten years old ' 3 Abortion is not mentioned in this case, but in view of the widespread prevalence of this custom, abortion is presumably the means used to bring about this result. In Mexico ${ }^{4}$ and throughout South America abortion was commonly employed. "The use of the means of abortion is common and explains the small number of children', says Karl von den Steinen of the Bakairi. ${ }^{5}$ It is especially frequent in Brazil and among the Indians of the Chaco. ${ }^{6}$

8. Compared with the prevalence of abortion, infanticide was not very common among the agricultural tribes of the northern half of the Continent. It was occasionally practised among the Sioux, and more girls were killed than boys. ${ }^{7}$ Of the Creeks it is said that 'to destroy a new-born infant is not uncommon '.8 It was common among the Pimas. ${ }^{9}$ Infanticide is usually confined to the killing of deformed children, and in this form it is recorded of the Apaches, Mohaves, Navahos, Zuni, and the Tepecano. ${ }^{\mathbf{1 0}}$ Infanticide is more common in South America. It is found in Brazil among the Guanas ${ }^{\mathbf{1 1}}$ and the Mbayas. ${ }^{\mathbf{1 2}}$ The former are said to kill more girls than boys. It is also practised to a considerable extent in the Chaco. "Infanticide is quite common among the Lenguas, an interval of seven or eight years being always observable between children of the same family.' 13 Grubb states that the first child is always killed if a girl. ${ }^{\mathbf{1 4}}$ The killing of deformed children is reported from many parts, including Dutch Guiana ${ }^{15}$ and Peru. ${ }^{16}$

9. War plays no less a part in the lives of the agricultural than of the hunting tribes in America. It was perhaps more murderous among the famous tribes of the Atlantic coast and the great

1 Russell, 26th A. R. B. E., p. 186. ${ }^{3}$ Grinnell, Am. Anth., vol. iv, p. 15. 269. s Von den Steinen, Durch
${ }^{2}$ Stevenson, 23rd A. R. B. E., p. 296. 4 Bancroft, loc. cit., vol. ii, pp. 183 and Central-Brazilien, p. $123 . \quad{ }^{6}$ Ehrenreich, Königliches Museum zu Berlin, vol. i, Heft 2, p. 27 ; Azara, loc. cit., vol. ii, p. 116. 7 Schoolcraft, loc. cit., vol. iii, p. $243 . \quad 8$ Ibid., vol. ii, p. 272. $A$. R. B. E., vol. i, p. 99 . cit., vol. ii, p. 93.

10 Hrdlicka, loc. cit., p. 165.

Yarrow, 11 Azara, loc. 14 Grubb, loc. cit., p. 223. and Lowe, Narrative, p. 240.
13 Hawtrey, J. A. I., vol. xxi, p. 295.

15 Bonaparte, Suriname, p. 48.
${ }_{16}$ Smythe 
plains than anywhere else except possibly Brazil. 'As all nations of the Indians in their natural condition', says Catlin, 'are unceasingly at war with the tribes that are about them, for the adjustment of ancient and never ending feuds, as well as from a love of glory, to which in Indian life the battlefield is almost the only road, their warriors are killed off to that extent, that in many instances two and sometimes three women to a man are found in a tribe.' 1 Another author, himself a member of the tribe, describes the manner in which the young Ojebway Indian is brought up to regard war as his chief object in life. "When they are young a spirit of war is instilled into their bosom ; and in order to excite them to courage and ambition, the parents and the old wise men recount to them the wonderful exploits of the braves in former days, such as a single warrior stealing up secretly to a village, killing a number of the enemy, taking off their scalps, and making his escape before the remainder were apprised of the slaughter.' 2 So again, 'among the earliest songs to which a Dacotah child listens are those of war. As soon as he begins to totter about, he carries as a plaything a miniature bow and arrow. The first thing he is taught as great and truly noble is taking a scalp, and he wants to perform an act which is so manly. At the age of sixteen he is often on the warpath.' 3

The neighbouring tribes maintained feuds that must have been the cause of a regular and by no means inconsiderable amount of elimination. The Crows were at war with the Blackfeet, ${ }^{4}$ the Sioux with the Ojebway, ${ }^{5}$ the Minnetanes with the Shoshones. ${ }^{6}$ The Sioux are said to have killed women." Farther south the Yuchi and other members of the Creek Confederacy were very warlike. ${ }^{8}$ The Pueblo Indians were more peaceful, though they were subject to vigorous and frequent attacks from the Apache and other neighbours. ${ }^{9}$ The wars undertaken by the empires of Mexico and Peru were of rather a different nature. Both states pursued a regular policy of aggression towards the less advanced peoples who surrounded them. These wars partook more of the nature of raids upon the weaker nations for the purpose of robbery

1 Catlin, loc. cit., vol. ii, p. 119.

3 Neill, History of Minnesota, p. 68.

5 Neill, loc. cit., p. 70. 'Matthews, U.S. Geog. and Geol. Survey, Misc. Pub and pp. 78 ff. 7 . 7 Long, loc. cit., p. 29.

Publications, vol. i, No. 1, p. 84.
2 Jones, Ojebway Indians, p. 64.

- Catlin, loc. cit., vol. ii, p. 42.

uois see Perrot, Mémoires, pp. 9 ff.

s Speck, Univ. of Pennsyliania 
than of great sporting contests, as were on the whole the wars of the northern tribes. Throughout South America warfare seems to have been a regular feature of inter-tribal relation. It was usually very sanguinary. According to Wallace the Mandrucos fight with their neighbours every year. ${ }^{1}$ Much the same is reported by Church of the Araras, Mogos, Masas, and other tribes of the Amazon basin, ${ }^{2}$ and also by White of the north-western regions of the southern half of the Continent. ${ }^{3}$ The peoples of the Chaco were formerly at continual war with the Guaranis. ${ }^{4}$

10. There are frequent references to blood-feuds in the accounts of these races. Blood-feuds are only to be with difficulty distinguished from warfare; feuds between man and man and between family and family merge into those feuds between tribes that are called warfare. It will be sufficient here to note that a considerable amount of elimination must among certain tribes arise from that peculiar form of blood-feud which is connected with the belief that natural death is caused by some enemy. In Guiana 'a person dies and it is supposed that an enemy has secured the agency of an evil spirit to compass his death. Some sorcerer, employed by the friends of the deceased for that purpose, pretends by his incantations to discover the guilty individual or family, or at any rate to indicate the quarter where they dwell. A near relative of the deceased is then charged with the work of vengeance. ... If the supposed offender cannot be slain, some innocent member of his family-man, woman, or little childmust. suffer instead.' 5 The Uaupes of the upper waters of the Rio Negro " scarcely seem to think that death can occur naturally, always imputing it either to direct poisoning or to the charms of some enemy, and on this supposition will proceed to revenge it. This they generally do by poison.' ${ }^{6}$ It is difficult to ascertain how far these beliefs are acted upon; it seems nevertheless clear that among certain tribes a considerable amount of elimination must be attributed to this source.

11. It is not necessary to repeat here what was said in the former chapter with regard to disease. Our conclusion was that in all probability most diseases did not evolve until at or about the beginning of the third period. We therefore regard this

1 Wallace, Travels on the Amazon, p. 516. See also Church, loc. cit., p. 77. 2 Church, South America, pp. 78, 99, and 137. See also Von Martius, loc. cit., vol. i, p. $129 . \quad{ }^{3}$ White, J. A. I., vol. xiii, p. 244. 'Grubb, loc. cit., p. 105. Brett, Indian Tribes, p. 357.

W Wallace, loc. cit., p. 500. 
source of elimination as of relatively small importance among the races of this group whether in America or elsewhere. We may note a few examples of the evidence to the effect that these races were formerly on the whole remarkably healthy and long-lived. Catlin says that the Mandans were 'undoubtedly a longer lived and healthier race' than most civilized peoples. ${ }^{1}$ According to Le Beau the Iroquois were 'practically never ill'; in spite of a climate that he himself found trying, 'they were', he says, 'nearly all strong and robust', and had few diseases.' 'On the whole, the Yuchi, men, women, and children, are a remarkably healthy set of people.' 3 The Shawnees are 'very healthy and are exempt from many diseases '.4 Spix and Martius, with their extensive knowledge of the Indians of Brazil, say in general that 'the Indians are seldom sick and generally live to an advanced age '.5

12. There is among the agricultural races of America as among the hunting races, and, as we shall see, among all primitive races, a very high rate of child mortality. As before we find the chief causes to be lack of knowledge, lack of care, lack of suitable food and surroundings, and certain peculiar customs. ${ }^{6}$ It is not necessary to review the evidence in detail. Of the Sioux we are told that 'many die in infancy caused by exposure'.? Another observer uses the same phrase, and adds that 'their mothers, to inure them to suffering and to strengthen their constitution, do not take all the necessary care of them '.8 Lumholtz attributes child mortality in part to the eating of unsuitable food. ${ }^{9}$ Not only in North America but also in Brazil are new-born children regularly dipped in cold water. ${ }^{10}$

\section{Africa}

13. Pre-puberty intercourse is common throughout Africa both in the form of recognized early marriage and of irregular but tolerated early sexual relations. Among the Cross River natives

1 Catlin, loc. cit., vol. ii, p. 228. 3 Speck, loc. cit., p. 14. loc. cit., vol. ii, p. 249. American Indians, p. 238. nech, loc. cit., vol. ii, p. 295.
2 Le Beau, loc. cit., vol. ii, pp. 93 and 98.

4 Ashe, loc. cit., p. 270.

s Spix and Martius,

8 For the North American Indians see Handbook of

7 Schoolcraft, loc. cit., vol.iii, p. $238 . \quad$ \& Dome$\checkmark$ Lumholtz, Unknown Mexico, vol. ii, p. 90.

${ }^{10}$ Heriot, Travels, p. 343. Dumarest, speaking of the Cochiti, says that 'sometimes, in the midst of winter, the child's father will take him out and, after breaking the ice, ... will immerse him in the river. Formerly when, in winter, children came from bathing in the river, they were not allowed to approach the fire until their hair was thawed out' (Mem. Am. Anth. Ass., vol. vi, No. 3, p. 144). KochGrüberg states the mortality is heavy among the children of the Amazon tribes and that they are dipped in cold water (Zwei Jahre, vol. ii, pp. 59 and 150). 
' until she reach the age of puberty, a girl is permitted by her parents and by her betrothed to go about freely and have as many lovers as she pleases'.1 A considerable proportion of marriages among the Ibo-speaking people of Nigeria take place before puberty. ${ }^{2}$ In the Congo districts the habit is specially common. ${ }^{3}$ Sexual relations commence early among the Bushongo ; after reaching the age of puberty ' a girl is not supposed to have further sexual relations before marriage '.4 It is also recorded by van Overbergh of the Mangbetu, ${ }^{5}$ by Delhaise of the Warego, ${ }^{6}$ by de Rochebrune of the Onolove. ${ }^{7}$ Speaking of the Bangala, Weeks says that 'above the age of five years it would be impossible to find a girl who was a virgin' 8 ' The sexual morality of the Bahuana is conspicuous by its absence; the unmarried indulge as they please from a very early age, and the girls before puberty.'9 "Torday and Joyce, who are responsible for this statement, speak very similarly of the Bambala, ${ }^{10}$ and $d u$ Chaillu of the Mpongwe.11 The Masai and the Nandi in East Africa have a regularized system of intercourse before puberty. ${ }^{12}$ It also occurs among the tribes inhabiting the Baringo district ${ }^{13}$ and among the Swaheli. ${ }^{\mathbf{1 4}}$ Children of the Wapagoro, in what was German East Africa, are brought together by their parents when about seven years of age, and are separated for a period when menstruation begins-that is to say, about three years later. ${ }^{15}$ Pre-puberty marriage was formerly very prevalent among the Makonde ${ }^{16}$ and the Wanjamuesi ${ }^{17}$ of the same district. 'As regards the little girls over nearly the whole of British Central Africa, chastity before puberty is an unknown condition. Before a girl is become a woman it is a matter of absolute indifference what she does, and scarcely any girl remains a virgin after about five years of age.' 18 Similar testimony is given by Stannus ${ }^{19}$ and by Maugham ${ }^{20}$ for the same district.

1 Partridge, Cross River Natives, p. 254.

2 Thomas, Ibo-speaking Peoples, p. 62. ${ }^{3}$ Hutereau, Ann. Mus. Congo Belge, sér. 3, tome i, p. 4; Cureau, Sociétés Primitives, p. 109. Torday and Joyce, Ann. Mus. Congo Belge, sér. 3, tome ii, p. 110. 5 Van Overbergh, Coll. Mon. Eth., No. 4, p. 309. - Delhaise, ibid., No. 5, p. 157. He thinks, however, that the practice was introduced by the Arabs. ' Rochebrune, Rev. d'Anth., vol. iv, sér. 2, p. 281. ${ }^{8}$ Weeks, J. A. I., vol. xxxix, p. $442 . \quad{ }^{9}$ Torday and Joyce, J. A. I., vol. xxxvi, p. 288. $\quad{ }_{10}$ Ibid., vol. xxxv, p. $420 . \quad{ }^{11}$ du Chaillu, Explorations and Adventures, p. $162 . \quad{ }_{12}$ Johnston, Uganda Protectorate, vol. ii, pp. 824 and 878. ${ }_{13}$ Dundas, K. R., J. A. I., vol. xliii, p. 60. 14 Velten, Sitten und Gebräuche der Swaheli, p. $28 . \quad{ }^{15}$ Fabry, Globus, vol. xci, p. $221 .{ }^{16}$ Weule, East Africa, p. 305. ${ }^{17}$ Reichard, Z. G. E., vol. xxiv, p. $253 . \quad{ }_{16}$ Johnston, British Central Africa, p. 409, note. ${ }^{19}$ Stannus, J. A. I., vol. xl, p. 309. ${ }_{20}$ Maugham, Zambezia, p. 333 . 
14. The suckling period would appear to last nearer three than two years on the average. A few examples may be given. Ewespeaking people, two or three years ; ${ }^{1}$ Yoruba-speaking people, three years $;^{2}$ the Tenda, two and a half to three years $;^{3}$ in Liberia, three or four years $;^{4}$ the Kagero, two to three and sometimes five years ; ${ }^{5}$ the Hausa, two years $;^{6}$ the Bushongo, two years ${ }^{7}$ in the Congo Basin, at least two years on the average $;^{8}$ the Mangbetu, a year and a half or longer $;^{9}$ in West Africa, on the average two to three years ; 10 the Mayombe, two years or longer; ${ }^{11}$ in Uganda, at least two years ; ${ }^{12}$ the Nandi, two years; ${ }^{13}$ the Kuku, three years ; ${ }^{14}$ the Wamakonde, Wakaua, and Wamuera, three years $;^{15}$ the Baganda, three years $;^{16}$ the Wadschagga, two years: ${ }^{17}$ the Wanjamuesi, two to three vears; ${ }^{18}$ the Wazaramo, two to three years ${ }^{19}$ the Baronga, three years ; ${ }^{20}$ in the Madi district of Central Africa, three years ; ${ }^{21}$ in South Africa, two years, according to Lichtenstein, ${ }^{22}$ frequently until three years, according to Kidd. ${ }^{23}$

15. There is little or no evidence of any postponement of marriage among girls. The facts with regard to men will be given later. According to Denham and Clapperton, girls in Bornu ' rarely marry until they are fourteen or fifteen; often not so young '.24. Among the Bangala, girls marry at the age of sixteen to eighteen. ${ }^{25}$ 'In the primitive Bantu tribe every girl gets married, some, however, sooner than others.' ${ }^{26}$ 'The average age at marriage among girls south of Lake Nyassa is fifteen. ${ }^{27}$ 'For the whole of the Bantu tribes south of the Limpopo the average [age at marriage for girls] would probably be between fifteen and sixteen.' 28

16. Throughout the negroid and Bantu races of Africa sexual

1 Ellis, Ewe-Speaking Peoples, p. 206. ${ }^{3}$ Delacour, Rev. d'Eth., 1912, p. 45.

5 Tremearne, J. A. I., vol. xlii, p. 174. and Customs, p. 93. vol. ii, p. 112.

Eth., No. 3, p. 296.

s Cureau, loc. cit., p. 180. 10 Harris, Mem. Anth. S bergh and Jonghe, Coll. Mon. Eth., No. 2, p. 217. Uganda, vol. i, p. 187. Eth., No. 6, p. 205. 16 Roscoe, Baganda, p. 55. loc. cit., p. 257. Ba-Ronga, p. 19. 19 ${ }_{13}$ Hollis, Nandi, p. 65. 15 Fülleborn, Nyassa- und Rowuma-Gebiet, p. 61 . ${ }_{17}$ Gutmann, Globus, vol. xcii. Burton, Central Africa, vol. i, p. 117. 20 Junod, 21 Felkin, Trans. Edin. Obstet. Soc., vol. ix, p. 19. 22 Lichtenstein, Travels, vol. i, p. 260. 24 Denham and Clapperton, Narrative, p. 319. p. 417 . p. 310. $\quad 28$ Theal, Yellow- and Dark-Skinned People, p. 347. ${ }^{23}$ Kidd, Essential Kaffir, p. 19. ${ }_{25}$ Weeks, J. A. I., vol. xxxix, ${ }_{26}$ Junod, South African Tribe, p. 183. 27 Stannus, loc. cit., 
intercourse is almost invariably prohibited for some time after child-birth. As a rule the prohibition holds good so long as suckling lasts, and this we have seen to mean more nearly three than two years. In some cases the period is shorter, and there are instances where it only endures for a few months. There are also very rare examples of the exact opposite-namely, of a prohibition against breaking off intercourse. As the evidence given below shows, to this custom we must attribute very great importance.

A Ewe-speaking woman " may not admit the male ... while suckling' 1 Among the Yoruba-speaking people 'during the period of lactation the wife must not cohabit with her husband '.2 Intercourse during the suckling period is prohibited by the Kagero, ${ }^{3}$ the Hausa, ${ }^{4}$ and in Benin. ${ }^{5}$ In the Warri district of the Niger Protectorate it is 'customary for a woman to aroid cohabitation with her husband for nearly three years after pregnancy '.6 Mungo Park, who travelled through this district, observed that ' three years' nursing is not uncommon, and during this period the husband devotes his whole attention to his other wives ' .7 Other authors record similar facts for other West African races. The Moioa abstain for four years, ${ }^{8}$ the Gallinas until the child can talk and walk ${ }^{9}$ the Hobbés during lactation; ${ }^{10}$ Nassau, spealing generally of the Cameroon district, mentions three years, ${ }^{11}$ and Reade, speaking of Ashanti, mentions the period of lactation. ${ }^{\mathbf{1 2}}$ With regard to the Congo district, Cureau, ${ }^{13}$ Johnston, ${ }^{14}$ and Ward ${ }^{15}$ mention prohibition during the suckling period. Among the Bangala 'during the suckling period the husband has no sexual relations with his wife, or the child will become thin and weak and probably die ' ${ }^{\mathbf{1 6}}$ Another account of the same people mentions two years as the length of the period. ${ }^{17}$ Prohibition during lactation is also recorded of the Mayombe, ${ }^{18}$ the Ababua, ${ }^{19}$ and the Bayaka. ${ }^{20}$ Intercourse is only resumed among the Mandja ${ }^{21}$ and the Warega ${ }^{22}$ when the child can walk. The

1 Ellis, Eue-Speaking Peoples, p. $206 . \quad 2$ Ellis, Yoruba-Speaking Peoples, p. 185. $\quad 3$ Tremearne, J. A. I., vol. xlii, p. 174. "Ibid., vol. xxxvi, p. 93. ${ }^{5}$ Ling Roth, Great Benin, p. 39. ${ }^{\circ}$ Granville, J. A. I., vol. xxvii, p. $106 . \quad 7$ Mungo Park, Travels, p. 402.

of Nigeria, p. 239. 9 Harris, loc. cit., p. 36. 10 Desplagnes, Plateau Central Nigérien, p. 227. 11 Nassau, Fetichism in West Africa, p. 11. ${ }_{12}$ Reade, South Africa, p. $45 . \quad{ }_{13}$ Cureau, loc. cit., p. $378 .{ }_{14}$ Johnston, George Grenfell and the Congo, p. 671. $\quad{ }^{15}$ Ward, J.A.I., vol. xxiv, p. 289. ${ }^{16}$ Weeks, J. A. I., vol. xxxix, p. 418.17 Overbergh and Jonghe, Coll. Mon. Eth., No. 1, p. 199. ${ }_{16}$ Ibid., No. 2, p. $219 .{ }_{10}$ Halkin, ibid., No. 7, p. $260 . \quad 20$ Torday and Joyce, J. A.I., vol. xxxvi, p. 51. ${ }_{21}$ Gaud, Coll. Mon. Eth., No. 8, p. 154. $\quad{ }_{22}$ Delhaise, loc. cit., p. 154. 
Adio of the Upper Congo region form a curious exception ; soon after birth intercourse again takes place ; if it did not, the child would die, since the father would thereby show "no affection for the mother. ${ }^{1}$ The Kuku only interrupt intercourse for three or four months, ${ }^{2}$ although suckling lasts three years, ${ }^{3}$ and among the Nandi prohibition only extends over a period of three months, ${ }^{4}$ while lactation lasts two years. ${ }^{5}$ Similarly 'among the AwaWanga many believe that within five or six days of the birth of a child the parents must cohabit or the child will die ' ${ }^{6}$

The last-mentioned cases are rare exceptions; the customs that we saw to be normal in the west of Africa are also normal elsewhere in Africa. In Uganda ' a woman must live apart from her husband för two years, at which time the children are weaned '.7 A.Baganda woman 'lived apart from hor husband for three years while nursing her child ' 8 When a woman in the region of the Rovuma river 'bears a child she lives completely apart from her husband till the child is able to speak, as otherwise it is believed that harm, if not death, would come to the infant '.9 A year is mentioned as the length of the prohibited period among the Swaheli, ${ }^{10}$ and the duration of lactation as the period among the tribes of the Baringo district ${ }^{11}$ and among the Wanjamuesi.12 On the other hand the prohibited period is short among some of the tribes of what was German East Africa ; it is two months in Konde-land, ${ }^{13}$ three months among the Wapagoro, ${ }^{14}$ and ' a few months' among the Wagogo. ${ }^{15}$ In British Central Africa the wife does not resume sexual intercourse with her husband for two years, unless she is the only wife, when six months to one year is allowed to elapse. ${ }^{16}$ In the Miri district the period is six months, although suckling lasts for two years, ${ }^{17}$ and the same is recorded of the Atonga.18 Speaking of Baronga, Junod says that there is no cohabitation until the child is weaned, and mentions that this is so among all the Bantu races of South Africa. ${ }^{19}$ Kidd confirms Junod's statement, and says that most Kaffir women live in strict seclusion from their husbands while

\footnotetext{
1 Hutereau, loc. cit., p. 46.

2 Plas, loc. cit., p. 203.

s Ibid., p. 205.

4 Hollis, Nandi, p. 66. ${ }^{5}$ Ibid., p. 65. ${ }^{\circ}$ Hobley, p. 358. $\quad 7$ Wilson and Felkin, loc. cit., vol. i, p. 187.

p. 55. Ratzel (History, vol. iii, p. 16) gives three years. Journ., vol. iv, p. 73. 10 Velten, loc. cit., p. 73. vol. xl, p. $60 . \quad 12$ Reichard, loc. cit., p. 257.

A., vol. xxxiii, 8 Roscoe, Baganda, - Thomson, Geog. 11 Dundas, J. A.I., p. 352. ${ }^{14}$ Fabry, loc. cit., p. $223 .{ }_{15}$ Cole, J. A. I., vol. xxxii, p. 312. ${ }_{16}$ Stannus, loc. cit., p. 311 . ${ }_{17}$ Felkin, loc. cit., p. 31 . British Central Africa, p. $415 . \quad 19$ Junod, Ba-Ronga, p. 490.

18 Johnston,
} 
they are suckling their children, which frequently lasts for three years. ${ }^{1}$ In Loango the suckling period is the prohibited period. ${ }^{2}$

17. Among the races hitherto surveyed there is no evidence of the existence of any practice that renders sexual intercourse fruitless. There are numerous instances of magical practices that are supposed to have this result, but we have no reason to think that any of them are effective. In Africa we again meet with similar magical practices, and we must similarly account them to be without effect. In this area, however, we also meet with for the first time practices of quite a different nature. Many races in different parts of the Continent are acquainted with means of preventing fertilization. It is not necessary to describe how this end is achieved. ${ }^{3}$ Any one who is interested in the subject can turn to Junod's account. The methods employed in other parts of Africa are apparently similar to those there described. Knowledge of these methods extends throughout South Africa, ${ }^{4}$ the Congo, ${ }^{5}$ what was formerly German East Africa, ${ }^{6}$ and probably elsewhere. Generally speaking this practice is employed under two different circumstances; measures may be taken to prevent conception by those who are not married and who do not wish to be married for some time; they may also be used by married people under certain circumstances, as for example by parents in the Thonga tribe who may have intercourse when the child begins to crawl, but who must avoid conception until the child is weaned. ${ }^{7}$ Again, young married couples in the neighbourhood of Port Herald live in a special house so long as they are unable to build a house for themselves, and during this time no children must be conceived. ${ }^{8}$

18. Almost without exception the average number of children is everywhere recorded as small. Mungo Park, referring apparently to the Mandingoes, says that ' few women have more than five or six children' .9 In Northern Nigeria, four to five is given as the maximum number.10 Talbot collected some statistics for the Ekoi people; the average number born to sixty-one married women was 4.3.11 'The marriages', says Burton of the Egbas, ' are not very prolific' ${ }^{12}$ Assinien women very exceptionally

1 Kidd, Essential Kaffir, p. 19. $\quad 2$ Pechuel-Loesche, Loango-Expedition, p. 31. 3 Junod, South African Tribe, vol. i, p. $488 . \quad 4$ Junod, ibid. See Macdonald, J. A. I., vol. xix, p. $117 . \quad{ }_{5}$ Cureau, loc. cit., p. $189 .{ }^{\circ}$ Fülleborn, loc. cit., p. 552, note. ${ }^{7}$ Junod, loc. cit., p. 55. ${ }_{8}$ Fülleborn, loc. cit., p. 550. - Mungo Park, loc. cit., p. $403 . \quad{ }_{10}$ Tremearne, J.A.I., vol. xlii, p. 174.

11 Talbot, Shadow o the Bush, p. 12. 12 Burton, Abeokuta, vol. i, p. 207. 
have more than six children, according to Mondière ; ${ }^{1}$ in Liberia families are said to be small.' Among the Bangala 'it is rare for a woman to have more than two or three children ' ; ${ }^{3}$ another observer, speaking of the same people, remarks that 'there was much sterility'.4 Cureau, speaking generally of the tribes of the Congo Basin, says that families are small. ${ }^{5}$ Kuku women bear three children on the average. ${ }^{6}$ The number of children is given as three to five for the Baholoholo, ${ }^{7}$ three to four for the Bambala, ${ }^{8}$ and the same for the Warega $;^{9}$ among the Mangbetu families are not large, ${ }^{10}$ while ' on an average a Bayaka woman bears three children; families of more than four are rare'.11 Turning to the races of the eastern side of the Continent we find that the average Swaheli family is given as consisting of two children.12 Figures have been collected concerning forty-nine families of the Akikuyu, from which it appears that the average number of children is between three and five. ${ }^{13}$ The Bakene women 'are, as a rule, strong and healthy and have children, though few of them ever have so many as six, three being the average number for each wife'.$^{14}$ Two or three-at the most five-children are born to the Wanjamuesi mother. ${ }^{15}$ Among the tribes south of Lake Nyassa " the average number of children in a family is three to five '. ${ }^{16}$ The Makalaka ' race is not prolific, and the women ... seldom bear more than an average of three children ' ${ }^{17}$ Lastly, the Hottentots have ' seldom more than two or three children', and ' many of the women are barren ', 18 while similarly in Madagascar the natives 'do not, as a rule, have large families, and a considerable portion is childless ' .19

19. Abortion is practised by many races in various parts of Africa. Among the Tenda people married women rarely practise abortion, unmarried women more frequently ; ${ }^{20}$ Tremearne mentions it in speaking of some of the Nigerian tribes. ${ }^{21}$ It appears

1 Mondière, Rev. d'Anth., vol. iv, p. 75.

3 Overbergh and Jonghe, loc. cit., No. 1, p. 201. xxxix, p. 420.

5 Cureau, loc. cit., p. 138.

7 Schmitz, Coll. Mon. Eth., No. 9, p. 595. vol. $\mathrm{xxxv}$, p. 51 . cit., No. 4, p. 297.

12 Velten, loc. cit., p. 28.

- Delhaise, loc. cit., p. 157. 11 Torday and Joyce, J. A. I., vol. xxxvi, p. 51. 13 Routledge, Prehistoric People, p. 136. Owing to the fact that the families are incomplete, there is some uncertainty about these figures. Bantu, p. 151 14 Roscoe, Man, vol. ix, p. 118. 15 Reichard, loc. cit., p. 255. 17 Elton, Journal, p. 6. $\quad{ }_{18}$ Barrow, Travels, vol. i, p. 97. See also Theal, Yellow-and Dark-Skinned People, p. 86. 2) Delacour, loc. cit., p. 45.
2 Buttikoffer, loc. cit., p. 82. 4 Weeks, J.A.I., vol. 6 Plas, loc. cit., p. 208. s Torday and Joyce, J. A. I., 10 Van Overbergh, loc.

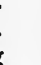


to be on the whole more common in the Congo district than elsewhere in Africa. 'The practice of provoking abortion is a very common one throughout Congoland (though ignored, for example, by the Bayaka), but most of all in the North and Centre.' 1 With regard to particular races in this district, we find abortion mentioned as common among the Bangala, ${ }^{2}$ Bahuana, ${ }^{3}$ Warega, ${ }^{4}$ Ababua, ${ }^{5}$ and Onolove ${ }^{6}$; it exists but is less common among the Mangbetu, ${ }^{7}$ and the Bushongo. ${ }^{8}$ In what was German South-west Africa abortion is very frequently employed. ${ }^{9}$ It would appear to be less frequently practised in the eastern half of the Continent. It is known among the Akamba ${ }^{10}$ and the Swaheli.11 In what was formerly the German Nyassa district ${ }^{12}$ and in British Central Africa it is said to be not uncommon ${ }^{13}$; the same is said of the Zambezi Valley. ${ }^{14}$ Abortion is 'almost universally practised by all classes of female in Kafir society'.$^{15}$ It is mentioned in connexion with Madagascar. ${ }^{16}$

20. Almost every tribe throughout Africa kills children under certain circumstances which they believe to be unpropitious. Examples of such circumstances are peculiarities in the process of birth, the birth of twins, or the cutting of the upper teeth before the lower. Motherless children are also sometimes killed. The cumulative effect of these habits cannot be considerable, and we may disregard them. It is an almost invariable rule that infanticide-in the sense in which we use the term when speaking of the Australians-is unknown in Africa. The only exceptions are to be found among the Hottentots and in Madagascar-in both cases the races practising infanticide not being of Negroid or Bantu stock. The former are said to have killed their female children fairly frequently ${ }^{\mathbf{1 7}}$ in Madagascar infanticide was very common. ${ }^{18}$ Among the various unpropitious circumstances mentioned above, there is one which is widely recognized in Africa, and which is of interest to us. Children who are abnormal or

1 Johnston, George Grenfell and the Congo, p. 671.

vol. xxxix, p. 449 ; Overbergh and Jonghe, loc. cit., No. 1, p. 201. ${ }_{3}$ Torday and Joyce, J.A.I., vol. xxxvi, p. 228. 5 Halkin, loc. cit., p. 259. Hutereau (loc. cit., p. 101) says that it is not often employed. ' Rochebrune, loc. cit., p. 283. $\rightarrow$ Van Overbergh, loc. cit., No. 4, p. $298 . \quad 8$ Torday and Joyce, J. A. I., vol. xxxvi, p. 111. ' Lübbert, Mitth. der Forschungsreisenden, vol. xiv, p. 88. 10 Hobley, Akamba, p. 58. "Velten, loc. cit., p. $29 . \quad 12$ Fülleborn, loc. cit., p. 352. ${ }_{13}$ Johnston, British Central Africa, p. 417; Angus, Azimba and Chipitaland, p. 324. 14 Maugham, Zambezia, p. 339. ${ }_{15}$ Maclean, Kaffir Laws and Customs, p. 62. ${ }_{16}$ Ellis, History of Madagascar, vol. i, p. 55. ${ }_{17}^{17}$ Kolben, Cape of Good Hope, vol. i, p. 144.

18 Ellis, loc. cit., vol.i, p. 155 ; Iittle, loc, cit., p. 60. 
deformed are nearly always killed. Among other instances this practice is recorded of the Hausa, ${ }^{1}$ Kagero, ${ }^{2}$ the Congo tribes generally - the Fangs being especially mentioned in this respect ${ }^{3}$ - the Mandja, ${ }^{4}$ the Basonge, ${ }^{5}$ the Ababua, ${ }^{6}$ the Bushongo, ${ }^{7}$ the Wanika, ${ }^{8}$ the Wakamba, ${ }^{9}$ in the Lenda district, ${ }^{10}$ in the Lind Hinterland, ${ }^{11}$ in British Central Africa, ${ }^{12}$ in Portuguese East Africa, ${ }^{13}$ among the Kaffirs ${ }^{14}$ and the Hottentots. ${ }^{15}$

21. The nature and frequency of warfare varies very greatly in different parts of the Continent. Although among some few tribes and in some districts war is as murderous as in America, it is on the whole far less effective as an agent of elimination than in that country. - The position is somewhat complicated by the fact that the more northern Negroid races have long been in contact with Hamitic and Semitic tribes; the latter have made war on the former and greatly influenced the history of the Negroid races. Movements have been set on foot and passed like waves over the whole Continent.

War is not a very serious matter among the Gallinas of Sierra Leone ${ }^{16}$ or the Ewe-speaking people of Togoland. ${ }^{17}$ It was very different in Dahomey ${ }^{18}$ and Benin ; ${ }^{19}$ the Amazons of the former region have often been described.20 Tremearne gives an account of the head-hunters of Nigeria, whose habits must have been the cause of a great deal of elimination. ${ }^{21}$ Throughout the whole of this region, especially in Ashanti ${ }^{22}$ and Benin, human sacrifice is practised on a large scale, and results in a considerable loss of life. ${ }^{23}$ Passing farther south towards the Congo region, we find that warfare is a moderately important factor of elimination. ${ }^{24}$ Weeks describes family, town, and district fights-the last two developing out of the former ; considerable loss of life and material

1 Tremearne, Hausa Superstitions, p. 93.

2 Ibid., Head-Hunters of Nigeria, p. 239. $\quad{ }^{3}$ Cureau, loc. cit., p. 177 ; Ward, J. A. I., vol. xxiv, p. 291. Torday (Camp and Tramp, p. 142), when speaking of the Bayaka, mentions them as an exception to the general rule that Congo tribes kill misshapen children. 'Gaud, loc. cit., p. 257. $\quad{ }_{5}$ Van Overbergh, loc. cit., No. 3, p. 241. Halkin, loc. cit., p. $260 . \quad{ }^{7}$ Torday and Joyce, Ann. Mus. Congo Belge, sér. 3, tome ii, p. 113. ${ }^{8}$ Krapf, Travels, p. $193 . \quad$ Hildebrand, Recht und Sitte, p. 293. ${ }_{10}$ Livingstone, Labours and Travels, p. 577. 11 Fülleborn, loc. cit., p. 62. 12 Johnston, British Central Africa, p. 417. 13 Maugham, Portuguese East Africa, p. $270 . \quad 14$ Barrow, loc. cit., vol. i, p. 157 ; Kidd, Essential Kaffir, p. 202 ; Shooter, Kaffirs of Natal, p. 88. vol. i, p. $48 . \quad 16$ Harris, loc. cit., p. 27. p. 190 ; Maclean, Compendium, p. 62. 19 Ling Roth, Great Benin, p. 125.

21 Tremearne, Head-Hunters of Nigeria, passim.

${ }_{23}$ See Ellis, Ewe-Speaking Peoples, pp. $117 \mathrm{ff}$. ${ }_{15}$ Theal, History and Ethnography, ${ }_{17}$ Ellis, Ewe-Speaking Peoples, 18 F. E. Forbes, Dahomey, p. 15. 20 See Forbes, loc. cit., p. 23. 23 Beecham, Ashantee, p. 207. ${ }_{24}$ Cureau, loc. cit., p. 348. 
damage appears to follow. ${ }^{1}$ Du Chaillu describes what he calls 'blood feuds 'in the Shekiani tribe; 'frequently a dozen villages are involved, ... the killing and robbing goes on for months and even for years '.2 Again, 'when war has rarely broken out in the country [of the Bakalai] there is no rest or safety. No man or woman in any village can take a step in any direction, day or night, without fear of death. . . . At last whole districts are depopulated; those who are not killed desert their villages.' ${ }^{3}$ Burrows, describing the tribes of the Upper Welle district, says that they all live 'in a perpetual state of internecine warfare'. ${ }^{4}$ War and human sacrifice have a perceptible effect upon the numbers of the Banyala; ${ }^{5}$ fighting is frequent among the Bambala, but is not very sanguinary. ${ }^{6}$ Of the Bahuana we hear that ' wars are frequent, and in some cases last for years ', 7 of the Bayaka that, though 'frequent', wars 'do not seem to have any appreciable effect upon the population ' 8 . The Baganda are a warlike people, and fighting occurs yearly with the neighbouring tribes. Though a regular cause of the loss of life, such wars are not so serious as the intermittent civil wars "which also broke out from time to time in Uganda between rival princes who laid claim to the throne. These latter wars were by far the most disastrous that could happen to the country; and during the few weeks they lasted, untold damage was done and a great loss of life took place.' 9 In this district the presence of the Masai tribe was a continuous source of murderous war. ${ }^{10}$ Among the Akanda ' no warriors but those who had killed a Masai were supposed to be able to marry '; 11 between the Akikuyu and the Masai perpetual war was waged. ${ }^{12}$ Of the tribes of the Baringo district it is said that they are all good fighters, and that war is a sanguinary affair. ${ }^{13}$ The pastoral Bahima are, on the other hand, a peaceable people. ${ }^{14}$ "It is doubtful whether (in Central Africa) great loss of life occurs in any of the wars among the natives.' 15 In South Africa the military qualities of the Zulus

1 Weeks, Congo Cannibals, p. 222.

2 Du Chaillu, loc. cit., p. $161 . \quad 3$ Ibid., p. $386 . \quad$ Burrows, loc. cit., p. 38. For the Azanda and Abandia see Hutereau, loc. cit., p. 36 and p. $44 . \quad{ }_{5}$ Coquilhat, Hart Congc, p. 287 ; Overbergh and Jonghe, loc. cit., No. 1, p. $413 . \quad{ }_{6}^{6}$ Torday, loc. cit., pp. 97 ff. ; Torday and Joyce, J.A.I., vol. xxxv, p. $416 . \quad{ }^{2}$ Torday and Joyce, J.A.I., vol. xxxvi, p. $289 . \quad$ Ibid., vol. xxxv, p. 49. $\quad$ Roscoe, Bayanda, p. 346. ${ }^{10}$ Hollis, Masai, passim. 11 Hobley, Akamba, p. 45. See also Dundas, J. A. I., vol. xliii, p. 505. $\quad{ }^{12}$ Routledge, Prehistoric People, p. $13 .{ }_{13}$ Dundas, J. A. I., vol. xl, p. 51. 14 Roscoe, J. A. I., vol. xxxvii, p. 108.15 Johnston, British Central Africa, p. 470. 
are well known; all through this region in former days warfare was regularly waged, and was a constant source of elimination. ${ }^{1}$ Of the Hottentots we hear that the tribes ' were constantly at war with each other' ${ }^{2}$ from another account it would seem that the fighting was neither very prolonged nor sanguinary. ${ }^{3}$

22. Belief in witchcraft as the cause of death is common throughout Africa; furthermore as a result of this belief the supposed offender is often slain. There seems to be no doubt that among many tribes this factor of elimination is of some importance. Speaking of Kalabar, Hutchinson says : 'They cannot believe, or at least they will not try to understand, how natural causes create diseases, but attribute them and subsequent death to "ifod" or witcheraft. Hence a plan is adopted to find out the perpetrator by fixing on a number of persons, and compelling them as the alternative of the Egbo law of decapitation, to take a quantity of a poisonous nut, which is supposed to be innocuous if the accused be innocent, and to be fatal if guilty.' 4 Even among the relatively advanced Baganda, 'death from natural causes rarely presented itself to the native mind as a feasible explanation for the end of life; illness was much more likely to be the result of malice finding vent in magical art' 5 Torday mentions the Bayaka as an exception to the general rule in that they accept illness as a cause of death, though ".trials for witchcraft are not unknown '.6 Though the belief is widespread that the cause of death is to be sought in magic, the amount of elimination varies considerably from place to place. Formerly in British Central Africa 'it was so general that deaths due to it were in the larger villages matters of daily occurrence '.' Among the South African tribes 'the number of persons who perished on charges of dealing with witcheraft was very great' 8 In West Africa it was undoubtedly common. ${ }^{9}$ For the Congo district, Burrows ${ }^{10}$ and Du Chaillu, ${ }^{11}$ for Central Africa, Werner ${ }^{12}$ and Macdonald,13 for South Africa, Junod,14 and for Madagascar, Parker, ${ }^{15}$ bring similar evidence. Speaking of the Congo region,

1 Theal, Yellow- and Dark-Skinned People,p. 344. vol. i, p. 38 .

3 Kolben, loc. cit., vol. i, p. 282.
2 Same author, History,

- Hutchinson, Western Africa, p. 150. See also same author's Ten Years' Wanderings, p. 54. '5 Roscoe, Baganda, p. 98. Torday, loc. cit., p. 137. $7{ }_{7}$ Maugham, British Central Africa, p. $276 . \quad 8$ Theal, Yellow-and Dark-Skinned People, p. 205. - Wilson, Western Africa, pp. 115 and 179; Beecham, loc. cit., p. 227. 10 Burrows, loc. cit., p. 43. Native Races, p. 168. 11 Du Chaillu, loc. cit., p. 271. 13 Macdonald, loc. cit., p. 106.

12 Werner, 14 Junod, South African Tribe, vol. i, p. 417.
${ }_{15}$ Parker, G. W., J. A. I., vol. xii, p. 478. 
Weeks says that three forms of death are recognized; (1) by act of God ; (2) by another's witchcraft ; (3) by one's own witchcraft. Thus to die by the swamping of a canoe is a case of the first, to die by being swamped and eaten by a crocodile is an example of the second, because ' no crocodile will upset a canoe unless told to do so by a witch'. Deaths from the second cause and executions following upon them appear to be frequent. ${ }^{1}$

23. With respect to disease the African races are in a somewhat different position when compared with the other races of this group. Owing to the fact that they have never been wholly cut off from Eur-Asiatic civilization, some diseases have penetrated among them which are not found, for instance, among the American agricultural races who were cut off from the rest of mankind long before the origin of the third period. It is a very difficult matter to decide what diseases are African in origin and what diseases have found their way into Africa from the north. It is possible that sleeping sickness and black-water fever are African in origin. It is certain, however, that most of the diseases which cause a large amount of elimination among the African races at the present time have been imported from Asia. Among the latter are dengue fever, small-pox, bubonic plague, cholera, Asiatic relapsing fever, dysentery, typhus, and syphilis. ${ }^{2}$ Nevertheless in spite of the fact that disease is of more importance in Africa than in America, there is a considerable amount of evidence to the effect that upon the whole before the advent of the white man the African races were healthy and long-lived. Thus Theal says that the Bantu races of South Africa were formerly 'subject to few diseases ', 3 and Roscoe, that the Baganda 'were happy and healthy before the introduction of European civilization '.4 In earlier days the Basuto were remarkable for their healthiness ; ${ }^{5}$ they lived to a great age. We are also told that the Hottentots suffered from few diseases. ${ }^{6}$

24. We find many records of a high death-rate among children. Undoubtedly this is largely due nowadays to diseases that have been introduced into Africa within the last few hundred years. The evidence seems to show, however, that, when we discount

1 Weeks, Congo Cannibals, p. 341.

2 On this subject see Davidson, Geographical Pathology; Clemow, Geography of Disease; Hirsch, Geographical ind Historical Pathology; and Johnston, Negro in the New World, pp. $15 \mathrm{ff}$. 3 Theal, Yellow- and Dark-Skinned People, p. 174.

- Ellenberger, Basuto, p. 295.

4 Roscoe, Baganda, p. 174.

- Barrow, loc. cit., vol. i, p. 109. 
this factor, child mortality is still high, and is due to ignorance and want of care on the part of the mother. Both Harris ${ }^{1}$ and Talbot, ${ }^{2}$ speaking of West Africa, remark upon the high rate of infant mortality, and connect it with want of care. 'As soon as an infant is able to walk, it is permitted to run about with great freedom,' says Mungo Park. ${ }^{3}$ According to Du Chaillu, 'they know nothing scarcely of the care of children, and lose a great proportion through mistake in treatment in infancy'.4 In the Congo region, "that a great portion of the children die-once they are born-is evident from the Baptist Mission records, extending over more than twenty years. This is chiefly due to unsuitable, indigestable, or insufficient food. Grenfell has some notes on the preposterous attempts to feed infants a few weeks old with manioc paste.' 5 Among the Mangbetu many children die, 'as they are left to look after themselves at about two or three years of age ${ }^{6}{ }^{6}$ Weeks $^{7}$ and van Overbergh ${ }^{8}$ also record high infant mortality for other tribes of this region. It is interesting to observe that Delhaise, speaking of the Warega-an isolated and primitive people-says that disease is rare among the children, though the death-rate is high. ${ }^{9}$ According to Gutmann, many children die in infancy among the Wadschagga, owing to the very unsuitable methods of feeding. A mother takes food from her mouth and presses it into that of her child. ${ }^{10}$ Similarly a high infant death-rate and want of care is recorded of the Zulus, ${ }^{11}$ Basutos, ${ }^{12}$ and Kaffir ${ }^{13}$ races generally.

\section{Oceania}

25. Intercourse before the age of puberty is not very common among the races of Oceania. In New Zealand, however, it was frequent. 'It often happened that a girl would have intercourse with a youth before she arrived at puberty. At times marriage took place and was consummated at this early age.' 14 This state of things is confirmed by several other authors. ${ }^{15}$ Dumas says it

1 Harris, loc. cit., p. 68.

2 Talbot, loc. cit., p. 12.

${ }^{3}$ Mungo Park, loc. cit., p. $403 . \quad$ Du Chaillu, loc. cit., p. 163. Johnston, George Grenfell and the Congo, vol. ii, p. 672.

$?$ Weeks, J. A. I., vol. Xxxix, p. 418

- Delhaise, loc. cit., p. 157.

and Amatongas, p. 198.

${ }_{10}$ Gutmann, loc. cit., p. 3.

an

12 Casalis, Basutos, p. 193.

cit., p. 172.

${ }_{14}$ Best, Man, vol. xiv, p. 32.

11 Leslie, Zulus

13 Holden, loc. vol. ii, p. 16 ; Angas, Australia and New Zealand, vol. i, p. 314 ; Tuke, Edinburgh Medical Journal, vol. ix, part 1, p. 224 ; Tregear, J.A.I., vol. xix, p. 101. 
formerly occurred in Hawaii, ${ }^{1}$ and from an account given by Kubary it seems to have been practised in the Pelew Islands. ${ }^{2}$ Although Brainne does not actually say so, his account seems clearly to indicate that intercourse occurs before puberty in New Caledonia, ${ }^{3}$ and the same may be deduced from Codrington's account of Banks's Island ${ }^{4}$ and from Danks's description of the marriage customs of the New Britain group. ${ }^{5}$ Krieger mentions pre-puberty intercourse in British New Guinea, ${ }^{6}$ while Murray says that among the Baru and other tribes girls are married when between seven and ten years old, adding that marriage is consummated immediately. ${ }^{7}$ According to Seligman among the Sinaugolo ' connection often takes place before menstruation is established '.8 The same is reported as occurring among the Javanese $^{9}$ and very occasionally among the Topebatos of Celebes. ${ }^{10}$

26. The average length of the suckling period would seem to be at least two years and is probably longer. A few facts may be given. In Samoa it lasts two years, ${ }^{11}$ in the Solomon Islands two years or more, ${ }^{12}$ in New Caledonia more than three years, ${ }^{13}$ in Fiji two or three years, ${ }^{14}$ in the Bismarck Archipelago often up to three years, ${ }^{15}$ in what was German New Guinea about three years, ${ }^{16}$ in Sarawak three to five years, ${ }^{17}$ among the Bontoc Igorot slightly less than two years, ${ }^{18}$ and among the Ainu four or five years. ${ }^{19}$

27. Of postponement of marriage among girls there is again practically no evidence. In the Society Islands girls were married when between twelve and sixteen years of age. ${ }^{20}$ In the Western Islands of the Torres Straits girls marry a few years younger than the men, of whom the age of marriage is given as between twenty and twenty-five. ${ }^{21}$ Very occasionally, as in the above cases, there would seem to be some insignificant postponement of marriage.

1 Dumas, Collection des Thèses, p. 18. $\quad{ }^{2}$ Kubary, Journal des Museum Godeffroy, vol.i, p. 53. See also same author, Ethnographische Beiträge, p. $148 .{ }^{3}$ Brainne, Nouvelle-Calédonie, p. 250. 'Codrington, Melanesians, p. $235 . \quad{ }^{5}$ Danks, J. A. I., vol. xviii, p. 288. ' ${ }^{\circ}$ Krieger, Neu-Guinea, p. 297. ' Murray, Papua, p. 195. ${ }^{8}$ Seligman, J. A. I., vol. xxxii, p. $302 .{ }^{9}$ Epp, Holländisch-Ostindien, p. 393. $\quad{ }^{10}$ Kreutz, Zeit. Soc. Wiss., vol. ii, p. 201. ${ }_{11}$ Pritchard, Polynesian Reminiscences, p. 141. ${ }_{12}$ Ribbe, Zwei Jahre, p. 144. ${ }_{13}$ Bernard, NouvelleCalédonie, p. 288. See also Glaumont, Rev. d'Eth., vol. vii, p. 80; Lortsch, Globus, 1885 , p. 107; and Moncelon, Bull. Soc. Anth., vol. ix, p. 361. ${ }^{14}$ Thomson, Fijians, p. 176. ${ }_{15}$ Thurmwald, Forschungen, p. 123. ${ }_{16}^{16}$ Hagen, Unter den Papuas, p. 233. $\quad{ }_{17}$ Ling Roth, Sarawak, p. 100. ${ }_{18}^{18}$ Jenks, Ethnological Survey Publications, vol. i, p. 61. ${ }_{19}$ Hitchcock, A. R. B. E., 1890, p. $465 .{ }_{20}$ Moerenhaut, Voyages, p. 62. ${ }^{21}$ Cambridge Anthropological Expedition, vol. v, p. 247. 
In general, however, it is clear that practically all women are married from the beginning of and throughout the mature period.

28. The important taboo upon sexual intercourse for a period after child-birth is common. The Maori cease to cohabit "after childbirth till the child is weaned '.1 'Throughout the Western Islands (of the Torres Straits) cohabitation ceases early in pregnancy and is not resumed for some time, the baby sleeping between the husband and wife. This restriction is in force in Maburag until the child spontaneously endeavours to move about. As a matter of fact another child is seldom born until the previous one is some three or four years old.' 2 In Savage Island 'the child was usually suckled about twelve months, during which period there was strict sexual abstention between the parents ' 3 According to Kubary the prohibited period lasted ten months in the Pelew Islands, ${ }^{4}$ and according to Krämer, six months in Samoa.5 A married woman among the Sinaugola 'is supposed to forego cohabitation during the period of suckling ' ${ }^{6}$ In the neighbourhood of Finschafen in New Guinea, intercourse is not resumed until the child can walk and speak. ${ }^{7}$ With regard to the Solomon Islands, Ribbe speaks of a 'long period' of prohibition ; ${ }^{8}$ with reference to New Caledonia, Glaumont speaks of several months ${ }^{9}$ and another observer of a considerable time in this connexion. ${ }^{10}$ "After the birth of a child (in the Bismarck Archipelago) the husband was not supposed to cohabit with his wife until the child could walk.' 11 'During the whole of this time [two or three years], unless he had more than one wife, a Fijian was obliged to lead a life of celibacy.' 12 The same author says that 'in Tonga and the Gilbert Islands the separation is rigidly enforced '. ${ }^{13}$ Another account places the length of the period of separation in Fiji at three or 'even four years '.14

29. Here, as elsewhere, we find numerous references to practices intended to render conception impossible. A good example is found in Seligman's account of the Sinaugolo. 'There is generally a woman in the village or one of the surrounding villages who is

1 Tregear, loc. cit., p. 103. p. 199. 3 Thomson, $S$ Museum Godeffroy, vol. i, p. 54.

2 Cambridge Anthropological Expedition, vol. v,

- Schellong, Zeit. für Eth., vol. xxviii, p. 19.

- Glaumont, loc. cit., p. 80.

Melanesians, p. 37.

is Seeman, Viti, p. 191. \&ibbe, loc. cit., p. 144.

1. Lambert, Néo-Calédoniens, p. 104. " Brown, is Ibid., p. 178. 
supposed to be gifted with a power inherited from her mother of causing women to become "hageabani", literally incapable of having more children. Suppose that a woman considers that she has had enough children, she will by stealth seize an opportunity of consulting such a woman and will pay for services. The woman gifted with the power sits down behind and as close as possible to her patient, over whose abdomen she makes passes while muttering incomprehensible charms. At the same time herbs or roots are burnt, the smoke of which the patient inhales.' 1 Such practices are obviously purely magical, and are quite ineffective. There are many definite statements with regard to particular races that no effective practices are known. In a few cases it is asserted that conception can be and is prevented. Krieger, for instance, says that in what was German New Guinea methods of preventing conception are known. ${ }^{2}$ Pfeil gives a circumstantial account of a method said to be employed in New Ireland. ${ }^{3}$ It seems clear, nevertheless, that the prevention of conception can be of but little importance in this region.

30. There is the same remarkable unanimity of evidence with regard to the small average size of families in Oceania as elsewhere. In New Zealand ' families are usually small in number'. ${ }^{4}$ According to Dieffenbach, ' families are not large; there are rarely more than two or three children ', ${ }^{5}$ while Brown says 'they have very few children. Large families are never seen among them; perhaps two would be a high average compared with the number of marriages.' 6 There are rarely more than three children in a family in the Western Islands of the Torres Straits $;^{7}$ in the Eastern Islands the number varies from two to six, leaving childless marriages out of account. ${ }^{8}$ In the Sandwich Islands the average is said to be three. ${ }^{9}$ 'There were few instances of large families' in Samoa ; 'four or five would be the average.' 10 Three is said to be the average among the Aru Islanders, ${ }^{11}$ while in the Pelew Islands marriages are reported to be often childless. ${ }^{12}$ 'I have never known', says Melville of the Marquesas, 'of more than two youngsters living together in the same home, and but

1 Seligman, J. A. I., vol. xxxii, p. 303.

3 Pfeil, Studien und Beobachtungen, p. 31.

5 Dieffenbach, loc. cit., p. 33.

J. A. I., vol. xix, p. 359 .

p. 108.

p. 83.
Godeffroy, vol. i, p. 54 .
2 Krieger, Neu-Guinea, p. 165. - Angas, loc. cit., p. 314. - Brown, New Zealand, p. 40. 'Haddon, - Cambridge Anthropological Expedition, vol. iii, 10 Turner, Samoa, 12 Kubary, Journal des Museum 
seldom even that number.' 1 Tautain, who collected some figures for these islands, found the birth-rate to be very low. ${ }^{2}$ In the Kingsmill Islands 'a woman seldom has more than two children, and never more than three ' 3 Dr. Seligman is under the impression that ' childless marriages are not very uncommon' among the Koitu and Motu of New Guinea ; ${ }^{4}$ and Stone says of the latter that 'as a rule their progeny is not numerous '.5 Krieger remarks upon the small families in New Guinea, attributing the fact to abortion and infanticide. ${ }^{6}$ In the Bismarck Archipelago 'families as a rule are not very large. . . . A large number of the women have no children.' 7 Three is the average number in New Ireland; a family of four or five is considered large. ${ }^{8}$ In New Caledonia there are seldom more than three in a family. ${ }^{9}$ Fijian women are not prolific. ${ }^{10}$ Iing Roth, surveying the literature of Sarawak, notes that the small size of the families has often been remarked upon, and quotes the statements of Houghton and Whitehead."11 According to the former, 'in general there are more than two children in a family; on an average three or four, very seldom only one child '. ' The families of the natives', says Whitehead, 'are very small ; in one or two instances I have known them to contain eight or more by one mother, but many women have only three or four, most one or two children; and it is by no means uncommon to find them childless.' Brooke estimated ' four or five births to every married woman '.12 Wallace was impressed by the same fact, and took some pains to investigate the matter. "From inquiries at almost every Dyak tribe I visited, I ascertained that the women had rarely more than three or four children, and an old chief assured me that he had never known a woman have more than seven.' ${ }^{13}$ So, too, according to Bock, 'a Dyak family seldom consists of more than three or four children '. ${ }^{14}$ Hagen estimates four children born per fertile married woman as the average among the Orang Kubı of Sumatra ; ${ }^{15}$ while Marsden, writing in the eighteenth

1 Melville, Narrative, p. 213. 3 Jenkins, Voyage, p. 404. New Guinea, p. 93.

2 Tautain, L'Anthropologie, vol. ix, p. 418. confirms this (Deuts p. 37. Neu-Guinea, vol. i, p. 150).

- Seligman, Melanesians, p. 80.

Stone,

- Krieger, loc. cit., pp. 165, 293, and 390. Neuhaus 8 Pfeil, loc. cit., p. 32. See also Stephan and Graebner, Neu-

Mecklenberg, p. 16. Rev. d'Eth, vol. ii, p. 330. p. 178. vol. ii, p. 335 . 11 Ling Roth

- Lortsch, loc. cit., p. 107; De Vaux, 'Les Canaques', 10 Blyth, Glasgow Medical Journal, vol. xxviii,

Head Hunters, p. 211. is Wallace, Malay Archipelego, vol. i, p. 141.

12 Brooke, Sarawak, 14 Bock, 
century, was struck by the small average fertility; 'women are by nature unprolific ' in Sumatra, was his opinion. ${ }^{1}$ Two or three is the average in Nias ; ${ }^{2}$ " the average number of persons in one family in Java, where it is perhaps as large, if not larger than elsewhere, is estimated at only four or four and a half ' 3 Kreutz has compiled some figures for Celebes. He makes two and a half children to be the average per married woman among the Tolage and four among the Topebatos." The Ainus 'are not at all prolific' $;^{5}$ while according to another author, 'not many children are born, usually three or four ${ }^{\prime}{ }^{6}$

31. Abortion and infanticide are common, and there are many places where both practices are employed. Abortion is often spoken of among the Maoris ; it would appear to be fairly common. ${ }^{7}$ In the Murray Islands of the Torres Straits 'abortion was very common ${ }^{\prime} ;{ }^{8}$ and in the Western Islands it is very frequent according to Haddon, ${ }^{9}$ and similar facts are recorded of the Eastern Islands. ${ }^{10}$ Abortion was known in Hawaii ; ${ }^{11}$ in the Kingsmill or Gilbert Islands it was extremely prevalent, ${ }^{12}$ and also in Samoa..$^{13}$ It was practised at times in Rotuma ${ }^{14}$ and in Savage Island. ${ }^{15}$ Abortion seems to be fairly common in Fiji. ${ }^{16}$ It is said to be 'fairly frequent' in the New Hebrides ; ${ }^{17}$ according to Jamieson, ' a certain proportion of women die in endeavouring to procure abortion '.18 There is very similar evidence from New Caledonia ${ }^{19}$ and the Solomon Islands; it was probably more prevalent in the latter than in the former, especially among the south-eastern islands of the group. ${ }^{20}$ In the Bismarck Archipelago it is reported to be frequently practised, ${ }^{21}$ and so also in the Aru

\footnotetext{
1 Marsden, History of Sumatra, p. 219.

2 Modigliani, Viaggio, p. 554. ${ }^{3}$ Bickman, Travels, p. $278 . \quad{ }^{4}$ Kreutz, loc. cit., p. $40 .{ }_{5}$ Batchelor, Ainu, p. $19 .{ }^{\circ}$ Hitchcock, loc. cit., p. $465 .{ }^{7}$ Dieffenbach, loc. cit., vol. ii, p. 12 ; Goldie, Trans. and Proc. N.Z. Inst., vol. xxxvii, p. 110; Tuke, loc. cit., p. 735. ${ }^{8}$ Hunt, J. A. I., vol. xxviii, p. $9 . \quad{ }^{\circ}$ Haddon, J. A. I., vol. xix, p. 359. $\quad{ }^{10}$ Cambridge Anthropological Expedition, vol. vi, p. 197. 11 Dumas, loc. cit., p. 18.12 Jenkins, loc. cit., p. 404; Krämer, loc. cit., p. 335 ; Thomson, Fijians, p. $211 .{ }_{13}^{13}$ Krämer, loc. cit., vol. ii, p. 53 ; Turner, Samoa, p. $79 . \quad{ }_{14}$ Gardiner, J.A.I., vol. xxvii, p. 480. ${ }_{15}$ Thomson, Savage Island, p. 141.

18 Pritchard, Polynesian Reminiscences, p. 423 ; Waterhouse, King and People of Fiji, p. 327 ; Thomson (Fijians, p. 180) thinks that it has been introduced lately. On the other hand Blyth says that it was formerly more prevalent than it is now (loc. cit., p. 181).

${ }_{17}$ Hagen and Pineau, Rev. d'Eth., vol. vii, p. $332 . \quad{ }_{18}$ Jamieson, Aust. Med. Journ., new series, vol. vii, p. 53. 19 Bernard, Nouvelle-Calédonie, p. 288 ; Rochas, Nouvelle-Calédonie, p. 200.20 Codrington, Melanesians, p. 229 ; Ribbe, loc. cit., p. 144 ; Parkinson, Int. Arch. Eth., vol. xiii, p. 8 ; Elton, J.A.I., vol. xvii, p. 93. ${ }_{21}$ Brown, Melanesians, p. 33 ; Danks, J. A. I., vol. xviii, p. 291 ; Pfeil, loc. cit., p. 313 ; Stephan and Graebner, loc. cit., p. 18.
} 
Islands 1 and the island of Flores. ${ }^{2}$ Similar evidence comes from all parts of New Guinea. ${ }^{3}$ It is specially mentioned for the Gulf of Geelink, ${ }^{4}$ among the Baru tribe, ${ }^{5}$ near Dorej, ${ }^{6}$ among the Mafulu people, ${ }^{7}$ among the Koitu and the Motu, ${ }^{8}$ and the Southern Massim. ${ }^{9}$ It is also practised in Nias, ${ }^{10}$ Central Celebes, ${ }^{11}$ among the Bontoc Igorot, ${ }^{12}$ and in the Mitchell group. ${ }^{13}$

32. Infanticide was very prevalent in New Zealand-far more so than abortion. 'Infanticide is frequent among the New Zealanders '; ${ }^{14}$ according to another observer 'it was formerly very common' $;{ }^{15}$ or again, 'it was formerly very prevalent '.16 We hear of the 'wholesale destruction of human life through infanticide' ${ }^{17}$ There is evidence that girls were more often killed than boys. ${ }^{18}$ Infanticide occurred in the Western Islands of the Torres Straits ; ${ }^{19}$ among the Eastern Islanders 'after a certain number had been born, all succeeding children were destroyed ' ${ }^{20}$ In the Gilbert Islands it is very prevalent; ${ }^{21}$ in Samoa and Tonga, ${ }^{22}$ on the other hand, it is either absent ${ }^{23}$ or very rare. ${ }^{24}$ It appears to have been fairly common in Savage Island, ${ }^{25}$ in Tikopia (Barwell Islands), ${ }^{26}$ and in Nissau. ${ }^{27}$ 'Infanticide was committed on a large scale' in Rarotonga ${ }^{28}$ and in Funafuti, ${ }^{29}$ but it probably reached its greatest extent in Tahiti. The famous secret society known as the Areoi is said to have enjoined the killing of all children upon its members. In any case it was very prevalent in the island, and not confined to the Areoi. ${ }^{30}$ "The first missionaries have published it as their opinion that not less than two-thirds of the children were murdered by their own parents.' 31 Infanticide was not known in the

1 Ribbe, loc. cit., p. 194.

2 Riedel, Rev. Col. Inter., vol. ii, p. 71. - Krieger, loc. cit., p. 165 (German New Guinea), p. 292 (British New Guinea), and p. 390 (Dutch New Guinea). Ibid., p. $392 .{ }_{5}$ Murray, Papua, p. 194. 'Rosenberg, Malayische Archipel, p. 454. ${ }_{7}$ Williamson, Mafulu People, p. 176. 'Seligman, Melanesians, p. 135. Ibid., p. 568. 10 Modigliani, loc. cit., p. 554. 11 Kreutz, loc. cit., p. 201. 12 Jenks, loc. cit., p. $60 .{ }_{13}$ Turner, Samoa, p. 280.16 Angas, Australia and New Zealand, vol. i, p. $312 . \quad{ }^{15}$ Taylor, Te Ika a Maui, p. 338. 16 Tuke, loc. cit., p. 221. ${ }_{17}$ Polack, Manners and Customs, vol. ii, p. 92. See also Meade, Ride, p. 163, and Dieffenbach, loc. cit., vol. ii, p. 16. ${ }^{18}$ Brown, New Zealand, p. 41 ; Earle, Narrative, p. 243. 19 Haddon, J. A. I., vol. xix, p. 359 ; Cambridge Anthropological Expedition, vol. vi, p. 107. 20 Cambridge Anthropological Expedition, vol. vi, p. 107. 21 Tuituila, Journ. Pol. Soc., vol. i, p. 267. ${ }_{22}$ West, Ten Years, p. $270 . \quad{ }^{23}$ Turner, Samoa, p. 79 ; Brown, Melanesians, p. 47. 24 Krämer, loc. cit., vol. ii, p. 53. ${ }_{25}$ Thomson, Fijians, p. 141. 28 Rivers, Melanesian Society, vol. i, p. 313. 27 Thurmwald, Zeit. für Eth., vol. xlii, p. 111. ${ }^{28}$ Gill, Coral Islands, vol. ii, p. 13. ${ }^{20}$ Edgeworth David, Funafuti, p. $195 .{ }_{30}$ Lutteroth, Insel Tahiti, p. $12 . \quad 31$ Ellis, Polynesian Researches, vol. i, pp. 249 ff. ; same author, Tour through Hawaii, p. 325. 
Caroline Islands ${ }^{1}$ (with the exception of Pelew). It was apparently as common in the Sandwich Islands as in Tahiti. ${ }^{2}$ It was also practised in Fiji, but girls were destroyed in preference to boys. ${ }^{3}$ Glaumond states that it is 'very common' in New Caledonia, ${ }^{4}$ and this is confirmed by Bernard ${ }^{5}$ and Moncelin. ${ }^{6}$ The last named mentions that girls are killed in preference to boys. It is moderately common in the New Hebrides, and again more girls are killed than boys $;^{7}$ according to Meinecke it is not so frequent in Tala as in Fate. ${ }^{8}$ Infanticide is 'very common' in Banks's Island, ${ }^{9}$ in Radack, ${ }^{10}$ in Vaitapu, ${ }^{11}$ and in the Marquesas. ${ }^{12}$ It would not seem to be very prevalent in the Solomon Islands, except in Ugi, where both Elton ${ }^{13}$ and Guppy ${ }^{14}$ report it to be common. Otherwise, in the rest of the group it seems to be rare, ${ }^{15}$ and is absent in San Christoval. ${ }^{16}$ It was formerly common in the Bismarck Archipelago. ${ }^{17}$ There is evidence of the existence of the habit in various parts of New Guinea ; according to Seligman it is 'common' among the Southern Masisim; ${ }^{18}$ among the Northern Massim it is practised if there is a large family of girls. ${ }^{19}$ The same is said of the Mafulu people. ${ }^{20}$ Otherwise it would seem, generally speaking, that infanticide is somewhat rare in New Guinea. Murray suspects its existence among the Baru tribe, ${ }^{21}$ and Erdweg among the inhabitants of Tumleo. ${ }^{22}$ Newton says that he only knows one district in British New Guinea where it is frequently practised. ${ }^{23}$ So, too, among the Dyaks it is decidedly uncommon. ${ }^{24}$ Lastly, it may be noticed that there exists 'in some parts of the Solomons and New Hebrides a most remarkable state of things, all the children are killed, chiefly by infanticide, it would appear, and substitutes purchased ${ }^{\prime 25}$

1 Kotzebue, Voyage, p. 211.

${ }^{2}$ Ellis, Narrative, pp. 324 ff. ; Angas, Polynesia, p. 144 ; Dumas, loc. cit., p. 19. The last-named author says that it was undoubtedly more prevalent before the arrival of Europeans than later. 3 Waterhouse, loc. cit., p. 328.

- Glaumond, loc. cit., p. 79.

5 Bernard, loc. cit., p. 288 . $\quad$ Moncelin, loc. cit., p. 357. ' Somerville, J. A. I., vol. xxiii, p. 4. According to Paton, 'Infanticide is systematically practised' (New Hebrides, p. 452). "Meinecke, Z. G. E., vol. ix, p. 340. ton, Melanesians, p. 229. 10 Kotzebue, loc. cit., p. 173. ${ }^{11}$ Turner, Samoa,

Codring. p. 284. $\quad{ }_{12}$ Hale, U.S. Exploring Expedition, vol. vi, p. $15 .{ }_{13}^{13}$ Elton, J. A.I., vol. xvii, p. 93. ${ }_{14}$ Guppy, Solomon Islands, p. 42. 15 Elton, loc. cit., p. 93 ; Somerville, J. A. I., vol. xxvi, p. 393 ; Parkinson, loc. cit., p. 8; Ribbe, loc. cit., p. $144 .{ }_{16}$ Verguet, Rev. d'Eth., vol. iv, p. 206. ${ }^{17}$ Brown, Melanesians, p. 36 ; Pfeil, loc. cit., p. $18 . \quad{ }_{18}^{18}$ Seligman, Melanesians, p. 568. 19 Ibid., p. 705. loc. cit., p. 194. ${ }_{20}$ Williamson, Mafulu People, p. 176. 21 Murray, Wien, vol. xxxii, p. 281. ${ }_{23}$ Newton, New Guinea, p. 189. Forests of the Far East, vol. i, p. 48; Brooke, loc. cit., vol.ii, p. 337 loc. cit., vol. i, p. 268; Romilly, Western Pacific, p. 68. 
33. Warfare occurs everywhere in Oceania ; 1 ' apparently there is not a single case in which it is definitely recorded to be absent. In some islands it is as murderous as anywhere in America, though on the whole it cannot be regarded as anything like such an important cause of elimination as in that Continent. The Maories were especially skilled in the art of war which 'carries off a large number of their strongest men, and has often proved so destructive to a tribe, that it has been broken up entirely and has disappeared '.2 In the Murray Islands there is frequent fighting and raiding of the neighbouring islands and coasts $;^{3}$ and the same is true of all the islands of the Torres Straits, though it is commoner in the western than in the eastern islands." "A life for a life' is, we are told, the principle underlying warfare among these people $;^{5}$ in the Sandwich Islands elimination from this cause must have been very considerable; we hear, for example, of 'the sanguinary character of their frequent wars ' ${ }^{6}$ It is much the same in Tahiti; 'their wars were merciless and destructive '; ${ }^{7}$ ' occasions of hostility were also at times remarkably trivial, though not so their consequences.' 8 War seems to be equally frequent in Samoa, though possibly less murderous. ${ }^{9}$ Brown believes that 'the wars of the Samoans tended for a long time to check the natural increase of the population'.10 'There was never any difficulty in finding a reason, if a fight was desired (in Rotuma), as any pretext could be seized.' 11 Such fights were sometimes followed by very considerable slaughter. ${ }^{12}$ 'War, either offensive or defensive, was their continual delight [in Rarotonga]. A state of peace was rarely known to continue long between the tribes. . . These quarrels invariably led to fighting, in which the warriors of each tribe engaged with the utmost desperation and cruelty.' ${ }^{13}$ War was the favourite occupation of the Kingsmill Islanders, ${ }^{14}$ and in the Pelew Islands it was their ' daily concern '.15 Warfare is said to have been especially

1 Moerenhaut, loc. cit., vol. ii, p. 30, says that 'Tous ces peuples étaient très fréquemment en guerre '. J. A. I., vol. xix, p. $110 . \quad 3$ Hunt, loc. cit., p. 10. 4 Cambridge Anthropo${ }_{2}$ Dieffenbach, loc. cit., vol. ii, p. 16 ; Tregear, vol. v, p. $298 . \quad 6$ Ellis, Narrative, p. 4 ; Dumas, loc. cit., p. $19 . \quad 7$ Ellis, Polynesian Researches, p. 293 ; Lutteroth, loc. cit., p. $17 .{ }_{8}$ Ellis, Polynesian Researches, p. 294 . Turner, Samoa, p. 189 ; Pritchard, loc. cit., p. 55 ; Angas, Polynesia, p. 270. $\quad 10$ Brown, Melanesians, p. 173. 11 Gardiner, J.A.I., vol. xxvii, p. $470 . \quad 12$ Ibid., p. 474. ${ }_{13}$ Gill, Coral Islands, vol. ii, p. $12 . \quad 14$ Jenkins, loc. cit., p. $407 . \quad{ }^{15}$ Kubary, Journal des M useum Godeffroy, vol. i, p. 62 ; Wilson, Pelew Islands, p. 334; 
developed in the Marshall Islands. ${ }^{1}$ According to Williams, ' Fiji is rarely free from war and its attendant evils' ; ${ }^{2}$ ' natural deaths are reduced to a small number among the heathen Fijians, by the prevalence of war and various systems of murder which custom demands.' 3 On the other hand, it is interesting to note that Thomson thinks that the destructive nature of warfare in Fiji is exaggerated as elsewhere in Oceania. He gives the following account of his own experiences in another island as an example of what he found warfare really to mean. 'As we travelled along the coast we found that every village had its frontier; a stream mouth, or a sapling stuck upright in the sand, beyond which none would venture. The natives did their best to dissuade us from crossing these boundaries by representing their neighbours as thirsting for the blood of strangers. But on the other side of the frontier we found a meek folk, lost in wonder that we had come through the last stage of our journey unscathed, so cruel and ferocious were its inhabitants. Every man lived in active terror of his neighbours, and went armed to his plantation, but this did not prevent him from being a most skilful and industrious husbandman, or from living to a good old age. The fear being mutual, there was scarcely any war; an occasional attack upon a woman or upon an unarmed man served to keep the hereditary feud alive.' 4 It may very well be that the murderous nature of warfare has often been exaggerated, and that as a matter of fact the true state of affairs often approximates more closely to the picture given by this author.

In the New Hebrides fighting is said to be fairly frequent ; ${ }^{5}$ in New Caledonia it is certainly common, though perhaps not very serious. ${ }^{6}$ So too in the Solomon Islands there is ' unceasing war ' ? According to Romilly, 'in a battle the victorious party, if they can surprise their enemies sufficiently to admit of a wholesale massacre, kill not only the men, but also all the women and children '.8 A very similar general impression is gained from descriptions of Now Guinea. "The Western section of the Koita, especially the Arauwa and the Rokurokuna, were formerly

1 Seligman, Melanesians, p. 671.

- Williams, Fiji, p. 43.

Ibid., p. 203. He calculates the annual loss as from 1,500 to 2,000 , and adds that this should be increased in order to include widows strangled on the death of their husbands (p. 53). $\quad{ }^{4}$ Thomson, Fijians, p. 86. ${ }_{5}$ Hagen and Pineau, loc. cit., p. 336.

6 Lambert, loc. cit., pp. 173 ff. ; Moncelon, loc. cit., p. 358 ; de Rochas, p. 304 ; Brainne, loc. cit., pp. $244 \mathrm{ff}$. Verguet, loc. cit., p. 215. 
involved in almost continual warfare with Kabadi ; apart from this long continual struggle the Koita appear to have carried on little inter-tribal fighting. . . . A considerable number of people fell in these encounters.' 1 Other accounts of New Guinea give the impression that, however frequent the fighting, the losses were not large. ${ }^{2}$ Among the Dyaks, on the other hand, warfare is not only frequent but also sanguinary $;^{3}$ women and children are killed at times. 4 Fighting occurs between the Ainu clans, and is the cause of much loss of life. ${ }^{5}$

34. It is not necessary to discuss the prevalence of feuds in general, as they are not to be distinguished from warfare. But in addition to the special case of murder following upon natural death, one form of homicide deserves mention as it is of considerable importance in this region, though not uncommon elsewhere-especially in Nigeria. Head hunting is popularly connected with Borneo, but it is also practised in New Guinea and the neighbouring islands. The taking of a head is counted a proof of manliness; in Kiwai Island, Fly River, it enables a young man to secure a wife more easily. ${ }^{6}$ Thomson saw thirteen skulls over the door of a house in an inland village in Normanby Island. ${ }^{7}$ Woodford found whole villages devastated owing to the prevalence of this habit. ${ }^{8}$ Speaking of the New Hebrides, Hickson says that ' in the olden times a fresh human head was an indispensable preliminary to any marriage negotiations' .9 Among the Sea Dyaks (Ibans) it is certainly a form of sport; the other inhabitants of Borneo are said only to practise it as a part of warfare. ${ }^{10}$ It is not true to say that in Borneo the taking of a head is indispensable for a youth who wishes to marry, 11 though considerable prestige clearly attaches to it. ${ }^{12}$ Without doubt the practice is responsible for a large number of deaths, ${ }^{13}$ and the head of a woman or child is equally valuable as that of a man. ${ }^{14}$ The habit is also very prevalent among the Bontoc Igorot. ' For unknown generations these people have been fierce

1 Seligman, Melanesians, p. 121.

2 Abel, New Guinea, pp. 129 ff. ; Williamson, Mafulu People, p. 180; Reche, Südsee-Expedition, 1908-10, p. 289. 3 Ling Roth, Sarawak, vol. ii, p. 117; Hose and McDougall, Pagan Tribes, pp. $158 \mathrm{ff}$. Ling Roth, Sarawak, vol. ii, p. 120.

p. $15 . \quad-$ Chalmers, J. A. I., vol. xxxiii, p. 123.

5 Batchelor, loc. cit., Guinea, p. $23 . \quad{ }^{8}$ Woodford, Head Hunters, p. 154 ; Guppy, loc. cit., p. 16 ; Romilly, Western Pacific, p. $73 . \quad$ Hickson, New Hebrides, p. $275 . \quad{ }_{10}$ Hose and McDougall, loc. cit., p. 187. ${ }^{11}$ Ibid., p. 187. ${ }^{12}$ Gomes, Sea Dyaks, p. 5. ${ }^{13}$ Brooke, loc. cit., vol. i, p. 121 ; Ling Roth, Sarawak, vol. ii, p. 143. 14 Brooke, loc. cit., vol. i, p. 121 ; St. John, loc. cit., vol. i, p. 68. 
head hunters. Nine-tenths of the men in the pueblos of Bontoc and Samoki wear on the breast the indelible tatoo emblem which proclaims them takers of human heads.' 1 Women and children over five years of age are killed. ${ }^{2}$

The failure to recognize natural death as such is as prevalent in this region as in the others which we have surveyed. Speaking of New Guinea, Romilly says ' in the native opinion there is no such thing as a " natural death". If a man escapes a violent death and dies of fever or pneumonia, it is said that he has been bewitched and that a devil has killed him.' 3 So, too, von der Sande in his description of Humboldt Bay records that 'the opinion is generally held that death is always caused by the evil desire of other persons ' $;{ }^{4}$ in consequence numerous murders follow.

35. With regard to the prevalence of disease it is sufficient to say that disease was certainly less common than in Africa. It is probably true that disease was of as little importance as a factor of elimination as in America. ${ }^{5}$

The evidence with regard to infant mortality shows that it is again considerable, and due to ignorance and want of care. Speaking of New Guinea, Newton says that ' the rate of infant mortality is high, but it is often due, so we suspect, to the absolute belief that a child must have some taro to eat-where taro is the staple food-if it is to thrive. . . Infants not twenty-four hours old have had taro given them.' ${ }^{6}$ Kreutz found that many more children died in the second than in the first year; this he attributed to the fact that, whereas while in the first year they are well looked after, in the second year, when they begin to crawl about, they often die from want of attention.? Turner states that not less than two-thirds of the children in Samoa died in childhood from carelessness and mismanagement, ${ }^{8}$ and of the Bontoc Igorot we are told that children are brought up without any sign of knowledge as to how they should be treated from the point of view of health. ${ }^{9}$

1 Jenks, loc. cit., p. 172.

8 Ibid., p. 182.

${ }^{3}$ Romilly, From my Verandah, p. 52.

- Von der Sande, Nova Guinea, vol. iii, p. 270. Neuhaus, loc. cit., vol. i, p. 131, attributes considerable importance to this factor.

5 It is again noticeable that in the accounts of the older observers the general good health of these races is remarked upon. See, for instance, Kotzebue, loc. cit., p. 129 (for the Sandwich Islands) and p. 170 (for Radeck); Cheyne, Western Pacific Ocean, p. 9 (for Island of Pines).

- Newton, loc. cit., p. 189. For New Ireland see Stephan and Graebner, loc. cit., p. 18, where the high child mortality is attributed to exposure. loc. cit., p. $202 . \quad{ }_{8}$ Turner, Samoa, p. $135 . \quad$ Jenks, loc. cit., p. 61. For the whole question of child mortality among primitive races see Gerland, UUber das Aussterben der Naturvölker, p. 24 to p. 39. 


\section{Asia}

36. It is not worth while to deal at length with the remaining peoples who are to be classed with this group. For the most part they are herders of reindeer; the more western races have long been in contact with Eur-Asiatic civilization; even the Chuckee were discovered in the first half of the seventeenth century, ${ }^{1}$ while the Yukaghis were converted to Christianity two hundred years ago. ${ }^{2}$ It is interesting to note, however, that generally speaking the conditions among them are similar to what we have found elsewhere.

Suckling lasts three years among the Koryak ${ }^{3}$ and the Tunguses, ${ }^{4}$ while Yakut mothers sometimes suckle their children until the latter are five years of age. ${ }^{5}$ There is no evidence of postponement of marriage ; the early age at which it takes place has been remarked on for most of these races. ${ }^{6}$ As regards the number of children, Krasheninicoff says of the inhabitants of Kamtchatka that 'in general these people are not fruitful'? Sograff comments on the small number of Samoyed children. ${ }^{8}$ Jochelson found an average of over five to married women above forty years old. ${ }^{9}$ Abortion and infanticide are common in Kamtchatka ${ }^{10}$ Stellers comments especially on the prevalence of abortion which is very frequently employed.11 The Samoyeds destroyed deformed children. ${ }^{12}$ There are the usual comments on the general good health of these people. Both Ostyaks and Samoyeds 'enjoy the best of health and attain a very old age '.13 Infant mortality is large. ${ }^{14}$

1 Bogoras, Jesup North Pucific Expedition, p. 690. ' ' 'Jochelson, ibid., vol.ix, p. 110. ' Ibid., vol. vi, p. $413 .{ }^{3}$ Sumner, J. A. I., vol. xxxi, p. 79. 'For instance, by Sumner, loc. cit., p. 79, for the Yakuts; by Jackson, Great Frozen Lnnd, p. 82, for the Samoyeds; and by Georgi, Bemerkunjen, vol. i, p. 265, for the Tunguses.

' Krasheninicoff, History of Kamtchatka, p. 216. De Lesseps (Travels, rol. i, p. 133) puts the average at four to five children. It may be noticed that according to Pallas (Rcise, vol. iii. p. 77) the Samoyeds do not cohabit for two months after the birth of a child.

Sograff, Arch. für Anth., vol. xir, p. 293.

p. 414 .

p. 349.

p. 513. 13 Finsch, Reise, p. 538. See Georgi, loc. cit., p. 263, for the Tun-

10 Krasheninicoff, loc. cit., p. $21 \%$.

- Jochelson, loc. cit., vol. vi, guses and Bogoras, loc. cit., p. 33, for the Chuckee. it See Finsch, loc. cit., p. 538, for the Ostyaks and Samoyeds; Jochelson, loc. cit., vol. vi, p. 423, for the Koryak ; and Sumner, loc. cit., p. 79, for the Yakuts. 


\section{THE REGULATION OF NUMBERS AMONG PRIMITIVE RACES}

1. We have now to ask what light these facts throw upon the quantitative aspect of the problem. Remembering that among all the races concerning which facts have been given there exists that form of primitive social organization the nature of which has been referred to, we may first examine briefly the theory of population as it applies to society in which there is co-operation; for the existence of this primitive form of social organization implies co-operation. We may then go on to apply what we learn from this review of the theory of population to the facts, so far as they concern the races of the first and second groups. We may next ask how far we can apply what we learn from primitive races to prehistoric races up to the opening of the third period, and finally we may inquire how it is to be supposed that the transition took place from the conditions under which the pre-human ancestorlived to those under which the earliest societies, of which we can indirectly gain any knowledge, must be supposed to have lived.

Malthus was the first writer to set out a theory in detail and to support it with evidence. ${ }^{1}$ Of the origin of his book some account has been given in the first chapter. In this book Malthus, according to his own account, attempted to. show three things-that population was limited by the means of subsistence, that it almost always increases when the means of subsistence increase, and that there are three checks upon increase-vice, misery, and moral restraint. By 'vice' and 'misery' he meant disease, war, poverty, and so on. By ' moral restraint' he meant restraint from sexual intercourse. This last check was not mentioned in the first edition of the Essay ; it was introduced for the first time in the second edition.

It is important to observe the nature of the argument put forth

${ }^{1}$ On this subject see Cannan, Theories of Production and Distribution, ch. v. Professor Cannan's work has been used in this and in the two following sections. 
by Malthus. The whole theory is one of the comparative rapidity of the increase of population and of the increase of food. Population, he said, when unchecked, increases in a geometrical ratio, food only in an arithmetical ratio. Therefore there must always be checks at work limiting population. He made a survey of the social conditions in different countries, and pointed to the evidence of the existence of various forms of vice and misery ; where moral restraint was practised, there was less vice and misery; where no moral restraint was practised, vice and misery reached their greatest prevalence. Furthermore the checks taken together must always be effective; it was, according to Malthus, merely a question of what kind of checks should be in operation. It was desirable in his view to increase 'moral restraint' in order to decrease 'vice' and 'misery'. It also follows that, if the checks were always effective, there could be no such thing as over-population. The conception of over-population, properly speaking, did not enter into the theory at all. It belongs to the later theory, which is based upon the productiveness of industry -an idea which finds no place in his book.

In the later editions Malthus made certain reservations about the impossibility of subsistence increasing faster than in an arithmetical ratio. Facts incompatible with the theory regarding the increase of population in America had come to light, and he admitted the possibility of the increase of subsistence in a geometrical ratio in new countries under certain conditions. $\mathrm{He}$ maintained, however, that in general subsistence could not increase faster than in an arithmetical ratio, and that his theory was therefore in essential features still correct. The ratios were at the basis of his theory, and sum up the whole essence of the argument. It has frequently been said, however, that Malthus did not attach much importance to the ratios. Professor Nicholson, for example, says that he used them 'not strictly-but as the basis for a simile'.' But Professor Cannan has shown that there is no foundation whatever for this view, and quotes a passage from Malthus exhibiting the importance which the latter attributed to this part of his theory. ${ }^{2}$

That subsistence can only increase in an arithmetical ratio, or in other words that the periodical additions to the average annual p 143.

Nicholson, Political Economy, vol. i, p. 182, note.

2 Cannan, loc. cit., 
produce cannot be increased, has long ago been disproved by statistics. Such figures as caused Malthus to make his reservations about new countries have been forthcoming for old countries also. In his own lifetime the census showed the falsity of the arithmetical ratio for so 'old' a country as England. It is not necessary to go into the facts, which are well known and beyond dispute. It is only the result of the proof of the fallacy contained in the arithmetical ratio part of the theory that concerns us. The result was that the whole argument collapsed, founded as it was on the comparative rapidity of the increase of population and of food. As Professor Cannan says: 'The Essay on the Principle of Population falls to the ground as an argument, and remains only a chaos of facts collected to illustrate the effect of laws which do not exist. Beyond the arithmetical ratio theory, there is nothing whatever in the Essay to show why subsistence for man should not increase as fast as an "unchecked population ". "With every mouth God sends a pair of hands," so why should not the larger population be able to maintain itself as well as the former?' 1

2. The answer to this question was in process of being discovered during the lifetime of Malthus. In the early years of the century attention was drawn to the high price of corn, to the position of agriculture, and especially to the fact that less rich land was being brought under cultivation. From the discussion which ensued, and to which Malthus contributed, there arose the idea of decreasing returns to agriculture. It has been pointed out that it must always have been recognized in practice that it did not 'pay' to employ more than a certain amount of labour on a given area of land. In these years, however, for the first time the principle which underlay this fact was made clear. West and Ricardo were chiefly responsible for bringing the matter to light. The development of the theory need not occupy us. It may be formulated shortly here. 'Whether we consider an acre of land or a whole country, after a certain point is reached, the return to a given amount of labour and capital will diminish. It will do so, however, only under the supposition that the arts of agriculture, using the phrase in the broadest sense, remain stationary.' 2 The law is not limited to agriculture. It is applicable to all industry. Whenever some agent in production, upon

'. Cannan, loc. cit., p. 144.

${ }^{2}$ Nicholson, loc. cit., vol. i, p. 163. 
which an industry is absolutely dependent, is strictly limited, after a time equal increments of capital and labour will not produce equal returns. In other words the returns to the same doses of capital and labour will diminish. 'This, however, will only occur when, as stated above, the supply of some agent in production is restricted; otherwise when an industry enlarges there will, as a rule, be an increasing return. The more labour, apart from diminishing returns, the better.

When speaking of agriculture it was said that diminishing returns only supervene when there are no improvements in the arts of agriculture. This is true in general of all other industries. Any increase in the arts of production will in general admit of more labour and capital being profitably employed; if there are . no further improvements, there will after a time come a point when diminishing returns will again supervene. The following out of the working of these laws in detail would call for a long and intricate discussion. Since the problem of quantity is only touched upon in its broadest outlines, such a discussion is not necessary here. The broad fact which emerges, and which alone is relevant here, is that, since the laws in general are applicable to all industry, there will be, taking into account on the one hand the known arts of production and on the other hand the habits and so on of any people at any one time in any given area, a certain density of population which will be the most desirable from the point of view of return per head of population. There will, in fact, under any given circumstances always be an optimum number; if the population fails to reach that number or if it exceeds it, the return per head will not be so large as it would be if it attained that number. This conception is of such importance that its formulation in other words by a modern economist may be quoted. 'At any given time, or, which comes to the same thing, knowledge and circumstances remaining the same, there is what may be called a point of maximum return, when the amount of labour is such that both an increase and a decrease in it would diminish proportionate returns. . . . If we suppose all the difficulties about the measurements of the returns to all industries taken together to be somehow overcome, we can see that at any given time, or knowledge and circumstances remaining the same, just as there is a point of maximum return in each industry, so there must be in all industries taken together. If 
the population is not large enough to bring all returns up to this point, returns will be less than they might be, and the remedy is increase of population; if, on the other hand, population is so great that the point has been passed, returns are again less than they might be, and the remedy is decrease of population.' 1

This idea of an optimum number is one which can be developed in great detail. It is only necessary here to notice that when, as in the higher economic stages, the arts of production on the one hand are improving and the habits and so on of any people are on the other hand constantly altering, the most desirable density is in consequence frequently changing. In the lower stages, when progress in skill is slow and social conditions more or less stationary, the optimum number may remain about the same over long periods of time. Further, with regard to progress in skill, inasmuch as the productivity of labour is enhanced by every improvement in the arts of production, the result of such progress will be that the return per head will, as a rule, increase. Such progress will thus, as a rule, allow of a larger population which will have a larger income per head.

This idea of an optimum density of population is wholly different to that put forward by Malthus. To him the problem was one of the relative increase of population and of food ; with us it is one of the density of population and of the productiveness of industry. To Malthus the position was much the same in all ages ; in his view population, except under unusual circumstances, had in any country at any given time always increased up to the limit of subsistence, and was in process of being checked-chiefly by vice and misery. In the modern view increase in skill has brought to an increasingly dense population a larger income per head. The chief cause of the largest possible income not on all occasions being reached is that at times the density of population increases beyond the optimum number for the given conditions, though at other times the failure of population to reach the desirable level may produce the same result.

Though the problem of diminishing returns was being discussed as the successive editions of the Essay appeared, the idea was

1 Cannan, Wealth, p. 68. This is frequently misunderstood. Thus we read that 'occasionally, from accidental circumstances, England was for a short time under-populated, and these were the periods when ... the labourer was well off' (Inge, loc. cit., p. 90). The evidence alluded to would tend to show that there was previously a condition of over-population. In any case a people as a whole is not better off when under-population arises. 
not applied by Malthus to the population question. This was undertaken by later writers. The development of the modern theory need not detain us ; it is of interest, however, to observe that J. S. Mill, whose influence over contemporary thought was so great, never shook off the deep impression made upon him early in life by the Malthusian theory. He exhibited a remarkable dread of over-population, and in his Principles of Political Economy seems to have regarded diminishing returns as so often arising that only rarely could an increase in population be advantageous. He appears in fact to have thought that after a certain stage all further increase in population was harmful, and to have looked upon 'the degree of industry which is required for the maximum productiveness of industry as something fixed once and for all '.1 Mill's view, therefore, though founded upon the productiveness of industry, differs from the modern view to which it has given place. It has since been clearly established that there is no maximum density desirable from the point of view of productiveness; so long as skill increases, other things being equal, so long will the desirable density increase. The influence of Mill's writings has had the effect of keeping alive in England longer than elsewhere the pessimistic view of the problem which the teaching of Malthus had originally given rise to. ${ }^{2}$ So strong were Mill's opinions that he regarded the limitation of the family by virtuous restraint as one of the most desirable of social reforms. $\mathrm{He}$ was led in the following passage to express views that are strongly in contrast with those of the many who now deplore the decline of the birth-rate among the upper social classes of to-day. 'Little improvement', he said, 'can be expected in morality until the producing of large families is regarded with the same feelings as drunkenness or any other physical excess. But while the aristocracy and clergy are foremost to give the example of this kind of incontinence, what can be expected of the poor ? 3

3. This conception of an optimum number holds good wherever there is social co-operation between groups of men living within definite areas. As we have seen a primitive form of social organization exists among all these races. This implies in itself a certain

1 Cannan, Theories of Production and Distribution, p. 181. Beaulieu, Question de la Population, ch. iii. vol. i, p. 180 .

2 See LeroyQuoted by Nicholson, loc. cit., 
degree of co-operation. It remains to show that these races are all, without exception, divided into groups which are strictly limited to definite areas-contrary to the still common notion that they wander where they please-and we may further note that the inhabitants of these areas co-operate to a greater or less degree in the search for food, and that there is a social obligation upon each man to do his share. We may review the evidence, beginning with the first point, which is of such importance that it may be treated at some length.

It is both misleading and dangerous to apply terms carrying modern legal conceptions to these races; unfortunately, any terms that may be used are to some extent biased. We have, however, somehow to describe the results of investigation into the customs of these races, and it has been found that among all these races, without exception, groups of men are recognized as, if not owning, then as enjoying the usufruct of certain very clearly defined areas. According to ling Roth it is not clear whether the Tasmanian tribes had any definite hunting grounds or not. ${ }^{1}$ Bonwick confidently asserts that it was so, ${ }^{2}$ and Wheeler thinks that the conditions in Tasmania were probably the same as in Australia. ${ }^{3}$ From Australia we have abundant evidence; the facts recorded from different parts of the continent vary considerably, and we must either believe that customs differed from place to place, which is not at all unlikely, or that many observers are mistaken, which, considering the positive nature of their assertions, is not very probable. Wheeler has reviewed the evidence and sums up his conclusions as follows: 'Our information shows the existence in some, at any rate, of the areas of Australia of what must be held to be private ownership in land, but it does not follow that the whole of the tribal territory was so allotted. The unit would generally be the individual family, but there are a few indications that the ownership might even be vested in single persons within the family, other than the head. The clearest mentions of individual or of family ownership seem to come from the south-eastern area, where the physiographic conditions are most varied, and where, in consequence, fishing rights become important. But it seems likewise that the rights of families or of individuals, as also those of local

1 Ling Roth, Tasmania, p. 116. Tribe in Australia, p. 35.

${ }^{2}$ Bonwick, loc. cit., p. $83 . \quad{ }^{3}$ Wheeler, 
groups, were, in general, subject to tribal over-rights, though we have no clear information on this point.' 1

There is no doubt, in the first place, that the tribes were everywhere restricted to clearly defined territories. ${ }^{2}$ Doubt only arises with regard to the smaller groups. The usual mode of living is in small groups of one to three families, which usually form a portion of a local group, but which may exceptionally be actual local groups. Only in the more fertile districts is the number of families living in contact greater. ${ }^{3}$ These local groups, into which the tribes are divided, seem generally to have their elearly defined areas within the tribal territory. Thus Brown, speaking of the Kariera tribe of Western Australia, says that 'the country of a local group, with all its products, animal, vegetable, and mineral, belongs to the members of the group in common. Any member of the group has the right to hunt over the country of his group at all times. He may not, however, hunt over the country of any other local group without the permission of its owners.' 4

In some parts no further subdivision of the land was recognized. 'I could not find', says Brown of Western Australia, 'any evidence of the individual ownership of any part of the soil or of any of its products. The whole of the territory of the group and everything in it seem to belong equally to all the members of the group.' 5 In other parts wo hear of family ownership ; Stanbridge says of Victoria that the tribal land 'has been from time immemorable parcelled out among its families and transmitted by direct descent to the present generation '.6 Further, some accounts speak of individual ownership. The natives of King George's Sound 'who live together have the exclusive right of fishing and hunting upon the neighbouring grounds, which are, in fact, divided into individual properties, the quantity of land owned by each individual being very considerable'? Eyre speaks of the parcelling out of territory among the individual

1 Wheeler, loc. eit., p. 45. Malinowski (loc. cit., p. 152) comments on this tribal over-right, which, he says, is shadowy.

2 See, for instance, Brown, J. A. I., vol. xliii, p. 144, for Western Australia; Spencer and Gillen, Native Tribes, p. 7, for Central Australia; and Stanbridge, Trans. Eth. Soc., new series, vol. i, p. 286, for Victoria. $\quad{ }^{3}$ Malinowski, loc. cit., p. $156 . \quad$ Brown, J. A. I., vol. xliii, p. 145. Spencer and Gillen describe the ownership by local groups of quarries where stone suitable for making instruments is found (Native Tribes, p. 590). - Ibid., p. 146. See, too, Spencer and Gillen, Northern Tribes, p. 27. bridge, loc. cit., p. 286. See also Parker, Aborigines, p. 12. 'Brown, Geog. Journ., vol. i, p. 12. 
members of the tribe. 'Every male has some portion of land, of which he can always point out the exact boundaries. These properties are subdivided by a father among his sons during his own lifetime, and descend in almost hereditary succession.' 1 As further examples of the ideas held by the Australians on the subject of property, it may be noticed that Smyth describes individual property in trees which passes by inheritance, ${ }^{2}$ while Lumholtz says that ' if a native finds a hive of honey in a tree, but has not an immediate opportunity of chopping it out, he can safely leave it till some other day; the discoverer owns it, and nobody else will touch it, if he has either given an account of it or marked the tree, as is the custom in some parts of Western Queensland '.3

Wherever we turn we find similar evidence of the recognition of distinct areas over which groups have more or less exclusive rights. ${ }^{4}$ Every tribe certainly has its own clearly defined territory; it seems further very probable that in most places the tribal territory is divided among local groups, if indeed the subdivision does not go farther. The Bushmen were formerly divided into tribes occupying 'well defined tracts of country, which they looked upon as their own ancestral hunting ground '. Their respect for property is shown by the fact that, when a man found a bees' nest, he put his mark upon it, and thenceforward it became 'the sacred property of the finder'. ${ }^{6}$ Klutschak describes distinct territories for the Eskimo tribes, who confine their seasonal migrations to these definite areas. ${ }^{7}$ Subdivision of land does not seem to go farther than subdivision among the villages; the inhabitants of a village had the right to refuse to allow any strangers to settle permanently in the neighbourhood. 'If a new family wished to settle at an inhabited place, the newcomers had to await the consent of the people already settled there, which was given by certain signs of civility or welcome, the strangers having meanwhile put their boat ashore, but not yet begun bringing up their goods. If these signs were not given they pushed the boat off again and went on to look for another place.' 8 It is so well known that the American Indians recognized clearly defined tribal boundaries that we need not attempt to

\footnotetext{
1 Eyre, loc. cit., vol. ii, p. 297. 3 Lumholtz, Among Cannibals, p. 147. p. $35 . \quad$ Stow, loc. cit., p. 35.

p. 227. $\quad{ }^{8}$ Rink, loc. cit., p. 31.

${ }^{2}$ Smyth, loc. cit., vol. ii, p. 145. 4 See Grosse, Die Formen der Familie, ' Ibid., p. $86 . \quad 7$ Klutschak, loc. cit.,
} 
illustrate the fact by means of references. ${ }^{1}$ It is perhaps not so well known that there are also many indications of family and even of individual property in land. In the first place we often hear of the right of the inhabitants of a village to clearly defined tracts. 'Each tribe had its village sites and contiguous hunting and fishing grounds; as long as the people lived on these sites and regularly went to their hunting grounds, they could claim them against all intruders.' 2 Of the Carrier Tribes, Harmon says that 'the people of every village have a certain extent of country, which they consider their own, and in which they may hunt and fish ; but they may not transcend these bounds, without purchasing the privilege from those who claim the land '.3 Of the Western Tinneh, Hill Tout records that the heads of the clan own the hunting grounds, "the limits of which were always very clearly defined .4 Among the more nomadic Eastern Tinneh we hear that bands used the same hunting grounds; these areas, however, were not regarded as belonging exclusively to them. ${ }^{5}$ Among the Salish of the Interior, ' all hunting, fishing, root and berry grounds were common property and shared in by all alike'; whereas among those of the coast the food grounds were the property of the septs and local groups. ${ }^{6}$ From other accounts it appears that family and individual ownership were recognized in some places. This applies especially with regard to the fishing stations of the Pacific Coast tribes, where 'varying lengths of shore were held as private fishing rights by heads of families, and these rights were passed from father to son and were always respected '.? Swanton gives an especially interesting account of the practices of the Haidahs of Queen Charlotte Islands. 'Each Haidah family had its own creek or creeks, or portion of a creek, where its smoke houses stood. Some of the smaller creeks are said to have no owners ; and, on the other hand, some families are said to have had no land. In the latter cases they were

1 "Captain Cook found among the Ahts "very strict notions of their having a right to the exclusive property of everything that their country produces", so that they claimed pay for even wood, water, and grass. The limits of their tribal property are very clearly defined, but individuals rarely claim any property in land '(Bancroft, loc. cit., vol.i, p. 191). See also Dellenbaugh, North Americans, pp. $410 \mathrm{ff}$.

2 Handbook of American Indians, Article 'Land Tenure'. loc. cit., p. 255.

- Hill Tout, British North America, p. 147. See also Ban. croft, loc. cit., vol. i, p. 118.

Ibid., p. 157.

- Ibid., p. 159. - Handbook of American Indians, Article 'Property'. See Teit, loc. cit., vol. ii, p. 255, for a description of this among the Lillooet Indians; also Bancroft, loc. cit., vol. i, p. 230, and Niblack, loc. cit., pp. 298 and 337. 
obliged to wait until another family was through before picking berries, and had to pay for the privilege. Any family might pick berries on the land belonging to another after the owners had finished picking, if it obtained the consent of the latter and paid a certain price.' 1 Referring to the Sitkin Indians, Elliot says that 'the coastline, and especially the margins of streams and rivers, are duly divided up among the different families. These tracts are regarded as strictly private property.' 2 Krause further gives an account of what can only be called individual private property in land among the Thlinkeets. ${ }^{3}$ The remarkable system in vogue among the Veddahs deserves notice. 'The whole Veddah country was divided into small hunting regions, of which each family possessed one.' The arrangements were most elaborate; the size of the tracts varied in accordance with the goodness of the land, and each included a portion of hill country to which each family thus had access during the rainy season without trespassing upon the ground of other families.' 4 Cooper has very fully reviewed the evidence for the Fuegians. It has been stated that communism existed among them. This is not correct. 'While all Fuegians are nomads,' he says, 'yet a Yaghan, for instance, is chary of poaching on Alacalufan or Onan territory. Even within recognized tribal territory the existence of more or less definitely marked off family hunting grounds is explicitly attested for the Onas by Professor Furlong and Dr. Dabbene and implicitly by Dr. Gallardo.' 5

4. Passing now to the races of the second group it is everywhere found that tribal territories are recognized, and it has been shown that, compared with hunting and fishing races, there is an increase in the number of cases in which land is held by smaller groups, if not by individuals, and a corresponding decrease in the number of cases in which communal ownership is recognized. ${ }^{6}$ The details of the variation in land tenure are not relevant, and a few descriptions of the conditions among different tribes will serve as examples

\footnotetext{
1 Swanton, Jesup North Pacific Expedition, vol. v, p. 71. Province, p. 54.

Krause, loc. cit., p. 167. Before leaving the Indians it is worth noticing that each family had, as a rule, its own store of food. Many different methods of storing food are known; the more migratory tribes of the Salish and the Tinneh stored food either in detached sheds elevated on posts several feet from the ground, or where the soil was unusually dry in well-like holes. See Hill Tout, British North America, p. 108.

- Sarasins, loc. cit., vol. iii, pp. 475 ff. See also Seligman, loc. cit., p. 106. - Cooper, loc. cit., p. 178.

'Hobhouse, Wheeler, and Ginsberg, Institutions of the Simpler Peoples, pp. 243 ff.
} 
of the manner in which groups of men among these races drew their means of subsistence from clearly defined areas. 'Among the Navahos a section of territory was parcelled out and held as clan land and, as descent in the tribe was traced through the mother, was spoken of by members of the clan as " my mother's land ". Upon such tracts the woman worked raising maize, \&c., and the product was recognized as their property.' 1 Of the North-American Indians generally we are told that 'occupancy gradually established a claim or right to possess the tract from which a tribe or individual derived food. This occupancy was the only land tenure recognized by the Indian ; he never of himself reached the conception of the land as merchantable. . . . As long as a person planted a certain tract the claim was not dis. puted, but if its cultivation were neglected, any one who chose might take it. Among the Zuni, according to Cushing, if a man, either before or after marriage, takes up a field of unappropriated land, it belongs strictly to him, but is spoken of as the property of his clan, or on his death it may be cultivated by any member of that clan, though preferably by near relatives, but not by his wife or children, who must be of another clan.' 2 So, too, among the Omahas maize was cultivated in patches of one-half to three acres in size; property in these patches was recognized so long as cultivation was continued. Afterwards any one could take them. ${ }^{3}$ In Mexico there was an elaborate system of land tenure. ${ }^{4}$ Conditions in South America were very similar to those in North America. Spix and Martius speaking of Brazil say: 'The savages consider the lands they have put under cultivation to be in some measure the property of their tribe. . . . One or several families unite to clear a part of the virgin forest and plant maize, manioc, cotton, or bananas. ... The same ground is cultivated every year, because it would be too difficult every yeas to clear new portiono of the forest. . . . A field cultivated for several years is considered to belong to a family, and the neighbours recognize these rights.' 5

The rights over land are very similar in Africa. Bartle Frere says that 'it is clear, from the accounts of early Dutch and other travellers in South Africa, that every Hottentot tribe had its

'Handbook of American Indians, Article 'Property'.

2 Ibid., Article 'Land Tenure'. $\quad{ }^{3}$ Fletcher and La Flesche, 27th A. R. B. E., p. 269. Dorsey (loc. cit., p. 366) gives further details.

5 Spix and Martius, vol. i, p. 83.

4 Joyce, Mexican Archaeology, p. 116. 
territory, into which strangers might not intrude for pasture or hunting, without the leave of the whole tribe; each kraal had its pasture lands distinct, over which the people of that section of the tribe moved their mat huts, as the need of their herds, for grass or water, rendered advisable. As each kraal had more or less of a family constitution, it is difficult to say how far the pasture lands were held in common, or as the property of the individual occupant.' 1 Of the Ewe-speaking people, Fllis says that among most tribes there is no private property in land but that the family in occupation of any tract cannot be disturbed. ${ }^{2}$ Of the Yoruba-speaking people the same author says that land, belonging to the community collectively, is vested in the chief, who distributes among households and families as required. No man can be depossessed of land once allotied to him, and the usufruct descends to his children, but the land cannot be sold. ${ }^{3}$ 'It is doubtful', says Talbot, ' whether any part of the Ekoi country remains at present unowned.' 4 According to Scott Elliot the rights of property in land of the different families in Sierra Leone are carefully maintained, especially in times of famine..$^{5}$ In Northern Nigeria a ' man is free to mark out a farm on any unoccupied land', and has a claim to it as long as he cultivates it. ${ }^{6}$ Among the Bangala of the Upper Congo 'the boundaries of a town are well defined, and the lands belonging to a town are well known to all other towns in the neighbourhood. If an animal is killed on ground owned by a town other than that to which the huntsman belongs, he has to send a portiongenerally the head-to the chief of the town which claims the ground.' 7 Further, 'every woman had her farm, which was her exclusive property, and not even a fellow wife had any rights over it ' 8 The hunting grounds of the Bushongo are well defined ; the usufruct of the soil belongs to individuals, and trees are private property. ${ }^{9}$ Among the Mangbetu 'every tribe has an accurate knowledge of the boundary of the territory over which it can hunt, move about, and establish its villages '.10 Hobley states that among the Akamba there is individual property in land which passes on the death of the father to the sons. ${ }^{11}$ The

1 Bartle Frere, J. A. I., vol. xii, p. 260.

3 Ibid., Yoruba-Speaking People, p. 188.

5 Scott Elliot, J. A. I., vol. xxiii, p. 82. Weeks, J. A. I., vol. xxxix, p. $123 .{ }^{8}$ Ibid.,
Joyce, Ann. Mus. Congo Belge, sér. 3, tome ii, p. 91 .
cit., No. 2, p. 465 . cit., No. 2, p. 465.

2498
2 Ellis, Ewe-Speaking People, p. 217.

4 Talbot, loc. cit., p. 262.

6 Tremearne, J. A. I., vol. xlii, p. 187.

8 Ibid., p. $129 . \quad 9$ Torday and 10 Van Overbergh, loc. 11 Hobley, Akamba, pp. 82 and $136 \mathrm{ff}$. 
limits of the properties are very well defined and well known among the Ababua; cultivation renders the title good.1 The Baganda 'lived in their gardens or plantations. These gardens were often joined one to the other, and a number of people lived in a community, often forming four or five miles of continuous garden with families living each on their own plot.' 2 Even the fallow land among the Akikuyu was 'all in private ownership', and could not be brought into cultivation without the owner's consent. ${ }^{3}$ In British Central Africa a man making a garden is ' perfectly free to choose so long as the ground is not in cultivation, or has not been bespoken by some one else; and, once marked, no one can interfere with it '.4 Of the same region we are told that 'these negroes have clear ideas of property. The waste land is usually considered to belong to the chief, but plantations and enclosures belong personally to the individual who originally made them. . . . Natives have clear ideas of large or small estates, or of their kingdom; and in the case of the former they are marked by the planting of certain trees of thick growth, while of course streams and mountains are recognized as boundaries and natural limits of territories.' 5 Of the Thonga tribe in Portuguese East Africa we hear that 'each man has his own field which he tills '.6 Speaking of the Bantu races south of the Zambezi, Theal says that "the chief apportioned to each head of a family sufficient ground for a garden according to his needs, and it remained in that individual's possession as long as it was cultivated '.' So Conder says of Bechuanaland, 'the land belongs to the chief. He divides it among his head men and they in turn among their people. There is no division of grazing land. The mealie fields are practically the property of the cultivator so long as they are tilled. I found each patch to belong to an individual, and to be divided generally by untilled land from the next patch.' 8 Among the Ovaherero the whole land is common property, but the rights of tribes and also of individuals to particular spots are recognized so long as they are occupied. Whoever first appropriates a spring and the surrounding pastures can maintain his right. ${ }^{9}$

1 Halkin, loc. cit., p. 493.

Prehistoric People, p. 39.

British Central Africa, p. 471.
7 Theal, Yellow- and Dark-Skinned People, p. 230. p. 86 .
are much the same; see Parker, J. A.I., vol. xii.
2 Roscoe, Baganda, p. 15.

4 Werner, loc. cit., p. 179.

3 Routledge,

5 Johnston, 
The conditions in Oceania are in their main elements, which alone are relevant, similar to those in America and Africa. In the Pelew Islands every family had its plot of land, which was considered as private property so long as it was occupied and cultivated.' In New Zealand ' land was held primarily by tribal rights ; and within this tribal right each free warrior of the tribe had particular rights over some portion'.2 In Sarawak 'each tribe had its limits, which have been handed down from father to son for ages, so that every old man of a tribe knows the exact extent of its district ' 3 In British New Guinca we hear of "properly regulated and well-defined property rights, certainly not bounded by surveyed lines commonly used to indicate European land claims, but marked and known by natural features such as surface conditions afford ' 4 It may also be noticed that rights over certain areas are recognized by the peoples of Northern Asia, such as the Tunguses and the Yakuts. ${ }^{5}$

5. Passing to the second and third points we find that within these areas there is evidence even among the most primitive hunters of co-operation in the search for food and of strict rules for the division of the available food. Coupled with this we find that there is a social obligation which is strongly enforced for every man to do his share. Thus, where hunting and fishing can only be carried out by joint parties, strict rules exist for the division of the catch among those who have taken part and among their dependents. This may be illustrated by a few examples. Howitt gives many details of the rules in force in Australia with regard to the division of food. These rules not only differ from place to place but also for the various forms of game. Thus among the Kurnai 'a wombat is cooked, then cut open and skinned. The skin is cut into strips and divided with parts of the animal thus. The head to the person who killed the animal. His father the right rib; mother the left ribs and backbone, which, with some of the skin, she gives to her parents. Her husband's parents receive some of the skin. The elder brother gets the right shoulder, the younger the left. The elder sister gets the right hind leg, the younger the left hind leg, and the rump and the liver are sent to the young men in the camp.' ${ }^{6}$ 'If a man only killed enough game or procured enough food for

1 Wilson, Pelew Islands, p. 297.

Roth, loc. cit., p. 419.

Unter Tungusen, p. 46.
${ }^{2}$ Tregear, loc. cit., p. 106.

Thomson, British New Guinea, p. 194.

6 Howitt, Native Tribes, p. 759.
3 Ling

5 Müller, 
himself, his wife and his children, then he need not divide with others; but if he found that his father had no food, he would give them what he had and go out and look for more.' 1 ' All the males in the Chepara tribe are expected to provide food, if not sick. If a man is lazy and stays in camp, he is jeered at and insulted by the others.' 2

The principal features of the Australian customs as described above are to be found among all hunting and fishing races, though such elaborate rules as are usual in Australia are somewhat uncommon elsewhere. The Bushmen may have had their rules for the division of game; all we are told is to the effect that they shared food. 'When one feasted they all partook ; and when one hungered they all equally suffered.' ${ }^{3}$ We have evidence both of the sharing of food and of the dividing up of game among the Eskimos. Details of the latter are given by Nansen; ${ }^{4}$ with regard to the former we have frequent references to the division of food within the villages among all who need it. ${ }^{5}$ Of the Eskimos we are also told that ' it might be considered a law that every man, as far as he was able to do it, should practise the trade of a hunter on the sea, until he was either disabled by old age or had a son to succeed him. This duty neglected, he brought upon himself the reprehension not only of the other members of his own family, but also of the wider community.' 6 Rules for the division of food were almost universal among the Indians ; when a hunter of the Hare tribe kills an animal, he is only allowed the tongue and ribs ; the rest is distributed according to a system. ${ }^{7}$ So, too, among the Lillooet a regular partition of the game took place, of which one of the features was 'that the persons who had the game had no preference over others ' .8 Such phrases as 'studied equity in the distribution of necessaries ', 9 which is applied to the Seri Indians, indicate the existence of similar rules. ${ }^{10}$

Conditions are similar among the races of the second group. In Fiji 'public opinion took care that no man in the community

${ }^{1}$ Howitt, Native Tribes, p. $765 . \quad{ }^{2}$ Ibid., p. 767. ${ }^{3}$ Stow, loc. cit., p. 41 . See also Theal, Yellow- and Dark-Skinned People, p. 48. ${ }^{4}$ Nansen, Eskimo Life, p. 113. 5 See, for instance, Klutschak, pp. $231 \mathrm{ff}$.. and Whymper, Voyages et Aventures, p. 346. 'Rink, loc. cit., p. 31. ' Bancroft, loc. cit., vol. i, p. 121 . ${ }^{8}$ Teit, loc. cit., vol. ii, p. 256. $\quad$ MacGee, 17th A. R. B. E., p. 273.

10 Hill Tout (British North America, p. 159) describes an exceptional case of a Salish tribe in which 'even the food was held and the meals taken in common, the presiding elder or headsman calling a certain family each day to provide the meals for all the rest, every one taking it in turn to discharge this social duty'. 
shirked his work'.1 In New Ireland, 'should a man neglect his family, a mode of punishment very similar to one practised by schoolboys among civilized races is adopted. A double row of men, women, and children - the whole population of the villagearmed with stiff birches is formed; and at a signal from the chief the delinquent is obliged to run a certain number of times through the lines and receive a general castigation from the rods of the villagers '.2 Of the Pawumwa Haseman says ' all work together. ... If any one refuses to assist in planting, the chief forces him to work. I saw one Indian with a long scar on the side of his head and neck, the result of punishment for laziness.' ${ }^{3}$

6. It is thus clear that within any group in any primitive race, the members of which co-operate together to obtain their food from a definite area to which they are confined, the principle of the optimum number holds good. There is, that is to say, taking into account the abundance of game, the fertility of the land, the skilled methods in use, and all other factors, a density of population which, if attained, will enable the greatest possible average income per head to be earned ; if the density is greater or if it is less than this desirable density, the average income will be less than it might have been. Obviously it must be a very great advantage for any group to approximate to this desirable density. There are three possibilities open to any group. The desirable number may be approached, it may be exceeded up to the point where men can only just exist, or it may not be reached. Extreme departures from the optimum number must be very disadvantageous ; if numbers increase until they are limited by starvation only, then no benefit arises from the use of any skilled methods that may be known. Under such circumstances all inventions in the methods of hunting, fishing, and cultivating the ground profit nothing. Social conditions must also inevitably be unstable where starvation alone limits numbers.

This being so, how are numbers regulated? We may observe to begin with that there is a number of factors at work among all these races which incidentally limit increase. This they may do either by decreasing fertility or by increasing elimination. To the former class belong pre-puberty intercourse and prolonged lactation, to the latter war and lack of care of children. Two

1 Thomson, Fijians, p. 229. Am. Anth., vol. xiv, p. 338 .

${ }^{2}$ Angas, loc. cit., p. 373.

3 Haseman, 
characteristics of these factors are noteworthy. The effect they have upon the limitation of increase is incidental ; it is the chance accompaniment of the practice of certain customs or of certain habits. In the second place the working of any single factor, so far as it reduces fertility or increases elimination, is fairly regular ; in any primitive race at any given time such habits as prolonged lactation and early intercourse, when they occur, cause a certain definite reduction of fertility ; and such customs as those of war and those connected with the upbringing of children cause a certain definite amount of elimination. The nature of the factors present and the degree of their incidence varies considerably from race to race, but it follows from what has been said above that in any primitive race over a considerable length of time the amount by which fertility is decreased and the amount of elimination which is caused remain fairly constant.

There is another class of factors the primary and not the incidental function of which it is either to reduce fertility or to cause elimination. These factors are prolonged abstention from intercourse, abortion, and infanticide. The view put forward here is that normally in every primitive race one or more of these customs are in use, and that the degree to which they are practised is such that there is an approach to the optimum number. With regard to this view we may first deal with the question as to the prevalence of these customs. We may then refer to the evidence as to the nature and origin of these practices. We shall then be in a position to ask how it may be supposed that they are so practised as to bring about that amount of restriction of increase which will result in an approach to the optimum number being made.

7. The evidence regarding the practice of these customsprolonged abstention from intercourse, abortion, and infanticidehas already been given in the last two chapters. This evidence is also summed up in the Appendix to which the reader is invited to turn. No attempt has been made to conduct an exhaustive inquiry ; but it is claimed that there is ample evidence of the widespread prevalence of one or more of these practices. There is no indication of the correlation of any one practice with any one economic stage. As far as the evidence goes, any practice may be in use in any economic stage. To the meaning of this we shall refer later. It is further claimed that, when the influence of contact with Europeans is taken into account, the evidence of the existence 
of these customs is still more impressive; for the observations upon which we have to rely have for the greater part been made after these races had been in contact with Europeans, and such contact is followed by a diminution of these practices.

One of the first changes brought about by this contact is connected with the introduction of diseases previously unknown. These diseases are often peculiarly fatal, causing a very high deathrate, and it is clear that, unless the practices of abstention from intercourse, abortion, and infanticide were largely abandoned, the race would perish. There is no difficulty in understanding how these practices would actually be abandoned soon after the introduction of disease. The proximate causes of these practices are largely, as we shall see, the difficulty of transporting many young children and the undesirability of having more than one child during the period of lactation. If disease began to carry off many children, the immediate causes for these practices would largely or entirely disappear and so would the customs themselves. ${ }^{1}$

Apart from the introduction of disease, the effect of contact is in other ways to reduce the extent of these practices. Contact has often resulted in warfare between aboriginal races and European settlers-as in Tasmania, Australia, and America, or as between the Bushmen and the Boers. The largely increased death-rate would, as in the case of disease, be followed by a diminution of the extent of these practices. Further, under other conditions, as for instance in Polynesia, the efforts of missionaries have long been directed to putting a stop to these customs. ${ }^{2}$ We may also note that there is often a bias on the part of observers to underestimate the extent of the employment of infanticide and abortion. Many observers are attracted by the races who come under their notice and seem to think that these practices are incompatible with the kindly nature or pleasant disposition of the people they describe-that in fact the attribution to them of such customs as

1 Some authors have assumed without producing any evidence that these practices-especially infanticide-have increased, if they have not been initiated, after contact with Europeans. It is, therefore, of interest to note that according to Rivers there is definite genealogical evidence from Tikopia of the disappearance of infanticide (Melanesian Society, vol. i, p. 352).

2 According to Neuhauss for instance, both abortion and infanticide were formerly prevalent in parts of New Guinea where they are now rare owing to missionary teaching. No accurate account has been given of these tribes in their former state, and thus quite possibly a modern observer might well merely record the practices as being 'rare'-a statement which would be misleading for our present purpose but which is of the kind likely to be relied on by those who do not admit the wide prevalence of these customs (Deutsch Neu-Guinea, vol i, p. 31). 
a normal feature of existence is a kind of libel-and if they report them at all, they persuade themselves into thinking that they are infrequent and abnormal. We have only to remember the objection taken to the interpretation of the finds in neolithic graves in England as evidences of infanticide-an objection based on the dislike of believing that our ancestors could have been guilty of such a habit. Or, again, we may remember that Tacitus, when desirous of holding up the Germanic tribes as an example to the Romans of his day, declared that they never committed infanticide-the implication being again that infanticide is to be regarded as a degenerate and unworthy custom. ${ }^{1}$

Nevertheless, in spite of all such tendencies working for the rapid disappearance of these customs, and in spite of the bias against believing in their existence, there is, as the Appendix shows, ample evidence that one or more of these practices are recorded for almost every people.

8. We may now consider the evidence regarding the origin and nature of these practices. We shall find that abortion and infanticide arise owing to the difficulty of providing for more than one child at a time. We shall also find that these customs are practised as normal features of social life and in such a manner as to keep the number of children at a fairly constant average figure. There is frequently a belief that a particular number of children is the right number of children. Further, it will be seen that the number of children to be preserved is a matter for consideration in which the wishes of not only the parents but also of the relations and of the community in general have to be taken into account, and that the practices are enforced by social pressure.

A large amount of evidence has been given to the effect that the number of children is always small-the number, that is to say, after these practices have taken effect. This is in itself evidence, it may be noticed, that these practices do result in reducing the number of children to a small constant average. Some additional evidence may now be noted.

According to Curr infanticide in Australia ' resulted principally from the difficulty, if not the impossibility, of transporting several children of tender age from place to place on their frequent marches ',2 and later he observes that 'infanticide was common

1 As will be pointed out later, this statement of Tacitus is undoubtedly incorrect.

2 Curr, Recollections, p. 252. 
among the Bangerang. ... They themselves gave as their reason for it the impossibility of the woman carrying more than one infant in their constant wanderings.' 1 'Every child which was born before the one which preceded it could walk was destroyed, because the mother was regarded as incapable of carrying two,' ${ }^{2}$ says another observer of the Narrinyeri tribe. Considerable information is given by Howitt for various Australian tribes. ' Infanticide is practised by the Mining to some extent, the mode of killing being by starvation. After a few days of short commons the child becomes peevish and troublesome, and in consequence more neglected, being placed by itself away from the camp and fires, and it is said to be afflicted with Mupurn (magic). When death ends its sufferings Mupurn is the cause. The reason they give for this practice is that if their numbers increase too rapidly there would not be enough food for everybody. Yet they are very fond of their offspring, and very indulgent to those they keep, rarely striking them, and a mother would give all the food she had to her children, going hungry herself.' 3 ' In the Tongaranka tribe the practice of infanticide was common, because a baby was frequently too much trouble to look after, and it was often the mother who killed it. But it was not done until the family consisted of three or four; but after that too much work in hunting had to be done to keep the family in food. ... In the Mukjarawaint tribe the children belonged to the grandparents, though the parents had care of them. If, for instance, a boy was born and then a girl, the father's parents might take them, or the mother's parents, and so also with another couple of children. If, then, another child was born and one of the grandparents took it, it would be kept. If not, it was killed, there being too many children. The grandparents had to decide whether a child was to be kept alive or not. If not, then either the grandfather or father killed it, by striking it against the mother's knee, and then knocking it on the head. ... According to Buckley, if a family increased too rapidly in the Wadthawing tribe, as, for instance, when a woman had a child within twelve months of the previous one, there was a consultation in the tribe as to whether it should live or not. . . Infanticide in the Kurnai tribe arose through the difficulty of carrying a baby when there were other children,

${ }^{1}$ Curr, Recollections, p. 263. Native Tribes, p. 748.

2 Taplin, loc. cit., p. 14.

3 Howitt, 
especially when the next youngest was not able to walk.' 1 Of the aborigines of the River Darling we are told that 'it seems to have been the custom to kill many of the children directly after birth, to save trouble and privation in time of drought, when long distances must be travelled. in the search for food and water ', and the same proximate cause of infanticide is mentioned by Beveridge. ${ }^{3}$ Of the women in the Port Lincoln district it is said that they put forward as the cause of infanticide the fact that they ' cannot suckle and carry two children together' ${ }^{4}$ similar reasons are said to be given by the women of Central Australia. ${ }^{5}$ Speaking of the natives of Port Darwin Foelsche says that the reason for infanticide is that 'too many children encumber the parents in travelling about for food ' ${ }^{6}$ It is also worthy of note in connexion with Howitt's statement about there being at times a dread of over-population that, according to Spencer and Gillen, this is not the case at any rate in Central Australia ; ${ }^{7}$ according to Curr, however, ' in many tribes there is a great fear of a want of food arising from over-population' .8 Among the tribes of Port Lincoln ' the number of children reared by each family is ... . very limited, rarely exceeding four', and 'if a mother has children in rapid succession . . . the young infant is killed '.9 In the Dieyerie tribe about 30 per cent. of the children are destroyed at birth. ${ }^{10}$ In the neighbourhood of Port Darwin children are killed 'where a woman has more than three or four'.11 In Central Australia 'each mother only rears, upon an average, two children'.12 Among the Northern tribes of Central Australia the number of children is kept down by infanticide to two or three. ${ }^{13}$

Similar evidence is forthcoming for other races of this group. Thus of the Puelches Guinnard says that 'among these almost primitive creatures, children are not nearly so numerous as might be imagined, for the existence of the new born infant is submitted to the judgment of the father and mother, who decide on its life or

1 Howitt, Native Tribes, p. 749.

${ }^{2}$ Bonney, J.A.I., vol. xiii, p. 125.

${ }^{3}$ Beveridge, loc. cit., p. 26.

- Wilhelmi, loc. cit., p. 181 . See also Eylmann, loc. cit., p. $261 .{ }_{5}^{5}$ Spencer and Gillen, Native Tribes, p. $608 .{ }^{\circ}$ Foelsche, loc. cit., p. $192 . \quad$ Spencer and Gillen, Native Tribes, p. 264.

${ }^{8}$ Curr, Australian Race, vol. i, p. 76. In another work, however, the same author says that he often spoke to the natives on this subject, and is 'sure that the idea of over-population never entered their heads' (Recollections, p. 263). Smyth (loc. cit., vol. i, p. 52) thinks that there may be some fear of over-population.

- Schürmann, loc. cit., p. $223 . \quad 10$ Gason, loc. cit., p. 8. ${ }^{11}$ Foelsche, loc. cit., p. $192 . \quad 12$ Eyre, loc. cit., vol. ii, p. 376.

${ }^{13}$ Mathew, Two

Representative Tribes, p. 165. 
death '. Charlevoix says of the Abipones that 'they seldom rear but one of each sex, murdering the rest as fast as they come into the world, till the eldest are strong enough to walk alone. They think to justify this cruelty by saying that, as they are almost constantly travelling from one place to another, it is impossible for them to take care of more infants than two at a time; one to be carried by the father, the other by the mother.' 2

Turning to races of the second group in Funafuti every mother was allowed to keep alternate children, but the second, fourth, and so on had to be destroyed." 'A Tikopian family is usually limited to four children, any excess of this number being killed by burying them alive in the house or just outside it ; occasionally five or six may be kept alive but never more. If the first four children are girls one or more of these may be killed in the hope that the succeeding children may be boys, in which case the lives of the boys would be spared.' 4 In New Guinea, in the later years of marriage, abortion is used in order to lessen the number of children, since a large family would mean too much work for the parents. ${ }^{5}$ In the Kingsmill Islands ' a woman seldom has more than two children, and never more than three ; when she discovers herself to be enceinte for the third or fourth time, the foetus is discharged by a midwife ' .6 In the Sandwich Islands, ' however numerous the children among the lower orders, parents seldom rear more than two or three, and many spare only one; all the others are destroyed, sometimes shortly after birth, generally during the first year of their age '.7 ' They consider three children a burden,' says the same author in another passage, 'and are unwilling to cultivate a little more ground, or undertake the small additional labour necessary to the support of their offspring during the helpless period of infancy and childhood.' 8 'No married pair (among the Gilbert Islanders) are allowed by their law to have nor bear more than four children, that is only four children get the chance of life. The woman has the right to rear or to endeavour to rear one child. It rests with the husband to decide how many children shall live, and this depends on how much land there is to divide.' 9 In Fiji 'infanticide is more prevalent among

1 Guinnard, loc. cit., p. 143.

3 Edgeworth David, Funafuti, p. 195. p. 313.

See also Krämer, loc. cit., p. 335. p. 327.
${ }^{2}$ Charlevoix, loc. cit., vol. i, p. 405.

4 Rivers, Melanesian Society, vol. i, Jenkins, loc. cit., p. 404. Ellis, Narrative, p. 324. 
the poor classes than the rich '.1 Speaking of the Murray Islands Hunt says that ' after a certain number had been born, all succeeding children were destroyed, lest the food supply should become deficient ' ${ }^{2}$ Codrington says of the Melanesians that 'abortion and infanticide were very common. If a woman did not want the trouble of bringing up a child, desired to appear young, was afraid the husband might think the birth before its time, or wished to spite her husband, she would find some one to procure abortion. ... Infanticide was more prevalent in some islands than others.... The old women of the village generally determined whether a new-born child should live; if not promising in appearance or likely to be troublesome, it was made away with.' ${ }^{3}$ Among the Mafulu of British New Guinea a woman must not give birth to a child unless she can give a pig to a village feast, and consequently children are often destroyed either by abortion or infanticide, both of which are common.4 Of the Western Islands in the Torres Straits we hear that ' few women rear more than three children, and besides most of those born before marriage are doomed to be killed immediately after birth, unless the father-which is seldom the case-is desirous of saving the child. . . Even of other infants some, especially females, are made away with in a similar manner when the mother is disinclined to support it.' 5 Of the Eastern Islands it is said that 'after a certain number had been born, all succeeding children were destroyed, lest the food supply should become insufficient '.6

Rengger, who remarks upon the small number of children among the Guaranis, traces it to the regular practice of abortion after a certain number had been born.? "Infanticide is quite common among the Lenguas, an interval of seven or eight years being always observable between children of the same family. Not only are babies, which are born in the interval, immediately killed, but abortion is also practised.' 8 Among the Creek Indians ' to destroy a new-born infant is not uncommon in families that are grown so numerous as to be supported with difficulty '.9 Of the Cheyennes it is said that 'it has been the custom that a woman should not have a second child until her first is ten years old.

1 Waterhouse, loc. cit., p. 327.

3 Codrington, loc. cit., p. 229.

2 Hunt, J. A. I., vol. xxviii, p. 9.

- Williamson, Mafulu People, p. 177.

- Cambridge Anthropological Expedition, vol. v, p. $198 . \quad{ }^{6}$ Ibid., vol. vi, p. 107.

7 Rengger, loc. cit., p. 329. $\quad$ Hawtrey, loc. cit., p. 295. 'Schoolcraft,

loc. cit., vol. v, p. 272. 
When that age is reached, the man is likely to go with his wife and child to some large dance or public gathering, and there ... to announce publicly that now this child is going to have a little brother or sister.' 1 There is sometimes evidence, for instance among the Sioux ${ }^{2}$ and the Brazilian tribes, ${ }^{3}$ that abortion is committed after consultation with the husband. Among the Pima Indians 'sometimes a mother nursed a child until it was six or seven years old, and if she became pregnant in the meantime she induced abortion '. ${ }^{4}$ In Fiji there was no cohabitation until the child was two years old. 'This separation ... was in bygone times invariably enforced' and abortion was practised when there had been enough children. ${ }^{5}$ Speaking also of Fiji Seeman says that ' relatives of a woman take it as a public insult if a child should be born before the customary three or four years have elapsed, and they consider themselves in duty bound to avenge it in an equally public manner '.6 Similarly Gutmann says of the Wadschagga that it is considered most disgraceful if a woman, who is still suckling a two years child, again becomes a mother, and that in consequence abortion is frequent.? In German New Guinea, according to Krieger, only three children are as a rule brought up owing to a fear of scarcity of food. ${ }^{8}$ In Radeck ' every mother is allowed to bring up only three children; every fourth and succeeding one she is obliged to bury alive herself '. ${ }^{9}$ Infanticide was ordered by law in Vaitapu and not more than two children were allowed in a family. ${ }^{10}$ Of the Roro-speaking tribes of New Guinea we are told that 'formerly it was not customary for a woman to have children until her garden was bearing well'.11 In the New Hebrides 'infanticide was sadly prevalent. As the burden of plantation and other work devolved on the woman, she thought she could not attend to more than two or three children, and that the rest must be buried as soon as born.' 12 In the New Britain group 'after marriage children are not borne by the woman for a period of from two to four years. I am informed that this is the result of a popular dislike to speedily becoming a mother on the part of the women,

1 Grinnell, loc. cit., p. 15. reich, loc. cit., p. 27. loc. cit., p. 181.

Krieger, loc. cit., p. 16 loc. cit., vol. iii, p. 173. sians, p. 270. infanticide was 'systematically practised'.
${ }^{2}$ Keating, loc. cit., vol. i, p. 394.

4 Russell, 26th $A . R . B . E$., p. 186.

6 Seeman, Viti, p. 191.
3 Ehren-

5 Blyth,

7 Gutmann, loc. cit., p. 3.

9 Kotzebue, 11 Seligman, Melane-
Paton, loc. cit., p. 452 , ${ }_{10}$ Turner, Samoa, p. 284. 12 Turner, Samoa, p. 333 . According to Paton, loc. cit., p. 452, 
who use various means to procure abortion and use them successfully. . . . A considerable period elapses between the birth of one child and the birth of another. The general term is about three years. One child is always well out of hand before another appears.' 1 We may also note that, according to Rivers, infanticide is frequent among the Todas and is practised not when food runs short but as a regular custom. ${ }^{2}$

It will be remembered that, when setting out in the last two chapters the evidence for the extent of abortion and of infanticide, these practices were in many cases stated to be committed when there was a certain number of children thus corroborating the evidence given to this effect above.

9. We are now in a position to discuss the manner in which numbers are regulated among these races, leaving until later the discussion as to how the position among these races was derived from that among species in a state of nature. Everywhere groups of men are, as we have seen, confined to definite areas. Among the few things which the men composing these groups do know with accuracy are the limits within which their food must be obtained. Further they co-operate in the search for food. It follows, therefore, that within any such area there is-taking all the relevant facts into account-an optimum number. The advantages to any group of approaching this number are immense ; a wide departure from this number can only be socially disastrous. We found that within any group there is a number of factorsहome of which reduce fertility and others of which increase elimination-and that the average amount of restriction of increase resulting from the action of these factors was fairly constant. We also found that there were certain other factors-prolonged abstention from intercourse, abortion, and infanticide-which are everywhere present and considerably restrict increase. If, as we shall see later, there is reason to think that some approach to the optimum is everywhere attained, it is clear that the former factors cannot of themselves alone sufficiently restrict increase. It is therefore to the latter factors, the primary function of which it is to restrict increase, that we must look when we seek for the mechanism by which numbers are brought near the desirable level.

It is clear how these factors originate. Among more or less nomadic peoples abortion and infanticide are practised because

1 Danks, loc. cit., p. 291.

${ }^{2}$ Rivers, Todas, p. 401. 
of the difficulty of transporting and of suckling more than one child at a time. Abstention from intercourse arises as a taboo. The problem we have to face is how these practices could come to be of the necessary intensity. Now men and groups of men are naturally selected on account of the customs they practise just as they are selected on account of their mental and physical characters. Those groups practising the most advantageous customs will have an advantage in the constant struggle between adjacent groups over those that practise less advantageous customs. Few customs can be more advantageous than those which limit the number of a group to the desirable number, and there is no difficulty in understanding how-once any of these three customs had originated-it would by a process of natural selection come to be so practised that it would produce an approximation to the desirable number. There would grow up an idea that it was the right thing to bring up a certain limited number of children, and the limitation of the family would be enforced by convention.

Though, however, adjustment is understandable as the result of a natural selection of customs, the evidence shows that there is even among the most primitive races at times at least some deliberation as to whether a child shall be allowed to live. In the more advanced races there is increasing evidence of deliberation. It cannot be supposed that in deliberation of this kind there is any grasp of the true position regarding the importance of the optimum number, but it may be supposed that under these circumstances the actual position at the moment as to whether there are too many or too few in the group does weigh when taking the decision. To all members of such a group, confined as they are within the knowledge of them all to a limited area, the disadvantages of too many mouths must be obvious. Therefore even among the more primitive races there may be some semi-conscious adjustment of numbers by means of one of these methods. However this may be, it is clear that, even if there is no semi-conscious deliberation among the lower groups, there is to some extent an automatic adjustment to the needs of the moment. Suppose disease or severe weather, for instance, to produce a higher rate of infant mortality, then abortion and infanticide, inasmuch as they are practised because of the difficulty of transporting more than one child, will be less practised.

10. Leaving aside for the moment the evidence that an approxi- 
mation to the desirable number is in this manner attained, we may note that in order that the system should be effective, something more is necessary. The conception of an optimum number involves the idea of a standard of living. The attainment of the optimum number indicates that the highest standard, which is possible taking all the circumstances into account, has been reached. In order that the standard of living may be maintained, it is not merely enough that numbers should be restricted; the younger generation must become proficient in the skilled methods which make this standard possible of attainment, and in particular it is important that the young men should not marry unless they are both energetic and skilful-unless, that is to say, they are both willing to keep up and are capable of keeping-up the standard of living previously attained. There is abundant evidence to the effect that pressure is exerted upon the younger generation. It is commonly recognized that among primitive races the girls marry at or soon after puberty. It is not so often recognized that the young men not infrequently do not marry until some years later. The inefficient and the physically incapable sometimes do not marry at all. In such facts we may see evidence of the pressure exerted by social conditions and conventions. We find also that not only are young men carefully instructed in the skilled methods, but that the parents of the bride anxiously inquire as to the bridegroom's energy and capability of supporting a family. The conditions regarding marriage have been much studied, and in marriage by service and marriage by purchase we can observe the pressure which forces a young man to show himself proficient in the skilled methods which are in use among his people. Obligations on the would-be bridegroom to work and save up the purchase money or to serve his future parents-in-law make it necessary for the young man to learn skilled methods and to exhibit energy and competence before marriage, whereby it is in any case rendered unlikely that new families will be set up which will adopt a lower standard of living than that of the previous generation. At the evidence for these facts we may now glance.

In Australia betrothals generally take place in infancy and marriage follows later ; these betrothals are usually arranged as exchanges, but the system shows some features of marriage by purchase. ${ }^{1}$ Considerable postponement of marriage is often

1 Malinowski, loc. cit., p. 48. 
observed, especially among the less skilled members of the community ; thirty is mentioned as a not uncommon age for marriage. ${ }^{1}$ Among the Eskimos there is a very strong feeling that a boy must show himself proficient in the difficult arts of hunting and fishing as practised by them. A young man seldom marries until he is over twenty, though often betrothed when an infant. ${ }^{2}$ Before that age he cannot learn all the methods of hunting game, managing a kayak, and so on. Marriage is considered impossible because he would not be in a position to maintain his family, and it is the necessity of showing that he is in this position before marriage is allowed which is remarked upon by all observers of Eskimo life. ${ }^{3}$ The usual form of marriage in America is by purchase. ${ }^{4}$ As a rule, the amount to be paid is relatively large, and the boy must either spend some time in getting it together or else obtain it from his father. At other times marriage is by service. Thus among the Kenai the bridegroom performs a year's service, ${ }^{5}$ and among the Haidahs boys were often betrothed at an early age and went to live with the girl's family and worked for them until marriage. ${ }^{6}$ A Seri bridegroom leaves his own family and enters that of the bride for a year; ' he must display and exercise skill in turtle fishing, strength in the chase, subtlety in warfare and all other physical qualities of competent manhood ' $'$ Among the Jakun a husband ' is expected to provide a hut, cooking pots and other necessary articles such as will suffice to enable house-keeping to be started with reasonable comfort' 8 Among the Fuegians 'as soon as a youth is able to maintain a wife by his exertions in fishing and bird catching, he obtains the consent of her relations, and does some piece of work, such as helping to make a canoe, or preparing sealskins '. 9

1 Curr, Australian Race, vol. i, p. 110 ; Dawson, loc. cit., p. 35 ; Smyth, loc. cit., vol. ii, p. 291 ; Malinowski, loc. cit., p. 259 ; Lumholtz, Among Cannibals, p. 184. ${ }^{2}$ Crantz, loc. cit., p. 158 ; Boas, 6th $A . R . B . E$. , p. 579.

${ }^{3}$ Crantz, loc. cit., p. 158; Boas, 6th $A . R$. B.E., p. 579 ; Klutschak, loc. cit., p. 233 ; Nansen, Greenland, vol. ii, p. 230 ; id., Eskimo Life, p. 139 ; Murdoch, 9th $A . R . B . E .$, p. 104. 'There is a superstition', says Dall, speaking of the Eskimos of Alaska, " that a youth must not marry until he has killed a deer, otherwise he will have no children' (loc. cit., p. 196).

' Handbook of American Indians, Article 'Marriage'. $\quad{ }^{5}$ Bancroft, loc. cit., vol. i, p. 134. For the Kutchins see Richardson, Arctic Searching Expedition, vol. i, p. 407, and for the inhabitants of Cadiack, Lisiansky, Voyage, p. 198. - Swanton, Jesup North Pacific Expedition, vol. v, p. 50.7 MacGee, 17 th $A$. R. B. E., p. 280 . For further details of marriage by service among the Indians see Carver, loc. cit., p. 373, and Domenech, loc. cit., vol. ii, p. 300. ${ }^{8}$ Skeat and Blagden, Malays, vol. ii, p. 70. vol. ii, p. 182. See also Bridges, loc. cit., p. 201.

9 King and Fitzroy, loc. cit., 
In this connexion it may be noticed that we often hear of the scorn poured upon the weak and unsuccessful; it appears to be the case that such men sometimes never marry. ${ }^{1}$ Among the Kutchins "poor men, whose abilities as hunters are small, and who have been unable to accumulate herds, remain bachelors ' ${ }^{2}$ One of the most difficult things an Eskimo has to learn is how to catch seals. 'The poor wretch' that cannot do so 'is despised to the last degree, and is obliged to subsist on women's diet, such as scolpings, which he can fish for on the ice, mussels, periwinkles, dried herrings, \&c.' 3 With regard to postponement of marriage, from twenty-three to twenty-five is the usual age of marriage for men among the Thompson Indians, ${ }^{4}$ from twenty-one to twentyfive for men among the Lillooets, ${ }^{5}$ and twenty-five among the Abipones. ${ }^{6}$

With regard to the races of the second group, among the Topebatos men marry when about eighteen. They have both to present gifts to, and to do work for, their future parents-in-law. ${ }^{7}$ The bridegroom also works for his future parents-in-law among the Bontoc Igorot. ${ }^{8}$ In Fiji 'young men of the lower orders married rather late in life for a primitive people, rarely, it appears, before the age of twenty-five'.9 Later the same author says that ' marriages were often delayed for years when the bridegroom's family were too poor to acquire property commensurate with their pride '. ${ }^{10}$ In the Maldive Islands 'although a man is allowed four wives at a time, it is only on condition of his being able to support them '.11 In the Caroline Islands 'a suitor serves for his wife in the house of his father-in-law elect as Jacob did with Laban and frequently has his pains for nothing '.12 Marriage by purchase is common in New Guinea and often results in a postponement of marriage while the purchase money is being collected. ${ }^{13}$ Speaking of the New Britain Group, Danks says ' some I have met who have never

\footnotetext{
1 See Carpenter, Intermediate Types, passim. vol. $i$, p. 383. $\quad{ }^{3}$ Crantz, loc. cit., vol. $i$, p. 163.

2 Richardson, Joc. cit., ${ }_{4}$ Teit, loc. cit., vol. $i$, p. 321. $\quad 5$ Ibid., vol. ii, p. 265.

${ }^{6}$ Ratzel, loc. cit., vol. ii, p. 124. According to Man (loc. cit., p. 81) men among the Andamanese marry at from eighteen to twenty-three years of age; Portman (loc. cit., p. 369) says twenty-six. Deniker (Rev. d'Eth., p. 301) says that the Ghiliaks do not marry until between twenty and twenty-five years old. The Aetas of the province of Cagayan have to pass a bow and arrow shooting test before marriage (Blumentritt, Z. G. E., vol. xxvii, p. 65). The custom of wrestling for wives has been recorded for various races (Lubbock, Primitive Man, p. 106).

7 Kreutz, loc. cit., p. 202.

Fijians, p. 172.

${ }^{8}$ Jenks, loc. cit., p. 68.

12 Christian, Geog. Journ., vol. xiii, p. 114.

11 Rosset, J. A. I., vol. xvi, p. 168.

13 Krieger, loc. cit., pp. 172, 297.
} 
married, but the cause lay in their inability to raise the shell money with which to purchase a wife '.1 In Sumatra marriage by purchase is said to constitute a certain hindrance to marriage, in spite of which, however, there are few celibates. ${ }^{2}$ Among the Negritos of Zambala the amount of the purchase money is large and ' there is no doubt that the gifts made represent almost all the wealth of which a young man and family can boast ' 3 In the Western Islands of the Torres Straits men marry when between twenty and twenty-five years old, ${ }^{4}$ marriage being by purchase. ${ }^{5}$ In certain cases where head huuting is practised prowess in this art must be shown before marriage. ${ }^{6}$ In certain parts of Borneo ' it is a rule among all the tribes that no youth can regularly wear a mandan, or be married or associate with the opposite sex, till he has been on one or more head hunting expeditions '.?

Marriage by purchase is often found in Africa. The necessity of collecting the purchase money frequently involves some postponement of marriage. Formerly among the Thonga a man generally married when about twenty-five years old, ${ }^{8}$ but there was some variation in the age owing to the varying difficulty experienced in getting together the necessary cattle. ${ }^{9}$ Sooner or later nearly all the men among this tribe get married; ${ }^{10}$ and this is true of all Bantu peoples. 'The kind of individual called a bachelor does not abound among the Bantu. 'The wretched, the invalids, the weak-minded only, are deprived of the legal marriage which for the black man is and remains the one object in life.' 11 Sometimes in South Africa 'a young man too poor to acquire a wife by the transfer of cattle would make an arrangement with the father of the girl to live with her and serve him '.12 Among the Baronga young men do not marry for several years after puberty. ${ }^{1 \text { s }}$ Werner's description of marriage among the Zulus is of particular interest. 'The price paid by the Zulus (under the name of Lobola) and others cannot properly be called purchase, being rather in the nature of a settlement or guarantee that the suitor is able to support a wife; it is held by her family in trust for her and her children.' 14 The same author, speaking generally of British Central

\footnotetext{
1 Danks, loc. cit., p. 288.

bei den Kannibalen, p. 247.

2 Marsden, Sumatra,pp. 218, 219 ; Brenner, Besuch ${ }^{3}$ Reed, loc. cit., p. 56 .

Anthropological Expedition, vol. v, p. 247.

loc. cit., p. 275 (of the New Hebrides).

8 Junod, South African Tribe, p. 100.

5 Ibid., p. 230.

${ }^{4}$ Cambridge

7 Bock, Head Hunters, pelo.

9 Ibid., p. 102. 10 Ibid., p. 101.

11 Ibid., p. 125.

13 Junod, Ba-Ronga, p. 30.

12 Theal, Yellow-and Dark-Skinned People, p. 220.

14 Werner, loc. cit., p. 129.
} 
Africa, says that "young men may have to wait for some years, owing to lack of means or other reasons. In the country under the Angoni chiefs they are called on ... to "serve their time", herding the chief's cattle, and later, perhaps, going to war.' 1 The men of the Akikuyu are described as 'not marrying very young '.2 Roscoe enumerates the various articles required to make up a bride's price among the Baganda, and adds that 'they were difficult to obtain and represented a large sum to a poor person, so that it took him a long time to collect them ; a man frequently spent twelve months begging among his relatives and friends the amount asked ; for though as a rule he had secured some of the things before he went to ask for the lady, there would still be a balance to find '. 3 Among the Wapagoro regular sexual intercourse is practised by children. At puberty they are separated and the boy must then begin to collect the purchase money, and not until he has finished may intercourse be resumed. ${ }^{4}$ Plas notes postponement of marriage among the Kuku owing to the amount of the purchase money. ${ }^{5}$ An interesting account of the Akamba is given by Dundas. 'During his lifetime a man so divides his stock that he allots a portion to each of his wives. On his death the portion of each wife goes to her son or sons. ... If the cattle left are not numerous enough to buy a wife for each son they are loft with the eldest son until the increase suffices for the purchase of' a wife for him. When the increase is again large enough it is given to the second son to buy a wife, and so on until each has a wife.' 6 According to Johnston, 'the Masai warrior is not allowed by the elders of his tribe to marry until he has reached about thirty years of age and has accumulated a fair amount of property or else so distinguished himself by .his bravery as to merit an early retirement ' $?$ Among the Bangala, if a man is accepted by a girl he has to pay the 'bespoke' money, after which 'the girl is reserved for him until such time as he can pay the whole or larger part of the marriage money', and, while he is collecting this money, he will build a house if he does not already own one. ${ }^{8}$ Further, 'a man can marry as many women as he can afford to pay marriage money for, but to each he must give a house ${ }^{\prime},{ }^{9}$ and

1 Werner, loc. cit., p. 128.

2 Routledge, Prehistoric People, p. 124. 3 Roscoe, Baganda, p. 88. The price of course varies with varying economic conditions, as Dundas notes for the Wawanga (J.A.I., vol. xliii). "Fabry, loc. cit., p. $221 . \quad{ }_{5}^{5}$ Plas, loc. cit., pp. 215, 219.

vol. xliii, p. 516.

vol. xxxix, p. 440.

? Johnston, Uganda, vol. ii, p. 822.

6 Dundas, J. A. I.,

${ }^{8}$ Weeks, J. A. I., 
as a result of the high marriage price, if his family cannot help him, a man 'cannot save enough to procure a wife until he is thirty or even older'.1 Cureau states that in the Congo basin a bride's relatives are much interested in estimating a suitor's capabilities of supporting a family and of, in general, doing well.2 Among the Ekoi people, ' by a native custom if a man wishes to marry an Ekoi maiden he must serve her people for some considerable time, usually from two to three years. His work mostly consists in helping to clear bush for next season's farms, but other services may be required of him. During this time he is expected to make presents to the relations of his future wife.' 3

Very similar institutions are found in America. Among the Nandowensis, "when one of their young men has fixed upon a young woman he approves of, he discovers his passion to her parents, who give him an invitation to come and live with them in their tent. He assiduously accepts the offer, and by so doing engages to reside in it for a whole year, in the character of a married servant. During this time he hunts, and brings all the game he kills to the family, by which means the father has an opportunity of seeing whether he is able to provide for the support of his daughter and the children that might be the consequence of their union.' 4 Among the Ojebway Indians marriage is also by service and the future father-in-law is described as being anxious that the suitor should be a good hunter. ${ }^{5}$ Of the Natchez it is said to be ' rare for young men to marry before they be fiveand-twenty. Till they arrive at that age they are looked upon as too weak, without understanding and experience.' 6 According to Dorsey, men of the Omaha tribe did not marry formerly until between twenty-five and thirty." Among the Attakapas, "if a savage desires to marry a girl whose father is still living, he approaches the latter; the latter then inquires if he is a brave warrior, a good hunter ', if he can make weapons, and so on. ${ }^{8}$ In Mexico men married about twenty. ${ }^{9}$ In British Guiana marriage is by service, and the bridegroom must show that he is capable of

${ }^{1}$ Weeks, J.A.I., vol. xxxix, p. 417.

2 Cureau, loc. cit., p. 417.

3 Talbot, loc. cit., p. 105. It will also be remembered that contraceptive practices are of importance in Africa, and that there is evidence that in the neighbourhood of Port Herald a young couple must not have children until they have built a house for themselves. See p. 177.

4 Carver, loc. cit., p. $373 . \quad 59$. Pratz, loc. cit., p. 199.

7 Dorsey, loc. cit., p. $259 . \quad{ }_{8}$ Bossu, Voyages, p. 247. $\quad$ Joyce, Mexican Archaeology, p. 162. See also Bancroft, loc. cit., vol. ii, p. 251. 
taking a man's part ; within a certain time he must clear a piece of land of a given area. ${ }^{1}$ Another author gives further details : 'Before he is allowed to choose at all he must prove that he is a man and can do a man's work.' There are various tests. Among others 'he clears a space in the forest to be planted with cassaba, and brings in as much game and fish as possible, to show that he is able to support himself and others' ${ }^{2}$ In Peru a man had to be twenty-four years old before he could marry. ${ }^{3}$ Von Martius states that the system of marriage by purchase in Brazil is to be regarded as a symbol that the bridegroom can support a family. Men among the Guanas marry when over twenty. ${ }^{5}$

11. The evidence so far adduced shows that the mechanism whereby numbers may be kept near to the desirable level is everywhere present. When inquiring into the nature of the mechanism, we found certain indications that it was effective. The regularity with which certain customs are practised, the small average number of children, and other facts afford strong but not conclusive evidence that an approach is normally made to the optimum number. We may now ask what further evidence there is as to the position in this respect.

Conclusive evidence is not available. It is only when we are able, as among some of the races in the third group, accurately to measure the average income over a period of years during which numbers are changing that we can arrive at a definite result. With regard to other races we have to adopt methods which, though less precise, nevertheless afford important evidence. We may inquire into the general conditions of life and ask whether there are indications of the approach to the highest standard of living within reach, or whether living is more usually reduced to the bare level of subsistence. We may ask whether famine and starvation frequently occur, what the average physical condition of the people is, whether old age is often reached, whether, in short, the conditions are such as we should expect to find them when the optimum number was approached, or as we should expect to find them when the numbers reached the maximum which could just be kept alive.

The conception of savage life which formerly prevailed was that

1 Schomburgk, Reisen, vol. ii, p. $251 . \quad 2 \quad{ }^{2}$ im Thurm, loc. cit., p. 221. 3 Rivero and von Tschudi, Peruvian Antiquities, p. 185.

loc. cit., vol. i, p. 109.

- Von Martius, 
primitive races were always in a condition of semi-starvation. ${ }^{1}$ In this connexion it has to be remembered that the conception of the optimum number has regard to all the conditions, and that among these races, taking the degree of skill, social customs, and so on into account, there would be, even if the optimum number was attained; times of scarcity, if not of famine. The existence of times of scarcity, therefore, is no evidence that, so far as numbers are concerned, these races have not attained the best conditions possible for them. It is undoubtedly the fact that they are physiologically adapted to withstand periods of scarcity in a manner that civilized men are not adapted, and it is interesting to observe that, according to the results of certain experiments, occasional periods of semi-starvation are far less harmful than is continual under-feeding. ${ }^{2}$

Descriptions of the Australians suggest the picture of anything but an emaciated people in a condition of semi-starvation. In one place Spencer and Gillen speak of them as well nourished $;^{3}$ in another place they describe a typical Arunta as 'by no means poor in physique; in fact he might often serve a sculptor for a model, and, when walking behind a native, you are continually struck with his proportions and beautiful carriage'.4 Thes $\theta$ authors go on to say, however, that 'there are times when he is hard pressed and during a long continuance of drought his life is not a happy one '.5 So, too, Schürmann says of the tribes of Port Lincoln : " the male sex exhibits a great deal of unstudied natural grace in their deportment, their walk is perfectly erect and free, motions of the body easy and gestures natural under all circumstances.' 6 Further, we are told that 'their food, if of indifferent quality, was at least wholesome and readily procurable, six hours a day abundantly sufficing for that purpose, so that hunger was little known', 7 and that 'in most of the districts with an indigenous population game is so abundant compared to the number of inhabitants, as to enable every one to procure for himself and his family as many pounds of meat a day as his heart desired '.8 Perhaps this is too optimistic a view ; there are certainly many references to lean times when food is difficult to procure. 'In few

${ }^{1}$ See Herbert Spencer, Principles of Biology, vol. ii, p. 515. ${ }^{2}$ Morgulis, American Naturalist, vol. xlvii, p. 477 . 3 Spencer and Gillen, Native Tribes, p. 44. 'Same authors, Across Australia, vol. i, p. $191 .{ }^{5}$ Ibid., vol. i, p. 197. 6 Schürmann, loc. cit., p. 209. 259. Semon, loc. cit., p. 217 . See also Smyth, loc. cit., vol. i, p. 122. 
parts of Australia', says Thomas, 'can the native count on anything like regular supplies of food ',1 and we hear of those powers of enduring hunger and thirst so common among these races and so incomprehensible to Europeans. ${ }^{2}$

Burchell is enthusiastic about the Bushmen; 'as we rode onwards, I could not cease admiring the beautiful symmetrical form of our Bushman guide, who walked and sometimes ran before us, with a gait the most easy and free that I ever beheld. All the limbs, unshackled by clothing, moved with a grace never perhaps seen in Europe. The contemplation of his well-proportioned, although small and delicate figure, his upright manly port, his firm and bold step, and the consciousness of liberty which beamed in his countenance afforded us indescribable pleasure.' 3 Nevertheless the Bushmen had 'periods of fasting' 4 and were 'often destitute of food for several successive days during seasons when both roots and game were scarce' .5 They were also 'capable of remaining a long time without food, and could then devour immense quantities of meat without any ill-effect ' .6

In most countries a lean season occurs periodically once or more every year. Such lean seasons are more exacting in some regions than others. The Australians manage to subsist for the most part without storing up food $;^{7}$ in other countries the storing of food becomes a necessity. ${ }^{8}$ We often find that where food has to be stored up the stock runs low towards the end of the lean season. The Eskimos have to depend for many months upon the food they have preserved, and if for any reason, as for instance the late formation of the floe, their calculations are upset, they may be placed in a very trying situation. ${ }^{9}$ Of them we hear stories, as of other races, regarding their powers of withstanding hunger ; 'a man who has eaten nothing for three days, at least nothing but sea grass, can manage his little kayak or canoe in the most furious waves ' ${ }^{\prime 0}$

1 Thomas, Natives of Australia, p. 88. See also Eylmann, loc. cit., p. 293. 2 See, for instance, Palmer, J. A. I., vol. xiii, p. 281. ${ }^{3}$ Burchell, loc. cit., vol. i, p. $422 . \quad$ Stow, loc. cit., p. 91. 5 Ibid., p. 180. Theal, loc. cit., p. 36. See also Moffat, loc. cit., p. 57, and Campbell, Personal Narrative, p. 88. Thomas (loc. cit., p. 117) speaks of storing up of food. The Kurnai do not store up food, but the Dieri, who are closely allied to them, do so on a small scale (Fison and Howitt, loc. cit., p. 108).

8 The beginnings of this custom are found among the Fuegians. Darwin says that "when they find a stranded whale, they bury large portions in the sand, and during the often recurrent famines travel from great distances for the remnants of the half putrid mass ' (Voyage, vol. i, p. 327).

- Boas, 6th $A . R . B . E .$, p. 427. See also Rink, loc. cit., p. $186 . \quad{ }^{10}$ Crantz, loc. cit., vol. i, p. 134. 
Of the Kutchins of the Peel River we are told that they are ' an athletic and fine-looking race, considerably above the average stature, most of them being upwards of six feet in height and remarkably well proportioned'.1 'The Indians north of the Columbia are, for the most part, good-looking, robust men, some of them having fine, symmetrical forms. They have been represented as diminutive; with crooked legs and uncouth features. This is not correct ; but, as a general rule, the direct reverse is the truth.' 2 The Montagnais are described as 'tall, strong, erect, well-proportioned and agile ' $;^{3}$ on the other hand, we hear that they are liable to shortages of food, are able to go without food for three days together at a time, and are guilty of great excesses of eating and drinking when food is plentiful. ${ }^{4}$ Hardisty says that the Loucheux can always obtain food except under very unfavourable circumstances. ${ }^{5}$ 'It sometimes happens that the Ahts are in straits for want of food, when the fish do not appear until late in the spring,' 6 but ' they can bear the want of food a long time without becoming exhausted'? The account of the Californians given by the acute Jesuit missionary Baegert is of particular interest. ' Notwithstanding the barrenness of the country, a Californian hardly ever dies of hunger, except perhaps now and then an individual that falls sick in the wilderness and a great distance from the mission, for those who are in good health trouble themselves very little about such patients, even if these should happen to be their husbands, wives or other relations, and a little child that has lost its mother or both parents is also occasionally in danger of starving to death. . . . The food of the Californians is certainly of a mean quality, yet it keeps them in a healthy condition, and they become strong and grow old in spite of their poor diet.' 8 ' Californians can endure hunger easier and much longer than other people; whereas they will eat enormously if a chance is given.' 9 Three days without food appeals differently to an observer in a mission station on the one hand and to a prisoner

1 Bancroft, loc. cit., vol. i, p. 127. In the spring, when the winter stores are exhausted, they usually experience a lean season (ibid., p. 129).

2 Swan, North-west Coast, p. 154.

3 Le Jeune, Jesuit Relations, vol. vi, p. 229.

Ibid., pp. 233, 277, and 285.

5 Hardisty, loc. cit., p. 311. Other accounts describe lean periods among the northern tribes as fairly common, during which 'they often subsist for a great length of time upon a very little food ' (Harmon, loc. cit., p. 284). See also Morgan, Houses and House Life, p. 56.

6 Sproat, loc. cit., p. 53.

? Ibid., p. 22.

8 Baegert, loc. cit., p. 366.

- Ibid. 
among the Indians on the other. 'So protracted was the hunger we experienced,' says Cabeça de Vaca, ' that many times I was three days without eating anything. The natives also endured as much, and it appeared to me a thing impossible that life could be so prolonged.' The : ame author goes on to say, however, that ' they are a merry people considering the hunger that they suffer; notwithstanding they never cease to dance nor to observe their festivities and ceremonies. To them the happiest part of the year is the season of eating prickly pears ; for then they have hunger no longer, and pass all the time in dancing and they eat day and night. . . . It occurred to us many times while we were among this people, and there was no food, to be three or four days without eating, when they, to revive our spirits, would say to us not to be sad, that soon there would be pears and we should enjoy plenty, and drink of the juice, and that our bellies would be very big, and we should be content and joyful, having no hunger.' 1

It is submitted that the conditions indicated by the type of evidence of which examples have been given above are not compatible with the state of existence on the bare means of subsistence. ${ }^{2}$ With this evidence there should be considered the facts given in the sixth chapter regarding the good health and advanced age to which these races generally attain.

Turning to races of the second group, in the Eastern Islands of the Torres Straits 'nutritious food is generally very scarce at the end of the dry season'.3 The Dyaks usually experience a season when it is difficult to procure food. ${ }^{4} \mathrm{We}$ are told that famine was unknown in Fiji, ${ }^{5}$ but that from November to February there was sometimes a scarcity when the last yam crop had been consumed and the next crop had not ripened. ${ }^{6}$ So too among the Baganda 'no one ever went hungry while the old customs were observed ', but there are at times lean seasons. 'When food is abundant they have their three meals daily; when it is scarce they content themselves with two, and hope for the rain and a plentiful supply of fruit.' 8 Cureau, describing

- Cabeça de Vaca, p. 63.

2 Similar evidence is forthcoming for many other races. For the Andamanese see Man, loc. cit., pp. 342 ff. ; for the Payaguas, Azara, loc. cit., vol. ii, p. 142 ; for the Ghiliaks, Deniker, Rev. d'Eth., vol. ii, p. 295 ; and for the Fuegians, Hyades and Deniker, loc. cit., pp. 122, 339.

' Cambridge Anthropological Expedition, vol. iv, p. 180.

Sarawak, vol. i, p. 422.

$?$ Roscoe, Baganda, p. 12.

- Thomson, Fijians, p. 332

8 Ibid., p. 6.

- Ling Roth,

- Ibid., p. 335. 
the races of the Congo Basin, gives a general description of the conditions which corresponds very closely with that just quoted for the Baganda." In British Central Africa " "the time of hunger" comes after the rains, when the last year's corn is eaten, and the new is not yet ripe-about March '.2 Of the Kagero of Northern Nigeria we are told that the 'people are naturally more healthy and better conditioned in December, say, than in August', because towards the end of the year there is plenty of food from the harvest whereas later in the wet season there is usually a scarcity. ${ }^{3}$ So of the Mandja of the Northern Congo we are told that 'generally by the month of October, the Mandja family has consumed its reserves of food; thereupon follows want for two months ' 4 In North America Catlin describes times of scarcity among the Mandans ${ }^{5}$ and im Thurm describes similar conditions in Guiana. ${ }^{6}$

Of the fine development of physical form there is abundant evidence. The inhabitants of Timor Laut are 'handsomefeatured fellows, tall, erect, and with splendidly formed bodies '.7 One of the earlier travellers, Kotzebue, was very much struck with the physical features of the inhabitants of the Sandwich Islands ${ }^{8}$ and of Radeck. ${ }^{9}$ So, too, in Africa the Akikuyu are ' exceedingly strong, muscular, healthy and well set up ' 10 'The Damaras, generally speaking, are an exceedingly fine race of men. Indeed it is by no means unusual to meet with individuals six feet and some inches in height, and symmetrically well proportioned withal. Their features are, besides, good and regular ; and many might serve as perfect models of the human figure.' ${ }^{11}$ As in the case of the races of the first group, we are told that formerly these people lived to a great age. 'Under natural conditions the Bantu were a longer lived people than the Europeans.' 12 Catlin was greatly impressed with the physical fitness of the North American Indians. He never saw 'a more hardy and healthy race of men', while the women were 'exceedingly healthy and robust'.13 According to Heriot, 'the North Americans are in general robust, and of a healthful temperament,

${ }^{1}$ Cureau, loc. cit., p. 252. J. A. I., vol. xlii, p. 180. vol. ii, p. 124.

${ }^{8}$ Kotzebue, loc. cit., p. 129.
People, p. 19 .

People, p. $19 . \quad{ }_{11}$ Anderson, Lake Ngami, p. 49. Dark-Skinned People, p.
loc. cit., vol. ii, p. 228.
I Ibid., p. 170
2 Werner, loc. cit., p. 181.

4 Gaud, loc. cit., p. 21. Forbes, Timor Laut, p. 9. 10 Routledge, Prehistoric 12 Theal, Yellow-and

13 Catlin, 
calculated to live to an advanced age '.1 Du Pratz speaks highly of the physical characteristics of the Natchez and much similar evidence could be quoted. ${ }^{2}$ Azara, for instance, says that the Payaguas lived to an advanced age ${ }^{3}$ and also mentions especially the fine physical features of the Mbayas ${ }^{4}$ and of the Charruas. ${ }^{5}$ Of the Mandrucos Wallace says that 'their figures are generally superb ; and I have never felt so much pleasure in gazing at the finest statue, as at the living illustrations of the beauty of the human form. The development of the chest is such as I believe never exists in the best formed Europeans, exhibiting a splendid series of convex undulations, without a hollow in any part of it.' ${ }^{6}$ Humboldt remarks of the Chaymas that both men and women 'are very muscular, but fleshy and plump'?' So, too, Spix and Martius, speaking of Brazilian tribes, say that 'the Indians are seldom sick and generally live to an advanced age ' 8

Lastly it may be observed as evidence of some weight that, as between different races, the higher the skill and the greater the natural richness of the surroundings, the higher, so far as we can judge, is the return per head. Though the return cannot be measured, all that we know of the conditions of life point to this conclusion, whether we contrast the agricultural races in general with the hunting and fishing races, or whether we contrast such hunting and fishing races as those of the north-west coast of America with the Fuegians.

12. The conclusions derived from a study of the first two groups are therefore to the effect that an approximation to the desirable number is normally attained by the practice of one or more of the three customs mentioned. As a result, the small average size of the family is arrived at-the size being just about that which allows for the average mortality from various causes later in life, so that in the next generation there will be as nearly as possible the same number of adults as in the former generation. It may be that, as an occasional coincidence, just that amount of reduction of fertility and just that amount of elimination necessary to bring about approximation are effected by means of those factors which were described as having incidentally these results. But such a coincidence must be very rare and the

1 Heriot, loc. cit., p. 350.

2 Du Pratz, vol. ii, p. 161 . loc. cit., vol. ii, p. 142 . - Ibid., p. 107. 5 Ibid., p. 8. Narrative, p. 478. 7 Humboldt, Personal Narrative, vol.iii, p. 233. and Martius, loc. cit., vol. ii, p. 249.

${ }^{3}$ Azara, -Wallace, 8 Spix 
evidence shows that the practice of one or more of the other class of factors is widespread, if not universal. Further, it has been shown that a more or less automatic adjustment is attained by means of variations in the intensity of the operation of these latter factors, whereas it is difficult to see how it could come about by variations in the former factors only, and, however unprogressive social organization and general conditions may be, some adjustment must be necessary from time to time.

It has been observed that there is no apparent connexion between the practice of any of these customs and the different economic stages, and it may be stated that an investigation undertaken to see if any connexion could be detected was without result. This conclusion is not surprising. The problem of how to control numbers is one which is presented to all races at all times. We have grouped races according to their economic status; but there is no reason to expect that under any one economic system a particular method of controlling population would be adopted more than any other method-no reason, for instance, why infanticide rather than abortion should be practised under one system rather than under another. As has been said, the problem is always present, and the method adopted must originally have depended upon some factor quite independent of the economic stage; abortion, for instance, might have been practised in one country because of the presence of some herb which experience showed to be effective, while in another country a taboo upon sexual intercourse for magical reasons might have been developed into a taboo during lactation.

This survey of primitive races is limited to the elucidation as to what is normally the position as regards the regulation of numbers. The evidence is not sufficiently detailed to allow us to judge whether in any one particular instance there was or was not a close approximation to the desirable number before contact with Europeans had changed their conditions. It is merely suggested that there was in general a tendency for such an approximation to occur and it may further be deduced that, since the desirable number remained the same over a great length of time, rendering the approximation, so to speak, easy of attainment, the adjustment to the optimum number normally came about. And in this connexion the limitation to clearly defined areas may be borne in mind. The very fact of the universal 
recognition and careful maintenance of these areas is an indication that such was the normal condition. Migration is abnormal, and this fact is apt to be forgotten for two reasons. First, when reviewing the course of history, migrations stand out as the chief, if not the only, known facts and the huge intervals of time between one movement and another are forgotten. Secondly, our knowledge of primitive races is largely derived from observations made when migration had been set on foot owing to contact with Europeans, as, for instance, in America. Migration may be a disturbing factor, upsetting the adjustment of numbers; but it is an abnormal condition and hence it is disregarded here.

And here, in answer to the objections that a greater prevalence of infanticide and so on is assumed than there is evidence for, we may, bearing in mind the many reasons why the evidence is deficient, reply that this assumption is not unreasonable. If such an assumption is not made, the position among these races is not comprehensible. It may be granted, for instance, that there is evidence of the practice of infanticide on a large scale among certain Australian tribes; but it may be pointed out that similar evidence is lacking in the case of other tribes. Allowing that a far more detailed examination of the evidence is desirable than there is space for here, especially with regard to the nature of the evidence and the date to which it refers, we may ask what it is supposed was going on among those tribes who did not, as it may be alleged, practise infanticide-abortion and abstention from intercourse being uncommon or unknown in Australia. The fecundity of all Australians is presumably very similar ; it would be very remarkable indeed if it were not. The factors tending to lessen fertility and to produce elimination do not differ so very much from one tribe to another. It must follow that, if one tribe practised infanticide on a large scale and maintained its numbers nevertheless, another tribe which did not do so must have been rapidly increasing. This rapid increase is not compatible with the strict maintenance of well-defined territories and with all that we know of normal inter-tribal relations, and it is submitted that the assumption made here, namely that infanticide or some other custom was almost always practised, provides the only explanation of the position. ${ }^{1}$

1 There is evidence of an Australian tribe asking for an extension of territory. So rare, however, is evidence of this kind that it only serves to emphasize that the strict maintenance of territories was the normal condition. 
Lastly it may be recalled that there are many indications that fecundity is somewhat less among these races than among modern races. However this may be, it has always to be remembered that the power of increase is nevertheless huge. It has been, for instance, calculated that between the years 1906 and 1911 the population of the world was increasing at such a rate that it would double itself in about sixty years. At this rate of increase the estimated population of the world in 1914 - namely, 1,694,000,000 -would be produced by the progeny of a single pair in 1,782 years. ${ }^{1}$ Furthermore, this is occurring under conditions in which the increase is everywhere obviously and severely checked in very many ways; therefore, even if the fecundity among these races is less than among civilized races, it must not be thought that there is thereby brought about any considerable alleviation of the problem as to how numbers should be controlled.

13. We have now to ask how far the conclusions to which we have come regarding primitive races are applicable to prehistoric races. In the first place, they are clearly only applicable to races among whom social organization has become established; for it is only when men begin to reap the advantages of co-operation that the conception of the optimum number arises. As we have seen, it is not possible to say when primitive social organization arose. It must certainly have been present in the Upper Palaeolithic; the evidence of the presence of a large body of tradition is otherwise incomprehensible. As suggested, it may very well be that we should look for the origin of social organization in the Lower Palaeolithic. Among the Tasmanians, whose skill was not much in advance of that of Acheulean man, there was a primitive form of social organization. Nevertheless, wherever the beginnings of social organization are to be placed, we must suppose that a long period of time elapsed before it assumed that rigid form characteristic of primitive races. Giving all due weight to this consideration, we must assume that in, and perhaps before, the Upper Palaeolithic era the position with regard to numbers was in all essentials similar to that among primitive races. It is not uncommon to meet with statements to the effect that man was a wanderer until he began to practise agriculture. This is a mistake if it implies that after social organization had arisen definite areas for different groups of

1 Knibbs, Census of Australia, Appendix A, vol. i, p. 31. 
men were not recognized. There can be no direct proof of this ; but the fact that, wherever we look, absolutely without exception we find among all primitive races the recognition and careful maintenance of such areas, just as much among hunting and fishing as among agricultural races, must lead us to suppose that among the more skilled hunters of pre-history similar conditions normally obtained. It is altogether unreasonable and without foundation to suppose that the recognition of areas is a development subsequent to the separation of primitive races from the main line of social evolution. If then the recognition of areas, which probably arose with the development of social organization, was a characteristic of the later prehistoric races, we are led to conclude that the desirability of attaining to and maintaining the optimum number must have had the same consequences as among primitive races. - It must, in other words, have resulted in the practice of infanticide, of abortion, or of abstention from intercourse. For there is no reason to assume that the factors bearing upon fertility and elimination reached a considerably greater intensity than among primitive races. We have, however, no reason to assume that any one of these habits was practised rather than another; for there is, as we have seen, no correlation between these factors and stages in economic development. All that we assume is that in one way or another adjustment was brought about, and in corroboration of this we may note, as will be pointed ont in the next chapter, that as prehistoric races emerge into the light of history there is abundant evidence of the practice by them of one or other of these customs.

14. Lastly we may ask how we are to view the change from the conditions under which the pre-human ancestor must have lived to the conditions which we have found reason to attribute to prehistoric races. The conditions to which species in a state of nature are subjected, and to which therefore we assume the pre-human ancestor to have been subject, were described in the second chapter. We found that the fecundity of any species was connected with the sum of all the unavoidable dangers as we called them to which the young of the species are exposed. A limit is set to the development of the strength of fecundity beyond a certain point by the fact that it cannot be to the advantage of any species that its fecundity should increase considerably beyond the point which ensures the survival of the 
species, as such an increase would intensify the struggle between the members of the species-this intensification of the struggle not bringing any corresponding advantages.

We are ignorant with regard not only to the form of the prehuman ancestor, but also with regard to the conditions under which he lived. We must suppose, however, that his fecundity, like that of any other species in a state of nature, was of a strength that enabled a sufficient proportion of his offspring to survive the unavoidable dangers. As we have seen, that which marks the setting out of man on the path which led to the dominion over all other species was the growth of his intellect. The most obvious consequence of this increase in intellectual power must have been to enable man to protect himself against many of these dangers. We do not know to what dangers he was subject, but they must in all probability have been many and serious in view of his relatively poor equipment with means of defence. Yet we have only to look at the Tasmanians to find that, when a degree of skill had been reached not far superior to that of Lower Palaeolithic man, he had freed himself from most of these dangers. Parasites were not then, so far as we can tell, a serious menace, and against the attacks of other species he could defend himself with almost complete success. Whether his fecundity had come to differ from that of the pre-human ancestor by that time we cannot tell; in any case the fecundity of prehistoric man was evidently in the main a legacy of the pre-human ancestor-a degree of fecundity that had been evolved in the face of quite other conditions. Since the time of prehistoric man fecundity has increased-this increase being apparently in the main in the nature of a modification due to changed conditions of life.

It appears that we must regard the growth of intellect as having enabled man to avoid the serious consequences which a fecundity in excess of that necessary to ensure survival would otherwise have brought about. Excessive fecundity, not therefore being a disadvantage, was not reduced by selection. It must be remembered that human fecundity is only relatively excessive ; actually man is a slow-breeding animal, and when we speak of this relatively excessive fecundity, we are not to think of such a degree of fecundity as would occupy so prominent a place among the bodily functions as to render man less adaptive than he would have been had it been less. Fecundity, in other 2498 
words, could only have become disadvantageous had it led to greatly increased competition among the members of the species without any corresponding advantage. This might perhaps have happened, had not the growth of the intellect indirectly produced new causes of elimination and intensified some of the formerly existing factors. Thus the change in food and in the mode of life was evidently productive of a higher infant mortality; such changes were due to the growth of the intellect, and the higher mortality consequent upon them, if not, as is possible, an actual advantage, was at least countenanced by natural selection. ${ }^{1}$ Such changes therefore were, other things being equal, tolerated, as were also abstention from intercourse due to taboos, prepuberty intercourse, prolonged lactation, and so on. The practices of abortion and infanticide together with prolonged abstention from intercourse-also due to the intellect-enabled man completely to escape any disadvantages which might have arisen from his relatively excessive fecundity-- the reserve, so to speak, of fecundity being possibly an advantage.

In some such manner as this it seems that we have to regard the present position of man with reference to fecundity. The strength of fecundity is in the main a legacy from ancestors who were subject to wholly different conditions. It is excessive relatively to his present needs; this excess has, however, not been a disadvantage; for the consequences which might have been deleterious have been avoided owing indirectly to the modifications of the conditions of life and directly to the rise of certain customs all ultimately traceable to the growth of the intellect.

1 There is some evidence that miscarriages are not uncommon among these races (for Australia see Grey, loc. cit., vol. ii, p. 249, and for the Loucheux Indians see Hardisty, loc. cit., p. 312). If this is so, it would appear also to be due to changed conditions to which primitive races had not learnt to adapt themselvesthere being no advantages in their so doing. 


\section{$\mathrm{X}$}

\section{HISTORICAL RACES}

1. IN this chapter we deal with the factors bearing upon fertility and elimination in the third of the three groups described in Chapter V. In one important respect this and the following chapter are contrasted with the preceding chapters. In the latter we were dealing, not with the facts derived from the peoples whom it was our object directly to investigate, but with the facts derived from primitive races with the object of throwing light upon prehistoric races. Here we are dealing with the facts relating to the peoples whose position it is our object to consider. The setting out of the facts relating to these races presents great difficulties. The amount of information is huge, but for the most part it is far from being of a satisfactory nature, and the rapid changes which have occurred within the historical period increase the difficulties. Only a brief review of the subject, designed to illustrate the more important features, is attempted.

The races under consideration are divided into four sub-groups. Sub-group 1 includes the civilizations of Egypt, Mesopotamia, Greece, and Rome up to the fall of the Western Empire. In these countries we have some knowledge of the progress from Neolithic culture to a culture exhibiting a far greater command over nature. From this culture is derived the later civilization of Europe, which we consider under sub-groups 3 and 4-the former beginning with Europe after the fall of the Western Empire and ending roughly about A. D. 1760, the date given by Marshall as marking the beginning of the industrial system, the latter beginning about 1760, and including all European races and their derivatives in America, Australia, and so on. Generally speaking, therefore, sub-group 1 includes the ancient empires, sub-group 3 Europe from the Roman Empire to the rise of the industrial system, and sub-group 4 the epoch of the industrial system.

There remain those races which have passed beyond the culture of the races included in the second group, but which have been 
out of the main stream of the evolution which led to the industrial evolution. In sub-gromp 2 are included India, China, Persia, Japan, and also the partly nomadic Arabs and certain other Asiatic peoples. In late years the customs of the races in this sub-group have been influenced by contact with Europeans; changes due to this contact will be disregarded, as were similar changes in the races belonging to the first and second groups.

In one very important respect the conditions with regard to all races in the third group differ from those in the preceding groups. Disease is a factor of the greatest importance, and we may consider in the first place the facts regarding the prevalence of disease in all groups. What little there is to be said regarding war, famine, and child mortality in all the races of this group may also be considered together. With regard to the other factors we find that in many respects the conditions in the first two sub-groups are similar and are contrasted with those in the latter two sub-groups. Therefore, after considering disease and the other factors mentioned for all groups taken together, it will be convenient to deal with the sub-groups separately, taking the ancient empires and the Asiatic peoples first, and mediaeval and modern Europe afterwards.

2. Something was said in Chapier VI with regard to the evolution of disease. It was there shown that in all probability most diseases evolved relatively late in history. The more settled conditions of the Neolithic Age may have provided the first suitable surroundings for the evolution and spread of disease. The taking of metals into use was followed by an increase in the density of population when conditions became yet more favourable. The use of metals was followed by the rise of the first civilizations. It was then that men first came to dwell in towns and cities, and it is interesting to observe that the earliest cities were very compact. Mr. Hall, speaking of Minoan civilization, says that the towns were very cramped, ' more so than the most confined of European mediaeval cities ' 1 On the rise therefore of these early civilizations the conditions were strongly contrasted with those which, we imagine, must have been prevalent before, and favoured the spread of disease as it had never been favoured before. From the rise of the early civilizations until within the last hundred years, con

1 Hall, Ancient History of the Near East, p. 110. So, too, Memphis was apparently a very cramped city. 
ditions have remained favourable. They may perhaps have been most favourable in European towns in mediaeval and later times. According to Rogers, 'the habits of the people were favourable to pestilence. Every writer during the fifteenth and sixteenth centuries who makes his comment on the customs and practices of English life, adverts to the profuseness of their diet and the extraordinarily uncleanliness of their habits and persons. The floor of an ordinary Englishman's house, as Erasmus describes it, was inconceivably filthy, in London filthier than elsewhere, for centuries after these events. The streets and open ditches of the towns were polluted and noisome beyond measure. The Englishman disdained all the conditions of health.' 1

'Turning to the evidence, we find that facts are very scanty for the earliest times of this period. Such evidence as we have points to the prevalence of disease during the early civilizations. We know that pestilence swept over Egypt-the references to the subject in the Bible being familiar. But precisely what diseases were prevalent are not known; the descriptions of the symptoms are seldom sufficiently accurate even in mediaeval times to make it possible to identify the diseases. ${ }^{2}$ From other evidence it appears that in any case tuberculosis, leprosy, plague, and bilharzia were common in Egypt. There is much evidence to show that most diseases only reached Europe in relatively late times. Plague, for instance, probably first reached Europe in A.D. 542. It is possible, however, that the pestilence which ravaged most of Europe between A. D. 164 and 188 was plague. ${ }^{3}$ Generally speaking, the evidence points to the conclusion that many diseases came from the Fast, where they in all probability originated. Plague, cholera, and small-pox all seem to have had their origin in India. This conclusion is in harmony with what we know of the origin of civilization, for it is where men first came to dwell in close proximity that we should expect to find that disease originated.

With regard to mortality from disease very little accurate information is available until modern times. It is quite impossible to say, for instance, whether disease was the cause of a higher

1 Rogers, Six Centuries, vol. i, p. $336 . \quad 2$ On this subject see Hirsch, Handbook of Geographical and Historical Pathology; Hecker, Epidemics of the Middle Ages; Clemow, Geography of Disease. ${ }_{3}^{3}$ The famous 'plague' of Athens, 430 to 428 B.c., was not plague in the technical sense; it was probably typhoid or small-pox. 
mortality in the Middle Ages than in the times of the Early Empires. All we know is that it was in both cases an important factor of elimination. Within the last century disease has come to be in an increasing degree under the control of scientific methods. Some diseases such as small-pox have been almost banished from this country ; others, such as diphtheria, have been rendered less lethal. Of the Early Empires there are of course no exact facts. Macdonell, working on figures from the Corpus Inscriptionum Latinarum of the Berlin Academy, found that there was a very low expectation of life in ancient Rome as compared with the present day. Whereas in England at the age of fifteen the expectation of life for boys is forty-five years and for girls forty-eight years, in Rome it was twenty and fifteen years respectively ; and again, whereas in England at the age of thirty the expectation of life for men is thirty-three, and for women thirty-six years, in Rome it was nineteen and fourteen respectively. He also found that, though the death-rate was less high, the position was much the same in the provinces of Hispania and Lusitania as in Rome. In Africa, however, the expectation of life was higher, but this may perhaps be due to the presence of a large number of colonists. ${ }^{1}$ There is abundant evidence of a high rate of mortality in Europe up to the opening of the last century. We must remember, says Rogers, that 'in the Middle Ages the risks of death from disease were far greater than they are at present, that medical skill was almost non-existent, that the conditions of life were eminently unwholesome, that the diet of the people, during fully one-half of the year, though abundant, was insalubrious ' $;{ }^{2}$ and in another place he says that 'in the large towns the deaths, to judge from the return up to the eighteenth century, greatly exceeded the births'.3 Theilhaber, quoting Goldstein, gives figures which corroborate this view for Basel and Frankfort. ${ }^{4}$ Halley constructed one of the earliest tables of mortality for the city of Breslau for the years 1687 to 1691, and they show that the mortality was " considerably higher than that shown by modern statistics'.5 Price constructed a table for Northampton for the years 1735 to 1780 , and it shows a death-rate of $249 \cdot 31$ per thousand for the ages $0-2$, which is far higher than at the present day. 6

1 Macdonell, Biometrika, vol. ix, p. 369.

2 Rogers, loc. cit., vol. i, p. 118. 3 Ibid., p. $336 . \quad$ Theilhaber, Das sterile Berlin, p. 27.

Mortality Laws and Statistics, p. $3 . \quad{ }^{\circ}$ Ibid., pp. 4, 110. 
Finally, though disease has nowadays very largely come under scientific control, by far the greater number of deaths is due to disease. Thus in 1917 there were 498,922 deaths among the civilian population of England and Wales. Of these, 20,480 were due to violence (not suicide), 2,495 to suicide, 2,485 to ill-defined or unknown causes, and 2,598 took place during pregnancy. Of the remainder all were due to defined diseases. Enteric fever (typhoid and paratyphoid) accounted for 977, measles for 10,538; whooping cough, 4,509; tuberculosis for 59,934; syphilis for 2,127 ; cancer for 41,158 ; pneumonia for 39,832 ; diphtheria and croup for 4,498 ; meningitis for 4,761 ; organic heart disease for 52,692 ; bronchitis for 38,907 ; other respiratory diseases for 7,031 ; diarrhoea for 13,311; nephritis and Bright's disease for 14,298 ; and congenital debility for $23,850 .^{1}$

3. Warfare is the second factor-disease being the firstwhich among these races is remarkable in that its effects are not regular. It is wholly impossible to estimate the loss of life in war until modern times. Contemporary statements nearly always hugely exaggerate the numbers engaged in battle and the number slain. 'In the first Battle of St. Albans we have been told that five thousand persons were slain. It is almost certain that not much more than half that number were in action.' 2 This is probably equally true of nearly all such statements. With regard to elimination through warfare there are two facts to be borne in mind. The first is that, even if the contemporary statements were correct, war is by no means so important a factor as it seems at first sight. A practice which resulted in the conscious limita. tion of each family by one child would have greater results over a period of years than there is ever claimed as the direct result of warfare in reducing numbers over a similar period. The second is that the indirect elimination following upon warfare is of far greater importance than the direct elimination. Famine and disease following warfare were until recent times responsible for many more deaths than warfare itself. When we are told that during the Thirty Years' War the population of Würtemberg was reduced from 500,000 to $46,000,{ }^{3}$ it has to be remembered that disease and famine played a greater part than loss of life in battle. Further, when the direct effects of warfare as a whole

1 Annual Report of the Registrar-General for $191 \%$. p. 332.

${ }^{3}$ Niebuhr, Lectures in Ancient History, vol. ii, p. 231. 
are contrasted with those of disease, it is apparent that they are relatively insignificant. Elimination from disease, though irregular, is always in progress within this period ; there are lengthy epochs when there is little or no loss through war. Again, the great epidemics are far more destructive of life than the great wars; Hecker, for instance, after a lengthy examination of the various accounts, came to the conclusion that about a quarter of the inhabitants of Europe perished during the Black Death. ${ }^{1}$

The nature which warfare assumed in this period is obviously connected with the rise and consolidation of large states. Warfare becomes a matter of policy ; it is no longer a cause of a regular degree of elimination. There is among the races under review in this chapter every degree between the kind of warfare typical of the races previously reviewed and that typical of this period. The nomadic races of Asia have at least until recent times maintained a form of regular warfare. Vambéry states that there is 'inveterate and irreconcilable enmity' between two tribes of Turkomans. ${ }^{2}$ We are told that among such a primitive people as the Nagas the tribes were formerly in a state of constant hostility. ${ }^{3}$ Among these people also head-hunting was formerly common and the victims sometimes included women. ${ }^{4}$ 'One individual showed Mr. Carnegie his apron which recorded the deaths of twenty-five individuals-men, women and children-slain by his own hand.' 5 Generally speaking, however, we have in this period to think of warfare as typically of a different nature to that which we have noticed before, but whether it on the whole accounts directly for a higher degree of elimination, it is not possible to say.

The features of warfare which are of importance here are its relatively slight effect in producing elimination, its irregular action, and its important consequences in bringing about disease and famine. With disease we have already dealt. Famine appears here for the first time among the more important factors of elimination, and its appearance is obviously connected with the higher organization of society which, if broken down, as by warfare, may give rise to famine. ${ }^{6}$ Social organization may be

1 Hecker, loc. cit., p. 30. The proportion of the population which perished is often said to have been greater. Rogers (loc. cit., vol. ii, p. 223) puts it at onethird of the population of England.

3 Hodson, Naga Tribes, p. 113. C Crooke, Northern India, p. 41. p. 313 . p. 47. Thus in irrigated countries, if the system of irrigation is allowed to fall into decay or if it is damaged directly or indirectly by war, famine may result. See Cresswell, Man, vol. xv, p. 68. 
broken down by other causes than war, as by some failure in government. Famine may also result from exceptionally bad weather in any period; but it would seem that when a certain stage of organization has been reached the danger is greater than before or afterwards. There were great famines in England in the years 1194 to 1196,1257 to 1259 , and 1315 to 1316 , since when famines have been unknown. These famines were all connected with bad weather, but the fact that they have not occurred since is to be attributed rather to superior social organization and increasing knowledge than to better climatic conditions. The sudden and prolonged changes of climate which occur in certain places, as for instance in Peninsular India, tax the resources even of modern knowledge and skill. In the case of famine, more so even than in the case of warfare, the loss of life is apt to be overestimated. Famine somehow strikes the imagination and gives it a prominence in history which it does not deserve. It is in reality a minor factor.

Before leaving the subject of warfare, it is interesting to note, in view of what will be said later regarding the influence of Christian teaching in assisting to put a stop to abortion and infanticide, that the early Church was also opposed to warfare. According to Clement of Alexandria, Tertullian, Origen, and Lactantius, all warfare was unlawful for the converted. Under Domitian a Christian soldier who refused to fight was executed. There was a controversy between Celsus and Origen on this matter. The view of the latter was that the prayers of Christians were more effective than their swords. This attitude, however, was soon abandoned, and by the time of Constantine the army was largely Christian. ${ }^{1}$

4. In the groups already discussed we have found child mortality to be an important factor. We traced it to certain factors such as carelessness, ignorance, and practices such as bathing newborn babies in cold water. Within the historical period child mortality also plays an important part. Though there is little or no evidence of the practice of such customs as those referred to, ignorance and carelessness are familiar enough among the less educated classes at the present day, and in addition disease exacts a very heavy toll of infant life. In fact, whereas among primitive races the actual cause of the death of children appears

${ }^{1}$ Lecky, European Morals, vol. ii, p. 248. 
generally to be exposure or debility following on the results of ignorant and careless treatment, among the races of this group the actual cause of death is usually disease to which children have been more or less predisposed by the treatment they have received.

There is little exact evidence as to the conditions among the historical races, but the general impression gained from the literary evidence is that infant mortality was high. The high rate of child mortality among Asiatic races is well known. In the period 1902 to 1911 the average deaths of children for one thousand births was in the United Provinces 352, in Bombay 320, in Burma 332, and in the Punjab 306. ${ }^{1}$ Referring to these figures, Wattal comments on the 'extremely unsanitary conditions of child-birth and the appalling ignorance prevalent on the subject'.2 With regard to ignorance it is everywhere the same story. Of the modern Egyptians we are told that 'the children are foul in the eyes, and are allowed to munch such indigestible dainties as beans and bread as soon as they have - teeth with which to bite, while the mother protests to the lady doctor that nothing but milk has ever passed the child's lips, though its little hand may actually be holding a hunch of raw potato ' ${ }^{3}$

Of the Middle Ages again there is no exact evidence, but all we know points to a very high child mortality. Some figures for the eighteenth century from the country have been quoted and doubtless there was a considerably higher death-rate in the towns. ${ }^{4}$ From the general conditions and from the prevalence of disease it is obvious that it could not have been otherwise.

Within the last century there has been in most European countries a considerable decrease in child mortality. The degree of mortality varies greatly from country to country. For the same period as that for which the Indian figures were given the figures are for England and Wales 127.3, France 132.4, Germany $186 \cdot 6$, Hungary 207.6, New Zealand $64 \cdot 4$, Sweden $84 \cdot 4$, Australia $87 \cdot 5$, and Scotland $116 \cdot 1.5$ Some further reference to this subject and particularly to the differences between the various countries will be made in Chapter XI.

5. With this reference to the importance of disease, war,

1 Wattal, Population Problem. p. $20 . \quad 2$ Ibid., p. 21. p. 224. For Chinese Tibet see Wilson, Abode of Snow, p. 193. J.R.S.S., vol. lxiii, p. $610 . \quad 5$ Wattal, loc. cit., p. 20. 
famine, and child mortality throughout the historical period we may pass to the consideration of the remaining factors, dealing first with the ancient empires and Asiatic peoples and afterwards with Europe from the fall of the Roman Empire. We shall find that considerable differences exist regarding the importance of the various factors as between these two divisions. Roughly speaking, with the exception of the importance of disease, conditions in the ancient empires and in Asiatic countries are similar to those in previous groups. In Europe after the introduction of Christianity we shall find that certain factors become of little or no importance, whereas others appear for the first time, and finally in the modern period we shall discover further changes. It is therefore convenient to consider the conditions in the first two sub-groups taken together and afterwards those in the latter two sub-groups.

All the evidence goes to show that celibacy and postponement of marriage were very rare in the ancient empires and are very rare among Asiatic peoples. The only instances to the contrary are such cases as those of the later days of the Roman Empire ; such cases are wholly exceptional and what there is to be said about them will be deferred to the next chapter. Generally speaking, every one married at or soon after the age of puberty. In Greece ' in various places criminal proceedings might be taken against celibates ' 1 Though the figures on which he worked were small, Macdonell found that marriages in Rome took place between the ages of ten and twenty. ${ }^{2}$ In all these countries we find examples of religious celibacy. In Egypt ${ }^{3}$ and Chaldea ${ }^{4}$ there were celibates of koth sexes for religious reasons, in Rome there were vestal virgins, in Persia Sun Priestesses who did not marry, and in India and Tibet there are similar examples. ${ }^{5}$ But unlike Christian celibacy, these instances of celibacy were of no importance whatever as regards the question of numbers. The religious celibates never formed more than an insignificant fraction of the whole population. In general, as we shall see later, marriage was strongly encouraged by religions other than Christianity for the mass of mankind. The one other important exception would seem to be Buddhism, which not only forbade marriage to the sacerdotal caste,

1 Westermarck, Moral Ideas, vol. ii, p. 403. See on this subject Schömann, Griechische Alterthümer, vol. i, p. 271, and Plutarch, Lycurgus, p. 15. donell, loc. cit., p. 369 .

3 Müller, Das Sexuelle Leben, p. 7. 2 Mac-

5 Westermart . Society, the girl members of which swear never to marry (Giles, China, p. 69). 
but discouraged marriage among all those who would attain to wisdom. It would appear, however, that celibacy, though possibly widespread in the earlier days of Buddhism, soon ceased to be practised except by a small proportion of the adherents of Buddha. 'It is said that in Nepal, under the modern Gurkha rule, the celibate occupies a lower position than the married monk, to whom the services in the temples are committed. It is said, too, that the Lamas of Sikkim and other northern countries constantly have children living with them, though they do not admit them to be their own. Yet for all that celibacy is the rule, and, nominally at any rate, the great majority of Lamistic monks in Western Asia are unmarried coenobites, who live together in monasteries.' 1

Much evidence could be given as to the view taken of celibacy and of the duty of marrying early. 'The Oriental', says Polak, 'does not understand how any one can remain a celibate who has the chance to marry.' 2 'Le célibat', says Fustel de Coulanges, ' devait être à la fois une impiété grave et un malheur; une impiété, parce que le célibataire mettait en péril le bonheur des mânes de sa famille; un malheur, parce qu'il ne devait reçevoir lui-même aucun culte après sa mort et ne devait pas connaître ce qui réjouit les mânes. C'était à la fois pour lui et pour ces ancêtres une sorte de damnation.' 3 Doughty describes the reasons for the absence of celibacy among the Arabs. ${ }^{4}$ The same conditions are found in China. 5 Of Corea, Ross says "the male human being who is never married is never called a " man", whatever his age, but goes by the name of "yataw", a name given by the Chinese to unmarriageable young girls, and the man of thirteen or fourteen has a perfect right to strike, abuse, order about the "yatow" of thirty who dares not so much as open his lips to complain'.6 'Among natives of India', says Kerr, 'it is considered an indispensable duty to enter into the marriage state.' ? 'A Hindoo male', according to Wattal, 'must marry and beget children-son, if you please-to perform his funeral rites lest his spirit wander uneasily in the waste places of the earth. The very name of son, "Putra ", means one who saves his father's soul from the hell called " Puta". A Hindoo maiden unmarried at puberty

1 Monier-Williams, Buddhism, p. 269. 3 Fustel de Coulanges, Cité antique, p. 50. p. 321. Sce also Bertherand, Médecine et Journ. Eth. Soc., new series, vol. ii, p. 19. Natives of India, p. 202.
2 Polak, Persien, vol. i, p. 205. ' Doughty, Travels, vol. i, Hygiène des Arabes. 5 Gardner,

6 Ross, Corea, p. $313 . \quad 7$ Kerr, 
is a source of social obloquy to her family and of damnation to her ancestors. Among the Mohammedans, who are not handicapped by such penalties, the marriage state is equally common, partly owing to Hindoo example, and partly to the general conditions of life in primitive society where a wife is almost a necessity both as a domestic drudge and as a helpmate in field work.' 1

Early marriage is considered to be a duty for all. Westermarck quotes Mohammed as saying: 'whenever a servant of God marries verily he perfects half his religion'.2 According to the Laws of Manu it is a religious duty incumbent upon all. ${ }^{3}$ In the Vedic Age marriage was much encouraged and offspring greatly desired. ${ }^{4}$ Confucianism and Zoroastrianism similarly encouraged marriage. 'A youth', says Dubois, speaking of India, ' who was not married before he was eighteen was considered by them to be sinning against the command of the Creator, which says " Increase and Multiply ".'5 'Almost all Chinese,' says Gray, ' robust or infirm, well formed or deformed, are called upon by their parents to marry so soon as they have attained the age of puberty.' ${ }^{6}$ 'Marriage and the upbringing of offspring became a duty incumbent upon every Chinese who is normally fit for mảrriage ', 7 say two modern Chinese authors. Similar statements are to be found regarding Burma, ${ }^{8}$ Upper Siam, ${ }^{9}$ Persia, ${ }^{10}$ the Mongols, ${ }^{11}$ and the Kalmucks. ${ }^{12}$

6. Marriage before the age of puberty is not uncommon and is very prevalent in India. It is doubtful how far intercourse takes place before maturity. In the North-West Provinces of India it is stated that in most cases there is no cohabitation before puberty. ${ }^{\mathbf{1 3}}$ This statement is confirmed by Risley; he further states that in Bengal cohabitation begins at once. ${ }^{14}$ In India in 1901, 243,500 girls were married under the age of five, 2,030,000 between the ages of five and ten, and 6,585,000 between the ages of ten and fifteen. $^{15}$ Eram, speaking generally of the East, states that prepuberty marriages are not rare. ${ }^{16}$ We hear that 10 per cent. of

1 Wattal, loc. cit., p. 3. 3 Ibid., p. 400. Hindu Manners, p. 214. China, p. 85, and Giles, China, p. 189.
C Crawford, Journal, vol. ii, p. 240. 10 Polak, loc. cit., vol. i, p. 201 . loc. cit., vol. i, p. 305 . People of India, p. 185. ${ }_{13}$ Crooke rooke, North. Western Provinces, p. 228. ${ }^{14}$ Risley, People of India, p. $185 . \quad 15$ Leopold and Weise, Sexual-Ordnung, p. 347. In
the Vedic Age, however, child marriages were apparently unknown (see Bennet, Antiquities of India, p. 114).
2 Westermarck, Moral Ideals, vol. ii, p. 400. Williams, Buddhism, pp. 363, 364.
Gray, China, vol. i, p. 186. See also Douglas, ${ }^{7}$ Leong and Tao, Life in China, p. 10. 9 Bock, Temples and Elephants, p. 186.
11 Huc, Sonvenirs, vol. i, p. 297. 16 Eram, Accouchements en Orient, p. 69. 
the Kirghiz marry before the beginning of menstruation. ${ }^{1}$ Among the Annamites ${ }^{2}$ and the Todas ${ }^{3}$ intercourse sometimes occurs before puberty. Macdonell's figures for Rome, already referred to, show that marriage must sometimes have taken place before puberty.

7. The ancient Egyptians suckled their children for two or three years. ${ }^{4}$ The suckling period is prolonged in Arabia. ${ }^{5}$ In the Koran mothers are recommended to suckle for two years. ${ }^{6}$ Among Asiatic races lactation is usually extended for a con. siderable length of time. The Japanese sometimes do not wean their children until the fourth year. ${ }^{7}$ The poorer classes in Persia suckle until the third year. ${ }^{8}$ In Upper Siam 'infants are generally suckled three years '. ${ }^{9}$ In Turkey lactation is also prolonged. ${ }^{10}$ Generally speaking, the evidence is to the effect that the suckling period is always prolonged-the average being perhaps somewhere about two to three years.

8. In sub-group 1 many examples are found of restraint from sexual intercourse being imposed upon married persons at certain seasons. Examples from Egypt are given by Müller.11 According to the Laws of Manu separation from the wife was obligatory at certain periods-for instance, at new and full moon and on certain days of the month. ${ }^{12}$ Similar conditions existed in Persia. ${ }^{13}$ Such restrictions, however, are not of much importance. Of prolonged abstention from intercourse there is little evidence. In China, according to Gray, 'a husband is not expected to cohabit with his wife after she has conceived, nor after the child is born, during the whole period that it is being nourished at the mother's breast. Any violation of this rule is supposed not only to cause the child to become sickly but to provoke the displeasure of the ancestors and to bring misfortune upon all members of the family. Wealthy Chinese are generally very careful in the practice of such abstention.' 14 It is quite clear, however, from what we know of Chinese fertility that this custom is not widely practised among the people as a whole. Smith speaks of " the objection of the Arabs to inter-

1 Wassilief, Zentralblatt für Anthropologie, vol. x. Anth., vol. ii, p. 465. ancienne, vol. iii, p. 142.

Notes on the Bedouins, p. 96. p. 285. See also Warnick, Archiv für Gynaekologie, vol see Matignon, Dix Ans, p. 318. Temples and Elephants, p. 260. 1 Müller, Das Sexuelle Leben, p. 6. 14 Gray, China, vol. i, p. 185.
2 Mondière, Mém. Soc. - Lenormant, Histoire 5 Doughty, loc. cit., vol. i, p. 237 ; Burckhardt, 7 Faulds, Nine Years,
8 Polak, loc. cit., p. $216 . \quad 9$ Bock, 10 Rigler, Die Türkei, vol. i, p. 212. 12 Ibid., p. 29. 
course with a nursing mother which was supposed to hurt the suckling '.1 In Turkey also there is said to be no intercourse during lactation. ${ }^{2}$ There are thus indications of the practice of this important custom, and it may be that it existed in such a degree as to have exerted considerable influence. ${ }^{3}$

9. There is a general impression that the knowledge of contra. ceptive methods has only been acquired in modern times. This, however, is not correct. We have already seen that there is evidence of their use in the second group. In the third group there is evidence of the knowledge of certain methods in early times. Theilhaber gives some particulars regarding their employ* ment among the Jews, German tribes, Arabs, the Franks before their conversion to Christianity, the Greeks, and the Romans. ${ }^{4}$ Methods are employed at the present day in China ${ }^{5}$ and in India, ${ }^{6}$ though there is some doubt as to how far they are effective. According to Wattal there is some statistical evidence from India which may be interpreted either as a result of such practices or as a result of abstention from intercourse. ${ }^{7}$ There is not sufficient evidence to allow of any exact estimate being made of the extent of these practices. Generally speaking, however, it would appear that it is not until we arrive at the latest period of human history that we find these practices to be of considerable importance.

10. With regard to the size of families in early times there is little exact evidence. Among Asiatic peoples at the present day we have some evidence of the degree of fertility, whereas hitherto the evidence has been only that of the number of children surviving after infanticide and infant mortality had taken their toll. This evidence from the Asiatic races we may now turn to discuss, but we may note before doing so that the evidence for the remaining races of the first sub-group gives a rather different impression than that which we obtain when studying primitive races; upon the whole we get the impression that families were at least not so markedly small as among primitive races. ${ }^{8}$

Fertility is often said to be higher in the East than in Europe. So far as India is concerned this is not so. The error has arisen because attention has been paid to the crude birth-rate. When

1 Smith, Kinship, p. 283.

2 Rigler, loc. cit., vol. i, p. 212.

3 It is possible that there are traces of this custom in Persia (see Polak, loc. cit., vol. i, p. 216).

- Theilhaber, Das sterile Berlin, p. 10. See also Theilhaber, 'Die Geburten-Beschränkung', Neue Generation, 1913. s Collineau, Revue Men. suclle de l'Ecole d'Anthropologie, 1899.

7 Wattal, loc. cit., p. 28.

- Webb, Pathologia Indica, p. 258. 8 Aristotle, Politics, ii. 9. 
the crude birth-rate is corrected for the number of married females of reproductive age, then the true birth-rate is found to be lower than in Europe. 'The total number of births registered in England and Wales during the year 1911 was 881,138, which when calculated on the total population gives a crude birth-rate of 24.4 per thousand. The total number of births registered in India during the same year was $9,209,703$, which when calculated on the total population gives a crude birth-rate of 38.59 per thousand. It would seem therefore that the fertility in India is higher than in England. But this is not so. The total number of females of reproductive age (15 to 45$)$ in England and Wales at the census of 1911 was $8,988,745$ and if we calculate the births per thousand of such females the figure stands at 98 . The total number of females of those ages in India in 1911 was 71,535,861 and the corresponding Indian figure is 128 . If, however, we calculate the births on the number of married females of reproductive ages the Indian figure stands at 160 while the English figure is 196.' 1

The fertility in other Asiatic countries is often said to be very high; it is asserted to approach 50 per 1,000 in China. Exact figures are, however, lacking. There is no doubt that the corrected birth-rate would be much less. Among nomadic people the evidence is to the effect that the number of children is small, and this is probably connected with the practice of abortion, which is common for instance among the Arabs.

11. We have now to give some account of the factors which have a bearing upon elimination in the third group. Of these the first is abortion. Classical literature is full of references to the subject. Plato and Aristotle both permitted abortion. 'No law in Greece or in the Roman republic or during the greater part of the Empire condemned it.' 2 'A long chain of writers both pagan and Christian represent the practice as avowed and almost universal. They describe it as resulting, not simply from licentiousness or from poverty, but even from so slight a motive as vanity which made mothers shrink from the disfigurement of child-birth. They speak of a mother who had never destroyed her offspring as deserving of signal praise and they assure us that the frequency of the crime was such that it gave rise to a regular profession.' 3

2 Wattal, loc. cit., p. 7.

3 Ibid., p. 21 .

${ }^{2}$ Lecky, European Morals, vol. ii, p. 21. 
The practice was more common in Rome than in Greece. ${ }^{1}$ It was deplored, nevertheless, by many authors including Ovid, Juvenal, and Seneca. Some details of the methods employed are given by Theilhaber. ${ }^{2}$

In sub-group 2 we find the practice to be widespread. Eram comments upon its frequency throughout the East. ${ }^{3}$ Wilkins says that 'the crime of procuring abortion is one of the commonest in India', and quotes Dr. Chevers as saying that abortion is 'an act of almost daily commission' and has become 'a trade among certain of the lower midwives'. It is especially common in Bengal-perhaps 10,000 being destroyed monthly. ${ }^{4}$ According to Matignon, in China abortion is legal and usual during the threeyear suckling period. ${ }^{5}$ It is also common in Annam, ${ }^{6}$ in Japan, ${ }^{7}$ Persia, ${ }^{8}$ and Turkey. ${ }^{9}$ Infanticide was condemned by Mohammed but not abortion, which is frequent among the Arabs. ${ }^{10}$ In Turkey it is very common ; ${ }^{11}$ it is said to be brought about after the second birth if the husband consents. ${ }^{12}$ The fact that in later times we hear of enactments against abortion among the Burgundians, Ripuarians, Visigoths, Bavarians, Saxons, Frisians, and Lombards seems to point to the conclusion that the practice was common before their conversion to Christianity. ${ }^{13}$

12. There is some reason for believing that infanticide was common among the Egyptians at the time of Moses. ${ }^{14}$ 'The ancient Jews seem to have themselves practised infanticide, for as late as the time of Isaiah they are reproached with the habit of "slaying the children in the valleys under the clefts of the rocks ". In 2 Chron. xxviii. 3 and xxxiii. 6 the same statement is made. It is true that these cases may have partaken of the nature of human sacrifices rather than of ordinary infanticide, but the two generally prevailed togèther, and we have express testimony that both of them existed at that time among all the

1 Westermarck, Moral Ideals, vol. i, p. 415.

2 Theilhaber, Das sterile Berlin, p. 11. See also von Siebold, Versuch einer Geschichte der Geburtshilfe. 3 Eram, loc. cit., p. 45. It was forbidden by the Laws of Manu (viii. 37). 4 Wilkins, Hinduism, p. 429. See also Shortt, Transactions Obstetrical Society, vol. ix ; Webb, loc. cit., p. 259; and Jellinghaus, Zeit. für Eth., vol. iii, p. 365. 5 Matignon, Dix Ans au Pays du Dragon, p. 318. See also the same author's La Superstition, le Crime et la Misère en Chine, and Collineau, loc. cit., p. 352. - Mondière, loc. cit., vol. ii, p. 487. $\quad$ Warnick, loc. cit., p. 574. See also MacLennan's quotations from Miss Bird, loc. cit., p. $105 . \quad{ }_{8}$ Polak, loc. cit., vol. i, p. 217. $\quad 2$ Ploss and Bartels, loc. cit., vol. i, p. $846 . \quad 10$ Rique, Gaz. Méd., vol. xviii, p. 161. For the Tartars see Niemojowski, Siterian Pictures, vol. i, p. 161. 11 Rigler, loc. cit., vol. i, p. 206, and vol. ii, p. 229. 12 Polak, loc. cit., vol. i, p. 218.

1 Buckle, Miscellaneous Works, vol. ii, p. 240. 
surrounding nations, Phoenicians, Aramaeans, Syrians, and BabyJonians as well as among their kindred the Carthaginians.' 1

Glotz has an interesting chapter on the subject of infanticide in Greece. In prehistoric times in Greece the head of the family could, as among all people of 'Aryan origin', dispose of his children as he wished. ${ }^{2}$ After examining the myths it is clear, according to Glotz, that infanticide was a daily occurrence during the period at which myths were being formed. ${ }^{3}$ In historical times it was universal. Most often infanticide was ordered by the father, who generally on the fifth, sometimes on the seventh or tenth, day after the birth publicly proclaimed whether he would keep the child or not. 4 If he decided not to keep the child, it was exposed, and there is reason to think that most of the exposed children died. ${ }^{5}$ Infanticide was enjoined by the ideal legislation of Plato and Aristotle and by the actual legislation of Lycurgus and Solon. Summing up his examination of this subject Glotz says : 'L'opinion de la Grèce ancienne est donc à peu près unanime. Reçue dans la vie privée, cette pratique a été admise en droit par les législatures et fondées en raison par les maîtres de la pensée.' 6

In very early times infanticide was general in Rome. Later the right of the father to destroy his children was somewhat restricted. 'The power of life and death, which in Rome was originally conceded to the father over his children, would appear to involve an unlimited permission of infanticide; but a very old law, popularly ascribed to Romulus, in this respect restricted the parental rights, enjoining the father to bring up all his male children, and at least his eldest female child, forbidding him to destroy any well-formed child till it had completed its third year, when the affections of the parents might be supposed to be developed, but permitting the exposition of deformed or maimed children with the consent of their five nearest relations.' 7 There is evidence to show that this permission was frequently utilized. The plots of Plautus and Terence sometimes turn upon the reappearance of children supposed to have been destroyed. "Pliny says that infanticide is really a lamentable necessity " seeing that the fertility of some women is so over-abundant in

${ }^{1}$ Sutherland, loc. cit., vol. i, p. $132 . \quad{ }^{2}$ Glotz, Etudes sociales, p. 187. 3 Ibid., p. 188. 'Ibid., p. 19l. Possibly, however, there was no public ceremony unless the father had decided to keep the child. ${ }^{5}$ Ibid., p. 212.

- Ihid., p 224, 'Lecky, European Morals, vol. ii, p. 26. 
children that it needs some such practice to counterbalance it" while . . . Seneca saw nothing reprehensible in it.' 1 Suetonius has several allusions to the matter which prove that infanticide was accepted by the Romans in a very matter-of-fact spirit. For instance, in describing the public grief for the death of Germanicus, he mentions that many women exposed their infants. The opening of the fourteenth episode of the Golden Ass of Apuleius describes how a husband before going forth on a journey directed his young wife that the coming babe if a girl was to be destroyed; the whole being related as a perfectly natural and common occurrence. ${ }^{2}$

Among the Arabs it was very prevalent before the time of Mohammed, by whom it was forbidden. Instances are given by Smith to show the extent of the practice: ${ }^{3}$ Discussing the matter in another place Smith says that Wilken "doubts whether among the Arabs the practice was carried to such an extent as to do more than keep the sexes balanced-men being more exposed than women to violent deaths; but there is evidence that, at any rate in some places and at some times, there was a strong pressure of public opinion against bearing any daughter, even though she were the only child of her parents. If we take along with this the fact that wealthy and powerful men had often several wives, there can, I think, be no question that, at least in some parts of the country, wives must have been so scarce that the mass of the tribesmen must have been driven to practise polyandry.' 4 The evidence from China is somewhat conflicting ; this is probably to be accounted for by the variations in habits in different parts of the country. Norman has collected evidence to show its wide extent. 'The testimony', he says, ' of a Chinese teacher is as follows: "Infanticide is very common among the poor, and even people in pretty easy circumstances. There is hardly a family in which at least one child has not been destroyed, and in some families four or five are disposed of." . . . Another man, who is now a member of the Christian Church, says that in his village there is hardly a family that has not destroyed two or three children. . . . A lady contributor to the North China Daily

1 Sutherland, loc. cit., vol. i, p. 136.

2 Ibid., p. 137.

${ }^{3}$ Smith, Kinship, p. 279. See also Doughty, loc. cit., vol. i, p. 239, and Wilken, Da.s Matriarchat, p. 53. $\quad$ Smith, Kinship, p. 129. For evidence of infanticide in Mingrelia see Chardin, Travels, p. 144. The Gagas put all childien to death and stole others (Battel, Strange Adventures, p. 326). 
News furnished the following statistics : "I find that 160 Chinese women, all over 50 years of age, had borne 631 sons and 538 daughters. Of the sons 366 or nearly 60 per cent. had lived more than 10 years ; while of the daughters only 205 or 38 per cent. had lived 10 years. The 160 women had, according to their own statement, destroyed 158 of their daughters; but none had ever destroyed a boy. As only four women reared more than three girls, the probability is that the infanticides confessed to are considerably below the truth. I have occasionally been told by a woman that she had forgotten just how many girls she had had more than she wanted. The greatest number of infanticides confessed to by any one woman is eleven."' 1 Gray states that infanticide prevails to a huge extent and gives an example of a young husband who had had three sons and four daughters. Of the latter three had been killed. ${ }^{2}$ According to Douglas the practice prevails among the poorer classes 'to an alarming extent ' ${ }^{3}$ Abeel speaks of infanticide in China as 'very common ' ${ }^{4}$ 'In Pekin,' he says, ' after deducting more than one-half for the natural deaths the number of exposed children is, according to Barrow, about 4,000 a year. . . . In some Provinces not one in three is suffered to live.' 5 The practice is said to be specially prevalent in South China.6 Milne says that in Canton 'infanticide was rare. Dr. Williams, however, states that though the practice is rare in Canton it is common in Amoy. He thinks that in general the proportion of children killed is not great, but mentions two provinces wherein the practice prevails to an atrocious extent, twenty to thirty per cent. of the female children, therefore, tên to fifteen per cent. of the total number of infants born, being put to death.' 7 The important fact, upon which we shall comment in the next chapter, seems to be that in China infanticide is practised not so much as a regular habit as in times of distress. Moule thinks that infanticide is connected with times of want, and Douglas that it is in general only committed by the poorer classes in times of distress. Norman emphasizes the fact that it is chiefly committed by the poorer classes. Giles denies its existence altogether. ${ }^{8}$ It is therefore of particular interest to

1 Norman, Peoples and Politics, p. 290.

2 Gray, China, vol. ii, p. 50.

a Douglas, China, p. $106 . \quad$ Abeel, Journal, p. 108. ${ }^{5}$ Ibid., p. 109. Douglas, however, says that infanticide scarcely exists in Pekin (Society in China, p. 353). p. 142. - Moule, New China, p. 179. 'Sutherland, loc. cit., vol. i, - Giles, China, p. 97. 
find two modern Chinese authors speaking as follows. 'One must not assume that such a custom is prevalent; flagrant and sometimes dramatic accounts, of which we have heard so much, only hold true in certain districts and at times of famine.' 1 According to Faulds infanticide was formerly common in Japan. ${ }^{2}$

It is said that infanticide was formerly characteristic of fivesixths of British India. ${ }^{3}$ Recently it was common in Central India, Rajputana, Cutch Bhooj, Agra Province, Khondistan, among the Jats, and in the Punjab generally. The Mysore census for 1852 showed that in a population of $3,410,382$ there was a 10 per cent. excess of males in the adult population in spite of a 16 per cent. excess of female births. ${ }^{5}$ It is stated to exist among the Nagas ${ }^{6}$ though the evidence is conflicting. ${ }^{7}$ It was formerly 'very common all over the Jeypore country'. ${ }^{8}$ It was especially prevalent among the Khond people, ${ }^{9}$ where 'it was expressly sanctioned and promoted by their religious doctrine '.10 It was also common among the Todas. ${ }^{11}$ 'Lieut.-General Walker estimated that about 33,000 female children were annually put to death in Cutch and Gujerat, a rate amounting to about one-fourth of the total births and therefore to about half of the girls born. Watson and Kaye assert that " no criminality either by law or usage was ever attached among the Rajputs to infanticide. The child was smothered in milk or else opium was smeared upon the mother's breasts in quantities sufficient to cause immediate death." The Encyclopaedia of India asserts that in one of the districts of this Province, while there were 82,400 boys, there were only 35,137 girls at the 1874 enumeration, a discrepancy which clearly showed that more than onehalf of the girls had been destroyed.' 12

It is worthy of note that in addition to infanticide ' the wilful neglect of female children operates destructively in every town and village throughout the length and breadth of India '.13 This

1 Leong and Tao, loc. cit., p. 91.

2 Faulds, loc. cit., p. 285. For Tonquin see Richard, History of Tonquin, p. $757 . \quad{ }_{3}$ Wilkins, Hinduism, p. 431. - Risley, loc. cit., pp. 166 and 168; Dubois, Hindu Manners, vol. ii, p. 612; Crooke, loc. cit., p. 136 ; Browne, Indian Infanticide, p. 612 ; Wilkins, loc. cit., p. 431 ; Russell, Central Provinces, vol. iv, p. $419 . \quad{ }_{5}$ Wilkins, loc. cit., p. 432. -Godden, J. A. I., vol. xxvi, p. 179 ; Risley, loc. cit., p. 165.

Man, vol. xiv, p. 98.

8 Thurston, Ethnographic Notes, p. 504.

7 Hodson, 9 Macpherson, Memorials of Service, p. 132; Campbell, Personal Narrative, p. 139. 10 Macpherson, Religion of the Khonds, p. 65.

12 Sutherland, loc. cit., vol. i, p. 144.

${ }_{13}$ Wilkins, loc. cit., p. 431.

11 Rivers, Todas, p. 478. 
is especially noticeable where in recent days infanticide has much diminished. Thus in Rajputana girls are allowed to die when in the case of a boy medical aid would be summoned. ${ }^{1}$

Infanticide was extensively practised among the Teutonic tribes before their conversion to Christianity. ${ }^{2}$ The well-known statement of Tacitus that the Germans did not practise infanticide is certainly erroneous. Lecky suggests that the whole passage is to be taken rather as an indirect way of scolding his own people than as a sober statement of fact about-another. 'Guizot regards the picture which Tacitus draws as being analogous to the portrait Fenimore Cooper gives of the Red Indians.' 3 There is ample evidence that the practice was common. 'Grimm declares that " all the Teutonic Sagas are full of the exposure of children, and there can be no doubt that in the early days of heathenism it was lawful". Müller says that all the Teutonic races in early times had the right of exposing their children, but in the course of centuries it came to be exercised only by the parent. . . . It is related that, when in A. D. 1000 the Norsemen of Iceland were converted to Christianity, they stipulated that the right of slaying their infants should not be removed.' 4 There is evidence from graves that infanticide was practised in Neolithic times in England. 5 It is thus of particular interest to notice that, where we can catch sight of peoples emerging out of the prehistoric period, we find infanticide established as a practice; the conclusions derived from the direct evidence of the peoples of this group are thus linked on to the conclusion to which in the last chapter we came from indirect evidence. ${ }^{6}$

13. Turning to sub-groups three and four, disease, war, famine, and child mortality have already been dealt with. The remaining factors are those in respect of which there is a contrast between these sub-groups and those just considered. Furthermore, as

1 Dubois, loc. cit., vol. ii, p. 612. Infanticide was sometimes committed by the Kandyans (Bailey, Trans. Eth. Soc., vol. ii, p. 296), by the Koulous (Ujfalvy, Bull. Soc. Anth., vol. v, p. 227), by the Belochis (Barton, Sindh, p. 244), and in Svanetia (Phillipps-Wolley, Savage Svanetia, vol. ii, p. 92).

2 Stricker, Arch. für Anth., vol. v, p. 451. It was practised in the Vedic age (Kaegi, Rigveda, p. 16). $\quad{ }^{3}$ Sutherland, loc. cit., vol. i, p. 138. See Grimm, Deutsche Rechtsalterthümer, pp. $455 \mathrm{ff}$. Ibid., p. 138.

5 Lubbock,

Prehistoric Times, p. 176.

- Infanticide was practised by the extinct Guanches of the Canary Islands (Ploss, Das Kind, vol. ii, p. 259). Dasent says of the Norsemen that it was the father's right to rear his children or not at his will. As soon as it was born the child was laid upon the bare ground; and until the father came and looked at it ... its fate hung in the balance' (Story of Burnt Njal, vol. i, p. xxv). 
regards these factors there are certain important differences as between what we may call mediaeval and modern races.

Of pre-puberty intercourse there is no evidence. Among modern races it is certainly negligible. If it occurred at all in the mediaeval period it was very uncommon, and we may therefore regard this factor as of no account. Hitherto we have found that, though far from being universal, it is a fairly common practice. Its disappearance thus makes one distinction between these races and those which we have previously studied.

The length of the suckling period marks a similar distinction. It is not possible to lay down any hard and fast distinction; even at the present day in certain country districts it is prolonged, as, for instance, in Hungary for several years according to Gönezi. ${ }^{1}$ Generally speaking, however, the tendency has been for a reduction to the conditions which now rule in Western Europe.

14. A far more remarkable difference between these races and those hitherto considered is provided by the conditions regarding celibacy and the postponement of marriage. Hitherto we have found that, with the exception of a very few individuals who chiefly for religious reasons did not marry, all women, if indeed not married before puberty, got married soon after. Such postponement of marriage as occurred was confined to men, and therefore neither celibacy nor postponement had any effect upon numbers. At this point we come upon wholly new conditions.

The views of St. Paul with regard to marriage are well known and the attitude of all early Christians authors is similar though usually more pronounced. If marriage was tolerable, virginity was in any case preferable. 'For why', says Tertullian, 'should we long to bear children, whom, when we have them, we desire to send before us ... ourselves also longing to be removed from this most wicked world. . . . Therefore whether marriage be for the sake of the flesh, or of the world or of having descendants, not one of these necessities belongeth to the servants of God.'2 Such a passage as this can be paralleled in the works of almost any of the Fathers. They return to the subject over and over again. St. Augustine did not avoid the practical difficulty. 'But I am aware', he says, 'of some that murmur. What, say they, if all men should abstain from sexual intercourse, whence will the

1 Gönezi, 'Die auf die Geburt und das Säugen der Kinder bezughabenden Gebräuche', Zentralblatt für Anthropologie, vol. xii. $\quad{ }_{2}^{2}$ Tertullian, First Book to His Wife, p. 414. 
human race exist? Would that all would thus ; ... much more speedily would the City of God be filled and the end of the world hastened.' 1 There are many decrees of the early Council regarding celibacy. It was first enjoined by the Popes in $385 .^{2}$ Gregory VII was very zealous on behalf of celibacy, and owing to his efforts it became very general in Europe in the eleventh century though it was not rigidly enforced until the thirteenth century among the clergy. ${ }^{3} \quad$ Much earlier, however, in certain countries celibacy had come, under the influence of Christian teaching, to be of importance. It was initiated by Paul the Theban and St. Antony after the Decian persecution ${ }^{4}$ and according to Schönberg there were at one time in a single diocese in Egypt 20,000 men and 10,000 women celibates. ${ }^{5}$ It was felt to be necessary to take steps to limit the extent of the practice, and in 381 the Council of Saragossa forbade virgins to take the veil unless over forty years of age. ${ }^{6}$ Thus under the influence of Christianity celibacy became for the first time in human history a factor of importance. That the celibate is to be preferred to the married state is emphasized by the Council of Trent.?

Also in this period for the first time in the history of the world postponement of marriage became of importance. The evidence as to the age at marriage is unfortunately far from exact. There is, however, a very large amount of evidence which shows that, at least in the society typical of most European countries from the tenth century onwards, marriage was, except among the privileged classes, always somewhat, and often very long, postponed both for men and women, though more for the former than the latter. This postponement was brought about by the pressure of social conditions, customs, and laws. To these conditions and customs it will be necessary to refer in the next chapter, and it will suffice to say here that, generally speaking, in the country. there was a limited number of households and that, until death made vacancies, there was no house for those wishing to marry, who in consequence had to wait, and that in the towns guild restrictions bound apprentices for a large number of years during

1 Augustine, On the Good of Marriage, p. 285. vol. i, p. $62 .{ }^{3}$ Ibid., pp. 260 and 301.

2 Lea, Sacerdotal Celibacy, berg, Volkswirtschaftslehre, p. 868.

- Lea, loc. cit., p. 107.

7 Concilium T'ridentinum, Sessio 24, Can. 10. 'Si quis dixerit statum coniugalem anteponendum esse statui virginitatis vel coelibatus, et non esse melius ac beatus manere in virginitate aut coelibatu, quam iungi matrimonio, anathema sit (quoted by Lea, loc. cit., vol. ii, p. 204). 
which they might not marry, and that at many times in many places the poor were prohibited from marrying.

There is very little statistical evidence. The matter has been investigated by Rubin, who worked with the Danish figures for the sixteenth, seventeenth, and eighteenth centuries. 'It was the universal rule', he says, 'that as soon as the obstacles ... were surmounted, every one proceeded to get married.' 1 Apart, therefore, from religious celibacy, there was no voluntary celibacy such as we find at the present day. But these obstacles in the case of the dependent class were serious, and marriage was long postponed by men in this class, though postponed for a shorter time by women than by men. 'Even though the social and economic structure of the community of old restrained one section of the population-the dependent section-from marriage, the other part of the population, the independent section, married far earlier than nowadays. There was perhaps ... some holding back in certain circles of the best society. But for ordinary independent people marriage at an early age was a matter of course. Those who could marry early, then, did so. But those who were unable to marry till late in life-when they no longer held the position of journeymen, labourer, \&c.-yet married.' 2 With regard to women there was ' a state of affairs resembling that found in the case of men, a compulsory condition of celibacy for certain sections of the population. ... In certain classes of the population there were a good many more unmarried women at something over twenty years of age than there are now.' 3 Summing up his conclusions the same author says ' in spite of the fact that in the independent section of the community marriage took place, as a rule, at an earlier age in the eighteenth century than it does now, the average age of marriage was yet higher at that time, because the more numerous dependent class married later', and adds that 'there must have been a difference between the age of the bride and bridegroom considerably greater than in the marriages of our time ' ${ }^{4}$

The state of things indicated above is that typical of mediaeval Europe and lasted up to the industrial revolution. It is not to be supposed that there was any abrupt change from the conditions prevalent before; on the contrary the conditions described
1 Rubin, loc. cit., p. 598. p. 609.
1 Ibid., p. 606.
${ }^{3}$ Ibid., p. 608.
4 Ibid., 
developed slowly as European mediaeval society assumed its typical aspect. The growth of these conditions is connected with the disappearance of abortion and infanticide, and, as we shall see, there is evidence that there was a lengthy period during which these customs continued to be practised after the introduction of Christianity.

The transition from the conditions of what we have called the mediaeval period to those of the modern period was relatively sudden. The economic changes connected with the rise of the industrial system in the latter half of the eighteenth century did away with the obstacles to marriage which have been mentioned. As a result the average age at marriage was lowered. In England between 1867 and 1888 it was for men twenty-six and for women twenty-four. The question of the age at marriage in the modern period need not detain us because it was not the variations in that factor which have controlled numbers. There have been variations, but these variations have been compensated for by other factors.

Celibacy for religious reasons, which at certain times and places was of great importance in the mediaeval period, has ceased to be of any importance in the modern period. On the other hand, for the first time in history voluntary celibacy on grounds other than religious has come to be of importance. 'In the course of this century [nineteenth century] Wappaus found that in Saxony 14.6 of the unmarried adult population died single; in Sweden 14.9 per cent., in the Netherlands 14.2 per cent., and in France 20.6 per cent.' 1 The returns of the thirteenth United States ${ }^{2}$ Census showed that of the females $35-44$ years of age 10 per cent. were single.

In this connexion there is another matter which may be mentioned here. It will be referred to in detail later on. Whereas in the mediaeval period the independent class married early and the dependent class considerably later, in the modern period this has been reversed. It is now the wage-earning class which marries earlier than the independent class.

15. Contraceptive methods were known in the mediaeval period, ${ }^{3}$ but there is no evidence that either these practices or restraint from intercourse between married persons were of any importance. According to Rubin, "so far as the eighteenth century ... is

1 Westermarck, Human Marriage, p. 145. Applied Eugenics, p. 136. Mulierum, p. 233.

- See, for instance, Albertus Magnus, De Secretis 
concerned, it may be taken as a fact that in the main the limit of fertility of marriage was set by nature alone.' 1 This applies equally to the whole of the period. In this respect, therefore, this period resembles those which preceded it.

Whatever effects may have to be attributed to these factors in the former periods, it is within the modern period that they first become of primary importance. Something was said in the First Chapter as to the history of the so-called Neomalthusian propaganda, and it was pointed out that it began relatively early in the century. It is obviously very difficult to obtain any definite facts regarding the extent of these practices, but a very great body of evidence points to the conclusion that, not only have these practices come to be of great importance, but also that it is by means of variation in their use, together with deliberate restraint from intercourse between married persons, by which population has come to be regulated.

With regard to definite evidence the results of an inquiry carried out by the National Birth-Rate Commission may be quoted. Of 481 schedules issued, 366 gave definite replies regarding the limitation of births. In 288 cases the marriages were specifically stated to be limited, and in 203 of these 288 cases there were further particulars. In 105 cases (51.7 per cent.) limitation appears to have been due merely to restriction of intercourse to periods when conception was believed to be unlikely or to abstention from intercourse. There were 98 cases in which contraceptive methods were apparently employed. ${ }^{2}$

16. We now come to a consideration of the three practices which we found to be of such importance in the first two groups. Of prolonged restraint from intercourse imposed as a social custom there is no trace. It was certainly not characteristic of the mediaeval period; it is possible that in the earlier portion of the period, of which in these respects we have little definite knowledge, the practice may have been continued from the times previous to conversion to Christianity, just as we shall find that abortion and infanticide were continued in some cases. It is important also to notice that abortion and infanticide, at least in the form of well-recognized social customs, are also absent. For the first time in history none of these practices are in common use. We may shortly consider how they came to disappear.

\footnotetext{
1 Rubin, loc. cit., p. 614.

2 The Declining Birth-rate, p. 20.
} 
'Abortion', says Lecky, ' was probably regarded by the average Roman of the later days of Paganism much as an Englishman in the last century regarded convivial excess, as certainly wrong, but so venial as scarcely to deserve censure. The language of the Christians from the very beginning was widely different. With unswerving consistency and with the strongest emphasis, they denounced the practice, not simply as inhuman, but as definitely murder.' 1 The evidence goes to show that the Christian objection to abortion was the chief factor in bringing this practice into discredit. The Christian attitude was due to the belief that children in the womb possessed souls. St. Clement of Alexandria, for instance, was of opinion that such children had guardian angels. It is probable that abortion was not stamped out altogether; it may have continued to some extent throughout the whole mediaeval period; it is known to be practised to a considerable extent among the less educated classes in the more advanced countries at the present day. ${ }^{2}$ Figures have been given which show that abortion is at the present day a considerable factor in France. But it is clear that from being a factor of the first importance it has come to be altogether a secondary factor and what is more to be regarded definitely as criminal.

The first protest against infanticide was made by Philo in the first century A. D. ${ }^{3}$ The Christians opposed infanticide as they did abortion, but it does not appear that Christian influence played as large a part in putting down this practice as it did in the case of abortion. Speaking of the later days of the Empire, Lecky says that 'the legislators then absolutely condemned it and it was indirectly discouraged by laws which accorded special privileges to the fathers of many children.... Pagan and Christian authorities are united in speaking of infanticide as a crying vice of the Empire and Tertullian observed that no laws were more easily or constantly evaded than those which condemned it. A broad distinction was popularly drawn between infanticide and exposition. The latter, though probably condemned, was certainly not punished by law; it was practised on a gigantic scale with absolute impunity, noticed by writers with most frigid indifference, and, at least in the case of destitute parents, con-

1 Lecky, European Morals, vol. ii, p. 21. Leroy-Beaulieu gives a very high estimate of the number of abortions which occur yearly in France (Question de la Population, p. 330). p. 223. 
sidered a very venial offence.' 1 Finally in 374 Valentinian made all infanticide a capital offence and particularly enjoined the punishment of exposition. There is much evidence to show that many centuries elapsed before the practice was finally suppressed in Europe. It was apparently common in France up to the time of Charlemagne, who made it a capital offence. A law of the Spanish Visigoths in the seventh century punished both abortion and infanticide with death. ${ }^{2}$

The disappearance of both these practices is connected with the evolution of the typical conditions of mediaeval society which are marked, as we saw, by postponement of marriage and religious celibacy. As the former went out the latter came in, and we shall find that numbers came to be regulated by the peculiar methods of the latter period as they had previously been regulated by those of the former.

17. Mention may perhaps be made of the influence of venereal disease. Gonorrhoea has long been prevalent; there is some reason to think that it was known in Assyria. Syphilis is of more recent introduction into Europe and may be of more recent evolution. Though syphilis is a frequent cause of abortions, still-births, and infant mortality, it does not produce sterility. The presence of gonorrhoea tends to prevent conception in women and, if not cured within a certain time, it may cause permanent sterility. A considerable proportion of the sterile marriages at the present day may, as indicated in the Fourth Chapter, be due to gonorrhoea. Though no great influence is to be attributed to venereal diseases when the whole question is broadly viewed, it may be remembered that within the third period they have been prevalent and thus further distinguish that period from the first two periods. ${ }^{3}$

1 Lecky, European Morals, vol. ii, p. 27.

2 The fact that penance was imposed upon the mother who killed her child by the Council of Mentz in 852 suggests that the practice was not uncommon at that time (Westermarck, Moral Ideas, vol. i, p. 411). 3 See The Declining Birthrate, pp. 58-62. 


\section{$\mathrm{XI}$}

\section{THE REGULATION OF NUMBERS AMONG HISTORICAL RACES}

1. Ar the beginning of the Ninth Chapter it was shown that under any given conditions there is a certain density of population which is the most desirable. What was there said applies whenever men enjoy the benefits of co-operation. In the first and second groups, most men are engaged in the production of food, and, except in some races in the second group, the distinction between rich and poor, the division of labour, and other complications of social life are not far advanced. Where such complications are much increased, as in the third group, it may seem that so simple an idea as that of an optimum number is no longer even in general valid. It must suffice here to state that such complications do not destroy the general validity of this view. In fact the quotation from Professor Cannan given in the Sixth Chapter has direct reference to modern conditions, and the principle there laid down was intended by him to apply to the economic conditions of the present day, though it is, as he says, also applicable to any society once co-operation has arisen.

Among the complications of social life to which we may refer are those connected with property, the division of labour, and the division into classes. The latest development connected with property-the introduction of the capitalist system and the appearance of a large class of wage-earners-does not change the general position. One remarkable modern development of the division of labour results in one country devoting itself largely to manufacture while another may devote itself to the production of food. Of all such complications there is only one which so modifies the idea of an optimum number as to require mention in a broad survey. That complication is the division into classes. Where there is a more or less well-marked division into classes performing more or less well-defined kinds of labour, there is nevertheless generally such an ebb and flow between them that there can hardly be any question, broadly speaking, of over- 
population in any one class. This, however, may not always be so, and in particular at the present day it may be that in the lowest social class there is over-population though not in the nation as a whole. It may be that in this class there is a failure to attain to such a standard of living as is within reach owing to over-population, and whether this does in fact happen will have to be discussed later.

In the Ninth Chapter, when discussing the data of the first and second groups, we showed how approximation to the desirable number might be brought about and we further came to the conclusion that normally there was such an approximation. It is proposed here to treat the data for the third group in the same fashion, though the treatment will be even slighter than in the case of the other groups. Subsequently we shall discuss the chief causes of failure of adjustment in this and in the preceding groups. Lastly we shall touch upon the questions of migration and war which have often been held to be caused by over-population.

2. The difficulties in dealing with the first sub-group are so great that we shall only devote a very few words to the consideration of the problems that arise. The facts regarding the social conditions in Egypt, Assyria, and other great empires are very scanty. It is only with regard to Greece and Rome that we have any considerable amount of information. Our conclusions as to what was in the main the position with regard to the ancient empires will be rather in the nature of a deduction when we have completed our sketch of all the races in the third group.

How adjustment may be conceived to have come about in these races is in the absence of knowledge of their social conditions not possible to illustrate. We know, however, that the social life of these races was based upon the cultivation of the land. It is true that in addition to the cultivator we find the artisan and the wage-earner. ${ }^{1}$ The question as to how the increase of the artisan, merchant, and wage-earning classes may be kept down to the desirable level will be discussed when dealing with the third and fourth sub-groups, and, as in general the conditions are probably much the same everywhere, we may omit the consideration of what happens among these classes in this sub-group. With regard to the cultivators we have already

1 Among these races the evolution of the capitalist system had begun and in Babylonia had gone a considerable distance (Sayce, Babylonians and Assyrians, p. 127). 
seen, when dealing with the second group, how, when either family or village communities are limited to a certain area, we can understand that the undesirability of such an increase as would cause the average earnings to fall below the possible maximum not only might, but actually does, result in a limitation of increase. So again here among the cultivators, we can suppose that the danger of an undue increase was brought to notice in the same fashion. We shall also discuss in some little detail what happens among the cultivators in the third sub-group, and thus we can omit the consideration of what facts there are for this sub-group and merely ask what methods there are whereby increase could have been checked.

Of fertility we have little evidence. It has to be remembered that on general grounds fecundity was if anything greater than among the races of the former groups. Lactation was apparently often prolonged and pre-puberty marriage may not have been uncommon. Disease and war were very important as factors of elimination, and moreover they were both erratic in their action. It is no longer possible to think of a certain average degree of elimination from these causes which varied little from year to year. On the other hand there is no difficulty in supposing that abortion and infanticide were employed in varying degrees to meet the situation. It may be observed that abortion in Rome and infanticide in Greece were practised systematically, and not merely when pressure had arisen but in order that it might not arise. Thus Hesiod recommends the cultivator not to bring up more than one son at home, for thus ' wealth will increase in the house'.1 What practices were in use among the Egyptians, Assyrians, Babylonians, Hittites, and other peoples we do not know in any detail, though there is considerable evidence of the prevalence of infanticide. There may have been a tendency to practise these customs to some average degree, but there is no difficulty in supposing that following upon war and pestilence there would be such a relaxation as soon to bring numbers up to the former level. The question as to how far we may assume these methods to have been effective will be left for discussion until later in the chapter.

3. There is a great variety of races in the second sub-group; they fall under the headings of the more or less nomadic peoples, 1 Myres, Eug. Rev., vol. vii, p. 30. 
the more primitive peoples of India, who in many respects are comparable to the races of the second group but who have been to some extent influenced by Eur-Asiatic culture and the chief races of India and China. Those races which fall under the second and third headings are predominantly agricultural ; 75 per cent. of the population of Bengal, for instance, is supported directly or indirectly by agriculture. Whether, as in many parts of India, the village community system obtains, ${ }^{1}$ or whether, as in China, there is family or individual ownership of the land, ${ }^{2}$ what was said above regarding the possibility of the desirable number making itself felt also applies here. So also does what was there said about the position of the artisan and wage-earner. As a typical example of the manner in which the pressure is felt where the family system obtains in an agricultural community, we may take the conditions in China as described by two Chinese authors. 'In a village the well-to-do family is a rare exception, and the typical family is the working-class family. The father is, as a rule, a husbandman, and the sons follow his footsteps. If they do not possess a piece of land of their own they cultivate either the land of the ancestral hall, of the village temple, or that of any private owner. The mother, daughter, and daughter-in-law do the household work together, and also add considerably to the family income by such employment as may be carried on in the home. The earnings of all the members of a family are given to the mother in a hotch-potch for the maintenance of the corporate whole. The family from our point of view is a living organism which possesses a spirit quite apart from the individuals who form it. Each member does not live and work for himself, but for the family to which he belongs. Every other member has a claim on his earnings.' 3 Another author, after describing this system, tells us that 'any member of the family who should disgrace himself in any way, as by becoming an inveterate gambler and permanently neglecting his work, or by developing the opium vice to great excess, would be formally cast out, his name being struck off the ancestral register. Men of this stamp generally sink lower and lower, until they swell the ranks of the professional beggars, to die perhaps in a ditch.' 4

With regard to the nomadic peoples we always find that they

1 There are often a limited number of families in each village (Barnett, Antiquities of India, p. 105).

3 Leong and Tao, loc. cit., p. 10.

2 For particulars see Gray, China, vol. ii, p. 108. 2498

4 Giles; loc. cit., p. 189. 
are restricted to clearly marked territories. Robertson Smith, after describing the local groups among the Arabs, goes on to say that " the nomadic Arabs, whose way of life supplied the type in which all Arabian society was mainly moulded, are not to be thought of as roaming quite at large through the length and breadth of the peninsula. Each group, or confederation of groups, had its own pastures, and still more its own waters, beyond which it could not move without immediate risk of a hostile encounter.' 1 Somewhat similar conditions are recorded of all nomadic people. ${ }^{2}$

4. When we consider the factors which bear upon fertility and elimination, we find that, as in the first sub-group, war and disease are of importance-especially the latter-and that, further, the amount of elimination to which they give rise is very irregular from one period to another. For some thousands of years India appears to have been subject to the passage of scourge after scourge, sweeping away hundreds of thousands of the population. Famine at times is the cause of many deaths, and is due in part, as it is in varying degrees among all races, to climatic factors. It happens, however, that in India these conditions are such as to produce famine more often than anywhere else. Peninsular India is 'subject to excessive variations [of rainfall] distributed quite irregularly and over very long periods. In such regions years of adequate rainfall and abundant yield may follow in successive decades at a time during which a considerable population settles and opens up the country; then follow a few years of drought with high temperature and aridity; and unless migration and storage works are practicable and are executed in time, the land is deserted.' 3 Famine is also due at times to some failure in social organization or to some failure to keep up the previous level of skill, as, for instance, to maintain irrigation works.

Fecundity, as we have seen, is probably, on the whole, higher among these races than among the races of the second group. Pre-puberty marriage in India probably has a considerableinfluence in reducing fertility, at least among the Hindu part of the population. With regard to practices limiting fertility and causing elimination, we have seen that infanticide and abortion are wide-

1 Robertson Smith, Kinship and Early Marriage, p. 36. 2 For Tartar tribes see William de Rubruck, Journey, pp. 53 and 188. 3 Dickson, Climate and Weather, p. 148. For a study of somewhat similar conditions in Australia see Taylor, The Australian Environment. 
spread and perhaps to some small extent contraceptive practices or prudential restraint are employed by all these races. It may also be noticed that there is plenty of evidence that in former times in any case marriage customs existed which ensured that before marriage took place the bridegroom was competent to support a family at the recognized standard of living. "The days are still remembered when no young Munda could marry before he was able to construct a plough with his own hands, nor would a Munda girl be given away in marriage before she could, with her own hands, weave mats with palm leaves and spin cotton.' 1

5. There is no reason for thinking that over-population occurs among the nomadic people such as the Arabs ; presumably the methods we have seen to be in use are effective. There has been much discussion as to whether over-population exists in India, and there is a very great difference of opinion on the matter. Hitherto, in order to throw some light on this point, we have had to rely on general evidence ; here, for the first time, there is some exact evidence of the kind which should enable a definite answer to be given. What we want to know in the case of India is whether the real income per head is increasing or not with the increase of population. Unfortunately the evidence in India is somewhat contradictory. It has recently been summed up by Wattal. $^{2}$ He shows that some of the arguments in favour of the view that there is no over-population are of very doubtful validity. It has been said, for instance, that large areas are sparsely populated; in this connexion it has to be remembered that the soil is poor in these areas. It has also been said that the complaints about insufficiency of labour point to the same conclusion. There are, however, many reasons to account for this fact. On the other hand, Wattal points out that the acreage per unit of the agricultural population hardly exceeds one and a half acres and further shows a tendency to fall. $\mathrm{He}$ also points out that in Europe 250 persons to the square mile is thought to be the limit of density where agriculture is practised, whereas in India from four to five times that number in many places find their support on an area of this size. He finds the explanation of this not in any superiority of soil or of skill but in the lowness of the standard of living. Four persons subsist on an income that would hardly support one person in Europe. The lowness of the standard of

1 Roy, Mundas, p. 346.

2 Wattal, loc. cit., ch. v. 
living, the small area of land per head, and the tendency for the amount of land per labourer to decrease points strongly to the conclusion that over-population does occur in many parts of India.

It is also probable that many parts of China are over-populated. The apparent overcrowding and the low standard of living lend support to this view, and in this connexion it is interesting to note that infanticide is practised not so much as a regular custom but at times when, numbers being so great, no further increase could be supported. Moule says that infanticide is 'local and spasmodic' rather than 'chronic and national', and notices that it is especially connected with ' want from flood, or famine or civil war'.1 There is much other evidence to the same effect. ${ }^{2}$ It will be remembered that some evidence was given in the last chapter pointing to the great prevalence of infanticide; it is necessary to remember that such conditions are consistent with a state of over-population. The prevalence of such a custom in no way shows that numbers are as a result being kept down to the desirable level. These customs may bring about this result, or they may, in a country in the position in which there is reason to believe that many parts of China now are, merely eliminate those for whom there is no place at all. Almost the same amount of infanticide, which would limit numbers to a desirable level in a country where such a level was being maintained, would, in a country where numbers were already excessive, merely cut off those for whom enough food to support existence could not be found.

What, then, is the cause of the over-population which almost certainly exists in parts of India and China? The irregularity of the factors of elimination is itself no bar to the adjustment of population, but this irregularity may indirectly have an important bearing upon the outlook of man and ultimately upon the behaviour of the mass of the population. Great scourges must tend to produce a hopelessness of outlook. When time and again war and disease sweep through a country, there must arise a tendency for the standard of living to be lost sight of. Gradually there may come about a condition of things when there is no hope or fear. European influence may have had an ill effect. The lessening of elimination through disease and war does not of

1 Moule, New China and Old, p. 179. See also Bland, Recent Events and Present Policies in China.

2 See p. 260. 
necessity in itself tend to bring about any over-population. On the contrary, it tends rather to remove the causes which produce degraded social conditions and the consequences referred to above which flow from them. But in other ways European influence is not beneficial. Those customs, for instance, which insist upon the bridegroom possessing a certain degree of skill break down, and unless a more or less distinctly formulated ambition takes their place, there is nothing to ensure that the necessary effort to secure the highest standard of living that is possible will be made.

That which is common to these races, where over-population is suspected, is the absence of hope and fear alike, of ambition and of a standard of living; they are contented to subsist on what will just support life. Such conditions are fatal to the attainment of the desirable number. Abortion and infanticide may still be practised, but as a rule only in the presence of absolute need, not as regular customs before the need arises. To the bringing about of these conditions the factors mentioned above contribute, but they probably never represent the whole cause. In these cases we seem always to find that political misfortunes have overtaken these peoples. They have suffered from oppression in one form or another and gradually the old customs have been lost; hope and ambition have faded from the outlook. In consequence of oppression the mass of the people has by degrees sunk to a degraded condition in which neither the former customs are practised nor is an individual effort, as a rule, made towards the attainment of the best which the skilled methods available, surroundings and so on, make possible.

It is also probable that there is over-population in Egypt; it is probable, that is to say, that with the recent increase of the fellaheen population there has been a decrease in the income per head. Any one who has had an opportunity of watching the behaviour of the fellaheen side by side with that of members of some race such as the Somalis cannot fail to have been very much impressed. The fellaheen cultivate one of the richest countries on earth, and they have within their grasp and to some extent within their use a considerable degree of modern skilled methods. At an Egyptian port these men were to be seen at work during the war. From time to time Somalis disembarked with shiploads of camels. The Somalis live in a country which relatively 
to Egypt is infertile and have little knowledge of skilled methods. Stepping ashore for the first time in a modern port, bewildered as was inevitable with his surroundings, the Somali, nevertheless, showed himself a man. $\mathrm{He}_{e}$ carried a certain pride with him. The fellaheen grovelled to him; the Somali obviously despised him. The Somali is a man who has not suffered from a tradition of oppression as has the Egyptian fellah ; it is easy to understand how among the Somalis conditions would arise and would be maintained whereby excess of numbers would not reduce them to a bare level of subsistence, whereas among the fellaheen numbers probably tend to be regulated by the ability of the land to keep men alive in spite of the possibility of the return per head being far higher in Egypt than in Somaliland.

6. Passing to the third sub-group we come for the first time upon conditions substantially different to those with which we have hitherto met. All those factors, which in previous groups we have noticed as having a bearing upon fertility, have either diminished in importance or more often have vanished altogether. Lactation was not prolonged, there was no pre-puberty marriage, and there is no evidence of restraint from intercourse between married persons being imposed as a social custom. Though the knowledge of contraceptive practices may have been present there is no evidence of their extensive use. It has also to be remembered that fecundity, if anything, was increasing. On the other hand disease was the cause of a very high death-rate, higher almost certainly than in any previous age.

The most striking difference, however, between this and former sub-groups is the absence of abortion and infanticide. Further, we find what we have never found before, or rather never in such a degree as to be of any importance, postponement of marriage and celibacy ; in other words, for the older methods there has been substituted a new method whereby fertility may be reduced. We have now to inquire how the realization of the desirability of some limitation brought about the most important factorpostponement of marriage-and how effective it was. With regard to religious celibacy, which was of less importance and ceased to be of any importance in England during the sixteenth century, we may merely notice its existence. Under conditions, such as we shall describe, and which rendered marriage difficult, monastic institutions were obviously a refuge for many for whom 
there was little prospect of marriage. ${ }^{1}$ The estimates of the number of religious celibates are too vague and conflicting to be worth analysing. It can only be regarded as a secondary factor representing the most extreme result of the working of the pressure which we are about to describe.

7. The following sketch is in the main confined to conditions in England since the thirteenth century, and we may first notice that in that century ' almost every one not only possessed land, but cultivated it '.2 Yet the serf, in addition to the pressure to postpone marriage which was felt by all cultivators, was definitely restricted with regard to marriage. Besides being unable to move away from his manor, " the serf was disabled from marrying his daughter without licence and fine. Very numerous instances are found of these kinds of payment, under the name of mercheta, in the earliest times. Similarly fines are paid for marrying a daughter outside the manor, for marrying a nief, i.e. a female serf, who was possessed of property, and men of another manor for marrying a female serf from her lord's manor. I have found traces of this custom,' says Rogers, 'though they become very infrequent far on into the fifteenth century.' 3 Such facts are to be regarded as the legal enforcement among serfs of that amount of postponement which was on the average enforced by circumstances upon cultivators in general. As villein tenements were usually indivisible, the question arises as to what happened when there was more than one son. "The indivisibility of villein tenements is chiefly conspicuous in the law of inheritance; all the land went to one of the sons if there were several ; very often the youngest inherited; and this custom, to which mere chance has given the name of Borough English, was considered as one of the proofs of villeinage. It is certainly a custom of great importance, and probably it depended on the fact that the elder brothers left the land at the earliest opportunity and during their father's life. Where did they go to ? It is easy to guess that they sought work out of the manor as craftsmen or labourers ; that they served the lord as servants, craftsmen and the like; that they were provided with holdings, which for some reason did not descend to male heirs; that they were endowed with some demesne land, or fitted out to claim land from the waste.

1 See Marshall, Principles of Economics, p. 186. vol. i, p. 47.

3 Ibid., p. 45.

2 Rogers, Six Centuries, 
We may find for all these suppositions some supporting quotations in the records. But still it would be hard to believe that the entire increase of population found an exit by these by-paths. If no exit was found, the brothers had to remain on their father's plot, and the fact that they did so can be proved, if it needs proof, from the documents. The unity of the holding was not disturbed in this case ; there was no division.' 1 Here we see the pressure at work. The tenement would in general be of the size which could well support a family, and a tenement might not be divided, thus ensuring that there was no overcrowding of families until life could only just be supported.

8. Far more important than any particular disabilities regarding marriage which attach to serfs are the conditions making very difficult any increase in population which are always found among cultivators. When a village has as many hands as it requires, the number of houses is not increased. Speaking generally of the Middle Ages Pollard says that 'the number of holdings was almost stationary and the number of families fixed '.2 The number of hands in a village found to be required would be about that which experience has shown to produce the largest average income. Any further increase is made very difficult, if not impossible. ' Country life was as elsewhere rigid in its habits ; young people found it difficult to establish themselves till some married pair had passed from the scene and made a vacancy in their own parish ; for migration to another parish was seldom thought of by an agricultural labourer under ordinary circumstances. Consequently whenever a plague or famine thinned the population, there were always many waiting to be married, who filled the vacant places.' 3

Such always are the conditions among cultivators in a settled country ; it is forced to the notice of every one that not more than a certain number of hands are required and postponement of marriage is thus imposed upon the younger people. Neither land nor houses are available for them at an early age. 'Before the Reformation, not only were early marriages determinately discouraged, but the opportunity for them did not exist. A labourer living in a cottage by himself was a rare exception to the rule; and the work of the fields was performed generally ... by servants

1 Vinogradoff, Villeinage in England, p. $246 . \quad 2$ Pollard, Factors in Modern History, p. 135.

3 Marshall, Principles of Economics, p. 186. 
who lived in the families of the squire or farmer, and who, while in that position, commonly remained single, and married only when by prudence they had saved a sufficient sum to enable them to enter some other position.' 1 Miss Davies, working on figures for the parish of Corsley, found reason for thinking that at any one time there was usually a number of younger people who were debarred from marrying and were waiting their turn, so to speak, until they could step into places vacated by members of an older generation. ${ }^{2}$

9. In addition to those who cultivated the land there were merchants and craftsmen who for the most part lived in the towns. 'The essence of the mediaeval town was the formation of gilds of merchants and craftsmen' and. 'within the limits of the corporation gilds had the monopoly of manufacture or trade'. ${ }^{3}$ Membership of a guild was a birthright or an inheritance, and newcomers could only enter after a long period of apprenticeship. The result of this system of apprenticeship was to bring about the postponement of marriage, and thus to limit the undue increase of population. 'The position of a son who acquired a holding when his parent died is analogous to that of an apprentice who cannot set up as a master till given permission by the proper authorities. It is quite plain that in the eyes of the ordinary man in the sixteenth century one of the advantages of a system of compulsory apprenticeship was that it prevented youths marrying at a very early age, e.g. an Act (2 and 3 Philip and Mary) forbids the admitting of any one to the freedom of the City of London before the age of twenty-four, and enacts that apprentices are not to be taken so young that they will come out of their time before they are twenty-four. The reason alleged for this rule is that distress in the city of which "one of the chief occasions is by reason of the over-hasty marriages and the over soon setting up of householdes by the younge folke of the city ... be they never so younge and so unskilful ".' Again a petition of the weavers states (Hist. MSS. Com. Cd. 784, p. 114): " whereas by the former good laws of their trade none could exercise the same until he had served an apprenticeship for seven years and attained the age of twenty-four, now in these disordered times many apprentices having forsaken parents and masters ... refuse to

\footnotetext{
${ }^{1}$ Froude, Henry VIII, p. 3, note. ${ }^{2}$ Davies,
p. 31.
} 
serve out their time, but before they are 18 or 20 years old betake themselves to marriage.' 1

Rubin, whose conclusions regarding the age at marriage in the sixteenth, seventeenth, and eighteenth centuries have already been quoted, sums up the position in these words: ' The domestic servant class then, as now, was unmarried, but that class was much more numerous than at present. Subordinates in the industrial class and in handicrafts were not, as in our own time, free and independent, but lived for the most part in the houses of their masters, and, at any rate, were accustomed to wait until they became masters before marrying; the chances of attaining the position of master were greater than now. The same rule applied to other journeymen in various employments, whether in town or country.' 2

Thus in the town as well as on the land the pressure was at work. The result was twofold. Marriage was made difficult, and many sought refuge in lifelong celibacy in religious institutions. Again, a standard of skill was insisted upon which tended to ensure that young husbands would be able to support a family as well as ensuring that they would not have a family at all until there was a place for them. And it must be assumed that the number of families felt to be desirable was somewhere about that which given all circumstances produces the maximum benefit from co-operation. It may also be observed that we can in evidence of the above kind detect the recognition of a standard of living-the essential feature in any community in which an approach to the desirable number is to be attained. For this standard of living is only the income per head which, given all the circumstances, is the highest within reach.

10. Regulations prohibiting the marriage of the poor were not uncommon in this period. 'In Bavaria ... the regulations of the Government and the Police Ordinances of 1616 forbade the marriage of servants, day labourers, and others without property, and the punishments by which those were threatened by the legislation of 1751 who, without the permission of the superior authorities, entered into wedlock, and were afterwards unable to support themselves without begging or the like, extended to corporal chastisement and the like.' 3 The Poor Law Commission of 1834

\footnotetext{
1 Tawney, Agrarian Problem, p. 105, note.

Rubin, loc. cit., p. 597.

2 Rubin, loc. cit., p. 598.
} 
obtained reports regarding the regulations in various European countries. Of Denmark we hear that ' all those persons who are in receipt of parish relief are not allowed to marry unless by permission granted on special grounds by the Commissioners '.1 In Würtemberg no subject of the state could marry until he possessed 'the rights of a member of a community or a settled non-freeman. But even such a one must prove to the magistrates before his marriage that he possesses sufficient means of subsistence. The want of such means of subsistence is considered as existing, (a) in every one who is not primarily qualified in the exercises of a liberal art or science, or for exercising on his own account, commerce, a profession, agriculture or some other business sufficient for the support of a family, or possesses sufficient property for the independent support of a family; and (b) in every one who, at the time of the intended marriage, is the subject of political or police investigation, for vagabondage, prodigality, habitual idleness, notorious propensity to drinking, or repeated fraud, repeated theft or systematic begging, or who has been punished for the same, during the two years immediately preceding, or who in the course of the three preceding years (except in the case of misfortune not incurred by his own fault) has received assistance from public funds for his own support, or is in receipt of such at the time of the intended marriage.' 2

11. Somewhat similar conditions to those which led to postponement of marriage in England were operative elsewhere. Looking at this period in general there is little doubt that the restrictions upon increase were upon the whole effective. There were fluctuations in the nearness of approach to the desirable number; at times for some reason too great an increase became noticeable. Adjustment, however, soon followed. 'There was reason to believe that towards the middle of the fifteenth century there was a considerable increase in population, unaccompanied by any great improvement in the means of production, and consequently a relative over-population in many European ccuntries. The frequent complaints of poverty and lack of employment which led eventually to stringent measures against foreign competitors,

1 Report for Inquiry into the Administration and Practical Operation of the Poor Laws, Appendix F, p. 283.

2 Ibid., p. 520. For further information on this subject see the Handworterbuch der Staatswissenschaften, Article 'Eheschliessung'. The Danish Poor Law Act of 1891 prohibits the marriage of the poor under certain circumstances. 
confirms the evidence from other sources to show that the gilds were being overstocked with journeymen who could hardly hope to attain the position of householders and employers. In the callings where the old-world organization was effective there was an increasing tendency, during the fifteenth century, to restrict the avenues by which the freedom to exercise a craft could be obtained; larger fines were demanded on admission, while the social qualifications of those who were eligible as apprentices were raised.' 1 At other times certain social changes caused over-population. Such was the case in the sixteenth century owing to the enclosures. "According to one calculation made in 1548 three hundred thousand men had been thrown out of work by the decay of agriculture-or about ten per cent. of the whole population.' 2 What is important, however, is that in general the determination of the people to maintain the standard of living brought about adjustment-which at this period usually took the form of making marriage more difficult.

There were also instances of a definite failure over a long period to achieve adjustment. Such a case is that of Ireland in the eighteenth century. The potato was introduced about 1585 , and when taken into cultivation throughout the country could have enabled a larger population to subsist at a higher standard of living. So degraded, however, did the social conditions become, that the introduction of the potato merely enabled a larger population to subsist at the lowest standard which would support life. The conditions of that period have been described in the following passage by Goldwin Smith: 'The mass of the people were socially and economically in a state the most deplorable, perhaps, which history records as having existed in any civilized nation.... The Irish gentry were probably the very worst upper class with which a country was ever afflicted. Their habits grew beyond measure brutal and reckless. Their drunkenness, their blasphemy, their ferocious duelling, left the squires of England far behind. . . . Over the Roman Catholic poor on their estates these "vermin of the kingdom ", as Arthur Young calls them, exercised a tyranny compared with which the arbitrary rule of the old chiefs over their clans was probably a parental authority used with beneficence

1 Cunningham, Growth of English Industry and Commerce, vol. i, p. 442. 2 Pollard, loc. cit., p. 144. 
and justly repaid by gratitude and affection. ... All moral restraints on the growth of population were removed by the compulsory ignorance into which Protestant ascendancy and the penal laws had plunged the Catholic peasantry and by the abject wretchedness of their lot. ... The island became utterly overcharged with population.' 1

In this period it is not common to find over-population on this scale for so long a time. In all countries there were periods when population was excessive, but as a rule the circumstances were seldom such that the vigour of the people did not before long bring about a return to a higher standard.

12. In the fourth sub-group there is available a far larger amount of information, and more exact estimates as to the position are rendered possible than could be the case before. We have first to ask whether there is evidence of over-population. Where, as in this period, there has been a rapid increase in numbers the question is more easy to answer than when asked in connexion with periods which, compared with the present conditions, were relatively static. We can form a judgement based upon the relative increase in wealth and in population and upon the average real wages over a number of years. The following figures show that wealth has grown more quickly than population. ${ }^{2}$

Wealth of

Great Britain.

$\mathfrak{f}$
Population.

$\begin{array}{lllll}1861 & . & . & . & 28,927,485 \\ 1871 & . & . & . & 31,484,661 \\ 1881 & . & . & . & 34,884,848 \\ 1891 & . & . & . & 37,732,922 \\ 1901 & . & . & . & 41,458,721 \\ 1911 & . & . & . & 45,216,665\end{array}$

The following figures show that real wages have risen during the period. ${ }^{3}$ With regard to the drop in real wages in the last years shown in this table it has to be remembered that such minor fluctuations may be due under complex modern conditions to a variety of causes. The condition of credit, the share taken of the total income by capital and many other factors, the action of some

1 Two Centuries of Irish History, edited by Bryce, p. 21. Sir Horace Plunkett estimates that Ireland can now support about 2,500,000 agriculturalists or 500,000 families (Ireland in the New Century, p. 30). The population in 1790 was estimated at over $4,000,000$. In the sixteenth century it was probably less than $1,000,000$.

${ }_{2}$ Quoted by J. A. Hobson in evidence before the National Birth-Rate Commission, The Declining Birth-rate, p. 285. Income, p. 18.

3 Bowley, Distribution of National 
of which, there is good reason to think, was the cause of the decline in real wages noticeable in the early years of the present century, all affect real wages.

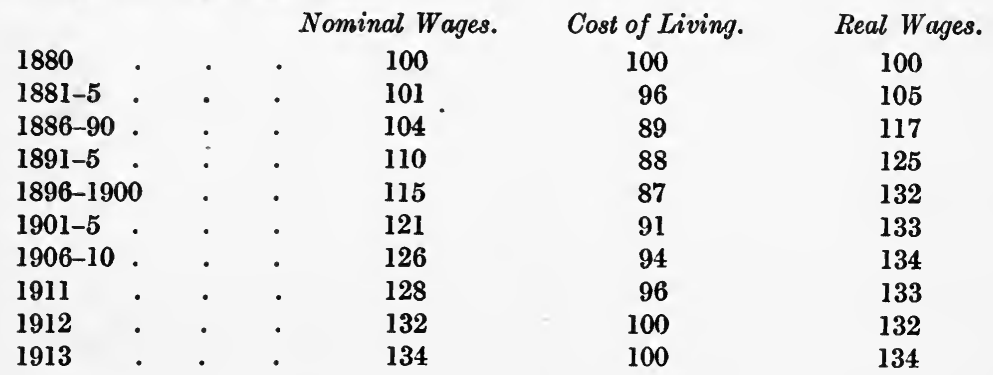

All the evidence points to the same conclusion-namely, that throughout this period there has, on the whole, been an increase in the average real income per head, and that therefore there has been no over-population in England. On the whole the same conclusion applies to other countries in which the industrial conditions are more or less similar. It is well known that during this period there have been very remarkable fluctuations in the birth-rate. In England it was stationary from about 1840 to 1880. Since that date it has declined by about one-third. ${ }^{1}$ It seems clear that during this latter period the rapid increase of the former period ceased to be economically advantageous. In other words the decline has been in response to changing economic conditions. What we have now to ask is how the desirability of a limited increase under the conditions existing in this period can have been so realized that the adjustment of numbers to economic requirements took place.

13. The factors bearing upon fertility and elimination are in this sub-group in the main the same as those in the former subgroup. The factors which did or may have limited fertility in the earlier groups are again absent. Postponement of marriage is, as in the third sub-group, an important factor and in addition for the first time contraceptive practices play a large part. The importance of contraceptive practices, together with the decline in the birth-rate, due to the start made in this period towards the mastering of disease, are the chief differences to be noticed when comparing conditions in this sub-group with those in the former. It may also be noticed that the factors of elimination are more

1 The decline in Austria dates from 1883, in Germany, Hungary, and Italy, from 1885, in Norway from 1900. 
regular in their operation; epidemics of disease are rare and war does not now, as so often formerly, indirectly cause a large deathrate. The methods of limiting increase are confined to those which reduce fertility with the exception of the practice of abortion, which is still of some importance in the lower social classes. It is important to observe that, although the postponement of marriage has throughout the period had an important bearing upon fertility, it has not been, as in the former sub-group, by raising or reducing the age of marriage that the change in fertility has been chiefly due.1 This change has been due to diminished fertility which is to be chiefly accounted for by the conscious limitation of fertility in the form either of abstention from intercourse between married persons or of the use of contraceptive practices. We are not able to measure the prevalence of the different forms of conscious limitation and thus definitely to associate them with the declining birth-rate. We do not find, however, any such increase in other factors, such as venereal disease, which diminish fertility, as will account for the facts. We have no reason for supposing that fecundity has diminished, and as we have strong reasons for thinking that conscious limitation has been increasingly practised, 'we must attribute the changes to this cause. ${ }^{2}$

14. In this sub-group we may roughly distinguish between property owners, professional classes, peasant proprietors, and wage earners. The distinguishing feature has been the rise of a very large wage-earning class not possessed of property. The peasant proprietor, who, though a property owner, is to be distinguished from other property owners, is of little importance in England. In other countries, however, the class of peasant proprietors is of importance, and we may first ask how the desirability of limiting increase is brought home to them.

As we have seen, wherever men owe their living to the produce of a definite area of land, whether ownership is vested in families or in village communities, the situation is in the main always much the same. That more than a certain number cannot

1 Thus the mean age for marriage of all husbands in England only increased from 28.43 years in 1896 to 28.88 years in 1909 , while the mean age for all wives only increased from $26 \cdot 21$ in 1896 to $26 \cdot 69$ in 1909 .

${ }^{2}$ For evidence that the decrease in fertility is due to artificial restraint see Stevenson, J. R.S.S., vol. lxxxiii, p. 431. There are reasons for thinking that contraceptive methods play a smaller, and restraint from intercourse a larger, part than is generally supposed (see Dr. Greenwood's remarks on the paper quoted above, p. 440, and also Dudfield, J. R. S. S., vol. Ixxi, 1908, p. 25). 
maintain the standard of living upon a certain area must be obvious. It may be more or less definitely realized by each individual ; it is also enshrined in the social conventions of such people. The conditions in French rural districts have been much studied, and there is a large amount of evidence pointing to the conclusion that families are consciously limited owing to the realization that large families are not economically advantageous. Legal regulations and social customs as to the inheritance of landed property are of considerable importance. in France and other countries in this connexion, but there is no space to enter upon the subject here. The conditions are, generally speaking, clear; any one who is acquainted with the conditions of life in the country districts of France, Switzerland, or Norway, for instance, has probably observed what is going on. Somewhat as has been described for the Middle Ages in England, the number of houses, and consequently the number of families, is not increased. Further, when marriage takes place the number of children is clearly limited by considerations based on the economic conditions. There is, of course, always an opportunity for the young people to go to the towns and work for wages, and we must now ask what the conditions are among the wage-earning classes.

Hitherto, with the exception of the artisans of the Middle Ages, we have always found men in groups of varying size supporting themselves upon a restricted area. In the case of the wageearners it is more difficult to understand how the desirability of limitation can become translated into customs or habits whether consciously or unconsciously followed, because, first, we are no longer dealing with a small group confined in the knowledge of all of them to a certain area, and, secondly, because restrictive customs in the nature of taboos have little chance of establishing themselves to-day. There are no restrictions of importance on the entrance to various trades ; there is at least nothing comparable to the restrictions imposed by the guilds. A young man of the wage-earning class finds no hindrance to marriage and further finds that the maximum rate of earning he is ever likely to attain to is within his grasp at an early age. Why therefore should he not marry early? Compared with the mediaeval period he does marry early. As Rubin says, "the journeymen and servants of former times were as a rule unmarried, while in our times skilled workmen and factory employees, on account of the 
high level of their earnings (and the fact that they are received in money, not in kind), and because, for the greater part, as stated earlier, they attain no better position by waiting-are married as journeymen or labourers.' 1 We have now to ask why the wage earners should, if they do not limit fertility by postponing marriage, limit fertility by conscious restriction of their families.

This is not an easy question to answer. As there are no barriers to marriage, so to the production of a large family there are no barriers in the form of social conditions or customs which must be conformed to. There is apparently no hindrance to the production of as many children as can be fed-that is to say, to the increase of population to the level at which life can just be supported. Such an increase, however, does not take place. To understand why it is so we must first realize how powerful the desire to better the social conditions and to raise the standard of living has been among the wage earners. Mr. and Mrs. Hammond have lately described the conditions under which the village labourer existed in the earlier years of the last century. ${ }^{2}$ During the agricultural depression which followed the Napoleonic Wars the most determined effort was made by the governing classes to induce the labourer to lower his standard of living in the very mistaken hope that such a change would ease the position. Above all things the labourers were urged to abandon the use of wheaten bread and to accept some substitute. All kinds of arguments and many forms of pressure were employed. The labourers, however, were obstinate. They clung to their standard of living and in England, at least since the Industrial Revolution, this determination to maintain, and if possible to raise, the standard has been powerfully manifested by the wage-earning class. ${ }^{3}$ Returning now to the question we have to answer, we may say that this determination becomes translated into a system of family limitation, and that the decline in the birth-rate which is due to conscious limitation has been in correspondence with changing economic conditions. The previous rate of increase was no longer desirable in the interest of the wage-earning classes, and it has been checked. Nevertheless it has not been checked by a fully conscious realization of the position. At the most there has only been a semi-

a Rubin, loc. cit., p. 606.

Pigou, Wealth and Welfare, p. 28.

2 Hammond, Village Labourer, ch. vii.

3 See 2498 
conscious understanding of what is involved. In the main we have to think of this determination as achieving its end by an unconscious adaptation of social habits and practices to the needs of the time. There have been other factors at work tending in the same direction. The influence of factory legislation in restricting the employment of children has been to render large families of less economic advantage. Again among more highly paid sections of the wage-earning classes certain influences, which are not economic at all but rather social, have also tended towards reduction in fertility.

It may be observed that, where the determination to maintain the standard of living is not strongly manifested, there overpopulation may occur. Thus in England, and in all industrial countries, there is at the bottom of the social scale a thriftless, unskilled, and casually employed class in which the limitation of families is little, if at all, practised. Among this class the desire to improve conditions is seldom shown and in consequence the birth-rate is not limited. If the members of this class ' restricted considerably their rate of growth, there is', says Hobson, 'reasonable ground for holding that they would make a double economic gain, being paid at a higher rate, for more efficient and more regular work.' 1 .

The conditions among the professional classes need not detain us. In addition to feeling, as do the working classes, the benefit in a general way of restriction, there are many other factors which come in. Of these perhaps the most important is the question of the age at which the maximum income is attained. As these classes form only a small proportion of the population, and as we shall have to discuss in the following chapter the causes of the lower rate of increase in these classes than among the wage-earning class-having in view the possible bearing upon certain problems of qualitative change-we can omit any further discussion here, merely observing that no question of over-population arises. Possibly there may be relative underpopulation in something the same way as there is over-population in the lowest grade-whether this is so or not depending largely upon the ease with which the professional classes are recruited from below.

1 Hobson, 'Evidence before the National Birth-Rate Commission', The Declining Birth-rate, p. 289. 
15. The discussion of historical races has been brief and was only entered upon in order to round off the subject. There are other points in connexion with the adjustment of numbers to which allusion must be made. It will be convenient, however, first to review shortly our conclusions regarding the problem of adjustment as a whole and then to ask in respect of the various periods in turn what we are led to think that the position must have been.

Reasons have been given for thinking that, if any general change in fecundity has taken place in the course of history, it has been in the direction of increase. In any case fecundity is very largethe theoretical power of increase, that is to say, is very great. Some figures were given at the end of the fourth chapter in illustration of this fact. There is a tendency noticeable in nearly all discussions of questions of quantity to under-estimate the power of increase. Another calculation previously given may therefore be recalled. It has been shown that at the present average rate of increase of the population of the world-a rate of increase which is obviously everywhere very severely restricteda single pair would produce in 1,750 years descendants equal in number to the present population of the world.

In connexion with the under-estimation of the power of increase, is the over-estimation of the relief given by any factor which allows of a growth of population-increase in skill or migration, for example. It is constantly assumed that, when such a factor can be detected in operation, there is, so to speak, a complete outlet for fecundity. This is the idea which lies at the basis of such statements as those which speak of 'surplus population' as being drawn off by migration. When, however, a calculation is made, it is seen that the relief given is, except under very unusual circumstances, almost negligible. It was shown on p. 105 that under the circumstances mentioned a population of $1,000,000$ would remain stable so long as to each married woman there was born an average of two children. But if the average was $2 \frac{1}{2}$ children, then in a hundred years the population would be $3,050,000$. It follows, therefore, that most unusually favourable circumstances only make room for a fraction of the possible increase and that migration likewise only draws off an insignificant fraction of the possible additions to the population.

Starting with such considerations and taking into account the fact that, wherever social co-operation exists, there must 
be within any area a certain desirable density of populationthe optimum number-it has been argued that there will come about an approximation to this number owing to the practice of certain habits and customs restrictive of increase. It has further been argued that, except under most unusual circumstances, habits and customs having primarily, and not merely incidentally, this result, must everywhere exist. This is seldom realized. Professor Myres, for instance, after remarking upon the fertility of ancient Egypt and of Assyria, says that ' anything like infanticide was out of the question ' .1 It is clear from the context that he is thinking not of infanticide in particular but of any practices restrictive of increase-the implication being that in a fertile country, where skill is increasing, there is a sufficient outlet for the increase of population resulting from the power of human increase. Again, it has been asserted that the Bantu races do not commit infanticide-at least on a large scale-as do many other primitive races, because, inasmuch as they have been in movement for an unknown length of time, the attrition following upon the war that is always in progress at the fringe of the movement is sufficient to absorb the 'surplus population'. But it has been forgotten that the Bantu races practise prolonged abstention from intercourse-a custom quite as effective as abortion and infanticide-as may be seen when the evidence as to the small average number in a family is considered $;^{2}$ for the average number in a Bantu family is as small as among primitive races in general, and it cannot be held that attrition through war falls seriously upon others than adults. It therefore follows that war among these people affords but an insignificant amount of relief.

16. Turning now to the methods of adjustment, we may take the first two groups together and afterwards the third group. War and migration, however, may be put aside and considered separately at the end of the chapter. As regards the first two groups it is necessary to say no more than this: evidence has been produced to show that everywhere among primitive races either abortion, infanticide, or prolonged abstention from intercourse are practised in such a degree and in such a manner as to have as their primary result the restriction of increase.

The question as to how far these practices are effective in

1 Myres, Eugenics Review, vol. vii, p. 21. is not uncommon.

2 Also abortion, as we have seen, 
bringing about an approximation to the desirable number is not easily answered. As regards those primitive races which have come under European observation there is no definite evidence because, before exact observation could be made, the conditions of life had wholly changed. But, putting aside the fact that the evidence, such as it is, does suggest an approximation, it may be observed that conditions were such as to render an approximation easy. Skill increased so slowly that for long periods of time the desirable number remained about the same; the factors of elimination, such as war and disease, were not erratic in their action; social organization was not complex in the economic sense and therefore the danger of break-downs followed by changes in the desirable number was absent. Thus we may conclude that, in all probability, in primitive society an approximation was normally attained.

In the third period we meet with wholly different conditions. Social organization becomes elaborate in the economic sense, war and disease become erratic in their operation, and, relatively to what was the case before, skill increases rapidly. As a result the desirable number frequently changes, becoming on the whole larger, particularly in those periods with which we are best acquainted. There has in consequence grown up an idea that throughout human history population has been increasing, whereas in fact it is far more correct to regard population as normally having been stable. It may not improbably turn out that the third period is in this repect peculiar and we may be approaching a period when population will again normally be stable. However that may be, the changes mentioned tend to make adjustment more difficult; on the other hand, there have been at the same time at work changes tending to facilitate adjustment among which growing freedom from convention and increasing sensitiveness to the economic situation may be particularly noticed. As regards the methods of adjustment, evidence has been adduced to show that, up to the opening of what we have called the mediaeval period, one or other of the same methods were in use that were in vogue before, that all these methods ceased to be practised in the mediaeval period and were replaced by postponement of marriage, and that finally in the modern period deliberate restriction has replaced postponement of marriage. 
The question of the effectiveness of adjustment in this period has been touched upon. In general it may be said that underpopulation is rare. Under-population seldom arises and then generally as a result of the abnormal incidence of war or disease. It sometimes happens that for a long period an area once densely populated maintains but a sparse population and this is not infrequently taken to indicate under-population. But such situations generally arise when for one reason or another-such as the destruction of capital during prolonged war, or the breakdown of artificial works upon which the food-supply dependsthere has been a decrease in the density desirable. Thus the Mesopotamian watercourses were neglected by the Mongol conquerors in the thirteenth century and in consequence the optimum number for that area declined. Such a situation should therefore not be taken as indicating, without further inquiry, under-population.

Over-population is less rare. It may be due to the neglect of ancient practices having as their effort the restriction of increase without the taking into use at the same time of new practices. This sometimes happens when a higher and a lower civilization come into contact and has been one of the causes of over-population in India. It may also arise when on the passing away of old practices some influence may militate against the taking up of new practices. In this manner the influence of the Catholic Church, which has been directed against contraceptive practices, may have tended to produce over-population in Ireland. More commonly, however, over-population arises in this period as the result of a spirit of apathy and listlessness. Under such circumstances, which are usually the result of social oppression or political misfortunes, no effort is made to keep up the standard of living and in consequence the machinery designed to restrict increase breaks down.

Taking the third period in more detail, we have as regards the last section of that period definite evidence to the effect that approximation is fairly close. There are minor fluctuations in the nearness of approach to the desirable number. That, however, which it is in any broad view of the whole problem of quantity desirable to emphasize, is the nearness of approach-the fact that in any country at any given time within this period the numbers present are, roughly speaking, 
those economically desirable. We shall, indeed, find later, when dealing with war and when referring again to this subject in the next chapter, that minor differences in the nearness of approach as between countries in close proximity may be of considerable importance.

Within the mediaeval period, in spite of the very high deathrate from disease, over-population occurred in England from time to time. The fact that wages rose after the Black Death, and that, after the decline in the population which took place in the last years of the seventeenth century and in the opening years of the eighteenth century, the average man was more prosperous, points to this conclusion, as does the fact that frequent attempts were made to render the restrictions upon marriage more severe. It seems probable that there was a similar tendency towards over-population in most European countries, and the reason would appear to be that postponement of marriage did not bring about a sufficient restriction of increase; postponement of marriage was not in fact a sufficient substitute for abortion, infanticide, and abstinence from intercourse, in spite of the prevalence of disease. Under-population was less frequent; when it occurred it was sometimes due in part to a spirit of luxury and selfishness following upon a period of expansion and prosperity, as in the later history of Spain. There may for similar reasons be underpopulation among the richer classes in Europe and America to-day.

With regard to the preceding period we have considerable knowledge of the conditions in Greece and Rome. The history of both countries presents very similar features. - In the earlier times practices restrictive of increase comparable with those found among primitive races were operative. There followed a vigorous epoch of colonization and then a period of decadence. These periods of decadence have been much discussed. In these discussions it is sometimes forgotten that the destruction of capital in civil war on a large scale, especially in the last century B. c. in Rome, must have diminished the numbers desirable. But in spite of this it is clear that we are here again in the presence of examples of decline, at least among the richer classes, owing to the presence of selfishness and luxury ; this is certainly the conclusion to which a study of Polybius leads as regards Greece, while the admirable works of Sir Samuel Dill render the 
position in Rome equally clear. ${ }^{1}$ Our knowledge of Egypt, Assyria, and Babylonia is insufficient to enable any judgement to be passed on conditions in these countries.

18. Our conclusions, therefore, are broadly as follows. Within the first two periods failure to approach the optimum number is rare. Within the third period departures away from the desirable number are less rare but are usually checked so long as the tone of society remains healthy and vigorous. In an oppressed society over-population not infrequently arises; in a selfish and luxurious society there may at times be underpopulation.

In the main, changes in numbers come about in response to economic requirements. We should not therefore attribute directly to changes in the quantity of population great historical events. Thus we may agree with Mr. Keynes when he says that ' some of the catastrophes of past history, which have thrown back human progress for centuries, have been due to the reactions following on the sudden termination, whether in the course of Nature or by the act of man, of temporarily favourable conditions which have permitted the growth of population beyond what could be provided for when the favourable conditions were at an end ' ${ }^{2}$ But we cannot agree when he says that 'the great events of history are often due to secular changes in the growth of population and other fundamental economic causes, which, escaping by their gradual character the notice of contemporary observers, are attributed to the follies of statesmen or the fanaticism of atheists. Thus the extraordinary occurrences of the past two years in Russia, that vast upheaval of Society, which has overturned what seemed most stable-religion, the basis of property, the ownership of land, as well as forms of government and the hierarchy of classes-may owe more to the deep influences of expanding numbers than to Lenin or Nicholas; and the disruptive powers of excessive national fecundity may have played a greater part in bursting the bonds of convention than either the power of ideas or the errors of autocracy.' 3 In the first passage it is suggested that consequences of great import may follow when events so derange social organization that the numbers

\footnotetext{
1 The excessive practice of infanticide in Tahiti and elsewhere in Oceania may be regarded as an example of selfish indulgence. See p. 190. So, too, the Gagas put all children to death and stole others (Battel, Strange Adventures, p. 326). 2 Keynes, Economic Consequences of the Peace, p. 215. Ibid., loc. cit., p. 12.
} 
previously maintained cannot be supported. To this we may agree ; the attribution of the catastrophe is not directly to changes in numbers. In the second passage it is suggested that national fertility may be so much in excess of requirements as to disrupt social organization. To this we cannot agree. In general increase is in response to economic requirements and, when it is in excess and is not within a reasonable time curbed, the conditions which result are not those which, though they may have important consequences, produce the kind of result here attributed to them.

19. Both war and migration are frequently said to be the consequences of over-population. We may take migration first.

It may be as well to give some typical expressions of this point of view. Mr. Haddon says, ' when reduced to its simplest terms a migration is caused by an expulsion and an attraction, the former nearly always resulting from a dearth of food or from over-population, which practically comes to the same thing. Sooner or later a time comes when the increase of the population of a country exceeds its normal food supply.' 1 In the Report of the National Birth-Rate Commission we read that 'a pressure of population in any country brings as a chief historic consequence overflows and migrations into neighbouring and other accessible countries ' ${ }^{2}$ Professor Myres, speaking of the Greek migrations of the eighth to the sixth centuries B. c., attributes them to the fact that 'population had overtaken the means of subsistence' ${ }^{3}$

It is not altogether clear what is meant by such statements. Very frequently it seems to be implied that there commonly exists a condition in which there is more than enough food in an area occupied by an existing population, that sooner or later population catches up, so to speak, the food-supply and that migration afterwards follows any further increase of population. We may first look at this point of view. All that has so far been said goes to show that only for very short periods and under very unusual circumstances can there be under any conditions of social organization a state of under-population. Even if we put

1 Haddon, Wanderings of Peoples, p. 1.

2 The Declining Birth-rate, p. 43.

3 Myres, Eugenics Review, vol. vii, p. 31. These authors are only repeating what has often been said in former times, as, for instance, by Bacon in the following passage : 'Look when the world has fewest barbarous people but such as commonly will not marry, or generate, except they know means to live (as is almost everywhere at this day except Tartary) there is no danger of inundations of people. But when there be great shoals of people which go on to populate, without foreseeing means of life and sustentation, it is of necessity that once in an age or two they discharge a portion of their people upon other nations' (Essay on the Vicissitude of Things). 
aside the idea of an optimum number, we must regard the population, which any area is capable of supporting, as strictly limited. Given the vast power of increase that we know always to be present, there cannot commonly be a condition of under-population-a condition, that is to say, in which population has ceased to increase before it was checked by the food limit. For we are not dealing with the spread of population into unoccupied areas ; that occurred perhaps before there was any social organization. We are dealing with a period in which all portions of the earth's surface, relative to the degree of skill attained, had long been fully occupied. Though increase of skill may at times enable regions previously quite infertile to be occupied, this is altogether an exceptional case ; increase of skill normally merely allows of an increase of density in the same area. General considerations point to the normal condition being of necessity either one in which population has increased up to the level of subsistence or one in which it has increased to the optimum level, and the evidence presented shows that as a rule some approximation to the latter condition is attained. General considerations on the one hand and the evidence on the other both render wholly unacceptable the idea that commonly there is a condition of under-population which is followed by migration when the pressure due to the catching up of the amount of food available by the increase of population begins to be felt. Further, no support is given to this view by the facts known regarding any particular migration. What evidence is there, with regard to the Greek migrations referred to by Professor Myres, that for some unknown length of time, for some unknown reason, the increase of population had not reached the limit made possible by the food-supply? Unless there is definite evidence of peculiar circumstances in some peculiar combination, such a theory cannot be held to account for migration. The idea of population catching up the means of subsistence and bringing about a crisis followed by migration is the product of an altogether unhistorical view of the matter. ${ }^{1}$

We do know, indeed, that at times a condition of under-population arises chiefly owing to the irregular action of certain factors

1 These remarks also apply to those cases in which countries at times appear to be empty. Attention is, for example, sometimes drawn to the fact that England appears to have been an 'empty' country in the Middle Ages. The emptiness is only apparent. Relative to the available skill and all other relevant circumstances England was fully populated in the Middle Ages; the tendency was rather towards over- than under-population. 
of elimination. Disease has been known to remove one-third of the population of a country in two or three years. There does not, however, appear to be a single instance in which migration can be-traced to the recovery from such a catastrophe. What appears usually to happen is that there is a certain relaxation of the pressure which before either hindered marriage or caused abortion or infanticide to be practised; population increases until the pressure again makes itself felt. And it may be observed that it is not following upon exceptional conditions such as these that migration is supposed by the authors quoted to take place. It is supposed to take place because not infrequently there is a condition of under-population for which there is in fact no evidence whatever. This view further clearly implies an overestimate of the relief afforded by migration. The calculations given on a previous page apply here.

The view criticized above is sometimes so expressed as not to lay stress upon under-population as the condition which ultimately gives rise to migration. It is merely asserted that over-population is in some manner the cause of migration, and we may now examine this view. Let us recall what we found to be the condition in those countries in which over-population had undoubtedly occurred. We found the distinguishing feature of the social conditions of those countries to be the absence of hope, of a spirit of enterprise, and of a determination to maintain a standard of living. It most emphatically is not where such conditions are prevalent that migration takes place. All that we know of migration points to precisely the opposite. It is not from countries in the condition of India and China at the present day or of Ireland in the seventeenth and eighteenth centuries that waves of migration arise. Migrating races exhibit the opposite characters. With them we associate enterprise, hopefulness, courage, and so on. The conclusion cannot on general grounds be doubtful. Migration does not arise where a condition of over-population has come about. Further, if we examine the evidence of particular migration in no case can it be shown that migration has begun as a direct result of those conditions. ${ }^{1}$ It is true that compared with the

1 Except perhaps in the case of the so-called Irish migration to America after the famine in the middle of the last century. Without doubt, however, there was in this migration a large political element-the nature of which is referred to below. Further there were many peculiar features in the position, and the migration was scarcely a migration in the broad historical sense. 
numerous migrations of which we have knowledge, there are but few cases concerning which we have detailed information of the social conditions. But where this information does exist, in no instance do we find evidence that migration began after a condition of over-population had come about.

It may be that what is implied by those who put forward the view that migration is somehow connected with over-population is somewhat different. It may be implied that migration occurs when what we have called the optimum number has been attained. If our general conclusions on this subject are borne in mind, it is clear that something more than this is required as an explanation. Migration occurs irregularly at long intervals ; adjustment of population to some point approximating to the desirable level is always going on. Clearly it cannot be in the ordinary process of adjustment that some impetus to migration occurs ; there must be some particular predisposing factor or factors at work. What are these factors? It would seem to rest with those who put forward the theory to show what they are, and this has not been done.

On the other hand, there is another explanation which appears to account at least in part for nearly every migration of which we have some knowledge of the circumstances, and it may be that an explanation on similar lines accounts at least in part for most of the later migrations in history. There is nothing novel in this explanation; it is admitted as an explanation of many migrations by Mr. Haddon. ${ }^{1} \mathrm{He}$ admits that many migrations have been undertaken for political or religious reasons and instances the voyage of the Mayflower and the Islamic and Buddhistic movements. That which is common to these explanations is that migration is undertaken in response to some idea. There is no question of over-population at all, and in all those cases in which we have any detailed knowledge of the conditions connected with migration we find that we can point to some idea as the motive force. Why, therefore, when details are not known, should another explanation be sought? It is suggested that essentially the same explanation is the most reasonable explanation of all migrations, at least in historical times. With regard to the earlier movements we find that migrations appear to occur where a high level of skill has been reached in some area. Where races are in contact with others of a markedly

1 Haddon, Wanderings of Peoples, p. 3. 
lower degree of skill there arises a tendency for the former to eject the latter. The immediate motive is desire to possess their land where the land is fertile relative to the skill of the first mentioned race. Thus the Bantu people have pushed back the Bushmen until the latter were left with regions infertile relatively to the Bantu culture. Throughout human history there must have been this tendency for migrations to follow the great steps in the acquirement of power over nature. But there is no reason to think that, even in early times, migrations only arose as a result of increase of skill. What we know of even the most primitive races, such as the Australians, shows that we can imagine movements to have been initiated quite apart from any differences in skill. The accounts given of the respect in which the older men are held among the Australians renders it possible to understand how an impulse to movement might be initiated by them which, once having taken shape, might have far-reaching consequences. We have further accounts of the existence of a spirit of restlessness among primitive races. This restlessness proves on analysis to be nothing more than the currency of an ideaan idea that some benefit would arise if a movement took place.

We have, however, to remember when dealing with this matter that many if not most of the migrations of pre-history were probably not migrations at all in the usual sense of the term. They are probably better thought of as driftings of people ; they may have occupied very long periods of time and have been connected with slow changes in climate. At any one time the movement may have been quite imperceptible, and, when this was so, such driftings are seen clearly enough to have had nothing to do with over-population if the immensity of the power of increase is borne in mind.

Some reference may now be made to the much-discussed theory to the effect that historical migrations are due to climatic changes. Ellsworth Huntington has put forward in a number of publications the view that not only have important climatic changes occurred within the historical period, but that these changes have been what he calls 'pulsatory'-that is to say, there has been an alteration between humid and arid conditions in many parts of the world. ${ }^{1}$ This view has been contested. ${ }^{2}$ In particular doubt

\footnotetext{
1 See especially his Pulse of Asia, and Civilization and Climate, ch. xi. $\quad 2$ See Gregory, Geographical Journal, vol. xliii.
} 
has been cast upon the 'pulsatory' nature of such changes as have taken place. Whether this view as to climatic change is correct or not is of no particular importance for our present purpose ; for on the one hand it has been shown that certain migrations, particularly those in Central Asia, where the evidence as to the pulsatory nature of the change is said to be most clear, are explicable as due to political changes, ${ }^{1}$ while on the other hand it does not appear to be possible in general to correlate the migrations of history, which are essentially rapid movements, with slow changes of climate. It seems likely that population would adjust itself to slow climatic changes without difficulty or with as little difficulty as it adjusts itself to other changes which alter the optimum density. Extreme climatic changes very slowly brought about, as doubtless took place in pre-history, might result in driftings, ${ }^{2}$ but the less extreme changes in climate during the historical period have in all probability been without any pronounced effect upon movement.

Enough has been said to show that, whereas there is much that can be adduced both on theoretical grounds and after a review of the evidence against the theory attributing migration to over-population, there is much to recommend the extension of the explanation already admitted in many cases. But even if migration is not a result of over-population, it may seriously affect the adjustment of population, once it has been set on foot. And it is to the observation of the secondary disturbing effect of migration on population that we may attribute the error of tracing migration to over-population.

When, however, all allowances are made, the easy manner in which the common theory of migration is assumed to be true and is used by writers of great authority is very surprising. Mr. Hogarth, for instance, in a well-known and very delightful book alluding to the Chaldean or fourth great wave of migration from Arabia, is led to explain all these Arabian migrations as follows: 'The great Southern Peninsula', he says, 'is for the most part a highland steppe endowed with a singularly pure air and an uncontaminated soil. It breeds, consequently, a healthy population whose mortality, compared with its death-rate, is unusually high; but since the peculiar conditions of its surface preclude

1 Peisker, Cambridge Mediaeval History, vol. i, p. 328. 22 Frequently, no doubt, such driftings were directed towards regions previously uninhabited and uninhabitable. 
the development of its internal food supply beyond a point long ago reached, the surplus population which rapidly accumulates within it is forced from time to time to seek its sustenance elsewhere.' 1 Let us consider this theory for a moment. To begin with, so far as relatively to the civilizations of the valleys of the Nile and of the Euphrates disease was rare and the death-rate on that account low, so far the position in Arabia merely approximated to that normal among primitive races. Therefore the healthy conditions taken by themselves do not necessarily form an incentive to migration. The remainder of the argument rests upon an over-estimate of the relief afforded either by increased skill or by migration. It is implied by Mr. Hogarth that, could additional skill have been applied to the increase of food, the 'surplus population' would have been absorbed; but that it was not so absorbed and found relief in migration. The calculations already given show how illusory is the idea that relief can be thus afforded. In such arguments the strength of human fecundity is always under-estimated. Further the theory assumes a condition of things within Arabia which is very difficult to understand. The Chaldean migration took place about 800 в. с., the third or Aramean, about 1500 B. c., the second or Canaanite, about 2500 B. c., and we may add a fifth or Islamic in the seventh century A.D. The shortest interval between these movements is 800 years. We are asked to suppose that for several hundred years there was an increase of population over and above that which could properly be supported; for evidently it is not supposed that the increases occurred only shortly before the migrations. Now we know that abortion and infanticide were practised regularly in Arabia. The increase therefore must have been small compared with the possible increase, and yet these methods were not according to the theory effective in producing that position which it must be the object of every vigorous society to attain. A very small increase in the degree to which these practices were performed would have brought about this result, and is it not far more reasonable to suppose that an approximation to this desirable position was normally attained rather than to suppose that there was a chronic failure the results of which could only have been socially disastrous?

There are two other considerations which may be adduced in

1 Hogarth, Ancient East, p. 78. 
favour of the view here advanced. Migration, in the first place, takes place rather from vigorous communities than from countries where social conditions have long been depressed by over-population. In the second place, the migrations in question are susceptible of another explanation. Regarding the only one of these migrations of which we detailed knowledge-the Islamic-we know that it was prompted by the currency of an idea, and why should we not assume that the previous migrations were so prompted rather than fall back on the theory of over-population which raises so many difficulties ?

20. It has often been said that war is a 'biological necessity'. 'Wherever we look in nature', says General von Bernhardi, 'we find that war is a fundamental law of development. This great verity, which has been recognized in past ages, has been convincingly demonstrated in modern times by Charles Darwin. $\mathrm{He}$ proved that nature is ruled by an unceasing struggle for existence, by the right of the stronger, and that this struggle in its apparent cruelty brings about a selection eliminating the weak and the unwholesome.' 1 This has been a favourite contention of German publicists. ${ }^{2}$ It is not necessary to show here that such views rest upon a fundamental misunderstanding as to what is implied by the term 'struggle for existence'. The error has been recently exposed by Mr. Chalmers Mitchell, who in particular has pointed out that there is nothing in the relationship between species in a state of nature which can in any sense be called war and further that modern nations are not units of the same order as species. ${ }^{3}$

It has also been held that war originates from the necessity for the search for food. 'La guerre', said Comte, 'constitue à l'origine le moyen le plus simple de se procurer les subsistances.' 4 There is no foundation whatever for this view based either on what we know of species in a state of nature or of the conditions obtaining among primitive races. The same may be said of the theory that 'eagerness to acquire property was originally the cause and object of war'.$^{5}$

The idea that over-population is the cause of war is sometimes carried back to some such 'biological' origin as that indicated

1 Quoted by Mitchell, Evolution and the War, p. 3.

2 It is only fair to say that at least one German author has recently demonstrated the falsity of this view (see Nicolai, Biology of War, p. 34). Philosophie positive, vol. iv, p. 506.

Mitchell, loc. cit., ch. i.

5 Nicolai, loc. cit., p. 34.

- Comte, 
above; in disproof of such views no more need be said. More often we meet with statements such as the following: 'The population question pushes Germany on. For the most part it is inland peoples that have most severely felt that pressure of a growing population. Islanders and coast dwellers can expand over the seas. But when inland peoples outgrow their bounds, they must burst them.' 1 Such a statement is a striking example of the over-estimation of the relief afforded by war and by migration and of the under-estimation of the power of fecundity. What has been said above regarding migration is again applicable here.

If our contention that over-population is not the cause of migration is well founded, then the argument that war is also due to over-population falls to the ground. Precisely the same conditions which are supposed to push nations on to fight are also supposed to push them on to migrate. As we shall see later, this by no means implies that questions of population do not at times enter into the situation when war breaks out.

What then is the cause of war ?. It is probable that the instinct of pugnacity led to fighting when men lived in groups of familiesat least between the males for the headship of the family. It is further probable that, as social organization slowly evolved, sporadic fighting of this kind was continued and led to fighting between groups. But in very early times tradition began to overlay and obscure the manifestations of instinct; the manner in which this occurs will form an important part of the discussion in the second part of this book. It is enough to say here that among primitive races tradition can be of such a nature as altogether to overlay the instinct of pugnacity, as among certain. peoples, who though not devoid of the instinct scarcely ever fight because of the existence of powerful social conventions and customs inhibiting its expression. Thus, though war may form an outlet for pugnacity, it can no longer be said that it is the direct consequence of pugnacity. War, in fact, gradually becomes a custom. That this is now essentially the nature of war needs no proof. It is obviously at the present day a mode of action whereby the highly organized governments of modern nations try to achieve some political end. ${ }^{2}$

1 Holland Rose, Origins of the War, p. 47.

2 Evidence has lately been brought forward to show that not only is war a custom 2498 
It has, however, to be remembered that warfare takes a place among the factors of elimination and that the greater the amount of elimination through war, the less need there is for the practices of abortion and infanticide. But this is quite a different thing from saying that warfare results from over-population or from the pressure of population. It merely implies that, given the power of increase, warfare is a factor which may perform to a small degree the elimination necessary in order that numbers should not exceed the desirable limit and is therefore toleratedother things being equal-as a factor in social life by the natural selection of customs.

It has nevertheless to be allowed that, when war breaks out, the position as regards population may form an element in the situation-just as the passions which have been aroused may do so though they cannot be regarded as the cause of modern war. Let us consider the late war. Broadly speaking in all European countries increase in population was in response to economic requirements. Yet there were what we have called minor fluctuations away from the desirable economic density. France and Germany represented two opposite tendencies towards under- and over-population respectively. These relatively slight differences were greatly exaggerated in popular opinion owing to the attention paid to the birth-rate. The Germans were thinking and talking of expansion whereas the French were stay-athome people. The Germans looked across the frontier and thought they saw a half-empty country which they could well develop with their 'surplus' population, while the French thought they but also that it is a custom of relatively late origin (Perry, ' Ethnological Study of Warfare', Mem. and Proc. Man. Lit. and Phil. Soc., vol. lxi). It is suggested that it is bound up with organization under kings and chiefs, and that it was introduced at a more or less definite time by a certain race. The evidence adduced would seem not to be adequate. The theory belongs to a series of attempts to show that very many customs and social institutions now widespread evolved in some one centre and spread thence. Though Professor Elliot Smith and his school may with justice insist on the fact that the multiple origin of similar customs has been too lightly assumed, it is equally unreasonable to insist upon tracing all similar customs and institutions to the same source. Those who are acquainted with the numerous and wonderful examples of convergence in the animal kingdom - the independent evolution of tracheae in the Arachnids and in the Insects, to take only one example-will have little difficulty in accepting the view that, in response to similar environmental conditions, similar customs may from time to time have been independently evolved. Other authors have urged that the apparent absence of weapons among the cultural remains of early races is evidence that they did not practise warfare (Havelock Ellis, Philosophy of Conflict, p. 49). But when so much doubt surrounds the question as to how the most common implements were used, it is dangerous to attribute much woight to this argument. 
saw a teeming population ready to burst its bonds and overwhelm them. Again, as between England and Germany it appeared to many Germans that, while Germany had little or no opportunity of expansion, Great Britain had owing to her overseas dominions ample opportunity. But increase is governed by the conditions within a country, and the fact that emigration from Great Britain to Greater Britain was possible did not enter into the situation as regards the control of increase in Great Britain ; very many people in this country, however, as well as in Germany, if they did not actually think that it did so, at least held opinions which were in fact founded upon this supposition. Thus though in actual fact England broadly speaking restricted her population as did Germany in accordance with the economic situation within the country, yet the position of England with reference to Greater Britain had an influence upon the situation, and many Germans thought that unless there was an outlet for 'surplus population' Germany would eventually be over-populated, whereas the fact is that, as we have seen, so long as conditions remain healthy in a country, over-population does not arise. ${ }^{1}$

This subject could be elaborated at great length. Upon analysis it would always be found that, though certain aspects of the population problem and certain mistaken views of the position may and do predispose nations and governments towards war, it is not true strictly speaking to say that the population question is in any sense a cause of war. War has now merely become a mode of action whereby an organized state tries to achieve certain political ends. It is within the power of mankind to renounce this mode of action. There is nothing in the nature of man or of social organization which renders war inevitable. 'Dieu ne leur a donné ni des canons de vingt-quatre ni des baïonnettes, et ils se sont fait des baïonnettes et des canons pour se détruire.' 2

1 It has been remarked that those countries which began the war were on the whole the countries with the highest birth-rate. The conclusion has been drawn that pressure of population brings about war. On the same lines as above, this conclusion may be shown to be unacceptablc.

2 Voltaire, Candide - the opinion of the Anabaptist Jacques. 


\section{XII}

\section{SOME MODERN PROBLEMS}

1. The present period, which may be dated from 1760 in England and from rather a later date in most European countries, has been marked by an unparalleled increase of population. The rate of increase has for some years been slowing down. This outburst of population is sometimes referred to as though there was something mysterious about it. It was, in fact, merely the response to increase in skill, and similarly the declining birth-rate is, at least for the most part, merely the response to the fact that skill is no longer increasing so fast. ${ }^{1}$ The fact that the upper social classes began to restrict their increase before the lower social classes is partly due to the fact that they are more sensitive to economic requirements. This point is almost always overlooked in discussions of the question of differential fertility, and when forming any judgement on this subject the possible loss in quality has-though it is a very difficult problem-to be balanced against the gain in arriving at an adjustment of quantity. There may be more to be said for Mill's opinion quoted on a previous page than most eugenists would be willing to admit.

Before we go on to notice some of the many problems that arise, something may be said as to the future. So deeply have men been impressed by this increase that the most pessimistic prophecies have been published as to the fate in-store for the human race. Numerous calculations have been made to show that within a certain length of time there will only be standing room. Mr. Knibbs has calculated that, if the rate of increase of the population of the world obtaining in the five years preceding 1911 was to be continued, there would in 500 years be 246,114 millions. $\mathrm{He}$ is appalled by the prospect. "No artifices of cultivation,' he says, 'nor any possible diminutions of human stature, so as to decrease the necessary quantity of food per

1 As an example of this increase in skill it may be noted that between 1840 and 1895 the quantity of product of various crops in America per unit of labour increased fivefold (Quaintance, American Economic Association Publications, 3rd Series, vol. v, 1904, p. 21). 
person, can possibly relieve the gravity of the situation. In no case can the increase of 1 per cent. per annum be maintained for five centuries.' 1 This pessimism is baseless ; he appears to regard increase as something inevitable that cannot be stopped-forgetting that throughout the greater part of human history numbers have been for all practical purposes stationary, and that increase only exceptionally outruns economic requirements. ${ }^{2}$ There is no room, it is true, for mere complacency. Though the desirable density may as a rule result from economic pressure without conscious effort, it does not follow that it would not be better if adjustment was consciously brought about. And it may be that if and when men begin consciously to adjust numbers, they may have to take some criterion other than the economic into account. To this point we may now turn.

2. So far we have proceeded on the assumption that the only criterion was the economic criterion-that the only test whereby the desirable number might be ascertained was the test founded on the average income per head. There does not seem to be any reason for supposing that there is any limit to the increase of skill in the production of food, and that therefore there is any limit to the desirable number so long as the criterion remains economic. This suggests that at some point mankind will have to introduce another method of estimating what density is desirable, as it is clear that the economic advantages of increase somewhere come into conflict with other ideals as to desirable social conditions. In other words, at some point a larger income would not be worth having if it necessitated too large a population.

It would require a lengthy discussion of many points and the weighing up of many factors before it would be possible to come to any definite conclusion on this subject. It may be observed that there are those who with some apparent reason doubt whether, taking the broadest view of human welfare, a denser population than that now existing in Belgium can be thought desirable. It also seems reasonable to hold that our great cities, and in particular London, have passed the limit that allows of

1 Knibbs, Scientia, vol. xii, p. 495.

2 Mr. J. A. Hobson's opinion, which is in conformity with that of the great majority of economists, may be noted: 'there is', he says, 'no evidence that the world's population is outrunning the natural resources; but on the contrary the presumption is that for their fuller utilization a larger population is necessary and thereby could be maintained with a higher standard of living (The Declining Birth-rate, p. 75). 
the development of the most healthy social conditions. It is a matter of doubt whether such agglomerations of population are or will remain necessary even if the total population of the country was to increase. There are two considerations which are relevant here. First it may be objected that in spite of the high average density of population in this and other countries, it is still easy to find lirge areas which are sparsely populated, and that their existence shows that there can be as yet no question of overcrowding beyond what is the socially desirable limit. But it must be remembered that the great mass of the population scarcely ever moves out of the densely populated areas, and that if they attempted to get out into the country, if only for holidays-as with increased leisure and higher incomes they will soon attempt to do-there would soon be little left of the solitude which can now be easily found.

The other consideration that is very relevant in this connexion is the average income per head. ' Before the war', says Professor Bowley, "the home income would not have yielded more than $£ 230$ gross annually per family of five, or $£ 170$ net after all rates and taxes were paid and an adequate sum invested in home industries.' 1 No one can regard this position as satisfactory. On all hands it is agreed that it must be increased even by those who with justice insist upon the fact that any general improvement must be accompanied by a decrease in the emphasis now laid upon material comfort. Income can, of course, be increased without increasing population, but probably it cannot be increased so fast.

3. There are at least two other points of view from which the density desirable may be considered. England, for instance, is in a peculiar position with regard to her food-supply, a fraction only of which is produced in the country while the rest is imported. Only by this method can England maintain her present population. How far in the case of war, or in a lesser degree in the case of social or economic disturbances elsewhere, such a position is compatible with national safety is certainly a matter for careful consideration. It seems scarcely likely that the decision would ever be taken to limit population on this account alone in view of the economic sacrifices which it would entail.

In addition to treating the question as it affects safety in the 1 Bowley, Division of the Product of Industry, p. 49. 
sense just alluded to, it may also be treated from the purely military point of view. The desire for a dense population so frequently expressed from the sixteenth century onwards, as noted in the first chapter, is largely based on the fact that the larger the population the more potential soldiers there are. Harrison, for instance, wrote as follows: 'Some also do grudge at the great increase of people in these days thinking a necessary brood of cattle far better than a superfluous augmentation of mankind. But if it should come to pass that any foreign invasion should be made, which the Lord God forbid for his mercy's sake, then should these men find that a wall of men is far better than stacks of corn and bags of money, and complain of the want when it is too late to seek the remedy.' 1 This attitude was especially noticeable in Germany before the war. A German author comments on the 'mad craze for numbers', and goes on to add that ' at the back is rather the desire, which I admit is often vague, for as many soldiers as possible '. ${ }^{2}$ It is by no means absent in England. 'In the second Report of the National Birth-Rate Commission we read as follows: 'But in the event of a war similar to that which we have just experienced, what would happen to us with a greatly reduced birth-rate? Surely all we have would be taken, and we must become slaves-as we should be to-day if we had entered on the struggle with Germany without adequate man-power. Moreover what would happen to our Empire?'3 It may be doubted whether there has been produced by such considerations in recent times even in Germany any effect upon numbers; probably economic forces have overruled such considerations altogether. It is possible that they may have had some influence upon military nations in earlier days. It is to be imagined and certainly to be hoped that they will not mould views on this subject in future.

4. As has been indicated, such departures as there are among European nations at the present day from the desirable density are of the nature of minor fluctuations. Europe is not from the point of view of productiveness over-populated (or was not in 1914 ; it is very difficult to sum up the position in this respect since the loss of capital owing to the war). There is no ground

1 Harrison, Description of England, Bk. II, ch. x, p. 215.

2 Nicolai, loc. cit., p. 48. ${ }_{3}^{3}$ Problems of Population and Parenthood, p. 73. A French historian says that 'la densité de la population fait la force des nations' (de Morgan, Premières Civilisations, p. 111). 
for the pessimism of Sir Thomas Holdich, who says that 'Asia affords no asylum for overcrowded Europe '. 1

It may be observed that the phenomenon of unemployment as generally seen in industrial countries is not evidence of overpopulation. It has been shown by Beveridge that unemployment, such as now occurs in England, is in no way connected with over-population. It is due to certain maladjustments in the industrial system, such as the decay of industries, the seasonal and cyclical fluctuations of trade, and the position as to the normal reserve of labour. The industrial system so functions that, unless special measures are adopted, there is always a certain amount of unemployment. Unemployment is thus ' a problem of industry' as Beveridge has called it." It may also be observed that the remarkable differences in the return per head as between different countries are in any case very largely due to the amount of skill employed and not to the nearness of approximation to the desirable number. ${ }^{3}$

At any given time in any nation it is desirable either that population should be stable or that there should be a certain ratio of increase. It is clear that increase may be brought about under many different conditions. A high birth-rate and a high death-rate may give the sarne ratio of increase as a low birth-rate and a low death-rate. These conditions have been much studied; the consideration of them falls outside this book. But we may

${ }^{1}$ Holdich, Political Frontiers, p. 256. It is interesting to note that the wheateating population of the world increased less rapidly in the twenty-five years preceding 1906 than the wheat area of the world. At the beginning of this period there were 283 people (more or less wheat-eaters) for every hundred acres of wheat, whereas in 1906 there were 264 such people for the same area (Agricultural Statistics, Cd. 3832, vol. xvi, part 4).

2 Beveridge, Unemployment, ch. i.

3 The average income per head in 1914 is given by Stamp as follows (J. R. S.S., vol. Ixxxii, p. 491):

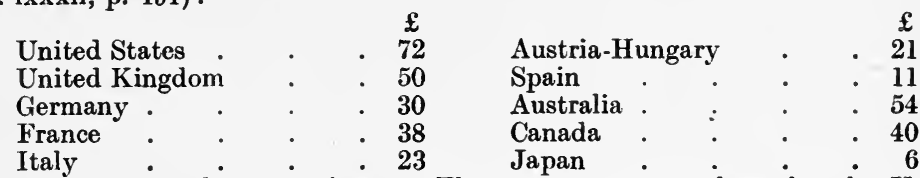

The figures are only approximate. The most accurate-those for the United Kingdom and for Australia-are not likely to be inaccurate to a greater extent than 10 per cent., while the least accurate-those for Japan-may be inaccurate to a greater extent than $\mathbf{4 0}$ per cent. Rowntree, commenting upon the low wages in Belgium as compared with England, attributes them, not to over-population, but to a low standard of education, low degree of efficiency and productivity, to the fact that only a small proportion of workers are engaged in the production of high-class goods, and to the feebleness of the trade unions (Land and Labour, pp. 75 ff.). 
note that, as far as numbers and the direct effect of the environment are concerned, but not necessarily so far as quality is concerned, a low birth-rate and a low death-rate represent more healthy conditions than a high birth-rate and a high death-rate. There is a certain correlation between the birth-rate and the death-rate, though it is often exaggerated. In Japan, for instance, there has recently been a tendency towards a decrease in the birth-rate and an increase in the death-rate. It is necessary to beware of those sweeping generalizations which assert that a high birth-rate means a high death-rate and vice versa. It may also be noted that the decrease in the birth-rate has been attained in different European countries in very different ways. Thus, whereas in England the decrease has been general throughout the country, in Germany it has been almost entirely confined to the towns. ${ }^{1}$

Here, however, the discussion is limited to general principles ; problems of this nature cannot be further inquired into. We have already noted that minor fluctuations in the nearness of approach to the optimum number as between neighbouring countries may increase, if it does not give rise to, friction between them. We may further note here that such consequences only follow when there are differences of nationality involved. When within an area inhabited by one nationality some part of it is becoming under-populated-say the urban districts-population flows in from other districts and in this case from the country. But when one country is tending towards under-population and a neighbouring country towards over-population, there is but little flow of population and but little adjustment. Various difficulties, some legal, others sentimental, check the movement towards the less fully populated country. Nevertheless, there is some movement. Thus there were resident in France in 1911 1,132,696 foreigners. ${ }^{2}$ But this immigration only went a little way towards bringing up the population towards the level which was apparently economically desirable, and there thus arose a position which aggravated the tension between France and Germany.

There are certain ' new' countries where curious conditions obtain. Their position cannot be judged in the same manner as can that of a country which has long been settled. The occupation

1 Newsholme and Stevenson, loc. cit., p. 55.

${ }^{2}$ Leroy-Beaulieu, Question de la Population, p. 55. 
of such countries can only proceed at a certain pace, and if it went more quickly we should have a peculiar condition of relative over-population. There is reason to think that population in many of these countries has not grown as quickly as it might have done, and that there is, in fact, a condition of relative underpopulation.

5. This leads us to refer to the methods of limiting increase in use at the present day. It is clear that some methods are necessary. Even if it should be thought advisable that population should increase, it cannot possibly be desirable that it should increase as fast as is possible. It therefore falls to those who disapprove of certain methods to suggest others. This problem is seldom faced by those who would dissuade men and women from certain practices. The attempt to advise in these matters without regarding the problem as a whole is strongly to be deprecated. ${ }^{1}$

Abortion is condemned on every hand, and we are thus left with postponement of marriage, restriction of intercourse between married persons, and the use of contraceptive methods as the means of bringing about the limitation of families. There is much to be said for the postponement of marriage for a certain period after the age of puberty. That education should be completed, and that a certain experience of life should be gained, before the choice of a partner is made and the responsibilities of setting up a home are undertaken, are obviously desirable. In order, however, that postponement of marriage should be effective, it would require that the average age at marriage should be so late as to produce many undesirable consequences. It seems certain that, not only under present conditions, but under almost any conditions of social life that we can picture, late marriage must be accompanied by a system of prostitution and irregular sexual habits which nearly every one agrees in deploring. Apart from this almost overwhelming argument against late marriages, there are many other objections. It is not a good thing on the whole for children that their parents should be beyond a certain age. ${ }^{2}$ Much can be urged with force against late marriage on the lines that after a certain age the outlook and habits of celibates

1 The high birth-rate in certain parts of Ireland-said to be due to the influence of the Roman Catholic Church in discouraging the use of contraceptive methodsmay be leading to over-population. $\quad 2$ Thus Marro found that children of older parents were more melancholy than those of younger parents (La Pubertd studiata nell' uomo e nella donna). 
so change as to render them less fitted to bring up a family. As few will be found to advocate late marriage as the only method; we may leave the matter at this point.

With regard to restriction of intercourse, the most important question is whether it can possibly be effective. Very serious doubts arise on this account when it is proposed to rely on this method alone. Late marriage might be in practice effective because sexual desire would find its satisfaction apart from marriage. Abstention from intercourse implies that sexual desire will not find its satisfaction; on this account it is likely that it can only be effective when accompanied by a system of prostitution that we have already agreed to regard as undesirable. Apart from this there are strong arguments against this method founded on the undesirable psychological results of abstention. It is in fact perhaps scarcely possible to think of a marriage system as satisfactory in which what we must regard as the physical object of marriage is not realized.

We are thus led to speak of the use of contraceptive methods and we may glance at two sides of the matter-ethical and physiological. It is a notable fact that all religious bodies, so far as their opinions can be discovered, are strongly opposed to this method. ${ }^{1}$ This objection is founded upon many considerations, of which the most striking is perhaps that the method allows of, or rather that it encourages, self-indulgence. Those whose attention has been drawn to the problem of the causes of the progress and decay of civilization will not fail to feel the force of any argument against the spread of habits which encourage selfindulgence. But it may be suggested that the normal exercise of any physical function can scarcely be called self-indulgence. There is a mean in the satisfaction of physical appetites, on the one side of which is self-indulgence and on the other side asceticism. It is a question whether those who disapprove of these methods because they encourage self-indulgence are not in fact demanding an ascetic life. As a form of self-discipline there may be much to recommend ascetic practices, but that is another matter. The fact is that in the use of these methods there is nothing which necessarily encourages self-indulgence. They make it possible to exercise a normal function; they do not necessarily lead to its over-use. This would seem to be without doubt the most tangible 
objection to these methods. The other objections are on the whole rather of a religious than of an ethical nature, and as such fall outside the scope of this book. ${ }^{1}$

In addition to the ethical there is the physiological aspect of the matter. There are several different methods employed to prevent conception. It is a matter of great importance to know which of them can be employed without causing ill health. With regard to this little is known and opinions vary. More than this need not be said.

It is no part of the object of this book to advocate any particular methods $;^{2}$ it is only intended here to show what problems arise at the present day from the quantitative aspect of the whole matter. It may be suggested that upon the question of the methods of limiting population it is desirable that there should be some consensus of opinion. Man has not only to settle how far he is going to limit population, but how he is going to do it.

6. We may now glance at certain questions which if they are to be referred to at all in this part of the book are most easily introduced here. These questions are not directly concerned with the quantitative aspect of the problem; their importance lies chiefly in their bearing upon the qualitative aspect.

It is a familiar fact that at the present day the birth-rate is higher among certain sections of the population than among others. There is reason to believe that this was also the case in some of the older civilizations-notably in Rome. We may first look at the facts and afterwards at the causes. Many investigations have shown that there is a higher fertility among the lower social grades. Heron found that there is a high average correlation between the birth-rate and conditions which indicate a lower social grade, such as the number of pawnbrokers per 1,000 inhabitants, the amount of child employment, pauperism, overcrowding, and other indications of poverty and lack of

1 The arguments for and against are fully and fairly set out in Problems of Population and Parenthood, pp. 44-8.

2 Personally I agree with the views of the Dean of St. Paul's on this matter. Speaking of the use of these methods, 'it seems', he says, 'a pis aller which highminded persons should avoid if they can practise self-restraint. Whatever injures the feeling of "sanctification and honour" with which St. Paul bids us regard these intimacies of life, whatever tends to profane or degrade the sacraments of wedded love, is so far an evil. But this is emphatically a matter in which every man and woman must judge for themselves, and must refrain from judging others' (Outspoken Essays, p. 74). 
culture. ${ }^{1}$ Whether a differential birth-rate results or not in a differential contribution to the next generation depends on whether there is a differential survival rate, and if so, whether it compensates for or not the differences in the birth-rate. The following extract from the Report of the National Birth-Rate Commission throws light upon this point. Using material provided by the Registrar-General for England and Wales giving the births for 1911 classified according to the occupation of the father, the following is the position :

' Classifying into groups arranged in descending order of social grade we have :

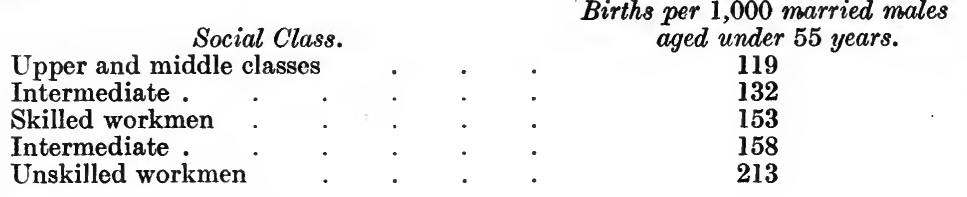

The rate of mortality in the first year of life is also provided in these groups, and follows the same order, viz. $76 \cdot 4,106 \cdot 4,112 \cdot 7$, $121.5,152.5$. If, however, we multiply the birth-rate by the difference between unity and the proportion of deaths, i. e. the proportion of survivals in the first year, the resulting effective birth-rates are still in the same order, viz. 110, 118, 136, 139, 181, after the hazards of the first twelve months have passed. We must conclude, therefore, that the initially higher birth-rate of the lower classes is not so reduced by heavier infant mortality that their effective birth-rates are brought into approximate equality with those of the wealthier classes. We have no material allowing us to extend the comparison to later years of life; but equally we have no reason to suppose that such an extension would change the order.' 2

All available evidence goes to support this conclusion, namely, that the higher the social status the lower is the fertility, and that this initial difference is not removed by subsequent differential mortality. This is apparently true not only for England but also for other countries in which the economic situation is similar, and is a relatively recent phenomenon. ${ }^{3}$

'Heron, Drapers' Company Research Memoirs, No. 1, 1906. See also Stevenson, loc. cit.

2 The Declining Birth-rate, p. 9.

${ }^{3}$ It is also noticeable in India (Wattal, loc. cit., p. 24). It will be remembered that in Denmark in the latter part of the mediaeval period there was some slight tendency according to Rubin for marriage to be postponed among the very highest social class; among the independent class generally marriage in those days, however, not only took place earlier than now but earlier than in the lower grades of society. 
7. In looking for the causes of this differential increase as between various sections of the population, we have to remember that, in accordance with what has been said in the last chapter, we suppose the position in regard to population in the nation as a whole to be ruled by economic factors. If there is an increase, then we suppose that increase to be somewhere about that which the economic situation demands in order that the greatest income per head should be gained. It follows that generally speaking the conditions we find among the mass of the industrial population excluding the lowest class approximate to those which are demanded; but it is possible that, just as the increase among the lowest class is excessive, so the increase among the higher social class may be too small judging from the economic standpoint. We have here to ask what factors there are which bring about a lower fertility among the upper classes, recollecting that this low fertility may not be wholly due to an approximation to economic requirements, but may represent a failure in adjusting increase to the economic situation.

Among the factors which bring about a low fertility in these classes, those connected with differences in education and the age at which the maximum income is obtained are the most important. Education is continued in these classes up to an age at which men in the industrial classes are earning almost the maximum wage to which they will ever attain. Even when education in the ordinary sense of the word is finished, there usually follows a period when the salary gained is low, or when perhaps a premium has to be paid in order to obtain a start in whatever profession is chosen. Doctors and barristers seldom begin to earn until some years after their education is complete. Further, when they do begin to earn, the income is often for many years low, and may in some cases not reach the maximum until late in life. Among the industrial population conditions are wholly different, and the maximum rate of wages is soon reached. Whatever forces, therefore, may be playing upon the industrial population, there are in addition the above forces playing upon the professional classes which account for the lower rate of increase among them.

There are also many other factors at work in the same direction. In the life led by the upper social classes there are more numerous ways in which the desire for relaxation, change, and pleasure may 
find satisfaction than among the lower classes. Life is in many ways more varied. This has an influence upon fertility in two ways. When a marriage takes place among the upper social classes it involves to a far more considerable extent than among the lower classes a renunciation of much that has given pleasure hitherto. When a man marries and ' settles down' he gives up, as a rule, many activities from which he had derived pleasure before. Further, after marriage the possibility of indulging in many of the pleasures which this varied life offers is often incompatible with the bringing up of a family. Children are often in the way. Again, though children are no longer the economic asset among the lower classes which they were before the passing of the Factory Acts, the possession of children is generally looked upon among those classes as a support in old age when the power of earning has gone. Children, on the other hand, among the upper classes have rather to be provided for, and in any case are seldom regarded as a support. ${ }^{1}$

Very many other factors might be mentioned, but enough has been said to show how in the main the different fertilities may be explained. The insufficient income of many of the less well-paid groups of the higher classes is often alleged as a cause of low fertility. This view is well founded when, as often occurs, social conventions force a so-called higher standard of living upon them -a standard which merely involves the expenditure of more money than among the industrial classes in preserving appearances without adding to welfare. When, however, this is extended to classes in which income has reached, say, between $£ 600$ and $£ 1,000$ a year, it cannot be allowed that income is truly insufficient. It is in such cases only insufficient when more than is reasonable is expended in the way of material satisfaction. A man cannot be heard to say that he cannot afford a large family as otherwise he will not be able to keep a motor-car-so long, that is to say, as the motor-car is not necessary to his profession.

7. This differential fertility occurs in countries where the population is less homogeneous than in England, and thus assumes importance in another aspect. For whether we assume the

1 Among the industrial classes conception before marriage is not uncommon. It is said that many men are not desirous of legal marriage until it is probable that they will have children. This is not to be regarded as evidence of loose sexual morality so much as evidence of foresight and of thought for old age when, if they have children, they will be more or less secure against extreme poverty. 
differences between the elements in the population to be due to inherited characters or to varying social traditions-a question to be discussed later-a change in the proportion of the different elements will be of great importance. According to Hill, who has investigated this matter in America, the percentage of white women of native parentage under 45 years of age bearing no children is $13 \cdot 1$, while the percentage of foreign women is $5 \cdot 7$. The average number of children for white married women of native parentage is $\mathbf{2 \cdot 7}$, and for similar women of foreign parentage 4.4. Among the foreign women the average number of children for a married English woman is $\mathbf{3} \cdot 4$, for a German $4 \cdot 3$, for an Italian 4.9 , for a Pole $6 \cdot 2.1$ The above is only one example of conditions which obtain in many countries raising difficult problems.

The same problem arises in an even more important form when there are in the same country races as different as the white and the negro. In the United States and in South Africa the problem assumes greater importance than elsewhere. In the latter country the negroes are increasing faster than the white element of the population. In America this has not been the case in the last hundred years; but the fact that the white element has maintained its relative position is due to the fact that in addition to the increase in the native-born white population there has been an increase due to immigration. ${ }^{2}$

8. The question of the relative rate of increase of the population of different countries is a somewhat different matter. It is due to many factors, one of the less important of which is that the population of various countries does not approach equally closely to the number desirable in each country. The chief factor is the variation in the arts of production in use in each country. It is only an extension of this fact that the natural resources of different countries have been more exploited in some cases than in others. In Russia the arts of production in use are less advanced than in more western countries. The introduction of western methods into such a country enables a rapid increase of population to take place. During the same period the increase can only be

1 Hill, Amb. Stat. Ass., vol. xiii, p. 590. An allied problem is that connected with the proportion of various racial elements among the immigrants into a new country. Thus between 1900 and 1913 Great Britain contributed roughly one-third and the United States and continental Europe two-thirds of the immigrants into Canada.

2 Tucker, Progress of the U.S.A., p. 98. 
slow in countries which already use these methods, and thus, so long as the knowledge of the arts of production is not equal in all countries, different rates of increase are liable to arise when knowledge spreads into a more backward country. ${ }^{1}$

Such changes are altering the composition of the population of the world. The following figures have been given for Europe. Out of 1,000 inhabitants there were in the years named the following proportion of Teutonic, Romance, and Slav elements :

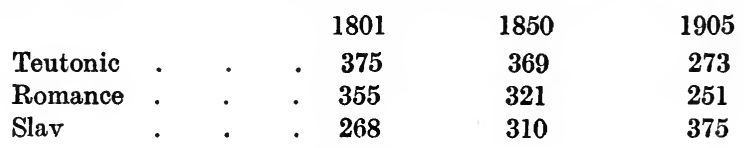

1 Leroy-Beaulieu estimates that, if the degree of skill in production now found in Western Europe was extended throughout the world, the population economically desirable would be from two to three times that of the present population of the world (Question de la Population, p. 174). 


\section{XIII}

\section{THE QUALITATIVE PROBLEM}

We now approach the second part of the problem, and turn from a consideration of the quantity to a consideration of the quality of the population. In the second and third chapters we found that reproduction was a necessity, and that to the fact of reproduction can be traced the origin of problems both of quantity and of quality. In the third chapter we further found that the position of animals and plants in a state of nature as regards problems of quality was in its broadest aspect simple. The changes in the forms of organisms, which, when viewed as a whole, we call evolution, are due to changes in the physical basis from which new generations arise. The long procession of organisms, usually of increasing complexity, from the simplest type to the immediate ancestor of rational man, thus owes its being to a long procession of changes in the germ-cells.

When treating of the quantitative problem among men, we considered a large amount of evidence which bears also upon quality, and this evidence, supplemented as and where necessary, will enable us to make some estimate of the importance of qualitative changes among men. This is the object of the remaining chapters. We have to try and estimate how far human history is comparable with changes among species in a state of nature-is due, in other words, to germinal changes. There are two other possible causes of change among men-the direct influence of the environment and the influence of tradition-and in order to estimate the importance of germinal change, it will be necessary also to consider what importance is attributable to these two factors. To one of them-namely to tradition-we shall find reason to attribute great importance. Tradition, in fact, comes ultimately to be of more importance than germinal change among the underlying causes of history. But tradition is profoundly influenced by the quantity of population among other factors, and, therefore, to the extent to which this is so, the determining factor in human history is still bound up with the population problem. 
As history ceases to be strictly correlated with changes in quality, it comes to be influenced by a factor the nature and strength of which is largely determined by the quantity of population. Thus the two aspects of the whole problem are linked together in the case of man in a manner that does not obtain among species in a state of nature.

The attempt to deal with this problem will be undertaken in the following manner. In Chapter III reference was made to the influence of the environment upon growth and upon the adult form. In Chapter XIV this subject will be developed so far as species in a state of nature are concerned, and the results of the inquiry will be applied to man in Chapter XV. The subject-matter of Chapter XV will be confined to the influence of the environment upon human bodily and mental characters. The influence of the environment as a stimulus affecting the direction in which mental characters are used will be left to a future chapter.

We next turn to inquire what influence changes in the germcells have had. Before we can make any progress it is necessary to ask what characters-physical and mental-have their basis in the germ-cells, and an inquiry into this subject will occupy Chapter XVI. In Chapters XVII and XVIII we ask what evidence there is of selection and other factors throughout human history which we have reason to suppose have a bearing on change in the average germinal constitution of the human species. This will among other things necessitate an examination of the facts in Chapters VII, VIII, and X in order to ascertain what bearing they have upon selection. Having thus some knowledge of what is inherited, and having discussed the occurrence of such factors as in human history may have brought about changes from one generation to another in the character inherited, we shall be in a position in Chapter XVII to attempt an estimate of the results which the factors-selection and others-have had upon physical characters. We shall find that as regards these characters the position is more or less clear ; we shall find, that is to say, that the influence of selection and other factors, considered in conjunction with the influence of the environment discussed in Chapter XV, does enable us in the main to understand how the physical evolution of man has come about. With regard to mental characters to be discussed in Chapter XVIII, we shall find that the task cannot be similarly completed. With a far less 
degree of certainty. than in the case of physical characters we shall arrive at some idea as to how mental characters have evolved. This, however, still leaves unanswered the problem as to how far the evolution of mental characters accounts for what we call history. To attack this problem it is necessary to consider how the environment acts as a stimulus upon the mind, conditions the degree to which and the direction in which the mind is used, and thus leads to the building up of tradition. In Chapter XIX we consider the nature of human mental endowment and the manner in which tradition is built up and handed on. In Chapter XX we consider the nature of the environment under which human mental activities have been exerted. This will enable us in Chapter XXI to survey the broad facts of history and to come to some conclusion as to the parts played respectively by the changes in human mental endowment, by the direct influence of the environment upon the mental faculties, and by its influence as a stimulus upon the exercise of these faculties. In a concluding chapter the results of the whole inquiry will be summed up. 


\section{XIV}

\section{THE INFLUENCE OF THE ENVIRONMENT AMONG ANIMALS AND PLANTS}

1. A BRIEF inquiry into the part played by the environment among animals and plants forms a necessary introduction to a similar inquiry as regards man such as we shall undertake in the next chapter.

In the second chapter something was said as to fertilization and as to the development of the zygote or fertilized egg to the adult form. It has been shown by Herbst that, if this process of development is to result in a normal adult, all the factors comprising the normal environment must be present. Herbst ascertained the exact composition of sea-water at Naples. ${ }^{1}$ Using as his material the larvae of sea-urchins, he changed slightly in many different ways the composition of the water. The experiments were very exhaustive. At every stage in the development he observed the results of abstracting one or more of the constituents of sea-water-the normal environment of the larvae. Commenting upon the results of these experiments, Jenkinson says: "Whatever may be the ultimate explanation of the facts, there can be no doubt whatever that the most complete demonstration has been given of the absolute necessity of many of the elements occurring in ordinary sea-water, its normal environment, for the proper growth and differentiation of the larva of the seaurchin. Nor is this all. Some of the substances are necessary for one part or phase of development, some for another, some at the very beginning, others only later on. Thus potassium, magnesium and some degree of alkalinity are essential for fertilization, chlorine and sodium for segmentation, calcium for the adequate cohesion of the blastomeres, potassium, calcium and the hydroxyl-ion for securing the internal osmotic pressure necessary for growth, while without the sulph-ion and magnesium the due differentiation of the alimentary tract and the proper

1 These experiments have been summarized by Jenkinson (Experimental Embryo$\log y$, pp. 141 ff.). 
formation of the skeleton cannot occur; the secretion of the skeleton depends on the presence of some sulphate and alkalinity, the skeleton requires calcium carbonate, cilia will only beat in an alkaline medium containing potassium and magnesium, and muscle will only contract when potassium and calcium are there.' 1

Summing up the results of these and other experiments, Jenkinson says: 'Every factor, or nearly every factor [of the environment], is necessary for this or that phase or part of the process, some for the whole. Light of a certain wave length will accelerate development; light of another kind, or, in some instances, darkness, will retard it, or will stop it altogether ; a certain degree of heat is indispensable; oxygen is required for respiration, water for growth; some eggs demand constant agitation, others complete rest; fertilization or segmentation or gastrulation, or some one or other of the later phases of development may depend absolutely on the presence of some particular chemical element; remove the factor in question, whatever it may be, and that particular process will not occur, and the specific typical end which is reached in normal development will not be attained.' 2

Putting aside until later the question as to what is implied by the term normal environment, we may now ask what the relation is between heredity and environment. To regard a developing organism as subject to the action of two forces, one tending to thrust it in one direction and the other in another, is to view the matter in a wholly false light. Heredity and environment are, as is clear from the evidence given above, complementary one to the other ; without a germinal constitution there can be no organism; without appropriate stimuli an organism cannot develop. Similarly it is misleading to speak of the relative importance of the two factors unless the terms are very clearly defined. To see that careful definition is necessary, we have only to remember that, because, with the exception of the anaerobic bacteria, all organisms require free oxygen, environment might be held to be all-important. Here, however, we are not concerned with the problem as to the conditions under which we might speak of the relative importance of the two factors ; we have merely to note that they are essentially complementary.

2. We may now observe that, just as a normal adult only

$$
{ }^{1} \text { Jenkinson, loc. cit., p. } 151 . \quad 2 \text { Ibid., p. } 157 .
$$


develops under normal stimuli, abnormal stimuli experimentally induced may be followed by every kind of result from the most extreme to the most insignificant changes both of form and of life-cycle. We may note the results of a few of the vast number of experiments which have been made. With regard to plants it has been shown that ' each developmental stage depends upon special external conditions, and in cases where our knowledge is sufficient, a particular stage may be obtained at will. In the Green Algae, as in the case of the Fungi, we may classify the stages of development into purely vegetative growth (growth, cell-division, branching), asexual reproduction (formation of zöospores, conidia), and sexual process (formation of male and female sexual organs). By modifying the external conditions, it is possible to induce algae or fungi . . . to grow continuously for several years, or, in the course of a few days, to die after an enormous production of asexual or sexual cells. In some instances even an almost complete stoppage of growth may be caused, reproductive cells scarcely being formed before the organism is again compelled to resort to reproduction. Then again the sequence of different stages of development may be modified as we desire.' 1

Another kind of experiment shows that foliage shoots can be converted into runners and vice versa ; it is, for instance, possible to induce a germinating tuber of the potato to form foliage shoots under the influence of a higher temperature. The transfer of plants from one environment to another is often followed by remarkable changes. MacLeod, for instance, states that a species of Philodendron which has large leaves pierced with round holes was cultivated for many years in the Botanical Gardens at Ghent in a greenhouse which was rather cool and dry. The holes were found to be rare; at times it was not possible to observe a single perforation in any of the leaves of a specimen. In every other respect the plants were healthy. The plants were later transferred to a greenhouse that was warm and moist and after a few months the new leaves were found to be abundantly perforated. ${ }^{2}$ Bonnier made some very interesting observations on the dandelion. $\mathrm{He}$ found that the plant, when sown at a high altitude in the Pyrenees, produced very short stems with hairy, dark green leaves and

\footnotetext{
1 Klebs, 'Influence of the Environment', in Darwinism and Modern Science, p. 227. 2 MacLeod, Quantitative Method, p. 12.
} 
a compact flower. On the other hand, seeds gathered from such plants growing at a high altitude and sown in the neighbourhood of Paris produced after three years elongated stems with less hairy and brighter leaves, or, in other words, plants very similar to those grown from seeds obtained in the neighbourhood of Paris. The modifications acquired during a given time by a lowland plant grown at a high altitude, or by a highland plant grown at a low altitude, took about the same time to disappear on returning the plants to their original climates. ${ }^{1}$ Similarly ' Schubeler sowed seeds of various plants in different latitudes in Norway and proved that the brilliancy of the flowers increased with the latitude. So great was the difference that it was difficult to conceive that they were produced from the same batch of seeds.' 2 Observations on European peach-trees transported to Réunion were made by Bordage. Such trees lost their deciduous habit and became evergreen, though in some cases it took twenty years before the change was complete. ${ }^{3}$ Lastly the red primrose ' reared at a temperature of $30^{\circ}-35^{\circ} \mathrm{C}$. (with-moisture and shade) has pure white flowers, but the same plants reared at $15^{\circ}-20^{\circ} \mathrm{C}$. have red flowers. If the white-bearing plants are brought into a cooler place, the flowers that are already in bloom remain white, but those that develop later in the cooler temperature are red.' 4

We may also notice the results of some of the experiments upon developing animals. Stockard experimented with the fish Fundulus heteroclitus. He subjected the eggs both before cleavage began, and after the two- and four-celled stages had been reached, to solutions of magnesium salts in sea-water. The eyes of a large percentage of the embryos were abnormal. In some cases there was a single median eye ; in other cases there was a median eye showing signs of a double structure. ${ }^{5}$ 'In a long series of experiments Féré has shown that monstrosities can be produced by exposing the hen's egg to the unfavourable influence of a large variety of substances. Vapour of ether, alcohol, essential oils, nicotine, mercury and phosphorus, injection of alkaloids such as morphine, nicotine, strychnine and others, of bacterial toxins (those of tubercle and diphtheria), of peptone, dextrose, and

${ }^{1}$ Vernon, Variation in Animals and Plants, p. $312 . \quad{ }^{2}$ Quoted from Henslow by Vernon, ibid. $\quad 3$ Thomson, Animal Life, p. 407.

Mechanism of Mendelian Heredity, p. 38. $\quad$ Stockard, Journal of Experimental Zoology, vol. vi, p. 334. 
glycerine, several alcohols, certain salts ... are all harmful, retarding and distorting the embryọ to a greater or less extent.' 1 Many experiments show the effect of differences in food, temperature, and humidity upon developing organisms. Agar carried out some experiments on a small water-flea, Simocephalus. This animal is enclosed in a kind of shell composed of two valves, somewhat like a mussel. Normally the edges of the valves nearly meet, so that if a section is cut across the animal transversely the shape of the body enclosed in the valves is oval. When the food was varied in a certain way, Agar found that the edges of the valves were turned outwards so that the shape of a transverse section was no longer oval but bell-like. He also found that the length was reduced by exposure to a high temperature. ${ }^{2}$ Experimenting with a beetle, Tower found that both the colour and the colour pattern could be modified by changes in temperature and humidity. ${ }^{3}$ Similarly Morgan showed that a species of the fruit fly exhibited a peculiar formation of the abdomen in the presence of moisture; when raised in dry conditions the abnormality disappeared. ${ }^{4}$ Poulton obtained some remarkable results when working with the larvae of moths. 'Larvae surrounded by the leaves on which they fed, became, in the majority of species, light brown or light grey in colour. If, however, an abundance of twigs had been mixed with the leaves of the food plant, they became dark in colour. The larvae of the Peppered Moth afforded the most striking result of all, for when reared among green leaves and shoots they became bright green without exception, whilst in the presence of dark brown twigs they nearly all assumed a corresponding colour.' 5

A Japanese experimenter exercised rats for 90 to 180 days; he found that this long-continued exercise markedly increased the weight of the heart, kidneys, and other organs on an average to about 20 per cent. 6 A thickening has been observed in the stomach of a gull fed on grain for a year and this change is said to take place under natural conditions in the herring gull, which feeds in winter on fish and in summer on grain. Conversely if graminivorous birds are fed on a carnivorous diet, the gizzard assumes the form of a carnivorous bird's stomach. Cuvier found the length of the intestine of the wild boar is as 9 to 1 and of

\footnotetext{
1 Jenkinson, loc. cit., p. 132.

2 Agar, Phil. Trans., Series B, vol. cciii,p. 319

3 Tower, Investigation of Evolution, pp. $168 \mathrm{ff}$.

Morgan, loc. cit., p. 39

- Vernon, loc. cit., p. 219.

- Thomson, Animal Life, p. 383.
} 
the domestic boar as 13.5 to 1 , a difference which is in part probably due to diet. ${ }^{1}$ The colour of birds' plumage is affected by their diet. Hempseed causes bullfinches and other birds to become black. Cayenne pepper causes yellow to change to orange red. In the New York Zoological Gardens it has been shown that some birds, such as the bobolink, may be so dieted that they keep their breeding plumage throughout the year and will sing their spring song in mid-winter. ${ }^{2}$ Warren produced marked changes in the common water-flea, Daphnia, by keeping them in a confined space for many generations. 'Semper and de Varigny found that when freshwater snails were reared in vessels of a shape that allowed them abundant water but very little surface in which to take exercise they developed into dwarf forms. Every precaution was taken to procure abundant food, perfect aeration and thorough removal of waste products. De Varigny's experiments were particularly careful and point convincingly to the conclusion that the condition of dwarfing was the restricted area for exercise.' 3 Lastly it may be remembered that, as is well known, parasites may induce remarkable changes in their hosts.

3. From what was said in the third chapter it follows that the results of artificial subjection to abnormal stimuli just described are to be interpreted as examples of the fact that different responses are given to different stimuli by the same or approximately the same germinal constitution. But large and conspicuous changes of this nature are not visible only as the result of artificial circumstances. Many species are normally subject to more or less definite and sudden changes in the surroundings, and some of them exhibit more or less definite responses to such changes. This is especially obvious in the case of sessile organisms the outward form of which is clearly modified by the surroundings. Thus 'the water ranunculus, when growing submerged beneath the surface of a pond, produces leaves the blades of which are cut up into a number of fine thread-like segments. As soon as the top of the plant reaches the surface of the water, those leafrudiments which are just commencing their existence, proceed to develop in a totally different fashion. The leaves to which they give rise possess a wide and unpointed blade, which floats

1 Vernon, loc. cit., p. 294.

2 Thomson, Animal Life, p. 391. In the case of the Porto Santo rabbit examined by Darwin the change in colour was found to be due to the environment. 3 Ibid., p. 383. 
upon the surface of the water. The two sets of leaves are as utterly different in their appearance as it is possible for leaves to be. Yet the effect of the external conditions upon the young leaf-rudiment determines which of the kinds is to appear.' 1

Examples may also be found in the life-history of free-living organisms. One is afforded by the common honey-bee. As is well known, a queen bee differs markedly from a worker bee in shape. Both queens and workers arise from fertilized eggs; whether a queen or a worker develops from any one egg appears to depend wholly on the environment-a larva that gives rise to a queen receiving in the first place different and presumably more nutritious food than that received by a larva giving rise to a worker, and, in the second place, inhabiting a cell which differs in size and shape from that inhabited by a worker larva. Another is afforded by the life-history of the plant lice (Aphids). At certain seasons of the year winged forms appear. It has long been suspected that the appearance of winged forms depends on some environmental stimulus. That this is so has been rendered practically certain by the work of Shinji, who has shown that aphids reared on plants watered with certain solutions are winged almost without exception. ${ }^{2}$

Among mammals the assumption of a winter coat by the lemming, ptarmigan, and variable hare is a similar phenomenon. Sir John Ross has told how a Hudson Bay lemming was protected from the cold on board his ship by keeping it in the cabin. It retained its normal summer coat during the winter. On exposing it in a cage on deck to a temperature of $30^{\circ}$ below zero, the fur on the cheeks and a patch on each shoulder became perfectly white during the first night. After another day's exposure 'the patches on each shoulder had extended considerably, and the posterior part of the body and the flanks had turned a dirty white. ... At the end of a week it was entirely white except in a dark band across the shoulders prolonged posteriorly down the middle of the back.' 3

A curious example of the importance of the environment before birth is afforded by the difference between the mule and the jennet. The former is the product of a cross between a stallion and a she-ass, the latter between a jackass and a mare. There

1 Lock, Recent Progress, p. 317. Biol. Bull., vol. xxxv.

2 Shinji, 'Wing Development in Aphids', ${ }^{3}$ Quoted by Vernon, loc. cit., p. 242. 
is every reason to believe that it is not the fact that horse-ancestry is in one case traced through the father and in the other case through the mother that gives rise to the difference; the difference can only. be attributed to the fact that in one case the period before birth is passed within a mother of one species and in the other case is passed within a mother of another species.

4. We are now in a position to discuss what is meant by the term 'normal environment'. There is a more or less definite range of variations of the environmental stimuli to which species in a state of nature are subject. The range may differ greatly from species to species, but remains more or less constant for any species. So long as the variations fall within this range, the environment may be described as normal. Such variations will be followed by different responses on the part of similar germinal constitutions, and the variations among the members of any species under a normal environment are. due to the combined influence of differences in the environment and of differences in the germinal constitutions. From time to time in a state of nature organisms are subject to variations of the environmental stimuli which fall outside the normal range, and there are thus produced extreme modifications similar to those which can be experimentally induced. Thus Gemmill on examining a large number of fish embryos found monsters with a single eye very similar to those experimentally produced by Stockard. ${ }^{1}$

It may next be observed that there is a marked difference in the degree to which sessile organisms on the one hand and freeliving organisms on the other hand respond to differences in the normal environment. The former are much more susceptible to differences-or at least to certain differences. Sessile organisms, for instance, differ from one another very greatly in form and such variations are known to be chiefly due to differences in the environmental stimuli. Free-living organisms do not vary in this manner. The reason is fairly clear. All species are adapted to a particular niche in nature, and among the former the mode of adaptation of necessity takes the form of susceptibility to surrounding conditions; a tree or a sponge must be able to adapt itself to its actual surroundings. A free-living organism is, on the other hand, adapted by its specific form to its niche in nature

1 Gemmill, Teratology of Fishes, p. 44. The cyclopia found was attributed by Gemmill only partly to environmental causes. 
and, in order that it may reach and maintain this form, it must exhibit a relative lack of susceptibility to surrounding conditions.

5. The broad features of the situation among species in a state of nature are now fairly clear. By outward inspection it cannot be ascertained whether any particular characteristic is of the nature of a modification or of a mutation. But we know that among sessile organisms outward form is largely of the nature of modification, whereas among free-living organisms, which interest us more closely because conditions are more nearly comparable to those obtaining among men, modifications play a much smaller part. A large number of measurements has been made of certain features of particular species in a state of nature as, for instance, by Allen for the squirrel and Weldon for the common shrimp. Though on general grounds we may have reason to suspect how far the variations recorded are of the nature of modifications, we can arrive at no certain answer. Observations made on members of a species in a state of nature, some of which are subject to conditions that differ from those to which the rest of the members are subject, are of more assistance for our present purpose. Thus the tiger ranges from tropical to temperate regions. Tigers from the former regions exhibit certain differences from those from the latter regions in respect of the condition of the coat. It is possible that these differences are purely environmental. Again certain marine molluses from cold waters exhibit differences when compared with members of the same species from warmer waters; these differences may again be purely envirommental. In those cases in which a species has within recent years spread to a new environment, it is often found the degree of variation has increased. Thus Bumpus found that the egg of the common sparrow is more variable in the United States than in England ; ${ }^{1}$ it has also been noticed that the variation of the common periwinkle is greater in America than in England-both these species having been recently introduced into America. ${ }^{2}$ It is also of interest to observe that Montgomery found greater variations among migratory than among nonmigratory species of birds and the greatest variation among those that had the widest range. ${ }^{3}$ Though, until the matter has been put to the test of experiment, nothing can be affirmed as to the

1 Vernon, loc. cit., p. 213.

${ }^{2}$ Ibid., p. 215 . Similarly the snail, Helix. aspersa, has a variety (tenuis) with a thin shell which is found where calcareous material is scarce.

${ }^{3}$ Vernon, loc. cit., p. 261. 
nature of these differences, it is probable that in part at least these differences are environmental. Such observations help to exhibit the degree of importance that we are led to attribute to modifications among free-living animals in a state of nature. It must also be borne in mind that departures from the normal range of variations of the environmental stimuli are not so very infrequent and that more or less extreme modifications, as result, for instance, from the attacks of parasites, do occur.

Lastly we may go a step farther and ask what happens when the environment changes. This inquiry, if pursued, would lead us beyond the scope of this chapter. A reference, however, to this problem may assist to render the relation of a species under natural conditions to its environment rather more clear. Change of environment may be due either to the migration of some or all the members of a species to a locality where the environment is different, or to an actual change in the environment in the same locality. If the change is at all marked, then there will be a different response on the part of the germinal constitution to the new conditions. Let us suppose that the diet is changed and that some members of a species of bird take to a diet of grain having previously existed on a diet of fish. There will be a different response in that the stomach will assume a different form. But it is most unlikely that the old germinal constitution will be that which gives the best response to the new conditions. Almost certainly a somewhat different type of germinal constitution will be that which will give the best response to the new conditions, will be that, in other words, from which there will arise the form of stomach most suited to dealing with grain. If and when, therefore, a mutation arises exhibiting this changed type of germinal constitution, it will be favoured, and in this manner a new variety and ultimately a new species may be formed. It has thus to be remembered that, although the germinal constitution to some extent responds differently to different stimuli, it is very unlikely that any average type of germinal constitution will give the best response to any other environment than that to which the species is now subject. It does not therefore follow that, because the responses of the germinal constitution are various, there are not factors making for a change in the germinal constitution when the environment changes.

Summing up what we have said, we have to think of every 
species as living under a more or less clearly defined environment into which very many elements enter; whether the variations in these elements are great or small, there is an average condition, and to that average condition a certain type of germinal constitution gives the best response. In the case of a free-living animal in the adult form the germinal constitution does not respond readily to ordinary variations from the normal, though it has to be remembered that extreme influences such as those caused by parasites may cause marked reactions. Further, it has always to be borne in mind that during development all animals and plants are particularly susceptible to environmental changes. It is not possible to make any precise statement as to how far the differences we see under natural conditions are modifications and how far mutations. All that we can say is that the part played by the environment in producing modifications is on the whole smaller in the case of free-living animals than in the case of sessile animals and of plants. 


\section{XV}

\section{THE INFLUENCE OF THE ENVIRONMENT UPON MAN}

1. The way has now been cleared for a consideration of the influence of the environment upon man. The relation of the ancestors of man to the environment must have been the same as that between any wild species and its environment. In the course of his history man has moved away from this position until his relation to the environment has become so different from that described for other animals and plants that we can no longer speak of a normal environment. This has not come about because man is not subject to the same laws as are other organisms. It has come about because his relation to the environment has been modified in many ways.

The conception of the normal environment involved the idea that, much as the different elements composing the environment might vary, there was some more or less clearly defined limit to their variations. In one sense this remains true for man; but the variations are so much greater in degree and in kind that there is a clear distinction between the conditions obtaining among civilized races and those obtaining among any wild species. Let us glance for a moment at what has happened. It is obvious that man has varied his surroundings in every respect, not only with regard to what we may call external circumstances, but also with regard to nutrition and to customs and habits that we sum up under the name of use. The most obvious changes in external circumstances are those connected with the spread of man to every corner of every continent. Man has become subject to every extreme of heat and cold, humidity and aridity, of barometric pressure and all that goes to make up olimate. There is in addition a vast mass of artificial influences, due in the first place to various methods of protection adopted against climate. Every description of house is known, involving all degrees of access to fresh air. Dress again varies almost infinitely - the variations all being of possible influence upon the development of mental and 
physical characters. The aggregation of men into towns involves exposure to smoke, noise, and vibration among other factors. Modern industrial conditions in particular expose workers to varied surroundings. Human diet has become equally varied. The cooking of food was an innovation involving great changes in the factors which play upon the digestive organs. Innumerable animals and plants have been drawn upon by man as food. Perhaps more important than the variations in what man eats are the variations in what he drinks. Under the heading of use come all those habits such as reading, washing, smoking, and shaving. Various occupations bring with them various degrees of muscular activity or involve its reduction to next to nothing. There are various modes of riding and various ways of sitting. Lastly the prevalence of disease has introduced another factor which has a profound influence upon mental and physical characters.

This varying of the environment has come about gradually, slowly at first and with increasing speed latterly, until at the present day, of four men having their homes in the same town one may do clerical work involving no exercise, another may labour in a cotton mill where it is warm and moist, a third may perform hard physical labour in a mine in semi-darkness where the air is full of a partioular kind of dust, and a fourth may work on board ship exposed to all the rigours of the Atlantic. To such differences may be added all the differences between meat-eaters and vegetarians, smokers and non-smokers, alcoholic drinkers and abstainers, and so on. Contrast the variations in the environment of modern man with the variations in the environment of any species in a state of nature and it will be apparent why it was said above that the relation of man to his environment was clearly distinguishable from that of any species in a state of nature to its environment.

We are about to consider in this chapter the influence of the environment upon the physical basis of life ; we shall be concerned, in other words, with its function as the complement of the germinal constitution. The discussion will be limited to the notice of such factors as are in operation; of the possible effects of the environment there is no need to speak. From two other points of view also the environment is of importance ; as a factor in selection it will be considered in Chapters XVII and XVIII; as the subject. 
matter, so to speak, upon which mental processes work, it will be considered in Chapters XIX and XX. It is important to distinguish the influence of the environment in this latter respect from the sense in which it is considered in this chapter. The failure to do so has vitiated many contributions to the subject. It is felt that the environment is somehow of great influence in social life and it is not realized that, if in the respect in which it is considered in this chapter we find reason to conclude that its influence is not great, there is another field in which its influence may be established.

When considering the influence of the environment on man, it is convenient to distinguish between physical and mental characters. Mental characters may be considered under the head of the intellect, the disposition, and the temperament. It must, however, be clearly understood that this is merely a distinction convenient for our present purpose which is not based upon, and does not imply, any fundamental distinction. Mental characters, such as the instinct of gregariousness, and physical characters, such as head form, are for our purpose merely characters resulting from the play of certain stimuli upon a certain germinal constitution.

2. It is remarkable how little on the whole is known with regard to the influence of the environment on man. For the most part we have to depend upon observations as distinguished from experiments. Though a few experiments have been made, some deliberately but others accidentally, we are for the most part in the position with regard to man that we should be with regard to other animals, had we only such observations to go on as those quoted respecting the size of marine molluscs, the variation in the coat of the tiger, and the changes in the sparrow and the periwinkle when introduced into America. Though such observations may strongly suggest certain conclusions as to the part played by the environment, no definite or precise results can be reached.

We may first consider some evidence bearing upon the influence of particular factors such as exercise and climate. We may disregard those rare cases of extreme modifications deliberately induced, such as the distortion of the shape of the head or of the feet by pressure. These extreme modifications approximate to mutilations such as amputation of the finger-joints or the knocking out of the incisor teeth. 
It is well known that exercise has considerable effect upon the development of the muscles. It is also well known that there is a clearly defined point for each individual beyond which exercise will produce no further effect. Though exercise has a considerable effect upon other órgans, especially certain internal organs, it is probable, not only that it has more effect upon the muscles than upon any other system of organs, but that the effect upon the muscles is relatively at least as great as, if not greater than, the effect upon any organ of any other factor that we shall consider except disease. Owing to differences in the amount of exercise the bodily development of man in a modern community varies very considerably; owing to such modifications clerical workers differ from blacksmiths, whether or not there are also germinal differences. So too owing to differences in habits men of one race differ from men of another race. Darwin, for instance, refers to the thin legs and thick arms of the Payagua Indians who spend a large part of their lives in canoes. ${ }^{1}$ It is certain that all such physical differences are not due to germinal differences and that environment in the shape of use is in part the cause. The position is similar with regard to certain peculiarities observable among races that have adopted unusual modes of squatting.

Little is known as to the effects of use upon mental characters. To some degree no doubt the intellect is developed by use, though, perhaps, the degree to which this is so is apt to be exaggerated in popular estimation owing to the fact that modes of thinking acquired through education add to the efficiency of the faculty, which result is mistaken for the effects of use. That the effects of exercise are not great is shown by the experience of the Workers' Education Association. This institution gives advanced courses to men often of middle age who have received but little intellectual training in early life. It does not appear that the strength of their intellects is much less than it would have been had they received a university training. It seems at any rate certain that the differences in the amount of use now obtaining between the various professions and classes in England have less effect generally upon mental than upon physical characters. Given, for example, two men of equal intellect, one of whom received the best educational training of the day, and the other of whom received the training given to the working classes, it does not

1 Darwin, Descent of Man, p. 33. 
seem that at the end of the training there would be anything like the same difference as regards strength of intellect between them as there would be between two men, one of whom received an athletic training and the other of whom did not.

3. Physical changes are not infrequently observed to follow upon changes in the environment, though, when these changes are complex, it is often impossible to say with which features of the environment the changes are connected. Thus ' the Anthropological Committee of the British Association long ago showed the beneficent effects of the Factory Acts, which rescued young children from the hardships of daily toil. Boys of nine years in 1873 had a height and weight equivalent to the height and weight of boys of ten years old in 1833.' 1

It is known that stature has increased in certain European countries during the last century. Soren Hansen gives the following figures for Denmark : ${ }^{2}$

\begin{tabular}{|c|c|c|c|c|}
\hline 1852 to 1856 & & $165 \cdot 42$ & $\mathrm{~cm}$. & era \\
\hline 1879,1888 & & $167 \cdot 78$ & ", & , \\
\hline 1891,1900 & & $168 \cdot 43$ & , & , \\
\hline 1904,1905 & & $169 \cdot 11$ & , & , \\
\hline
\end{tabular}

Similarly the stature of the Dutch has increased from $165.5 \mathrm{~cm}$. in 1866 to $167 \mathrm{~cm}$. in 1883 and to $168 \mathrm{~cm}$. in 1899 . A number of reasons has been suggested to account for this marked increase, the higher standard of living, the decrease in the incidence of disease which is known to inhibit growth, and the smaller number of children in a family. This last factor is certainly of importance at the present day. From some observations made in an English manufacturing town Ewart concludes that when children are born at a longer interval than two years they are on an average three inches taller and three pounds heavier than children born at a shorter interval. ${ }^{3}$ It is a fair assumption that in families, where the income is small, the fewer the children the more favourable would be the environmental conditions.

The physical inferiority of people living in poorly endowed surroundings is often thought to be at least in part due to modifications induced by hard conditions. Thus physical inferiority is noticeable in districts in Europe that are markedly poorly endowed, as for instance in the area between limoges and

, Mitchell, loc. cit., p. 47. 2 Hansen, 'Increase in Stature', p. 23, in Problems in Eugenics. 3 Ewart, 'The Influence of Parental Age on Offspring', Eugenics Review, vol. iii. 
Périgueux in France. So far as this is so, the causes may have to be sought in climatic differences as well as in other differences more directly comparable with those which exist between welland ill-treated children in the same country.

4. The influence of climate upon man has long attracted attention. Statistical evidence has been produced showing that the shape of the head-a characteristic usually considered not to be in any way susceptible to such differences in the environment as occur between one climate and another-is modified by climate. Boas produced figures showing that the cephalic index (a measure obtained by calculating the relation of breadth of the head to the length which is put at 100) of Sicilians born in America to be 80, while that of Sicilians born in Sicily is 78, and of Hebrews born in America to be 81, while that of Hebrews born in Eastern Europe, whence the immigrants came, is 83 . It would appear that the Hebrews who are broad-headed in Europe become narrowerheaded in America, and that the Sicilians who are narrow-headed in Europe become broader-headed in America. There would thus seem to be an approximation in America to a cephalic index the mean of which lies between 80 and $81 .^{1}$ These results have been severely criticized from many points of view. Sergi claims to have shown that the results are the "pure effect of illusion due to the statistical methods employed by the author'.$^{2}$ It has also been suggested that the results are due to selection. In this connexion it is interesting to note that both Ammon and Levi obtained somewhat similar results and that they both attributed them to selection. The former, working with figures from Baden, found that the inhabitants of cities tended to become longer-headed and concluded that the short-headed type tended to die out under the conditions of city life. ${ }^{3}$ Levi, working on Italian figures, came to the same conclusion and attributed it to the same cause. ${ }^{4}$ Others have suggested that the methods of nursing children may affect the shape of the head and that the changed habits of the immigrants in America may account for the changes in the shape of the head. Though it has certainly not been proved that the environment can in the manner suggested change the shape of the head, the question cannot be regarded as settled.

1 Boas, Changes in Immigrants, p. 5.

2 Sergi, 'Variation and Heredity', p. 18 (in Problems in Eugenics). Few anthropologists, it may be added, accept Boas's conclusions. $\quad{ }^{3}$ See Ammon, Natürliche Auslese beim Menschen and Zur Anthropologie der Badener.

${ }^{4}$ Levi, Anthropometria Militare. 
Observations have been made on the cephalic index of Jews which may perhaps be held to lend support to the views of Boas. Huntington gives the following table: ${ }^{1}$

\section{Cephalic Index of Cephalic Index of \\ other Races.}

Country.

$\begin{array}{lccc}\text { Caucasus . } & . & . & 87.5 \\ \text { Galicia } & . & . & 83 \cdot 6 \\ \text { Baden . } & . & . & 83 \cdot 5 \\ \text { Little Russia } & . & . & 82 \cdot 9 \\ \text { Turin . . } & . & . & 82.4 \\ \text { Lithuania . } & . & . & 81 \cdot 7 \\ \text { Russian Poland } & . & . & 81.9 \\ \text { White Russia } & . & . & 80.9\end{array}$

$87 \cdot 4$

$84 \cdot 4$

$81 \cdot 1$

$83 \cdot 2$

$84 \cdot 9$

$80 \cdot 6$

$80 \cdot 9$

$82 \cdot 5$

\section{Difference}

$0 \cdot 1$

$0 \cdot 8$

$0 \cdot 6$

$0 \cdot 3$

$2 \cdot 5$

$1 \cdot 1$

$1 \cdot 0$

$1 \cdot 6$

It would appear that there is a tendency for the cephalic index of Jews to vary as does that of the surrounding types. Fishberg has attributed this fact to intermarriage ${ }^{2}$ and this may be in part at least the explanation, though in the present state of our knowledge the possible influence of the environment cannot be altogether excluded.

The uncertainty surrounding the matter is in fact a good example of the state of our knowledge regarding many similar problems affecting man. ${ }^{3}$ Boas himself, it may be noticed, only believes in a 'strictly limited plasticity' 4 of head-form; it is indeed evident that this and other physical characters which distinguish the races of man are for the most part of the nature of mutations and not of modifications. Europeans who migrate to tropical climates and the inhabitants of tropical climates who come to live in Europe retain the greater part of their distinguishing physical characters.

The manner and degree in which tropical climates influence Europeans is a matter of considerable interest. There is as yet but little known on the subject. Sir Patrick Manson, writing in 1907, said that 'although many attempts have been made to trace and explain the effect of temperature on the physiological processes of the human body, more especially in reference to the pathological proclivities to which atmospheric heat and cold may

1 Huntington, World Power and Evolution, p. 173. Am. Anth. Ass., vol. i, 1905.

2 Fishberg, Mem.

systrom has elaborated a theory according to which the habits and customs of races profoundly affect the shape of the head, but it cannot be said that he brings forward any convincing evidence ('Utber die Formenveränderungen des menschlichen Schädels', Arch. für Anth., Bd. 27).

- Boas, loc. cit., p. 76. 
conduce, it cannot be said that any important conclusions have been attained.... But though we may not be able to indicate precisely the way in which our bodies are physiologically affected by extremes of atmospheric temperature, especially prolonged high temperature, our sensations, the loss of physical and mental energy, the modification of physical characteristics undergone by white races when placed for several generations in tropical conditions, and the dark skins of all tropical races indicate that the white races on first arrival are not in all respects adapted for tropical conditions, that they are somehow prejudicially affected thereby, and that while living in tropical countries they are more open to certain pathological risks than are the natives of those countries.' 1 Since the year in which Sir Patrick Manson wrote further information has been obtained on this subject. Our knowledge is, however, still scanty. With regard to actual facts it is known that the pulse, rate of breathing, and temperature of the body do not vary when measured in Europe and when measured among white men under tropical conditions. The number of red blood-corpuscles and the amount of haemoglobin in the blood is the same and metabolism is not less intense. On the other hand the rate by which a nervous impulse travels along a nerve decreases in Europeans the longer they live in the tropics. The muscles and the connective tissue become more elastic. The well-known pallor of Europeans living in the tropics is due to a thickening and softening of the epidermis, which becomes opaque. ${ }^{2}$

It is very difficult to arrive at any conclusions as to what the effects of a tropical climate upon Europeans really are. It is necessary to discount the effect of tropical diseases and of the habits and customs of Europeans living in the tropics. On the whole it is probable that the popular notion of the considerable and generally injurious nature of the modifications undergone by Europeans living in the tropics is exaggerated. It is said that, for example, in Java, when sanitary conditions are good and reasonable habits adopted, the death-rate among European children is less than in Europe. Nevertheless a tropical climate does have an injurious effect upon Europeans. There is no doubt that Europeans in the tropics are more irritable and in general

1 System of Medicine, edited by Allbutt and Rolleston, vol. ii, part ii, p. 2. 2 See Kohlbrugge, 'Influence of a Tropical Climate on Europeans', Eug. Rev., vol. iii. 
more highly strung than in their native land. Clearly in some fashion the nervous tone is injuriously affected by residence in tropical climates. In a similar manner nervous tone is affected by many elements in the environment in civilized countries, such as vibration, noise, and so on, leaving aside the effect of food, drink, and disease. To the importance of disease in this respect we shall return later. As regards noise, vibration, and so on, but little is known-their influence being possibly considerably greater than is usually suspected.

5. Ellsworth Huntingdon has in recent years in a number of publications elaborated a theory according to which climate has been one of the main factors in determining where civilization shall develop and flourish. As this theory depends upon the supposed direct influence of the environment on man, it may be noticed here. He has made observations which are interpreted as showing that there are optimum climatic conditions under which the maximum energy is exhibited. These conditions arise when the average temperature of day and night together lies between $58^{\circ} \mathrm{F}$. and $71^{\circ} \mathrm{F}$. and when there is a certain degree of moisture. His observations, made in America, were based on the output in piece-work factories and on similar data and tend to show that not only do all European races, including the Finns, display most energy under these optimum conditions, but also the Japanese and the negroes. He then proceeds to show that where these optimum conditions prevail in the world, there to-day are to be found the highest forms of civilization. Upon these data he raises a very far-reaching theory to the effect that throughout history civilization has arisen and flourished only where there has been an approximation to these climatic con. ditions. To the obvious difficulty that former civilizations have often flourished in countries the climate of which at the present day is far from these optimum conditions, he replies that climate has changed, a theory which he has for some years strongly advocated. 1

It may be said that there is nothing novel in the idea of optimum climatic conditions. ${ }^{2}$ In the previous section it was pointed out that Europeans in tropical climates suffer from

1 Huntingdon, Civilization and Climate. See also the same author's 'Climate and Evolution of Civilization ', in Evolution of the Earth and its Inhabitants, edited by Lull. $\quad 2$ Thus Montesquieu remarked upon the fact that greatest vigour is found in the colder climates (Esprit des Lois, Part III, Bk. XIV, ch. î). 
injurious mental disturbances. What is remarkable is that the optimum conditions for negroes should be the same as for Europeans. It will require more proof than has yet been advanced before this can be accepted. Further, the changes in climate which the theory demands have not been proved. Professor Gregory has reviewed the question and his conclusions do not support those of Huntingdon-at any rate not in such a manner as to render the theory tenable. ${ }^{1}$ Nevertheless, whatever the fate of the theory in its present form may be, its enunciation has raised many interesting questions and has incidentally helped to show how little we know at present regarding the effect of the surroundings on man.

6. When men move from one climate to another they come under the influence not only of changes in temperature and moisture but also of food and sometimes of altitude and other factors about the effects of which there is a considerable amount of information. A vegetarian diet is said to produce changes in the gut; but changes in beverages are probably of far greater importance than changes in food. The effects of alcohol have been closely studied, chiefly with regard to its influence upon nervous tone. Nervous tone is affected in an important manner by many drugs, as for instance by opium, and in a lesser degree by tea and coffee. Changes in nervous tone are of such importance that its susceptibility to various influences has to be borne in mind. It is quite possible that the introduction of a new form of beverage into a country might have a perceptible effect upon the average condition of nervous tone and thus have a bearing upon the course of history.

Altitude is known to have various effects upon physical characters. The fact that the larger lung capacity of those who live at high altitudes diminishes on descent to the plains, as recorded by Darwin of the Quicha Indians, is evidence that this character is in part at least environmental. 2 The effect of high altitudes has lately been studied in much detail. It is known that there is among other changes an increase in the number of red blood-corpuscles in the blood. ${ }^{3}$

1 Gregory, Geog. Journ., vol. xliii. There have been many studies of the influence of climate upon temperament. See, for instance, Dexter, Weather Influences. 2 Darwin, Descent of Man, p. 35. 3 Acton and Harvey, 'Increase in the Number of Erythrocytes', Biometrika, vol. viii. See also the results of the Monte Rosa and Pike's Peaks Expeditions, Phil. Trans., vols. cciii and ccvi. 
7. Attempts have been made to obtain more precise information by the use of statistical methods. Thus the correlations have been measured between the state of children's eyesight and fifteen environmental conditions. The mean of the correlations was found to be 0.04 - only one reaching $0 \cdot 1.1$ Again the association between various intellectual and physical characters and conditions, which were taken as representing a good or bad environment, has been measured. A slight association was found between intelligence in boys and few people per room and no association between eyesight, condition of glands and hearing, and bad economic and moral surroundings. ${ }^{2}$ Somewhat different results have been reached by American workers, who find that by employing psychological tests a fairly well-marked difference can be detected between children in the same school whose parents belong to different social classes and who would therefore be subject to different home conditions. ${ }^{3}$

The interpretation of these results is difficult. Before any definite conclusion could be reached with regard to any one character, it would be necessary to measure the effect of every factor in the environment upon that character. As the matter stands there is strong but not conclusive evidence of the small influence of the surroundings, so long as we suppose that innate differences do not exist between the subjects measured. But if innate differences exist, then the interpretation of the absence of any marked degree of correlation must be that the common elements of the environment supplied by the community are of greater importance than the innate differences. We shall find reason to conclude in later chapters that small innate differences do exist, and, if this is so, then the interpretation of these results is not at variance with the general, though necessarily vague, conclusions derived from the evidence previously given, i.e. that the influence of the environment as represented by the variations actually occurring in the elements of the surroundings hitherto mentioned is small.

8. There is another class of factors which may be summed up under the heading of disease. It was remarked that disease may produce notable results among species in a state of nature.

1 Barrington and Pearson, Eug. Lab. Mem., No. 5, $1909 . \quad 2$ The results of these investigations have been summed up by Miss Elderton in a pamphlet entitled The Relative Strength of Nature and Nurture.

3 See, for instance, Bridges and Coles, 'Relation of Intelligence to Social Status', Psychological Review, vol. xxiv. 
Compared, however, with the state of things among men, and especially in the later stages of history, disease is rare among such species. Among men it comes to assume a peculiar importance.

Disease results both from the attacks of parasites and from other causes. The various classes of disease will be referred to in the next chapter. We are here concerned only with the results, and we may think of the disease as affecting particular organs in the body and as affecting the general functioning of the organs. Every organ in the body is liable to be attacked by disease and the modifications produced are in many cases familiar. Thus all physical and mental characters may be directly and to almost any degree modified by disease. Again it is known that the result of disease in children is to inhibit growth, and that the growth thus lost is not subsequently made up. Disease may thus be said to draw upon the capital and not upon the income of children. ${ }^{1}$ We may here confine ourselves to some notice of the effect of disease on the general functioning of the bodily organs which though not so familiar is more important from our point of view.

The functioning of the bodily organs has been found in late years largely to depend upon certain glands-known as the endocrinous or ductless glands-in a manner and to a degree altogether unsuspected. The thyroid gland, for example, manufactures a secretion which is essential to the proper growth and normal metabolic functions of the whole body. If it is removed from a child, the whole body is stunted and mental deficiency results. Certain maladies, such as goitre, cretinism, and others are known to be connected with a diseased condition of the thyroid. Profound modifications of both physical and mental characters may thus follow when these glands cease to function normally, which is known to be the case in certain specific diseases and may arise in other ways which are not fully understood.

Temperament depends upon the general functioning of the bodily organs and upon the actual condition of the nervous system. Disease, whether it takes the form of a failure of the ductless glands to function as they should, or some other form, always affects the general functioning of the body and thus has a direct bearing upon temperament. Thus 'we know now',

1 Galton, Inquiries into Human Faculty, p. 236. 
says Mr. McDougall, 'that defect of the functions of this organ (thyroid) may reduce any one of us to a state of mental apathy bordering upon idiocy, and that its excessive activity produces the opposite effect and may throw the mind into an over-excitable condition verging upon maniacal excitement. Again we know that certain diseases tend to produce specific changes of temperament, that phthisis often gives it a bright and hopeful turn, diabetes a dissatisfied and cantankerous turn. It is clear that in some such cases of profound alteration of temperament by bodily disorder the effects are produced by means of the chemical products of metabolism, which, boing thrown out of the diseased tissues into the blood and reaching the nervous system by way of the blood-stream, chemically modify its processes. It is probable that every organ in the body exerts in this way some influence upon our mental life, and that temperament is in large measure the balance or resultant of all these many contributory influences.' 1

Thus in addition to the more obvious direct effects of disease upon mental and physical characters, there are the more subtle and profound effects upon temperament, and in our general summing-up we shall find reason to attribute no small importance to temperament in its influence upon progress. It thus becomes of importance to observe that in particular regions of the world certain chronic and non-lethal diseases are very common. Clearly the result of disease upon temperament is greatest when it takes the form, not of kill or cure-which is on the whole the case in temperate climates-but of chronic non-lethal maladies which are common in tropical climates, especially in Africa. The hookworm disease is an African disease which has been introduced into America. The hook-worm (Ancylostomum duodenale) is an internal parasite which attaches itself to the lining of the intestine and causes bleeding and anaemia. Death may ensue, but the patient usually lives a long time. It is a most debilitating disease ; it is said to affect some 50 per cent. of the inhabitants of some tropical and sub-tropical countries. Even as a mild infection it is the cause of much invalidity and heavy economic loss. The laziness and degeneracy of the 'poor white trash', who are said to belong to the purest-blooded English stock in the United States, are due not so much to the environment ${ }^{2}$ and heredity

1 McDougall, Social Psychology, p. 118. social or traditional environment.

2 Here environment clearly means 
as to the ravages of these parasites. In a public lecture, delivered before the University of Pennsylvania, in 1915, Allan Smith, citing the factors which have caused the Southern States to lag in American progress, mentioned that the hook-worm disease 'stands with malaria as worse than wars and the devastations of battles and worse than all other pathogenic agencies in combination'. Through the influence of these diseases ' the men and women of the South, bred from the best colonial stock, offspring of pioneers, with the blood of English gentry and of continental cavaliers in their veins, sank lower and lower in physical degeneration and squalor, were derided and denounced as lazy and shiftless and condemned in popular opinion as worthless and a disgrace '. 1

There are many other tropical and sub-tropical diseases of a somewhat similar nature. Among them may be mentioned the 'yaws'-marked by various eruptions probably due to a spirochaete-the guinea-worm, which lives buried in connective tissue; bilharzia, a trematode worm which lives in the bladder and infects nearly 50 per cent. of the inhabitants of Egypt ; and elephantiasis, due to a worm related to the guineaworm. The manner and degree in which these various diseases affect the nervous tone varies very much. In general the result is debility and a lowering of the nervous tone. So too malaria is a cause of debility, and the main reason for the decadence of Ancient Greece has been sought in this disease. ${ }^{2}$

9. The results of the inquiry so far, though illuminating, are indefinite. This is largely because we are ignorant regarding innate differences. The consideration of the evidence as to identical twins offers a way out of this difficulty. It is known that there are two kinds of twins. There are the so-called identical twins, between whom there is a very close resemblance, and other twins between whom there is no greater resemblance than between any two children of the same parents. It was formerly thought that there was a sharp distinction between the two kinds of twins-identical twins arising from a single ovum, which completely separated into two halves during early development, each half giving rise to one child, ordinary twins arising from the simultaneous fertilization of two ova. If this was so, then identical twins would always have approximately the same

1 Loiper, 'Some Inhabitants of Man',p. 151 (in Animal Life and Human Progress, edited by Dendy).

See Jones, Malaria and Greek History. 
germinal constitution, whereas other twins would have germinal constitutions as different as on the average are those of the offspring of the same parents. That identical twins do thus at times arise is practically certain, but it has recently been shown that the supposed sharp distinction between identical and other twins does not exist. 1 This may be explained by various assumptions. It may be supposed that some ova are binucleate or that some ova are penetrated by two spermatozoa; again the earlier or later separation of the blastomeres may have a bearing upon the position. Under any of these circumstances twins intermediate between identical twins and ordinary twins would arise-twins, that is to say, not having approximately similar germinal constitutions, but germinal constitutions more nearly alike than those of ordinary twins. However this may be, it is sufficient for our purpose to note that so-called identical twins are always more alike in their germinal constitutions than are ordinary twins and may quite frequently have practically similar germinal constitutions. It, therefore, follows that differences between identical twins must be very largely of the nature of modifications. It is thus of great interest to ask in what these differences consist.

Galton collected data regarding thirty-five cases of identical twins. He summarizes the information about them up to the time at which they left the family circle as follows. 'In a few of these not a single point of difference could be specified. In the remainder, the colour of the hair and eyes were almost always identical ; the height, weight, and strength were nearly always so. Nevertheless I have a few cases of a notable difference in height, weight, and strength, although the resemblance was otherwise very near. The manner and personal address of the thirty-five pairs of twins are usually described as very similar, but are accompanied by slight differences of expression, familiar to near relatives though unperceived by strangers. The intonation of the voice when speaking is commonly the same, but it frequently happens that the twins sing in different keys.' 2 He goes on to say that 'both twins are apt to sicken at the same time in no less than nine out of the thirty-five cases. Either the illnesses,

1 See Fisher, 'Genesis of Twins', Genetics, vol. iv.

2 Galton, Inquiries into Human Faculty, p. 219 . Curiously enough there was no similarity to be detected in handwriting - an interesting commentary on the value to be attributed to deductions drawn from handwriting as to character. 
to which I refer, were non-contagious, or, if contagious, the twins caught them simultaneously; they did not catch them the one from the other. This implies so intimate a constitutional resemblance, that it is proper to give some quotations in evidence,' ${ }^{1}$ and he proceeds to give detailed evidence. Later he remarks on the "similarity in the association of ideas. No less than 11 out of the 35 cases testify to this. They make the same remarks on the same occasions, begin singing the same song at the same moment and so on.' 2 With regard to tastes and dispositions Galton says that ' in 16 cases-that is in nearly onehalf of them-these were described as closely similar; in the remaining 19 they were much alike, but subject to certain named differences. These differences belonged almost wholly to such groups of qualities as these : the one was more vigorous, fearless, energetic, the other was gentle, clinging, and timid; or the one was more ardent, the other more calm and placid; or again the one was the more independent, original, and self-contained; the other the more generous, hearty, and vivacious. In short the difference was that of intensity or energy in one or other of its protean forms ; it did not extend more deeply into the structures of the characters. The more vivacious might be subdued by ill health, until he assumed the character of the other; or the latter might be raised by excellent health to that of the former. The difference was in the key-note, not in the melody.' 3 Galton sums up the evidence as follows: 'It follows from what has been said concerning the similar dispositions of the twins, the similarity in the association of their ideas, of their special ailments, and of their illnesses generally, that the resemblances are not superficial, but extremely intimate. I have only two cases of a strong bodily resemblance being accompanied by mental diversity, and one case only of the converse kind.' 4

It has to be remembered that up to this period the twins had been reared under very similar conditions indeed; Galton in fact says that they had been reared 'exactly alike'. 5 He goes on to ask what changes were produced when they left the family and went out into the world. He sums up the result of his inquiries into this point as follows: "Here are 35 cases of twins who were "closely alike" in body and mind when they were
1 Galton, loc. cit,. p. 226.
Inbid., p. 233.
2 Ibid., p. 231.
3 Ibid.
4 Ibid., p. 232. 
young, and who have been reared exactly alike up to their early manhood and womanhood. Since then the condition of their lives has changed; what change of nurture has produced the most variation? . . . They (the 35 cases) showed me that in some cases the resemblance of body and mind had continued unaltered up to old age, notwithstanding very different conditions of life; they showed me that in other cases the parents ascribed such dissimilarity as there was, wholly or almost wholly to some form of illness.' 1 'We may, therefore, broadly conclude that the only circumstance, within the range of those by which persons of similar conditions of life are affected, that is capable of producing a marked effect on the character of adults, is illness or some accident which causes physical infirmity.' 2 Galton then turns to consider the details regarding twenty cases of unlike twins and he finds that in spite of similar surroundings no growing resemblance can be traced. ${ }^{3}$

10. In attempting to sum up our conclusions on this subject, we may first ask what influence is to be attributed to the environment and then ask what bearing such influence has upon the main problem under review in these later chapters. It has always to be recollected that changes in the environment may have the most extreme results. Some examples were given of experiments upon developing animals, and it was shown that in the case of fish, for example, monsters of various kinds can be produced. So too doubtless extreme modifications could be produced in the case of man and are occasionally produced by untoward surroundings and by such customs as those of the distortion of the head and of the feet. What we want to know, however, is not what modifications can be produced, nor what exceptional modifications sometimes arise, but what changes are induced by the variations in the environment which usually occur.

The answer is that, putting aside disease among the factors and leaving out for the moment temperament among the characters, such variations as occur are of little importance. This applies to both physical and mental predispositions and includes the effect of not only such factors as climate and so on but also of such factors as are summed up in a good or bad home environment.

1 Galton, Inquiries into Human Faculty, p. 233. p. 237. The general results of Galton's work have been confirmed in all important respects by Thorndike's elaborate investigations ('Measurements of 'Twins' Archives of Philosophy, Psychology, and Scientific Methods, vol. i, 1905), 
It is probable that physical predispositions are on the whole more susceptible to such changes as occur than are mental predispositions. There is no reason, for instance, to think that the normal variation of any factor which influences mental predispositions produces as much effect as does use upon muscular development. But it is also further clear that, though we must, keeping aside for the moment questions connected with disease and with temperament, think of such modifications as occur as not of much importance, nevertheless these modifications are of greater importance than among species in a state of nature closely related to man. Consider for a moment muscular development. Side by side in the same street may live a man whose daily work is wholly sedentary and whose muscles are in consequence undeveloped, and a man who works in a mine or engineering shop and develops his muscles accordingly. Nowhere among vertebrate animals in a state of nature are such differences found.

Though but little is definitely known on the subject, it seems that temperament is more susceptible to changes in the environment than are other mental or physical characters. Changes of climate, for example, appear to produce more marked changes in temperament than in any other character. Temperament is certainly very susceptible to the influence of disease, which may also profoundly affect all characters both mental and physical. It is probably in its effects upon temperament that disease has its chief importance. We may even have to recognize in the effects produced by certain tropical diseases a serious hindrance to progress in tropical countries. It is difficult, however, to disentangle from the effects of disease in this sense the effects which it produces in the sense awaiting consideration in later chapters. Clearly the wide prevalence of disease, where such is the case, must form an important factor in the surroundings which the mind has for the subject-matter of its activitiesbut on this and on allied subjects there will be more to say later.

We have already in what has just been said referred to one kind of modification in its effect upon progress. Upon this subject in general it may in the first place again be emphasized that, so far as is at present known, there are no grounds for believing that modifications of the kind considered in this chapter give rise to mutations. From this it follows that the results 
of modifications are not cumulative. Unless in every successive generation the modification is induced, it will not reappear. Secondly these modifications on the whole tend, as in the case of the effect of disease upon temperament, to be connected with a condition of things that acts as a drag upon progress rather than as a spur to progress. The reason for this is clear in the light of what has been said in the last chapter. It was there shown that the germinal constitution among species in a state of nature has come to be through the action of selection of such a kind that under certain stimuli it gives rise to a certain form. This form is that which is best adapted to meet the normal features which characterize the niche in the organic world which the species occupies. Large modifications only become apparent when the species or certain members of it are no longer subject to their normal environment. To meet the new surroundings a somewhat different germinal constitution would give a better response. In other words the most satisfactory conditions are those under which considerable modifications do not arise.

Lastly we may again refer to a point that has already been alluded to. Great stress has been laid by certain authors, for instance by Professor Ridgeway, upon the influence of the environment upon man. Ridgeway in one passage speaks as follows : 'My argument was, and is, that as the ice sheet receded, man passed upwards from the south or south-east into Europe, and settled in the three southern peninsulas, gradually spreading northwards over the Alps and extending eventually up to the Baltic. As they gradually spread upwards, under the influence of the environment (and in the environment I, of course, include food), they grew less dark, those of them who settled permanently along the axis of the Alps tending to have shorter skulls, while those who had passed upwards earliest became the most blonde and tallest people in the world.' 1 It is clear from other passages that Ridgeway is including two very different things under the phrase-the influence of the environment. He is thinking of the production of modifications such as have been studied here, and also of the elimination of certain types under the influence of the environment. He does not make it as clear, as is perhaps desirable, to which of these two factors he attributes most importance. But what is more relevant to our argument is that to include

' Ridgeway, J. A. I., vol. xl, p. 13. 
the results of selection under the heading of the influence of the environment is somewhat misleading. Selection is a wholly different matter and, unless it is carefully distinguished from other factors, confusion of thought results. The effects which follow from elimination owing to climate and food should not be summed up under the heading of the influence of the environment; in this book they are considered in Chapters XVII and XVIII. 


\section{XVI \\ HEREDITY IN MAN}

1. Having touched upon the influence of the environment in producing modifications, we have now to approach the subject of the selection of characters, both mental and physical. It is proposed to consider in this chapter what it is that is inherited, and in the two following chapters to consider the results of selection.

What is meant by saying that any character is inherited is clear from former chapters. There are certain predispositions in the germinal constitution of every individual-predispositions to give rise to certain characters under certain conditions and to other characters under other conditions. There is a predisposition, for instance, in the species of primrose referred to in Chapter XIV to give rise to red flowers at a certain temperature and to white flowers at a different temperature, the colour of the flower being equally inherited in both cases. We may therefore take as our starting-point the fact that there are in the constitution of every individual many such predispositions.

From the results of breeding experiments upon Mendelian lines certain conclusions have been reached which carry us farther. These experiments have shown that certain unit-characters can be isolated which behave in a certain manner when crossed, and from the manner in which they behave it is deduced that they depend upon unit-factors in the germinal constitution which are invariable. The number of unit-characters which have been detected in any one species is not large; it is true that in one species of insect about two hundred unit-factors have been found, but even this number can only form a small proportion of the total number if it is the case that the germinal constitution consists entirely of unit-factors which on crossing give the Mendelian ratio. This is the working hypothesis which is put forward as the explanation of inheritance, and which it is the object of investigators now to test. We may consider for a moment what it is that this hypothesis implies. 
We have to think of the germinal constitution as containing a very large number of factors-predispositions in other words. The characters to which these factors give rise may be the characters which we see when we examine any individual, but more often they are not. There are, for instance, cases in which the colour of a flower or of the coat of a mammal are unit-characters but more often such visible characters are the product of two or more unit-factors. There are many cases now known in which the visible character is in this fashion due to the presence of several unit-factors. With regard to this hypothesis in general all that can be said is that at present no facts are known which are definitely in contradiction to it. It is the only hypothesis which holds the field. ${ }^{1}$

Up to the present very few unit-factors have been distinguished in man. It has been found that brachydactyly-a peculiar malformation of the hand-presenile cataract, tylosis-a thickening of the hands and of the soles of the feet-epidermolysis bullosa-a blistering of the skin-and night blindness behave as simple Mendelian characters. So too does eye colour, pigment in front of the iris being dominant to its absence-in other words, brown, green, and hazel being dominant to pure grey and blue. There is also some reason for thinking that musical ability is a recessive character. On the hypothesis outlined above it must be supposed that most of the visible characters of man are the product of several unit-factors which have not yet been identified. What appears to happen when matings take place between people of different colour, stature, and so on is that there is a blending of characters in the offspring. Such appearances are not incompatible with the hypothesis-the assumption being that many unit-characters are concerned.

This question as to the ultimate nature of inheritance has been introduced here because of its inherent interest. It is not as a matter of fact strictly relevant. What we require to know is rather what predispositions are present in the germinal constitution. It is this which is essential ; the precise manner in which the predispositions are represented in the gametes, whether by

1 The 'Ancestral Law of Heredity', which attributes on the average half the germinal constitution to the parents, a quarter to the grandparents, an eighth to the great-grandparents, and so on, is not incompatible with Mendelian inheritance as a general statistical result. The conception of the nature of inheritance involved in this theory is, however, incompatible with the Mendelian conception of unit-characters, which conception, it may be said, is the only one which fits the facts. 
unit-factors or not, is not essential to the present inquiry. We require to be able to give some answer to such questions as whether disease, intellect, and temperament are represented in the germinal constitution or not, and we can find an answer to these questions without concerning ourselves with the ultimate nature of inheritance.

2. Since studies of inheritance on Mendelian lines do not at present enable us to say what human characters are inherited, we are dependent for our knowledge of this subject chiefly upon biometry. The method pursued by biometricians is as follows. The particulars with respect to any character-say stature-are noted both for the parents and for the children. The average degree of resemblance between parents and children can thus be measured and expressed numerically. If the resemblance was complete, if, that is to say, in respect of any character children exactly resembled their parents, the fact would be expressed by saying that the correlation was equal to unity. When the resemblance is less, the fact is expressed by representing the degree of resemblance as a fraction. It must be emphasized that this method merely measures the average degree of resemblance; it does not without further inquiry tell us how far that resemblance is due to inheritince. So far as such degrees of resemblance are found, it is quite possible, supposing that we had no further knowledge of the subject at all, that they might arise as follows. It might be that all men had the same germinal constitution, and that the degree of resemblance was due to the fact that fathers and their children were brought up under more or less similar surroundings. Though this fact should be borne in mind, such an explanation of the resemblances found in the cases that will be quoted in what follows is as a matter of fact shut out, and we may accept the correlations as a measure of the degree of likeness due to inheritance.

The correlations between parents and children and between children of the same parents have been determined in respect of many characters-both mental and physical-and found to be about $0 \cdot 5$. Thus, in respect of height the correlation between father and son was found to be $0 \cdot 514$, between father and daughter $0 \cdot 510$, between mother and son $0 \cdot 494$, between mother and daughter 0.507 , between brother and brother 0.511 , between sister and sister $0 \cdot 537$, between brother and sister 0.553 . So too the average parental 
correlation in respect to eye colour is 0.495 , and the average fraternal correlation is $\mathbf{0 . 4 7 5}$. With regard to ability the fraternal correlation is $0 \cdot 46$, the correlation between sisters 0.47 , and that between brother and sister $0 \cdot 44$.

All that has been said with regard to the nature of fertilization and development lead to a similar conclusion. In the processes of maturation and fertilization we can detect a mechanism whereby the bearers of inherited qualities are transmitted from parent to child. In development we see how, when the appropriate stimulus plays upon the fertilized egg, an adult member of the species grows up. It is only when we suppose that within the fertilized egg there are certain predispositions in respect of every character which are derived from the parents that we can in any way understand how adult individuals come into being. ${ }^{1}$ And it must be emphasized that, however much opinions may differ regarding the precise nature of the mechanism of inheritance, there is no difference regarding the fact of inheritance. All biologists are agreed on this subject up to a point; the matters in debate are not strictly relevant here.

3. There are certain points, however, which, in view of the form which the discussion in the following chapters will take, require further treatment. As regards physical characters, it is sufficient to think of all such characters whether great or small as inherited. But with regard to disease it may be as well to consider further to what degree it can be said to be inherited.

What we call disease falls under two headings: disease due to the attacks of parasites and disease due to structural defects or weaknesses. Parasitic infections are of post-conceptional acquirement; they may be acquired, it is true, before birth, but such an acquirement is strictly analogous to the 'catching' of a disease after birth. In the sense, therefore, that parasites are not transmitted from the parent to the ovum, as the germinal constitution is transmitted, disease is never inherited.

It has been shown, however, that in the case of certain diseases, such as tuberculosis, a definite susceptibility to contract the disease is inherited. In other words, given an equal exposure to infection, some men do not 'catch' the disease, some only

1. The fact that the germinal constitution of any one individual belonging to a species, in which biparental reproduction is taking place, differs almost always from that of any other member of the species is due on the Mendelian hypothesis to the chance mixture of factors in the zygote. 
experience a mild form, and others die rapidly of it. It is probable that there are innately given degrees of susceptibility to all diseases which are caused by the invasion of the body by small, chiefly unicellular, parasites, which produce their harmful effects by the secretion of toxins. The same does not apply to the diseases caused by the attacks of the larger multicellular parasites, such as tapeworms, which, at any rate to a large extent, produce their harmful effects directly by causing lesions of the tissues. As to how far we have to think of different kinds of susceptibility to different diseases, or how far diseases go in classes, so that susceptibility to one disease goes with susceptibility to another disease and vice versa, little is known, but it is probable that in some cases at least there is a linkage of susceptibilities, that, for instance, susceptibility to scarlet fever is to some extent linked with susceptibility to measles. We must suppose that there are structural peculiarities which underlie these different susceptibilities. Though various hypotheses have been put forward, we are still ignorant as to their nature. But whatever the exact nature of these structural differences, it is clear that they are inherited like any other physical character.

We have therefore to note that. (a) susceptibility to specific infections is inherited. If gross anomalies, such as polydactyly and syndactyly (malformations of the hand) are to be accounted as diseases, then we may note that $(b)$ gross anomalies are inherited. (c) Minor anomalies are also inherited. Under this head come haemophilia, due to some peculiarity in the blood-vessels, which results in their not contracting as they should and to the absence of coagulating power in the blood, albuminuria, due to some defect in the filtering apparatus in the kidney, albinism, myopia, icthyosis, and others, all of which are clearly due to structural peculiarities. Further, we may note that $(d)$ ' other conditions due, it would seem, to disturbances of metabolism, underlying which may very possibly be finer anatomical variations, have for long been noted as tending to be inherited; such as obesity, diabetes, gout, and chronic rheumatism ' 1 Lastly (e) certain nervous diseases are inherited. We may distinguish two classes of nervous disease-the homeomorphic and the heteromorphic. 'In the former the offspring show the same lesions and

1 Adami, 'Inheritance and Disease', p. 26 (in A System of Medicine, edited by Osler and McCrae). 
symptoms as the parents. These are cases more particularly of lack of development or of premature atrophy of certain groups of nerve cells.' 1 In the latter the parent may suffer from one kind of disease, while the offspring may exhibit one or more of a group of other diseases. This is attributed to a lack of development of the highest nerve-centres as a whole. There is a lack of " perfect stability and co-ordination of various parts so that according to the strains to which the individual members of family are subjected, now one, now the other series of centres may show itself unable to respond adequately, and one or the other form of mental disturbance and nervous disease may result. Here are to be included the condition of insanity, familial epilepsy and the neuroses.' 2 In other words, we sometimes find stocks in which there is a nervous weakness, which may manifest itself in very various ways, including hysteria, epilepsy, inability to control impulse, delusion, and so on. Alcoholism is sometimes spoken of as though it was to be regarded as an irresistible impulse to drink. Probably we should rather imagine that a condition of general nervous weakness may at times manifest itself in the form of a loss of control with regard to the use of alcohol. According to Mott " there can be no doubt that neurasthenics, epileptics, imbeciles, degenerates, eccentrics, and potential lunatics-all those indeed with an inherent narrow margin of highest control - possess a marked intolerance to the effects of alcohol ' ${ }^{3}$ Certain facts are, however, very puzzling. Suicide, for example, would seem to be a manner in which general nervous weakness may manifest itself. Nevertheless, the tendency to suicide appears sometimes as a very definite and peculiar disease which manifests itself generation after generation at a certain age. Perhaps in these cases we should rather see an example of how the same outward circumstances-here the knowledge of the family history -tends to cause a general nervous weakness to manifest itself in the same way, rather than an example of a specific nervous weakness. ${ }^{4}$

4. What we know as temperament stands half-way between physical and mental characters. As we have seen, temperament depends upon influences exerted by the functioning of the bodily organs on the nervous system and upon the peculiarities of the

\footnotetext{
1 Adami, loc. cit., p. 26.

2 Ibid.

${ }^{3}$ Quoted by Thomson, Heredity, p. $275 . \quad$ Tredgold found that 80 per cent. of the mentally deficient had a bad nervous inheritance (Mental Deficiency, p. 40).
} 
nervous system itself. Though on this account temperament or nervous tone is peculiarly susceptible to environmental influences, a certain condition of nervous tone is innately given. We must suppose the actual structure of the nervous system to be largely inherited, and we must suppose that the manner in which the bodily organs function is largely due to their innate organization. We have thus to think of a certain temperament as always given in the germinal constitution. An extreme example is an innate defect of the thyroid gland which can produce any degree of mental apathy. Again, such characters as excitability, rapidity of response, and differences in respect of fatiguability and recuperation are largely inherited.

Habit is best considered under this heading. The essential fact about habit is that, if the nervous system is stimulated in a certain fashion, so that a stimulus passes along certain paths, the next stimulus of the same kind will produce an impulse which passes more easily along those paths. Thus in time the same result, whatever it may be, is achieved by the application of a weaker stimulus. This fact, which is of great importance, suggests an actual modification of structure-an actual fashioning of a path. Whether this is so or not, we do not know. What is noticeable here is that the ease with which habits are formed undoubtedly differs from man to man, and we have again to postulate predispositions in the germinal constitution which tend towards a definite degree of development of the power of the formation of habits.

5. The greatest difficulties arise when dealing with the purely mental characters. There is a general agreement among biologists that mental characters are inherited just as physical characters are inherited. As we have pointed out, there is evidence to this effect derived from studies on biometric lines. The difficulties arise when we attempt to give more precision to the statement that mental characters are inherited. One method of approaching this problem is to analyse mental behaviour, and to ascertain if possible what faculties there are which cannot be explained in terms of other faculties. Such faculties are the least that we must suppose to be given in the germinal constitution. It does not, of course, follow that irreducible characters are Mendelian characters. In all probability they are due to the presence of many Mendelian factors. This analysis is a matter of great 
difficulty, and there is at present no agreement among psychologists as to what ultimate faculties of the mind are given out of which the characters we observe are compounded. Modern analysis leads to the idea of mental process as the activity of a subject. But though we may not think of the mind merely as a bundle of faculties, we have to attribute certain faculties to the subject, and the question arises as to what these faculties are.

There is less difficulty with regard to the affective and conative faculties, that is to say, those that are connected with feeling and striving, than with regard to the cognitive faculties. Psychologists are to some extent agreed as to what instincts can be recognized. McDougall, for instance, gives the following list, associating in each case with the impulse an emotion representing the conative or affective aspect. ${ }^{1}$ (1) Instinct of flight and emotion of fear. (2) Instinct of repulsion and emotion of disgust. (3) Instinct of curiosity and emotion of wonder. (4) Instinct of pugnacity and the combative emotion. (5) Instinct of selfassertion and the emotion of elation. (6) Instinct of self-abasement and the emotion of subjection. (7) Parental instinct and the tender emotion. To the remaining instincts which he names a special emotion is less definitely attached. These instincts are those of reproduction, sexual jealousy, female coyness, gregariousness, aequisition, and construction. This list is not exhaustive; there are other instincts of less importance, such as the instinct which tends to make a boy at a certain age leave his home. The list, however, includes all the more important instincts.

There are a number of other emotions often described as primary which McDougall describes as complex. Thus, according to his view admiration is a combination of wonder and selfabasement. When fear is added, we have the emotion of awe, and when gratitude is added we have the emotion of reverence. Gratitude is itself a combination of tender emotion and selfabasement. In a similar fashion loathing, fascination, and envy can be explained. According to this view, therefore, we should not think of separate predispositions towards these complex emotions as existing in the germinal constitution; we should think of them as determined by the predispositions towards the simpler emotions.

1 McDougall, Social Psychology, ch. iii. 
McDougall further describes three general or non-specific tendencies which have sometimes been classed as instincts. ${ }^{1}$ Sympathy, or the sympathetic induction of the emotions, describes the fact that instinctive behaviour incites similar behaviour in the observer. Suggestion is defined as a process of communication resulting in the acceptation with conviction of the communicated proposition in the absence of logically adequate grounds for its acceptance. Imitation, which has often been used to include sympathy and suggestion, is in the limited sense adopted by McDougall, the tendency to copy the bodily actions of some one else. Finally there is the tendency to play.

Apart, therefore, from temperament and the faculties connected with cognition, we have to recognize predispositions towards certain instincts and certain general tendencies. Without question they differ in strength from man to man; without question such differences are in large part attributable to varying predispositions in the germinal constitution, and we must presume that such differences are in some way connected with differences in nervous structure or organization. The subject is one of great difficulty, and nothing can be affirmed regarding it with any certainty. The ultimate modes of feeling seem to be those of pleasure and displeasure, and of excitement and depression. It is scarcely possible to stop at this point, and it seems that we have to go on and attribute the more specific forms of feeling or emotions to primary faculties incapable of further analysis. So, too, conation or striving seems to be distinguishable into striving towards and into striving away from an object. Again as with feeling it appears that we have to go farther and presume certain more clearly-defined faculties as indicated above.

6. The difficulties are still greater with regard to cognition. Analysis seems to bring us to three ultimate faculties-those of judgement, of comparison, and of association. ${ }^{2}$ Judgement consists in affirming or denying, and upon this faculty all higher reasoning is built. Memory should perhaps be considered as a special aspect of this faculty-as the power to think of an object over again, and to affirm or deny it to be the same object. This faculty, together with the faculty of comparing, enables that process of distinction and systematization to be performed upon which all the higher developments are based. To these 
faculties of analysis and synthesis are to be added the faculty of association, whereby objects come to be thought of in groups according to the sequence in which they came to be presented to the mind quite apart from their intrinsic affinities or distinctions. Thus, in addition to the faculties whereby the mind attains some grasp of the constitution of the world of objects, there is a faculty which in some degree mirrors the history of the world.

It is probable that in addition to these ultimate faculties, there may be many other faculties which cannot be reduced to aspects of the former, and have therefore to be considered also as primary. However that may be, what is important for our present purpose is that the faculty commonly known as ability is undoubtedly innate. It may be very variously composed of specialized forms of these three primary faculties, and perhaps of other faculties combined in very many ways. Every degree of ability is known and there are many forms in which it is specialized, such as in musical, artistic, and other directions. It has been shown that a high development of general ability is correlated with a high development of any special ability. In other words, we have to think of a genius with some marked talent as possessing on an average a high general level of ability. There is ample evidence of the inheritance of ability, both derived from the examination of large numbers of cases and from the analysis of family histories. This evidence also shows that musical and other forms of special ability are inherited. We have therefore to think of predispositions in the germinal constitution, which give rise to abilities of all kinds, although a more correct analysis may refer them to some combination of the ultimate faculties enumerated above, much as certain complex emotions are to be referred to the combination of certain primary emotions, and not thought of as given as such in the germinal constitution.

There are other characters which do not appear to be covered by any extension of the term ability. Such, for instance, are will and self-control. In its essence will seems to be the rising up of some dominating impulse which controls or harmonizes the feelings. The weaker impulse somehow overcomes the stronger. The will is variously explained, and in modern psychological writings often in such a manner as to exclude the idea of a special faculty. It is not necessary here to go into these explanations of 
the origin of will. It is clear that, if it has to be referred to the working of complex faculties which are themselves explicable in the terms of more ultimate faculties, it is in a sense innately given. Undoubtedly will is in a sense inherited. However much will may be a product of the environment in the second sense, there is clearly an innate tendency towards the development of a will of a certain form and strength. Whether therefore it is an ultimate faculty or not, it is due in some measure either to a definite innate predisposition or to such a combination of other innate predispositions as give rise to the manifestation of a will of a certain nature.

Finally, we may look at the whole problem of the inheritance of mental characters from another point of view. It is known that certain areas in the brain associated with particular functions are differently developed in different individuals, and that there are differences in the speed at which the nervous impulse travels which are doubtless due to anatomical peculiarities and so onthat there are, in fact, differences in the physical basis which underlies the manifestation of mental characters. It is thus easy to understand in a general way how mental characters which are based upon the nervous organization are inherited. A man may inherit a brain in which certain regions are of relatively large size, or a nervous organization by which impulses are swiftly conducted, and thus we can understand how quickness of response, power of concentration, readiness of association, type of mind (whether emotional or intellectual) are inherited.

7. This brief inquiry into what is given in the germinal constitution leads to the conception of large numbers of predispositions which under the stimulus of the environment develop into the characters that we observe. This is true at least of physical characters. In physical characters we observe the result of the play of the environment upon certain predispositions. With regard to mental characters the position is somewhat different. Into mental characters, as presented to us-into ability, for instance-there enters the influence of the environment in the second sense. When we are judging the ability of a man from a practical point of view, we are judging a character into the make-up of which has entered not only certain predispositions and a certain environment-using environment in the sense of the complement to inheritance-butalso the results of the influence 
of the environment in the second sense, such as habit and what may be regarded as tools, namely, modes of thought and so on. In order to get at the characters we must disentangle or allow for these tools. This difficulty does not arise in the case of physical characters. When measuring the physical strength of two men we are not likely to allow one man to throw an object with a throwing-stick and another to use his own unaided strength. But we are apt when comparing ability to forget that one man may have learnt to distinguish between the categories when another may not have done so. To this difficulty we shall refer again later.

These predispositions have their basis in the germinal constitution and are therefore inherited. It is probable that we should regard the germinal constitution as consisting of unit-factors. However that may be, it is clear that either a differential birthrate (reproductive selection) or a differential death-rate (lethal selection), will change the average germinal constitution of the race among which they are operative. Bearing this in mind, we may inquire when and where in human history we can detect reproductive and lethal selection at work. And we must remember that in the first place not merely obvious factors such as war have been and are at work, but also that subtle changes in social life and social organization may have profound effects, and that in the second place not merely obvious characters may be favoured or eliminated, but the least striking and most minute (though not necessarily the least important) characters both mental and physical may be similarly affected. 


\section{XVII}

\section{THE EVOLUTION OF PHYSICAL CHARACTERS}

1. Having discussed what is given in the germinal constitution, we are now in a position to inquire into the question of changes in the germinal constitution. Such changes may affect physical or mental characters. In this chapter we are concerned with physical characters alone. Compared with the evolution of mental characters the evolution of physical characters is a subsidiary matter; as this evolution, however, has gone hand in hand with the evolution of mental characters and furthermore possesses an importance of its own, all reference to it cannot be omitted though the subject will be treated very briefly. As a preliminary to this inquiry we may note certain facts about the selection of physical characters in general.

We know practically nothing as to the cause of mutations. We have, therefore, to take mutations for granted, and to inquire how certain stocks with particular predispositions are favoured and others eliminated. In this process both lethal selection and reproductive selection play a part. Among species in a state of nature lethal selection is more important then reproductive selection; among men the importance of reproductive selection tends to increase until in the latest period it assumes an importance approaching that of lethal selection.

Hitherto we have only referred in passing to the existence of tradition among men. Tradition will be found to form a serious complication when we come to deal with the selection of mental characters. With regard to physical characters it also complicates the position, but only to a relatively unimportant degree. In the first place it makes the environment very varied, especially in industrial societies where men living next door to one another may be subject to very different conditions in their daily occupations. Secondly, the fact that men protect themselves against external conditions-for instance, against cold-may lead to a group of men who are protected surviving, where a less wellprotected group perishes, though the former may conceivably be 
naturally less resistent to cold than the latter. In the broad and rapid survey that we shall make these complications can be disregarded; the existence of tradition does not introduce, so far as physical characters are concerned, that peculiar complication which is the cause of much difficulty when we come to deal with mental characters-namely, the combination of what is acquired through tradition with the underlying character itself in such a way that the manifestation of the character is connected in a varying degree with its innate strength. We shall thus, when dealing with mental characters, have to attempt to strip off the acquirements. When dealing with physical characters we can always get down to the character at once. There is no difficulty in separating the arm from the tool which it employs, and we thus get directly at the character which has developed as the result of the influence of certain stimuli upon a given predisposition. It is, on the other hand, difficult to separate the intellectual characters from all those traditional elements which combine with them in their outward manifestations-to measure, for example, the strength of the instinct of curiosity, which involves discounting all those elements in the tradition which may inhibit or emphasize its expression.

With regard to the strength of selection there is no exact knowledge, with the exception of some work which has been done upon statistics for modern communities. The occurrence of selection in the past is in fact merely a deduction from what we know regarding innate predispositions and regarding elimination and differential fertility. It has been shown, however, that lethal selection does occur at the present day. Professor Karl Pearson has calculated that selection accounts for a very large percentageperhaps 60 per cent.- of the deaths at the present day, ${ }^{1}$ and Mr. Snow, summing up the results of an inquiry into this subject, states that 'natural selection in the form of a selective deathrate, is strongly operative in man in the early years of life '. ${ }^{2}$ As it is universally agreed that, if anything, the intensity of natural selection has decreased with civilization, we may take it as certain that it was operative to as great, or to a greater, extent during

1 Pearson, 'Groundwork of Eugenics', Eugenics Laboratory Lecture Series, 1909 , p. 25.

2 Snow, Studies in National Deterioration, No. 7, p. 34. See also Beeton and Pearson, 'Inheritance of the Duration of Life', Biometrika, vol. i, 1901, and Elderton and Pearson, ' Further Evidence of Natural Selection in Man ', Biometrika, vol. $x, 1915$. 
the emergence of man and during what we have called the first and second periods of his history.

2. During the intermediate period, which was of relatively immense length, the greater part of human bodily evolution was accomplished. Only two or three worn and fragmentary remains have as yet been discovered from this period. It is at least apparent from them that the amount of physical evolution which has been accomplished since the end of that period is slight compared with what was accomplished within that period. We may assume that lethal selection was strongly at work. Changes in mode of life-the assumption, for instance, of a terrestrial for an arboreal existence and the adoption of an upright posturemust have involved lethal selection. These changes of habit must also have brought ancestral man into contact with new enemies. The spreading of man into new climatic zones was doubtless followed by selection, and must again have involved contact with new enemies. So, too, selection followed upon the first great steps in the acquirement of skill--the making of clothes, the use of fire, and so on. In addition to lethal selection, differential fertility acting through polygamy must have been at work. In part no doubt differential fertility merely reinforced lethal selection; but in part also it may have taken the form of sexual selection and have favoured other types.

But when we come to details we find that we are ignorant regarding the causes of even the largest changes. Some guesses have been hazarded. The increase in the capacity of the skull is connected with the evolution of the intellect and may be left for consideration in the next chapter. The decrease in the size of the jaw and the corresponding decrease in the size of the teeth were perhaps connected with a change in diet. The loss of the hairy covering may have been connected with sexual selection or it may have been favoured because it removed a lodging-place for parasites.

3. As we pass from the intermediate to the first period of human history we reach a region of less uncertainty. We are ignorant as to the physical characters of man at the close of the intermediate period, but we know that in the earlier part of the Upper Palaeolithic there was existing one variety-the Grimaldi race-which bears certain resemblances to the negroid type, and that in the latter part of the same period there were existing 
several varieties closely resembling types of modern European man. It seems, therefore, that, so far as this period is concerned, we have to account not merely for the evolution of the main types but also for the evolution of the less easily distinguishable varieties of man. The splitting up may have begun in the former period but it probably did not go far. As to how this evolution took place certain conclusions may first be drawn from what we know must have been the general conditions of life. Secondly, from what we know as to the position among races of the first group, rather more definite conclusions may be added concerning the nature and direction of selection.

It has already been remarked that in all probability man spread into various climatic zones before the beginning of the first period of history. The consequences of this spreading of man must have been twofold. Mankind became segregated into groups, the surroundings of which differed, first in that they were subject to different climatic environments, and secondly in that they were forced to pursue different modes of life. It is clear that, whereas in some tropical regions man would be supporting himself by hunting and collecting in such a way as to require a certain type of bodily exertion, in a temperate region he would be supporting himself by a very different form of exertion. This difference between the needs of daily life would result in the favouring of different types in the two regions. The type best adapted to gain a living in one region would be different to that best adapted to gain a living in another region. ${ }^{1}$

Of far more importance in the production of varieties of men than the factors already noted are differences in climate to which man has become exposed. How great these differences are is familiar and need not be laboured. We know little with regard to the manner in which different types of man are suited to different types of climatic zones. But it is clear that in general the races of men are innately adapted to different climates. There is no historical reason why in all parts of North and South America the European races should not have ousted the American Indian as they have done in the United States. In Mexico and other regions, however, where the climate differs most markedly from that in Europe, Europeans have not succeeded in establishing

${ }^{1}$ Pruner-Bey ('Mémoire sur les Nègres', Mém. Soc. Anth., vol. i, p. 334) has shown in detail how the peculiarities of negro structure are to be regarded as adaptations to the surrounding conditions. 
themselves as they have elsewhere in the Continent. In Mexico Indian blood largely predominates over European, and the explanation must be that Europeans are not so well physically adapted to the climate as are the original inhabitants. In this there is nothing surprising. We can understand how certain types of respiratory, excretory, and circulatory organs might be better adapted than others to certain conditions of temperature, moisture, actinic rays, and so on, though we may be ignorant as to what types of circulatory and other organs are best fitted to any particular climatic zone. It would be very remarkable if it were not so, if, that is to say, any type of constitution was equally well fitted to any kind of climate. In this manner to differences in habits and in climate we may attribute the origin of the varieties of mankind.

Nevertheless, differences between races are far from being wholly due to selection on these grounds. Sexual selection is of importance. There grows up within each race a more or less clearly defined type of physical beauty, and differential fertility working through polygamy would evidently favour such types. That differences in colour to some extent represent adaptations to climate is clear; in part such differences are probably to be explained as due to varying ideals of beauty, but there are probably yet other factors entering into the evolution of racial types.

Recently Professor Keith has made an interesting contribution to the problem of racial differences. Reference has already been made to the ductless glands. It is now known that variations in the functioning of these glands have a profound influence upon the bodily organs. Keith has remarked that, if the peculiarities characteristic of the chief racial types of man are considered together, it appears that they are attributable to different degrees of development of these glands in different races. It should be observed that this suggestion does not involve the conclusion that racial differences are of the nature of modifications. It is merely supposed that the innate development of these glands is different in different races-that there is a predisposition in one race to the peculiar development of one gland and in another race to the peculiar development of another gland. The value of the suggestion lies in the fact that it shows how many racial peculiarities, for which there are at least no obvious explanations, may be merely the accompaniments of a difference in the development 
of one or more of these glands. It must be supposed that one or more of the results of the developments of a gland are of direct value in facing the peculiar conditions, whether climatic or otherwise, to which the race is subject, and that the other consequences of the development of the gland are in any case not prejudicial. These other consequences may possibly take the form of noticeable peculiarities of colour or of bodily structure which thus turn out in themselves not to be of survival value but merely to be, so to speak, accidental peculiarities.

Some quotations from Professor Keith's exposition of this suggestion may make the matter more clear. 'When we compare', he says, "the three chief racial types of humanity - the Negro, the Mongol, and the Caucasian or European-we can recognize in the last-named a greater predominance of the pituitary than in the other two. The sharp pronounced nasalization of the face, the tendency to strong eyebrow ridges, the prominent chin, the tendency to bulk of body and height of stature in the majority of Europeans, is best explained, so far as the present state of our knowledge goes, in terms of pituitary functions.' 1 After remarking that the interstitial glands are largely the cause of secondary sexual differences he goes on to say: 'I am of opinion that the sexual differentiation-the robust manifestation of the male characters-is more emphatic in the Caucasian than in either the Mongol or Negroid racial types. In both Mongol and Negro, in their most representative form, we find a beardless face and an almost hairless body, and in certain negro types, especially in Nilotic tribes, with their long, stork-like legs, we seem to have a manifestation of the abeyance in the action of the interstitial glands. At the close of sexual life we often see the features of a woman assume a coarser and more masculine appearance.' ${ }^{2}$ Later he remarks that the evidence points to the original human colouring as black. Now, the supra-renal bodies cause a clearing away of pigment and 'there can be no doubt that the suprarenalbodies constitute an important part of the mechanism which regulates the development and growth of the human body and helps in determining the racial characters of mankind. We know that certain races come more quickly to sexual maturity than others, and that races vary in development of hair and of pigment, and it is therefore reasonable to expect a satisfactory explanation
${ }^{1}$ Keith, Nature, vol. civ, p. 302.
2 Ibid., p. 303. 
of these characters when we have come by a more or less complete knowledge of the supra-renal mechanism.' 1 The thyroid acts directly upon the skin and the hair and also upon the skeleton. - This is particularly the case as regards the base of the skull and the nose, the arrest of growth falls mainly upon the basal parts of the skull with the result that the root of the nose appears to be flattened and drawn backwards between the eyes, the upper forehead appears projecting or bulging, the face appears flattened, and the bony scaffolding of the nose particularly when compared with the prominence of the jaw is greatly reduced. Now these facial features which I have enumerated give the Mongolian face its characteristic aspect, and, to a lesser degree, they are also to be traced in the features of the negro. Indeed, in one aberrant branch of the negro race-the Bushman of South Africathe thyroid facies is even more emphatically brought out than in the most typical Mongol. You will observe that, in my opinion, the thyroid-or a reduction or alteration in the activity of the thyroid-has been a factor in determining some of the racial characteristics of the Mongol and Negro races. I know of a telling piece of evidence which supports this thesis. Some years ago there died in the East End of London a Chinese giant-the subject one must suppose of an excessive action of the pituitary glandsthe gland which I regard as playing a predominant part in shaping the face and bodily form of the European. The skeleton of this giant was prepared and placed in the museum of the London Hospital Medical College by Col. T. H. Openshaw, and any one inspecting that skeleton can see that, although certain Chinese features are still recognizable, the nasal region and the supraorbital ridges of the face have assumed the more prominent European type.' 2

4. If we turn now and look at the conditions of life among primitive races, we find that natural selection and differential fertility tend to work towards the preservation of existing types rather than towards further evolution. The Australians may have existed in their present home for many thousands of years without undergoing any considerable change of type, and we have every reason to suppose that, if they had been left untouched by white or other races, the racial type would have remained substantially the same for thousands of years to come. The same

1 Keith, Nature, vol. civ, p. 303.

2 Ibid., p. 404. 
holds good if we consider any primitive race in its normal surroundings.

That this is so is clear if we glance at the chief causes of elimination. The heavy child mortality recorded of all these races is put down to neglect and exposure; it must on the whole result in the elimination of the physically weak. Such customs as the bathing of new-born babies in cold water seem designed to ensure this result. Among the adults who on the whole are but little protected against climate there must always be a certain tendency towards the elimination of those less able to withstand the relatively harder seasons. There are also many factors making for the cutting-off of the malformed and the congenitally deficient. Among those races which normally practise infanticide, deformed children are always killed, and among those races, such as the Bantu races of Africa, who never regularly practise infanticide as a custom, abnormal children are nearly always done away with. Similarly the destruction of witches tends towards the same end though they are more often marked by mental than by physical peculiarities. Again the fact that abortion and infanticide are practised to a greater degree among the less fortunate and the lower social classes of the races in the second group has the same result, as in general the stronger and more successful members of the race are those who do not need to practise these customs to the same extent. Finally, the general conditions of life among these races-more particularly among the races of the first group-is such as to bring about a continual elimination of the less physically fit. Speaking of the Seri Indians McGee remarks upon the 'elimination of the weak and helpless', and says further that 'a parallel eliminative process is common among American aborigines; the wandering bands frequently undergo hard marches under the leadership of athletic warriors with whom all are expected to keep pace, which leads both to the desertion of the aged and the feeble'. He calls it ' a merciless mechanism for improving the fit and eliminating the unfit '. 1

As remarked above, the effect of polygamy must be to intensify the action of selection. In this particular case the general result of polygamy must be to preserve the average features of the race ; for it will be, so to speak, the all-round man, the man best adapted to the climate, to the peculiar conditions of life and so

1 McGee, loc. cit., p. 157. 
on who will leave most descendants. It is thus of interest to investigate the frequency of polygamy among the races of the first two groups. This point has been inquired into by Professor Hobhouse and his fellow authors. Polygamy was distinguished by them into general and occasional polygamy, and though it is very difficult to arrive at any exact figures their general conclusions may be summed up as showing that, in all the subdivisions considered by them, general and occasional polygamy was found in from 80-95 per cent. of the cases. General polygamy increases among the races here placed in the second group, though there is no strong correlation between the degree of polygamy and the economic stage. We observe in fact a wide prevalence of polygamy among all races of any economic stage, and upon the basis of these figures we must regard the differential fertility arising from polygamy as a factor of great importance.

It seems, therefore, that within the first and second periods there were two tendencies at work-towards the evolution of varieties and towards the maintenance of the various types. The evolution of varieties was due in the first place to the spreading of man. As soon as spreading was complete, a tendency towards a cessation of progress must have set in. That further evolution took place was due chiefly to migration, with the results of which we have yet to deal. Once varieties had been evolved, apart from migration, and apart from changes in climate and progress in skill, which only slightly and at long intervals modified the surroundings, there was little basis for further evolution. Man had mastered his surroundings up to a point and had made his position secure in the circumstances under which he lived and the tendency was towards the preservation of the types which had achieved these results in different places.

5. In the third of our three periods there has been a remarkable change. We find that there has been a gradual moving away from the conditions under which in normal times there is in the races of the first and second groups a rigorous elimination of those types which depart from the mean. Lethal selection has come largely to take the form of selection through disease. ${ }^{1}$ Though polygamy gradually ceases to be a factor of importance, other forms of differential fertility become prominent.

1 For evidence that disease leads to a selective death-rate see Popenoe and Johnson, loc. cit., pp. 124 ff. 
We may glance at the chief features of these changes. In the last section we mentioned various factors-infanticide of the deformed, customs with regard to the treatment of children, the neglect and exposure of children, the general conditions of life, and so on-all of which have as their result the cutting-off not merely of the deformed and the monstrosities but also of all departures from the type best fitted to contend with the climatic environment and the conditions of daily life. Some of these factors-such as infanticide-disappear; the action of others becomes, if anything, reversed; not only do the less well-fitted types have a better chance of survival than before, because the conditions are less rigorous, but they suffer little or no disadvantage owing to the fact that the conditions have been artificially rendered almost as favourable for the less-fitted as for the better-fitted. To take two examples, not only are men with defective eyesight not eliminated, but they are by the invention of spectacles placed in as good a position as those with perfect eyesight. Similarly a woman with a narrow pelvis is, owing to the advance in surgical skill, enabled to bear children and to transmit her peculiarity to her daughters.

It should not be forgotten that, in spite of the gradual lessening of the rigour of selection, selection owing to climate and the general conditions of life still continues. But another factor has within this period come to assume a preponderating importance, and that is selection through disease. It was pointed out that diseases may be roughly distinguished into those due to the attacks of parasites and those due to structural defects. It is to diseases of the first kind that the greater part of selection, which occurs in the third period, is due. Some figures were given in an earlier chapter showing how large a proportion of deaths at the present day is due to one or other of these diseases. In the last chapter it was shown that men differ in their susceptibility to these diseases, and selection has thus very largely come to take the form of the elimination of the more susceptible, and of the favouring of the naturally immune and of those who have a power of resisting disease and of acquiring immunity. There has therefore come to be an increasingly heavy premium upon the type of constitution which can resist disease, and a strong constitution in this sense is not necessarily the same as a strong constitution among primitive races where the premium is rather upon muscular strength, 
perfect development of the senses of seeing and hearing, and upon resistance to climatic conditions. We have thus to think of the whole course of bodily evolution as changing in the third period so as to meet a new danger rather than as continuing on the former lines so as better to contend with the old difficulties.

It is noteworthy that selection in this period has not been on account of diseases of the second type due directly to structural defects. These diseases are seldom lethal until after maturity has been reached, and the tendency has rather been, as in the case of eyesight, towards the increased chance of survival of those suffering from these defects. Thus, though selection has turned in this period towards the weeding-out of those susceptible to the attacks of parasites, it has come to tolerate those who exhibit defects in structure as distinguished from the peculiarities of structure which must be presumed to constitute the physical basis of susceptibility.

All this is familiar enough. It is not so often realized that the disappearance of polygamy in what we have called the mediaeval and the modern sections of the third period works in precisely the same manner. But the disappearance of polygamy does not mean that reproductive selection ceases to be of importance. In mediaeval and modern times celibacy, postponement of marriage, and restriction of families have come to be practised in varying degrees by different stocks. There is some trace of differential fertility in the earlier periods owing to causes other than polygamy, but it is only in the mediaeval and modern periods that they become important. It is doubtful how far, if at all, the religious celibate class of the mediaeval period differed in physical characters from the average. It would appear that during this period postponement of marriage led to the producing of less children by the lower social classes than by the upper. Therefore, so far as this epoch is concerned, it is not apparent that differential fertility had any considerable effect upon bodily characters one way or the other. Possibly the net result of postponement may have been to favour the better stocks.

Within the latter part of the modern period restriction of families has assumed very great importance. It has been calculated that, as a consequence of the fact that restriction is more practised by certain sections of the population than by others, 50 per cent. 
of the married population provide 75 per cent. of the next generation. It thus becomes most important to determine whether the various sections of the population are innately different as regards their physical characters. Largely owing to our ignorance respecting the direct influence of the environment, it is not possible to arrive at present at any precise answer. But there can be little doubt that on the whole the most fertile sections of the population are the less physically fit sections.

It is necessary, however, to view these facts in their proper perspective. Though differential fertility of this kind may have been at work among some of the ancient empires in their later years, it is chiefly a very modern phenomenon characteristic of modern European countries and their derivatives only within the last fifty years. Therefore, however important a problem it may be for modern communities, it is a factor which has had but little effect on human history as a whole. It may be noticed that restriction of increase is not the only form of reproductive selection in modern communities. Sexual selection is also at work. ${ }^{1}$

6. Such has been the direction in which, through selection and differential fertility within races, changes have taken place. But changes also take place through the conflict of races, and the nature of these changes demands some notice. Warfare plays a part in the normal existence of nearly all primitive races. It is sometimes, as among the American Indians, a bloodthirsty affair, but more often than not it is a relatively unimportant cause of elimination. It is not easy to arrive at any conclusion regarding the results of warfare as an agent of selection. Upon the whole, among primitive races, so far as physical characters are concerned, there is probably a tendency towards the preservation of the physically strong and fit. But it has to be remembered that missile weapons were early introduced, and that, as Thucydides remarked, missile weapons kill the strong man in the prime of life as well as the weak. Again, success in warfare very largely depends upon characters other than physical-such as the possession of skill. All that can be said with certainty is that in the first and second periods warfare was not a cause of elimination of the fit which it has come to be in the third period. This latter fact has been lately brought home to the civilized nations

1 See Popenoe and Johnson, loc. cit., ch. xi. 
of the world in so obvious a manner that it need not be further discussed.1

Warfare following upon migration may exterminate whole races. The Bushmen were apparently on the verge of extinction at the hands of the invading Bantu peoples. In this manner a peculiar physical type may be lost and another may become of relatively greater prevalence. Migration may also be followed by any degree of racial mixture. Many rash statements have been made by historians as to the biological results of crossing. The consequences actually observed to follow upon a mixture of races are to so large a degree the result of a conflict of traditions-a subject with which we shall deal in a later chapter-that the purely biological effects of crossing may be very largely obscured. Bearing this in mind we may briefly refer to the conclusions to be derived from recent biological work which bear on the problem. Roughly speaking there are two possible kinds of crosses between races. First there are crosses between the most clearly distinguished varieties such as white and black. Heterosis, or hybrid vigour, will be exhibited in a marked fashion in the first generation. Heterosis, the underlying cause of which has only recently become apparent, is always at its height in the first cross. The increase of vigour, however, is not long maintained in subsequent generations. Further, each type, such as those which we are considering, has a series of character complexes, built up through ages of selection and compatible with one another, and by crossing such complexes are broken apart. The chance of gain, on the other hand, through the favourable re-combination of characters is small. On the average, therefore, the result of such a cross is unfavourable. There may also be crosses between races exhibiting less differences. Again, heterosis will be visible on crossing. But in distinction to the results of the former kind of cross, the other results may not be unfavourable. Great variability may follow such a cross and this is on the whole advantageous. Valuable character re-combinations may also come to light. Thus we may say that, so long as there is not too great a difference between the races which cross, the results are

1 There has been much discussion as to the effect upon the population of France owing to the Napoleonic wars. The facts have been summed up by Havelock Ellis (Essays in War Time, pp. 33 ff.), and it appears that without any question the average physical constitution of the French was lowered; most kinds of infirmities, for instance, became more frequent. 
usually genetically favourable; there will be the advantage of hybrid vigour, though this is always temporary, and there may very possibly be the advantage of valuable character re-combinations. ${ }^{1}$

Migration is important not only because it is connected with war and racial crossing but also because of the selection which follows upon transfer to new climatic conditions. To the nature of these changes allusion has already been made; it has been pointed out that races adapted to maintain themselves in one environment cannot as a general rule maintain themselves in a very different environment. To new surroundings, which only differ slightly from the old, they may become adapted by selection, and much selection on these lines must have taken place as a result of racial movements in Europe. It is probably now taking place among Europeans who have migrated to America. Dublin and Baker, for instance, have shown that the death-rate varies considerably among the different racial elements who have immigrated, some elements being probably better adapted to the new environment than others. ${ }^{2}$ Similarly selection may be going on within modern races owing to the rapid urbanization of industrial countries. Urban conditions may be more favourable to some types than to others. But it has not yet been shown in what direction these changes, if they are in progress, are taking.

Summing up our conclusions we may say that the great changes in human bodily form were accomplished in the intermediate period when the splitting up into varieties may also have begun. This splitting was continued in the first period and the modern types were formed before the end of that period. The chief characteristic, however, of the first and second periods was the maintenance of the types evolved, though this was interfered with by migration which brought about elimination of certain types, racial mixture, and further climatic adaptation. Finally, in modern times there has been a lessening of the stringency of selection, which previously tended to maintain the existing

1 Recent research bearing on this problem has been summed up by East and Jones in their book Inbreeding and Outbreeding.

${ }^{2}$ Dublin and Baker, Amer. Stat. Soc., vol. xvii, 1920. Nevertheless, as shown by an important memoir by Hrdlicka, a new variety is not being produced, at least with any rapidity, under the influence of the American environment. Hrdlicka studied representatives of the old white American stock, whose ancestors had been for four generations in the United States, and concluded that no new sub-type has yet emerged. In fact the faithful preservation of the traits of the original immigrants is the outstanding result of the study. 
varieties, and a turning of lethal selection to the building up of immunity against disease.

7. There are one or two other points connected with germinal change about which a word may be said. Of the origin of mutations we know nothing, but since in all probability the ultimate cause of mutations may have to be sought in some kind of environmental change, it should be borne in mind that, as man is subject to an immense variety of environmental stimuli, mutations may be more frequent in man than in any species in a state of nature. It has been suggested that the germinal constitution may be adversely affected by certain factors, especially by the use of alcohol. Of this there is no certain evidence. ${ }^{1}$ It has been supposed that there may be some connexion between the difference in age of the parents on the one hand and the germinal constitution of the offspring on the other. It has been supposed, for instance, that the offspring are innately more vigorous when the parents are at a certain age. So far as investigation has gone at present, it has not been established that there is any such connexion - of sufficient importance at least to deserve consideration here. In other words, the fact that among certain races young men have wives of considerably greater age than themselves and older men young wives, does not to any noteworthy extent affect the germinal constitution of the race. Again it has been stated that the first-born are innately inferior to the later-born children. ${ }^{2}$ This conclusion has been criticized. ${ }^{3} \mathrm{As}$, however, the preponderance of first-born children among the offspring as a whole would not appear to be markedly greater at any one period of history than at another, the inferiority of the first-born, if it exists, would make no difference between men of different races at the same or at different times.

The correlation of characters one with another should not be forgotten. The favouring of one character may involve the favouring of quite other characters. Of this what was said regarding the ductless glands is an example. It has been supposed that liability. to disease is correlated with pigmentation and that, owing to selection through disease, a change in the average pig-

1 An admirable summing up of what is known'as to the effect of alcohol on the germinal constitution will be found in Popenoe and Johnson, loc. cit., ch.ii.

2 Pearson, 'Problem of Practical Eugenics', Eugenics Laboratory Lecture Series, No. 5.

3 Greenwood and Yule, J. R.S.S., vol. Ixxii, 1914. See Pearson's reply 'On the Handicapping of the First-born', Eugenics Laboratory Lecture Series, No. 10. 
mentation is taking place. Attempts to establish this correlation have, however, failed. It is of particular interest to notice that no correlations have been found between physical and mental characters ; this is so even as regards intellect and size of head.

8. A survey of the facts regarding the bodily evolution of man, and of the conditions under which it has taken place, shows that there is at least no more difficulty in understanding how it has come about than in understanding how the varieties of any other species have come into existence. Given mutations, the action of selection and of differential fertility provides an explanation which is in general satisfactory, though we may be a very long way from understanding how any one particular change came about. There are in fact greater difficulties met with from time to time in trying to reach a satisfactory explanation of evolution among other animals than when dealing with man. The evolution along particular lines of organization, the evolution of teeth and horns among mammals, for instance, raises a difficulty which seems in the present state of our knowledge perhaps to require the assumption that at times there is a tendency for the continual occurrence of mutations in the same direction. Such a difficulty does not occur in the case of human bodily evolution.

It has been suggested that the facts regarding the course which the evolution of living organisms has taken requires the assumption that large mutations have occurred from time to time and have established themselves as varieties. There is no doubt that large mutations do occur and have established themselves in the course of the evolution of the varieties of animal types known to us, and they may have done so in the case of man. There is, however, no reason to imagine that the origin of any particular change in man was due to a large mutation. It has been stated that the number of chromosomes is different in the white man and in the negro, and upon the basis of this statement it has been suggested that one variety had arisen as a mutation from the other. This statement, however, was apparently based upon erroneous observation. Again, it has been suggested that the Pygmy type arose as a mutation. But it has been observed that some $\mathrm{Ba}$ Twa pygmies of the Congo who three generations ago left their forests, settled near the Bushongo, and took to agriculture have become markedly taller. Inter-marriage is said to be out of the question and therefore we are led to suppose in the new environment 


\section{EVOLUTION OF PHYSICAL CHARACTERS}

a taller type has been favoured. ${ }^{1}$ If this is so, if a taller type can be selected by degrees in one environment, the shorter type may equally well have been selected in another environment.

So far, therefore, as the history of man outlined in the fifth chapter is the history of the development of his bodily structure, so far the explanation of history is to be sought in changes in the germinal constitution, and the changes undergone are strictly comparable with the changes among species in a state of nature, though owing to the more varied environment outward differences in the case of man may be rather more often due to environmental influences than in the case of species closely related to man.

Ridgeway, loc. cit., p. 18. 


\section{XVIII}

\section{THE EVOLUTION OF MENTAL CHARACTERS}

1. WHEN we originally asked how far changes in the quality of the population accounted for the facts given in the sixth chapter, it was at once obvious that the interest centred in mental changes. Now that we have gained some idea as to what is given in the germinal constitution and as to the influence of the environment in general, we are in a position to come more closely to the main problem. Three questions present themselves. We have to inquire into the facts regarding the stage of mental evolution reached at any one time. This involves making both an estimate as to the extent of the gap between the pre-human ancestor and Palaeolithic man and an examination of the differences in the mental characteristics of the chief living types of man which may be taken roughly to represent stages reached in the three chief periods. We have next to ask if we can account for these facts, and this involves an inquiry into selection, differential fertility, and such other factors as may assist to explain changes in the germinal constitution. Lastly we have to ask how far such changes as have taken place are correlated with the main features of human history.

To make an estimate of the stage of mental evolution reached by one race as compared with that reached by other races is not an easy matter. 'To begin with there is the difficulty of discounting the direct influence of the environment. Temperament we found to be peculiarly susceptible to environmental influence. Temperament affects the functioning of other mental characters in a very important manner. 'Effective mental ability', says Professor Punnett, ' is largely a matter of temperament, and this in turn is quite possibly dependent upon the various secretions produced by the different tissues of the body. Similar nervous systems associated with different livers might conceivably result in different individuals upon whose mental ability the world would pass a very different judgment.' 1 Apart, however, from this

1 Punnett, Mendelism, p. 208. 
difficulty and apart from the influence of disease, it is not probable that other factors in the environment have any marked direct effect upon either disposition or intellect.

There is a far more serious difficulty to which allusion has already been made. It arises from the fact that mental faculties as exhibited in daily life are overlaid by tradition and shaped by habit. There is no difficulty in getting down to any physical character. It is always possible to separate the limb from the tool which it uses. It is frequently very difficult to effect the corresponding separation between the faculty and the tool in the case of mental characters. Human mental characteristics, as manifested in daily life, represent the combination of acquirements with a certain basis. By the basis is meant that which develops as the result of environmental stimuli acting upon the predispositions. The basis of all mental faculties is, therefore, that which strictly speaking is comparable with physical characters. Mental characters as manifested in daily life represent this basis combined with tradition. This applies not only to the intellectual but also to the instinctive faculties. The manifestation of instinct is dependent upon the channels into which it has been led by tradition and upon the outlets which tradition provides for it.

In attempting to estimate the stage of evolution of mental characters reached at any one time, we must, as far as we can, allow for all that obscures their manifestation. There are several different ways in which this may be attempted and it will be found possible to reach a fairly definite conclusion with regard to the degree of development of mental characters as exhibited by two races as different as the white and black. But the inquiry into the differences between the more nearly related varieties, such as the different branches of the white race, present far more difficult problems, and by these methods we shall not be able to approach them with much hope of success. Further, these difficulties will be more strongly felt when we go on to inquire into the causes of the changes which have led to the evolution of the different types. Therefore, though in this chapter we shall reach certain conclusions as to the nature, causes, and results of mental changes in the earlier periods, we shall find that, owing to the difficulties mentioned, we are unable to reach any conclusion as to the later periods until we have inquired into the importance of tradition. It will thus be necessary to 
cut short this inquiry and turn to consider tradition in the following two chapters. Only when this subsidiary inquiry has been made shall we be able to complete our estimate as to the part played by changes in the germinal constitution so far as they affect mental characters.

2. The indications as regards mental development obtainable from a study of fossil remains are, of course, very vague. Such as they are, however, considerable interest attaches to them. In the later part of the first period, in the Upper Palaeolithic, that is to say, we meet with types of men whose cranial capacity and the formation of whose brain, so far as it can be judged from skulls, do not indicate any difference in mental capacity as compared with modern man. It must be emphasized that this is a very rough method of comparing intellectual capacity. Something, however, may be deduced from such observations, and it is probable that, though there may have been noteworthy differences between Cro-Magnon and modern European intellectual capacity, as we judge differences to-day, nevertheless looking at human evolution as a whole, we cannot escape the conclusion that by the end of the first period, before man had learnt to support himself otherwise than by hunting and fishing, by far the greater part of the journey from the condition of our prehuman ancestor to that of European man had been accomplished. This is a very important deduction, because, inasmuch as the greater part of the progress in skill had still to come, it means that progress in intellectual capacity and progress in skill did not go hand in hand.

What we should like to be able to do, but cannot at present do, is to follow the evolution of the cranial capacity and the shape of the brain of the ancestors of Upper Palaeolithic man. But all we have in the Middle Palaeolithic are representatives of the peculiar Neanderthal type, which apparently died out. Judging from the cranial capacity, the intellectual development of Neanderthal man must have been considerable and probably not inferior to that of the Australians. If Eoanthropus is to be assigned to the Lower Palaeolithic, it is the only skull of that period that we have. Judging not so much from the cranial capacity as from the formation of the brain, the intellectual capacity of Eoanthropus was clearly much less than that of Neanderthal man and of any other type of man now living. 
Pithecanthropus was certainly anterior to the Palaeolithic. $\mathrm{He}$ may, it is true, have used and even fashioned implements of a very primitive type. It is noteworthy that, so far as can be judged, in cranial capacity he stands about half-way between modern man and the hypothetical ancestor, and if we are going to make any deductions at all regarding so difficult a point, it is reasonable to assume that in general his intellectual capacity was about half-way between that of modern man and the prehuman ancestor.

On the basis of this evidence the broad outlines of the picture are as follows. At the very least the intellectual development of Early Palaeolithic man had reached a point half-way between that of the pre-human ancestor and that of modern European races. Almost certainly it had gone farther, perhaps considerably further. As regards man in the Middle Palaeolithic, we have details only regarding an aberrant type whose intellectual capacity, though considerable, probably did not reach that of the contemporary ancestors of the Upper Palaeolithic types of whom as yet we know nothing. The cranial capacity and other features of the Upper Palaeolithic types show no essential differences from modern man, though it has to be remembered that, as cranial capacity is but a rough guide, differences in mental capacity may have existed.

3. In order to gain a more exact idea of the stage of mental evolution reached in the first and second periods, we are obliged to turn to the evidence supplied by primitive races, just as we turned to them to fill in the gaps in our knowledge of the social habits of Palaeolithic man.

There are at least three methods whereby an attempt may be made to arrive at some idea of the intellectual development of primitive races as compared with modern European man. There is to begin with a very large number of observations and impressions recorded by men who in many cases have lived a long time among primitive races and have had unusual opportunities for judging them. Then attempts have been made to measure the intelligence of these races by the use of the Binet-Simon methods, and we may notice the results of some of these attempts. Lastly, in certain parts of the world for some generations white and coloured races have been living side by side under almost identical conditions and now compete together almost on an 
equality, and we may note the current opinion in those regions with regard to their respective intellectual capacities.

All the evidence regarding the Australians agrees in attributing to them a relatively high level of intellect. 'Most observers', says Thomas, "agree that up to the age of puberty, possibly longer, they have an extraordinary facility in the acquisition of knowledge.' 1 Speaking of the aborigines of Victoria, Smyth records that "black children brought up in the schools learn very quickly, and in perception, memory, and power to discriminate, they are, to say the least, equal to European children. A missionary, the Rev. F. A. Hagenauer, a gentleman of great ability, who has control of the aboriginal station at Lake Wellington, reports that the examination made by the Government School Inspectors shows that the aboriginal pupils taught by him are quite equal to the Whites. In his last report he states that the whole of the fifth class in his school has passed the standard examination (that appointed for children in State schools) and that they had received certificates.' ${ }^{2}$ Spiller has collected a large number of such opinions, ${ }^{3}$ and Semon says that 'on examining the accounts of missionaries who have had occasion to instruct children of Australian natives, it will be found that nearly all of them come to the conclusion that at the onset of instruction hardly any difference between faculties of black and white children in grasping the elements is to be remarked. There is such a capability of memory and sharpness of the senses that in reading, writing, drawing, topography, and geography they at first even excel the whites.' 4 Nearly all observers agree that there comes a point when the faculties of aboriginal children cease to develop. Thus Smyth says that 'With keen senses, quick perceptions, and a precocity that is surprising, he stops just short of the point where an advance would lead to a complete change in the characters of his mind ',5 and Mathew who remarks that 'in schools it has been observed that aboriginal children learn quite as easily and rapidly as children of European parents', goes on to say that ' while among Europeans the range of mental development seems almost unbounded, with the blacks its limit is soon attained ' ${ }^{6}$

1 Thomas, Natives of Australia, p. 25.

2 Smyth, Aborigines, vol. i, p. 22. 'Spiller, 'Mentality of Australian Aborigines', Soc. Rev., vol. vi. Australian Bush, p. 78. Eaglehawk and Crow, p. 78. 
Among the Andaman Islanders there is, according to Man, up to the age of 12 or 14 'as much intelligence as [among] ordinary middle class children of civilized races'; he goes on to quote another observer who found these people 'not deficient in brain power; it rather lies dormant and unused in their savage state', and gives the example 'of an aboriginal patient of 12 years of age, who had been entered in the Ross Orphanage School, and who, in his tender years, could yet read English and Ordu fluently, as well as speak and write in both these languages, retaining also a knowledge of his native tongue. He had besides a fair knowledge of arithmetic. I may add that this is not an exceptional case, for I would instance others, and one lad in particular who was his superior.' 1 These opinions could be paralleled many timês over. The Jesuit missionary Baegert, for example, had a high opinion of the intellectual abilities of the Californian Indians. - Like other people, they are possessed of reason and understanding, and their stupidity is not inborn with them, but the result of habit; and I am of opinion that, if their sons were sent to European seminaries and colleges, and their girls to convents where young females are instructed, they would prove equal in all respects to Europeans in the acquirement of morals and of useful sciences and arts.' 2

The opinions of observers of races which fall within the second group are all very similar. Some typical opinions may be given. Speaking of Bantu races south of the Zambezi Theal says that ' in Mission Schools children of early age are found to keep pace with those of white parents. In some respects, indeed, they are the higher of the two ... but while the European youth is still developing his powers the Bantu youth in many instances is found unable to make further progress. His intellect has become sluggish and frequently he exhibits a decided repugnance, if not an incapacity, to learn anything more. The growth of his mind, which at first promised so much, has ceased just at that stage where the mind of the European begins to display the greatest vigour.' 3 Later he says that 'the strong desire of the greater number is to live as closely like their ancestors as the altered circumstances of their country will permit, to make use of a few of the white man's simplest conveniences and of his

\footnotetext{
' Man, J. A. I., vol. xii, p. 95. Yellow- and Dark-Skinned People, p. 264.

s Theal,
} 
protection against their enemies, but to avoid his habits and to shut out his ideas. Compared with Europeans their adults are frequently children in imagination and in simplicity of belief, though not infrequently one may have the mental faculties of a full-grown man.' 1 Of the Bangala of the Upper Congo Weeks says that ' up to the age of 14 and 15, the boys and girls, especially the boys, are very receptive and easily taught; but after that age comparatively few make real advance in learning '.2 This is attributed partly to the fact that at this age other matters occupy their attention-working on their own account, looking round for a wife, and so on, but more especially to the fact that by this age they have learnt all that their fathers have to teach them and thenceforth settle down into a routine. Of the men of the races of Central Africa Johnston speaks as follows: 'his mental powers are not much developed by native training, but I am certain that he has in him possibilities in the present generation as great of those of the average Indian; and there is really no saying what he may come to after several generations of education. I think it is truly remarkable the way in which a little savage boy can be put to school and taught to read in a few months and subsequently become a skilful printer or telegraph clerk or even book keeper. The little boys are much sharper and shrewder than the grown-up male. When the youth arrives at puberty there is undoubtedly a tendency towards an arrested development of the mind.' 3 This latter tendency is attributed to the attention paid to sexual matters. This arrest of development is a feature in all descriptions of the mental characters of these races. Junod is inclined, however, to think that these descriptions are on the whole exaggerated.4 In order to enable us to form such judgement on this point a few further quotations may be given. Ellis, describing the Ewe-speaking peoples of Togoland, mentions the precocity of the children when compared with Europeans, and says that, 'at puberty the physical nature masters the intellect and frequently completely deadens it '. 5 The Bambala children ' are very precocious, and up to the age of puberty are often astonishingly intelligent; after puberty, however, they become exceedingly apathetic; sexual excess and continual intoxication with palm wine contribute largely to this result '. 6 Speaking

1 Theal, loc. cit., p. 265. British Central Africa, p. 408. Ewe-Speaking Peoples, p. 9.
2 Weeks, J. A. I., vol. xxxix, p. $131 . \quad 3$ Johnston, - Junod, South African Tribe, p. $100 . \quad{ }^{\circ}$ Ellis, - Torday and Joyce, J. A. I., vol. xxxv, p. 268. 
of the natives of Portuguese East Africa, Maugham refers to the great brightness and promise early displayed; as regards the later stages says that he has 'over and over again been the witness of the sudden and astounding change which takes place among the young male house servants as youth approaches manhood. Brightness and initiative disappear; they go about their duties in a most casual manner ; they are unable to remember the clearest and simplest instructions, and are constantly away without permission.' 1 Referring to this subject so far as the natives of the Congo are concerned, Weeks states that 'for generations boys on arriving at the age of 14 or 15 had learnt all their father had to teach respecting fishing, hunting, woodcraft, building, paddling, \&c. . . . Thus their intelligence had attained for generations the fullest development by the above age; now we have to help them over that crucial stage ; in some cases it is very difficult; but in other cases we can do so ; and in such there is no limit to the intellectual progress they can make. In many cases they have mastered a good working knowledge of French, Portuguese or English, both spoken and written, and as larger opportunities are given, a larger number of youths will make such mental progress as will encourage their friends and teachers.' 2 Bryant, who has had a wide experience of South African natives, sums up his impressions by confirming the common opinion that the native boy is, at an early age, if anything, superior to the European boy of the same age, and that at puberty his mental development is arrested. He thinks that Boer boys living in the backwoods and receiving practically. no training are, if anything, inferior to the African boys. $\mathrm{He}$ adds-what is of particular interest-the fact that the African boys, whose education begins early, do not show the same arrest of development as those whose education begins later. Nevertheless the African boy educated from an early age is surpassed by the European boy later on. We find also, he says, that 'in practically every case where a South African native has had the opportunity of receiving an education in one of the universities of Europe or America, that that native has invariably been able to hold his own against all white rivals and to pass as successfully as they the same examinations in Law, Medicine, or Arts.'

1 Maugham, Portuguese East Africa, p. 268. Cannibals, p. 76.

2 Weeks, Among Congo 
Such men, however, he adds, may have been selected as particularly fitted to profit by further education. ${ }^{1}$ Summing up his opinion upon this subject, Olivier says that it is not possible to 'justify a generalization that there is any particular human function for which coloured persons are by their African blood disqualified. In various categories of human activity we may maintain that, as a rule, black and coloured folk are not up to the normal standard of the white, and are difficult and disheartening to deal with. But in other categories they are more liberally endowed than the average white man, not only with sympathetic and valuable human qualities, but with talent and executive ability for their expression.' 2

The evidence has been taken from the accounts of African races ; very similar evidence could be presented for all races in the second group. No purpose would be served by so doing, however, because such evidence amounts merely to a repetition of opinion similar to those given above. Before attempting to analyse this evidence, we may glance at the evidence obtained on other lines.

4. Many investigations have been recently carried out to test the relative intelligence of children of modern Europeans and of primitive races. The method used is known as the BinetSimon method and consists essentially in subjecting children to a large number of carefully prepared tests upon the total result of which an estimate is made of the intelligence of each child. Some of the most instructive of these observations have been made in America, where white and coloured children receive a very similar education; the result of an investigation carried out by Miss Strong in that country may be described. She tested 225 white children belonging to two schools and 125 coloured children belonging to one school. There is a standard degree of intelligence for each year of age, and thus every child can be graded according as to whether it reaches the standard degree of intelligence, or is above or below it. The results of this particular investigation are summed up in the following table : ${ }^{3}$

$\begin{array}{lcccc} & & \text { Coloured Children. } & \text { White Children. } \\ \text { More than one year backward } & . & . & 29 \cdot 4 & 10 \cdot 2 \\ \text { Satisfactory } \quad . & . & . & 69 \cdot 8 & \mathbf{8 4 \cdot 4} \\ \text { More than one year advanced } & . & . & 0.8 & \mathbf{5 . 3}\end{array}$

1 Bryant, Eug. Rev., vol. ix., pp. 47-9. $\quad 2$ Olivier, White Capital and Coloured Labour, p. 59. $\quad 3$ Strong, Pedagogical Seminary, vol. xx, p. 501. 
That the inferiority of the coloured children is exhibited at all ages is shown by the following table: ${ }^{1}$

\begin{tabular}{|c|c|c|c|c|c|c|c|}
\hline & \multicolumn{2}{|c|}{ Below Age. } & \multicolumn{2}{|c|}{ At Age. } & \multicolumn{2}{|c|}{ Above Age. } \\
\hline & & White. & Coloured. & White. & Coloured. & White. & Coloured. \\
\hline \multicolumn{2}{|c|}{6 years } & $19 \cdot 4$ & $40 \cdot 0$ & $30 \cdot 6$ & $33 \cdot 3$ & $50 \cdot 0$ & $26 \cdot 7$ \\
\hline 7 & " & $13 \cdot 9$ & $39 \cdot 4$ & $61 \cdot 1$ & $58 \cdot 8$ & $25 \cdot 0$ & $11 \cdot 8$ \\
\hline 8 & , & 18.5 & $23 \cdot 0$ & $55 \cdot 5$ & 38.5 & $26 \cdot 0$ & 38.5 \\
\hline 9 & , & $32 \cdot 2$ & $71 \cdot 4$ & 41.9 & $21 \cdot 4$ & 25.9 & $7 \cdot 2$ \\
\hline 10 & , & $55 \cdot 1$ & $75 \cdot 0$ & $27 \cdot 6$ & $12 \cdot 5$ & $17 \cdot 3$ & 12.5 \\
\hline 11 & $"$ & $34 \cdot 6$ & $43 \cdot 7$ & $42 \cdot 2$ & $50 \cdot 0$ & $23 \cdot 1$ & $6 \cdot 3$ \\
\hline \multirow[t]{2}{*}{12} & , & $67 \cdot 5$ & $77 \cdot 0$ & 32.5 & $23 \cdot 0$ & - & - \\
\hline & & $34 \cdot 5$ & $51 \cdot 4$ & $41 \cdot 2$ & $33 \cdot 9$ & $24 \cdot 3$ & $14 \cdot 7$ \\
\hline
\end{tabular}

With regard to these results Miss Strong writes as follows: - This seems to lead to the conclusion that the coloured children are mentally younger than the white. There is a difference of nearly 15 per cent. in the satisfactory group, nearly three times as many are more than a year backward, and less than 1 per cent. are more than a year advanced. A course of study in the coloured school is practically the same as that in the white schools. To what extent the difference is due to racial inferiority, to what extent differences in the home environment, cannot be said. It is certainly not due to difference in school training.' 2

There are certain criticisms of weight to be made regarding the importance to be attached to estimates of intelligence due to this method. These need not detain us beyond noting that, although in general, inasmuch as the children use the same language, the mental equipment is similar, nevertheless there may be considerable differences in acquired habit as between white and coloured children which affect their responses to the test. There is a further criticism of some importance to be made regarding this particular investigation. It was entirely carried out by white examiners, and it may very well be that the coloured children did not respond as readily and acquit themselves as favourably as if they had been examined by members of their own race. There is reason to suspect therefore that the coloured children may not show up in as favourable a light as the white children quite apart from any colour bias that may possibly affect the results in the same way. In any case it seems certain that the results are not unduly favourable to the coloured children.

1 Strong, loc. cit., p. 503.

2 Ibid., p. 501. With regard to the disabilities under which the Negro labours in America see Booker T. Washington, Story of the Negro, vol. ii, pp. 114 ff. 
The results of several similar inquiries have been summarized by Popenoe and Johnson. 'The most careful study yet made', they write, 'of the relative intelligence of negroes and whites, is that of G. O. Ferguson, junior, on 486 white and 421 coloured pupils in the schools of Richmond, Fredericksburg, and Newport News, Va. Tests were employed which required the use of the higher 'functions' and as far as possible (mainly on the basis of skin colour) the amount of white blood in the coloured pupils was determined. Four classes were made: full-blood negro, $\frac{3}{4}$ negro, $\frac{1}{2}$ negro (mulatto), and $\frac{1}{4}$ negro (quadroon). It was found that ' the pure negroes scored 69.2 per cent. as high as the whites; that the $\frac{3}{4}$ negroes scored 73 per cent. as high as the whites; that the mulattoes scored 81.2 per cent. as high as the whites; and that the quadroons obtained 91.8 per cent. of the white score.' This confirms the belief of many observers that the ability of a coloured man is proportionate to the amount of white blood he has. ${ }^{1}$

Summing up a review of this subject Huntingdon writes as follows: 'So far as I am aware, every exact test which has been made on a large scale indicates mental superiority on the part of the white race, even when the two races have equal opportunities. For example, in Washington the coloured children remain in school quite as long as the white; they do not accomplish so much in the way of study and do not reach so high a grade. In the cities of the south, Mayo and Leram find that where the races are given essentially the same instruction, the proportion of whites who are promoted is greater than than of negroes. Moreover, the differences seem to increase with years, which suggests that the average coloured child not only stands below the average white child in mental development at. all ages, but ceases to develop at an earlier age. In the High Schools of New York, superiority of the white race is shown by Mayo's examination of the average marks. By the time the children reach the High School, the processes of promotion have weeded out a much larger proportion of coloured children than of white. Hence, the negroes form a specially selected group whose superiority to the average of their race is more marked than the superiority of the white High School children when compared with the rest of the race. Nevertheless, the average marks of the white children

1 Popenoe and Johnson, loc. cit., p. 288. 
are distinctly higher than those of the coloured.' 1 Here again, as in the case of Miss Strong's investigation, it is not improbable that the conditions are to some extent weighted against the coloured race.

5. A third method of arriving at some indication of the relative degree of mental capacity is to notice what has happened where for some generations the two races have lived under more or less similar conditions. 'One of the best places for comparing the two races is in the Bahama Islands', writes Huntington. For reasons which I shall present later, the process of making 'poor whites' has probably gone farther in the Bahamas than in almost any other Anglo-Saxon community. Part of the white population are like their race in other regions, but a large portion have unmistakably degenerated. Witness their intense and bigoted speech, their sunken cheeks and eyes, their sallow com. plexion, and their inert way of working. In spite of racial prejudice, there is no real colour line in the Bahamas. Persons with more or less negro blood are worthy occupants of the highest positions, and are universally accepted in the most exclusive social circles. The British Government gives the negro every possible opportunity. The state of affairs may be judged from the remarks of a 'poor white' sailor, who said to me: 'You want to know why I like the Southern States better than the North. It is because they hate a nigger and I hates him too. What kind of a place is this where they do everything for the nigger and nothing for the white man? It is bad enough to have to go to jail, but it's pretty hard for a white man to be taken there by a nigger constable.' In one Bahaman village I saw negro girls teaching white children in the public school. In that same village a number of the leading white men cannot read or write.

'When they were children, their parents would not send them to school with negroes. The despised negroes learnt to read and write but have now largely forgotten these accomplishments. The proud whites grow up in abject ignorance. To-day the same thing is going on. I visited two villages where the white children are staying away from school because they will not go to negro teachers. The homes of such whites are scarcely better than those of their coloured neighbours, and their fathers are called

1 Huntington, Civilization and Climate, p. 12. 
"Jim" and "Jack" by the black men with whom they work. Racial prejudice apparently works more harm to the whites than to the blacks. So far as occupations go there is no difference, for all alike till the soil, sail boats, and gather sponges.

'When the lumber industry was introduced into the islands, whites and blacks were equally ignorant of the various kinds of work involved in cutting trees and converting them into lumber. The managers did not care who did the work so long as it was done. They wanted three things : strength, docility or faithfulness, and brains. They soon found that in the first two the negroes were superior. Time and again persons in authority, chiefly American but also some of the more capable native whites, told me that if they. wanted a crew of men to load a boat or some such thing, they would prefer negroes every time. The poor white shirks more than the coloured man. He is not so strong, he is proud and touchy. Other things being equal, the negro receives the preference, but other things are not equal. The very man who praised the negroes generally added: "But you can't use a negro for everything. They can't seem to learn some things, and they don't know how to boss a job." The pay roll reflects this. Even though the negroes receive the preference the 400 who are employed earn on an average only about 60 per cent. as much as the 57 white men. If we take only the 57 most competent negroes their average daily wages are still only 88 per cent. as great as those of the native whites. The difference is purely a matter of brains. Although the white man may be ignorant and inefficient, with no more training than the negro, and although his father and grandfather were scarcely better, he possesses an inheritance of mental quickness and initiative which comes into evidence at the first opportunity.' 1

6. What conclusions are we to draw from this varied evidence ? Let us first ask how we should view the differences between the negro and the modern European. There seems to be no marked difference in innate intellectual power ; the differences are rather differences in disposition and temperament. The white man has initiative, inventiveness, versatility, and power of leadership ; the black man has humility, patience, loyalty, and self-sacrifice. It is remarkable with what unanimity observers of the negro race, in its own country speak of the high degree of intellectual

1 Huntington, loc. cit., p. 14. 
development. Certain points are noteworthy. The apparent arrest of development is associated with an absorption at a certain age in practical matters, in tribal habits and customs, in sex and in settling down to a normal married existence as much on the lines of their ancestors as is possible under the altered conditions. Several passages quoted above strongly suggest that what we are here witnessing is a turning away from the training felt to be strange and foreign, owing to the strength of the native tradition which claims all the affection and interest of the young man. In other words this arrest may not be so much an inevitable result of the kind of mental faculties which are inherited as a result of the coming into play of a peculiar tradition. This conclusion is supported by the fact that, when in Africa education begins earlier, when, that is to say, there is more of a break with native tradition, the arrest is less well marked. Further, in America, it is still less marked though it can be detected. The conclusion would thus seem to be that, though there may be some tendency for intellectual development to stop at a rather lower stage than among European races, it is nothing like so well marked as the observations of residents in South Africa would seem at first sight to show.

The evidence quoted has chiefly been drawn from observations upon the negro races. It must suffice here to say that what is known regarding the intellectual development of other races included in the first and second groups leads to the conclusion that, although there are signs of differences as between these races, yet these differences are not remarkable, and further that the degree to which so apparently low a race as the Australians differs from Europeans is not in any case much greater than the degree in which negroes differ from Europeans.

It remains to ask how far this evidence supplements that derived from a study of fossil remains. Judging from the fact that the general level of the development of the mental faculties of any primitive race is not separated markedly farther from that of modern European man than are the negro races in this respect from modern European man, it seems that we must allow to Upper Palaeolithic man on the average a degree of mental development at least equivalent to that of the negro. It is further only reasonable to suppose that the ancestors of Upper Palaeolithic man in the Middle Palaeolithic were little inferior in mental 
capacity, inasmuch as to Neanderthal man, to whom they were superior and whom they apparently exterminated, we must attribute a degree of intelligence little, if at all, inferior to that of the Australians. Of Lower Palaeolithic man we know much less. We do not know, for example, whether he buried his dead. But if we consider the variety of instruments fashioned and used by him, it is not unreasonable to attribute to him, in view of the nature of the general conclusions to which we are being led, a degree of intelligence again but little inferior to that of Middle Palaeolithic man.

Our conclusions can only be tentative; nevertheless such indications as we have all point the same way. It would seem that the major part of the progress in the evolution of the intellectual faculties had been accomplished far back in Palaeolithic times. Those living races which, with all due reservations and qualifications, may be held to represent in mental and bodily characters Palaeolithic races, differ from modern European man rather in disposition than in intellect. And it is important to note that it is in the growth of the intellect rather than in the growth of the other mental faculties that modern man is distinguished from his pre-human ancestor.

7. We may again follow the same procedure as in the last chapter. We may recall the general conditions to which man was subject in the intermediate and early periods and ask how the evolution of mental characters was influenced by them. We may further ask to what degree and in what direction the conditions of life among primitive races influence the selection of mental characters since in general we may assume the same influences to have been at work among prehistoric races. With regard to the intermediate period we have to remember that it was far longer than, perhaps many times as long as, the period which has elapsed since the rise of Palaeolithic industry. Within this period man descended to the ground, spread, if not into every continent, at least far over the surface of the world and came to dominate all other living organisms as no species had ever done before. Clearly he was enabled to achieve this result by his intellectual powers and by them alone. He did not develop any other means of attack or means of defence: he conquered by his intellect.

In a general way it is possible to understand how this came 
about. To begin with, the pre-human ancestor was favourably situated for such a development because he was a generalized and not a specialized type. Once specialization of form has set in, as it has among the anthropoid apes, there are two bars to such a development as occurred in man. The specialization of bodily form in order to cope with a peculiar environment renders the body less adaptable to any changes in the environment which may take place. Further, unless the body is of the nature of an instrument capable of being readily employed to perform various functions, the intellect is unable to manifest itself. We have only to think of the specialized fore and hind limbs of most mammalian types to see how poor an instrument the body would in those cases make in the service of the intellect.

Under these favourable circumstances, evolution took this momentous turn. We can understand how, since the pre-human ancestor depended for his survival almost solely upon his intellect, there would be a high premium upon intellectual development. It is reasonable to suppose that he lived in groups of polygamous families and that differential fertility thus intensified selection. There is, of course, never at any time only a single factor governing selection, and though undoubtedly intellect was the chief factor in selection, climatic and other factors were, as we have noticed, also in operation, bringing about those changes in bodily form that we have observed.

The instincts of the pre-human ancestor were doubtless, in common with those of other mammals, of a generalized type compared with what we find among some lower groups of animals. The tendency has been for them to become even more generalized, though not on the whole any less powerful. It seems likely, for instance, that the instinct of flight was definitely associated with the snakes among other objects from the attacks of which presumably the ancestor was at one time in danger. We assume this because we occasionally meet with cases in which this specialization of the instinct of flight still obtains. The instinct of flight has now become wholly generalized, being aroused by general conditions and not by specialized occurrences. If it is correct to think of the ancestor as living in groups of families, for the leadership of which there must have been continual struggles, we may conclude that the instincts of pugnacity and of self-assertion were favoured, and we may make other similar 
deductions in estimating the value of which, however, it must be remembered that we are very largely in the region of hypothesis and speculation. The one thing to which we may with certainty hold fast is the premium upon intellect.

8. At length the intermediate period gave place to the period marked by the origin of society of the primitive type, defined by the fact that men are bound together by rigid custom. Obedience to custom involves a considerable measure of control of the impulses. Before society of this kind could have arisen, mental evolution must have reached a still higher level.

Let us consider what is implied. To begin with, the dependence of man upon his intellect for his position in the world accounts, as we have seen, for the evolution of his mental faculties up to a relatively high level in the intermediate period. When this level had been reached, the next step was rendered possible. What was now required was that man should be able to enjoy the advantages of social co-operation. To this end law was necessary. McDougall quotes Bagehot as saying that 'law, rigid, definite, concise law, was a primary want of early mankind; that which they needed above everything else, that which was requisite before they could gain anything else', and that: ' in early times the quantity of government is much more important than its quality. What is wanted is a comprehensive rule binding men together, making them do the same things, telling them what to expect of each other, fashioning them alike, and keeping them so. What this rule is, does not matter so much. A good rule is better than a bad one, but a bad one is better than none.' 1 That man should acquiesce in the restrictions imposed by society involves prudence, which inhibits those impulses which would lead to a contravention of custom. Now, fear is a simple emotion, but prudence implies something more. It involves 'a capacity for deliberation and the weighing of motives in the light of selfconsciousness '.2 In order that society might become established, there was thus required a further evolution of the intellect, and, since the advantages to be gained by the establishment of society were so great, there must have been a heavy premium upon evolution in this direction.

The beginning of the first period in history thus marks the successful completion of a step in mental evolution raising man 
above the stage reached in the intermediate period. It is important to observe that all primitive races known to us are representatives of man after he had completed this step. So far, therefore, as their mental faculties throw light upon the mental evolution of man, it is the level of evolution reached after and not before the evolution of social organization that is illustrated.

We may now glance at the conditions characteristic of primitive races and ask how they bear upon the selection of mental characters. Primitive society we found to be characterized by hard and fast rules which must be implicitly obeyed if social organization is to be maintained. Obedience to these conventions is of the utmost importance; we may remember incidentally that, unless the conventions are observed, an approximation to the optimum population will not be made. There is in consequence a tendency towards the elimination of those exhibiting characteristics incompatible with due subservience to the prevailing tradition. Such characteristics are any marked developments of intellect or of disposition away from the average. The man who will not conform is not tolerated. However advantageous to the individual certain developments might be, such developments are not favoured because the importance of maintaining intact the group to which he belongs is of greater moment. Thus, as in the case of physical characters, the general tendency is on the whole against further evolution and towards the preservation of those types which have reached the position characteristic of any primitive race. And it is to be noted that, whereas bodily form is on the whole adapted to the physical environment, mental characters are adapted on the whole to the traditional environment. Men come to be selected in accordance with the needs of social organization, and as tradition grows in amount also in accordance with their capability of absorbing tradition.

It is difficult to point to any factors which markedly favour further evolution of mental characters. On reading the admirable accounts of the daily life and customs of the Australians as given by Messrs. Spencer and Gillen, or of a much more advanced people-the tribes of Borneo, as described by Messrs. Hose and McDougall-we observe, it is true, the respect paid to men of experience and of rather more than average intelligence. We can understand how men not up to the average level of intelligence would not in all respects fare as well as others, but we do not 
find that there are factors distinctly favouring unusual intelligence. Some progress there probably was. On the whole those men who attained the leadership were probably rather more intelligent than the average, though among the qualities which assisted such men to gain the leadership many other mental qualities played a more prominent part than intellect ; thus, by practising polygamy, or practising it to a greater extent than the rest, they left more than the average number of descendants-and, while perhaps raising the intellectual level of the race, more certainly altering the average mental endowment in respect of these other qualities.

Such an examination also shows that a somewhat different disposition is favoured in different cases. Thus a warlike type is favoured among the American Indians, for, though possibly the most warlike may be killed off the most quickly, this is more than counterbalanced by the ease which with such men get numerous wives. But such differences in no way conflict with the fact that the tendency is towards the preservation of a mean, which though it may vary somewhat, is in general outline the same among all these races.

There still remains to be noticed the result of conflict between groups, other than normal warfare, which at times led to the substitution, whole or partial, of a less intelligent by a more intelligent race, or at least of a less skilled by a more skilled race; for by this time the outward manifestation of intellect was to a considerable extent overlaid by tradition. But such substitution, though it changes the average germinal constitution of the species as a whole, scarcely accounts in any noteworthy degree for the evolution of a higher level of intellectual capacity. The conditions which did favour further evolution within these two periods are the same as those which, when present to a more marked degree and acting over a longer period of time, finally gave rise to the races of the third period, and to these conditions we may now turn.

9. There are certain regions of the world's surface which contain within them areas abutting one upon the other and differing sharply one from the other. Such a region is found in that part of Western Asia which embraces Asia Minor, Armenia, Syria, Arabia, Mesopotamia, and Western Iran. In such areas races live in close contact with other races of very different 
habits, customs, and modes of life ; hunting and fishing races, pastoral races, agricultural races, races predominately sea-faring, all come into contact and conflict. Under conditions of these kinds there is more tendency for advantage to accrue to intellect not only as between members of different races but as between members of the same race than in conflicts between races of similar culture. Where these conditions have ruled, therefore, there has been a tendency, though perhaps not a very marked tendency, for a premium to have been set upon intellect. It so happens, however, that this region of Western Asia is endowed above all other regions with plants and animals that can be domesticated and with other features offering rewards to skill. In this region there was thus an additional premium upon intellect, and it was apparently in this region that the races who initiated the third period made their appearance. To the favouring of intellect under these conditions we may thus attribute what superiority of intellect the races of this group exhibit over the races of other groups. But it is rather in disposition and temperament that the predominant races of this group differ from those of the preceding groups. In them we meet with a power of leadership, a resourcefulness, a versatility, that marks them off from the primitive races, and it is precisely these qualities which would be chiefly favoured in conflict and competition of the kind described. The outward manifestation of these qualities may indeed be largely a matter of tradition, but that they are also in part innate in the modern European we have seen to be the case, and the explanation of this further evolution is clearly to be found in the conditions to which they have long been subject.

Turning to the conditions within the races of the third period and neglecting the Asiatic races, those races which in fact have been left in a backwater out of the main stream of progress, we find ourselves faced with the difficulty that tradition has so far come to overlay the innate qualities of intellect and disposition, that we are only with great difficulty at present able to estimate the result of any factors which we may see at work favouring or otherwise particular types. We may note, however, that success both between and within races has fallen rather to character than to skill. There is, in other words, little reason for thinking that intellect, so far as it is measured by skill, has been markedly favoured. On the other hand, character as 
outwardly manifested has been clearly favoured. But how far we should see in this process a favouring of certain innate types of disposition and of temperament must be left for consideration until later. These reservations regarding the passing at present of any judgement apply with the greatest force to the results of the differential fertility between the social classes in many countries at the present day. But we may say that, as in the case of physical characters, there has now ceased to be any strong tendency towards the preservation of a mean type. Conditions allow of the existence of variations away from the meanbut, as regards intellect, unfavourable, equally with favourable variations.

10. We have seen that the evidence derived both from a study of fossil remains and from a study of primitive races leads to the conclusion that the evolution of human intellectual capacity early reached a relatively advanced stage. An inquiry into the forces which we must assume to have been at work shows that intellectual capacity was more favoured in the intermediate stage than after primitive society had come into being. It was favoured at first as the character which enabled man to obtain his dominating position in the world and afterwards again as the necessary trait which enabled him to enjoy the benefits of co-operation. When dwelling on the fact that the advance since the setting-up of primitive society has been relatively small, it must not be forgotten that the latter period has been far shorter than the former. The period of time which has elapsed since the ancestors of man set out, so to speak, to conquer the world, must at a minimum estimate be five to ten times as long as the period since the establishment of primitive society to the present day.

The general conclusion to which we are led is that of the whole degree of mental evolution which has taken place by far the greater part was achieved at a time when only a beginning had been made with progress in skill. To the question whether the historical process outlined in the fifth chapter is comparable with progress among wild species-is based, in other words, upon changes in the germinal constitution-these conclusions suggest the answer that the happenings in the intermediate period are very largely in any case to be accounted for in this manner. There can be little doubt that the increasing domination of man was largely proportional to the growth of his innate intellectual 
capacity. But to the question as to how far the happenings within the period since the origin of primitive society are connected with the further evolution of innate capacity we can at present return no answer. We may note the striking fact that within this period progress in skill has been immense and has latterly gone on at a greatly increased speed, whereas changes in the germinal constitution have been relatively small. This clearly suggests that there has been but little connexion between the course of history as usually understood and changes in the germinal constitution. But before we can pass any judgement, we must gain some idea as to the manner in which tradition contributes to the shaping of history. Only then shall we be in a position to estimate the part played by such changes in innate characters as have occurred, and to this task we may now turn. 


\section{XIX}

\section{THE NATURE OF TRADITION}

1. So far as bodily characters are concerned, we have seen that we have to look for the explanation of human history primarily in changes in the germinal constitution. With regard to mental characters the position is different. It is evident that, at least in the later stages of history, the outstanding features are not correlated strictly with changes in the germinal constitution underlying mental faculties. To some extent, possibly to a large extent, they are independent of such changes. The influence of the environment in the second sense has to be considered, and until it has been considered we cannot arrive at any conclusion as to the importance to be attributed to the former factor in bringing these events about. What is now required, therefore, is a study of the influence of the environment in the second sense. It will be very briefly undertaken in this and the following chapter. In this chapter we are concerned with the nature of tradition, and the manner in which it is passed on, stored up, and retained. In the next chapter we have to examine the influence of the various factors in building up tradition.

In Chapter II we saw that, as far as mental development is concerned, the distinguishing characteristic of man is the conceptual process of thought. Man is, of course, also possessed of all the simpler mental processes there described. We have here in particular to study the consequences which follow the attainment to this higher level of mental process. It has already been observed that the circumstances were favourable to the evolution of this faculty. They also enabled the fullest use to be made of it. ${ }^{1}$ In the first place the human instincts are very generalized. They provide a basis for all kinds of vaguely directed activities in response to vaguely discriminated impressions from a large class of objects. Since it is only through the instinctive faculties that the intellect gets to work, the generalized nature

1 See McDougall, Psychology, p. 171. 
of the instincts is a great advantage. Secondly the immature period is prolonged. In comparision with the higher animals the immature period occupies a far longer proportion of the normal term of life and thus there is provided a far longer period in which learning can proceed. Lastly there is the power of speech.

The question has been much debated as to whether there can be thought on the conceptual level without language. It would seem that conceptual thought can only exist without language when of a very rudimentary kind. 'Language is not merely an accompaniment of conceptual activity; it is an instrument essential to its development. It is an appropriate means of fixing attention upon ideally represented objects as distinguished from percepts.' 1 Language, on the other hand, so far as it is a means of communication concerning objects outside the actual range of perception, can only arise between persons capable of conceptual thought. And this is essentially the function of language. It reflects the conceptions by which empirical data are brought into relation. "Resemblances of quality are expressed by general terms, continuity of existence by individual names, the relation of ideas and the order of connexion in thought by the arrangement of words in a sentence.' 2 Language expresses that breaking up and re-combination of the elements of experience which we have seen to be the essential features of conceptual thought.

Language makes possible the influence of mind upon mind, and is the basis of all human social development. It fixes the mind of a thinker upon his own ideas, and, when in communication with another person, it fixes the mind of the hearer upon the ideally represented objects present in the mind of the speaker. Into its origin it is not necessary to go. But it may be observed that, just as the generalized nature of the instincts and the prolonged immature period enabled the fullest use to be made of the power of conceptual thinking, so the fact that the mouth and the throat, usually otherwise unoccupied, were ready at hand as convenient instruments of communication enabled speech to be developed without occupying other organs, such as the hands, which could be profitably employed at the same time for other purposes.

1 Stout, Manual of Psychology, p. 597.

${ }^{2}$ Hobhouse, Development and Purpose, p. 91. 
2. Before we go on to consider how tradition-the product of conceptual thought-is passed on and stored up, it should be noticed that we have not to regard the process of conceptual thought as one which sprang into being in its present form and continued as such ever since. There has been a movement of thought up from dim and rudimentary beginnings to its present stage. This movement may be considered as consisting essentially in the clarification of concepts. It is possible to examine the nature of this movement without reference to the question as to how far the later stages are dependent upon the evolution of innate mental characters. It may be that the higher stages of conceptual thought are possible only when the evolution of the intellect has proceeded beyond the point where lower stages of thought alone are manifested. This question may be neglected for the present and we may consider very briefly the nature of this movement, which is best illustrated if we contrast in a few words the stage which conceptual thought has reached among primitive peoples as a whole with the stage it has reached in the everyday life of the so-called civilized races of the present time, and in so doing we may follow Professor Hobhouse's recent exposition. ${ }^{1}$

With the origin of language arises the first sign of the power to grasp the data of experience in accordance with their affinities and so to build up conceptions of individuals, groups, and classes as the subject of rough-and-ready generalizations. "With regard to matters standing out very plainly in experience or very close to practical interests there is not room for much divergence in method. . . . But outside the limited area of readily tested belief lies a mass of more doubtful ideas of great significance in human life. In this region we find in the first stage that the movements of fancy under the sway of feeling take the lead in forming belief, and that the ideas formed are so obscure and inconsistent as to blur the deepest lines of distinction drawn for more developed thought in the logical categories. We may then consider the first stage in human thought to be one of which the process of organizing experience into common categories is incomplete, and the evidence for the truth of an idea is not yet separate from the quality which renders it pleasant.' 2 This is the stage charac-

1 Hobhouse, Development and Purpose, chs. vi, vii, viii, and ix. p. 96 .

2 Ibid., 
teristic of the most primitive peoples. The categories-particularly the category of substance-are not clearly defined. Thus vital functions may be confused with material substance, men and animals may be identified with their shadow, and a pain may be confused with a stone that can be extracted. So, too, it is thought that a man's qualities can' be obtained by eating him. Relations and qualities tend to become substances, substances deliquesce into a series of changes, and the general is confused with the particular. These tendencies of early thought underlie animism and magic, which are characteristic of all primitive races. Mr. Frazer, for instance, has distinguished two forms of sympathetic magic which he calls homoeopathic and contagious magic. Dancing and leaping to make the crops grow high is an example of the former; 'the sympathy that is supposed to exist between a man and any severed portion of his person, as his hair or nails, so that whoever gets possession of human hair or nails may work his will at any distance upon the person from whom they were cut', 1 is an example of the latter. 'Homoeopathic magic is founded on the association of ideas by similarity : contagious magic is founded on the association of ideas by contiguity. Homoeopathic magic commits the mistake of assuming that things which resemble each other are the same; contagious magic commits the mistake of assuming that things. which have once been in contact with each other are always in contact.' 2

There follows a second stage which Professor Hobhouse calls the stage of ' common sense'. It is characterized by the organization of ideas in accordance with the categories and by the differentiation of feeling from belief. At this stage the categories are no longer ordinarily confused and the difficulty of affirming what a man does not like to believe is no longer so strongly felt as in the previous stage. Experience is tested and good evidence is recognized as such. It is no longer believed that an enemy can be harmed by maltreating a likeness of him or that qualities like courage are substances that may be transferred. This is the stage of conceptual thought reached by the ordinary man in everyday life in a modern community when he is not specifically engaged in work of a scientific nature or in problems of religion or philosophy. Just as the simpler mental processes are present

1 Frazer, Golden Bough, London, 1911, part i, vol. i, p. $175 . \quad 2$ Frazer, loc. cit., part $i$, vol. $i$, p. 53. Taboo, it may be observed, is merely a kind of negative magic. 
among men who have reached the conceptual level, so the earliest stage of conceptual thought can be recognized where later stages have been reached, as, for instance, when grace is conferred by the laying on of hands in a modern community.

The beginnings of the common-sense stage may perhaps be found in the second period. Essentially it is characteristic of the third period, and it was only within this period that this stage came to its full development. When this stage is contrasted with that which preceded it, it is seen that it is the clarification of concepts which is the distinguishing feature of the movement of thought. In other words there has been an improvement in the instrument whereby the intellect works-comparable to any other process through which skill is increased. Just as the bow and arrow and the plough have been improved by a series of inventions, so has the instrument of thought been improved ; and just as further improvements in the plough and the bow and arrow after a certain stage has been reached may possibly depend upon further evolution of mental capacity, so possibly progress in the movement of thought may depend upon further evolution of the intellect. Again it should be observed that just as tools such as the plough are handed down as part of the tradition, so, too, the instrument of thought is handed down as part of the tradition and that, therefore, in studying in what follows the manner in which tradition is passed on and stored up, we are studying the methods whereby the instrument of thought as much as any other skilled process is transmitted.

Further stages in the movement of thought may be distinguished. It is not necessary to go into them here. . They arise within the third period, and relatively late within that period. They are characterized by the rise and development of science, philosophy, and religion-religion, that is to say, which has passed beyond the stage of folk-religion.

3. Let us now consider the passing on and storing up of the products of conceptual thinking. We may take the latter point first. Ideas may be stored up in language, customs, folk-lore, institutions, tools, and so on, using tools in the narrower sense of material implements. In one sense language is in itself a great storehouse of ideas quite apart from its function as a means of transmitting ideas regarding specific customs and rights. Slowly and painfully concepts have been elaborated, distinguished, 
clarified ; and as a child learns his native language, in a few years he acquires the products of the thinking of untold generations from whom he is descended. It is difficult to exaggerate the immense importance of this method of storing ideas. Very largely through language alone, though not without the acquirement of ideas otherwise stored, a man passes, by the time he has reached maturity, through a stage of thought corresponding to the primitive stage, to the common-sense stage, and even to the higher stages.

It is obvious enough that at the present day ideas are largely stored in books and the vast importance of writing and printinginventions of the third period ${ }^{1}$-is a commonplace. Similarly, ideas are stored in customs, institutions, rites, folk-lore, and so on. Behind all institutions, ceremonies for example, we must seek ideas. The original ideas which gave birth to customs have often been lost, but whether this is so or not, where an institution exists and is passed on there we are witnessing the perpetuation of an idea, if it is merely an idea that it is the correct thing to perform some simple action on certain occasions. Many valuable ideas are stored up in the making and use of tools and in the practice of skilled processes, especially those which are concerned with the provision of food. We can understand how by the exercise of thought improvements in some tool or in some method are now and again made, and, once made, how they are stored up. What is less easy is to understand precisely how the passing on of ideas so stored is brought about.

4. Something has already been said as to the manner in which by means of language ideas present in the mind of the speaker are transmitted to the hearer; and it has been pointed out that apart from this the learning of any language is in itself a process by which the ideas elaborated by former generations are acquired. Bearing in mind the manner in which ideas are transmitted by language, it is clear how, after the invention of writing and more especially after the invention of printing, ideas thus stored up are transmitted. But before these inventions, and indeed to a large extent after them also, ideas committed to memory by each generation are passed on by language. Among primitive people there is a vast store of ideas affecting conduct, belief, and 1 The beginning of writing-for instance the Maya script-dates from the
second period. 
every side of life which is so passed on. But the storing up of ideas is not merely a matter of memory and the passing on is not merely a matter of language. Rites, ceremonies, implements, and so on, are themselves storehouses of ideas, and the transmission of the ideas therein contained, as well indeed as the ideas solely retained in the memory of past generations, is accomplished in large part by a process of absorption.

This process of absorption is due to the presence of certain innate tendencies. These tendencies have sometimes been grouped under one head and called imitation-as, for instance, by Tarde and others who have studied the process and emphasized its great importance in social life. Following McDougall we may distinguish three innate tendencies. By sympathy is meant that the exhibition of emotional excitement on the part of the agent may induce a similar emotional excitement on the part of the patient. By imitation is meant that there may be a tendency for the patient to assimilate his bodily movements to those of the agent. Important as are these innate tendencies in facilitating the transmission of customs and so on-themselves ultimately the product of conceptual thought-suggestion assumes a far more prominent place.

McDougall's definition of suggestion has already been quoted. According to his view it is essentially the acceptance with conviction of a proposition in the absence of logically adequate grounds for its acceptance. He points out that a proposition is not necessarily communicated in formal language, and further distinguishes certain conditions which are favourable for the communication of propositions by suggestion. Chief among these are deficiency of knowledge relating to the topic in regard to which the suggestion is made, imperfect organization of knowledge, and the impressive character of the source from which the suggested proposition is communicated. These conditions are very prominent among children and in all primitive society. There is no doubt that it is largely by virtue of suggestion that children absorb the tradition of their race and time. Children have little knowledge and what they have is imperfectly organized; further propositions come to them from a source which is impressive and has prestige whether it is from parents or grown-up people or from the conventions ruling in society. Just as children in modern society absorb tradition by suggestion, so do men 
among primitive races where the conditions are even more favourable for transmission by this means. Thus, in order that the products of conceptual thinking of former generations should be transmitted, it is not necessary that there should be convictions based on logical grounds or that formal language should be employed. Tradition can be, and is to a large extent, ' absorbed'.

5. Tradition, having thus passed on, is retained, and in the process of retention habit plays an important part. Habit applies to all forms of mental process and to all forms of action. Once something has been performed in one way, it is easier to do it again in that way than in any other. ' This is the great principle', says McDougall, 'by which all acquisitions of the individual mind are preserved ', 1 whatever the mode of acquisition may be.

6. The products of the thinking of past generations are thus stored up, transmitted, and retained. Additions are made to the store, and improvements are made in the method of storing. Tradition, by which is meant this store, is thus cumulative. The acquirements of past generations are passed on to the present generation and, modified in some degree, are transmitted to future generations. New generations, therefore, so far as acquired knowledge is concerned, begin at the point where the former generation left off. It does not, of course, follow that additions are always being made to tradition; it may be for all practical purposes stagnant or much that has been acquired may be lost. ${ }^{2}$ It does, however, permit of accumulation, and this fact introduces an element of vast importance into the life of man as compared with his pre-human ancestors.

It is not, however, correct to say that this is a wholly new element. Tradition may be and is present before the level of conceptual thinking has been reached. Many animals, for example, live in herds and instinctively take shelter when one member of a herd utters a warning cry. If at some past time a new enemy has appeared, they will have learnt by experience to take shelter. Subsequent generations will raise the warning cry on the appearance of the enemy, because what we may think of as a tradition has grown up concerning the hostile nature of the species with which past generations were brought into contact. Thus

1 McDougall, Social Psychology, p. 111.

2 See on this subject, Rivers, 'Disappearance of Useful Arts', Festskrift tilleguad Edward Westermarck, 1912. 
we may think of a tradition among horses that motor-cars are not dangerous. When cars were first introduced horses tended instinctively to avoid them. Many horses learnt from experience that cars were harmless, and young horses, seeing motorcars for the first time in the presence of older horses, which have learnt the lesson, absorb the tradition that they are not dangerous. Tradition among animals can go even farther than this. To some degree it seems that the manner of nest building among birds is traditional and not instinctive. ${ }^{1}$ But compared with the vast importance of tradition among men, tradition among animals is an almost negligible feature in their lives, and for all practical purposes we can think of tradition as a peculiar characteristic of man due to the higher mental process to which he has attained.

7. In any race at any given time there is thus a vast body of tradition. These traditions govern both the degree and the direction in which the various mental processes function. What is meant will be clearer if we think of the influence of the mass of traditions upon physical characters. At any given time there are a number of tools and skilled processes known and employed and, in the first place, the degree in which the body is exercised will depend upon their nature. It may be that hunting with bow and arrow, or fishing in canoes, is the chief occupation of the men. The degree of physical exercise will be conditioned by these traditions and so too will, in the second place, the nature and direction of physical exercise. Similarly with regard to the intellectual faculties, the degree to which they are used will be conditioned by the form of language, by the necessity of learning what custom lays down as the acquirement of an average member of society, and by the opportunities and inducements generally to the employment of reasoning. The direction in which the inteflectual faculties work will also be closely conditioned by tradition. Having absorbed the traditions of the race and time, the direction which reasoning follows will always be very largely

1 There is a tendency to exaggerate the importance of tradition among animals. Thus it has been said that nest building is 'largely' traditional among birds. But the Curator of Birds in the New York Zoological Park raised a number of wild birds from incubated eggs, and found that these birds, although they had never had parental care or example, nevertheless learnt to fly, to build nests, and to perform all these activities-though they were sometimes slower in so doing than they would otherwise have been-that are at times said to be 'largely" traditional (see Lull, Organic Evolution, p. 170). 
governed by what has been acquired. Few men strike out new paths, and, when they do, the direction of the path is seen to be greatly influenced by the previous trend of thought. Tradition, as it advances, thus tends to proceed in grooves.

The manner in which tradition conditions the degree and nature of the manifestation of the instinctive tendencies is equally well marked. Among some races, such as the Eskimos, fighting is almost unknown. Though among such races the instinct of pugnacity is probably less strongly given in the germinal constitution than among other races, to a large extent the relative absence of fighting is certainly due to tradition. Among the Eskimos we find a great body of custom and ideas all of which tend to impress upon them that fighting is wrong. An emotion again may be greatly developed by some racial tradition. Self-assertion is much more developed among the members of the larger and more prosperous European races than among the smaller and weaker races. The attitude of the Englishman is very different from that of the Dane, who will tell you that Denmark is only a little country that wants to be let alone. Latterly the attention of the world has been drawn to the peculiar manner in which the teaching of various historians, moralists, philosophers and others has moulded the outlook of the Germans and come to direct the expression of their emotions. Thus in a most striking manner it has been made obvious how a peculiar body of tradition, ultimately the product of conceptual thinking, has directed the emotions and sentiments of a whole people into certain channels with such disastrous results. The direction of an instinct is often changed ; the tender emotion may be diverted among the childless to charitable works, and the instincts of celibates obviously become much changed. Any instinct indeed is capable of manifesting itself in very different forms according to the outlet which it finds, and the nature of the outlet is determined chiefly by tradition. 'On a, aujourd'hui', says Joubert, ' non seulement la cupidité mais l'ambition du gain.' 1 The simpler emotion has, that is to say, become more complex under the influence of the traditional environment.

8. Lastly we may note that the tradition acquired and present at any one time may form the basis for the selection of men and of groups of men. There are often, especially among the higher 
races, differences in tradition as between the groups and classes in the same race. The differences are usually much greater between different races. The tradition present in a race, whether because it includes a higher degree of skill, enables a greater degree of coherence to be realized, is the foundation of more vigorous endeavour, or because it is a combination of these and other elements, may enable one race, when in conflict with other races, to overcome those other races, should the latter be possessed of a tradition, which, taken as a whole, is, relatively to the conditions of the contest, less valuable. There is thus a process of natural selection based upon differences in tradition, just as there is a natural selection based upon differences due to modifications and also upon differences due to mutations. Selection of modifications, as we have seen, produces no permanent results. But selection of tradition, like selection of mutations, has results which may be permanent. In primitive society, where tradition within a race tends to be uniform, this selection of tradition chiefly comes into play in the conflict between races. In more advanced societies where there are considerable differences in tradition between the classes, it also comes into play within races.

The conflict between races is always in large measure a conflict between traditions whether or not the differences in tradition are a measure of more fundamental differences. In these conflicts a mass of tradition may be wiped out and lost for good or any degree of amalgamation of tradition may, take place.

At length within modern races there arises a competition of ideas of a rather different nature. Within civilized races there is no longer a mass of tradition which has to be accepted as a whole ; there are different ideas which may be said to compete. One idea may get the better of another within the minds of the majority without involving any elimination of men who hold any other idea, because men can now change their outlook-not, of course, in the case of the great majority by any logical process. Thus a struggle comes into being between ideas, customs, and institutions in modern communities which leads to change without involving human selection.

These considerations pave the way to an examination of the influence of the actual conditions under which tradition has been elaborated. We have seen that conceptual thinking develops with, and is furthered by, the use of speech. It does not develop 
merely to a particular level and there stop. There is a movement of thought which at least in its earlier stages takes the form of a clarification of concepts. The products of conceptual thinking are stored up and handed on in the form of tradition. This tradition is cumulative. Not only are additions made to it but the methods of storing are improved. Elements may also be • lost. Finally, forming, as it does, a very important element in the manifestation of human mental character, men, and more particularly races of men, may be selected in accordance with the degree and quality of tradition acquired by them. 


\section{$\mathrm{XX}$}

\section{THE ORIGIN OF TRADITION}

1. The study of the formation of tradition is a matter of great complexity. Many important aspects of the problem await elucidation. It is proposed here to reduce the discussion to the barest outline, and to confine it to the origin of skilled processes and in particular to the origin of skill in the provision of the essential requirements of daily life. We further take into account only the most important aspects of the environment-namely, the influence of fertility and the influence of contact of man with man and of race with race. All questions connected with innate differences in mental and physical characters will be for the present disregarded. We want to know-other things being equal-how skill will originate and grow, given such differences in the environment as regards fertility and contact as exist.

2. The first problem which confronts us is concerned with the differences in the environment arising from differences in fertility. To this term a special meaning is attached. Fertility as here employed corresponds to what is usually spoken of as the wealth of any country or district. The nature and abundance of the fauna and flora, the surface features of the land, the composition of the upper layers of the ground, the minerals and to some degree the climate, as well as other factors, all go to make up the fertility or wealth of any area. The differences in the factors which go to make up fertility as between different portions of the world's surface are well known; they are very great-areas closely approximated being at times sharply differentiated one from another whereas in other places conditions remain very similar over large areas.

The most important fact to be observed in this connexion is that there is no absolute standard of fertility. 'The term fertility', says Marshall, 'has no meaning except with reference to the special circumstances of a particular time and place.' 1 Fertility is in fact purely a relative term, and, now that we have for the

1 Marshall, Principles of Economics, p. 160.

D d 2 
moment put aside differences in mental and physical characters, fertility is relative for our purpose only to the amount of skill and such other elements of tradition as may be in existence. An area, which is very fertile for a race with some agricultural skill, may be far from fertile for a race with a knowledge only of hunting and fishing, and the presence of minerals obviously adds nothing to the fertility of an area to a race lacking knowledge of their use. The country inhabited by the Eskimos, who maintain a standard of living that, relative to the standard achieved by many races, is far from low, would be wholly infertile to a race not possessed of the peculiar skill distinctive of Eskimo culture.

It should be noticed that, so far as fertility is dependent upon animal and plant life, the fertility of different regions of the world's surface has varied in the period covered by the emergence of man, not merely relatively but absolutely. There have been climatic changes of which the glacial periods were the most remarkable. There have been changes in the boundaries of seas, such as the Mediterranean, which have profoundly affected the fertility of the neighbouring regions. The Sahara at one time was certainly not as barren as it is now. In any survey, such as that we are going to make, which was at all detailed, such changes would have to be taken into account. But in what follows they may be disregarded.

It may be observed here that in certain areas there is a destructive element in the total make-up of the surroundings. The variable climatic conditions of Australia and India which have been noticed in another connexion are of importance also in this respect. The hurricanes of New Caledonia are another example. So too are all those features in the Central African environment which destroy the products of man's handiwork. ${ }^{1}$ This destructive element is only an exaggeration of certain features which characterize all areas ; nevertheless where these features are exaggerated important results follow. There may be, for instance, two areas, otherwise equally fertile ; but if the destructive element is important in one area, it is probable that so high an economic stage will not be reached or maintained there as in the other, and further, for the same reason, there will be less tendency for any advance in tradition to be made. The reason for this will be clear when we have examined the influence which fertility has upon

1 See Cureau, loc. cit., p. 253. 
skill. For this destructive element is a kind of negative fertility, and just as fertility is on the whole favourable to progress in skill, so what is equivalent to its absence is a hindrance to progress in skill.

3. Fertility is thus relative to the tradition prevalent at any one time. Let us suppose that man has spread over the surface of the world and that the tradition present is everywhere very similar, being of a simple form so far as skill is concerned, and consisting in a rudimentary knowledge of hunting and fishing. It is clear that certain areas-the great deserts, the frozen wastes of the north-will be absolutely infertile; with this degree of skill only, life cannot be maintained there. The remaining areas will vary from those which are just sufficiently fertile to permit of life being maintained to those which are the most fertile relative to this particular degree of skill. Supposing for the moment that the degree of skill remains everywhere similar, it follows, from what has been said earlier, that population will be most dense and the return per head highest where the fertility is greatest. Other things being equal, differences in fertility are thus responsible for important differences in social life.

From these differences in fertility therefore consequences of the greatest importance follow. In the first place, where population is most dense, there, other things being equal, will be the greatest amount of contact between men. With the results that flow from contact we shall deal later, but it may be mentioned here that contact is in itself a stimulus leading to the origin of, additions to, and modifications of, tradition, and that the greater the amount of contact the more quickly and the more thoroughly is tradition transmitted. Therefore, in the first place, where the fertility is greatest, there will be the greatest opportunity for existing tradition to be quickly absorbed. In the second place, quite apart from the question of contact, where fertility is greatest there is also for other reasons the greatest stimulus to increase in skill. To illustrate this point let us remember in what fertility consists. The existence of areas of greater fertility implies that there are in such areas either a greater abundance of animals, plants, minerals, and so on which can be of use, or a greater variety, or both together. Let us further remember how inventions come about. For thousands of years in a variety of ways the fact that the seed if sown would produce its like in twenty-, fifty-, or 
a hundred-fold measure must have been pressing itself upon the attention of man when supporting himself by hunting and fishing only. How often the metal softened in the camp-fire before the method of fashioning a far more effective tool than stone was adopted, we cannot guess. Eventually the facts were observed, the practical applications to the needs of life undertaken, and valuable additions made to skill and embodied in tradition. Man in fact throughout the greater part of his history has not gone about consciously trying to better his lot. Rude and poor as life was, his necessity was not the mother of his inventions. Once, however, an invention was made it became a necessity, and it is more in accordance with the facts to invert the proverb and to regard invention as the mother of necessity. But where should we expect the facts to be noticed and the improvements to be made in skill? Obviously where they most often happened, where, in other words, fertility was greatest. For it is in those regions where there is the greatest abundance in quality and quantity of useful objects that there will be the greatest chance of their usefulness being observed, and that there will be derived the highest return per head from any improvements in skill.

The greater the fertility, therefore, the greater the incentive to progress in skill. This conclusion may seem to be in contradiction with the commonly accepted idea that, when a living is easily obtained, there is a tendency to stagnation. ${ }^{1}$ This notion is derived from observations and descriptions of countries where, so it is said, such is the bounty of nature that the hand has, so to speak, only to be stretched out in order to gather in the fruits of the earth. This idea is largely founded upon an under-estimate of the labour undergone, especially on the part of the women, and upon an exaggeration of the returns per head, and, as far as it is so derived, it is incorrect. But it is true that often in such regions there is a tendency to stagnation; such tendency, however, is in no way derived from the fertility; it arises because to the tradition there is added for quite other reasons a spirit of apathy and of listlessness. How this comes about may be discussed later but it may be mentioned here that a destructive element in the natural endowment of any area, such as not infrequently exists in tropical regions, is one cause of the existence of this spirit.

- Statements such as the following are commonplaces of anthropological literature. 'These [Brazilians] have found life too easy, as the latter [Australians] have found it too difficult' (Herbertson, Man and His Work, p. 3). 
4. Fertility then is relative, but, other things being equal, where fertility is greatest there we find the greatest incentive to increase in skill. From this follow certain important consequences. So long as progress in skill keeps along any one line, so long as it takes the form, for instance, of improvement in methods of hunting, so long, as a general rule, will the same areas remain the more fertile areas. But this does not always follow. It may be that some remarkable invention, that of the bow and arrow perhaps, renders areas, previously not the most fertile, the most remunerative. Hitherto it may have been that in such areas the hunting of game was for various reasons difficult until a higher degree of skill was reached, but this higher degree having been reached, these areas became more remunerative than those previously the most fertile. With the progress of skill there is thus a shifting of the centres of the highest fertility.

This shifting of centres becomes more marked where progress takes a different turn, where, for instance, agriculture arises. For this there are two main reasons. First, it generally happens that an area which is very fertile to a high degree of development of one kind of skill, as the north-west coast of America is fertile to hunting and fishing, is relatively infertile to another type of skill, as, for instance, agriculture, in any case in its lower forms. It is rare that any area is so highly endowed that at one and the same time it offers a large return to the development of a particular form of skill and is also relatively fertile to the first beginnings of another and ultimately far more remunerative type of skill. Secondly, it is not as a rule where skill is specialized that we find the beginnings of a new form of skill. Tradition tends to move in grooves and where attention is with success concentrated upon one type of skilled process, it is not there that we should expect to find the origin of quite another type of skilled process. This is only an application of a principle noted in another connexion, namely that evolution proceeds from the generalized and not from the specialized type.

In this manner the differences in natural endowment which distinguish one part of the world from another bring about a shifting of the centres of progress. There are other factors still to be considered which work in the same direction; but apart from them this shifting comes about. Though this shifting has been a feature of every stage of human history, it must have 
been most noticeable where the great steps in skill were madewhen agriculture superseded hunting, when animals were first domesticated, and when metals came into use.

5. To differences in fertility we have to add differences in contact as affecting progress in skill. Contact may be considered as varying in quality and quantity and may be thought of as influenced chiefly by two groups of factors which we may call geographical and economic. We may first ask how it is that differences in contact bring about differences in skill.

From contact two results follow. The spread of tradition is in varying degrees encouraged and progress in tradition is more or less stimulated. For the most part differences in the spreading of tradition are to be traced to differences in the quantity of contact, though differences in quality also play a considerable part, while differences in the manner in which progress is stimulated arise chiefly from differences in the quality of contact.

It is obvious that tradition can only be transmitted by means of contact. Later developments such as writing and printing allow of contact at a distance ; but for the most part it is personal contact with which we are concerned. It is on the whole true that, other things being equal, the more contact, the more swiftly and easily is the existing mass of tradition disseminated throughout any society, and that therefore the more fertile an area, the greater the chance of the spreading of tradition. But other things are by no means equal throughout history. At a certain stage, owing to the working of what we may sum up as economic factors, there comes about a profound change in the organization of society, the nature of which is described below. This change markedly favours the transmission of tradition and therefore we have to remember that the amount of contact ascertained by the density of population is by no means a fair measure of the degree in which the passing on of tradition is facilitated. Further consideration of this point may be left until we come to deal with the changes referred to. Geographical factors also have a bearing upon the transmission of tradition, but since their influence is chiefly pronounced in the stimulus to skill arising from the conflict of traditions of different quality we may leave them to be dealt with later as a whole.

Of more importance than the bearing of contact upon the transmission of tradition is the part it plays as a stimulus to 
tradition. The remarkable change which at a certain period supervenes in the organization of society and to which reference has been made above, has as far-reaching effects in affecting stimulus to skill as it has in facilitating the transmission of tradition. Again we may leave the consideration of this point until we have described the nature of this change. It is clear, however, that the presence of other men is on the whole a stimulus, though not in itself an important stimulus, so long as tradition is fairly uniform. Nevertheless the more contact there is, on the whole the more stimulus there is. 'There be thoughts', wrote Maitland, ' which only come to men when they be tightly packed.' 1 But it is when traditions of different quality come into contact that the stimulus becomes important. Where this happens, we have to distinguish two things. There is the passing over of the elements peculiar to each tradition to the other tradition and there is the stimulus which the mere contact affords.

The passing over of elements of one tradition to another and the stimulus afforded go more or less together. Under certain circumstances there is no passing over and there is no stimulus, or at least they are reduced to a minimum. This sometimes happens when two cultures varying very markedly one from the other come into contact-one being distinguished by the possession of a far higher degree of skill than the other. The less skilled race is driven from its territory, and if it survives it is because certain areas of its territory are relatively infertile to the higher skill of the conquering race. A remnant of the less skilled race survives in some corner of its former territory and reaches a modus vivend $i$ with the dominant race. This modus vivendi may take the form of an almost complete disregard of one race for the other; no influence is exerted by one on the other; little, if anything, is absorbed from the other tradition. Some such condition was reached as between the Veddahs, the Todas, the Central African pygmies, and the races respectively surrounding them.

In order that the contact should be effective it is necessary that the differences between the cultures should not be too great. It is most effective in such a region as Western Asia where in close proximity there are several areas varying markedly one from another. In such a region there will grow up, owing to the variations in fertility, somewhat different traditions. These 1 Quoted by Fisher, Soc. Rev., vol. i, p. 61. 
traditions coming into contact will rarely wholly overwhelm one another ; there will be a perpetual taking over by one tradition of some elements from the other so that each tradition will be enriched as it could not have been enriched had it depended entirely upon the environment which gave birth to it. Further, the fact that implements, skilled processes, customs, and institutions of a strange nature are now and again coming under the notice of each race acts as a stimulus to thought and invention quite apart from the advantage that may be gained by the absorption of what is valuable into its tradition. It is probable that in this stimulus to thought we have to recognize one of the most important factors making towards progress.

There is no space to pursue this subject here. It may perhaps be noticed that, when two cultures come into contact, all the elements do not spread with equal rapidity. It has been observed, for instance, that, when a tradition comprising greater skill comes into contact with a less full tradition, 'it is the recognition of the superiority of the natural objects and arts which prevails and makes possible the acceptance of other elements of an introduced culture.' 1 Certain general principles can be made out but there is much that requires elucidation. There is a considerable body of evidence in favour of the view that megalithic buildings have been derived from one centre. If this is so, it is a remarkable example of the fact that an art of an obviously impressive though not.useful nature can pass from one race to another without any noticeable assimilation of other elements of the culture where the art was first practised. Again, if this is so, it is not improbable that useful arts which in their way are equally impressive may have similarly proceeded from one centre. It is remarkable also that mythology can apparently spread from one culture to another. To whatever source we may attribute their origin there seems little doubt that in North America, for instance, tales and legends have spread from one side of the continent to the other after very different economic systems had been evolved and without any marked spreading of other elements in the traditions at the same time. ${ }^{2}$

6. Having thus indicated the manner in which contact influences the origin, growth, and transmission. of tradition we may turn

1 Rivers, 'Contact of Peoples', Essays and Studies presented to W. Ridgeway, p. 478 .

${ }^{2}$ Boas, J. A. I., vol. xl, p. 536. 
to consider what bearing geographical and economic factors have upon contact. The former problem may be passed over with a few words ; the latter will require a rather longer discussion. The former has been the subject of much attention and the main facts, which are all that concern us, are familiar; the latter has not received so much attention though it is of great importance.

Not only the spread of elements of culture but also the movement of peoples are governed by geographical factors. An isolated area is isolated from the slow permeation of elements of tradition, as well as from migratory races. To the degree, therefore, to which any area is isolated, it is removed both from those influences which favour the passing on of elements of tradition as well as those which form an incentive to skill. Isolation is never complete. The more isolated an area is, the less often have migratory races reached it, with the result that when they have done so, there has usually been a conflict between a culture so dissimilar in skill that the migratory race has often wiped out the original race. Apart from the visits of migratory races the beneficial results of contact are reduced to a minimum within an isolated area. The differences in traditions are small, and both the elements of culture which can be acquired and the stimulus which can be derived from contact are of little importance. What these geographical features are is fairly well known.

The first factor is isolation by sea. America was apparently peopled some time in the first period. Subsequently to the date of its original peopling it may be regarded as having been for all practical purposes isolated from the other continents and thus its inhabitants had been long cut off from contact with the rest of the human race when it was ' discovered' by Columbus. America was probably visited, perhaps more than once, by parties reaching the Pacific Coast and was certainly visited by the Norsemen, but this small amount of contact has exerted no influence upon the evolution of skill. America is the most remarkable instance of isolation by sea. Isolation by sea has exerted a profound influence upon Australia, New Zealand, and Oceania; and important also has been the semi-isolation of Africa, which continent, until great progress had been made in navigation, was only in contact with Asia by a narrow neck. Among primitive races deserts and mountains hinder contact in almost equal degree, and, though in one sense not geographical features, forests and 
vegetation, especially in tropical countries, exert a profound influence upon contact.

Other features in the environment may be regarded rather as facilitating contact. Rivers form great arteries of communication, and it is remarkable how small a hindrance they are to contact. Thus 'Christopher Gist, exploring the Ohio in 1751, found a Shawnee village situated on both sides of the river below the mouth of the Scioto, with about a hundred houses on the north bank and forty on the south. The small and unique nation of the Mandan Indians were found by Lewis and Clarke near the northern bend of the Missouri in 1864 in two groups of villages on opposite sides of the river. They had previously in 1772 occupied nine villages lower down the stream, two on the east bank and seven on the west.' 1 Freshwater lakes, though not salt lakes, have again been centres in which contact has been favoured, as was the case in Switzerland at one period and also in Mexico and Peru where the highest level of progress was reached in America.

In considering any area there are certain general features of importance quite apart from those which actually hinder or facilitate contact. In the case of any area there is what we may call its location, that is to say, its position relative to its surroundings. The position of any area in Africa with regard to its distance from the north-eastern corner, through which contact with inhabitants of other continents came about, is obviously of great importance, and similarly is the distance of any one area from the sea when navigation has been developed. To location we may add diversity of features which brings about the contact between different economic systems. This feature of diversity is chiefly relevant when considering large areas ; and in this respect Europe and Asia are far more favoured than America.

Lastly, differences in language are a potent influence in hindering contact though they are rather evidences than causes of isolation. When once established differences in language intensify the isolation due to other causes. It may be observed in passing that, contrary to what might be expected, the degree to which among primitive races 'foreign' languages are understood is considerable.

Though in the first two periods the importance of the various factors in hindering and facilitating contact has varied greatly, it is sufficient, when taking a broad view, to bear the general 
nature of these influences in mind. The tendency in the third period has been obviously to reduce vastly the importance of all hindrances to contact arising from features of the environment. Railways cross deserts and tunnels pierce mountains, and that amount of contact, small though it may be, which owing to the inventions of writing and printing now occurs between all parts of the world, can transmit more tradition than wide avenues of contact between neighbouring peoples previously made possible.

Here we can leave this matter. It has been worked out in much detail. All that is necessary for our purpose is to bear in mind the fact, which indeed is obvious, that contact is in various ways hindered and facilitated by geographical causes.

7. We have now to consider what is less familiar-the bearing of what we may call economic factors upon contact. These factors are themselves but an expression of the working of the economic system, and the economic system is correlated with fertility though modified by the degree and kind of contact allowed by the geographical environment. Therefore in considering the working of these economic factors, we are in fact considering another aspect of the manner in which the environment bears thus indirectly upon contact.

Progress in skill, as we have said, is correlated with increasing density of population, and, other things being equal, the greater the density of population, the more contact there is. But other things are not equal; the chief disturbing factors are those connected with the organization of society in their influence both upon the quality and quantity of contact. The vast importance of the step which led to the origin of primitive society has already been remarked upon. When we observe primitive society in more detail, it is seen that, compared with the type of organization which arose in the third period, this form of society essentially consists of a repetition of similar elements. ${ }^{1}$ A perfect type of this form of society would be one which consisted merely of a collection of families, not differing essentially one from another, each being a microcosm of the whole society. Actually we find that primitive society is usually composed of the repetition of larger elements than the family, which Durkheim calls ' clans'. This term is employed in order to mark the family and political

1 See Durkheim, Travail Social, chs. vi and vii. 
features which characterize these elements. The variations of the constitution of primitive society are very numerous, but it has essentially this form which Durkheim calls 'segmentary'. The solidarity of such a society rests solely upon the similarity between these clans; the binding forces, that is to say, which keep the society together, do not take their origin in the fact that these elements are complementary to each other and in combination form one organic whole; such coherence as there is arises solely from the fact that these elements, in so far as they are similar, coalesce together. Typically, then, we find in this stage of organization families living side by side very largely independent one of another but combining to form clans. These clans have as a rule no definite constitution; but at times, owing to internal dissensions or warfare, a leader or leaders may arise who put themselves at the head of these groups.

As has been said, variations in and developments of this type of organization may arise and, though among primitive races some approach to the higher type can be detected, the evolution of the higher type of organization is essentially a development which took place in the third period. This type of organization we call organic, as distinguished from segmentary, because in the first place the elements which go to form the whole do not simply cohere owing to their similarity; they are in the nature of more or less specialized organs all of which are necessary in order that the whole may exist. Each again has a particular rôle and each organ is formed of differentiated parts. These organs are not simply connected one with another like links in a chain; they are co-ordinated one with another and subordinated one to another into an organic system. The parts are dependent on the whole and the whole on the parts.

In an organic society, in fact, men are grouped according to their profession and not according to their descent. One of the marks of the segmentary type of society is that men are grouped according to their descent, real or fictitious, and it is this relationship which determines their position in society. In the organic type of society, that which determines a man's position is the function which he fulfils. In this form of society there are remnants of the older form of organization, as is seen in the recognition of areas, smaller elements such as parishes being united into larger areas such as boroughs, which are united in 
turn into provinces of which the country is composed. This, however, is not the form of organization which keeps the higher type of society together. That which forms its essential difference from the lower type is the organic nature of the relation of the parts - the complementary co-ordination of the various professions one with the other. It is this interlocking of complementary parts and not the coherence of similar segments which cement the form of society typical of the third stage.

8. The evolution from the segmentary to the organic form of social organization was relatively speaking a rapid process. The approach that can be made to the organic type within the segmentary type is only slight. Doubtless the new type developed within the old forms. Thus we see among the Hebrews the assumption of priestly functions by a single tribe-that of the Levites. But the development of the organic type cannot go far without breaking up the segmentary form of organization; the number and importance of functions does not correspond with the existing forms of organization and cannot long develop within them.

We have now to ask what brings about the evolution of the organic type of society and then what bearing these forms of organization have upon the growth and transmission of tradition. It is rather that the crumbling away of this segmentary organization of society brings to birth the organic type than that the growth of the latter is the cause of the disappearance of the former. This must be so because, as we have seen, the existence of the segmentary type is a barrier to the development of the organic type. This crumbling away is brought about by what Durkheim calls the growth of moral density. Growth in moral density comes about through the pressure of the increasing contact between men performing the same functions, and this causes the decay of the segmentary type of organization and brings about an organization resting upon function. The effect acts upon the cause and accelerates the process. The growth in moral density is connected with the growth in volume of society which is measured solely by the increase in population. But growth in moral density, though only made possible by growth in volume, does not of necessity go hand in hand with it. Growth of moral coalescence is not correlated absolutely with the increase in volume. The density of population can become very considerable, while the moral density remains relatively undeveloped. This 
result is sometimes attributable to the survival of certain elements of segmentary organization to which religious feelings have become attached and which have, therefore, resisted the forces which make for their dissolution. This is the case, for example, with regard to the maintenance of the family system in China, which is the great barrier to a development of moral coalescence. The most powerful motive in Chinese life is the devotion to the family system, and this motive prevents the progress of division of labour. Among the Hindus the caste system has the same effect. But it is not merely a survival from a former segmentary organization to which religious motives have become attached; it has actually undergone a development for the most part in opposition to the organic organization of society. 'The divisions', says Sherring, 'among the Hindus involving complete separation in respect of marriage and social intercourse, number not hundreds but thousands. In other words the Hindu brotherhood is split up into innumerable clans, holding not the smallest connexion with one another, acknowledging no common bond save that of idolatry. . . . Caste dissolves the social compact found in other countries, infuses the poison of deadly strife into the small village communities scattered in tens of thousands over the land, induces enmity between neighbours on the most trivial grounds, carries out its own childish rites and laws with Draconian severity, exercises the strongest power of disintegration the human race has ever been subject to, and only displays a spirit of binding and uniting in relation to those selfish creatures who belong to one and the same caste, and who are thereby kept apart from all the rest of mankind by an unnatural divorce.' 1

Thus primarily it is the breaking-down of the segmentary type of organization and the condensation of society which permits the division of labour. In the end the advance to the higher type of organization is attributable to the growth of population. It is first necessary that a relatively high degree of skill should be attained involving a certain density of population. Then there arises a tendency for the segmentary organization to break down and give way to the organic type. The breaking-down may be more or less imperfect and there may be factors which work against the full development of the organic type.

9. The degree to which the higher type of organization favours

1 Sherring, Hindu Castes and Tribes, vol.iii, p. 218. 
the increase of skill cannot be over-estimated. So long as all men are more or less self-supporting and perform all the functions necessary to maintain existence, the securing of food, the building of shelters, the fashioning of weapons, the making of clothes, their skill in any one direction must remain small. But as soon as certain men begin to concentrate upon certain functions the whole position changes. It then becomes possible for skill to reach a far higher level, and with the further division of functions there is practically no limit to the degree of skill which may be obtained. A concentration upon one function and the association of men so employed are themselves powerful stimuli to further progress. The facts are familiar and there is no need to elaborate the position. At length classification by function tends to override classification by any other standard, and all those engaged in any country in any one function become associated in such a manner that the stimulus is further emphasized and the possibility of any favourable new departure being lost is reduced to a minimum.

The importance of the organic type of organization in favouring the transmission and storing of tradition is equally well marked when compared with the segmentary type. The more society is divided into self-contained and self-sufficient segments, the less likelihood is there that any development in one segment will spread to the other segments. All events tend to be localized, and any promising departure in a new direction is unlikely to penetrate far and become embodied in tradition. New departures are more likely to be lost altogether than to be preserved. The more society is organized on the organic type, on the other hand, the more facilities there are for the transmission and storing of tradition. As the segmentary system breaks down, the transmission and storing of tradition themselves become special functions. Various means of communication are elaborated, the spreading of news becomes a function in itself, teaching becomes a profession, and libraries are established.

10. Such very briefly are the nature, causes, and results of the change in the organization of society so far as concerns our present purpose. There are certain points in this connexion to which some allusion may be made. We have already commented on the fact that the transformation to the higher type does not go hand in hand with growth in numbers. The two examples from India and China showed how little correspondence there may be 
between density of population and division of labour. Again, in modern communities there is an apparent anomaly. There are considerable differences as regards the amount and kind of tradition present in the different strata of society, and this may appear all the more anomalous because in such societies the division of labour has been developed to the greatest extent. Tradition, it would seem, should be very uniform. Uniformity of tradition, on the other hand, is a mark of primitive society. But this anomaly is only apparent. Among primitive races there is infinitely less tradition than among civilized races. Among the latter not only is the amount of tradition vast, but it is in the course of rapid evolution, and there is therefore in the first place a far greater possibility for differences in tradition to arise. Further, the very fact of the division of labour means that different forms of skill are cultivated deliberately by different elements of the community. Thence arise the differences in social customs and conventions which are apparent in the different strata of modern society. Where there is division of labour, there must be, as we have seen, differences in the acquirement of the tradition of skill, and we may think of the differences in the social and conventional tradition as originating in the differences in functions. Where there is ownership of property on a large scale, ownership may be regarded as a function in society, and clearly enough certain customs and conventions become connected with this function and distinguish the so-called upper classes. Differences in social tradition so arising do lead to some approach to a segmentary division of society. Once classes are set up, there is a tendency for a man to take his position rather according to his class than according to his function. Actually what happens is that the upper classes adopt certain functions, the sacerdotal or the military for example, and thus the differences in custom and convention become somewhat artificially perpetuated, perpetuated that is to say over and above the distinctions which would be the inevitable accompaniment of different functions. In this fashion class becomes of considerable importance in modern communities as a barrier to the transmission of tradition, and there is thus less coalescence between those performing different functions than the mere division of labour brings about. Interesting as are these modifications of the working of what we have called economic factors, they do not seriously affect the 
conclusions to be drawn in a broad survey. The transformation to the organic type of society is a mark of the third period, and therefore within this period the conditions became such as to stimulate the growth of skill and to facilitate the transmission and storing of skill in a manner never before approached.

11. Given, therefore, differences in fertility and in the geographical configuration as between one part of the world's surface and another, and given migration, we can understand how skill arises, how it is transmitted, and how it is stored. The factors considered are not the only factors, but they are the chief factors, and a consideration of them alone enables us to understand how in the main progress may be achieved-how, that is to say, to some degree command over nature may be attained. Further, in thus tracing the causes of progress in this narrow sense we are tracing in some manner the origin and growth of other elements in tradition, not directly concerned with command over nature. This can be illustrated by a reference to the results obtained by Messrs. Hobhouse, Wheeler, and Ginsberg. As we have seen, progress in skill falls easily into a series of stages-hunting and fishing, agricultural and metal-using. These authors further subdivided hunting people into two stages, agricultural peoples into three, and pastoral into two. They then investigated the correlation between these stages and the conditions relative to justice, the family, warfare, and so on. It was found that in the points indicative of the degree of social organization there is a certain correspondence with economic advance. 'This correspondence', they say they have found, 'in the development of government and of justice alike, in the fact that as we mount the scale there is more of government and more of the public administration of justice within society, and in the fact that the unit for government and for justice extends. Both intensively and extensively there is a growth of order corresponding roughly to the industrial advance. On the other hand economic development has no necessary connexion with improvement in the relation between members of a society. It does not imply greater considerateness or a keener sense of justice, and in some ways may be held even adverse to them. Thus in relation to marriage and the position of women, we find little change throughout the grades, and of those which we do find the most marked are specifically connected with the economic factor, viz. the extension of purchase and of general 
polygamy . . . Economic causes are again associated with the development of organized warfare and the substitution of the enslavement of prisoners for their slaughter, liberation, or adoption.' 1

In other words social institutions are correlated to a considerable extent with economic systems, and in tracing economic systems to differences in the ' wealth' of the environment and to differences in contact, we are at the same time accounting to the extent of this correlation for the existence of most social institutions. Those aspects of tradition which are not correlated with the economic system are obviously often explicable as due to the direct influence of the environment; for differences in the environment which have little or no bearing on the economic system may give rise to differences in these aspects of tradition. It has often been pointed out, for example, that the trend of legends is explicable as due to certain aspects of the environment; thus in Assyria legends were largely connected with floods. In large part, therefore, differences in fertility and differences in degree and kind of contact account not merely for progress in skill but also in large measure for many social institutions, and those aspects of social institutions which are not so to be explained are in part due to the influence of aspects of the environment which do not contribute directly to fertility, and in part to a complicated interaction of tradition. It was remarked that, so far as can be seen, the more conscious methods of adjusting the level of population to the optimum number are not correlated with the economic system. There is no evidence of infanticide and abortion, for example, being so correlated. The occurrence of these institutions among certain races irrespective of the economic conditions may be accounted for by differences in the environment which do not affect wealth. We have seen that certain methods must of necessity be employed, and the adoption of one method in one place and of another in some other place may be due, for example, to the presence in one area of plants by the use of which abortion can be brought about, or the presence in another area of an instrument designed for some other purpose which can be employed for this object. In other areas the practice of killing deformed children from superstitious motives may have been developed into a regular custom of infanticide, or the taboo upon intercourse for short periods may have become developed into a practice of abstaining for prolonged periods.

1 Hobhouse, Wheeler, and Ginsberg, loc. cit., p. 254. 


\section{XXI}

\section{THE RELATIVE IMPORTANCE OF TRADITION AND HEREDITY}

1. We are now in a position to attempt an estimate of the relative importance of germinal change on the one hand and of the accumulation of tradition on the other. ${ }^{1}$ The direct influence of the environment cannot be left altogether out of account ; it is, however, already apparent from what has been said on the subject that, although the direct influence of the environment may modify the course of history, it is not an important factor in comparison with the other two. That which renders the problem so difficult is the fact that the manifestations of human characteristics are shaped both by traditional changes and by germinal changes, and that each kind of change reacts on the other. Thus if we observe increasing subservience in the characteristics of a race, we must remember that previous elimination of the more self-assertive may manifest itself in this manner by moulding tradition through a long course of years, that the nature of the environment may set the current of tradition flowing in this direction, or that both factors may be at work; and that, so far as the second factor is at work, it may for reasons to be explained below cause the elimination of the more self-assertive and so accelerate the process. It is in fact very difficult to arrive at any precise conclusions regarding particular problems, but we shall, after considering certain problems, reach a fairly definite conclusion regarding the relative importance of these factors in general.

2. With these problems in view we may first consider the general characteristics of the intermediate period and then those of the three subsequent periods.

It is clear that at the beginning of the intermediate period the ancestors of man were living under those conditions which we

1 According to Delvaille (Histoire de l'Idée de Progrès, p. 405) Terrasson, writing in the eighteenth century, was the first author to emphasize the importance of tradition in the modern sense-particularly in his work La Philosophie applicable à tous les objets de la raison. 
have seen to be common to all species in a state of nature and that, therefore, if we are to speak of history at all, history was at this time an expression of germinal change alone. We know nothing directly as to what happened within this period, but from such hints as we can get we must apparently make certain deductions. We know that within this long period-many times as long as the whole period which has succeeded it-there was accomplished by far the greater part of the evolution of the human intellect. This is the conclusion we draw from the fact that the intellect of primitive races, which must be taken as representing man in the first period, differs in a small degree from that of modern man relative to the difference which we must suppose to have existed between the intellect of man in the first period and that of the pre-human ancestor. Again the picture we must draw of our ancestors in the intermediate period is that of a species acquiring domination almost solely by its intellect. Further we have seen that the advance to the stage of primitive society was only made possible by an important step in mental evolution-so important a step that none of our ancestors who did not take it have survived. The deduction to be drawn is clearly that in the intermediate period history was founded in the main on germinal change. If we find reason to conclude that germinal change has ceased to play so important a part in the following periods, it should be remembered that the sum of all these latter periods represents but a fraction of the length of the intermediate period. If we regard human history as a whole and date the beginning at the time when our ancestors began to move away from those conditions which govern the existence of all species in a state of nature, then we must conclude that germinal change has been the explanation of what has happened during far the greater part of history as defined above.

3. The course of history from the beginning of the first period to the present day presents certain remarkable features. It has to be remembered that the course of events in the first and second periods has been reconstructed from evidence gathered almost solely in Europe, and even then in large part from one country, namely, France. Europe was not the centre of progress, and it may be that the abrupt replacement of one stage of culture by another, of which we find evidence, would not be what we should find nearer the centre of progress. There the evolution of culture 
may have been more continuous. From time to time waves of migration swept westwards and a higher stage of culture overwhelmed the lower. It may be that the culture systems on reaching Europe followed lines of development of their own which, owing to circumstances being relatively less favourable, did not carry them so far as nearer their point of origin. Therefore the next wave as a rule brought a higher degree of skill which replaced the then existing European system.

However this may be, what we do undoubtedly find is a relatively slow rate of progress in the early part of the first period. Progress became more rapid in the Upper Palaeolithic and in the Neolithic. Finally at the opening of the third period progress was vastly accelerated and has gone on with ever-increasing rapidity subject to certain checks at various times and places. This speeding up of the evolution of skill is the chief outstanding characteristic of history since the opening of the first period, and with it we may associate the facts that progress has not been uniform as between different countries, and has been subject to set-backs within countries especially within the third period. Recalling what was said in the last chapter as to the influence of fertility and contact upon the growth of tradition, we may now note that the main outline at least of these outstanding events is apparently explicable as the result of the working of these factors. We may begin with a consideration of the facts regarding America.

America was apparently peopled from Asia at an early date.1 Putting aside the Eskimos, it is probable that the invaders possessed a simple and fairly uniform type of culture and that the cultural differences found at the time of the discovery as between races in different parts of the continent were indigenous. In other words America is a country in which a section of the human race was early cut off from contact with the remaindersuch contact as occurred with the Norsemen and may have occurred with the Pacific Islanders having been without importance. For many thousands of years cultural evolution had proceeded independently in this isolated area. At the time of the discovery it had in Mexico and Peru reached a fairly high level. A form of writing had even been evolved, and in general the level

1 See Am. Anth., vol. xiv, 1912, for a symposium of the views of American authorities on this point. 
of culture was such as we may compare with that attained shortly before the opening of the third period in Eur-Asia. It is possible that at this time the evolution of culture was on the verge of making a great step forward similar to that made in Eur-Asia in the third period. But in any case progress in America had fallen behind progress in Eur-Asia by some thousands of years, and it is thus interesting to compare the endowment of America with that of Eur-Asia.

It may first be noticed that the area is a large one and that it is diversified in that it contains within it many types of geographical and climatic environment. Nevertheless the shape of the area as a whole, and more especially perhaps the absence of relatively fertile areas in proximity differing sharply from one another such as we find in Eur-Asia, renders it less favourably disposed to facilitate contact than are parts of Eur-Asia. In this connexion it may be observed that the Isthmus of Panama is a barrier rather than a means of communication. Apparently the civilizations of Mexico and Peru had no knowledge one of the other. - The differences in respect of fertility are more remarkable. Generally speaking America is not notably less fertile than Eur-Asia, relative to skill in hunting and fishing. The northwest coast of North America is possibly more fertile relative to this type of skill than is any other area in the world. But when we come to examine the endowment of America as a whole relative to skill in the lower forms of agriculture and in the domestication of animals, we find that America is poorly endowed compared with Eur-Asia. Maize and rice are the only important indigenous cereals. All the other valuable cereals were absent from America. This is a very important fact because cereal culture is in many ways a far more profitable art than either root-culture or arboriculture. But America is poor not only in cereals ; many plants which play so important a part elsewhere in primitive agriculture are absent-the plantain, yam, banana, breadfruit, and date-palm, for instance. Among the important plants we may note the potato, coco-nut palm, manioc, arrowroot, and the cocoa plant. Brazilian arrowroot is, it may be observed, very easily propagated. 'Even if the plant is left in the ground when the root has been taken, new tubers grow from its joints after the first shower of rain.' 1 Some such plant as this in America

1 Payne, History of the New World, vol. i, p. 311. 
or as the yam elsewhere must have presumably been that first cultivated.

The poverty of America in animals fit for domestication is even more remarkable. If we except the reindeer, milch animals were entirely absent-a fact of great significance. The Indians had made the fullest possible use of such animals as there were. 'Setting aside the reindeer, an unprofitable animal on any soil which produces any better crop than moss, the Indians had domesticated every animal in the continent which was capable of domestication.' 1 Among them was the llama, an animal of restricted usefulness, as it cannot be used for draught and cannot be milked. The fact that there are several varieties of llama would seem to point to the fact that it had been brought under domestication many centuries before the discovery of America. Turkeys, dogs, pheasants, ducks, and geese were also domesticated as well as a few other animals of little importance.

When compared with Western Asia, America is seen to be poorly endowed in animals fit for domestication and plants suitable for agriculture. But it must be remembered that Western Asia is far richer than any other area. America is not poor compared with Africa or Australia. We may now glance at the endowment of these other regions beginning with Asia.

4. It is often a matter of the greatest difficulty to trace the original habitat of the species of animals and plants which after many centuries of domestication have given rise to the varieties now in use ; in some cases it is impossible ; the camel, for instance, has never been found wild. ${ }^{2}$ In some cases the evidence is vague and merely points to the original home as having been within some large and not very clearly defined region. Nevertheless an inquiry into the original habitat of the more important domesticated animals and plants leads to a very remarkable conclusion. We find that with few exceptions Western Asia was the home of the great majority of such species. The chief exceptions among animals are the elephant, buffalo, reindeer, and llama. The llama originated in South America, the reindeer is found in the circum-Polar regions, the elephant and buffalo are Indian. It is probable that the original home of the camel was somewhere in this Asiatic region, and, if this is so, then all milch animals, with

1 Payne, loc. cit., vol. i, p. 289.

2 The single-humped Arabian camel has never been found wild, but it is said that the double-humped Bactrian camel has been found wild in Turkestan. See Flower and Lydekker, Mammals, p. 297. 
the exception of the reindeer, whether belonging to the ovine, equine, bovine, or camel group, had their original home in the Asiatic region-a very important fact when the large part played by milch animals is borne in mind. It should be perhaps mentioned that certain species had a wide range and extended beyond this Asiatic region vaguely defined though it is. The wild ancestors of the ox and the pig were indigenous in Europe as well as in Asia. ${ }^{1}$ The richness of the Asiatic endowment as regards plants is no less remarkable. Practically all cereals of importance with the exception of maize and one or two others originated there. Wheat, barley, oats, rye, millet, and others of much importance at a certain stage of progress in skill are indigenous in that region.

5. Turning to Africa we find that the large majority of domesticated animals and of cultivated plants are not indigenous; when not introduced by Arabs or Europeans, they were derived from Egypt and into Egypt they had probably been brought from Asia. Of those animals now domesticated cattle, goats, sheep, and fowls were derived from Egypt, the dog from Arabia, the cat was brought by the Arabs, while pigs, muscovy ducks, turkeys, and pigeons were introduced by the Portuguese. Sorghum grain, millet, eleusine, colocasia (arum), yam, and the banana were introduced from Egypt; the sugar-cane, rice, wheat, oranges, limes, cucumbers, melons, gourds, onions, and hemp were introduced by the Arabs, while the coco-nut palm came from Asia and the date-palm from the Mediterranean basin. "The only doubtful exceptions are ground-nuts . . . which may be indigenous, and certain semi-cultivated beans.' 2 It may be observed that, although Africa is poor as regards animals and plants fit for domestication, the negro never domesticated with one or two exceptions such species as were capable of domestication. Thus the guinea-fowl and the coffee plant, both indigenous, were not domesticated by the negroes.

6. Of the natural endowment of other regions it is not necessary

1 Wild oxen were abundant in Europe in the time of Julius Caesar, and of them the Chillingham herd may be a remnant. Horses were abundant in Europe in Neolithic times. But it is probable that both oxen and horses were first domesticated in Asia. The ovine group was originally situated in the mountain region of Central Asia, though Ovis savigni, apparently allied to the Argoli, has been found fossil in the Forest Bed of Norfolk (Flower and Lydekker, loc. cit., pp. 355, 357, 367 , and 382).

'Johnston, British Central Africa, p. 429. 
to speak. The absence of all mammals, with the exception of marsupials, from Australia is well known, and the handicap resulting therefrom is obvious. There are, however, certain broad facts with regard to fertility and geographical configuration to which attention may be drawn here. With respect to the fertility of tropical regions in general there are two facts of importance. For the most part cereals are absent, and it is chiefly trees and roots among indigenous plants which lend themselves to domestication. Arboriculture and root-culture, however, do not in many respects give as good a return as does the culture of cereals. Further, fruits and roots cannot be stored as a rule ; their food value is not high, and in many ways the problems presented by the cultivation of roots and trees do not afford anything like the same stimulus to skill or require anything like the same advance in social organization and in settled and regulated conditions of life as does the cultivation of cereals. Again, in the general make-up of tropical conditions there is frequently an element of destructiveness. The rapidity with which in tropical Africa products of human handiwork are destroyed is well known, and to a greater or less degree something of the kind is a feature of all tropical regions.

7. Turning to geographical conditions in general as favouring or hindering contact we find America isolated and, as a land mass taken by itself, practically divided into two parts in which the conditions do not markedly favour contact--especially contact between different economic systems in close proximity. The remaining land masses may be thought of as radiating out from one centre. The central area is not only in general favourably located, but is in itself so formed as to favour contact. The diversity of its surface, the inland seas, the steppes, plateaux, mountain uplands, plains, and river valleys render it in this respect richly endowed. Forests were less of a barrier to contact in this region than elsewhere, and in this respect Western Asia was better situated than Europe. Tropical forests and jungles form far more serious barriers than do forests in temperate regions, and the discovery of the use of metals is of less effect in reducing the hindrances due to this barrier in tropical than in temperate countries. Looking at the tracts of land which radiate out from this centre we see how isolated is the greater part of Africa. Egypt is indeed almost a part of the Western Asiatic region, but 
it is nearly cut off from the rest of Africa by desert, being connected with the remainder of the continent only by the valley of the Nile which in its middle regions is almost barren while its upper reaches pass through huge tracts of marsh and swamp. As we approach the south of Africa we come to what in primitive times was a blind alley. So, too, in whatever direction we pass outwards from the heart of Eur-Asia, whether we cross the sea to Australia or travel to the eastern or western boundaries of the Eur-Asiatic land mass, we reach regions where, on account of what we may call their location and of other factors such as the presence of forest or jungle or on account of the configuration of their surfaces, contact is not favoured. We must in addition bear in mind that it is the central region which is the most richly endowed as far as fertility is concerned.

We may remember that there was another factor to which as a stimulus to the formation of skill and to the transmission and storing of tradition we found reason to attach great importance-namely, the replacement of the segmentary by the organic type of social organization. This great change took place in the Eur-Asiatic region and must be traced indirectly to the characteristics of that region as a whole. For we found that, before such a change could come about, a growth in the volume of population was necessary, and growth in volume is directly dependent upon fertility and all other elements in the surroundings which favour the increase of skill.

8. There is, therefore, at the least a very remarkable correspondence between the outstanding events of history since the opening of the first period and the distribution in space and time of the chief factors which influence, tradition. We may set events in America up to the time of the discovery against events in the rest of the world. We do not know with what degree of skill the original emigrants were armed; but since, generally speaking, the fertility of America relative to skill in hunting and fishing is not, if at all, inferior to fertility elsewhere, we have no reason to imagine that progress in the first period would be less rapid than elsewhere. Again the presence, though not in great variety, of easily cultivatable plants would facilitate much as elsewhere the transition to the most primitive form of agriculture. But the poverty in cereals and animals fit for domestication and the absence of milch animals indicate an environment which, 
relative to conditions in Western Asia, offers far less stimulus to progress. Further the general configuration of the land does not favour contact as in the latter region. Nevertheless considerable progress in cereal culture and in the domestication of animals had been made, and in Mexico and Peru, where the presence of lakes, the absence of formidable barriers, and the diversity of surroundings offered by the proximity of high and low land rendered contact most effective, there had grown up societies with a relatively high level of skill. But America had lagged behind Eur-Asia, and the degree to which this was so is only what would be expected if we suppose that the innate capacity of the inhabitants of both regions was once approximately equal and that, while some had wandered into less favourable surroundings, others had remained in or migrated to more favourable surroundings. It is at least clear that if we do set the general trend of events in America against events in Eur-Asia there is nothing incompatible with the theory that the constitution of the environment can account in the main for the differences in the growth and accumulation of skill.

Putting America aside, the same may be said of events elsewhere. The rich endowment of Western Asia has been emphasized, and this region appears to have been the centre of progress in the first period and was most certainly so in the second and during the earlier part of the third period. Everything leads us to suppose that at any given time up to a comparatively recent date the level of skill was higher in this region than elsewhere. From time to time waves of migration spread outwards-most often apparently into Europe and less often into Africa and Oceania-carrying with them a degree of skill so much higher than that existing in the outlying areas that an abrupt transition to another culture took place accompanied by varying degrees of extermination of, and mixture with, the races situated there. If we bear in mind the endowment of, and general conditions in, Africa and Oceania, the trend of events in these regions is again comprehensible on the same principles as those indicated above. It may also be observed that the principle of the shifting of the centre of progress owing to the change in relative fertility may be seen at work in Western Asia. At a certain period, for instance, after a particular degree of civilization had been reached, the great river valleys of the Nile and of the Tigris and the Euphrates, which formerly had been 
relatively less fertile, became the most fertile regions. Later the progress connected with the industrial revolution led to another remarkable shifting of the centre of advance-into regions, that is to say, where coal is abundant and easily worked. This shifting of the focus of progress is often overlooked by those who seek an explanation of the fact that the site of the earlier civilizations has not remained the site of the later civilizations. "The contrasts ', says Professor Elliot Smith, ' in the achievements of the various peoples cannot be explained away by lack of opportunities, in face of the patent fact that among the most backward races of the present day are some that first came into contact with, or were even the founders of, civilization, and were most favourably placed for acquiring culture and material supremacy.' 1 The shifting of the centre of progress is at least in part an explanation.

Within the third period there took place that remarkable change in social organization already described which so markedly stimulated the growth of skill and facilitated its transmission. This change had the most profound effects on events in Europe and Asia. With it we must associate a remarkable speeding up of the progress in skill which has been the chief feature of the last period of human history. The explanation of the acceleration of progress is thus ultimately based on the endowment of different regions in respect to fertility, location, and the facilities offered to contact. It may also be noted that the further trend of events in Europe and Asia is made comprehensible by this principle of the shifting of the centre of greatest fertility on the one hand and by the imperfect realization of the organic type of society on the other. In India and in China there have been forces working contrary to the full development of the organic type of organization in the shape of devotion, largely on religious grounds, to various forms of a segmentary division of society, and this may prove to be one of the chief reasons why in Eastern Asia we seem to be faced with a backwater out of the main stream of progress.

Further, as tradition became more complex, small differences in the environment often gave a favourable or unfavourable turn to the development of skill. Thus after the invention of writing countries were greatly favoured that possessed suitable

1 Elliot Smith, Presidential Address to the British Association, Section H, 1912, p. 577 . 
writing materials. In China writing, owing to the cumbersome form which it has taken, has never been of more than a very restricted usefulness. It has been suggested that the differences between the clarity of Greek thought and the vagueness of Indian speculation are in part due to the greater use made of writing in the former country. ${ }^{1}$

9. Thus in general if we survey the outstanding facts we find that explanations based on the influence of the environment in stimulating progress in skill suggest themselves. Racial differences do exist and play a part. We may gain some idea what this part is if we now turn to consider the nature of these differences, and we may first pay attention to the larger differences such as those which distinguish the negro from the European. These differences, it must be emphasized, are only large relative to the differences which exist between European races. Relative to the difference between the ancestor in the intermediate stage and modern man they are almost negligible.

Some analysis has been made of the differences between negroes and white men and with regard to these differences we may observe two things. In the first place they can only in part account for the differences in performance. Before coming into contact with Europeans, negroes had not passed beyond the stage of primitive thought; but it is evident that they are not innately incapable of so doing. The results of educating the negro have been to narrow the conception of the gap which separates him from the white man. Just as D'Alembert and Diderot would not believe that the Russians could be civilized up to the European standard, so a later generation believed that the negroes could not be so civilized; but 'negroes are now indisputably the equals of white men in categories in which one hundred years ago their masters would have confidently argued that they were naturally incapable of attaining equality'. ${ }^{2}$ Nevertheless there are differences; the negro is intellectually on the average somewhat inferior, and certainly possesses somewhat different emotional and temperamental characteristics.

In the second place from the end of the first period to the present day the evolution of mental characters shows little correspondence to the evolution of skill. Whereas progress in

1 Rhys Davids, Buddhism, p. 40. Labour, p. 57.

${ }^{2}$ Olivier, White Capital and Coloured 
skill has been vastly accelerated, progress in the evolution of mental characters has slowed down and may within the third period have'almost ceased. Selection within this last period has, it may be remembered, come largely to be on account of disease.

The nature, therefore, of racial differences and the trend of evolution of mental characters tend to confirm the conclusion derived from a study of the influence of fertility and of contactnamely, that the outstanding events are to be traced to environmental rather than to germinal changes. But germinal changes are not negligible. We have seen how these circumstances which favoured progress in skill in the earlier part of the third period also favoured mental evolution in just those directions in which the white race is superior. Germinal changes may thus be regarded as contributing to the progress which occurred at this time; evolution in the direction of self-assertion and other qualities, which characterize the white races, must have accelerated the cultural changes already in progress. When Mr. McDougall says that ' in so far as differences of cultural level are associated with differences of level of innate intellectual and moral qualities, cultural superiority must be regarded as the effect, rather than the cause, of innate mental superiority ', 1 the above considerations suggest another view. It would appear more probable that cultural changes and germinal changes went hand in hand, and that they were both products of the same environment; no doubt one kind of change reacted on the other, but there seems as little reason for holding that the former were the effect of the latter as for holding that the opposite was the case. Mr. McDougall's view meets with considerable difficulties when we extend our inquiry to the events within the third period. He attributes the cultural level of the early civilizations to previous mental evolution, but as he thinks that there has been no appreciable evolution since that time, the events of the last five thousand years including the continued progress in skill must be otherwise accounted for. It is surely more probable that this series of events beginning with the rise to the early civilizations and continuing to the present day is in the main based upon one determining cause. This we have found reason to identify with the influence of fertility and contact upon the development of tradition; to germinal change we attribute in the earlier epochs

1 McDougall, Group Mind, p. 119. 
no little influence but an influence which is contributorysupplementing for a time the process which continued when germinal change had largely ceased. So far as we find this conclusion to be supported by further inquiry, so far it is correct to say that races with the innate mentality of the negroes would not by themselves have reached the position attained by the white races, though it is not true that the white races progressed directly because of their superior innate mental endowment.

10. We may now inquire into the importance of the lesser racial differences. There are usually recognized in Europe three chief racial types-Nordic, Mediterranean, and Alpine. If innate mental differences exist, we should expect to find associated with each type certain peculiar traditional features; for, as we have seen, innate racial peculiarities tend to set the current of tradition flowing in certain directions, and the association of different institutions and beliefs with particular racial types would suggest that innate mental differences are making themselves felt, though, before this conclusion can be accepted, it must be shown that the play of environmental factors upon tradition has been fairly uniform for all these types. A considerable amount of evidence can be accumulated to show that such association exists. Thus the Nordic peoples are mostly Protestant and the Alpine and Mediterranean peoples mostly Catholic or Greek Church. The fact that during the Reformation a choice was set before most European nations as to what religion should be adopted-the issue hanging in the balance for some time in many places-seems to indicate that the conditions were more or less equalized and that the adoption of the Protestant religion by the Nordic type was influenced by certain innate characters attaching to this type-self-assertiveness and love of independence, for instance. 1 The South Germans, who are of the Alpine race, remained Catholic, while in the Netherlands the Nordic Dutchmen became Protestant. Similarly there is a certain correspondence in Europe between the distribution of types of political institutions and that of racial types, and much other relevant evidence could be brought forward. We have seen how sharply negroes are distinguished from Europeans in emotional disposition; similar differences, though on a much smaller scale, are visible as between European races. It can hardly be doubted that the Irish and the 
Scotch differ from the English in disposition and-though this is more doubtful-there may be intellectual differences. There may be some slight difference between the English and the French intellect; it is hardly likely that the French passion for logic and the English aversion to it are altogether traditional characteristics. It would, therefore, appear that there are innate mental differences between European races which tend not so much to thrust races along certain paths as in the course of generations to colour tradition and even at crises in national life, when a choice is presented, to determine which path shall be taken. These differences may be explained-though it is a somewhat speculative enterprise-by supposing that the ancestors of the various racial types were exposed to different surroundingsthe ancestors of the Alpine race to patriarchal institutions, of the Nordic race to more individualistic institutions-and that thus subservient and assertive types were respectively favoured. ${ }^{1}$

The influence, however, of innate racial differences between races so closely related as those of modern Europe must not be overrated. It is possible to point to many examples of the fact that the distribution of institutions, customs, and so on does not always correspond to that of racial types. In Belgium, to take only one instance, the Walloons are of Alpine stock, that is to say, they are racially similar to the great majority of Germans. But the Walloons are distinctly French in character and sympathy, whereas the Flemings-the other element in the Belgian population-are Nordic and are in certain respects more allied to the Germans with whom racially they have little in common. Either in such a case innate racial differences do not exist of the kind suggested, or, as is more probable, they exist but have been obscured by tradition. That tradition is the predominant factor in shaping those characteristics, of which we think when we have any nation in mind, can be seen when we look at two examples of race formation in modern times. Those characteristics which we find to be distinctive of the Boer race, for instance, can be traced to the peculiar turn given by the environment to the peculiar tradition-remarkable both in its religious and social aspects-brought by the first settlers. The racial elements represented among the Boers of to-day are well known-Dutch for the most part with some admixture of French and English blood. 
But it is not to this peculiar germinal constitution but to the motherhood of the South African veldt acting upon a peculiar tradition that what is distinctively Boer is to be attributed. So, too, there are no mental characteristics as manifested so definite as those which are associated with Americans. But it is impossible to think that they are the product of the influence of the various racial stocks which have gone to make the modern American population. Clearly enough these characteristics are almost wholly traditional, and it can without much difficulty be shown how the peculiar geographical, social, and political environment has given rise to them.

Can we further understand how these distinctive accumulations of tradition are maintained? Let us remember that a man requires not only a home in the usual sense of a roof over his head, but what we may call a home in the world of tradition, and that just as most men find a dwelling-place in the country in which they are born, so most men at the same time find a home in the world of tradition in the same country. Now in any area, where men have for a longer or shorter time acted together, there has been evolved a certain tradition under the influence of those elements in the surroundings enumerated above. If another group of men has been associated together in a very similar area, the tradition there elaborated will show a likeness to that in the former area. But there may be considerable differences. Small differences in the aggregate of influences sometimes have far-reaching effects, and again what we regard in our ignorance as 'chance' happenings may have, as we shall point out later, profound consequences. Small differences and apparently unimportant happenings give turns and twists to the direction in which tradition is built up, and the individual differences between one tradition and another become exaggerated by the tendency of tradition to move in grooves. The peculiarities of a tradition become embodied in the sum of all the institutions-using that term in the widest sense-characteristic of a race and, as each man passes under the influence of those traditions, so these peculiarities are maintained. As often pointed out language is of less importance than might be supposed. A common language does not imply a common tradition, and on the other hand a diversity in language does not imply a diversity in tradition. Institutions of another kind are of more importance. It is institutions like F 2 
the public schools of England which embody the distinctive elements in social tradition. The case of the Jews is especially noteworthy. There is no common language, no Jewish state, but one thing a Jew has in common with other Jews-his religion. Round the Jewish religion centres all that is distinctively Jewish, and the clinging of the Jew to his religion has resulted in the maintenance of a Jewish race amidst all the strange vicissitudes to which the Jews have been subject. 'Qu'est-ce qui a conservé le Juif à travers les siècles et l'empêché de disparaître au milieu des nations? C'est sa religion.... Or ces rites protecteurs, cette cuirasse ou cette carapace d'observances qui l'a défendu durant deux mille ans, et que rien ne pouvait transpercer, notre esprit occidental l'a entamée. . . . Si le judaïsme, débilité, venait à se décomposer et à se dissoudre, qu'adviendrait-il du Juif? Fermé et sauvegardé par sa religion, le Juif ne risquait-il point de s'évanouir avec le judaïsme ?'1

Again, it has been pointed out that men have often achieved fame as contributors to the civilization or literature of countries other than that to which they by race belonged. It has been said that no one could have been more French than the English Hamilton, the Swiss Rousseau, the Italian de Maistre, the German Heine, or the mulatto Dumas. Great contributions to the building up of what is typically British have been made by men who were not of British blood though of course distinguished British patriots. A string of names from Simon de Montfort to Disraeli can be quoted. On the other hand, 'natives of the British Isles have helped to create the armies and fleets, and to build up the politics of most European States. In the eighteenth century you might have found an Irishman directing as Prime Minister the fortunes of Spain, and another those of Naples, a third commanding the forces of Austria, and a fourth seeking to rebuild the French dominions in India. Scots as a rule restricted their attention to Protestant countries, but John Law in the early years of that century did wonderful things with French finance. The right-hand man of Frederick the Great was a Scot, and Scots took more than their share in the making of Russia-an article of almost exclusively foreign manufacture. Peter the Great himself had a mother of Scottish birth, and the fact made all the difference between him and his imbecile half-brothers. Napoleon

1 Leroy-Beaulieu, Israël chez les Nations, p. 77. 
himself was not a Frenchman by birth ; one of his marshals, an Italian, became king of Sweden, and founded the present Swedish line of monarchs.' 1

This reference to the nature of minor racial differences thus tends to confirm what was said above. Germinal differences are not to be disregarded, but we must reject such theories as those of Gobineau and Houston Chamberlain, who see in race the primary explanation of all national achievement. Mommsen poured scorn upon the Celts and Vacher de Lapouge attributed most of the misfortunes of France to the brachycephalic element in the population. ${ }^{2}$ But we are now sceptical of any such facile explanations of the course of history.

11. We can now come rather closer to the problem if we go on to examine certain instances of germinal change that we know to have taken place, and ask what effect is to be attributed to them and to such traditional changes as have accompanied them.

Again and again in the course of history certain stocks have been exterminated. This was apparently the fate of the Neanderthal race, and in modern times was the fate of the Tasmanians. But though in this manner the average germinal constitution of the whole species has been altered, and as a rule raised, there has been little or nothing in such events working towards the further evolution of the remaining stocks.

Intermingling of different racial stocks has been of frequent occurrence. $^{3}$ As we have seen, if the differences are large, vigour may be exhibited in the first cross but will soon disappear; unfavourable combinations of characters on the other hand are likely to arise, and the mulatto thus tends to be a genetically undesirable type. Nevertheless, the undesirable character of the mulatto is in large part traditional. The mulatto is neither of one race nor the other and he knows it. He is an outcast. There is no tradition which he naturally absorbs. He neither grows up with the pride of the white man nor with the feeling of community with his coloured relatives, whose position with regard to other races is generally accepted as something inevitable. In the world of tradition there is no home for him. There are no channels which enable his capacities, such as they are, to develop in a favourable manner, and we have thus to give greater weight

1 Pollard, Factors in Modern History, p. 15.

2 Vacher de Lapouge, Sélections sociales, pp. $293 \mathrm{ff}$.

3 For a discussion of this problem see Hoernes, Naturund Urgeschichte des Menschen, vol. i, pp. $119 \mathrm{ff}$. 
to tradition than to an unfavourable germinal constitution in producing the results which we see.

When the differences are less, there is again the advantage of hybrid vigour, though this again is transitory. More important is the chance of a favourable re-combination of oharacters. Many interesting speculations have been made, though they are all very fanciful, with regard to the fortunate blendings which have produced some races, the bringing together of practical capacities and imagination and the like. In general we may regard such crossing as genetically favourable and of considerable importance in history. Its frequent occurrence in Europe and Asia has probably favoured those regions as compared with India, where racial differences have perhaps been too great, and China, where they have perhaps been too small.

Nevertheless, it is probable that too great an effect has been attributed to the genetic results of crossing. When we come to consider traditional changes, we shall see how potent a factor is the contact of traditions which accompanies the intermingling of stocks and to which the greater part of the results observed have to be in all probability attributed.

It has been noticed that to an increasing extent from the first period onwards the selection of mental characters comes largely to be determined by tradition. The trend of tradition sets in a peculiar direction; tradition develops in a groove and the peculiarities tend to become exaggerated. Men are favoured in so far as they are innately adapted to the chief features of the tradition. In an oligarchic society like that of ancient Peru the naturally servile man fares better than the self-assertive man; among the warlike tribes of North America the servile man goes under. It may be argued that so distinctive a religion as the Mohammedan would only gain ascendancy where the average innate mental faculties were of a peculiar kind; however that may be, it is clear that once having gained ascendancy this peculiar tradition would favour a certain mental type and discourage others. Thus again we see the tendency of germinal change to reinforce the trend of traditional change rather than to determine traditional change.

We also saw that under a segmentary organization of society the tendenoy was towards the preservation of a certain type of mental constitution adapted to the peculiar tradition. Departures 
from the favoured type in any direction are cut off. When organic organization supersedes segmentary organization the whole position changes. It is no longer necessary that a peculiar tradition should be strictly maintained demanding a certain type of mental organization. There is favoured a tradition allowing of the fullest division of labour which not only permits but even encourages various types of innate mental capacity. Various forms of intellectual and emotional capacity thus find a place which would otherwise have gone under. But there are two sides to the picture; though artists and philosophers may survive, even if they do not exactly flourish, the feeble-minded survive also.

12. The selection of mental characters within the third period demands further attention. We have commented upon the incompatibility between group selection and the selection of individuals in the earlier periods. Favourable individual developments may not survive because they are not in harmony with the type necessary to maintain the group. Group selection was active in the earlier part of the third period, and resulted in the evolution of higher types. Later group selection took rather a different form, but though it allowed the survival of variations from the favoured type, it did not altogether cease. There was, however, apparently little tendency towards further evolution of groups as a whole; but germinal changes took place according as to whether circumstances allowed the survival of more departures in an upward or in a downward direction. We may glance at the results of lethal and of reproductive selection. The average germinal constitution of a race may be profoundly changed by the operation of factors that we may place under the heading of lethal factors, though they do not always operate by elimination. In a civilized country with its complicated machinery of social organization, the government may adopt a policy which greatly favours certain types and discourages others; apparently chance happenings may so turn the course of events as to produce the same results. Thus the Revocation of the Edict of Nantes drove out of France men who undoubtedly differed in their innate mental constitution from the average; so too did the setting up of the Inquisition in Spain. The bad management of the liberal movement in Germany in 1848 resulted in the setting up of a régime as a result of which a certain type of German, to whom 
the régime was uncongenial, tended to emigrate. The Bolshevist régime obviously favours a peculiar mentality. From such instances it is clear that the average germinal constitution may be changed in very different directions in neighbouring countries with great suddenness. But in seeking the explanation of what follows in these cases, it is clear that germinal change is following traditional change; it may accelerate traditional change, but it is only a contributory cause of the historical movement that we observe. The lack of enterprise that has marked the Spanish people during the last three hundred years was not caused by germinal change but by traditional change which moulded the outlook and disposition of the people ; germinal change doubtless followed in the same direction and contributed to the present position. The true importance of germinal change is not, as some authors would have it, that it sets movements in progress among civilized nations, but that it exaggerates the tendencies of traditional change, and renders it difficult for nations to get out of the grooves into which they have moved.

Just as germinal change may set in different directions owing to lethal selection, so it may do owing to reproductive selection. In the religious celibates of the Middle Ages we have probably to recognize a distinct mental type, though how far a more valuable type than the average it is impossible to say. Some of their peculiar qualities were doubtless valuable, but they presumably lacked other valuable qualities, since they were disinclined to face the difficulties of the normal social life of their time. Even if it is held that their peculiar qualities were on the whole distinctly valuable, we have, before a final judgement is passed as to the effects of celibacy, to remember that it may have had a beneficial influence on tradition. An authority upon the Middle Ages has said that 'It is more than probable that any real familiarity with the early Middle Ages will lead an unprejudiced student to the belief that the celibacy of the clergy was at that time essential to the setting apart of the clerical order, to the purification of the Church, and to its influence upon the world, that clerical celibacy was in fact a necessary stage in the spiritualization of European society '.1

Reproductive selection chiefly arises because certain classes make greater contributions to the following generation than other

1 Smith, Church and State in the Middle Ages, p. 83. 
classes. ${ }^{1}$ Generally speaking, throughout the Middle Ages, so far as is known, the so-called upper classes contributed more to succeeding generations than the so-called lower classes. The position is now reversed, and it would appear that in the later days of Greece and Rome also the upper classes contributed less to the population than the lower classes. It is possible that the same may have been the case in other ancient empires. The utmost importance has been attributed to the influence of this form of differential fertility upon the course of history. Mr. McDougall, for instance, writes as follows : 'Looking at the course of history widely, we may see in the differentiation of the social classes by the social ladder and in the tendency of the upper strata to fail to reproduce themselves, an explanation of the cyclic course of civilization.' ${ }^{2}$ Clearly a careful inquiry into this subject is needed. There are certain points to which attention may be at once drawn, but we shall not be able to reach any definite conclusion until we have examined farther into the nature of the modifications produced by differences in tradition between the different classes.

Here we may observe that it is altogether misleading to speak, as Mr. McDougall does, of the lower classes as being 'drained' by the operation of the social ladder, even in England at the present day where the chance of rising is as great, if not greater, than it has ever been before. A very limited number can and do rise. The ladder is not only steep and difficult to climb; it is also narrow and does not permit of many upon it at the same time. Further, so great is the prestige of class that very few of the descendants of those who have climbed ever sink back, and yet among them the regression to the mean is always in operation. In other words, supposing that the ancestors of those composing the upper social classes to have been distinguished by desirable mental qualities, their descendants, to whose disappearance so much is attributed, are not distinguished by a similar superiority to the average.

Granting for the moment all that has ever been claimed regarding the superiority of those who climb, the above considerations modify the importance to be attributed to differential fertility. It may further be noticed that, although Mr. McDougall

1 Where, as in America, different racial stocks exist, differential fertility assumes greater importance. See p. 320. $\quad 2$ McDougall, Group Mind, p. 260. 
and others speak of differential fertility as the origin of the cyclical course of civilization, what they in fact attribute to this cause are the periods of decline. Other causes are sought for to account for the equally remarkable periods of advance. Mr. McDougall, for instance, attributes the rise of Greek civilization to a ' happy blending' of two races. ${ }^{1}$ It may be said, on the other hand, that the facts point strongly to the conclusion that both the 'ups' and the 'downs' are part of the same phenomenon. The 'ups' are as remarkable as the 'downs'; one process seems just the reverse of the other. No one proposes to explain the 'ups' as due to the favourable results of differential fertility, and before we can accept unfavourable differential fertility as the chief cause of the 'downs' we may ask if the fundamental cause of both processes is not of another kind. If we should find this to be the case, it of course by no means implies that germinal change does not play a contributory part.

13. The greater part of the discussion in this ohapter has been devoted to an attempt to estimate the importance of germinal differences and of germinal changes; it is clear that in order to arrive at more definite conclusions we have to consider in rather more detail traditional differences and traditional changes. The influence of the direct effect of the environment must not be forgotten, as it frequently enters as a contributory factor into the moulding of the course of history. It may conveniently be considered here.

Temperament is a characteristic of great importance. Just as among men around us temperament is seen to influence each man's achievement, and largely to determine failure or success, so racial temperament is important in determining racial achievement. This characteristic is peculiarly sensitive to the direct effect of the environment, and it may very well be that changes in diet and so on may have influenced the course of history through their bearing upon temperament. At the present day the urban conditions under which so large a percentage of the members of western nations live-smoke, noise, vibration, and so on-may have a bearing upon temperament and so upon achievement.

Such considerations are somewhat speculative. More clear are the results of disease upon temperament. It is a remarkable fact 
that disease, which is chiefly noticeable within the third period, upon the whole then takes a lethal form from which the patient either dies or recovers more or less completely. In tropical regions, however, there are a number of diseases of the non-lethal chronic type, such as the hook-worm disease for instance, which affect a large percentage of the population and sap the energy - mental and physical -of those attacked. The prevalence of any such disease must act in an important fashion as a drag upon progress, and the spreading of such a disease into a country previously unaffected may quite conceivably put a stop to progress, and even bring about a condition of decadence. The decline of Greece has been attributed, for instance, to the introduction of malaria. In answering the question, therefore, why certain areas have lagged behind others, we must not forget the influence of chronic disease when it affects a large proportion of the population, and it is a curious fact that in general the working of this factor has been in the same direction as that of other factors which influenced tradition. Where, that is to say, there has been a low degree of fertility in the environment and little stimulus through contact, there also upon the whole the deleterious effect of disease has been most pronounced.

It may further be noticed that the direct effect of the environment has a bearing upon the moulding of tradition. The apathy and listlessness, for example, resulting from the prevalence of a disease like malaria form an element in the environment and give an unfavourable turn to the development of tradition.

14. Something has been said in the last two chapters as to the nature and formation of tradition, and in this chapter as to its influence in determining the main outlines of the course of history in the chief geographical divisions of the world. The part played by tradition may be best further illustrated by a reference to two problems to which allusion has already been made-namely, the cyclical course of eivilization and modern differential fertility. When we speak of the cyclical course of civilization we have in mind the alternations of periods exhibiting vigour and energy with periods exhibiting apathy. As within the same culture we see progress along one line and within one atmosphere. At times, more especially when the degree of skill is relatively low, there may be scarcely any progress and the condition is one of stagnation. But under such circumstances any turning back is rare. On the 
other hand, when the degree of skill is relatively high, we sometimes see periods of sudden advance often initiated almost at a definite date, and brought to a climax within a few-perhaps even in one-generations. There often follows a period of stagnation or even of decline. Professor Flinders Petrie has set out in a small book some striking facts regarding this tendency based largely on the history of Egypt. He shows how, taking what is left from various periods of artistic production, this tendency may be illustrated, and it would not be difficult to find many further illustrations in the history of any art in the last few hundred years in Europe, and in a less degree in the history of the various sciences. ${ }^{1}$ Sir Francis Galton, whose life work has done so much to illuminate the nature and importance of germinal characters, wrote as follows: 'I have studied the causes of civic prosperity in various directions and from many points of view, and the conclusion at which I have arrived is emphatic, namely, that ohief among these causes are a large capacity of labourmental, bodily, or both-combined with eagerness for work.' 2 Now the periods of advance are marked by the exhibition of these characteristics, and we may ask how far they are traditional in nature. Let us take as an example of a period of advance the Renaissance in England. 'Englishmen of the sixteenth century', says Sir Sidney Lee, 'breathed a new atmosphere intellectually and spiritually. They came under a new stimulus, compounded of many elements, each of them new and inspiring. To that stimulus must be attributed the sudden upward growth of distinctive achievement among them, the increase of the opportunities of famous exploits, and the consequent preservation from oblivion of more names of Englishmen than in any other century before. The stimulus under which Englishmen came in the sixteenth century may be summed up in the familiar word Renaissance. The main factor of the European Renaissance, of the New Birth of the intellect, was a passion for extending the limits of human knowledge, and for employing men's capabilities to new and better advantage than of old. New curiosity was generated in regard to the dimensions of the material world. There was a boundless enthusiasm for the newly discovered art and literature of ancient Greece. Men were fired by a new resolve p. 75 .

2 Galton, Eug. Rev., vol. i, 
to make the best and not the worst of life upon earth. They were ambitious to cultivate as the highest good the idea of beauty.' 1

Let us look at the other side of the picture when energy is lacking. There was a widely held opinion that the world would end in the year A.D. 1000. All classes shared this opinion and prepared for the end, and it can easily be understood how under these circumstances, whatever the innate capacities may have been, little capacity for labour or eagerness for work would be manifested. Again, in the later days of the Roman Empire there was abroad a spirit of lethargy and apathy. The great Empire was like a clock that had run down; the machinery was all intact, but there was no force to set it in movement. It is said that there was abroad in the minds of men prescience of some coming catastrophe, a feeling that the inevitable end was approaching. If such a mental horizon is contrasted with that set before an Englishman of the sixteenth century or a Greek in the age of Pericles, we can understand how eagerness for work as outwardly manifested is profoundly influenced by tradition.

When our knowledge of the circumstances is sufficiently precise, we can always detect the influence of a powerful stimulus in periods of progress. It often takes the form of a national purpose. 'A national purpose ', it has been said, ' is the most unconquerable and victorious of all things upon earth. It can raise up Babylon from the sands of the desert, and make imperial civilizations spring from a score of huts, and after it has wrought its will can leave monuments that seem as everlasting a portion of nature as the rocks.' 2 At times the stimulus may arise from an invention of great import, such as the discovery of the use of metals. More often, however, the stimulus takes the form not directly of invention but of friction between different ideas-the coming into the mental horizon not merely of new skilled methods but of strange and foreign ideas of all kinds. Thus contact of cultures is followed by more than the transfer of the elements of one culture to another; it is in itself a stimulus so powerful as to be of the greatest import in history.

In the past when there was little or no contact at a distance, stimuli were most often due to the physical contact of races whose tradition was not too dissimilar. 'Just as in the mental develop-

1 Lee, Great Englishmen of the Sixteenth Century, p. 28. tion and Reveries, p. 107.

${ }^{2}$ A. E., Imagina- 
ment of the individual a conflict of impressions invites selective attention, so in the spiritual development of society a clash of cultures awakes latent energies of a constructive kind.' 1 This fact has led to the error of attributing to war a more direct importance in stimulating energy than there is evidence for. ${ }^{2}$ Contact usually implied war, but contact is equally effective without war, as can be seen in the example of the Italian Renaissance. The stimulus in this case was largely derived from the rediscovery of the ideals and learning of ancient society, and can only be attributed to war in the far-fetched sense that Greek scholars were disseminated over Europe by the capture of Constantinople. And in more modern times stimulus is often derived from indirect contact in which war plays no part at all-as can be seen often enough in the history of art, in the influence, for example, of Chinese and Japanese art upon European painting.

The effect of a sudden stimulus may be to break down habit, and the importance of habit has been dwelt upon as the characteristic which enables tradition once acquired to be maintained. Such breakings down can be observed in the lives of men and women around us under the influence of sudden stress, and something similar may happen to a nation as a whole. Professor Graham Wallas has, for instance, dwelt upon the importance of the breaking down of habit as accounting for the excesses of the French Revolution. ${ }^{3}$

Though a stimulus may always be detected at work during periods of advance, it is by no means always possible to find evidence of favourable germinal change. There is frequently no evidence at all of germinal change at such periods. In the past no doubt contact often implied racial intermingling, and, though in the present state of biological knowledge we are justified in supposing that crosses between two races not too distinct would

1 Marett, Psychology and Folk-Lore, p. 73.

${ }^{2}$ As, for instance, by Renan in the following passage which contains nevertheless an element of truth : 'La guerre est, de la sorte, une des conditions du progrès, le coup de fouet qui empếche un pays de s'endormir, en forçant la médiocrité satisfaite d'elle-même à sortir de son apathie. L'homme n'est soutenu que par l'effort et la lutte. ... . Ie jour où l'humanité deviendrait un grand empire romain pacifié et n'ayant plus d'ennemis extérieurs serait le jour où la moralité et l'intelligence courraient les plus grands dangers' (Réforme intellectuelle et morale, p. 111).

${ }^{3}$ Wallas, Great Society, p. 80. 'In a settled and traditional society custom is of such overwhelming weight that a law can only act in accordance with it; a sudden change in the machinery of government would break down of itselfnay, in such a society laws can hardly be passed save those that the development of tradition demands' (Belloc, Life of Danton, p. 142). 
usually have favourable results, there is no sufficient foundation for attributing favourable results to all such intermingling as has been done by some authors-von Luschan, for instance. The conclusion would seem to be that germinal change is never more than a contributory cause of advance, and that traditional change is the whole explanation of some of such periods.

Periods of decline are, as we have seen, sometimes associated with unfavourable traditional changes. Tradition may be of such a nature as to sap rather than to encourage vigour. But directly unfavourable turnings of tradition, such as the belief that the world would come to an end at a certain date, are only occasional causes of decline. There is in the course which the development of tradition takes an almost inevitable tendency for periods of advance to be succeeded by periods of repletion and apathy. What appears to happen so often in the history of art after a time of advance may lead to an understanding of the tendency of events in general. If we watch the flowering of any school of art we reach a period, exemplified in the work of the followers of Raphael for instance, and perhaps, as some would say, in the work of Raphael himself, when the artists appear to be lost in the practice of the technical side of artistic production. The ideal, in order to express which the technique has been called into existence, has been lost. The original stimulus has in fact lost its power, and the means to the expression of the ideal have been mistaken for ends in themselves. The technique becomes a plaything, and, there being nothing to express, art takes the form of variations in technique, and becomes, in a word, conventional. This is what would appear to happen as regards progress in generaldue allowance being made in the case of skilled processes for the fact that, since they are designed to achieve practical ends, there is less chance of decadence. But in general we see the fading of the power of the stimulus, the coming of a time of repletion, when men are lost in the mass of what has under the influence of the stimulus been accumulated, and in the case of the arts tend to use the skill achieved as an end in itself. We may trace such a course of events in the history of Greek thought. The course of events may be profoundly modified in a multitude of ways by the appearance of a new stimulus and so on. But we may say that such is the inevitable working of the factors upon the formation of tradition, that periods of stagnation, of repletion, and 
sometimes of decadence tend to occur after periods of sudden advance.

It appears that we should attribute differential fertility, with its possibly serious effect upon the direction of germinal change which marks these periods, rather to the prevalence of apathy than, as Mr. McDougall and others would do, attribute to differential fertility the evidence of decline. For it is the upper classes upon whom the sense of apathy weighs, and it is they who contribute least to future generations. Further, we may remark that decline is not so mysterious a matter as is sometimes suggested. Such complex organizations are vigorous civilizations that, as has been said, "the wonderful thing is that they exist at all; what needs explanation is not so much the decay of some, but rather the long persistence of others.' 1

In concluding our reference to this matter, we may notice that in recent times, when the working of tradition is known to us in detail, changes can be traced as due to tradition to which the cyclical changes are similar though of greater magnitude. Attention has been drawn to the moulding of tradition in Germany in a particular direction which so powerfully affected the achievement of that race. These events cannot be explained as due in the main to germinal change, though possibly, as observed, the German régime initiated about 1850 may have resulted in the emigration of a certain type of German, and thus in this way germinal change may have played a minor part. So too England during the Boer War was very different to England at the election of 1906 , and the difference which was obviously traditional was enough to affect the achievement of the race. In such examples, which could be multiplied indefinitely, we see how the current of tradition may set in one way or another quite apart from germinal changes, and such changes of current may lead, when they hold good over generations, to periods of advance or of decay.

15. There remains to be discussed the importance of tradition in modern differential fertility. It is first necessary to examine rather more closely than has yet been done the manner in which mental characters as manifested are due to tradition, and then to ask how far the particular traditions, to which the upper and lower classes are respectively subject, account for the mental characteristics manifested by them. 
As pointed out, it is obvious that the direction in which the intellect works and the degree to which it works are very largely determined by tradition. Thought will be in the primitive or common-sense stage according to the nature of tradition. It is not so obvious that the manifestation of the disposition is equally governed by tradition. The negroes are innately placid, good tempered, and un-self-assertive as compared with white men. Yet Americans constantly complain of the 'sauciness' and truculence of the negro. These characters are evidently attributable to the surroundings to which the negroes in America are subject; the manner in which they are looked down upon and the irritating minor restrictions to which they are subject evoke in the negroes a reaction which results in their being called 'saucy' and truculent. In other words their disposition is so modified by tradition that, as manifested, it belies their innate characteristics. In an earlier chapter reference was made to the subservience of the Egyptian fellah. In the production of this characteristic a centuries-old tradition of oppression plays a large part, though the elimination of the more self-assertive individuals may have produced a low average level of self-assertiveness. If we compare an English public-school boy with an Egyptian fellah in respect to self-assertiveness and self-reliance, those characters as manifested form no guide to the underlying innate differences. Among the former self-reliance is strongly encouraged, among the latter it is inhibited. But if we compare English public-school boys one with another, and Egyptian fellaheen one with another, then the differences disclosed are to some considerable degree a measure of innate differences, because tradition is more or less equalized. Again, let any one observe the attitude of the British private soldier towards our coloured subjects, or for that matter what he can make of the attitude of the latter towards the English soldier, and he will not be in doubt as to the importance of tradition in producing the attitude of command and its opposite- the tendency to submission.

There is no doubt therefore as to the possibility of tradition affecting profoundly the manifestation of all mental characters. When we compare white with black races in their original home, we have to discount the whole tradition. European races, however, have a considerable amount in common, and when we compare them we have less to discount. When classes within the 2498 
same race are compared, there is still less to be discounted; they have both European and racial tradition in common; the differences come in at a higher level. Finally, when we compare individuals in the same class, we have only to discount family and individual differences in tradition. It is therefore clear that we have to gain some idea as to the nature of the differences in tradition to which the classes in England at the present day are subject.

16. We have already remarked upon the curious fact that, although the organization of society on organic lines greatly favoured the transmission of tradition, there have come to be large differences in tradition as between the different component elements of society. This we attribute to the fact that in the first place the different component elements specialize in different kinds of skill ; the vastness of the mass of tradition makes it impossible for every man to absorb all of it; so far as skill is concerned, each man absorbs that development of it, the specialization in which characterizes his function in society. This may account for differences as between classes with regard to skill, but why, it may be said, should it account for differences in manners, customs, and mode of life? Whatever functions may be performed by the different elements of society, do not the members of all alike now, in any case, enjoy the same privileges, have they not all homes, have they not access to the same interests apart from their professions? Manners and customs differ, and differ so much that a public-school boy finds himself out of his element as a private in a line regiment, and a university man finds it difficult to establish ordinary human relations with the men he meets in a working men's club such as he establishes without effort when in company of men of his class.

These differences are attributable to the fact that ownership of property has become a function in society. Further, there has come about the reservation to the property-owning class of certain professions - notably the military and the clericalwhich, though they do not afford a high rate of remuneration, do afford, as property-owning affords, ample opportunities for leisure. Property-owners as a class have had thus both the time and the means to cultivate the art of living. Some of the results are, as all will admit, of value. Some of the results are harmful ; but for the most part cultivation of the art of living results in 
the multiplication of conventions which are neither useful nor harmful, being mere matters of form. The wage-earning class, on the other hand, has neither the time nor the means to elaborate any code of manners. Working long hours for what amounts to little more than a bare subsistence, they could not have evolved such a code of manners nor have they absorbed any considerable part of it. There was no room for it as their lives were lived. Other consequences follow from property-owning. Propertyowning is power; in the property-owning class there arises a tradition of self-assertion, a habit of command, whereas in the wage-earning class there arises a tradition of self-abasement and a habit of subservience, traditions and habits so strong as largely to obscure natively given characters. We may note with interest, therefore, that it is on these lines, and not on a theory of germinal differences, that an eminent historian answers the question why military history shows that men of greater age and wider experience will on the field of battle follow to the death a boy of the upper class when they will not follow one of their own class. It may be objected that what has been said scarcely applies to the conditions of the present day and that subservience of the wage-earning class is not very marked at the moment. This is true, but it is a recent change. The reason why the change has come about is surely that the wage-earning class has obtained power and knows it. The change is an illustration of the power of tradition to dominate the outward expression of such important characteristics as assertion or subservience. No one can attribute the difference to germinal change. The wage-earning class has not absorbed the manners and customs of the property-owning class at the same time for a variety of reasons ; for one thing they have still little place for such conventions in their lives, and for another thing they tend to regard everything connected with the property-owning class as hostile, while we may also remember that many elements in the upper-class tradition depend for their existence upon the presence of servants.

An insight into the nature of these traditional differences may be gained in rather a different way. Many members of the upper class have attempted to interest themselves in the conditions of life among the less fortunate classes. It is doubtful how far the experience gained in the majority of cases is of value in throwing light upon the problems under consideration. But let any G g 2 
unprejudiced person go and live in the working-class quarter of any large town without any object in view other than to experience the conditions. Under such circumstances the conclusions which grow up in his mind will probably be something of the following nature. To all seeming the innate qualities of the boys and girls, in temperament, disposition, and intellect, are very much what they were in the boys and girls he was at school with or formerly associated with himself. The more intimate his acquaintance becomes with the conditions among which these boys and girls grow up, the more striking seem the peculiarities and limitations of the mental horizon outside of which they have little chance of penetrating compared with that which confronts children of his own class. The dullness and drabness, if there is nothing worse, of a home life in two or three rooms, relieved by what excitement can be found in the smaller streets of a large town, or later by picture palaces or an occasional football match, followed by marriage and the struggle to keep up a home under discouraging circumstances-all these features, which are not appreciated in their full weight until they are, as it were, felt by individual experience, seem adequately to explain the differences between the adults of one class and those of another. Any one who has had such an experience will, on the whole, be rather surprised that more sordidness does not exist, than be inclined to summon the hypothesis of innate inferiority to explain the sordidness that does exist.

17. Profound differences in tradition as between the social classes do therefore exist and, as far as we have gone, they might appear to account for all mental differences manifested; they must in any case account for a considerable part of them. But though we could prolong the discussion on the same lines and produce much evidence tending to the same conclusion, we could reach no precise result. We may go on therefore and ask whether there is any evidence of the existence of innate differences between the classes.

Mere observation of the boys and girls of the different classes does not indicate any differences. But mere observation is not enough. The problem may be approached in two ways. We may notice the results of inquiries made regarding the intelligence of children by modern methods, and we may also ask by what characters those who rise to a higher social status are distinguished, 
and further what innate differences may be supposed to underlie these apparent differences. The results of inquiries made to test the relative intelligence of children of parents of different social status are fairly uniform. Bridges and Coles, for example, found as the result of such an investigation that "there was very considerable dependence of intelligence upon "sociological conditions" '; they go on to say that "when children are classified according to the occupations of their fathers, a striking correlation is shown between intelligence quotient and occupation group. Hence if mental age rather than chronological age were used to determine the time for beginning school, the children of the professional group, for example, would begin school two years earlier than the children of the unskilled labour group; for the former mature intellectually much earlier than the latter.' 1 The interpretation of such observations bristles with difficulties. It is not clear what relation earlier maturity bears to adult intelligence. Though the direct effect of the environment is probably negligible, the possibility that tradition may influence the results is certainly not shut out. Indeed, the fact that the correlation is higher for boys than for girls suggests that tradition does come in. Nevertheless, a consideration of these results suggests that the whole explanation cannot be found in tradition and that we have here a sign of some superiority of intelligence in the children of parents of a higher social status. More than that cannot be said.

18. Let us approach the question in another way. Can we discover any characteristics distinctive of those who succeed in modern society, who rise, as it is called, from the ranks of the wageearning classes, and, if we can, what these characters are and how far we must assume the existence of innate differences to account for them? We may think of the upper social classes in England to-day as falling roughly into three divisions-the professional and the business classes and those whose fathers, grandfathers, or more remote ancestors came into possession of property by one means or another. In the case of a small proportion of the latter class, the position was won under conditions which differ more or less profoundly from these ruling to-day. This section is

1 Bridges and Coles, Psychological Review, vol. xxiv, p. 29. These authors do not commit themselves to any view with regard to the interpretation of their results - a fact which commends the investigation to students of these matters as indicating that the investigation was not undertaken with any bias (a condition of things unfortunately not common in studies of this problem). 
a small section, and we need not further consider it here. A large proportion of the so-called upper classes have attained their position under the conditions which now exist, or their forefathers have attained it under conditions which did not greatly differ from them. What characteristics are they, which, when manifested under these conditions, lead to success?

It has often been assumed that intellect is the chief factor in success. This assumption is not only made in ordinary conversation when a reference to the career of a successful man in any line is almost certain to be followed by the comment that ' he must be a very clever fellow' but in serious contributions to the problem. This assumption is certainly wrong. There are many elements in character which contribute more largely to success than the intellectual, such as capacity for work, energy, ambition, desire to dominate, tact, and so on. Furthermore, success in a profession is, to an almost as great an extent as success in business, attributable to such characteristics. Few more remarkable things-remarkable in being in conflict with ideas as commonly preconceived-are learnt from observation and experience than the length to which such characteristics in favourable association can carry a man even in the strictly learned professions where it might be supposed that intellect alone would carry off the prizes. There can be no doubt that those elements in character which are most important as regards success are such as we must attribute to differences in disposition and temperament rather than in intellect so far as we can attribute them at all to innate differences.

This is essentially what we want to know-what innate differences do exist. We have, when trying in the first place to fix upon the outwardly existing characteristics, to remember that tradition may give powerful twists and turns-sometimes of a desirable and sometimes of an undesirable nature-to the simpler characteristics mentioned above, such as ambition. In the business world, for example, the successful man is from many points of view the man who gets his head above the heads of other people, who gets more out of other people than they get out of him. But are we to imagine that there is something in him which impels him so to act with regard to his fellow men, or are we to imagine that the simpler characteristics of ambition and love of power lead to such conduct under the guidance of a par- 
ticular tradition without any definite desire on his part or even perhaps without any clear realization as to what it is precisely that he is doing? The second explanation is that which best fits the facts. It seems clear that, as tradition changes in the course of years, the activities of the energetic ambitious men are led this way and that. At present no doubt to a certain degree they are led in the direction of ' pushfulness '-by what M. Faguet has called 'le goutt d'arrivisme'. It is always dangerous to read into the activities of men the working of particular innate characteristics. Few things in history perhaps have been as harsh as the conduct of the generation which devised the game laws, and yet these very men did not merely quote the classics with unbounded enthusiasm but were also moved by genuine sympathy and altruism to abolish slavery, the horrors of which were known to them only by report. We should refrain, therefore, from deducing from the activities of the successful men and of the upper classes more than the presence of those simpler characteristics mentioned above as leading to positions of pre-eminence over other men.

These simpler characteristics are in themselves very complex manifestations of many elements, of which tradition is one. But in the case of this particular problem, as we are considering rise from a lower to a higher social status, tradition cannot be held to be the cause of the differences. There are, in fact, unquestionably innate differences in temperament and disposition which underlie the characteristics leading to success, and the temperamental differences which have been curiously neglected are probably the most important and form perhaps half the secret of success. Among the temperamental characteristics leading to success we may note low degree of fatiguability, high power of recuperation, rapidity of response, hopeful nature, vigour, energy, and healthy nervous tone. Among the characteristics of disposition we may note similarly the instincts of self-assertion, of emulation, and of acquisition. The power of forming habits is probably also a considerable aid to success under modern conditions.

What part does intellect play? Judging from the fact that it is certainly an aid to success, though not so powerful a factor as is usually thought, we may conclude that there is some, probably a slight, difference in intellectual capacity between the classes. Intellect, when it accompanies success, is rather more in the nature of an incidentally favourable factor than of a primary 
cause of success. The powerful instinct of self-assertion will make itself felt ; its possessor will probably arrive somewhere ; a powerful intellect may, in the absence of, or rather with the inadequate development of, other innate qualities, not advance its possessor's position in the world.

But though considerations based upon a testing of intelligence on the one hand and upon an analysis of qualities leading to success on the other do without question lead to the conclusion that there are differences in innate qualities as between the successful and the unsuccessful, there are grounds for thinking, for the reasons mentioned above, that the differences between the classes are not large. For we must remember that, so far from the lower classes being drained of men of certain qualities, a very small proportion of the men of these classes is ever enabled to set out on the path leading to success in business or in a profession. Also we must not forget, when considering success, the part played by fortunate accidents under complex modern conditions-mere chance happenings which, altering the whole course of a man's life, may determine whether he is successful or unsuccessful. Again, there is little or no relapse worth speaking of into the lower classes when once a status is gained. Such is the influence of place and status that for the most part, whatever may be the innate qualities, the descendants of men who won their way upwards maintain their position. Therefore the regression to the mean which is always at work must tend to lessen such innate differences as exist between the classes.

19. Lastly, there is a very difficult problem the existence of which must not be forgotten inasmuch as it affects in the most fundamental manner the judgement to be passed on the meaning of these differences. We have to ask what is the value of the differences which exist. Success alone cannot be taken as an indication of the value of the characters exhibited by the successful. There can indeed be no doubt that, other things being equal, a decrease in the average intellect would be an unfortunate thing. Similarly we may regard those temperamental qualities which were indicated as leading to success as on the whole of value. But it cannot be affirmed without more consideration that all temperamental qualities which distinguish the successful are desirable, and more particularly that a further development of the instincts of self-assertion, acquisition, and emulation would be 
desirable under present circumstances; it may even be that we might view a diminution in the average strength of some of the qualities which mark the successful at least with equanimity. The instincts of acquisition and self-assertion were of great value in the past, highly indeed as mankind has paid for the careers of men markedly endowed with these qualities. But, with the present stage of social evolution in Europe in mind, should it not be said that the instinct of acquisition when developed above the average is on the whole harmful, and may it not be that selfassertion is likely to be a source of difficulty in any form of co-operative commonwealth, and to some form of co-operation as replacing or modifying competition we seem to be tending.

We may see this whole problem of value in another light if we ask what it is that we value in the men of our race. Latterly we have had reason to dwell upon the qualities which we feel to characterize the men of our race at their best. Are they not self-respect together with modesty, tenacity together with tenderness? Any one who has served in the ranks of the army is not likely to allow that these qualities are on the whole less developed in the classes from which the ranks of the army are usually filled than in the rest of the nation. In this connexion we should perhaps remember that a sense of comradeship in facing the elemental facts of existence without material wealth may provide an impetus to the expression of these qualities, whereas an absorption in the less immediate necessities of life may militate against their expression. To the degree to which this is so, we should, when trying to estimate the innate capacities in the different classes, make the necessary allowances-in the case of the so-called upper classes for the circumstances adverse to the expression of these qualities and in the case of the so-called lower classes for circumstances which on the whole perhaps favour their expression. However this may be, we feel that these qualities are the most valuable which our race has produced and that upon their preservation and upon greater opportunities for their expression depends our best hope for the future. $\mathrm{He}$ would be a bold man, however, who suggested that the possession of these qualities in more than average amount was a characteristic of the successful. The same may be said of good taste, good manners, and of other qualities which, to say the least of them, contribute to the amenities of life. 
It is at least apparent how difficult a problem is the judgement to be passed on the effects of modern differential fertility. There are grounds for thinking that those who see in differential fertility the cause of the cyclical course of civilization both over-estimate the results and neglect certain aspects of the changes involved. Differential fertility is not a factor to be disregarded; the results which it is now producing demand the most careful investigation. Nevertheless, so far as our knowledge extends, we should view it rather as the result than as the cause of the cyclical course of history-the course which historical changes take being due primarily to changes in tradition. This conclusion is in harmony with all that has been said in this chapter as to the relative importance of change in tradition and of change in the germinal constitution. From the first period in history changes in tradition have come to override changes in the germinal constitution; the latter form of change is far from being negligible, but it is a contributory rather than a fundamental cause of the events of history. 


\section{XXII}

\section{CONCLUSION}

1. An attempt has been made to trace the origin of problems of population from their source and to indicate their nature and interdependence. They have been traced back to man's place in nature. The ancestors of man must at one time have been subject to the same conditions as those to which all species in a state of nature are subject. These conditions were investigated, and it was shown that fecundity is in the main determined by the sum of all the dangers to which the young of any species are exposed-allowance being made for the fact that a certain proportion of ova will not be fertilized. It was also shown that change or history-if history can be spoken of in this connexion -is due to germinal change alone, and it was further indicated how it may be supposed that germinal change comes about. The early stages in the moving away of the pre-human ancestor from these conditions elude our inquiry, though certain deductions may be made as to the main outline of what happened. For evidence as to the latter stages, until we reach historical times, we are chiefly dependent upon the method of using our knowledge of existing primitive races as throwing light upon prehistoric races.

2. Problems of population fall under two main headings, problems of quantity and problems of quality. But all problems of population are interwoven one with another and the method of solving any quantitative problem bears upon the quality of population. Thus the methods of regulating quantity affect quality by influencing germinal change, and in the later periods of history growth in quantity affects quality by its influence upon tradition. No one problem should be considered without reference to its bearing both upon quantity and quality. At the present day, for instance, differential fertility is almost always considered solely from the point of view of quality ; it is forgotten that the reduction in the birth-rate may be that which economic conditions demand and that it may of necessity 
have to begin among the upper classes. Though, therefore, differential fertility by producing unfavourable germinal changes is to be to that degree deplored, yet we have to remember that, so far as quantity is concerned, failure to meet economic requirements might be a much greater misfortune.

3. As regards quantitative problems we saw that from the first period of history onwards-from the time, that is to say, that it began to be possible for man to reap the benefits of co-operation-it was of the utmost importance for every group to approximate to the optimum number. This is the number which-taking into consideration the nature of the environment, the degree of skill employed, the habits and customs of the people concerned, and all other relevant facts-gives the highest average return per head. This number is not fixed once and for all. On the contrary it is constantly varying as the conditions referred to vary, and, as skill has tended to increase throughout history, so has the number economically" desirable tended to increase. The errors underlying the whclly different exposition given by Malthus have been indicated; for him there was no such thing as over-population. In his view population had at any one time increased up to the possible limit and was in process of being checked. In the modern view numbers may approximate to the desirable level, may not reach it, or they may exceed it, and if either of two latter positions arise, the return per head will not be as high as it might be.

The quantitative problem presents itself to all races at all times. There is no escaping it. The common notion. that it only presents itself at certain times and in certain places is based upon a failure to grasp the strength of fecundity. Almost without exception those factors, which incidentally restrict increase and produce elimination, are insufficient so to reduce fertility as to keep numbers down to the optimum level. There thus arises the need for factors which directly restrict fertility and cause elimination; among primitive races these factors take the form of abortion, infanticide, and prolonged abstention from intercourse. There is no correlation between these factors and the economic stage reached, and therefore we have no grounds for assuming any one factor to have been prevalent at any one stage in prehistory, though we must assume that one or more of these factors was always at work. This assumption is confirmed by 
the fact that, whenever we can catch sight of the emergence of prehistoric races into the light of history, we find one or other of these factors to have been present. Further there is every reason to suppose that normally such of these factors as are in use are effective and that therefore in the first and second periods of history some approximation to the optimum number was normally attained.

The third period is in many respects different from those that preceded it. In the first place the number desirable has been constantly increasing, so much so that increasing numbers are taken as being a normal feature of human society whereas, in fact, numbers throughout human history as a whole have been stationary. It may be that we are nearing a time when numbers will be again normally stationary, for though increase may remain economically desirable, it may cease to be so from a wider point of view of human welfare, when, that is to say, facts other than income per head are taken into account. In the second place there have been frequent failures to attain to the optimum number owing to the many disturbing influences at work. Chief among them are the fluctuations in the number desirable, the erratic action of certain causes of elimination, such as war and disease, and migrations.

Regarding the quantitative problem as a whole, it is evident that the necessity of solving it has had the most profound effect upon all societies at all times. It bears directly upon the relation between the sexes-around which so largely centres human welfare -and upon the most intimate and most valued aspects of the life of every adult-those connected with the family. In the past the solution has been unconsciously or semi-consciously achieved ; it has now come within the power of mankind after a due consideration of the position deliberately to decide what the best solution may be.

4. Turning now to the problems of quality, we found that change among species in a state of nature, and therofore among our pre-human ancestors, was due to germinal change alone. Just as man has moved away from the position in which all species in a state of nature are situated as regards quantity, so he has moved away from the position in which they are placed as regards quality. Human history, in other words, is not explicable as due to germinal change alone. Tradition becomes a factor 
of ever-increasing importance; the direct influence of the environment also assumes a greater importance than among species in a state of nature, though it remains relatively insignificant compared with changes in tradition and changes in the germinal constitution. Germinal change, however, retains almost its full importance so far as permanent changes in physical characters are concerned.

We have seen that physical characters as presented to us are the expression of the inter-action between certain germinal predispositions and a certain environment, and that, disease apart, such variations as usually occur in the environment do not in any notable manner affect these characters. Tradition does not enter directly into the expression of physical character ; it alters the environment, it is true, but that is another matter. Nevertheless when we come to investigate the smaller differences, such as those which are found as between members of different classes in a modern community, the greatest caution is necessary before the differences in the environment are ruled out as contributory factors in producing these differences. Broadly speaking, we may say that differences in stature, eye colour, eyesight, muscular power, and so on, are all, though in varying degrees, because different organs vary in their susceptibility to environmental differences, expressions of germinal differences. It follows that, so far at least as physical characters are concerned, the germinal constitution is of primary importance. Disease due to parasites is a question apart. The parasites might be eliminated, or conceivably preventive medicine might render susceptibility to disease of little account. Disease due to structural defects must be classed with other physical characters and, whether it be that we are considering health (immunity from parasitic disease excepted), stature, eye colour, or any other physical character, it is to the germinal constitution that we must look as the factor of chief importance.

It is not, however, of changes in physical characters but of changes in mental characters of which we think when we ask what it is that has caused those events the recording of which is the province of historians. There are three factors to be considered, germinal change, traditional change, and the direct effect of the environment, which latter factor we may pass over, merely recalling that it can at times-as in the case of chronic disease 
affecting at one time a large proportion of the inhabitants of a country-appreciably retard progress. It is never in the true sense a cause of progress. In thus relegating the direct effect of the environment to a very subsidiary place among the factors, we are not dismissing the environment as of little account in human history or in the lives of individual men and women. Though the direct effect of the environment on the germinal constitution is seldom clearly distinguished from its effect in moulding tradition, yet it is wholly distinct; and in fact, while attributing to the former little importance, we have found the latter to be of increasing importance until it comes altogether to dominate germinal change.

Nevertheless when this distinction is realized and an unbiased effort made to estimate the relative importance of these factors, it seems that the position is often curiously misunderstood. It is frequently suggested that the achievement of our race in the future and of mankind in general will somehow depend principally upon the course of germinal change, and that it is in the lives of men at the present day or at any given time that environment in its bearing upon tradition is of importance. But this is a misconception of the position. Bearing in mind the discussions in former chapters as to what is inherited and as to the direct influence of the environment, let us ask wherein the importance of germinal differences is to be sought, first as between men at the present day and afterwards in history as a whole. With regard to physical characteristics it is clear that, disease apart, men are what they are owing principally to their native endowment. Whether a man is tall or short, dark or fair, has blue or brown eyes, or what is, apart from the possibility of a correlation between these and more valuable characters, of more importance, whether he is healthy, vigorous, strong, endowed with good eyesight and hearing, in short with a sound constitution-this is a matter principally of native endowment, supposing the differences in the environment not to exceed those which now on the average occur. And the same applies to mental characters with an allowance made for the marked degree of susceptibility which temperament exhibits to changes in the environment. Whether a man has more than the average degree of intellect, is markedly assertive, pugnacious, or inquisitive, is capable of withstanding or recovering from fatigue, depends upon his native endowment. 
But it is at the same time true that achievement is in very large degree governed by tradition. This is true if the criterion of achievement is an historical criterion, as, for instance, when a man of one race is compared with another man of the same race belonging to a different epoch or when a man of one race is compared with a man of another race. It is not true when the comparison is made between two men of the same class within the same race because within classes in a race tradition is more or less equalized. And where tradition is equalized, there achievement is a measure of innate endowment, and it is within a class usually so equalized that at any given time the outward manifestation of mental characters is nearly as much a measure of native endowment as are those of physical characters. Therefore not what a man achieves as judged by historical standards, not whether his thought will follow primitive or common-sense lines, is dependent upon his endowment, but what he will make of the tradition of his time-his performance, in other words, compared with that of men around him.

Achievement, therefore, as judged historically, is in very large measure to be explained as due to the influence of the environment upon the origin and transmission of tradition. Up to a certain stage, however, and, when the whole of human history is taken into account, up to a late stage, achievement was in the main dependent upon germinal change. But this late stage is anterior by many thousands of years to the beginning of history in the usual meaning of that term. Beginning not later than the last period of the Palaeolithic, the explanation of the course of events is in the main to be sought not in germinal change but in the influence of environment upon tradition. The importance of germinal change in the later stages is by no means negligible. Germinal change, however, was not so much the cause of the course which events followed as a consequence of these events. The effect reacted upon the cause and accelerated the process. Finally when considering the latest phase of history-the latest phase, that is to say, when taking a broad view-we reach the following conclusion. We find that the great acceleration of the rate of progress which characterizes the history of the period is to be explained, not by a change in quality, but by the growth in quantity of the population which, though it does not of necessity lead to, is the indispensable condition precedent to, the break-down 
of the segmentary organization of society and to the rise of the organic type of social organization. This is the paradox of the population problem. Change among species in a state of nature is based upon germinal change alone; change among our prehuman ancestors was equally a matter of change in the quality of population ; but the explanation of the most outstanding fact in recent history broadly viewed is to be sought in a change in quantity rather than in quality of population.

The explanation of the course of events since late Palaeolithic times as due to tradition on the lines indicated in the last chapter is in general satisfactory, provided that on the one hand allowance is made for the fact that differences in race do imply differences in mental and moral qualities, which arising on the whole together with changes in tradition, reinforce the tendency to change along certain lines, and that on the other hand apparently chance happenings do give turns and twists to the course of events. The reconciliation of this latter phenomenon with the general trend of the argument is not difficult. Under certain conditions the death or even theindisposition of some prominent man or the whim of a powerful minister may appear to divert the course of events. Nevertheless in reality such events have only a passing effect and do not obscure the broad workings of the factors we have indicated.

The relation of the innate qualities to tradition may be illustrated by the use of a metaphor. Tradition may be likened to some vast structure which mankind is building. Each generation adds a few bricks to the structure. The part of the building to which any one man contributes, whether it is to the ground floor or to one of the upper stories, wholly depends upon the race and epoch to which he belongs; so too does for the most part the kind of brick he will lay and the methods he will employ in laying it. His contribution to the structure is governed by the plan of the building as elaborated by previous generations and by the bricks they have prepared and the methods of laying them they have introduced. But in any generation whether a man will lay a brick at all or whether he will do it energetically and intelligently as compared with his fellow-workers will depend upon the innate qualities with which he is endowed.

Our conclusion therefore must be something after this kind. Those who base upon germinal change their hopes for the physical condition of the human race in the future are building upon 
sound foundations. However much our power to control and regulate vital processes may increase-and it is clearly upon the verge of a very great increase-in the end a satisfactory physical condition can only be the product of a certain germinal constitution. On the other hand, those who think that germinal change in mental characters will effect the evolution of society and mould the course of history are upon the whole mistaken. The course of history is in the main dependent upon changes in tradition which are for the most part independent of germinal change. Just as the outstanding happenings in the last century-the turning of thought and conduct in Germany, for example, along certain lines, which ended in so great a catastrophe-were due to changes in tradition and not to changes in the germinal constitution, so whether the problems now pressing upon European society are to be solved or whether some greater catastrophe, reaching a climax in a long course of years or bursting suddenly upon us, is to be the outcome, will depend upon changes in tradition and not upon germinal change. The reason for this lies in the fact that the vast accumulation of tradition overlays he outward expression of mental character, determines the direction of intellectual activity and moulds the expression of the instinctive faculties. But as far as tradition is equalized, so far do innate mental differences manifest themselves as between man and man, and since tradition is more or less equalized, if not within races, at least within classes in the same race, to that degree is mental endowment of pre-eminent importance to the individual. 


\section{APPENDIX I}

There is presented below a summary of the evidence that in all parts of the world there existed among primitive races, before they had been subjected to European or other outside influence, customs the primary function of which was the restriction of increase. An ideal review of the matter would require a preliminary selection of certain areas where the conditions referred to could be thoroughly studied. After a mapping out of the races and tribes, in order that some idea might be obtained of the relative numerical importance of each, an exhaustive examination of the literature might be undertaken and the date of the observations noted. The credibility of the authors would have to be considered and some method of allowing for negative evidence elaborated. Nothing of the kind has been attempted here. It may be suggested that the problem should have been pursued at least somewhat further. Various attempts were made to analyse the evidence in other ways, but the difficulties met with were such that it seemed best to present the evidence as follows. Evidence of a reasonably credible nature as to the existence of these customs has been noted. In many of these cases other authors are silent as to these practices; in one or two cases the practices have been denied; but when the denial is either not apparently founded on careful observation or is of a distinctly later date, these instances have not been omitted. Where the weight of the evidence is against the existence of the practice, as in the case of the existence of infanticide among the Veddahs, any positive evidence is omitted. One author does record infanticide among the Veddahs, and it may be that it formerly existed.

It is submitted that the evidence as given below does show customs restrictive of increase to have been so widespread, in the form either of abortion, infanticide, or prolonged abstention from intercourse, as to have been practically universal. It is in fact submitted that, although for the many reasons set out in the text the evidence must be very incomplete, there is ample support for the theory which has been advanced.

In the following summary the letter ' $R$ ' stands for prolonged restriction of intercourse, ' $A$ ' for abortion, and ' $I$ ' for infanticide. Where the evidence records the practice to be rare, but there are reasons for thinking that the rarity may be recent, the reference has been included with the word 'rare' given in brackets. 
Group I.

\section{AUSTRALIA}

I. General References. Parker, Aborigines, p. 23 ; Lumholtz, Among Cannibals, p. 134.

Victoria. Curr, Recollections, p. 252.

Victoria and Riverina. Beveridge, Aborigines, p. 26.

New South Wales and Victoria. Mathews, Ethnological Notes, 1. 17.

Port Lincoln. Wilhelmi, Transactions of the Royal Society of Victoria, vol. v, p. 180 ; Schürmann, Aboriginal Tribes, p. 223.

River Darling. Bonney, J. A. I., vol. xiii, p. 125.

Southern Australia. Palmer, J.A.. ., vol. xiii, p. 280 ; Fison and Howitt, Kamilaroi and Kurnai, p. 190 ; Eylmann, Kolonie Südaustralien, p. 261 ; Smyth, Aborigines, vol. i, p. 52.

Mining Tribe. Howitt, Native Tribes, p. 748.

Tongeranka. Ibid., p. 749.

Mukjarawaint. Ibid.

Wotjos. Ibid.

Tatuthi. Ibid.

Wadthaurung. Ihid.

Narrinyeri. Taplin, Native Tribes, p. 13.

Queensland. Mathew, Two Tribes, p. 165.

Port Darwin. Foelsche, J. A. I., vol. xxiv, p. 192.

Central Australia. Eyre, Journals, vol. ii, p. 376 ; Spencer and Gillen, Native Tribes, p. 269.

Northern Tribes. Spencer and Gillen, Northern Tribes, p. 608.

Western Australia. Grey, Journals, vol. ii, p. 251.

A. North-west and Central Queensland. Roth, Ethnological Studies, p. 183.

Mythuggadi. Palmer, J. A. I., vol. xiii, p. 280.

Port Jackson. Collins, Edinburgh Review, vol. ii, p. 34.

Group I.

\section{TASMANIA}

A. Bonwick, Tasmanians, p. 76.

I. Ibid., pp. 79 and 85 ; Ling Roth, Tasmania, p. 167.

\section{Group I.}

\section{BUSHMEN}

I. Moffat, Missionary Labours, p. 58 ; Stow, Native Races, p. 51.

\section{Group I.}

\section{AMERICA}

R. General References. Weld, Travels, p. 373 ; Heriot, Travels, p. 339.

Yguazas. Cabeça da Vaca, Narrative, p. 62.

Abipones. Dobrizhoffer, Abipones, vol. ii, p. 97.

A. General References. Weld, loc. cit., p. 373; Robertson, History, vol. i, p. 297.

Eskimos. Wells and Kelly, U.S.A. Bur. of Education, 1890, p. 19 ; Bessels, Arch. für Anth., vol. viii, p. 112.

Hudson Bay. Ellis, Voyage, p. 198.

Knisteneaux. Mackenzie, Voyages, vol. i, p. 148.

Haidahs. Bancroft, loc. cit., vol. i, p. 169.

Puget Sound. Lord, Naturalist, vol. ii, p. 231.

Vancouver Island (Nootkas). Sproat, Savage Life, p. 94 ; Bancroft, vol. i, p. 197.

Thompson Indians. (Rare.) Teit, Jesup North Pacific Expedition, vol. i, p. 305, and vol. ii, p. 584.

Shushwap. (Rare.) Ibid. 
Western Washington and North-western Oregon. Gibbs, U.S. Geog. and Geol. Survey, vol. i, p. 199.

Chinooks. Bancroft, loc. cit., vol. i, p. 242.

Inland Tribes Pacific Coast. Ibid.

Omahas. (Rare.) Dorsey, 3rd A.R.B. E., p. 263.

Californians. Powers, loc. cit., p. 207.

New Mexico. Bancroft, loc. cit., vol. i, p. 590.

Guaycurus. Castelnau, Expedition, vol. ii, p. 450 ; Azara, Voyages, p. 146.

Payaguas. Rengger, Reise, p. 329.

Fuegians. Cooper, S. I. B. E., No. 63, p. 171.

I. Eskimos. Behring Straits. Nelson, 18th A. R. B. E., p. 289.

Central. (Rare.) Boas, 6th A.R.B.E., p. 580.

Smith Sound. Murdoch, 9th $\dot{A} . \dot{R} . \dot{B} . \dot{E} .$, p. 417 ; Bessels, Arch. für Anth., vol. viii, p. 112 .

King William Land. Murdoch, loc. cit., p. 417.

Greenland. Nansen, Greenland, p. 330.

Aleuts. (Rare.) Dall, Alaska, p. 399.

Malemutes. Bancroft, loc. cit., vol. ii, p. 81.

Kutchins. Kirkby, Church Missionary Intelligencer, vol. xiv, p. 115 ; Hardisty, 21st $A . R . B . E$. , p. 312 ; Mackenzie, Voyages, vol. i, p. 148.

Copper River District. Woldt, Reise, p. 393.

Chinooks. Bancroft, loc. cit., vol. i, p. 242 ; Lord, loc. cit., vol. ii, p. 231.

Koniagas. Bancroft, vol. i, p. 81.

Nootkas. Bancroft, vol. i, p. 197.

Haidahs. Gibbs, loc. cit., p. 198 ; Bancroft, vol. i, p. 169.

Kwakiutl. Bancroft, loc. cit., vol. i, p. 169.

Chepewayans. Keating, Narrative, p. 160.

Californians. Powers, loc. cit., p. 416 ; Bancroft, loc. cit., vol. i, pp. 390 and 413.

Yguazas. Cabeça da Vaca, Narrative, p. 62.

Abipones. Dobrizhoffer, Abipones, vol. ii, p. 97 ; Charlevoix, loc. cit., vol. i, p. 405.

Guaycurus. Church, South America, p. 248.

Puelches. Guinnard, Patagonians, p. 143.

Fuegians. (Rare.) Westermarck, Human Marriage, p. 313.

\section{Group II.}

R. Iroquois. Le Beau, Aventures, vol. ii, p. 200.

Illinois. Charlevoix, loc. cit., vol. vi, p. 5.

Crows. Holder, Am. Journ. Obstet., vol. xxv, p. 44.

Mexico. Bancroft, loc. cit., vol. ii, p. 281.

Moxos and Chiquitos. D'Orbigny, L'Homme Américain, vol. i, p. 47.

A. Sioux. Keating, loc. cit., vol. i, p. 394 ; Schoolcraft, loc. cit., vol. i, p. 204.

Crows. Holder, loc. cit., p. 44.

Apaches, Navahos, Pueblos, Pimas, Nahua, Otommi, and Aztec. Hrdlicka, S. I. B. E., Bull. 34, p. 163.

Menomini. Stevenson, 23rd A.R. B. E., p. 296.

Zuni. (Rare.) Ibid.

Mexico. Bancroft, loc. cit., vol. ii, pp. 183 and 269.

Brazil and the Chaco. Ehrenreich, Königliches Museum zu Berlin, vol. i, Heft 2, p. 27 ; Azara, vol. ii, p. 116.

Bakairi. Von den Steinen, Brazilien, p. 123.

I. Pimas. Yarrow, A. R. B. E., vol. i, p. 99.

Sioux. Schoolcraft, loc. cit., vol. iii, p. 243. 
Creeks. Schoolcraft, loc. cit., vol. ii, p. 272.

Guanas. Azara, loc. cit., vol. ii, p. 93.

Mbayas. Ibid., p. 116.

Lenguas, Hawtrey, J. A. I., vol. xxi, p. 295.

Guaranis. Rengger, Reise, p. 329.

\section{AFRICA}

R. Yoruba-speaking. Ellis, Yoruba-Speaking Peoples, p. 185.

Ewe-speaking. Ellis, Ewe-Speaking Peoples, p. 206.

Kagero. Tremearne, J. A. I., vol. xlii, p. 174.

Hausa. Ibid, vol. xxxvi, p. 93.

Benin. Ling Roth, Benin, p. 39.

Niger District. Mungo Park, Travels, p. 402.

Warri District. Granville, J. A. I., vol. xxvii, p. 106.

Moioa. Tremearne, Nigeria, p. 239.

Gallinas. Harris, Darkest Africa, p. 36.

Hobbés. Desplagnes, Plateau Nigérien, p. 227.

Cameroon. Nassau, West Africa, p. 11.

Ashanti. Reade, South Africa, p. 45.

Congo District. Cureau, Sociétés primitives, p. 378 ; Johnston, George Grenfell, p. 671 ; Ward, J. A. I., vol. xxiv, p. 289.

Bangala. Weeks, J. A.I., vol. xxxix, p. 418 ; Overbergh and Jonghe, Coll. Mon. Eth., No. 1, p. 199.

Mayombe. Overbergh and Jonghe, Coll. Mon. Eth., No. 2, p. 219.

Ababua. Hakin, Coll. Mon. Eth., No. 7, p. 260.

Bayaka. Torday and Joyce, J. A. I., vol. xxxvi, p. 51.

Mandja. Gaud, Coll. Mon. Eth., No. 8, p. 154.

Warega. Delhaise, ibid., No. 5, p. 154.

Uganda. Wilson and Felkin, Uganda, vol. i, p. 187.

Baganda. Roscoe, Baganda, p. 55.

Rovuma River. Thomson, Geog. Journ., vol. iv, p. 73.

Swaheli. Velten, Suaheli, p. 73.

Baringo District. Dundas, J. A. I., vol. xl, p. 60.

Wanjamuesi. Reichard, Z. G. E., vol. xxiv, p. 257.

British Central Africa. Stannus, J. $A . I$., vol. xl, p. 311.

Miri District. Felkin, Trans. Edin. Obstet. Soc., vol. ix, p. 31.

Atonga. Johnston, British Central Africa, p. 415.

South Africa. Junod, Baronga, p. 490 ; Kidd, Essential Kaffir, p. 19.

Baronga. Junod, Baronga, p. 490.

Loango. Pechuel-Loesche, Loango-Expedition, p. 31.

A. Tenda. Delacour, Rev. d'Eth., 1912, p. 45.

Nigeria. Tremearne, J.A.I., vol. xlii, p. 171.

Congo District. Johnston, George Grenfell, p. 671.

Bangala. Weeks, J.A.I., vol. xxxix, p. 449 ; Overbergh and Jonghe, Coll. Mon. Eth., No. i, p. 201.

Bahuana. Torday and Joyce, J. A. I., vol. xxxvi, p. 228.

Warega. Delhaise, loc. cit., p. 147.

Ababua. Halkin, loc. cit., p. 259.

Onolove. de Rochebrune, Rev. d'Anth., vol. iv, p. 283.

Mangbetu. (Rare.) Van Overbergh, Coll. Mon. Eth., No. 4, p. 298.

Bushongo. (Rare.) Torday and Joyce, J. A. I., vol. xxxvi, p. 111.

Akamba. Hobley, Akamba, p. 58.

Swaheli. Velten, loc. cit., p. 29.

Nyassa District. Fülleborn, Nyassa-Gebiet, p. 352.

British Central Africa. Johnston, British Central Africa, p. 417 ; Angus, Azimba and Chipitaland, p. 324.

Zambezi Valley. Maugham, Zambezia, p. 339.

South Africa. Maclean, Kaffir Laws, p. 62. 
Wadschagga. Gutmann, Wadschagga, p. 3.

Madagascar. Ellis, Madagascar, vol. i, p. 55.

I. Hottentots. Kolben, Cape of Good Hope, vol, i, p. 144.

Madagescar. Ellis, Madagascar, vol. i, p. 155; Little, Madagascar, p. 60 .

\section{OCEANIA}

R. New Zealand. Tregear, J. A. I., vol. xix, p. 103.

Torres Straits. Cambridge Expedition, vol. v, p. 199.

Savage Island (Niue). Thomson, Savage Island, p. 141.

Pelew Islands. Kubary, Journal des Museum Godeffroy, vol. i, p. 54.

Samoa. Krämer, Samoa-Inseln, vol. i, p. 38.

New Guinea. Seligman, J. A. I., vol. xxxii, p. 302 (Sinaugola); Schellong, Zeit.für Eth., vol. xxviii, p. 19 (Finschafen).

Solomon Islands. Ribbe, Zwei Jahre, p. 144 ; Glaumond, Rev. d'Eth., vol. vii, p. 80.

Bismarck Archipelago (New Britain Group). Brown, Melanesians, p. 37.

New Caledonia. Glaumond, loc. cit., p. 80 ; Lambert, Néo-Calédoniens, p. 104.

Fiji. Thomson, Fijians, p. 176 ; Seeman, Viti, p. 191.

Tonga. Thomson, loc. cit., p. 178.

Gilbert Islands (Kingsmill or Line Islands). Thomson, loc. cit., p. 178.

A. New Zealand. Dieffenbach, New Zealand, vol. ii, p. 12 ; Goldie, Trans. and Proc. N.Z. Inst., vol. xxxvii, p. 110 ; Tuke, Edin. Med. Journ., vol. ix, p. 735.

Torres Straits. Hunt, J.A.I., vol. xxviii, p. 9 ; Haddon, J. A. I., vol. xix, p. 359 ; Cambridge Expedition, vol. vi, p. 197.

Hawaii. Dumas, Îles Hawai, p. 18.

Gilbert Islands. Jenkins, Voyage, p. 404 ; Krämer, loc. cit., vol. ii, p. 53 ; Thomson, Fijians, p. 211.

Samoa. Krämer, loc. cit., vol. ii, p. 53 ; Turner, Samoa, p. 79.

Rotuma. Gardiner, J. A. I., vol. xxvii, p. 480.

Savage Island. Thomson, loc. cit., p. 141.

Fiji. Pritchard, Polynesian Reminiscences, p. 423 ; Waterhouse, Fiji, p. 327.

New Hebrides. Hagen and Pineau, Rev. d'Eth., vol. vii, p. 332; Jamieson, Aust. Med. Journ., vol. vii, p. 53.

New Caledonia. Codrington, Melanesians, p. 33 ; Ribbe, loc. cit., p. 144 ; Parkinson, Int. Arch. Eth., vol. xiii, p. 8 ; Elton, J. A. I., vol. xvii, p. 93.

Solomon Islands. Ribbe, loc. cit., p. 144.

Bismarck Archipelego. Brown, Melanesians, p. 33 ; Danks, J. A. I., vol. xviii, p. 291 ; Pfeil, Studien, p. 313 ; Stephan and Graebner, Neu-Mecklenburg, p. 18.

New Britain. Danks, J. A. I., vol. xviii, p. 291.

Aru Islands. Ribbe, loc. cit., p. 194.

Flores. Riedel, Rev. Col. Inter., vol. ii, p. 71

New Guinea. Krieger, Neu-Guinea, pp. 165, 292, 390, and 392 ; Murray, Papua, p. 194 ; Rosenberg, Malayische Archipel, p. 454 ; Williamson, Mafulu People, p. 176 ; Seligman, Melanesians, pp. 135 and 568; Neuhaus, Deutsch Neu-Guinea, vol. ii, p. 150.

Nias. Modigliani, Viaggio, p. 554.

Celebes. Kreutz, Zeit. Soc. Wiss., Heft 11, p. 21.

Luzon. Jenks, Ethnological Survey Publications, vol. i, p. 60.

Mitchell Group. Turner, Samoa, p. 280.

I. New Zealand. Angas, Savage Life, vol. i, p. 312 ; Taylor, Te Ika a Maui, p. 338 ; Tuke, loc. cit., p. 312. 
Torres Straits. Haddon, J. A. I., vol. xix, p. 359 ; Cambridge Expedition, vol. vi, p. 107.

Gilbert Islands. Tuituila, Journ. Pol. Soc., vol. i, p. 267.

Savage Island. Thomson, Fijians, p. 141.

Raratonga. Gill, Coral Islands, vol. ii, p. 13.

Tahiti. Lutteroth, Insel Tahiti, p. 12; Ellis, Polynesian Researches, vol. i, p. 249.

Pelew Islands. Kotzebue, Histoire, vol. xvii, p. 211.

Sandwich Islands. Ellis, Narrative, p. 324 ; Angas, Polynesia, p. 144 ; Dumas, loc. cit., p. 19.

Fiji. Waterhouse, Fiji, p. 328.

New Caledonia. Glaumond, loc. cit., p. 79 ; Bernard, Nouvelle-Calédonie, p. 288 ; Moncelin, Bull. Soc. Anth., vol. ix, p. 357.

New Hebrides. Somerville, J.A.I., vol. xxiii, p. 4 ; Paton, New Hebrides, p. 452.

Banks Island. Codrington, Melanesians, p. 229.

Solomon Islands (Ugi). Elton, J.A.I., vol. xvii, p. 93 ; Guppy, Solomon Islands, p. 42.

Bismarck Archipelago. Brown, Melanesians, 'p. 36 ; Pfeil, loc. cit., p. 18.

New Guinea. Seligman, Melanesians, pp. 568 and 705 ; Williamson, Mafulu People, p. 176 ; Newton, New Guinea, p. 189.

Funafuti. Edgeworth David, Funafuti, p. 195.

Tikopia (Barwell Island). Rivers, Melanesian Society, vol. i, p. 313.

Nissav. Thurmwald, Zeit. für Eth., vol. xl, 1908, p. 111.

Radeck. Kotzebue, Voyages, p. 173.

Vaitapu (Ellice Archipelago). Turner, Samoa, p. 284.

Marquesas. Hale, U.S. Exploring Expedition, vol. vi, p. 15.

Maupiti. Montgomery, Journal, vol. ii, p. 12.

Murray Islands. Hunt, J. A. I., vol. xxviii, p. 9.

\section{APPENDIX II}

Since the chapter on Human Fecundity was set up in type my attention has been called to a paper by Siegel (Münchener Medizinische Wochenschrift, 1916, p. 748). Though much has been said as to the supposed influence of the time of copulation with reference to the sexual cycle upon fertility, no definite information has been forthcoming. This paper, however, contains important data which bear upon this point. Owing to the fact that married soldiers only had occasional leave from the army during the war and then only for two or three days at a time, it has been possible to obtain information as to the period in the sexual cycle in which between two and three hundred children were conceived. Siegel finds that the likelihood of fertilization increases from the beginning of menstruation, reaches the highest point six days later, remains almost at the same height until the twelfth or thirteenth day, and then declines to the twenty-second day, after which there is absolute sterility.

If these data are confirmed, then those customs which encouraged copulation immediately after menstruation (as among the ancient Jews) or discourage it (as among the Hindus) must have an important bearing upon fertility. 


\section{LIST OF AUTHORITIES QUOTED}

A

A. E. Imagination and Reveries, London, 1915.

Abeel, D. Journal of a Residence in China, London, 1865.

Abel, C. W. Savage Life in New Guinea, London, 1902.

Acton, H. W., and Harvey, W. F. The Increase in the Number of Erythrocytes in the Blood, Biometrika, vol. viii.

Adami, J. G. Inheritance and Disease, in A System of Medicine, edited by Osler and McCrae, London, 1907.

- Medical Contributions to the Study of Evolution, London, 1918.

Agar, W. E. The Transmission of Environmental Effects from Parent to Offspring in Simocephalus vitulus, Phil. Trans., Series B, vol. cciii, 1913. Agricultural Statistics, Cd. 3832, vol. xvi, 1906.

Albertus Magnus.'De Secretis Mulierum, Amsterdam, 1665.

Ammon, O. Die natürliche Auslese beim Menschen, Jena, 1893. Zur Anthropologie der Badener, Jena, 1899.

Anderson, C. J. Lake Ngami, London, 1856.

Angas, G. F. Polynesia, London, 1847.

Angus, H. C. A Year in Azimba and Chipitaland, J. A. I., vol. xxvii.

Annandale, N., and Robinson, H. C. Fasciculi Malayenses, 1903.

Aquinas, St. Thomas. De Regimine Principum, Paris, 1509.

Armstrong, A. Personal Narrative of the Discovery of the North-West Passage, London, 1857.

Ashe, T. Travels in America, London, 1808.

Atkinson, J. J. : see Lang, A.

Augustine, St. On the Good of Marriage, in Seventeen Short Treatises (Library of the Fathers), Oxford, 1847.

Azara, Don Félix de. Voyages dans l'Amérique méridionale, Paris, 1809.

B

Baegert, J. An Account of the Original Inhabitants of the Californian Peninsula, $A . R . B . E ., 1863$.

Bailey, J. Wild Tribes of the Veddahs of Ceylon, Trans. Eth. Soc., 2nd Series, vol. ii, 1862.

Baker, G. W.: see Dublin, L. I.

Balls, W. L. Egypt of the Egyptians, London, 1915.

Bancroft, H. H. The Native Races of Pacific States of North America, London, 1875 .

Barnett, L. D. Antiquities of India, London, 1913.

Barrington, A., and Pearson, K. A First Study of the Inheritance of Vision, Eug. Lab. Mem., No. 5, 1909.

Barrow, J. Travels into the Interior of South Africa, London, 1806.

Bartels, M. : see Ploss, H. H.

Batchelor, J. The Ainu, London, 1901.

Battel, A. Strange Adventures, Pinkerton's Voyages, vol, xvi, London, 1851.

Beardmore, E. The Natives of Mowat, J. A. I., vol. xix, 1890.

Beau, C. le. Aventures ou Voyage Curieux et Nouveau, Amsterdam, 1738.

Beecham, J. Ashantee and the Gold Coast, London, 1841.

Beeton, M., and Pearson, K. Inheritance of the Duration of Life, Biometrika, vol. i, 1901. 
Belloc, H. Danton, London, 1911.

Benn, A. W. History of Rationalism, London, 1906.

Bernard, A. L'Archipel de la Nouvelle-Calédonie, Paris, 1895.

Bertherand, E. L. Médecine et Hygiène des Arabes, Paris, 1855.

Bessels, E. Einige Worte über die Innuit des Smith-Sundes, Arch. für Anth., vol. viii, 1875 .

Best, E. Maori Marriage Customs, Transactions and Proceedings of the New Zealand Institute, vol. xxxvi, 1903.

The Peopling of New Zealand, Man, vol. xiv, 1914.

Beveridge, P. The Aborigines of Victoria and Riverina, Melbourne, 1889.

Beveridge, W. H. Unemployment, London, 1912.

Bickman, A. S. Travels in the Eastern Archipelago, London, 1868.

Blagden, C. O. : see Skeat, W. W.

Blanckenhorn, M. : see Selenka, L.

Bland, J. O. P. Recent Events and Present Policies in China, London, 1912.

Blumentritt, F. Beiträge zur Kenntnis der Negritos, Z. G. E., vol. xxvii, 1892.

Blyth, D. Traditions and Customs of the Natives of Fiji, Glasgow Medical Journal, vol. xxviii, 1887.

Boas, F. The Central Eskimo, 6th A. R. B. E., 1884.

Ethnological Problems in Canada, $J . \dot{A}$. ., vol. xl, 1910.

Changes in the Bodily Form of Descendants of Immigrants, New York, 1912.

Bock, C. Head-Hunters of Borneo, London, 1881.

Temples and Elephants, London, 1884.

Bodin, J. Six Books of a Commonweale, London, 1866.

Bogoras, W. The Chuckee, Jesup North Pacific Expedition, vol. vii, 1904-9.

Bonaparte, Prince R. Les Habitants de Suriname, Paris, 1884.

Bonar, J. Malthus and His Work, London, 1885.

Bonney, F. Some Customs of the Aborigines of the River Darling, J. A. I., vol. xiii, 1884.

Bonwick, J. Daily Life and Origin of the Tasmanians, London, 1870.

Bosanquet, B. Philosophical Theory of the State, London, 1910.

Bossu. Nouveaux Voyages, Paris, 1768.

Botero, G. Delle Cause della Grandezza della Città, Milan, 1596.

Boule, M. L'Homme fossile de la Chapelle-aux-Saints, Ann. de Pal., vols. vi, vii, and viii, 1911-13.

Bowley, A. L. The Division of the Product of Industry, Oxford, 1919.

- Change in the Distribution of the National Income, 1880-1913, Oxford, 1920.

Brainne, C. La Nouvelle-Calédonie, Paris, 1854.

Brehm, A. E. Bird Life, London, 1874.

Brenner, J. von. Besuch bei den Kannibalen Sumatras, Würzburg, 1894.

Breton, W. H. Excursions in New South Wales, London, 1834.

Brett, H. W. The Indian Tribes of Guiana, London, 1868.

Breuil, $\mathbf{H}$. Les Subdivisions du Paléolithique Supérieur et leur signification, C. I. A., 1906.

— Sur la présence d'Éolithes à la Base de l'Éocène Parisien, L'Anthropologie, vol. xxi, 1910.

Bridge, T. W. Fishes, in Cambridge Natural History, vol. vii, Cambridge, 1904.

Bridges, J. W., and Coles, L. E. Relation of Intelligence to Social Status, Psychological Review, vol. xxiv, 1917.

Bridges, T. Manners and Customs of the Firelanders, A Voice from South America, vol. xiii, 1866.

Brooke, C. Ten Years in Sarawak, London, 1860.

Brown, A. R. Three Tribes of Western Victoria, J. A. I., vol. xliii, 1913.

Brown, G. Melanesians and Polynesians, London, 1910. 
Brown, R. Description of the Natives of King George's Sound, Geog. Journ., vol. i, 1832 .

Brown, W. New Zealand and its Aborigines, London, 1845.

Browne, B. J. C. Indian Infanticide, London, 1857.

Bryant, A. T. Mental Development of the South African Native, Eug. Rev., vol. ix, 1917.

Bryce, J. Two Centuries of Irish History, edited by, London, 1888.

Buchanan, J. North American Indians, London, 1824.

Büchner, C. Industrial Evolution, London, 1901.

Burchell, W. J. Travels in the Interior of South Africa, London, 1822.

Burckhardt, J. L. Notes on the Bedouins, London, 1831.

Burrows, G. Natives of the Upper Welle District, J. A. I., vol. xix, 1890.

Burton, R. F. Abeokuta and the Cameroon Mountains, London, 1863. Sindh, London, 1851.

The Lake Regions of Central Africa, London, 1860.

Buttikoffer, J. Einiges über die Eingeborenen von Liberia, Int. Arch. Eth., vol. i, 1888.

Cabeça da Vaca, A. N. Narrative, Washington, 1851.

Cambridge Anthropological Expedition to the Torres Straits, edited by Haddon, Cambridge, 1907.

Campanella, T. Civitas Solis, 1623.

Campbell, J. A Personal Narrative of Thirteen Years Service, London, 1864. Journal of Travels in South Africa, London, 1834.

Cannan, E. A History of the Theories of Production and Distribution, London, 1903. Wealth, London, 1914.

Carpenter, E. Intermediate Types, London, 1914.

Carver, J. Travels through the Interior Parts of North America, London, 1871.

Casalis, E. The Basutos, London, 1861.

Castelnau, F. de. Expéditions dans les Parties centrales de l'Amérique du Sud, Paris, 1850.

Catlin, G. North American Indians, London, 1842.

Chaillu, P. B. du. Explorations and Adventures in Equatorial Africa, London, 1861.

Chalmers, J. Natives of Kiwai Island, J. A. I., vol. xxxiii, 1903.

Chalmers, T. Political Economy in connexion with the Moral State and Moral Prospects of Society, Glasgow, 1832.

Chardin, J. Travels, in Pinkerton's Voyages, vol. ix, London, 1811.

Charlevoix, de. Histoire et Description générale de la Nouvelle-France, Paris, 1744.

Charlevoix, P. F. X. de. History of Paraguay, London, 1769.

Cheyne, A. Description of Islands in the Western Pacific Ocean, London, 1852.

Child, Sir J. A New Discourse upon Trade, London, 1694.

Christian, F. W. Exploration in the Caroline Islands, Geog. Journ., vol. xiii, 1899.

Church, G. E. Aborigines of South America, London, 1912.

Clapperton, H. : see Denham, D.

Clarke, W. : see Lewis, M.

Clemow, F. G. The Geography of Disease, Cambridge, 1903.

Codrington, R. H. The Melanesians, Oxford, 1891 .

Cole, H. The Wagogo of German East Africa, J. A. I., vol. xxxii, 1902.

Coles, L. E. : see Bridges, J. W.

Collineau, Dr. L'Infanticido et l'Avortement en Chine, Revue Mensuelle de l'École d'Anthropologie, 1899. 
Comte, A. Cours de Philosophie positive, Paris, 1889.

Conder, C. R. Native Tribes of Bechuanaland, J. A. I., vol. xvi, 1897.

Cooper, J. M. Analytical and Critical Bibliography of the Tribes of Tierra del Fuego, S. I. B. E., Bull. 63, 1917.

Coquilhat, C. Sur le Haut-Congo, Paris, 1888.

Coulanges, Fustel de. La Cité Antique, Paris, 1890.

Crantz, D. The History of Greenland, London, 1767.

Crawford, J. Journal of an Embassy, London, 1834.

Crawley, E. The Mystic Rose, London, 1902.

Creswell, K. A. C. Fluctuations in the Population of Irrigated Countries, Man, vol. xv, 1915 .

Crooke, W. Natives of Northern India, London, 1907. North-Western Provinces of India, London, 1897.

Croom, J. H. Mittelschmerz, Trans. Edin. Obstet. Soc., vol. xxi, 1896.

Cunningham, W. Growth of-English Industry and Commerce, Cambridge, 1910.

Cureau, A. Les Sociétés primitives de l'Afrique Équatoriale, Paris, 1912.

Curr, E. M. Recollections of Squatting in Victoria, Molbourne, 1883. The Australian Race, London, 1886.

Czaplicka, M. A. Aboriginal Siberia, Oxford, 1919.

D

Dale, G. Natives of the Bondei Country, J. A. I., vol. xxv, 1890.

Dall, W. H. Alaska and its Resources, London, 1870.

Danks, B. Marriage Customs of the New Britain Group, J. A. I., vol. xviii, 1889.

Darwin, C. Variations of Animals and Plants under Domestication, London, 1885.

The Descent of Man, London, 1871.

The Origin of Species, London, 1885.

Life and Letters, edited by Francis Darwin, London, 1887.

Dasent, G. W. The Story of Burnt Njal, Edinburgh, 1861.

Davenant, Sir. C. Political and Commercial Works, London, 1771.

David, Mrs. Edgeworth. Funafuti, London, 1899.

Davids, Mrs. Rhys. Buddhism, London, 1912.

Davidson, A. Geographical Pathology, Edinburgh, 1892.

Davies, M. F. Life in an English Village, London, 1909.

Dawson, C., and Woodward, A. S. On the Discovery of a Human Palaeo. lithic Skull, Quart. Journ. Geol. Soc., vol. Ixix, 1913.

Dawson, J. Australian Aborigines, Melbourne, 1881.

Déchelette, J. Manuel d'Archéologie préhistorique, Paris, 1912.

Delacour, A. Les Tenda, Rev. d'Eth., 1912.

Delhaise. Les Warega, Coll. Mon. Eth., No. 5, Brussels, 1909.

Dellenbaugh, F. S. The North Americans, New York, 1901.

Delvaille, J. Essai de l'Histoire de l'Idée de Progrès, Paris, 1910.

Denham, D., and Clapperton, H. Narrative of Travels and Discoveries, London, 1826.

Deniker, J. Etude sur les Kalmouks, Rev. d'Eth., vol. vi, 1883.

Les Giliaks, Rev. d'Eth., vol. ii, 1883. see Hyades, P.

Desplagnes, L. Le Plateau central Nigérien, Paris, 1907.

Dexter, E. G. Weather Influences, New York, 1904.

Dickson, $\dot{H}$. N. Climate and Weather, London, 1885.

Dieffenbach, E. Travels in New Zealand, London, 1843.

Dobell, P. Travels in Kamtchatka, London, 1830.

Dobrizhoffer, M. An Account of the Abipones, London, 1822.

Dodge, R. I. The Hunting Grounds of the Great West, Iondon, 1877. 
Domenech, E. Seven Years' Residence in the Great Deserts of North America, London, 1860.

Doncaster, L. An Introduction to the Study of Cytology, Cambridge, 1920.

Dorsey, J. O. Omaha Sociology, 3rd A.R. B. E., 1881.

Doughty, G. M. Travels in Desert Arabia, Cambridge, 1888.

Douglas, R. K. China, London, 1987.

Dublin, L. I., and Baker, G. W. The Mortality of Race Stocks in Pennsylvania and New York, Am. Stat. Ass., vol. xvii, 1920.

Dubois, J. A. Hindu Manners, Customs, and Ceremonies, Oxford, 1897.

Duckworth, W. L. H. Morphology and Anthropology, Cambridge, 1904.

Dudfield, R. Some unconsidered Factors affecting the Birth-rate, J. R. S. S., vol. lxxi, 1908.

Dumas, P. M. -Travels in Kamtchatka, London, 1830.

- Îles Hawaî. Collection des Thèses ; École de Médecine, Paris, 1861.

Duncan, J. Matthews. Sterility in Women, London, 1834. - Fecundity, Fertility, and Sterility, Edinburgh, 1866.

Dundas, C. History of Kitui, J. A. I., vol. xliii, 1913.

Dundas, K. R. The Wawanga, J.A.I., vol. xliii, 1913. Baringo District of British East Africa, J. A. I., vol. xl, 1890.

Dunlop, J. C. The Fertility of Marriage in Scotland, J.R.S. S., vol. Ixxvi, 1914.

Durkheim, E. De la Division du Travail social, Paris, 1911.

$\mathbf{E}$

Earle, A. Narrative of a Nine Months' Residence in New Zealand, London, 1832.

East, E. M., and Jones, D. F. Inbreeding and Outbreeding, Philadelphia, 1919.

Ehrenreich, P. Beiträge zur Völkerkunde Brasiliens, Königliches Museum zu Berlin, vol. i, 1891 .

_- Die Einteilung und Verbreitung der Völkerstämme Brasiliens, Petermanns Mittheilungen, vol. xxvii, 1891.

Elbert, J. Dubois' Altersbestimmung der Kendengschichten, Cent. für Min., Geol. und Pal., vol. xvii, 1909.

Elderton. E. M. The Relative Strength of Nature and Nurture, Galton Laboratory Lecture Series, No. 3, London, 1909.

Elderton, E. M., and Pearson, K. Further Evidence of Natural Selection in Man, Biometrika, vol. x, 1915.

Ellenberger, D. F. History of the Basuto, London, 1912.

Elliot, G. F. Scott. Native West African Customs, J. A. I., vol. xxiii, 1893.

Elliot, H. W. Our Arctic Province, New York, 1886.

-Ellis, A. B. The Ewe-Speaking Peoples, London, 1890.

Ellis, H. A Voyage to Hudson's Bay, London, 1748.

Ellis, Havelock. Studies in the Psychology of Sex, Philadelphia, 1910.

The Philosophy of Conflict and other Essays in War Time, London, 1919.

Ellis, W. History of Madagascar, London, 1833.

- Narrative of a Tour through Hawaii, London, 1827. Polynesian Researches, London, 1832.

Elton, F. Journal of an Expedition on the Limpopo River, Geog. Journ., vol. xlii, 1872.

Engelmann, F. Die Eingeborenen der Kolonie Südaustralien, Berlin, 1908.

Engelmann, G. J. First Age of Menstruation in the North American Continent, Trans. Amer. Gyn. Soc., vol. xxvi, 1901.

Epp, F. Schilderungen aus Holländisch-Ostindien, Heidelberg, 1852.

Eram, P. Quelques considérations sur les accouchements en Orient, Paris, 1860. 
Erdweg, M. J. Die Bewohner der Insel Tumleo, Mittheilungen der Anthropologischen Gesellschaft in Wien, vol. xxxii.

Eucken, R. Main Currents of Modern Thought, London, 1912.

Ewart, R. J. The Influence of Parental Age on Offspring, Eug. Rev., vol. iii, 1911.

Eylmann, E. Die Eingeborenen der Kolonie Südaustralien, Berlin, 1908.

Eyre, E. J. Journals of Expeditions of Discovery into Central Australia, 1845.

Fabry, H. Aus dem Leben der Wapogoro, Globus, vol. xci, 1907.

Falkner, T. Dessription of Patagonia, Hereford, 1774.

Faulds, H. Nine Years in Nipon, London, 1885.

Featherman, A. Social History of the Races of Mankind, London, 1881.

Felkin, R. W. Notes on Labour in Central Africa, Trans, Edin. Obstet. Soc., vol. ix, 1884.

see Wilson, C. T.

Ferdy, H. Die künstliche Beschränkung der Kinderzahl als sittliche Pficht, Berlin, 1897.

Ferguson, A. Essay on the History of Civil Society, London, 1767.

Field, J. A. The Early History of the Population Movement, American Economic Review, April, 1911.

Finck, H. T. Primitive Love, New York, 1899.

Finsch, O. Reise nach West-Sibirien, Berlin, 1879. Samoafahrten, Leipzig, 1883.

Fishberg, M. Materials for the Physical Anthropology of the Eastern European Jews, Mem. Am. Anth. Soc., vol. i, 1905.

Fisher, H. A. L. The Sociological View of History, Soc. Rev., vol. i.

Fisher, R. A. The Genesis of Twins, Genetics, vol. iv, 1919.

Fison, L. Land Tenure in Fiji, J.A. I., vol. x.

Fison, L., and Howitt, A. W. Kamilaroi and Kurnai, Melbourne, 1880.

Fitzroy, R. : see King, P. P.

Fletcher, A. C., and La Flesche, F. The Omaha Tribe, 27th A. R. B. E., 1905.

Flower, W. H., and Lydekker, R. An Introduction to the Study of Mammals, London, 1891.

Foelsche, P. Manners and Customs of some Tribes in the Neighbourhood of Port Darwin, J. A. I., vol. xxiv, 1895.

Foley. La Coquette Néo-Calédonienne, Bull. Soc. Anth., vol. ii, 1879.

Forbes, D. Aymara Indians of Bolivia and Peru, Journ. Eth. Soc., vol. ii, 1870.

Forbes, F. E. Dahomey, London, 1851.

Forsyth, J. Highlands of Central India, London, 1871.

Franklin, B. Observations concerning the Increase of Mankind, London; 1751.

Franklin, J. Narrative of a Journey, London, 1823.

Frazer, J. G. The Golden Bough, London, 1890.

Frederici, G. F. Forschungsreise nach dem Bismarck-Archipel, Mittheilungen aus den deutschen Schutzgebieten, Berlin, 1913.

Frere, Sir H. Bartle. Land Tenure in South Africa, J. A. I., vol. xii.

Fritsch, G. Die Eingeborenen Süd-Afrikas, Breslau, 1872.

Froude, J. A. Henry VIII, London, 1911.

Fülleborn, F. Das deutsche Nyassa- und Rowuma-Gebiet, Berlin, 1906.

G

Galton, F. Inquiries into Huma\% Faculty, London, 1883.

Gardiner, J. Stanley. Natives of Rotumna, J. A. I., vol. xxvii, 1898.

Gardner, C. T. On the Chinese Race, Journ. Eth. Soc., vol. ii, 1870. 
Garnier, J. Du Principe de la Population, Paris, 1885.

Gason, S. Aborigines of Australia, J. A. I., vol, xiv, 1895. Manners and Customs of the Dieyerie Tribe, Adelaide, 1879.

Gaud, F. Les Mandja, Coll. Mon. Eth., vol. vii, 1911.

Gautier, G. F. Madagascar, Paris, 1902.

Geisler, J. Die Oster-Insel, Berlin, 1883.

Gemmill, J. F. Teratology of Fishes, Glasgow, 1912.

Georgi, J. G. Bemerkungen einer Reise im russischen Reich, St. Petersburg, 1775.

Gerland, G. Über das Aussterben der Naturvölker, Leipzig, 1868.

Gibbs, G. Tribes of Western Washington and North-west Oregon, U.S. Geog. and Geol. Survey, vol. i, 1877.

Giles, H. A. The Civilization of China, London, 1911.

Gill, W. Gems from the Coral Islands, London, 1885.

Gillen, F. J.: see Spencer, B.

Ginsberg, M. : see Hobhouse, L. T.

Glaumond. Néo-Calédoniens, Rev. d'Eth., vol. vii, 1889.

Glotz, G. Etudes sociales et juridiques sur l'Antiquité grecque, Paris, 1906.

Godden, G. M. Naga and other Frontier Tribes, J. A. I., vol. xxvi, 1897.

Godwin, W. Political Justice, London, 1798.

Goldie, W. H. Maori Medical Lore, Trans. and Proc. N. Z. Inst., vol. xxxvii, 1904.

Gomes, E. H. Sea Dyaks of Borneo, London, 1907.

Gönezi, F. Die Gebräuche im Kom-Zala, Zentralblatt für Anthropologie, vol. xii, 1907.

Goodrich, E. S. Evolution of Living Organisms, London, 1919.

Gowland, W. The Metals in Antiquity, J. A. I., vol. xlii, 1912.

Graebner, F.: see Stephan, E.

Granville, R. K. Notes on the Jekris, J. A. I., vol. xxvii, 1898.

Graunt, J. Natural and Political Observations upon the Bills of Mortality, London, 1662.

Gray, J. H. China, London, 1878.

Greenwood, M., and Yule, G. U. The Determination of the Size of the Family, J.R. S. S., vol. lxxii, 1914.

Greg, W. R. Enigmas of Life, London, 1872.

Gregory, A. C. Aborigines of Australia, J. A. I., vol. xxvii, 1899.

Gregory, J. W. Is the Earth drying up ? Geog. Journ., vol. xliii, 1912.

Grey, G. Journals of two Expeditions of Discovery, London, 1841.

Grimm, J. Deutsche Rechtsalterthümer, Leipzig, 1899.

Grinnell, G. B. Cheyenne Women Customs, Am. Anth., vol. iv, 1902.

Grosse, F. Die Formen der Familie, Freiburg, 1906.

Grubb, W. B. An Unknown People, London, 1911.

Guinnard, A. Three Years' Slavery among the Patagonians, London, 1871.

Guise, R. E. Tribes of the mouth of the Wanigela River, J. A. I., vol. xxviii, 1899.

Guppy, H. B. The Solomon Islands, London, 1897.

Gutmann. Die Frau bei den Wadschagga, Globus, vol. xcii, 1907.

\section{H}

Haddon, A. C. The Wanderings of Peoples, Cambridge, 1911.

- Western Tribes of the Torres Straits, J. A. I., vol. xix.

Haeckel, E. The History of Creation, London, 1876.

Hagen, A., and Pineau, A. Les Nouvelles-Hébrides, Rev. d'Eth., vol. vii, 1888.

Hagen, B. Unter den Papuas, Wiesbaden, 1899.

Die Orang Kubu, Frankfurt am Main, 1908.

Hahn, E. Die Entstehung der Pflugkultur, Heidelberg, 1909.

Hahn, J. Ovahereró, Z. G. E., vol. iii, 1868.

Hale, H. United States Exploring Expedition, vol. vi, 1846. 
Hale, Sir M. The Primitive Origination of Mankind, London, 1667.

Halkin, J. Les Ababua, Coll. Mon. Eth., vol. vii, 1911.

Hall, C. F. Narrative of the Second Arctic Expedition, Washington, 1789.

Hall, H. R. Ancient History of the Near East, London, 1913.

Hammond, J. L., and B. The Village Labourer, London, 1911.

Handbook of American Indians, S. I. B. E., 1907.

Handwörterbuch der Staatswissenschaften, Jena, 1909.

Hansen. S. On the Increase of Stature in Certain European Populations, Problems in Eugenics, London, 1912.

Hardisty, W. L. The Loucheux Indians, A. R. S. I., 1866.

Harmon, D. W. A Journal of Voyages and Travels, New York, 1903.

Harris, J. H. Dawn in Darkest Africa, London, 1912.

Harris, J. M. Gallinas of Sierra Leone, Mem. Anth. Soc., vol. ii, 1866.

Harrison, W. Description of England, London, 1877.

Hartland, E. S. Primitive Paternity, London, 1909.

Harvey, W. F.: see Acton, H. W.

Haseman, J. D. Notes on the Pawumwa Indians, Am. Anth., vol. xiv, 1912.

Hawtrey, H. A. C. Lengua Indians, J. A. I., vol. xxi.

Heape, W. Abortion, Barrenness, and Fertility in Sheep, Journ. Roy. Agric. Soc., vol. $x, 1899$.

The Sexual Season of Mammals, Q. J.M.S., vol. lxiv, 1900.

Hearne, S. A Journey from the Prince of Wales's Fort to the Northern Ocean, London, 1795.

Hecker, J. F. K. Epidemics of the Middle Ages, London, 1844.

Henderson, R. Mortality Laws and Statistics, New York, 1915.

Herbertson, A. J., and F. D. Man and His Work, London, 1914.

Heron, D. On the Relation of Fertility in Man to Social Status, Drapers' Company Research Memoirs, No. 1, 1906.

Heriot, G. Travels through the Canadas, London, 1807.

Hickson, S. J. Naturalist in the New Hebrides, London, 1889.

Hildebrand, R. Recht und Sitte, Jena, 1896.

Hildebrandt, J. M. Ethnographische Notizen über Wakamba, Zeit. für Eth., vol. xx, 1878.

Hill, J. A. Comparative Fecundity of Women of Native and Foreign Parentage in the U.S.A., Am. Stat. Ass., vol. xiii, 1913.

Hirsch, A. Handbook of Geographical and Historical Pathology, London, 1883.

Hitchcock, R. The Ainos of Yezo, A. R. S. I., 1890.

Hobhouse, L. T. Development and Purpose, London, 1919.

Hobhouse, L. T., Wheeler, G. C., and Ginsberg, M. Material Culture and Social Institutions of the Simpler Peoples, London, 1915.

Hobley, C. W. Ethnology of the Akamba, Cambridge, 1910.

Kavirondo and Nandi, J. A. I., vol. xxxvi, 1903.

Hodden, W. C. Past and Future of the Kaffir Races, London, 1866.

Hodson, T. C. Female Infanticide in India, Man, vol. xiv, 1914. Naga Tribes of Manipur, London, 1911.

The Meitheis, London, 1908.

Hoernes, M. Natur- und Urgeschichte des Menschen, Vienna, 1909.

Hoffman, W. G. The Menomini Indians, 14th A. R. B. E., 1892.

Hogarth, D. G. The Ancient East, London, 1914.

Holder, A. B. Gynecic notes taken among American Indians, Am. Journ. Obstet., vol. xxv, 1892.

Holdich, Sir T. Political Frontiers and Boundary Making, London, 1916.

Hollis, A. C. The Nandi, Oxford, 1909. The Masai, Oxford, 1905.

Hose, C., and McDougall, W. The Pagan Tribes of Borneo, London, 1912.

Howard, G. E. History of Matrimonial Institutions, London, 1904.

Howitt, A. W. Native Tribes of South-east Australia, London, 1904. 
Howitt, A. W. : see Fison, L.

Hrdlicka, A. Early Man in South America, S. I. B. E., Bull. lii, 1912. Tuberculosis among certain Indian Tribes, $S . \dot{I}$. B. E., Bull. xlii, 1909. Skeletal Remains suggesting or attributed to Early Man in North America, S. I. B. E., Bull. xxxiii, 1907.

Recent Discoveries attributed to Early Man in South America, S. I. B. E., Bull. lxvi, 1917.

Physiological and Medical Observations, S. I. B. E., Bull, xxxiv, 1908.

Hubert, H., and Mauss, M. Mélanges d'Histoire des Religions, Paris, 1909.

Huc, M. Souvenirs d'un Voyage, Paris, 1860.

Humboldt, A. von. Personal Narrative of Travels, London, 1818.

Hunt, A. E. Notes on the Murray Islands, J. A. I., vol. xxviii, 1899.

Huntington, E. The Pulse of Asia, London, 1907.

World Power and Evolution, New York, 1919.

Civilization and Climate, New Haven, 1915.

Hutchinson, T. J. Impressions of Western Africa, Iondon, 1858.

-Ten Years' Wanderings, London, 1861.

Hutereau, A. Notes sur la vie familiale, Ann. Mus. Congo Belge, vol. i, 1909.

Hutton, S. K. Among the Eskimos of Labrador, London, 1912.

Hyades, P., and Deniker, J. Mission scientifique du Cap Horn, Paris, 1891.

Inge, W. R. Outspoken Essays, London, 1919.

Inglis, J. In the New Hebrides, London, 1887.

Jackson, F. G. The Great Frozen Land, London, 1895.

Jamieson, J. Natives of the New Hebrides, Aust. Med. Journ., vol. vii, 1885 .

Jellinghaus, T. Chota Nagpur, Zeit. für Eth., vol. iii, 1871.

Jenkins, J. S. Voyage of U. S. Exploring Squadron, Auburn, 1850.

Jenkinson, J. W. Experimental Embryology, Oxford, 1909.

Jenks, A. E. The Bontoc Igorot, Department of the Interior ; Ethnological Survey Publications, vol. i, Manilla, 1905.

Jennings, H. S. Behaviour of the Lower Organisms, New York, 1906.

Jeune, Le, Jesuit Relations and Allied Documents, Cleveland, 1897.

Jochelson, W. The Koyak, Jesup North Pacific Expedition, vol vi. The Yukaghin, Jesup North Pacific Expedition, vol. ix.

Joest, W. Guayana, Int. Arch. Eth., vol. v, 1893.

Johnson, R. H. : see Popenoe, P.

Johnston, Sir H. H. British Central Africa, London, 1897.

George Grenfell and the Congo, London, 1908.

The Negro in the New World, London, 1914.

The Uganda Protectorate, London, 1902.

Johnstone, J. Conditions of Life in the Sea, Cambridge, 1908.

Jolly, J. The Birth Time of the World, Science Progress, No. 33, 1914.

Jones, D. F. : see East, E. M.

Jones, F. Wood. The Origin of Man, in Animal Life and Human Progress, edited by Dendy, London, 1919.

Jones, P. History of the Ojebway Indians, London, 1861.

Jones, S. The Kutchin Tribes, A. R. S. I., 1866.

Jones, W. H. S. Malaria and Greek History, London, 1909.

Jonghe, E. de : see Overbergb, C. van.

Joubert, J. Pensées, Paris, 1911.

Joyce, T. A. Mexican Archaeology, London, 1914. 
Joyce, T. A. South American Archaeology, London, 1914.

- see Torday, E.

Junod, H. A. Les Ba-Ronga, Neuchâtel, 1893.

- Life of a South African Tribe, London, 1912.

K

Kaegi, A. The Rigveda, Boston, 1886.

Kate, H. Ten. Notes ethnographiques sur les Comanches, Rev. d'Eth., vol. iv, 1885.

Keane, A. H. The Botocudos, J. A. I., vol. xiii, 1884.

Keating, W. H. Narrative of an Expedition, Philadelphia, 1824.

Keith, A. Address to Section H, British Association, Nature, vol. civ, 1919.

Kelly, H. A. Medical Gynecology, London, 1912.

Kelly, J. W. : see Wells, R.

Kerr, J. The Domestic Life of the Natives of India, London, 1865.

Kerr, W. M. The Far Interior, London, 1866.

Keynes, J. M. The Economic Consequences of the Peace, London, 1919.

Kidd, D. The Essential Kaffir, London, 1904.

King, J. S. On the Practice of Female Circumcision, Journal of the Anthropological Society of Bombay, vol. ii, 1890.

King, P. P., and Fitzroy, R. Narrative of the Voyages of the Adventure and the Beagle, London, 1839.

Kingsley, M. H. West African Studies, London, 1901.

Kirkby, W. W. The Kutchin Indians, Church Missionary Intelligencer, vol. xiv, 1863.

Klebs, G. The Influence of the Environment on the Forms of Plants, Darwinism and Modern Science, Cambridge, 1909.

Klemm, G. Allgemeine Kultur-Geschichte der Menschheit, Leipzig, 1843.

Klutschak, H. W. Als Eskimo unter den Eskimos, Leipzig, 1881.

Knibbs, G. K. The Problems of Population, Food Supply, and Migration, Scientia, vol. xii, 1919.

Census of the Commonwealth of Australia, Appendix A, vol. i.

Koch-Grünberg. Zwei Jahre unter den Indianern, Berlin, 1910.

Kohlbrugge, J. H. F. The Influence of a Tropical Climate on Europeans, Eug. Rev., vol. iii, 1911.

Kolben, P. The Present State of the Cape of Good Hope, London, 1831.

Kotzebue, O. von. A Voyage of Discovery into the South Sea, London, 1821.

Krämer, A. Die Samoa-Inseln, Stuttgart, 1902.

Hawai, Stuttgart, 1906.

Krapf, J. L. Travels, Researches, and Missionary Labours, London, 1860.

Krasheninicoff, H. The History of Kamtchatka, London, 1864.

Krause, A. Die Thinklit-Indianer, Jena, 1885.

Kreutz, A. C. Zentralcelebes, Zeit. für Soc., vol. xi, 1903.

Krieger, E. Die Menstruation, Berlin, 1869.

Krieger, M. Neu-Guinea, Berlin, 1899.

Kubary, J. Die Palau-Inseln, Journal des Museum Godeffroy, vol. i, 1873-4. Ethnographische Beiträge zur Kenntniss der Karolinischen Inselgruppe, Berlin, 1883.

La Flesche, F. : see Fletcher, A. C.

Lambert, Le Père. Mours et Superstitions des Néo-Calédoniens, 1900.

Lang, A. Social Origins (including Primal Law by J. J. Atkinson), London, 1903.

Lapouge, C. Vacher de. Les Sélections sociales, Paris, 1896.

Lea, H. C. History of Sacerdotal Celibacy, London, 1907.

Lecky, W. E. H. History of European Morals, London, 1877. 
Lee, Sir S. Great Englishmen of the Sixteenth Century, London, 1904.

Leiper, R. T. Some Inhabitants of Man, in Animal Life and Human Progress, edited by Dendy, London, 1909.

Lenormant, C. Cours d'Histoire ancienne, Paris, 1837.

Leonard, A. G. The Lower Niger, London, 1906.

Leong, Y. K., and Tao, L. K. Village and Town Life in China, London, 1915.

Leroy-Beaulieu, A. Israël chez les Nations, Paris, 1893.

Leroy-Beaulieu, P. La Question de la Population, Paris, 1913.

Leslie, D. Among the Zulus, Edinburgh, 1875.

Lesseps, de. Travels in Kamtschatka, London, 1870.

Letourneau, C. Evolution of Marriage, London, 1890. La Guerre, Paris, 1895.

Lewis, M., and Clarke, W. History of an Expedition, Chicago, 1902.

Lichtenstein, H. Travels in South Africa, London, 1812.

Lisiansky, V. A Voyage round the World, London, 1814.

Little, H. W. Madagascar, London, 1884.

Livingstone, D. Missionary Labours and Travels, London, 1837.

Lock, R. H. Recent Progress in the Study of Variation, Heredity, and Evolu. tion, London, 1911.

Long, J. Voyages and Travels of an Indian Interpreter, London, 1791.

Long, S. H. An Account of an Expedition, in Early Western Travels, edited by Thwaites, vols. xiv and $\mathbf{x v}$.

Lord, J. K. A Naturalist in Vancouver Island, London, 1866.

Lortsch, A. Neu-Caledonien, Globus, 1875.

Lowe, F.: see Smythe, W.

Lübbert, A. Uं̉ber die Heilmethoden in Deutsch-Südwestafrika, Mittheilungen von Forschungsreisenden und Gelehrten aus den deutschen Schutzgebieten, vol. xiv, 1901 .

Lubbock, J. Prehistoric Times, London, 1890.

- Primitive Man, London, 1900.

Luden, H. Handbuch der Staatsweisheit oder Politik, Jena, 1811.

Lull, R. S. Organic Evolution, New York, 1917.

Lumholtz, C. Among Cannibals, London, 1889.

Unknown Mexico, London, 1903.

Luschan, F. von. Eisentechnik in Africa, Zeit. für Eth., vol. xli, 1909.

Lutteroth, H. Geschichte der Insel Tahiti, Berlin, 1843.

Lydekker, R. : see Flower, W. H.

\section{M}

Machiavelli, N. History of Florence, London, 1909.

Macdonald, D. Africana, London, 1882.

Macdonald, J. East African Customs, J. A. I., vol. xxii, 1892.

- Manners of South African Tribes, $J_{.} A . \dot{I}$., vols. xix and xx, 1890.

Macdonell, W. R. The Expectation of Life in Ancient Rome, Biometrika, vol. ix.

McDougall, W. Introduction to Social Psychology, London, 1908.

The Group Mind, Cambridge, 1920.

- Psychology, London, 1912. see Hose, $\mathrm{C}$.

McGee, W. J. The Seri Indians, 17th A. R. B. E., 1895.

The Siouan Indians, 15th $A . R . B . E ., 1893$.

Macgregor, W. British New Guinea, London, 1897.

Mackenzie, A. Voyages from Montreal, New York, 1902.

Maclean, J. Compendium of Kaffir Laws and Customs, Mount Coke, 1858.

MacLennan, J. F. Studies in Ancient History, London, 1896.

MacLeod, J. The Quantitative Method in Biology, Manchester, 1919. 
Macpherson, C. S. Memorials of Service in India, London, 1865. The Religion of the Khonds, London, 1852.

Maistre, J. de. Du Pape, Lyon, 1849.

Malinowski, B. The Family among the Australian Aborigines, London, 1913.

Man, E. H. On the Original Inhabitants of the Andaman Islands, J. A. I., vol. xii, 1883.

Manning, O. History and Antiquities of Surrey, London, 1884.

Manson, Sir P. Article in A System of Medicine, vol. ii, part 2, edited by Allbutt and Rolleston.

Marchant, J. A. R. Wallace, Letters and Reminiscences, London, 1916.

Marett, R. R. Psychology and Folk-Lore, London, 1920.

Marro, A. La Pubertà studiata nell' Uomo e nella Donna, Turin, 1900.

Marsden, W. History of Sumatra, London, 1783.

Marshall, A. The Principles of Economics, London, 1907.

Marshall, F. H. A. Fertility in Scottish Sheep, Transactions of the Highland Agricultural Society, vol. xx, 1908.

- Physiology of Reproduction, London, 1910.

- Oestrous Cycle in the Sheep, Phil. Trans., Series B, vol. cxcvi, 1904.

Martineau, H. Illustrations to Political Economy, London, 1832.

Martineau, J. Autobiography, London, 1887.

Martius, C. F. von. Beiträge zur Ethnographie Amerikas, Leipzig, 1867.

- see Spix, J. B. von.

Marx, K. Capital, London, 1887.

Mathew, J. Eaglehawk and Crow, London, 1899.

Two Representative Tribes of Queensland, London, 1910.

Mathews, R. H. Ethnological Notes on the Aboriginal Tribes of New South Wales and Victoria, Sydney, 1905.

Matignon, J. J. Dix Ans au Pays du Dragon, Paris, 1910.

-La Superstition, le Crime et la Misère en Chine, Paris, 1910.

Matthews, W. Hidatsa Indians, U. S. Geog. and Geol. Survey, vol. vii, 1877.

Maugham, R. F. C. Portuguese East Africa, London, 1916.

Maurault, J. A. Histoire des Abenakis, Paris, 1866.

Mauss, M. : see Hubert, $\mathrm{H}$.

Mayer, A. Über Sterilität, Leipzig, 1908.

Meade, H. A Ride through the Disturbed Districts of New Zealand, London, 1870.

Meinecke. Der Archipel der neuen Hebriden, Z. G. E., vol.i x, 1874.

Melville, H. Marquesas Islands, London, 1846.

Meyer, H. E. A. Manners and Customs of the Aborigines of Encounter Bay, Adelaide, 1879.

Mill, James. Article 'Colony', Encyclopaedia Britannica, Supplement to 4th and 5 th editions, 1818.

Mill, J. S. Autobiography, London, 1873.

Mirabeau, H. G. R., Comte de. Ami des Hommes, Avignon, 1758.

Mitchell, P. Chalmers. Evolution and the War, London, 1915.

Modigliani, E. Un Viaggio a Nias, Milan, 1890.

Moerenhaut, J. A. Voyages, Paris, 1837.

Moffat, R. Missionary Labours in Southern Africa, London, 1842.

Mohl, R. von. Die Geschichte und Literatur der Staatswissenschaften, Erlangen, 1855-8.

Handwörterbuch der Staatswissenschaften, Jena, 1909.

Moir, J. Reid. Pre-Palaeolithic Man, Ipswich, 1920.

Moncelon, L. Néo-Calédoniens, Bull. Soc. Anth., vol. ix, 1886.

Mondière, A. T. La Femme Annamite, Mém. Soc. Anth.. vol. ii, 1875. Les Nègres, Rev. d'Anth., vol. iv, 1881.

Monier-Williams, M. Buddhism, London, 1870.

Montelius, O. Sur la chronologie absolue de l'époque néolithique, C. I. A., 1906. 
Montesquieu, C. de S., Baron de. Grandeur et Décadence des Romains, Paris, 1846.

Montgomery, J. Journal of Voyages, London, 1831.

Morgan, C. L. Animal Behaviour, London, 1900.

Morgan, J. de. Les Premières Civilisations, Paris, 1909.

Morgan, L. H. Houses and House Life of American Aborigines, U. S. Geog. and Geol. Survey, vol. iv, 1881.

Morgan, T. H. The Mechanism of Mendelian Heredity, London, 1915.

Morgulis, S. Influence of Fasting upon Growth,. Americain Naturalist, vol. xlvii, 1913.

Mosnier-Clauzel, L. De quelques causes de stérilité, Montpellier, 1907.

Moszkowski, M. Probleme der Weltwirtschaft, Jena, 1911.

Ửber zwei nicht-malayische Stämme von Ost-Sumatra, Zeit. für Eth., vol. $\mathrm{xv}, 1908$.

Moule, A. E. New China and Old, London, 1891.

Müller, F. Unter Tungnsen und Yakuten, Leipzig, 1882.

Müller, J. Das Sexuelle Leben, Leipzig, 1902.

Murdoch, J. Ethnological Results of the Port Barrow Expedition, 9th A. R. B. E., 1884 .

Murray, J. H. P. Papua, London, 1912.

Musters, G. C. At Home with the Patagonians, London, 1873.

Myres, J. L. The Causes of the Rise and Fall in the Population of the Ancient World, Eug. Rev., vol. vii, 1915.

$\mathbf{N}$

Nansen, F. The First Crossing of Greenland, London, 1890. Eskimo Life, London, 1893.

Nassau, R. H. Fetichism in West Africa, London, 1904.

National Birth-Rate Commission. The Declining Birth-rate, London, 1916.

Problems of Population and Parenthood, London, 1920.

Neill, E. D. History of Minnesota, Philadelphia, 1858.

Nelson, E. W. The Eskimo about the Behring Strait, 18th A.R.B.E., 1896.

Neuhaus, R. Deutsch Neu-Guinea, Berlin, 1911.

Newsholme, A., and Stevenson, T. H. C. Human Fertility as shown by corrected Birth-rates, J. R.S.S., vol. lix, 1906.

Newton, H. Far New Guinea, London, 1914.

Niblack, A. P. The Coast Indians, A. R. S. I., 1888.

Nicholson, J. Shield. Principles of Political Economy, London, 1893.

Nicolai, G. F. The Biology of War, London, 1919.

Niebuhr, B. J. Lectures in Ancient History, London, 1852.

Niemojowski, L. Siberian Pictures, London, 1883.

Nordenskiöld, E. Grenzgebiet zwischen Peru und Bolivia, Zeit. für Eth., vol. xxxviii, 1906.

Norman, H. Peoples and Politics of the Far East, London, 1875.

Nystrom, A. Über die Formenveränderungen des menschlichen Schädels, Arch.für Anth., vol. xxvii, 1901.

Obermaier, H. Der Mensch der Vorzeit, Berlin, 1912.

Olivier, S. White Capital and Coloured Labour, London, 1916.

Orbigny, A. d'. L'Homme Américain, Paris, 1837.

Ordinaire, O. Les Sauvages du Pérou, Rev. d'Eth., vol. vi, 1887.

Ortes, G. M. Reflessioni sulla Popolazione, Milan, 1792.

Osborn, H. F. Evolution of Mammalian Molar Teeth, New York, 1907. Men of the Old Stone Age, London, 1916. 
Overbergh, C. van. Les Basonge, Coll. Mon. Eth., No. 3, 1908.

Les Mangbetu, ibid., No. 4, 1909.

Overbergh, C. van, and Jonghe, E. de. Les Bangala, ibid., No. 1, 1907. Les Mayombe, ibid., No. 2, 1907.

Pallas, P. S. Reise, Frankfurt, 1778.

Palmer, E. Some Notes on Australian Tribes, J. A. I., vol. xiii, 1884.

Park, Mungo. Travels in the Interior of Africa, London, 1817.

Parker, E. S. Aborigines of Australia, London, 1884.

Parker, G. W. Land Tenure in Madagascar, J. A. I., vol. xii, 1883.

- People of Madagascar, J. A. I., vol. xii, 1883.

Parker, K. L. The Euahlayi Tribe, London, 1905.

Parkinson, R. Abhandlungen und Berichte des Königlich-Zoologischen und Anthropologisch-Ethnographischen Museums zu Dresden, vol. vii, 1899.

- Neu-Guinea-Küste, Int. Arch. Eth., vol. xiii, 1900.

Partridge, C. Cross River Natives, London, 1905.

Paton, J. J. G. Paton; Missionary to the New Hebrides, London, 1894.

Paulitschke, P. Ethnographie Nordost-Afrikas, Berlin, 1893.

Payne, E. J. History of the New World, Oxford, 1892.

Pearson, K. The Groundwork of Eugenics, Eugenics Laboratory Lecture Series, London, 1909.

The Problem of Practical Eugenics, Eugenics Laboratory Lecture Series. No. 5, London, 1909.

On the Handicapping of the First-born, Engenics Laboratory Lecture Series, London, 1914.

- see Barrington, A.

- see Beeton, M.

- see Elderton, E. M.

Pechuel-Loesche, E. Die Loango-Expedition, Stuttgart, 1907.

Peisker, T. The Asiatic Background, in the Cambridge Mediaeval History, vol. i, Cambridge, 1911.

Perrot, N. Mémoires sur les Mours des Sauvages de l'Amérique Septentrionale, Paris, 1864.

Perry, W. J. An Ethnological Study of Warfare, Mem. and Proc. Man. Lit. and Phil. Soc., vol. Ixi.

Petrie, W. M. Flinders. Eaten with Honour, Contemporary Review, 1897.

- The Revolutions of Civilization, London, 1919.

Petroff, I. Report of the Population of Alaska, 10th Census of the U.S.A., 1880.

Petty, Sir W. Treatise of Taxes and Contributions, in Economic Writings, edited by Hull, Cambridge, 1899.

- An Essay concerning the Increase of Mankind, ibid.

Pfeil, J. Studien und Beobachtungen aus der Südsee; Brunswick, 1899.

Philips, R. C. The Lower Congo, J. A. I., vol. xvii, 1883.

Phillipps-Wolley, C. Savage Svanetia, London, 1883.

Pierson, N. G. Principles of Economics, London, 1883.

Pigou, A. C. Wealth and Welfare, London, 1912.

Pineau, A. Les Indiens de l'Etat de Panama, Rev. d'Eth., vol. vi, 1887.

- see Hagen, A.

Place, F. Illustrations and Proofs of the Principle of Population, London, 1822 .

Plas, J. V. Les Kuku, Coll. Mon. Eth., No. 6, 1910.

Ploss, H. H. Das Kind, Stuttgart, 1876.

Ploss, H. H., and Bartels, M. Das Weib, Leipzig, 1913.

Plunkett, Sir H. Ireland in the New Century, London, 1904.

Polack, J. S. Manners and Customs of the New Zealanders, London, 1840. 
Polak, J. E. Persien, Leipzig, 1865.

Pollard, A. F. Factors in Modern History.

Poor Law, Report for Inquiry into the Administration and Practical Operation of, Appendix F, 1832.

Popenoe, P., and Johnson, R. H. Applied Eugenics, New York, 1918.

Pöppig, E. Reise in Chile, Leipzig, 1835.

Portman, M. V. Notes on the Andamanese, J. A. I., vol. xxv, 1896.

Powers, S. The Tribes of California, U. S. Geog. and Geol. Survey, vol. iii, 1877.

Prejevalsky, N. Mongolia, London, 1876.

Pritchard, W. T. Polynesian Reminiscences, London, 1864.

Pruner-Bey. Mémoire sur les Nègres, Mém. Soc. Anth., vol. i, 1861.

Punnett, R. C. Mendelism, London, 1919.

\section{Q}

Quaintance, H. W. Am. Econ. Assoc. Pub., 3rd Series, vol. v, 1904.

Quesnay, F. Analyse du Tableau Économique, Paris, 1758.

$\mathbf{R}$

Raleigh, W. History of the World, Collected Works, London, 1751.

Ratzel, F. The History of Mankind, London, 1896.

Reade, W. W. South Africa, London, 1863.

Reche, O. Der Kaiser-Augustus-Fluss, Ergebnisse der Südsee-Expedition, 1908-10, Hamburg, 1910.

Reclus, E. Primitive Folk, London, 1891.

Reed, W. A. The Negritos of Zambala, Ethnological Survey Publications, vol. ii, Manilla, 1904.

Registrar-General, 80th Annual Report, 1917.

Reichard, P. Die Wanjamuesi, Z. G. E., vol. xxiv, 1889.

Reisner, G. A. Early Dynastic Cemeteries of Naga-ed-Dêr, University of California Publications, Leipzig, 1909.

Rengger, A. Reise nach Paraguay, Aarau, 1835.

Ribbe, C. Die Aru-Inseln, Festschrift zur Jubelfeier des 25-jährigen Bestehens des Vereins für Erdkunde zu Dresden, 1888.

- Zwei Jahre unter den Kannibalen der Salomo-Inseln, Dresden, 1911.

Richard, J. History of Tonquin, Pinkerton's Voyages, vol. ix, 1868.

Richardson, J. Arctic Searching Expedition, London, 1851.

Ridgeway, W. The Beginnings of Iron, Report of the British Association, 1907.

— The Early Age of Greece, Cambridge, 1901.

The Influence of the Environment on Man, J. A. I., vol. xl, 1910.

Riedel, J. G. F. The Island of Flores, Rev. Col. Inter., vol. ii, 1886.

Rigler, L. Die Türkei, Vienna, 1852.

Rink, H. Danish Greenland, London, 1877.

- Tales and Traditions of the Eskimo, London, 1875.

Ripley, W. Z. The Races of Europe, London, 1900.

Rique, C. Etudes sur la Médecine des Arabes, Gazette Médicale de Paris, vol. xviii, 1863.

Risley, H. The People of India, Calcutta, 1908.

Ritter, H. Land und Leute im Russischen Amerika, Zeit.für all. Erd., vol. xiii, 1862 .

Rivero, M. E., and Tschudi, J. J. von. Peruvian Antiquities, New York, 1853.

Rivers, W. H. R. History of Melanesian Society, Cambridge, 1914.

The Contact of Peoples, Essays and Studies presented to W. Ridgeway, Cambridge, 1913. 
Rivers, W. H. R. The Disappearance of Useful Arts, Festskrift tillegnad Edward Westermarck, Helsingfors, 1912.

The Todas, London, 1896.

Robertson, G. F. The Kaffirs of the Hindu Kush, London, 1906.

Robertson, W. History of America, London, 1778.

Robinson, H. C. : see Annandale, N.

Rochas, V. de. La Nouvelle-Calédonie, Paris, 1862.

Rochebrune, A. T. de. La Race Onolove, Rev. d'Anth., vol, iv, 1881.

Rogers, J. E. T. Six Centuries of Work and Wages, London, 1884.

Romilly, H. H. The Western Pacific, London, 1886.

From my Verandah in New Guinea, London, 1889.

Roper, A. G. Ancient Eugenics, Oxford, 1913.

Roscoe, J. Notes on the Bageshu, J. A. I., vol. xxxix, 1909.

The Baganda, London, 1911.

The Bahima, J. A. I., vol. xxvii, 1907.

The Bakene, Man, vol. ix, 1909.

The Northern Bantu, Cambridge, 1915.

Rose, J. Holland. Origins of the War, Cambridge, 1914.

Rosenberg, H. von. Der Malayische Archipel, Leipzig, 1873.

Ross, B. R. The Eastern Tinneh, A. R. S. I., 1866.

Ross, J. History of Corea, Paisley, 1880.

Rosset, C. W. Maldive Islands, J. A. I., vol. xvi, 1887.

Roth, H. Ling. Great Benin, London, 1903.

- The Aborigines of Tasmania, London, 1890. The Natives of Sarawak, London, 1906.

Roth, W. E. Ethnological Studies, London, 1897.

Routledge, W. S., and K. With a Prehistoric People, London, 1910.

Rowntree, B. S. Land and Labour, London, 1910.

Roy, S. C. The Mundas, Calcutta, 1912.

Rubin, M. Population and Birth-rate, J. R.S. S., vol. lxiii, 1900.

Russell, F. The Pima Indians, 26th $A . R$. B. E., 1904.

Rutot, A. Essai sur les Origines, Revue de l'Université de Bruxelles, 1911.

St. John, S. Life in the Forests of the Far East, London, 1862.

Sande, G. A. J. von. Nova Guinea, Leyden, 1907.

Sarasin, P. and F. Ergebnisse naturwissenschaftlicher Forschungen aus Ceylon, Wiesbaden, 1887-93.

Sartori, P. Die Sitte der Alten- und Krankentötung, Globus, vol. lxvii, 1895.

Sarytschev, G. Account of a Voyage of Discovery, Collection of Modern and Contemporary Voyages and Travels, vols. $\mathrm{v}$ and vi, London, 1807.

Sayce, A. H. Babylonians and Assyrians, London, 1910.

Scadenberg, A. Über die Nigrittos der Philippinen, Zeit. für Eth., vol. xii, 1880.

Schellong, O. Über das Familienleben und die Gebräuche der Papuas, Zeit. für Eth., vol. xxviii, 1896.

Schmitz, R. Les Baholoholo, Coll. Mon. Eth., No. 9, 1912.

Schoetensack, O. Der Unterkiefer des Homo heidelbergensis, Leipzig, 1908.

Schömann, G. F. Griechische Alterthümer, Berlin, 1897.

Schomburgk, R. H. Reisen in Guiana, Leipzig, 1841.

Schönberg, G. von. Volkswirtschaftslehre, Tübingen, 1896.

Schoolcraft, H. H. History, Condition, and Prospects of the Indian Tribes, Philadelphia, 1853.

Schürmann, C. W. The Aboriginal Tribes of Port Lincoln, Adelaide, 1879.

Schwalbe, G. Studien über Pithecanthropus, Zeit. für Morph. und Anth., vol. i, 1899. 
Schweinfurth, G. The Heart of Africa, London, 1873.

Seeman, B. Viti, Cambridge, 1862.

Selenka, L., and Blanckenhorn, M. Die Pithecanthropus-Schichten auf Java, Leipzig, 1911.

Seligman, C. G. The Sinaugolo, J. A. I., vol. xxxii, 1902.

The Melanesians, Cambridge, 1912.

Seligman, C. G., and B. Z. The Veddahs, Cambridge, 1911.

Semon, R. In the Australian Bush, London, 1899.

Semper, K. Die Philippinen, Würzburg, 1869.

Die Pelau-Inseln, Leipzig, 1873.

Semple, E. C. The Influence of the Geographic Environment, London, 1911.

Sergi, G. Variation and Heredity in Man, Problems in Eugenics, London, 1912.

Shelley, P. B. The Revolt of Islam, London, 1807.

Sherring, M. A. Hindu Castes and Tribes, London, 1872.

Shinji, G. O. Physiology of Wing Development in Aphids, Biol. Bull., vol. $\mathrm{xxxv}, 1918$.

Shooter, J. The Kaffirs of Natal, London, 1857.

Shortt, J. Criminal Abortion, Trans. Obstet. Soc., vol. ix, 1867.

Siebold, E. C. von. Versuch einer Geschichte der Geburtshilfe, Berlin, 1839.

Simson, A. Notes on the Zaporos, J. A. I., vol. vii, 1878.

Skeat, W. W., and Blagden, C. O. Pagan Tribes of the Malay Peninsula, London, 1906.

Smith, A. L. Church and State in the Middle Ages, Oxford, 1913.

Smith, C. E. Midwifery and Diseases of the Esquimaux, Edinburgh Medical Journal, vol. xiii, 1868.

Smith, E. R. The Araucanians, New York, 1855.

Smith, G. Elliot. Presidential Address, Section H, British Ass., 1912.

Smith, W. Robertson. Kinship and Early Marriage in Arabia, Cambridge, 1885.

Smyth, R. B. The Aborigines of Victoria, London, 1878.

Smythe, W.. and Lowe, F. Narrative of a Journey, London, 1836.

Snow, E. C. The Intensity of Natural Selection in Man, Studies in National Deterioration ; Drapers' Company Research Memoirs, No. 7, 1911.

Soetbeer, H. Die Stellung der Sozialisten zur Malthusischen Bevölkerungslehre, Berlin, 1886.

Sograff, N. J. Anthropölogische Skizze der Samoyeden, Arch. für Anth., vol. xiv, 1882.

Sollas, W. J. Presidential Address to the Geological Society, Quart. Journ. Geol. Soc., vol. lxvi, 1910.

- Ancient Hunters, London, 1915.

Somerville, B. T. Solomon Islands, J. A. I., vol. xxvi, 1897.

Some Islands of the New Hebrides, $J$. A. I., vol. xxiii, 1894.

Speck, F. G. Yuchi Indians, Anthropological Publications of the University of Pennsylvania, vol. i, 1909.

Spencer, B., and Gillen, F. J. Northern Tribes of Central Australia, London, 1914.

- Native Tribes of Central Australia, London, 1899.

- Across Australia, London, 1912.

Spencer, H. Principles of Biology, London, 1899.

Spiller, G. The Mentality of Australian Aborigines, Soc. Rev., vol. vi, 1913.

Spix, J. B. von, and Martius, C. F. von. Travels in Brazil, London, 1824.

Sproat, G. M. Scenes and Studies of Savage Life, London, 1868.

Stanbridge, W. E. Tribes in the Central Part of Victoria, Trans. Eth. Soc., vol.i.

Stangeland, C. E. Pre-Malthusian Doctrines of Population, Studies in History, Economics, and Public Law: Columbia University, vol. xxi, 1908. 
Steinen, K. von den. Durch Central-Brasilien, Leipzig, 1886.

Steinmetz, S. R. Entwicklung der Strafe, Leiden, 1894.

Stellers, G. W. Kamtschatka, Frankfurt, 1774.

Stephan, E., and Graebner, F. Neu-Mecklenburg, Berlin, 1907.

Stephen, A. M. The Navaho, Am. Anth., vol. vi, 1893.

Stephen, Leslie. The English Utilitarians, London, 1900.

Steuart, Sir J. Principles of Political Economy, London, 1767.

Stevens, H. V. Orang-Belendas, Orang-Djakum und Orang-Laut, Zeit. für Eth., vol. xxviii, 1896.

Stevenson, M. C. Zuni Indians, 23rd A. R. B. E., 1901.

Stevenson, T. H. C. The Fertility of various Social Classes, J. R. S. S., vol. Ixxxiii, 1920.

see Newsholme, A.

Stockard, C. R. Cyclopean Fish, Journal of Experimental Zoology, vol. vi, 1909 .

Stone, O. C. A Few Months in New Guinea, London, 1880.

Stout, G. F. Manual of Psychology, London, 1915.

Stow, G. W. Native Races of South Africa, London, 1905.

Stricker, W. Ethnographische Notizen über den Kindermord und die künstliche Fruchtabtreibung, Arch. für Anth., vol. v, 1872.

Sumner, A. The Yakuts, J. A. I., vol. xxxi, 1901.

Sumner, J. B. A. Treatise on the Records of Creation, London, 1816.

Sutherland, A. Origin and Growth of the Moral Instinct, London, 1898.

Svoboda, W. Die Bewohner des Nicobaren-Archipels, Internationales Archiv für Ethnographie, vol. v, 1892.

Swan, J. G. The North-west Coast, New York, 1857.

Swanton, J. R. Ethnology of the Haidah, Jesup North Pacific Expedition, vol. $\nabla$.

Social Conditions of the Tlingit Indians, 26th A.R.B.E., 1904.

Symposium, The Problems of the Unity or Plurality of the American Aborigines, $A m$. Anth., vol. xiv, 1912.

\section{$\mathrm{T}$}

Talbot, J. B. In the Shadow of the Bush, London, 1912.

Tannus, H. S. Some Tribes of British Central Africa, J. A. I., vol. xl, 1910.

Tao, L. K. : see Leong, Y. K.

Taplin, G. The Native Tribes of South Australia, Adelaide, 1879.

Tautain. La Dépopulation de l'Archipel des Marquesas, L'Anthropologie, vol. ix, 1898.

Tawney, R. H. The Agrarian Problem in the Sixteenth Century, Iondon, 1912.

Taylor, G. The Australian Environment, Melbourne, 1918.

Taylor, R. Te Ika a Maui, London, 1870.

Teit, J. The Thompson Indians, Jesup North Pacific Expedition, vol. i, 1900.

— The Shushwap, ibid., vol. ii.

The Lilooet Indians, ibid.

Temple, Sir W. Works, London, 1814.

Tertullian. De Anima, in Ante-Nicene Christian Library, Edinburgh, 1870. First Book to his Wife, in Library of the Fathers, London, 1848.

Theal, G. M. Yellow- and Dark-Skinned People of Africa, London, 1912. History and Ethnography of Africa, London, 1907.

Theilhaber, F. Die Geburten-Beschränkung, Neue Generation, 1913.

- Das sterile Berlin, Berlin, 1913.

Thomas, N. W. Natives of Australia, London, 1906.

- The Ibo-Speaking Peoples of Nigeria, London, 1913.

Thompson, J. P. British New Guinea, London, 1892.

Thomson, B. Natives of Savage Island, J. A. I., vol. xxxi, 1901. 
Thomson, B. The Fijians, London, 1908.

Savage Island, London, 1902.

Thomson, J. The Basin of the River Rovuma, Geog. Journ., vol. iv, 1882.

Thomson, J. A. Darwinism and Human Life, London, 1916.

- The Study of Animal Life, London, 1917.

Heredity, London, 1919.

Thorndike, E. L. The Measurements of Twins, Archives of Philosophy, Psychology, and Scientific Methods, vol. i, 1905.

Thurmwald, R. Forschungen auf den Salomo-Inseln, Berlin, 1912.

- Im Bismarckarchipel, Zeit. für Eth., vol. xlii, 1910.

- Nachrichten aus Nissau, Zeit. für Eth., vol. xl, 1908.

Thurn, Sir E. im. Among the Savages of Guiana, London, 1883.

Thurston, E. Ethnographic Notes in Southern India, Madras, 1907.

Southern India, Madras, 1906.

Torday, F. Camp and Tramp, London, 1913.

Torday, E., and Joyce, T. A. Bakuba, Ann. Mus. Congo Belge, sér. 3, vol. ii. Ba-Yaka, J. A. I., vol. xxxvi, 1906.

- Ba-Huana, J. A.I., ibid.

- Ethnography of the Ba-Mbala, J. A. I., ibid.

Tout, C. Hill. Stseelis and Skaulits Tribes, J. A. I., vol. xxxiv, 1904.

- British North America, London, 1907.

Tower, W. L. Evolution in Chrysomelid Beetles, Washington, 1906.

Tredgold, A. F. Mental Deficiency; London, 1920.

Tregear, E. Maories of New Zealand, J. A. I., vol. xix, 1889.

Tremearne, A. J. N. Notes on the Kagero, J. A. I., vol. xlii, 1912. The Tailed Head Hunters of Nigeria, London, 1912.

Tschudi, J. J. von. Reisen durch Sïdamerika, Leipzig, 1866.

see Rivero, M. E.

Tucker, G. Progress of the U.S.A. in Population and Wealth, New York, 1843.

Tuituila. The Line Islanders, Jour. Pol. Soc., vol. i, 1892.

Tuke, J. B. Medical Notes, Edinburgh Medical Journal, vol. ix, 1913.

Turgot, A. R. J. Sur le Commerce, Paris, 1753.

Turner, G. Samoa, London, 1904.

Turner, L. M. Ethnology of the Ungava District, 11th $A . R . B . E ., 1889$.

Turner, W. Y. Ethnology of the Motu, J. A. I., vol. vii, 1878.

$\mathbf{U}$

Ujfalvy, C. E. de. Voyages dans l'Himalaya, Bull. Soc. Anth., vol. v, 1882.

$\mathrm{V}$

Vambéry, A. Travels in Central Asia, London, 1864.

Vaux, L. de. Les Canaques, Rev. d'Eth., vol. ii, 1883.

Velten, C. Sitten und Gebräuche der Suaheli, Göttingen, 1903.

Verguet, L. Arossi ou San Christoval, Rev. d'Eth., vol. iv, 1885.

Vernon, H. M. Variation in Animals and Plants, London, 1913.

Vinogradoff, P. Villeinage in England, Oxford, 1892.

Voltaire, F. M. A. de. Candide (édition Nilsson), Paris, 1900.

Volz, W. Das geologische Alter der Pithecanthropus-Schichten, Neues Jahrbuch für Mineralogie, Geologie und Paläontologie; Festband zur Feier des 100-jährigen Bestehens, Stuttgart, 1907.

W

Wallace, A. R. Narrative of Travels on the Amazon, London, 1853. The Malay Archipelago, London, 1898.

Wallace, R. Dissertation on the Numbers of Mankind, London, 1753. 
Wallas, G. Life of Francis Place, London, 1898. The Great Society, London, 1914.

Ward, H. Congo Tribes, J. A. I., vol. xxiv, 1895.

Warnick, A. Gynäkologische Mittheilungen aus Japan, Archiv für Gynäkologie, vol. x, 1876.

Wartegg, E. von Hesse. Sitten und Gebräuche der Indianerstämme, Globus, vol. xxvii, 1888.

Washington, T. Booker. The Story of the Negro, London, 1909.

Wassilief, W. Beobachtungen über das Geschlechtsleben der Kirgesinnen, Zentralblatt für Anthropologie, vol. x, 1905.

Waterhouse, J. King and People of Fiji, London, 1868.

Wattal, P. K. The Population Problem in India, Bombay, 1916.

Webb, A. Pathologia Indica, Calcutta, 1848.

Weeks, J. H. Among Congo Cannibals, London, 1913. The Bangala of the Upper Congo, J. A. I., vol. xxxix, 1909.

Weld, I. Travels through North America, London, 1899.

Wells, R., and Kelly, J. W. Eskimo Vocabularies, U.S.A. Bureau of Educa. tion, Washington, 1890.

Werner, A. Native Races of British Central Africa, London, 1906.

West, T. Ten Years in South Central Polynesia, London, 1865.

Westermarck, E. The History of Human Marriage, London, 1901. The Origin and Development of Moral Ideals, London, 1906.

Weule, K. Native Life in East Africa, London, 1909.

Wheeler, G. C. The Tribe in Australia, London, 1910. see Hobhouse, L. T.

White, R. B. Races of the North-Western Provinces of South America, J. A. I., vol. xiii, 1888.

Whymper, F. Voyages et Aventures, Paris, 1871.

Wilhelmi, J. Manners and Customs of the Australian Natives, Transactions of the Royal Society of Victoria, vol. v.

Wilken, G. A. Das Matriarchat, Leipzig, 1884.

Wilkins, W. Modern Hinduism, London, 1887.

William of Rubruck, Journey of, Hakluyt Society Publications, London, 1905.

Williams, J. W. Obstetrics, London, 1912.

Williams, T. Fiji, London, 1858.

Williamson, R. W. Mafulu Mountain People of British New Guinea, London, 1912.

Mekeo People, J. A. I., vol. xliii, 1913.

Willoughby, C. Indians of the Quinault Agency, A. R. S. I., 1886.

Wilson, A. Abode of Snow, London, 1876.

Wilson, C. T., and Felkin, R. W. Uganda, London, 1832.

Wilson, H. An Account of the Pelew Istands, London, 1878.

Wilson, J. H. Western Africa, London, 1856.

Woldt, A. Capitän Jacobsen's Reise, Leipzig, 1884.

Woodford, C. M. A Naturalist among the Head Hunters, London, 1890.

Woodward, A. S. : see Dawson, C.

\section{$\mathrm{Y}$}

Yarrow, H. C. Mortuary Customs of North American Indians, 1st A. R. B. E., 1879.

Young, A. Travels in France, London, 1794.

Yule, G. U. : see Greenwood, M. 


\section{INDEX}

Ababua, restriction of intercourse, 175 ; abortion, 179 ; infanticide, 180 ; property, 210.

Abipones, lactation, 138 ; age at marriage, 140 ; abstention from intercourse, 140 ; infanticide, 149, 219 ; feuds, 152; health, 159 ; treatment of children, 160 ; age at marriage, 226.

Abortion, among hunting and fishing races, 145 ; among agricultural races, $168,178,189,196$; extent of, 215 ; origin of, 216; as regulating numbers, 222 ; among historical races, $256,268,314$.

Acheulean period, 121.

Acquired characters, not inherited, 74 ; contrasted with inherited characters, 68.

Adio, restriction of intercourse, 176.

Africa, evolution of tradition in, 442.

Aged, treatment of, among hunting and fishing races, 154 .

Ahts, lactation, 138 ; war, 151 ; treatment of aged, 155 ; food, 233 ; property, 296.

Ainu, lactation, 185; fertility, 189 ; war, 194.

Akamba, abortion, 179 ; property, 209 ; marriage customs, 228.

Akikuyu, fertility, 178 ; war, 181 ; property, 210 ; age at marriage, 228 ; health, 235.

Aleuts, fertility, 143 ; infanticide, 148.

Amazon tribes, war, 171.

America, evolution of tradition in, 439 ; racial characteristics, 451 .

American Indians, fertility, 99. See also under various tribes.

Andamanese, lactation, 138 ; fertility, 144 ; infant mortality, 160 ; intellect, 390 .

Animal behaviour, evolution of, $45 \mathrm{ff}$.

Annam, abortion, 256.

Anthropoid apes, evolution of, 109 ; mode of life, 110.

Apaches, contraceptive methods, 167 ; abortion, 168.

Arabs, celibacy, 252 ; lactation, 254 ; restriction of intercourse, 254 ; contraceptive methods, 255; abortion, 256 ; infanticide, 259 ; over-population, 275 ; migration, 302.
Aru Islands, fertility, 186 ; abortion, 189.

Ashanti, restriction of intercourse, 175 ; sacrifices, 180.

Asia, evolution of tradition in, 441.

Assiniens, fertility, 177.

Atonga, restriction of intercourse, 176.

Attakapas, age at marriago, 229.

Aurignacian period, 122.

Australians, fertility, 99 ; early intercourse 135; lactation, 137 ; initiation ceremonies, 138 ; age at marriage, 139 ; abortion, 145 ; infanticide, 147,216 ; war, 149 ; feuds, 152 ; treatment of sick, 154 ; disease, 157 ; health, 158, 231 ; property, 203 ; division of food, 211 ; marriage customs, 224 ; storing of food, 232 ; intellect, 389.

Awa-Wanga, restriction of intercourse, 176.

Aymaras, lactation, 165 ; fertility, 168.

Azilian-Tardenoisian, 123.

Aztecs, contraceptive methods, 167 ; abortion, 168.

Baganda, lactation, 174 ; restriction of intercourse, 176 ; war, 181 ; witchcraft, 182 ; health, 183 ; property, 210 ; marriage customs, 228 ; food, 233.

Bahamas, 396.

Bahima, war, 181.

Baholoholo, fertility, 178.

Bahuana, war, 171 ; early intercourso, 173 ; abortion, 179.

Bakairi, abortion, 169.

Bakalai, war, 181.

Bakene, fertility, 178.

Bambala, war, 171 ; early intercourse, 173 ; fertility, 178 ; intellect, 391.

Bangala, early intercourse, 173 ; age at marriage, 174; restriction of intercourse, 175 ; fertility, 178 ; abortion, 179 ; property, 209 ; marriage customs, 228; intellect, 391.

Banks Island, early intercourse, 185; infanticide, 191.

Bantu Races, polygamy, 102 ; age at marriage, 174 ; restriction of intercourse, 176 ; health, 183 ; intellect, 390.

Banyala, war, 181. 
Baringo District, early intercourse, 173 ; restriction of intercourse, 176 ; war, 181.

Baronga, lactation, 174 ; restriction of intercourse, 176 ; age at marriage, 227.

Basonge, infanticide, 180.

Basuto, health, 183 ; infant mortality, 184.

Bayaka, restriction of intercourse, 175 ; fertility, 178 ; war, 181 ; witchcraft, 182.

Bechuanaland, property, 210.

Behaviour, evolution of, 45.

Benin, restriction of intercourse, 175 ; war, 180.

Binet-Simon methods, 393.

Biometric methods, 358.

Bismarck Archipelago, lactation, 185 ; restriction of intercourse, 186 ; fertility, 188 ; abortion, 189 ; infanticide, 191.

Black Death, 248.

Blackfeet, war, 152, 170.

Boers, 450.

Bontoc Igorot, lactation, 185 ; abortion, 190 ; head-hunting, 194 ; infant mortality, 195 ; marriage customs, 226.

Borneo, fertility, 191 ; war, 194 ; headhunting, 194, 227.

Bornu, age at marriage, 174.

Botocudos, fertility, 144 ; infanticide, 149 ; health, 159.

Brazilians, early intercourse, 164 ; fertility, 168; abortion, 169, 221 ; health, 172, 236 ; infant mortality, 172 ; property, 208 ; marriage customs, 230.

Bronze Age, 125.

Buddhism, celibacy, 252.

Burma, marriage, 253.

Bushmen, early intercourse, 136 ; fertility, 143 ; infanticide, 148 ; war, 150 ; treatment of aged, 154 ; health, 158,232 ; property, 205 ; division of food, 212.

Bushongo, early intercourse, 173 ; lactation, 174 ; abortion, 179 ; infanticide, 180 ; property, 209.

Californians, early intercourse, 126 ; lactation, 138 ; age at marriage, 140 ; fertility, 140 ; infanticide, 149 ; war, 152 ; treatment of aged, 155 ; health, 159 ; infant mortality, 160 .

Cameroon district, restriction of intercourse, 175.

Caroline Islands, infanticide, 190 ; marriage customs, 224.

Carrier tribes, property, 206.

Casual Labour, over-population and, 290.
Catholic Church, contraceptive practices and, 294.

Celebes, early intercourse, 185; fertility, 189 ; abortion, 190.

Celibacy, primitive races, 226 ; historical races, 251, 263,278 .

Cells, nature of, 39.

Chaco Indians, abortion, 169 ; infanti. cide, 169 ; war, 171.

Chaldea, celibacy, 251.

Charruas, war, 152; health, 236.

Chellean period, 121.

Chepewayans, lactation, 138 ; fertility, 144; infanticide, 149; war, 152 ; treatment of aged, 155 .

Cheyennes, abortion, 169, 220.

Chichmics, lactation, 165.

Children, death-rate among hunting and fishing races, 159; among agricultural races, $172,183,196$; among historical races, 249.

- number of, see Fertility.

Chinese, fecundity, 100 ; celibacy, 252 ; marriage, 253 ; restriction of intercourse, 254 ; contraceptive methods, 255 ; fertility, 256 ; abortion, 256 ; infanticide, 259,276 ; over-population, 276 ; organization of society, 432.

Chinooks, fertility, 99 ; lactation, 138 ; abortion, 146 ; infanticide, 149 ; war, 151.

Chiquitos, fertility, 168.

Christianity, war and, 249 ; marriage and, 263 ; infanticide and abortion and, 268.

Chromosomes, as physical basis of heredity, 65 .

Civilization, cyclical course of, 459.

Climate, famine and, 249,274 ; migration and, 301 ; head form and, 341 ; tropical effects of, 342 ; selection and, 371.

Comanches, early intercourse, 137 ; fertility, 144 ; war, 152.

Confucianism, marriage and, 253.

Congo tribes, early intercourse, 173 ; lactation, 174; restriction of intercourse, 175; contraceptive methods, 177 ; fertility, 178 ; abortion, 179 ; infanticide, 180 ; war, 180 ; witchcraft, 182 ; infant mortality, 184 ; marriage customs, 229 ; food, 234.

Contact, influence upon tradition, 424 ; geography and, 427.

Contraceptive methods, agricultural races, 177, 186; historical races, 255, 266, 287, 315 ; Catholic Church and, 294.

Co-operation, in search for food, 211.

Copper Age, 125.

Copper River tribes, infanticide, 148.

Copulation, external, 42 ; internal, 43. 
Corea, celibacy, 252.

Creeks, infanticide, 169, 220.

Crees, war, 211.

Cro-Magnon man, 117.

Cross River tribes, early intercourse, 172.

Crow tribe, fertility, 99 ; restriction of intercourse, 166; abortion, 168; war, 170.

Cytoplasm, nature of, 39 ; relation to nucleus, 66.

Dacotahs, war, 170.

Dahomey, war, 170.

Damaras, health, 175.

Death, origin of, 38 ; causes of, among animals, 57 ; among plants, 58 ; ignorance of cause among hunting and fishing races, 153 .

Decreasing returns, 199.

Development of organisms, 41, 66, 325 .

Disease, evolution of, 155 ; among hunting and fishing races, 158; among agricultural races, 171, 183, 195 ; among historical races, 244 ; tropical, 348 ; twins and, 352 ; inheritance of, 359 ; selection and, 377 .

Ductless glands, 347, 372.

Dyaks, use of metal, 163 ; food, 233.

Egbas, fertility, 177.

Egypt, disease, 245, 247 ; treatment of children, 250 ; celibacy, 251 ; lactation, 254 ; restriction of intercourse, 254 ; infanticide, 272 ; over-population, 277.

Ekoi, fertility, 177 ; property, 209 ; marriage customs, 229.

Elimination, causes of among animals, 58 ; among plants, 59 ; under natural conditions, 75 .

Enclosures, population and, 284.

Environment, influence of upon animals and plants, 325 ; upon men, 336 ; influence upon progress, 458.

Eoanthropus, 115.

Eoliths, 118, 130.

Eskimos, fertility, 99, 143 ; early intercourse, 136 ; lactation, 137 ; age at marriage, 140 ; abortion, 145 ; in fanticide, 148; war, 150; feuds, 153 ; treatment of aged, 154 ; health, 158 ; infant mortality, 159 ; property, 205; division of food, 212 ; marriage customs, 225 ; -celibacy, 226 ; storing of food, 232.

Eugenics, attitude of Romans, 18 ; term due to Galton, 35 ; modern problems, 308.

Evolution, theory of, 17, 18, 34 ; of animal behaviour, 45 ; among species in a state of nature, 77 ; of Primates, 109 ; of man, 110, 128; of culture,
129 ; of disease, 155 ; of tradition, 438.

Ewe-speaking people, lactation, 174 ; restriction of intercourse, 175 ; war, 180 ; property, 209 ; marriage, 229.

Exercise, influence of, 329, 339.

Factory Acts, 290, 340.

Famine, climate and, 249.

Fecundity, early references to human, 25 ; definition of, 51 ; distinguished from fertility, 52 ; among species in nature, 63; how determined, 60 ; not influenced by the male, 88 ; relation to length of mature period, 90 ; to interval between births, 92 ; effect of captivity upon, 95 ; relation to number at a birth, 95 ; increase of human, 97 ; of primitive races, 99 ; in India and China, 100 ; opinion of Darwin regarding, 101 ; relation to polygamy, 102 ; to lactation, 102 ; to age at marriage, 103 ; to early intercourse, 103 ; to development of fat, 104 ; among men, 105 ; of prehuman ancestor, 241.

Fertility, distinguished from fecundity, 52 ; among hunting and fishing races, 141 ; among agricultural races, 167, $177,183,196$; among historical races, 255; Indian and European compared, 256 ; calculations regarding, 291 ; differential, 316 ; differential between classes, 316,457 ; between races, 320.

Fertilization, 40.

Feuds, among hunting and fishing races, 152 ; among agricultural races, 171 .

Fiji, lactation, 185 ; restriction of intercourse, 186 ; fertility, 188 ; abortion, 189,221 ; infanticide, 191, 219 ; war, 193 ; obligation to work, 212 ; age at marriage, 226 ; food, 233.

Flores, abortion, 190.

Food, co-operation in search for, 211.

Franks, contraceptive methods, 255.

Fuegians, early intercourse, 137 ; lactation, 138 ; fertility, 144 ; abortion, 146 ; infanticide, 149 ; war, 152 ; treatment of aged, 155 ; infant mortality, 160 ; restriction of intercourse, 166 ; property, 207 ; marriage customs, 225.

Funafuti, infanticide, 190, 219.

Gallinomero, infanticide, 149.

Gallinas, restriction of intercourse, 175 ; war, 180.

Gametes, nature of, 40.

Geological strata, time occupied by deposition of, 107.

Ghiliaks, lactation, 138 ; infant mortality, 160. 
Glacial epoch, duration of, 108 ; subdivisions of, 109.

Gonorrhoea, effect upon fecundity, 89.

Greece, discussion regarding population in, 19 ; celibacy, 251 ; contraceptive methods, 255 ; abortion, 256 ; infanticide, 258 ; malaria, 349.

Grimaldi man, 117.

Guanas, fertility, 168 ; infanticide, 169 ; age at marriage, 230.

Guaranis, fertility, 168 ; war, 171 ; abortion, 220.

Guatemala, early intercourse, 164.

Guaycurus, abortion, 146 ; infanticide, 149 ; war, 152.

Guiana, lactation, 165 ; infanticide, 169 ; feuds, 171 ; marriage customs, 229 ; food, 234.

Habit, inheritance of, 362 ; tradition and, 414, 462.

Haidahs, abortion, 146 ; infanticide, 149 ; war, 151 ; feuds, 154 ; property, 206 ; marriage customs, 225.

Hausa, lactation, 174; restriction of intercourse, 175 ; infanticide, 180.

Hawaii, early intercourse, 185 ; abortion, 189.

Head, shape of, and environment, 341 .

Head-hunting, 194.

Health, of primitive races, 158, 196, 231 ; of historical races, 245.

Heidelberg man, 114.

Hobbés, restriction of intercourse, 175 .

Hottentots, fertility, 178; infanticide, 179,180 ; war, 182 ; health, 183 ; property, 208.

Housing accommodation, population and, 280.

Hualaga tribes, fertility, 168.

Hudson Bay tribes, fertility, -143 ; abortion, 145.

Hungary, lactation, 263.

Hurons, early intercourse, 165.

Ibo-speaking people, early intercourse, 173.

Ice Age, see Glacial epoch.

Illinois, early intercourse, 165 ; restriction of intercourse, 166.

Imitation, inheritance of, 364 ; tradition and, 413.

Income, average per head, 310.

Increase, possible among animals and plants, 54 ; actual, 55 .

India, fecundity, 100; early intercourse, 104 ; infant mortality, 250 ; celibacy, 251 ; early intercourse, 253 ; contraceptive methods, 255 ; fertility, 255 ; abortion, 256 ; infanticide, 260 ; climate, 274 ; over-population, 275 ; caste system, 432.

Infant mortality, see Children.
Infanticide, hunting and fishing races, 146 ; agricultural races, 169,179 , 190,196 ; extent of under-estimated, 215 ; origin of, 216 ; importance of, in regulating numbers, 228 ; among historical races, 257, 268.

Ingaliks, lactation, 138.

Inheritance, relation of, to social problems, 18 ; physical basis of, 64 ; biparental, 71 ; environment and, 326 ; in man, 356 ; of physical characters, 359 ; of disease, 359 ; of mental characters, 361.

Initiation ceremonies, 138.

Instinct, nature of, 47 ; inheritance of, 363.

Intellect, inheritance of, 364; among prehistoric races, 387,398 ; primitive races, 389 ; negroes, 393 ; in the Bahamas, 396 ; evolution of, 399 , 409,438 ; influence upon progress, 447.

Intelligence, nature of, 47 ; development among animals, 50 .

Interdependence of organisms, 56.

Ireland, over-population, 284, 294.

Iron Age, 127.

Iroquois, restriction of intercourse, 166 ; fertility, 167 ; health, 172.

Jakun, marriage customs, 225.

Japan, rise of, 82.

Java, early intercourse, 185 ; fertility, 189 ; health, 343.

Jews, contraceptive methods, 255 ; infanticide, 256 ; civilization, 452.

Kabinapek, abortion, 146 ; infanticide, 149.

Kaffirs, see South Africa.

Kagero, lactation, 174 ; restriction of intercourse, 175 ; infanticide, 180 ; food, 234.

Kalabar, witchcraft, 182.

Kalmucks, marriage oustoms, 253.

Kamtchatka, age at marriage, 196 ; abortion, 196 ; infanticide, 196.

Kenai, marriage customs, 225.

Kingsmill Islands, restriction of intercourse, 186 ; fertility, 188 ; abortion, 189 ; infanticide, 190, 219 ; war, 192.

Kirghiz, early intercourse, 254.

Knisteneaux, abortion, 146.

Konde-land, restriction of intercourse, 176.

Koniagas, infanticide, $\mathbf{1 4 9}$.

Koryak, lactation, 196.

Kuku, lactation, 174; restriction of intercourse, 176 ; fertility, 178 ; marriage customs, 228.

Kutchins, infanticide, 148 ; celibacy, 226 ; health, 233.

Kwakiutl, war, 151. 
Lactation, influence upon fecundity, 102; among hunting and fishing races, 137 ; among agricultural races, $165,174,185,196$; among historical races, 254.

Land, claims of groups to areas of, 203 ; property in, 279.

Language, tradition and, 408, 412.

Laws, encouraging population, 22.

Lenda district, infanticide, 180.

Lenguas, lactation, 165 ; infanticide, $169,220$.

Liberia, lactation, 174 ; fertility, 177.

Lillooets, age at marriage, 140, 226 ; war, 151 ; division of food, 212.

Limitation of families, views of Mill, 202.

Limitation of the surface of the world, theories of population and, 20 ; influence upon erolution, 59.

Lind hinterland, 180.

Loango, restriction of intercourse, 176.

Loucheux, food, 233.

Macusis, fertility, 168.

Madagascar, fertility, 178 ; abortion, 179 ; witchcraft, 182.

Madi district, lactation, 174 .

Magdalenian period, 123.

Makalaka, fertility, 178.

Makonde, early intercourse, 173.

Malaria, 349.

Malays, 163.

Maldive Islands, marriage customs, 226.

Malemutes, infanticide, 148 ; war, 151.

Malthusian theory, anticipations of, 19 , 25 ; nature of, 28 ; reception of, 29 ; debt of Darwin and Wallace to, 34 ; summary of, 197 ; criticism of, 199.

Mandans, fertility, 167; health, 172 ; food, 234.

Mandingoes, fertility, 177.

Mandja, restriction of intercourse, 175 ; infanticide, 180 ; food, 234.

Mandrucos, war, 171 ; health, 236.

Mangbetu, early intercourse, 173 ; lactation, 174 ; fertility, 178 ; abortion, 179 ; infant mortality, 184 ; property, 209.

Manu, Laws of, 253.

Marquesas, fertility, 187 ; infanticide, 191.

Marriage, among animals, 62 ; influence of age at, 103 ; among primitive races, 139 ; postponement of, among hunting and fishing races, 139, 226 ; disparity in age at, 140 ; postponement of, among agricultural races, $165,174,185,196$; customs regarding, 224, 275 ; postponement of, among historical races, 251, 264, 279, 306 ; prohibition of, 281, 282.

Marshall Islands, war, 193.
Masai, early intercourse, 173 ; war, 181 ; marriage customs, 228.

Mayombe, lactation, 174 ; restriction of intercourse, 175.

Mbayas, infanticide, 169 ; health, 236.

Memory among animals, 50 .

Mendelian inheritance, nature of, 71 ; in man, 356.

Menstruation, relation to ovulation, 90 .

Mental evolution, among animals, 45 ; connexion with reproduction, 51 ; among men, 50 ; influence upon population problem, 80 .

Mercantile theory of trade, 22.

Mexico, lactation, 165; restriction of intercourse, 166 ; abortion, 169 ; war, 170; property, 208; age at marriage, 220.

Migration, over-population and, 297; selection and, 381.

Minnetanes, war, 170.

Minoan civilization, 244.

Miri district, restriction of intercourse, 176.

Mitchell Islands, abortion, 190.

Mittelschmerz, 94 .

Modifications, nature of, 68 .

Mohammedanism, celibacy, 253 ; lactation, 254.

Mohaves, infanticide, 169.

Moioa, restriction of intercourse, 175.

Monasticism, celibacy and, 279.

Mongols, marriage, 253.

Montagnais, health, 233.

Mousterian period, 121.

Moxos, restriction of intercourse, 166 ; fertility, 168.

Mundas, marriage customs, 275.

Murray Islands, war, 192 ; infanticide, 220.

Mutations, nature of, 68 ; origin of, 73 ; among men, 81, 383.

Nagas, war, 248.

Nahuas, contraceptive methods, 167 ; abortion, 168.

Nandi, early intercourse, 173 ; lactation, 174 ; restriction of intercourse, 176.

Nandowensis, marriage customs, 229.

Natchez, marriage customs, 229 ; health, 236.

Natural Selection, among species in nature, 75 ; lethal and reproductive, 367 ; strength of, 369 ; in intermediate period, 370 ; in first and second periods, 371 ; of tradition, 417.

Navahos, early intercourse, 164 ; contraceptive methods, 167 ; abortion, 168 ; infanticide, 169 ; property, 208.

Neanderthal man, 116.

Neolithic period 118, 123. 
Neomalthusianism, early history of, 31 ; Bradlaugh-Besant trial, 33 ; in foreign countries, 33 .

New Britain, early intercourse, 185 ; abortion, 222 ; celibacy, 226.

New Caledonia, early intercourse, 185 ; lactation, 185 ; restriction of intercourse, 186 ; fertility, 188 ; abortion, 189 ; infanticide, 191 ; war, 193.

New Guinea, early intercourse, 185 ; lactation, 185 ; restriction of intercourse, 186 ; contraceptive practices, 186 ; fertility, 188 ; abortion, 190 ; infanticide, 191 ; war, 193; headhunting, 194 ; witchcraft, 195.

New Hebrides, abortion, 189 ; infanticide, 191, 221 ; war, 193.

New Zealand, early intercourse, 184 ; restriction of intercourse, 186 ; fertility, 187 ; abortion, 189 ; infanticide, 190 ; war, 192 ; property, 211.

Nias, fertility, 189 ; abortion, 190.

Nigeria, fertility, 177 ; abortion, 178 ; war, 180 ; property, 209.

Nishinan, infanticide, 149.

Nissau, 190.

Nitrogen in the sea, 59 .

Nootkas, fertility, 99 ; lactation, 138 ; abortion, 146 ; health, 159 ; infant mortality, 160.

Nucleus, nature of, 39 ; function of, 66.

Oestrous cycle, 44.

Ojebway Indians, war, 170 ; marriage customs, 229.

Omahas, fertility, 144 ; property, 208 ; age at marriage, 229.

Onolove, early intercourse, 173 ; abortion, 179.

Optimum density of population, 200 , 213 ; approximation to, among primitive races, 230, 236, 292 ; among historical races, 239, 270, 293, 309; military needs and, 310.

Orthogenesis, 79.

Ostyaks, health, 196.

Otommi, abortion, 168.

Ovaherero, property, 210.

Over-population, fear of, in England, 23 ; among historical races, 275,283 , 284, 290, 304; migration and, 297 ; war and, 304 ; in the future, 308 ; unemployment and, 312.

Ovulation, see Menstruation.

Ovum, 40.

Palaeolithic period, 118

Patagonians, health, 159.

Pawumwa Indians, obligation to work, 213.

Payaguas, abortion, 146 ; health, 236.

Pelew Islands, early intercourse, 185 ; restriction of intercourse, 186 ; fertility, 187 ; war, 192 ; property, 211.

Persia, celibacy, 251 ; marriage, 253 ; lactation, 254; restriction of intercourse, 254 ; abortion, 265.

Peruvian tribes, fertility, 167 ; infanticide, 169 ; war, 170 ; age at marriage, 230.

Pimas, contraceptive methods, 167 ; abortion, 168, 221 ; infanticide, 169.

Pithecanthropus, 112.

Plough, invention of, 127 ; use of, 162. Polygamy, influence upon fecundity, 102 ; among primitive races, 132.

Poor law, marriage and, 282.

Port Herald, contraceptive methods, 177.

Primates, evolution of, 109.

Primitive races, value of evidence regarding, 85, 131 ; intellect and, 447.

Progress, definition of, 85; rate of, 130 ; in different regions, 438.

Protista, reproduction among, 39.

Pueblos, contraceptive methods, 167 ; abortion, 168 ; war, 170.

Puelches, lactation, 138 ; fertility, 144 ; infanticide, 149, 218; war, 152; infant mortality, 160.

Puget Sound Indians, lactation, 138; abortion, 146.

Pure Line inheritance, 69.

Queka Indians, treatment of aged, 155.

Racial crosses, $380,453$.

Radeck, infanticide, 191, 221 ; health, 235.

Rarotonga, infanticide, 190 ; war, 192.

Ratios of increase of food and of population, 26, 198 ; of human population, 105.

Reflex action, 45.

Religious writers, attitude to Malthus, 30.

Reproduction, necessity for, 38 ; nature of, 39 ; methods of, 41 ; among species in nature and men, 52.

Reproductive organs, size among primitive races, 97.

Rome, discussion of population problem in, 18, 49 ; expectation of life in, 246 ; age at marriage in, 251 ; early intercourse, 254 ; contraceptive methods, 255 ; abortion, 256,272 ; infanticide, 258.

Rotuma, abortion, 189 ; war, 192.

Rovuma River, restriction of intercourse, 176.

Salish, lactation, 138 ; age at marriage, 140 ; health, 159 ; property, 206.

Samoa, lactation, 185; restriction of intercourse, 186; fertility, 187; 
abortion, 189 ; infanticide, 190 ; war, 192; infant mortality, 195.

Samoyeds, fertility, 196 ; infanticide, 196 ; health, 196.

Sandwich Islands, infanticide, 101, 219 ; fertility, 187 ; war, 192 ; health, 235.

Sarawak, lactation, 185 ; fertility, 188 ; property, 211.

Savage Island, restriction of intercourse, 186 ; abortion, 189 ; infanticide, 190.

Selection, see Natural Selection.

Seri Indians, war, 152 ; treatment of aged, 155; division of food, 212 ; marriage customs, 225 ; elimination among, 375.

Sexual cycle in Mammals, 44 ; former condition in man, 92 ; effect of captivity upon, 94 ; peculiarity of, in man, 98.

Sexual instinct, 42, 43, 51 ; among primitive races, 97.

Sexual intercourse, before maturity, influence upon fecundity, 103 ; among hunting and fishing races, 135 ; taboo upon, among hunting and fishing races, 141 ; among agricultural races, $164,172,184$; taboo upon, among agricultural races, $165,175,186$; before maturity among historical races, 253, 263 ; taboo upon, among historical races, 267 ; restrictions upon, 315.

Shawnees, contraceptive methods, 167 ; health, 172.

Shekiani, feuds, 181.

Shoshones, war, 170.

Shushwap, lactation, 138 ; age at marriage, 144 ; war, 151 ; health, 159.

Siam, marriage, 253 ; lactation, 254.

Sierra Leone, property, 209.

Sioux, lactation, 165 ; fertility, 167 ; abortion, 168, 221 ; infanticide, 169 ; war, 170 ; infant mortality, 172.

Sitkins Indians, property, 207.

Social organization, origin of, 132 ; mental evolution and, 401 ; organic, 401 ; segmentary, 429.

Socialists, attitude to Malthus, 31 .

Society Islands, age at marriage, 185.

Solomon Islands, lactation, 185 ; restriction of intercourse, 186 ; abortion, 189 ; infanticide, 191 ; war, 193.

Sound Indians, fertility, 144.

South Africa, lactation, 174 ; abortion, 179 ; infanticide, 180 ; witchcraft, 182 ; infant mortality, 184 ; property, 210 ; marriage customs, 227 ; intellect, 392.

Spermatozoa, 40, 41.

Starvation, no evidence for, among primitive races, 231 ; among animals, 58,61 ; among plants, 59.
Stature, increase of, 340 .

Sterility, in males, 89 ; among Australians, 99 ; fat and, 104.

Suggestion, inheritance of, 364 ; tradition and, 413.

Sumatra, fertility, 188 ; marriage customs, 227.

Swaheli, early intercourse, 173 ; restriction of intercourse, 176 ; fertility, 178.

Sympathy, inheritance of, 364 ; tradition and, 413.

Tahiti, infanticide, 190 ; war, 192.

Tasmanians, early intercourse, 135 ; lactation, 137 ; fertility, 141 ; abortion, 145; infanticide, 146; war, 149 ; treatment of sick, 154 ; health, 158 ; infant mortality, 159 ; property, 203.

Tehuelches, age at marriage, 140.

Temperament, disease and, 347 ; environment and, 353, 438 ; inheritance of, 361.

Tendas, lactation, 171 ; abortion, 178.

Tepecanos, infanticide, 169 .

Texas tribes, fertility, 167.

Thirty Years' War, 247.

Thlinkeet, lactation, 138 ; war, 151 ; health, 159 ; infant mortality, 160 ; property, 207.

Thompson tribes, age at marriage, 140 , 226 ; abortion, 146 ; infanticide, 149; war, 151 ; infant mortality, 160.

Thonga, restriction of intercourse, 177 ; property, 210 ; age at marriage, 227.

Tibet, celibacy, 251 .

Tikopia, infanticide, 190, 219.

Timor Laut, health, 235.

Tinneh, early intercourse, 136 ; lactation, 138 ; age at marriage, 140 ; fertility, 143; war, 150; infant mortality, 150; property, 206.

Todas, infanticide, 222 ; early intercourse, 254.

Tonga, restriction of intercourse, 186 ; infanticide, 190.

Topebatos, age at marriage, 224.

Torres Straits, age at marriage, 185, 227 ; restriction of intercourse, 186 ; fertility, 187 ; abortion, 189 ; infanticide, 190, 220 ; war, 192 ; food, 234.

Trent, Council of, celibacy and, 264.

Tunguses, lactation, 196; property, 211.

Turcomans, war, 248.

Turkey, lactation, 254 ; restriction of intercourse, 255 ; abortion, 256.

Uaupes, feuds, 171.

Uganda, lactation; 174 ; restriction of intercourse, 176 ; war, 181. 
Under-population, 294, 295, 297.

Unemployment, over-population and, 312.

Unit-factors, 71.

Utilitarian school, attitude to Malthus, $29,32$.

Utis, contraceptive methods, 167.

Vaitapu, infanticide, 191, 221.

Vancouver Island, fertility, 99 ; age at marriage, 140 ; abortion, 146.

Veddahs, early intercourse, 137 ; age at marriage, 140 ; fertility, 145 ; infanticide, 149 ; infant mortality, 160 ; property, 207.

Venereal disease, 269. See also Gonorrhoea.

Wadschagga, lactation, 174 ; infant mortality, 184 ; abortion, 221.

Wagogo, restriction of intercourse, 176.

Wakamba, infanticide, 180.

Wamakonde, lactation, 174.

Wanika, infanticide, 180.

Wanjamuesi, early intercourse, 173; lactation, 174 ; restriction of intercourse, 176 ; fertility, 178.

Wapagoro, early intercourse, 173 ; restriction of intercourse, 176 ; marriage customs, 228.

War, among hunting and fishing races, 149 ; among agricultural races, 169 , 180,192 ; among historical races, 247; Christian Church and, 249 ; over-population and, 304 ; origin of, 305 ; selection and, 379 .
Warega, early intercourse, 173 ; restriction of intercourse, 175 ; fertility, 178 ; abortion, 179 ; infant mortality, 184.

Warri district, restriction of intercourse, 175.

Wazaramo, lactation, 174.

Wealth, of different areas, 419 ; influence upon tradition, 421 ; shifting of centres of greatest, 423 .

Welle district, war, 181 .

West African tribes, lactation, 174.

Wheel, invention of, 127.

Witchcraft, as cause of death, 182.

Work among primitive races, obligation to, 212.

Workers' Education Association, 239.

Woruk, infanticide, 149.

Writing, invention of, 127.

Yakuts, lactation, 196 ; property, 211.

Yguazas, abstention from intercourse, 141 ; infanticide, 149 ; war, 152.

Yoruba-speaking people, lactation, 174 ; restriction of intercourse, 175 ; property, 209.

Yuchi, war, 170 ; health, 172.

Zambala, marriage customs, 227.

Zaparos, treatment of aged, 155.

Zoroastrianism, marriage and, 253.

Zulus, war, 181 ; infant mortality, 184 ; marriage customs, 227.

Zuni, abortion, 169 ; infanticide, 169 ; property, 208. 


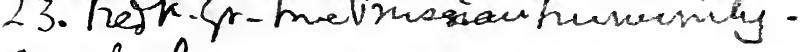

27. halthers -

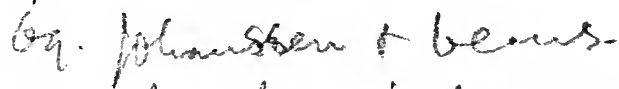

77. Drunfas taperwosin U.S.A.

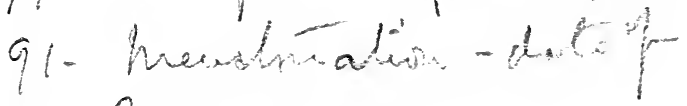

113. O thecourtipropens -

120. Eolins.

Humar stisi' crite usepul.

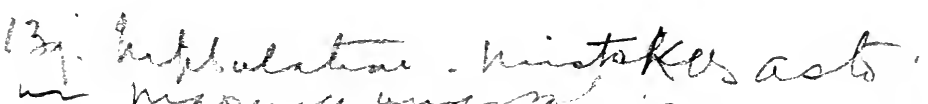

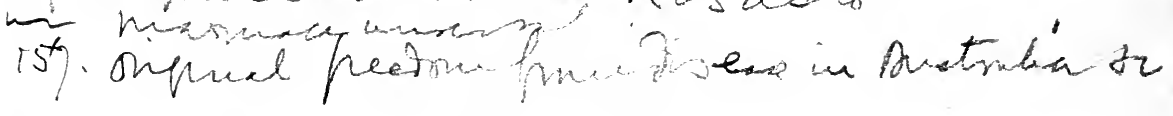

193. Fearnwar S. cen Shrs.

202. hillotharetheno.

as. Pencititans.

207 . Kearm for infaisteve

231. Austribien mirataured.

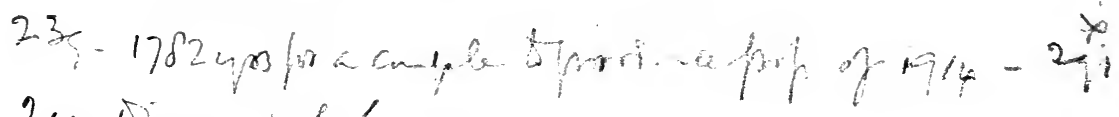

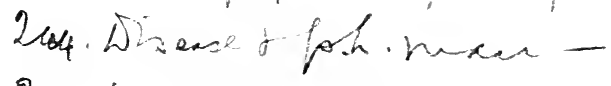

heq. hour keare ch.

23 . Ches masiaterion hin

256 . afotion in fordos.

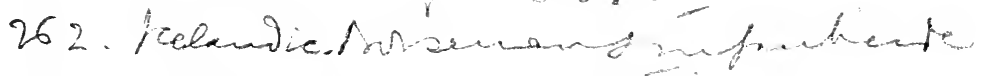

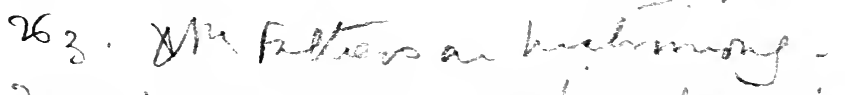

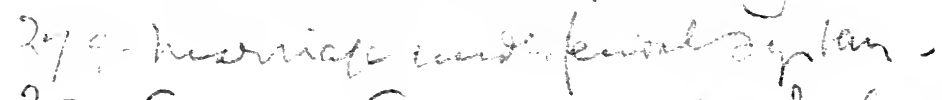

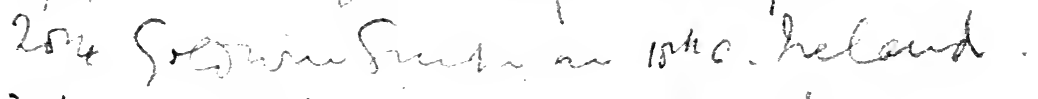

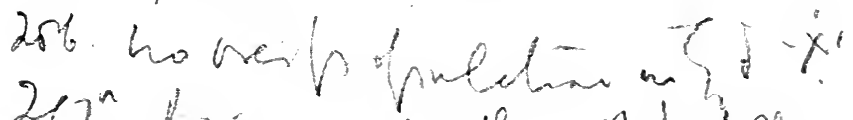

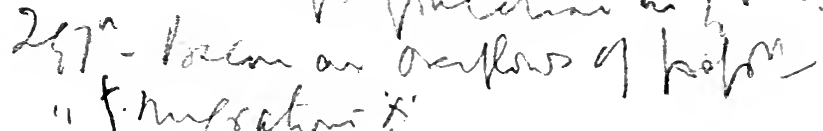

"f.hmeration- x

bor. Chimate-

3ou Members \& A malded 
houcised

CARR-SAUNDERS, A.M.

The population problem.
$0 \mathrm{HB}$ 851

. $\mathrm{C3}$ 


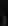

his

As

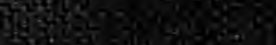

trontios

400 -

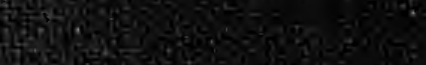

7050

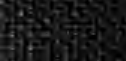

(5)

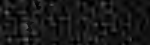

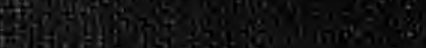

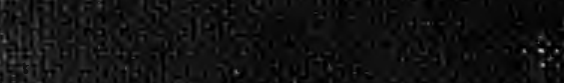

ong

and

Ging

4.

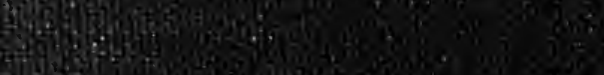

Hon

等

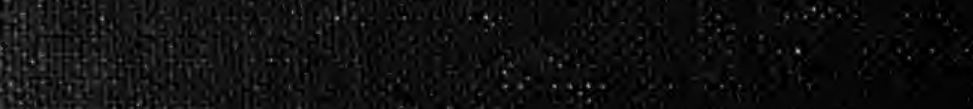

600

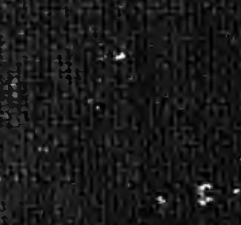

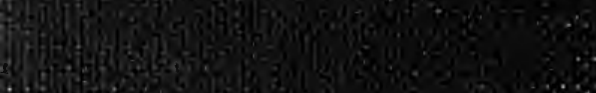

ang

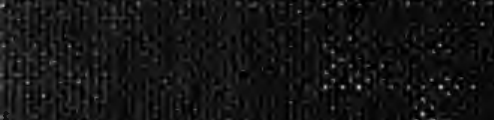

4.

(1)

势

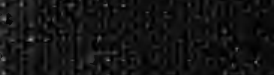

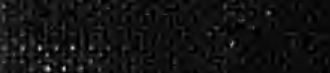

